
The Dibner Library of the History of

Science and Technology SMITHSONIAN INSTITUTION LIBRARIES

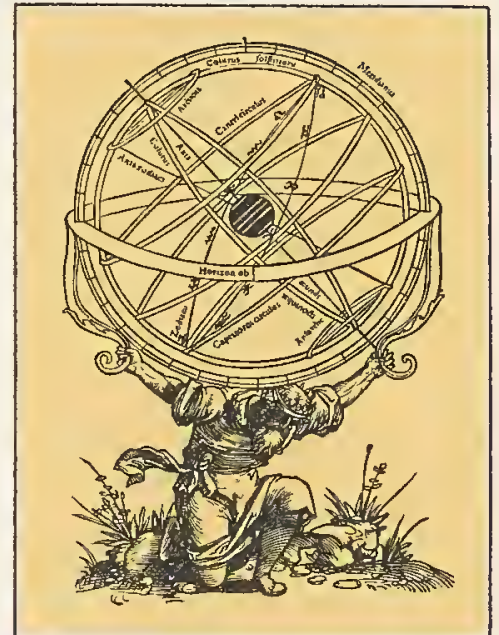






















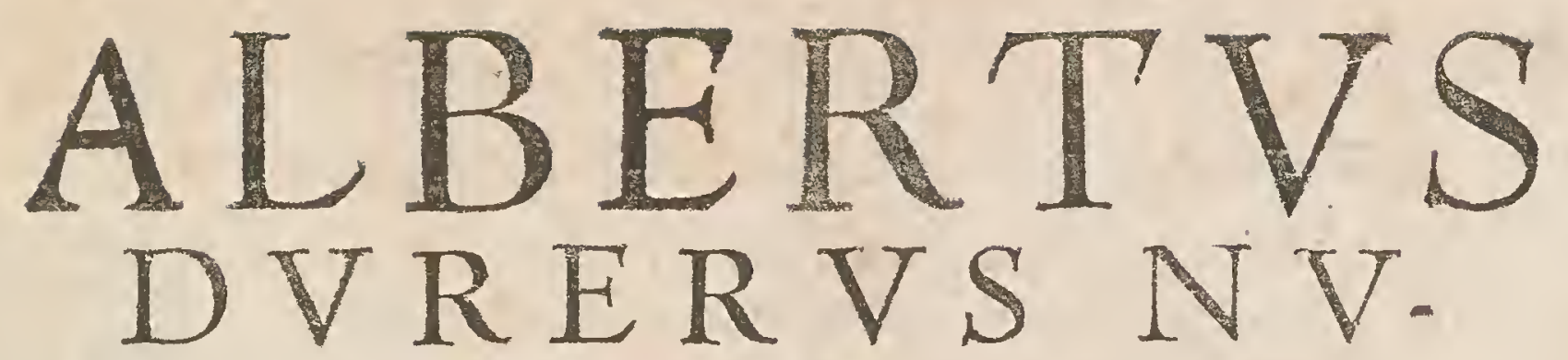

REMBERGENSIS PICTOR HVIVS

xtatis celeberrimus, verfus è Germanica lingua in Latinam, Pictoribus, Fabris ærariis ac lignariis, Lapicidis, Statuariis, \& vniuerfis demum qui circino, gnomone, li. bella,aut alioqui certa menfura opera fua examinant, propè neceflarius:adeò exactè Quatuor his fuarú

Inftitutionum Geometricarum libris, lineas, fuperficies \& folid" corpora tractauit, adhibitis defignationibus ad eam rem accommoda $a_{\bar{s}}$ tiffimis.

Denuo ad fcripti exemplaris fidem omnia diligenter recognita, emendatius iam in lucem exeunt.

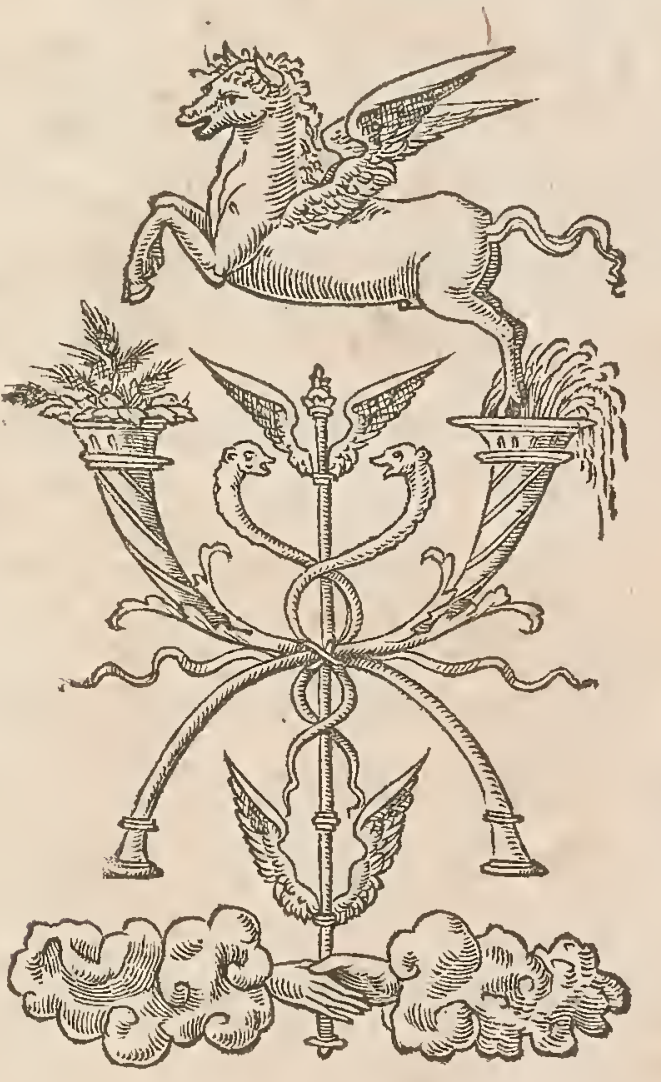

P A R IS IIS

Ex officina Chriftiani Wecheli; fub fcuto Bafilienfi. M.D.XXXV. 



\section{ALBERTVSDVRERVS BILIBALDO}

Birckeymerho Domino \& amico fuo pracipuo S.D.

N Germania noftra, Bilibalde excellếtiffime, adhibiti funt hactenus arti picture adolefcentés felicis ingeníi multi, qui fine omni artis fundaméto, fed quotidiana folú exercitatione inftituti, in ignorátia fua, velut arbor imputata adoleuerunt, quan quam aliqui ipforum ex longo vfu manum affecuti funt exercitatam, fic ve opera fua promptê fed inconfideratè, $\&$ pro fuo tantum arbitrio perfecerint. Quum veró magni $\&$ ingeniofi artifices, eorum tam inepta opera perfpexerunt, non immerito talium hominú riferunt cęcitatem:quú fanum iudicium nihil æquê ac pictưrà, nullo artificio, effi magna fedulitate, ac diligentia confectá, abhorreat. Quồ verò huiufmodi pictores fuum errorem non depręhenderint, folum in caufa fuit, g geometriam non didicerunt, fine qua nemo ab folutus artifex fieri aut effe poteft:quod tamé praceptoribus ipforum imputandum eft, qui hanc artem iplimet ignorarunt. Sed quium ipfa fit verum totius graphices fundamentum, vifum eft, itudiofis ty ronibus rudimenta quadam confcribere, quo ipfis anfam prabeam circino $\&$ regula vtendi, $\&$ in de veritatem ante oculos contemplandi, vt non folùm auidi ad artes fiant, fed etiam ad maiorem \& verum earum intellectum peruenire queant. Iam etfi noftro tempore, apud nos ars picture à quibufdam malèaidiát, qnòd fonitế ad idolatriá miniltrare dicatur, homo tamen Chriftanus per picturas, aut effigies, non magis ad fuperftitionem, quàm vir bon us gladio accin ctus ad latrocinium allicitur. Effet certe infulfus homo, qui picturas, ligna,aut lapides adorare vellet. Quare pictura ędificat magis, 8 commodat quàn offici at rei chriftianæ, modò honeftè, artificiofè $\&$ bené fit facta. Quo autem honore ac dignitate hęc ars apud Græcos $\&$ Romanos antiquitus habita fit, veteres $l i-$ bri fatis teftantur: quanquam poltea penitus deperdita $\&$ vltra mille annos latuerit, ac nun ctandem ante ducentos annos, per aliquot Italos rurfun in lucen prodierit. Nả facilè omnino artes amittuntur ac peréut: difficulter vero àc lógo tempore vix recuperantur. Quo circa fero neminem fapienten hunc meum laborem calumniaturum; quum ex bono animo $\&$ in gratiam omniu ingenu. as artes amantiü mihi fit fufceptus:nec folùm pictorıbus, fed $\&$ aurifabris, ftatuariis; lapicidis,fabris lignariis, \& breuiter omnibus $\mathrm{q}$ circino, regula, \& menfura vtuntur, vtilis fit futurus. Neque vero cogitur quifquam in his meis praceptionibus perperam bonas horas collocare, quanq̄ haud ignoré quicúọin in ipfis exercitatus fuerit, non tantùm artis fuę principia, fed quotidiana exercitati one exactum inde iudicinm percipiet, altiora inueltigabie, \& longè plura inue niet, quàm ego commonftraui. Quum veŕò, vir eximie, luce clari us fit, te omniú bonarum artium quafiafylum quoddam effe, tibi ex fingulari quodam erga te amore librum hunc dicare placuit, non quùd tibi magni quidpiam prxftitiffe videri cupiam : fed quòd inde propenfum meum erga te animum cognofcere queas : quumóq mea opera-tibi minus gratificari pofím, animo fakem parato beneficia, que in me confers, rependam Vale. 
D. ERASMIROTERODAMIIVDICI-

um de $A$ L BERTO DVRERO ex dialogo illius, Dere

ctalatini Gracióg fermonis pronunciatione infcripto,

excerptun.

\section{PERSON AE \\ VRSVS ET LEO. \\ I E O,}

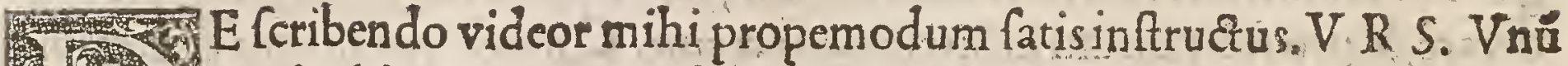
(7) illud addam, hacita tradêda pueris, ve feludere putent, non ftudere. Quidam enim hac tanta docent acerbitate, vt pueri prius dilcant odiffe literas, quàm noffe. A dhac iuuabit ad picturá interim nonnih1l exerceri puerum:ad eam artem plerique fua fponte feruntur, dum gaudent \& exprimere quod agnof cunt, \& agnofcere quod ab al iis expreffum eft. Vt auté qui mufices periti lunt, rectius pronunciant etiam non cantantes, ita qui ducen dis in omnem formam lineis digitos habet exercitatos, mollius ac felicius pin. get literas.Siquid fuper his req ras fubtilius exactiusóg, extat liber A L B E R. T । D V R E R I, Germanicè quidem, fed eruditıffime fcriptus, in quo prifcos huius artis heroas imitatus, nominatim Pamphilú natione Macedorem quú omniú literarum, tum Gcometrices \& A rithmetices egregiè peritum $\left(\mathrm{N}_{2}\right.$ fine his difciplinis artem abfolui poffe negabat) adh ac A pellem qui $\&$ ipfe ad Perfeum difcipulum de arte fua conferiplit, mulca praclare tradit de myfteriis graphices, ex Mathematicorum petita difciplinis: $\mathcal{Q}$ in his non pauca de figuris elementorum ac ductıbus proportioneóg literarum. L E O. Duteri nomen iam olim noui, inter pingendi artifices prim celebritatis. Quidam appellant horum temporü A pellem.V R S.Equidem arbitror finunc viueret A pelles,ve crat ingenuus $\&$ candidus, Alberto noftro ceffurum huius palmx gloriam.L. Qui poteft credi?V R S. Fateor A pellem fuiffe eius artis principe, cui nihl ob ics potuit à cxteris artificibus, nifi quòd nefciret manum tollere de tabula.Spe. ciofa reprahenfio. At A pelles coloribus, licet paucioribus, minuśg ambitiolis, tamen coloribus adıuuabatur. Durerus quanquá $\&$ alias admirandus, in monochromatis, hoc eft, nigris lineis, quid non exprimit?vmbras, lumen, fplëdorem, eminentias, depreffiones:ad hac, ex fitu rei vnius non vnam fpeciem fefe oculis intuentium offerétem. Obferuat exactè fymmetrias \& harmonias. Quin ille pingit \& qux pingi non poffunt, ign $\mathrm{cm}$, radios, tonitrua, fulgetra, fulgura, vel nebulas, vt aiunt, in pariete, fenfus, affectus omnes, denig totum hominis animum in habitu corporis relucentem,ac penèvocem ipfam. Hac fcliciflimis lineis îfque nigris fic ponit ob oculos, vt fi colorem illinas, iniuriam facias operi An hoc mirabilius, abfque colorum lenocinio praftare, quod Apelles praftitit colorum prafidio? $L$ EO.Non arbitrabar effe tă tum eruditionis in arte pin gendi, que nunc vix alit artificem. V R S. Non nouum hoc eft, infignes artifices tenui re effe. A t olim inter artes liberales numerabatur graphice, nec hane difcere licuit nifi claris, mox honeftis, caterum vetitum ne feruitiis traderetur. Principum eft ignominia, nop artis, fi caret fuis pramiis. \&c. 
omni virtutum genere ornato, non tantum maiorum imaginibus claro,

A LMARICO BOV C H A D O Santonum prafidi,

FRA NCISCI VALESII Galliarum regis chriftianiffimi à libellis \&c.Chriftianus Wechelus S.P.D.

閳:

A bet quidem natura rerum, vir præftantiffine, plurima qua admita tionem fui apud homines mereantur, verum mihi aliquando conditionem eius contemplanti fuccurrit ante alia mirari caufas viciffitidinis tam varia in rebus ac ingeniis nortalium, fertilitate fterlitate, pace bello, humanitate immanitate, reliquis, nunc apud ho, tuodo apud illos fe dem collocantibus \& ordine quodam mira vniuerfum orbem peruadentibus, vt certa quadam lege quemadmodum lucis ac tenebrarum ita bonorum quog ac malorum omnium omnes ex auo participes facere infitutum fib videdtot, more communis ac magnæ omnium matris; de cuius erga omnes xquabilitate nemo nifi immeritò conqueri poffit. Neque verò veteres illi collorum aftrorúg rimatores, rerum fenfui expofitarum ductu vim vniuerfi perfcrutantes fine fin gulari dei numine fiderum lationibus ac radiationibus cuncta obnoxia cen fuerunt, ne diis quidem fuis aut Ioue faltem illo Optimo Maximo à lege fatali Parcarumópftamine exemptis, quum digniorem aliam fubltantiam, cuius im perio \& imperfcrutabili volun tati fuperiora cum inferioribus fubderentur, humano intellectu affequi nó valerent, admiffi ad diuina quatenus pertingere po tuit imbecillitas humana:quorum opinio licet à noftræ religionis affertoribus vt impia \& Chrifti difcipulo indigna reiiciatur, idem tamen iter cum Paulo ad confilia diuina nitenti aut certe non ita diuerfum ingreffos dicas, ac cum codem exclamaturos nifi lux illa xterna illorum mentibus nondum affulffete fed qua deinceps reftabant auguftiora, magnitudine fua illorú modo ingenia obruiffent. Vt autem fateamur nos certas illas leges fiue fiderum reuolutionis, fiue tëporis curriculi decretive diuini nobis incogniti quibus tanta fubinde exi

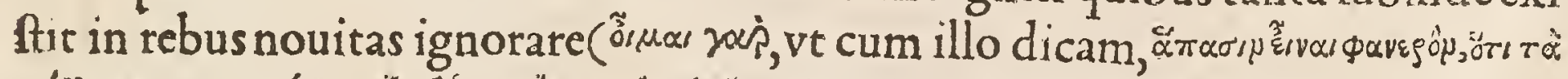

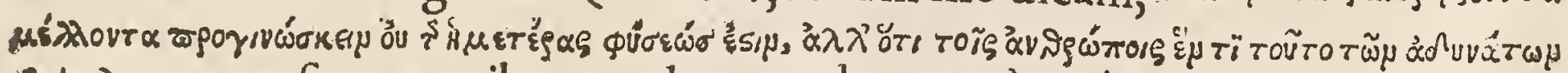
(sip) euentus fane omnibus paulum modo attendentibus planus ac in prom. ptu eft-Nam que nationes multis iam fxculis ob immanem feritatem immundiciémg nihil penè obtinere humani præter effigiem ac formam creditæe funt, iis prafertim qui fe vnos arcem $\&$ domicilium humanitatis tenere profitentur ac ob id barbarorum illis nomen indiderunt, ita fua ftudia paucis annis in diuerfum traducere coepere vt ditionum pernutatio inter Barbariem Humanita tém incumbitur, non à Grxcè latinég ftudiolis tantum, quin $\&$ ab artificibus mediáque è plebe hominibus, qui vernaculê verfa quacunque penè fiue à Gracis, $\mathfrak{l}_{\text {- }}$ ue latinıs fcriptoribus literis mandata funt, tanta diligentia excutiunt, vt iădudum nonnulla veteribus nó animaduerfa,neq̣ ea fane vulgaria, annotare cœepe rint:ex quibus quum inter Germanos quendă Albertum Durerú miris laudi- 


\section{A L M A ICO BOVCHARDO.}

bus ab hinc fere triennium non apud fuos tantùm vehi audirem, ob graphices

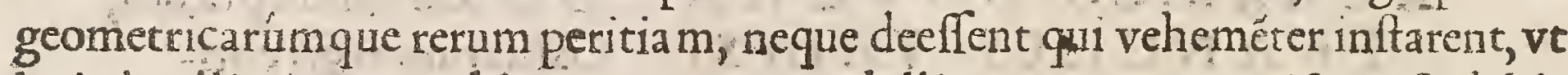
latinè gallicég vertendú curarem quicquid illius viri extaret, vifum eft ab his rudimentis geometricis exordiri, qux plurimos videbam admirari nominefru ctus, quem omne genus artificibus ex iis percipere promptum eflet. Nunc quan do fimilia frmilibus libenter aggregantur, vt eft in prouerbio, eximio artifici pri mx notx quxrĕdus erat patronus, in cuius tutela $\&$ beneuola confuetudine acquiefceret, cui quum vel fuo iudicio fi vocem xdere daretur, nullum tuo patro-' cinio optatius cotingere queat, manu noftra emiffum tux cliêtele tradidimus, proxenum futurum apud te fauoris erga me tui $\alpha$ beneuolentix, indicemo quo iudicium facere poffis, num excitatam iamdudü de Porportione humana reliquíf fuis fcriptis, quorum editionem adornamus, expectationem fuftinere polfit. Vale Parifis octauo idus Auguti. 



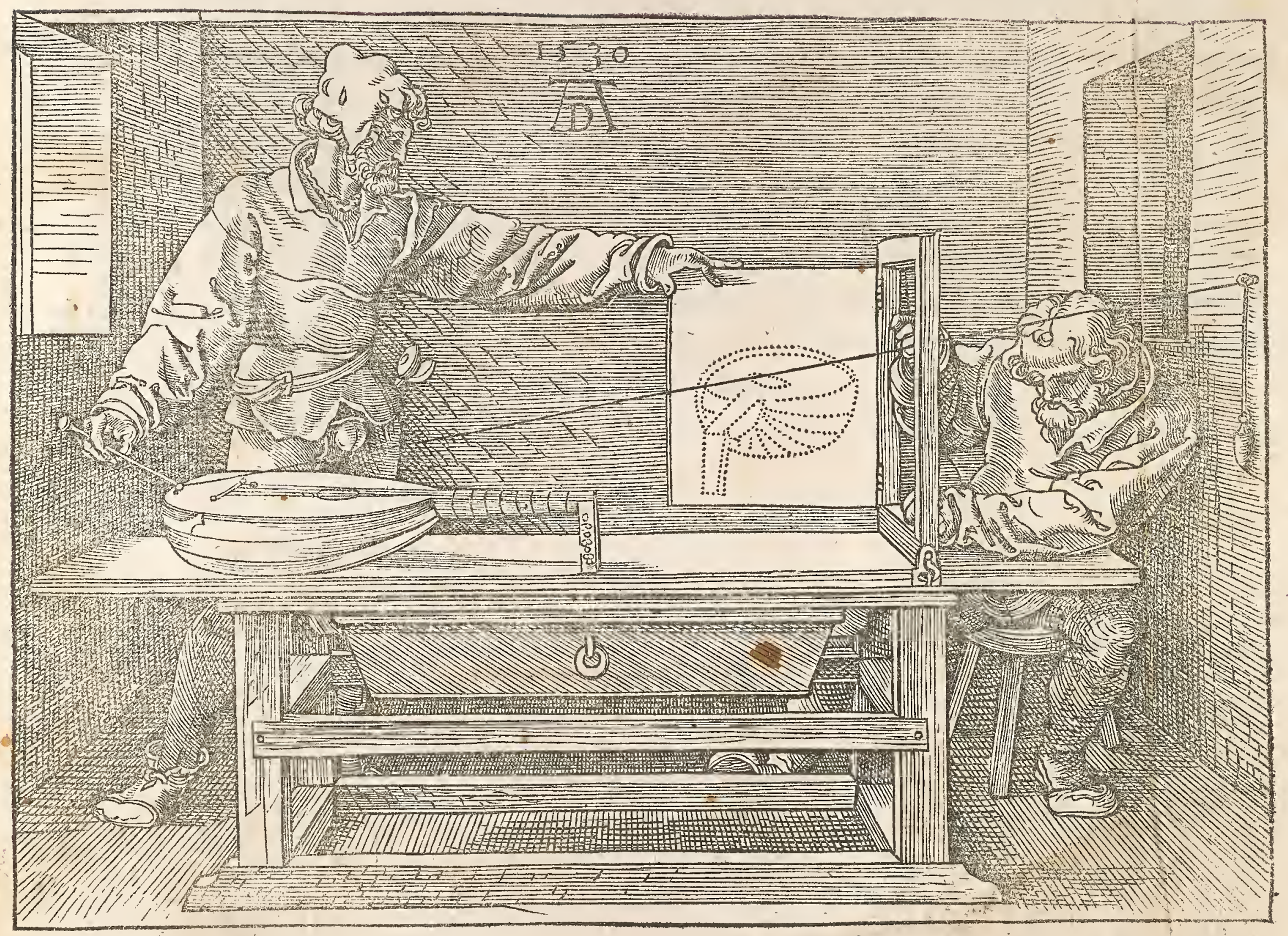




\section{A LBERTI DVRERI}

PICTORIS PRAESTANTISS. INSTITVTIOnum Geometricarum Liber Primus, qui eft de lineis.

E VCLIDES undecung; doctiffimus confcripfit Geometric elementa, que fi quis rectè intelligit traditio= nibus me is non opus babet, que iunenibus folum, $\mathcal{O}$. 26. Rimò quidem Geometriam docentem difcipulos inftituere de1. 10 . 1 1 23 \& quomodo rem propofitam fiue nuper inuentá , fiue à pradc17. cefforibus monftratam, menfurare debeant:tria igitur funt di12 - 12 menfionum genera:Vnum eft longitudo, fine laticudine \& pro2. funditate:deinde longitudo, qux etiamlatitudinem habet:tertio lon gitudo qua $\&$ latitudinem $\&$ profunditatem habet. Harum quantitatú omnium principiu $\&$ finis puncta funt:fed punctus eft res quæ neque latitudinế,neq̧ longitudinế, aut profunditatế habet, quare etiá indiuifibilis eft, 8 loçú non occupat. Poffumus tamen in animo noftro punctum locare quòcunq̧voluerimus, aut imaginari ipfum moueri furfum aut deorfum, vel in quamcunq; parté placuerit, quo tamen ipfi corpore noltro peruenire non poffumus. Sed vt iuuenes in praxi exercitatiores fiant proponam ipfis punctum in forma note ca lamo depictx, \& adfcribam dictionem,punctum, qua ipfum fignificatur.

$$
\text { Punctú ? }
$$

Quum vero punctú illud à primo fủoloco ad aliú trahitur, relınquit polt fe veftigiú quoddă, quod linea vocatur: $\&$ hęc linea lógitudo eft fine latitudine $8 x p$ funditate, \& poteft protrahi quantü libuerit:hác lineá hic repræefentabo tractus recto \& oblongo, calamo facto, cú nomine linea, Linea vt inuifbilis linea tractu illo recto animo intelligatur. In hunc enim modum internus intellectus fignificatur per opus externum. Quare omnia quacunque in his libris defcribam, figuris etiam declarabo, vt qua ego trado, iuuenes ante oculos videant, quo rectus capere ea $\&$ imaginari poffint . Iam notandum eft, quòd linex variis modis fcribi queunt, \& pracipuè triplices funt linex ex quibus multa fieri poffunt. Primó eft linea recta:deinde circumferentia, poltremó linea quxdam tortuofa, qux forté fortuna manu, aut de puncto in punctum tra hitur. (vt artes quedam docent) ex quo magnx diuerfitates proueniunt . Has lineas vt commodiùs intelligantur, hic fubfcripfi cum nominibus ipfarum.

Linea reeta. Circumferentia. Linea tortuofa.
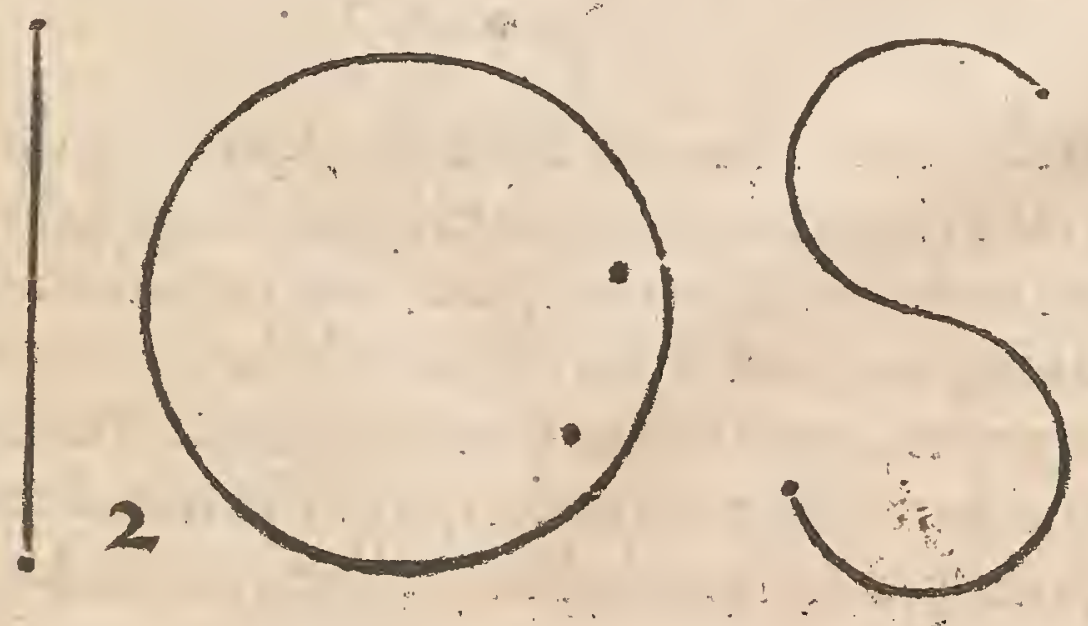


\section{A L B E R I D D R E R I}

7. 0 tádum eft, quòd tres illæ pradictx lineæ poffunt fcribi pro arbitrio, 1. W aut breues aut longx. Et fi locus \& tempus permitterent, poffer linea (2. recta protrahi in perpetuum, nihil tamen obftat quin cam intellectu noftro producamus in infinitum. Ea etiam licet vti tribus modis, per pendiculariter, tranfuerfè \& obliquè. Sed circúferentialicet vti integra vel par te ipfius, nec potelt produci longius, quàn à puncto vnde exire cœepit,donec ad idé rurfus reuertatur:quòd fi vltrà protraheretur, incurreret priorê circuitum: hęc circumferētia poteft effe aut craffa, aut tenuis, qux fi reflectatur furfum aut deorfum, fit ex ea linea tortuofa quam poffumus infinitis modis variare, atque ex ea conficere res mirabiles, in longum, latum, altum, \& profundum. Et quemadmodum certum eft, multas res arduas abfolui poffe vna tantùm linea, guod quidem ii qui linearum proprietates non perpendunt, ignorant, at $\phi_{\text {h }}$ ic etiam parum de eis tractetur, ita facilè diiudicandü erit, quid duabus, tribus aut pluribus lineis fieri poffit:prafertim cùm tria illa linearum genera, cum fuis differentiis concurrant:nam multx etiam linex funt, qux non nifi aliarum admini culo fieri poffunt. Sciendum quoque eft, quòd linex parallelx funt, qux femper equidiftanter currunt, fiue ducátur directé, circulariter, aut tortuofé. Item linex qua nô xquidiftanter ducuntur, conueniunt tandem, \& faciunt angulú acutú. Quare dua linex perpendiculares non erunt parallelę, eo quòd protractę, ad cé trü vniuerfi tandê concurrant. Sic faciút omnes linę̧, quę nó parallelicé currút aut enim ex vna parte coniúguntur, aut verfus alteram magis quoque aperiun tur:vt prius dictum elt. Quòd autem nos lineis perpendicularibus pro aquidiftantibus vtimur, in caufa eft, quòd hra tam longè, ad centrum videlicet terræ currant, antequam cóueniant:quocirca propter interualli magnitudiné diuerfitas nobis videtur infenfibilis. Atqui hec linearum genera hic protraxi. xquidiftáres rectę.eqd. circulares. eqduit.tortuof $x$. perpendiculares.
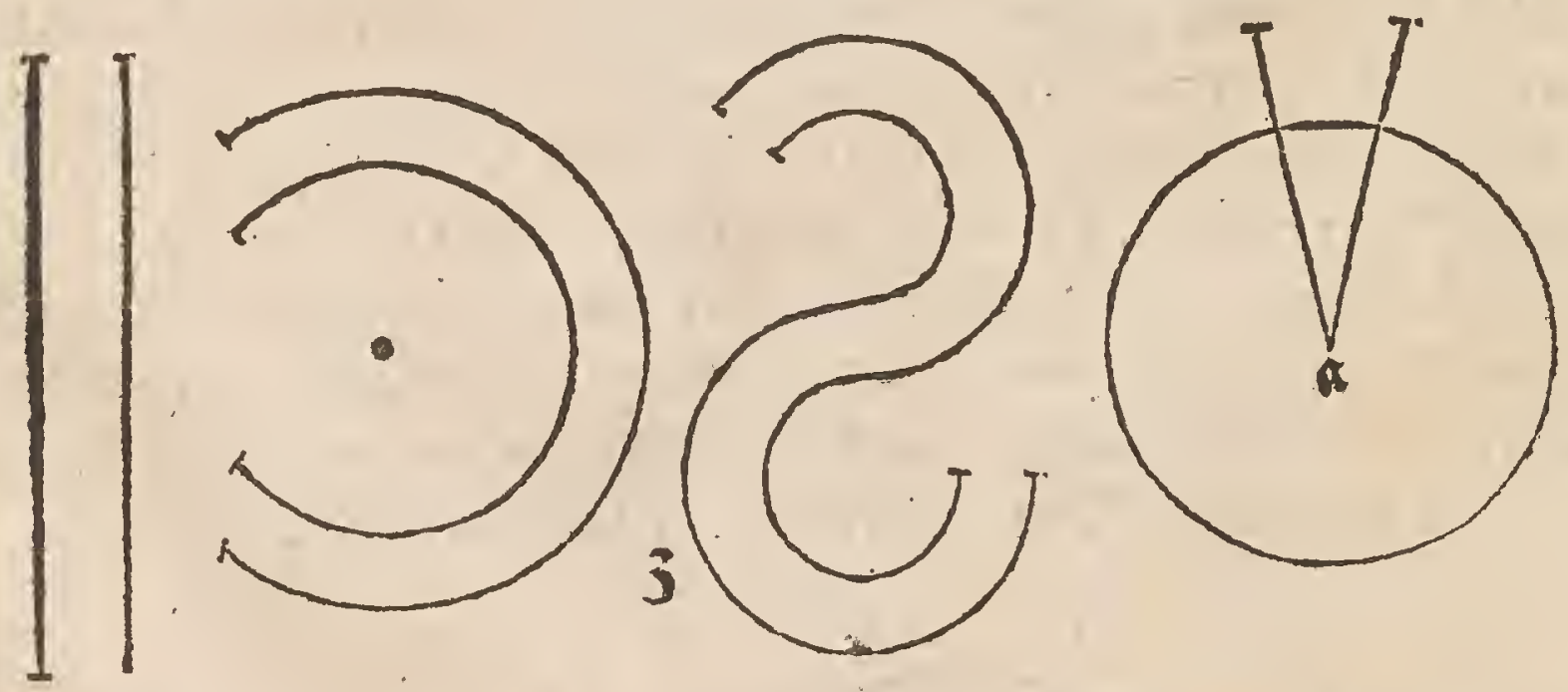

Vum intelligamus núc quid linex fint, $\&$ in qbus differăt, dicá dein ceps de earü lógitudine, qux etiam latitudinem habet, femper cum

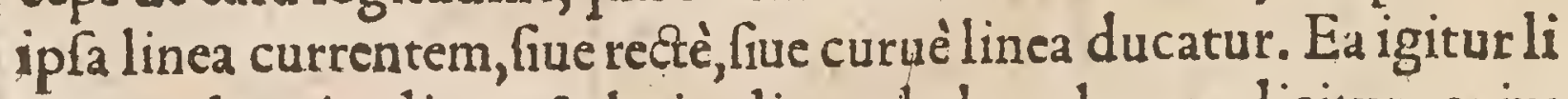
nea qua longitudinem \& latitudinem habet, planum dicitur, cuius etiam diuerfa funt genera, quemadmodú poltea dicetur. Poffemus planú ima ginari, quod ex omni parte finé non haberet, at de illo hic nó traçabimus, fed dehisfolum qua principiü $\&$ finem habent, $\&$ liness circundantur, vt formam aliquam 
aliquam recipiăt, quorum genera multa funt, ex quibus pauca quadam indicabo.Primùm igitur fuperficies plana eft, cuius media ab extremis non fubfultăt. Deinde eft fuperficies globofa, in modum hemifphrrii. Tertiò eft fuperficies concaua, quemadmodum ahenum. Quartò eft fuperficies tuberofa, locis quibuf dam eleuata, reliquis depreffa. Sunt etiam fuperficies quemadmodumlatus circulus, tortuofx , diverforum generum . Nam omnibus his rebus poffumus pro vtilitate in opibus noftris vti, quod fi non factü fuerit, fruftra obtúdimus caput. Nunc primùm defcribam fuperficiem planam, \& quadratam in hunc modum, Duco lineam tranfuerfam a b.eam moueo deorfum tantum, quantum ipfa longa eft,eritq quádratum perfectum. Sed fuperficiem planam circuli fic facio: Sit linea a b.cuius terminum a.relinquo immobilem:b.vero alterum terminum cir curnago, donec iterum redeat ad punctum, vnde moueri copit, $\&$ def cribet fignumb.circumferentiam plani circularis, quod fecit linea a b. $\&$ manebit punctum a.in medio, à quo diftantia ad circumferentiam vbiģ elt aqualis. $\mathrm{H}$ : niahic etiam protraxi.

Supficies fupficies fupficies fupficies fupficies fupficies quadrata. plana circuli. globora. cốcaua. curua. tuberofa.

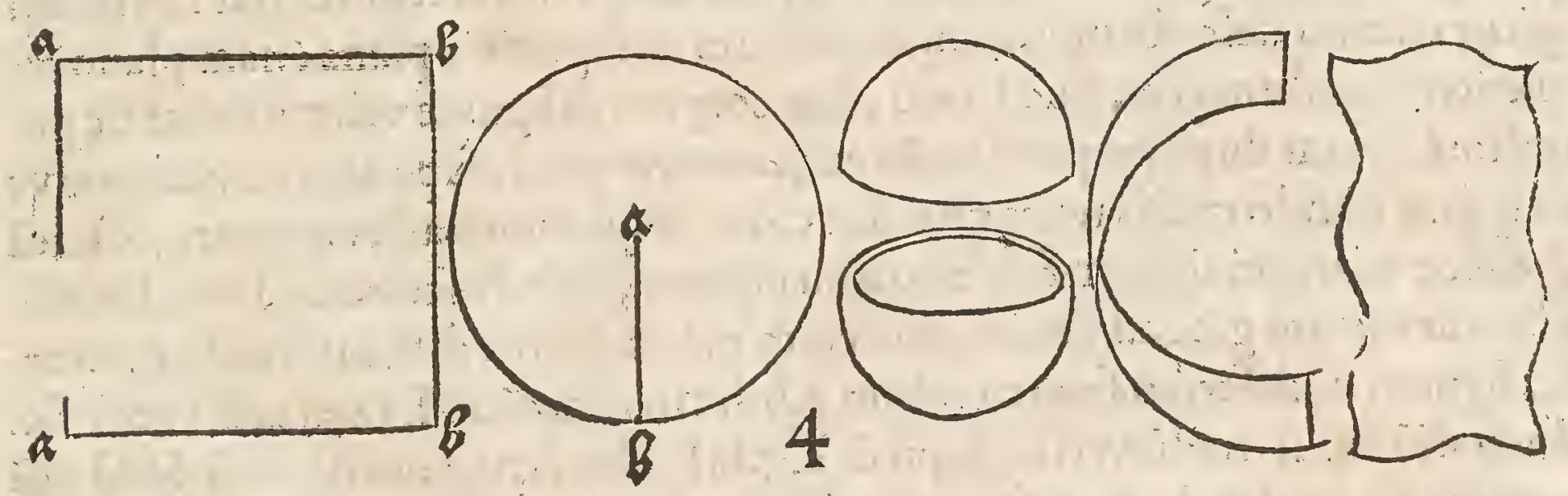

Vid longitudo \& latitudo, hoc eft, fuperficies fit, iam dictum eft:nunc (5) de longitudine, latitudine, atg profunditate, hoc eft, corporibus age(1. . mus, ex quibus, quo pacto aliqua fiant, docebimus. Primùm igitur acci 5. - pequadratum a.b.b.a.quod pauloante fecimus, $8<$ eleua illud rectiflimè tantum, quanta latitudo ipfrus eft, fietóp cubus æquilaterus, \& xquiangulus. Poftea reuertere ad planam circuli fuperficiem prius defcriptam, in qua femidiametrum b.a.protrahe in directum per totum circulum vfog ad circúferentiă, quam vbı attigerit, fac fignü c.\& erit b.a.c.diameter circuli, cuius parté finiftră nota puncto d.dextrá verò puncto e.Circa axem igitur b.c.immobilem, voluatur fuperficies b.e.c.d à puncto d.vf $\$$ ad e.faciet'̣́ circumferentiă b.e.c.d.globum abfolutiffmum, à cuius fupficie, ab omn i parte æquale interuallú eft ad cétrum a. $\&$ fic habes duo corpora folida, cubum fcilicet $\&$ globum'. At inter omnia corpora non eft perfectius globo. Hęc duo corpora fic oculis fubieci.

$A$ ii 


\section{Corpus quadratum, fiue Cubus. Globus, fiue Sphęra.}
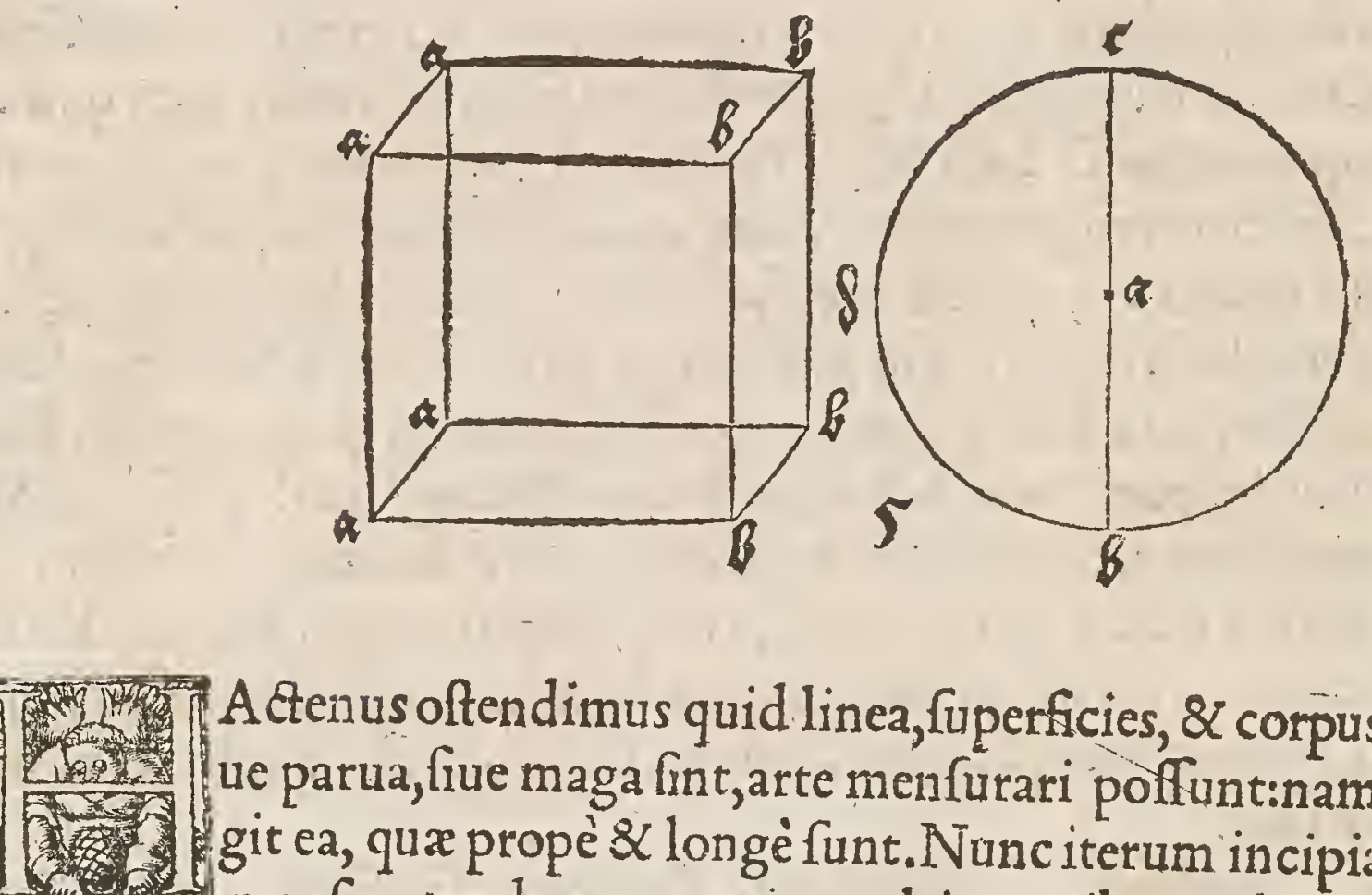

A ctenus oftendimus quid linea, fuperficies, \& corpus fit, qux omnia fi ue parua, fiue maga fint, arte menfurari poffunt:nam meufura contin git ea, qux propè $\&$ longè funt. Nanc iterum incipiam lineas quafdáa menfuratas ducere, qua in multis operibus vtiles erunt. Notum eft, quòd vna linea, variis modis protracta; diuerfas formas reprafentat: Primùm igitur circino defcribam lineam inuolutam in fuperficie plana (nam plano fre quenter vtend um erit, fiue lineas, fiue corpora defignare velimus) eam ficpro traho: Lineam duco perpend icularem, cuius terminus fuperior fit a.inferior ve rò b.eam diuido tribus notis qux fint c.d.e.in quatuor partes æquales, \& lineá d.e.feco etiam ad fignumf.in duo aqua fpacia, deinde fcribo ad linea latus finiftrum literam $\mathrm{g} .8 \mathrm{Q}$ ad dextrum literam $h$. Iam centro d. 8 interuallo d.a.verfus fignum $h$. defcribo femicirculum a.h.b : iterum cêtrof. interuallo verò f.c. duco aliú femicirculú verfus g.qui fit c.g.b.Poftea facio notá d. cétrú.\& ad quá titatem d.c.verfus h.duco femicirculum c.e. Nunc cêtrof. fpacio verò f.d.verfus g.duco femicirculum à d. vfogin e. Tandem pono vnum pedem circini fuper lineam a.b.in medio inter d.\& f.\& alterum in punctum d. fcribóg.yltimú femicirculum d.f.in latere, vbi eft h. A tq fic linea ifta abfoluta erit, qux quidé vtilis eft ad multa opera perficienda:quod vt melius intelligatur,produxi infrà ex punctis a. \& c.binaslineas tranfuerfas, \& inuicem parellelas, ab ipfa linea inuoluta verfus manum finiftram vt hic viderilicet.

Linea inuoluta circino defcripta.

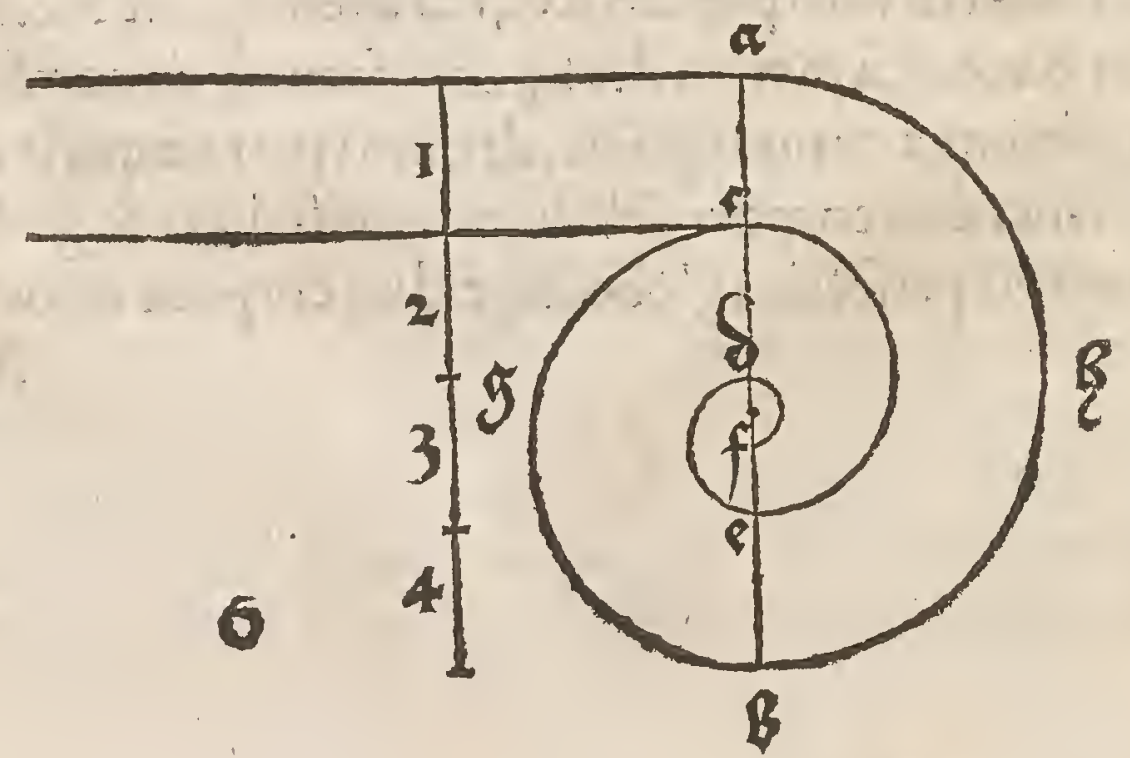




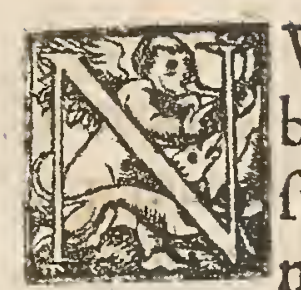

Vnc alia arte lineam fpira aut coclex in modum innolutam deforibemus, qux ad diuerfa opera erit vtilis, \& præcedentis loco vti ea por fumus, multáq arcana ex eius ductu difcuntur.Principium ipfius fut mitur à medio quodam puncto, circa quod reuolués longitudo eius, extenditur in immenfü, f́paciü tamen inter reuolutiones femper manet equale, praterḡ in prima. Hanc volutam fiue cocleæ lineá, fic facio:ego pono püctum a. fup quod defcribo circúferentiă tantæ quátitatis, ğtum cum linea futura excur rere lubet, eam circumferentiam diuido per duodecim puncta, in totidem inter ualla equalia.Poltea ex centro a.ducolineam rectam furfum, vfog ad circumferentiam, cuius terminus fit $b$.atque ibidem feribo $r_{2} .8$ ordinem punctorum nu

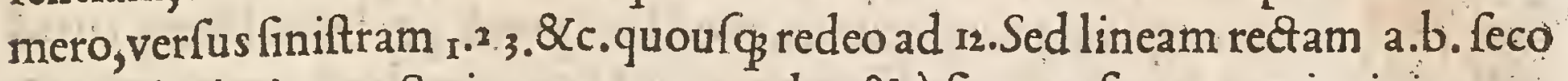
per viginti tria puncta in 24. partes equales, \& à figno a.fupputare incipio $1.2 . \xi_{0}$. $\&$ c.verfus b.Deiade accipio regulam rectam, \& tranffero in eam diuifiones pre dict $x$ linę $a . b$. quas etiam figno numeris arithmeticis, \& pono terminum regulęa.vbi numerus incipit, fuper a. centrum circuli $\&$ terminum $b$. pono fuper circumferentiam ad numerum ${ }_{1} .8$ vbi fignum 'r. regulę cadit, illic $f_{a}$ cio etiá notam $\mathrm{r}$. $\&$ fic fixo a. termino regul $x$ fuper a. centro circumago partem regule b: ad om nes diuifones circumferétie, notando cafus diuifionum regulx, $Q$ indicabunt partitiones regule omnia puncta fpiralis linex, per qux ducēda erit. Quare fi diligéter obferuaueris numerú non po teris errare. Sed cùm linea duas reuolutiones continuet, \& circúferentia duodecim folùm, regula verò currens vigintitres habeat diuifiones : animaduerte, vt re gulæ numerus ordine procedat: nam numero circumferentix $\mathrm{r}$. correfpondebunt. 13. 2, 14, 3, 15, 4, $16.5,17.6 .18 .7,19.8,20.9 .211022$. II . 23 Hac linea poteft multiplices circuitiones facere. Quare qui his o pus habet, multiplicet diuifiones regulx, \& puncta circumferenti\&

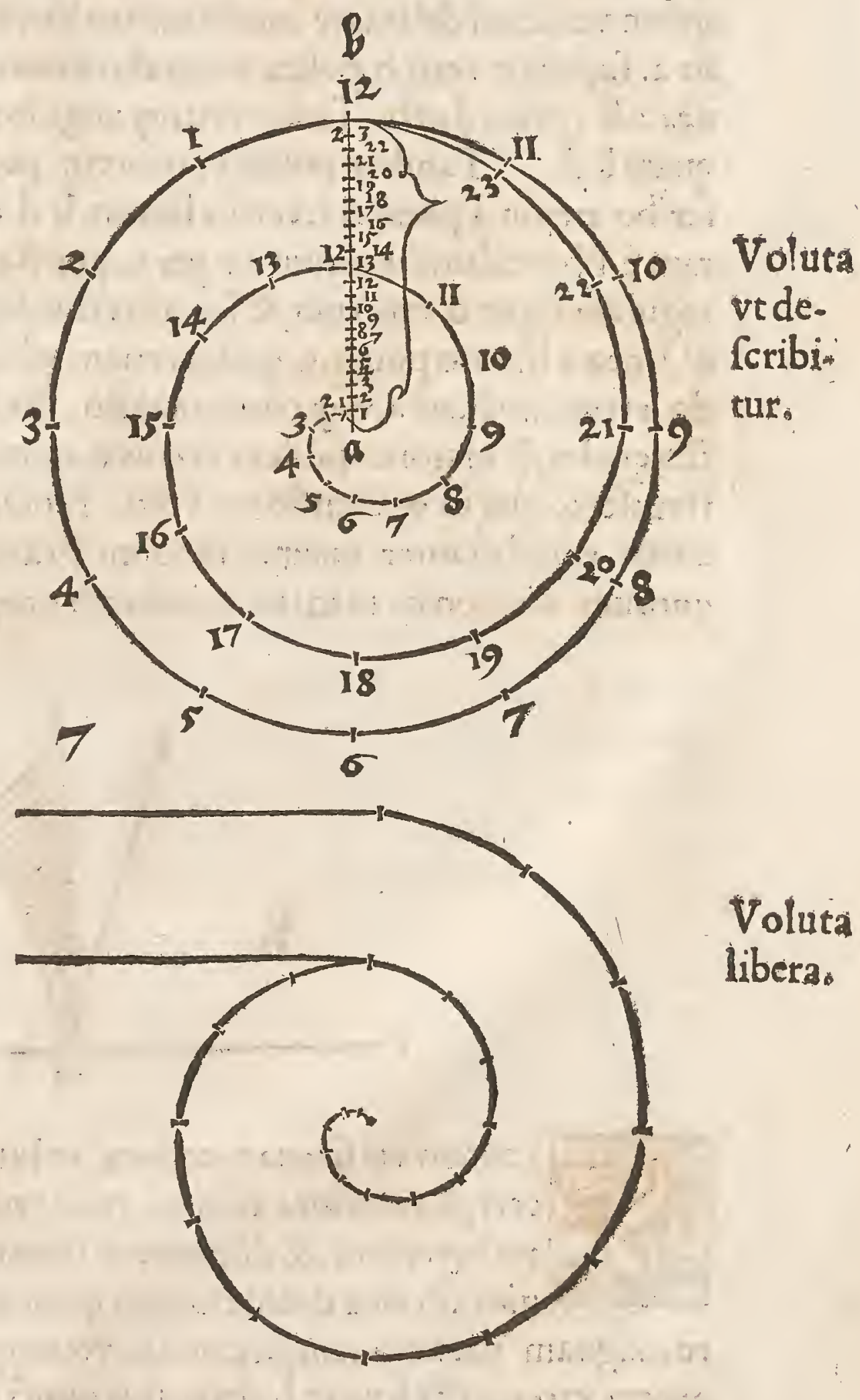
relinquat intacta:lá cóueniet circúferentiá cú regula diuifa, $\&$ omnibus fuis nu $A$ iii meris 
meris,per qua linea ad coclę fimilitudinem ducta eft, deponere, \& finere eam folam, vt abfolutè videri poffit. Quo pacto igitur linea ad inftar coclę protra-

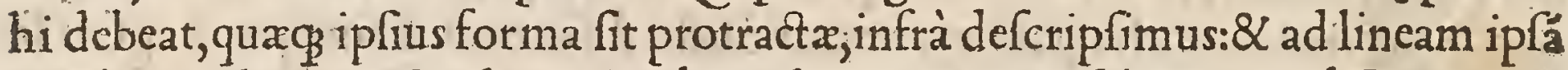
abfolutam duxi verfus fin iftram, binas lineas tranfuerfales $\&$ equidiftantes, fuperiorem quidem contingentem punctum b.terminum fcilicet exterioré lineæ fpiralis, inferioré verò contingentế punctû, vbı prima volutę reuolutio finitur. Has auté parallelas ptraxi, quo differentia huius cum pręcedête clariơ fieret.

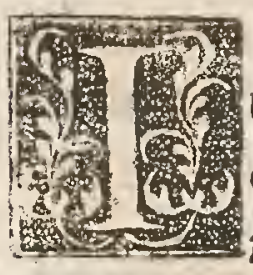

Am iterum volo pradictam volutam immutare per regulam ab.diuifam,per quam voluta ipfa ducenda crit: eam regulá cóueniet aliter diuidere, quàm prius fecimus, quod fieri potelt per binas lineas, curuå Ccilicet $\&$ rectam, quæ fe contingunt, $\&$ vna per alterá metitur, vt iam dicetur.Duco lineam perpendicularem, fecundum longitudinem regula , per quam volutam deliniare conftitui:eius linex perpendicularis terminus inferior fit a. fuperior verò b.poftea protraho lineam tranfuerfalem c.a.d. quax cum linea a $b$. prius ducta, faciat vtring angulos rectos, deinde fcribo lineam obliquam $\mathrm{b} d$. Tandem polito vno circini pede in puncto d.altero verò in a.defcribo arcum à puncto a.verfus lineam $b$ d.quam vbi arcus ille fecat, pono literam e. Nunc diuido arcum a e.per 23.puncta in 24. æqualia interualla, \& pono regulam fuper d centrum, \& fingulas diuifiones arcus a e.atg fectiones regula, 8 linex a b.noto punctis, quibus etiam adiicio numeros arithmeticos incipien do à termino b.ad a.vf continuando. Ex hoc clarum eft partes linex a b. effe inæquales, \& maiores quidem verfus b. minores verò verfus a : eas fic ad regulă transfero, qua in operando vti volo. Notandum interim quòd hæc linea non currit equidiftanter quemadmodum pracedens, 8 multa ex ea fieri poffunt. quorum hic non fit mentio. Iftiufmodi praparationes hic etiam protraxi:
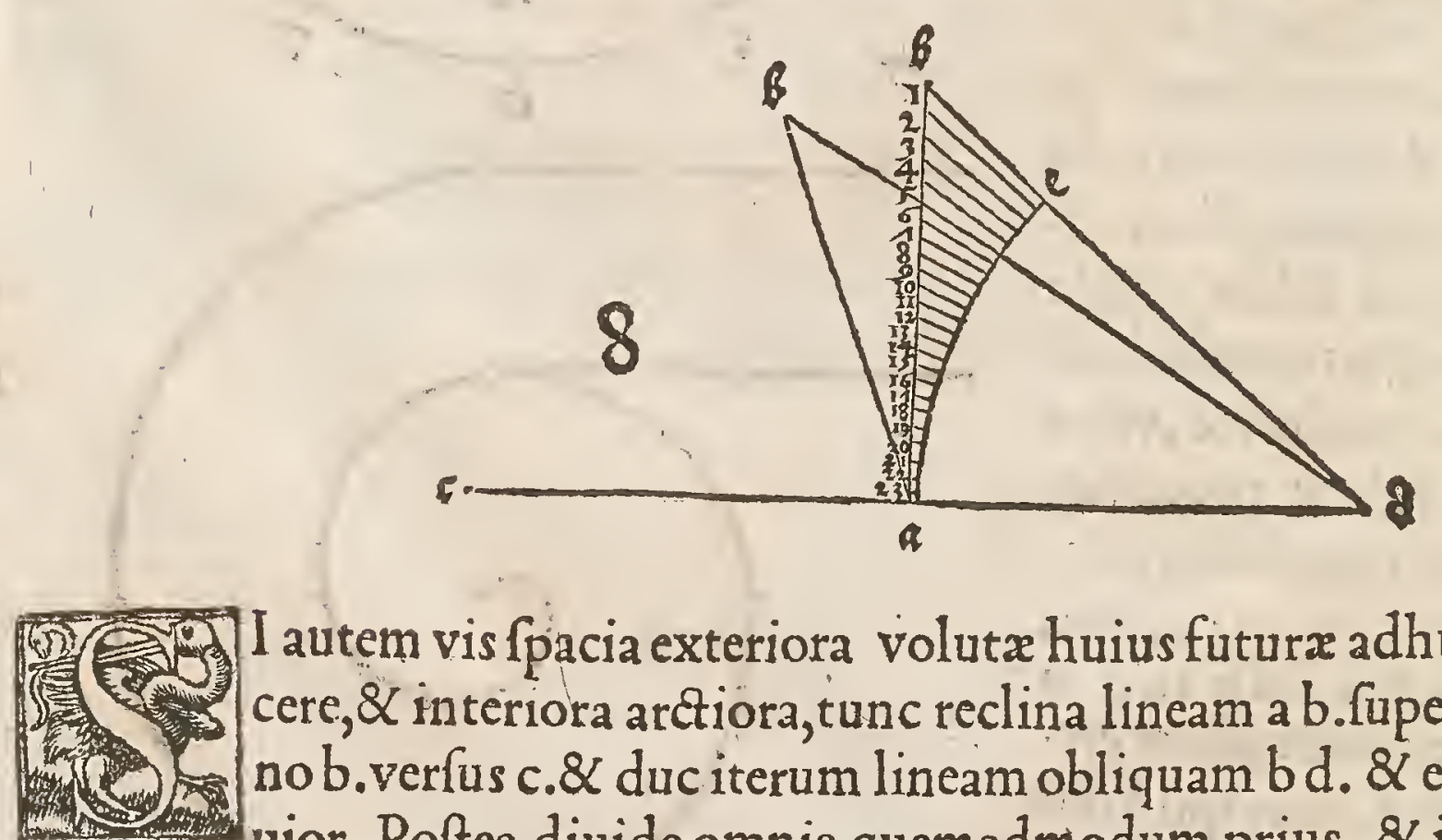

I autem vis fpacia exteriora volutx huius futurx adhuc ampliora $\mathrm{fa}$ : cere, $\&$ interiora arctiora, tunc reclina lineam a b. fupernè cum termino b.verfus c. $\&$ duc iterum lineam obliquam $b$ d. 8 erit arcus a e.bre uior.Poftea diuide omnia quemadmodum prius, \& inuenies in opere magnam vàriationem, hoc etiam vtcungs ex fuperiore figura patet. Quum autem variata illa linea a $b$. cum fuis punct is tranflata fuerit in regulam, "defcri be circulum, ad cuius centrum applicetur terminus a . regulg a b \& circum- 
ferentig applicetur terminus $b . \&$. fcribe fupra pü̊tum $b, x_{2}$. \& operare vt in vo luta præcedente docuimus, praterquam quòd illam à centro verfus circumferen tiam extraxifti:hæc à circumferentia verfus centrum inducenda erit. Quocirca progredere cum numero punetorum in circumferentia, numerando $1.2 .3,4$.verfus latus dextrum, $\&$ videbis differentiam inter hanc $\&$ pracedëtem, qux qp $^{\prime}$ ipfarum pulchrior fit. Hæc omnia hic, ve in antecedente, bis deliniaui.

Voluta variata cum lineamétis neceffariis.

Voluta variata libera.
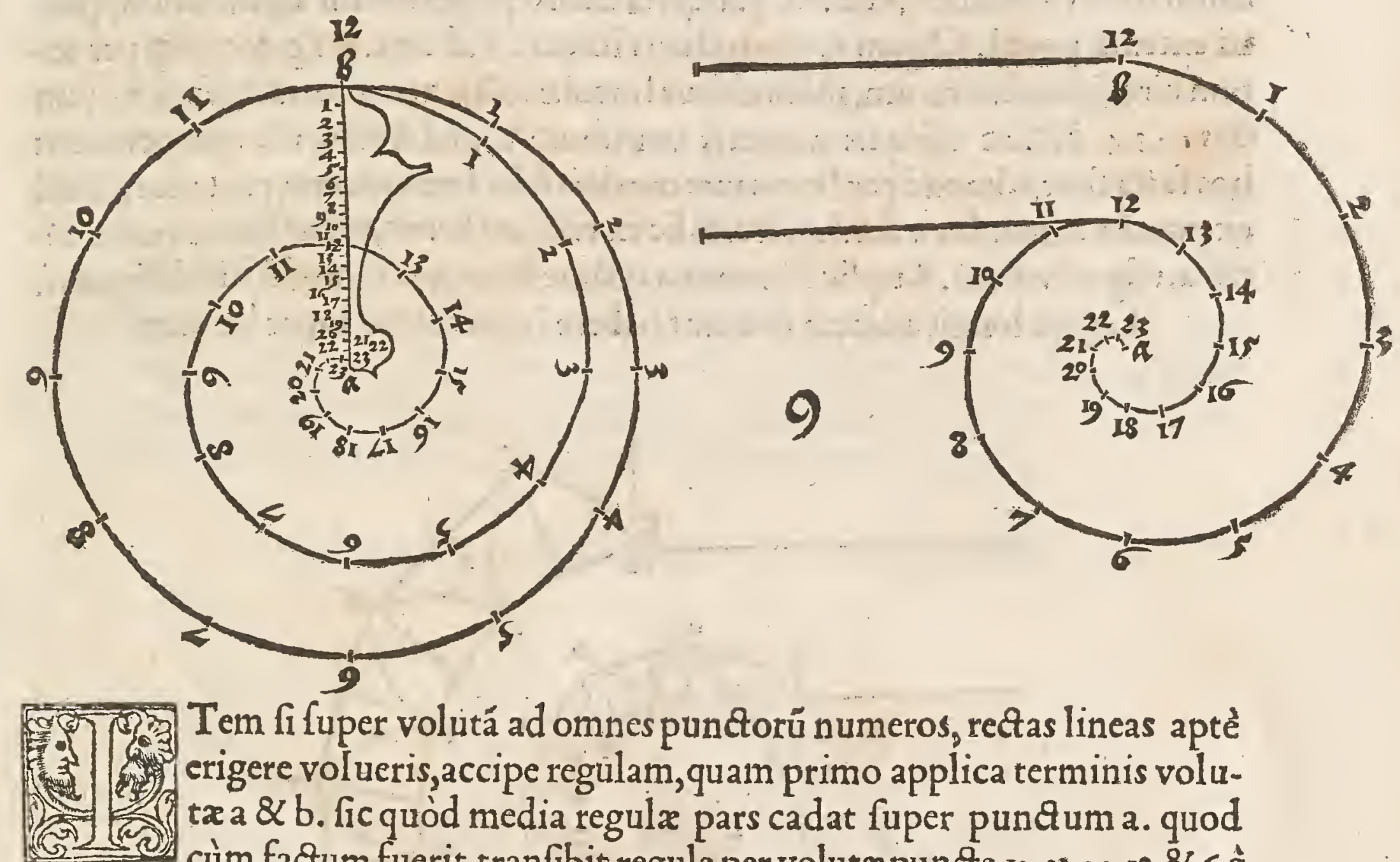

Tem fi fuper volutá ad omnes punctorú numeros, rectas lineas aptè erigere volueris, accipe regulam, quam primo applica terminis vol utxa \& b. fic quód media regulæ pars cadat fuper punqum a. quod cùm factum fuerit, tranfibit regula per volutx puncta I2.12.24.18. \& $6 . \hat{a}$ quibus punctis omnibus fecundum rectitudinem regulæ duc verfus partem exteriorem lineolas. Poftea immoto medio regulg fuper centro a.circúage extremi tates ad reliqua volutæ aut circumferentie puncta, \& femper adiumento regule ad omnes ipfius \& volutę fectiones, erige lineolas rectas, vt hic vides.

Quemadmodum linex fuper helicam pingendę fint.

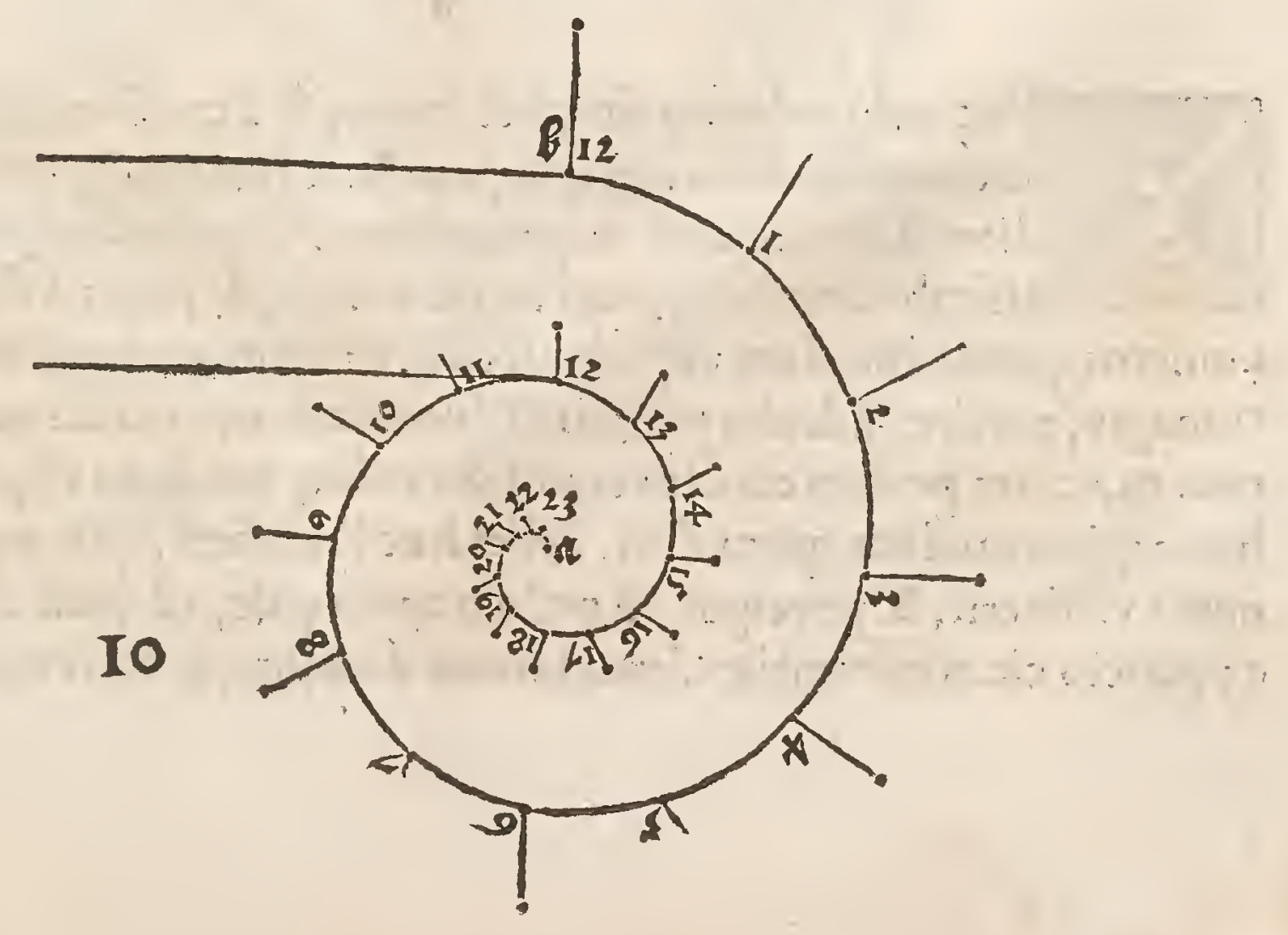




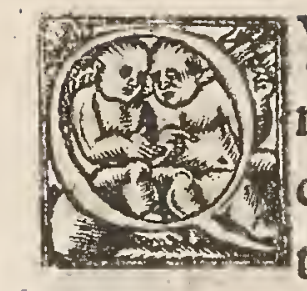

Vo pacto autem inuenire debeas longitudinem vniufcuiufq rectx $l i$ nex quar fuper volutam ponitur, in hunc modum operare. Accipe circinum; cuius vnum pedem pone in púctum ir.vbi eftlitera $b, \&$ al at fito cto r. fito, reliquo ex figno Iz. fcribe etian arcum verfus partem fuperiorem, $\&$ vbi illi duo arcus fe interfecant adiice literam $\mathrm{c}$. Sic fac inter omnia numerorút puncta ipfius volutx, vt inter I.\& a inter 2.\&3.\&c. Et figna fupernè fectiones at cuum literis alphabeti, vt d.e.f-g.atg ita deinceps per totum alphabetum, quo. nd extendi poteft. Quum verò producis lineas c d.d e.ef.\& fg.\&c. fic óp per totum circuitum literarum, abfcindútur lineolæ rectæ, qua protracte funt ex pun ctis r.2.3.4. \&c. ac reliquis numeris omnibus: Quod fi folia illa qua per arcus hos facta funt, cómodè per lineas intermedias diuidere volueris, protrahe primú ex puncto c.deinde ex d.e.f.g. \&c. \& fic ex reliquis literis, rectas lineas verfus cétrú a.vfqgad volută. Atģ fic ifta omnia ordine fiunt, quéadmodú hî́ defignaui, Quam longitudinem debeant habere linex erectx fuper helicam.
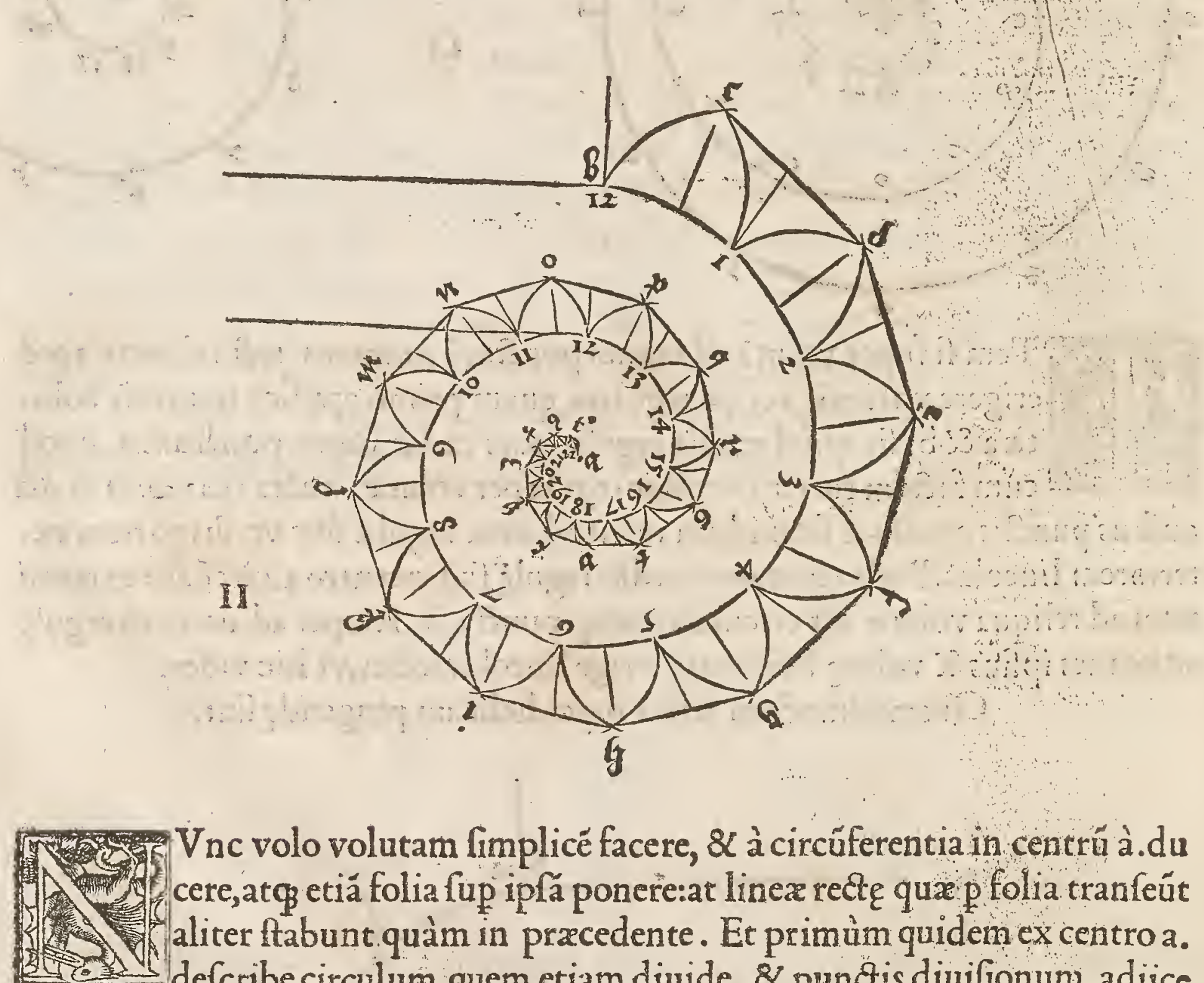

Vnc volo volutam fimplicé facere, $\&$ à circúferentia in centrú à.du cere, atqg etiá folia fup ipfă ponere:ar linea recte qua p folia tranfeút aliter ftabunt quàm in pracedente. Et primùm quidem ex centro $a$. defcribe circulum, quem etiam diuide, \& punctis diuifionun adice numeros, quemadmodum in priore fecifti. Lineam verò erectam a b. quam circumagis, partire vndecim punctis in duodecim $x$ qualia interualla, 2 circumi cum ea, vt iam pridem edoctus ês, puncta volute notando víg ad centrum a. In hunc igitur modum, quem dixi, debet hæclinea fieri, qux quidem ad diverfa opera vilis erit, \& pracipuè ad ped um epifcopale, ad quod abfoluencum duc ex puncto circumferentix $\sigma$. lineă rectam deorfum, $\&$ vtere medietate circumfe 
rentix, qux fignata eft numeris maioribus, vnà cum voluta, reliquá autem peripherix medietatem numero minore diftinctam omitte. Poftea accipe circinum, cuius vnum pedem pone in punctun circumferentix. \& \& alterum in punctú 7 . eìrdem circumferentià, à quo educ arcum circuli, deinde repone vnum pedem in punctú 7.8 altero protrahe arcú ex puncto $9.8 \mathrm{v}$ bi illi duo arcus fe interfecát, illıc fciribe literă c.atọ ex puncto circuitus s. duc lineam vígiad c. Eodem modo fac inter figna 9. \& Xr. \& fectioné fupnè nota d. Sed deinceps pofsto vno circini pede in púcto circúferentį ir al tero pduc ex figno volutx $\mathrm{r}$. arcû circuli, ac iterú vnopede manente in puincto volutx $\mathrm{x}$.altero duc arcum è puncto cir cumferentia ir.furfum, $\&$ ad fectionem illorum arcuum fcribeliterá e. Pari eti-

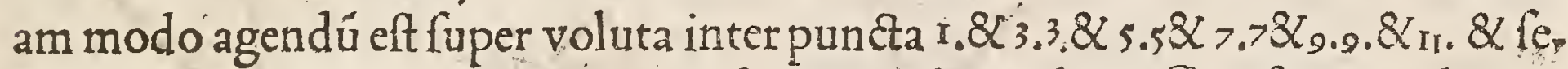
ctiones arcuum fupernéfigna literis f.g.h.i.K.fuo ordine. Quo facto trahe per folia volutx lineas rectas $\mathrm{e}_{\mathrm{I} 2} \mathrm{f}$ 2.g $4 . \mathrm{h} \mathrm{\sigma}$. i 8 . K $\mathrm{ro}$ : Tandem remanet adhucportio quadam volutæ, inter ${ }_{n}$. \& centrum a . cui fuperfcribe etiá duos arcas quorum fectio fit l. Eorum qux fuprà tradita funt, hic duas figuras feci, Primą cum? omnibus fuis lineamentis neceffariis, ex quibus conficitur.Secundam nudam. He volutatum defcriptiones ad multa opera defignăda funt vtiles, atque inte. rim etiam ad deliniandum ramale, quod \& hic poftremò protraxi.

Hac linea feruit ad defignandum pedum epifcopale.

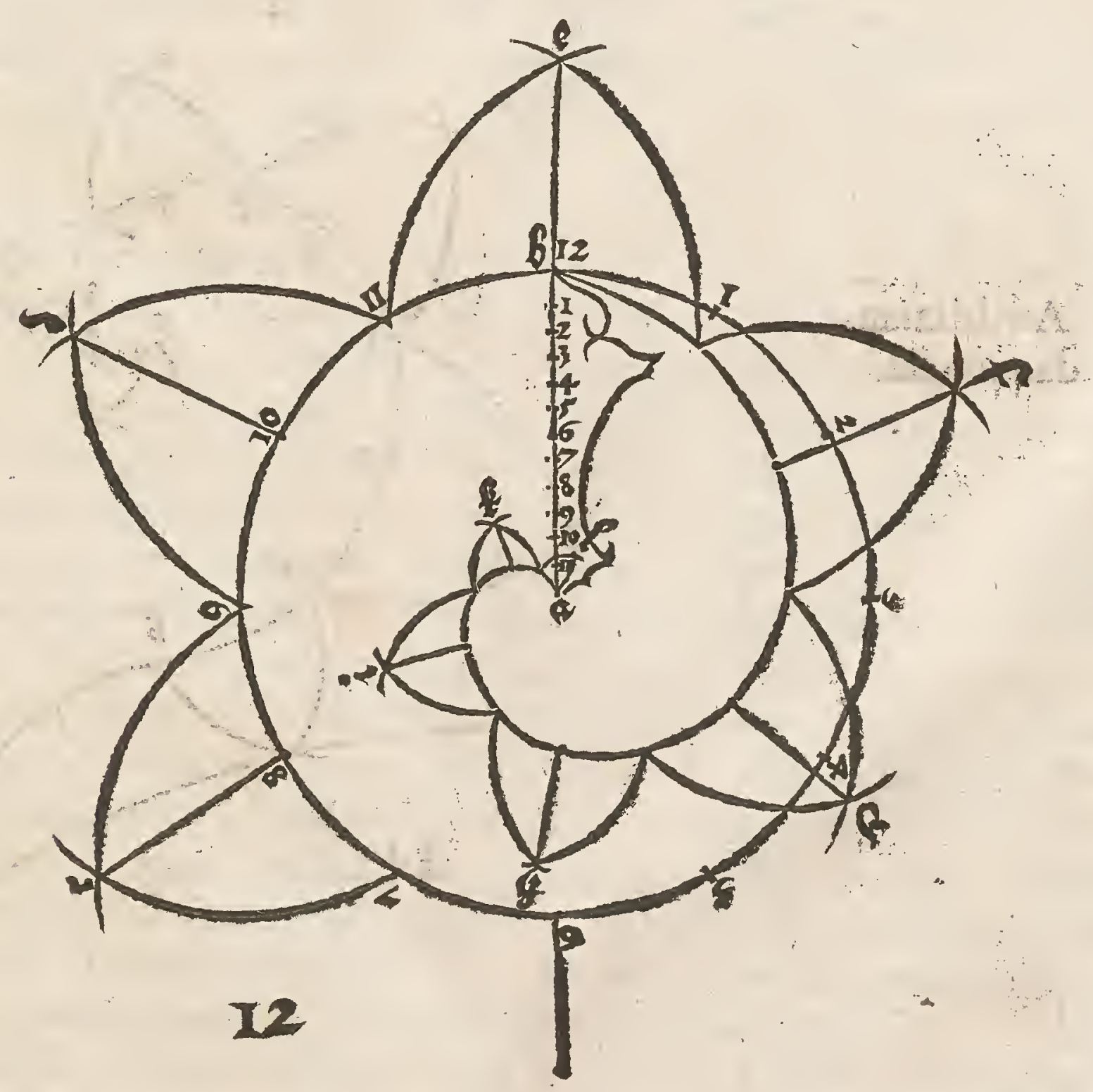


Linea pedi epifcopalis nuda.
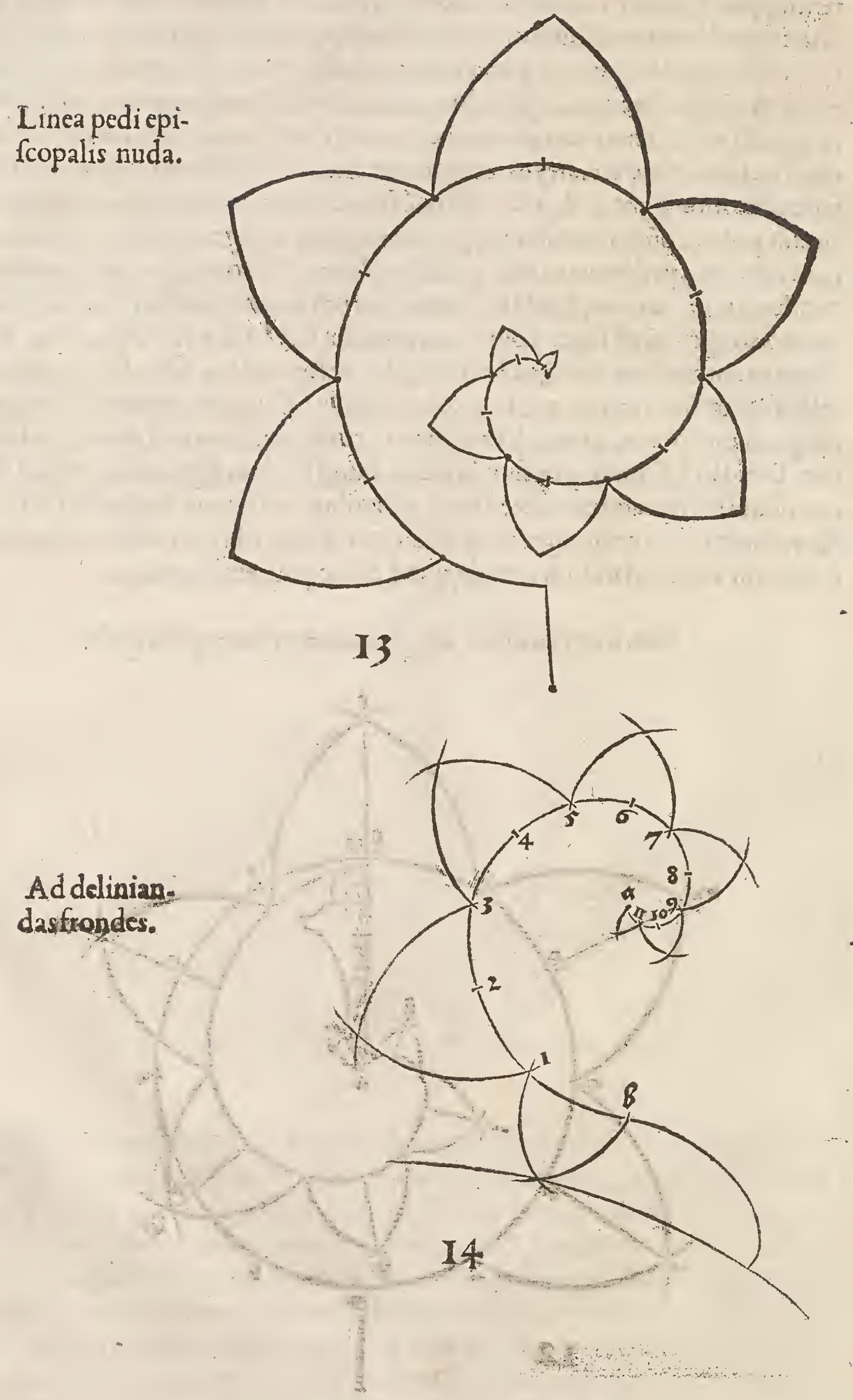
Otandum eft, quòd circumferentia circuli,per quam voluta defcribi. - tur, in tot partes diuidi poteft, in quot voluerimus . quo enim plures 42 fuerint partes, eo aretius menfurandum erit, $\&$ in quot partes circum ferentia diuifa fuerit, in tot partes erit $\&$ regula diuidenda, per quam volutam fimplicem facere vis. Si autê eam bis, ter, aut quater, circumducere ftatuifti, tum multiplica etiam puncta regula per duo, tria, aut quatuor: $\& \mathrm{crcum}$ curre totiescum ea, quoties puncta multiplicalti, inueniesóp poltulatum. Quòd fi times, quú partes regulæ multiplicas circumcurrendo per circúferentiam, pro pter numeros inæquales, te poffe errare, fac fic. Quum circúferentiă tuam diuififti (quod fir exếplı gratia) in duodecim partes, \& vis volutá bis, ter aut quater circumducere,partire regulam in quot volueris puncta, quibus adfcribe nume-

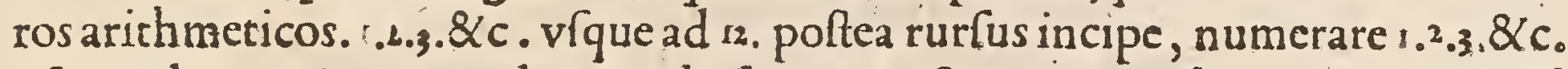
$v$ fque ad is.atq iterum eodem modo femper poft.12. repetendo .1...3. \& c.quoad perueneris ad finem vfque regulę. Et applica regulam fic circüfererentix, vt circủeundo numerus regula fêper correfpondeat numero circúferentix : co enim modo non poteris errare. Iam verò cùm voluta in fuperficie plana fit defcripta, confequenter eam è plano fuo in altum eleuare docebo. Certum autem eft cùm. quippiá côltruere volumus, primü fundamentú ipfius ftatuendü effe, fiue fit ędi ficiü aliquod, fiue quacúqg alia res:eadem ratione voluta noltra furfum duci nó poteft, nuli ipła prius, vt fundamétú, in plano fie polita. Quare ptrahe primùm pro tundanento, nudam illam præcedentế volută, vnà cum circumferentia lua ex qua facta eft,omiffis omnibus foliis:numeros tamen in ea coueniet, in bunc

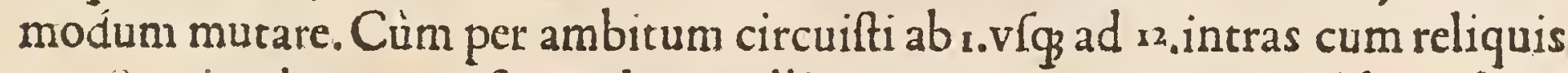

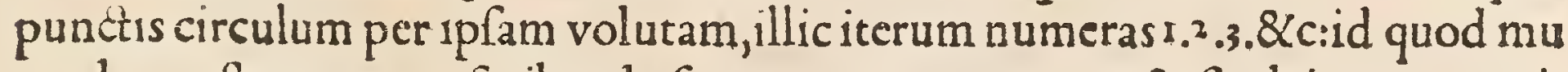
tandum elt, nam pro 1. fcribenda funt I3.pro 2.14. pro 3.15.\& fic deinceps continuando numerum vfque ad 33 . procedendum elt. lacto fic fundamento, ex pun. cto 6. duclineam reatam, furfum per centrum a.\& fignum x.tantę lógitudinis, quanta opush abes, \& ad terminum fuperiorem fcribe literam a. quòd punctus ille directè itet fupra centrum a. Deinde abfcinde perpedicularé a a.per lineam traniverfan $c$ d.mfernè ad punctun b. Quo facto, diuide lineam a b. fuperiore per 23 . puncta in viginti quatuor partes xquales. Ego tamen hic ordine prolongaho f pacia, fuperiora, quemadmodum pauloante pracepi:quare eundem modủ iterú repeto, nili quòd duas literas tráf́pono, ná a.pono fupernè, $\&$ b, infernè, atq̧ puncta diuilionú ${ }_{1 .}^{2} \cdot 3.8 \mathrm{c}$ incipio numerare in parte inferiore. Nunc quum lnea a b.hoc pacto diuifa cú fuis punctis $\&$ numeris, ftet in medio fundaméti erecta, pduco lineá ex púcto fundamếti r. furứ fecantē iprá lineă obliquă c d. deunde ex púctu I. linę̧a b.duco lineá tranfuerfá, verfus ıă protractá lineá erectã:\& vbi duę ill ̨̧ linę faciüt angulü, illic fcribo r.atọ hic eft primus púctus q incipit a cédere in voluta eleuata, fiue coclea. Sicfacio p omnia púcta $\&$ numeros fundaméti depreffi, $\&$ erectx lincę a b.in vtraq parte. Hoc en im modo fin. gula coclex puncta netantur, ab infimo figno b.vf̣́ ad fupremú a.poftea cốtinuo lineam tortuofam, $\mathrm{ab}$ vno puncto ad aliud. Item quando per hanc lineam coclea in tectum alicuius turris fit, debet infimus gradus multo longioreffe 


\section{A L B ER TI D VRERI}

fupremo, \& fic ordine perpetuo debet femper inferior fuperiore \& fibi incumbente longior effe:pari ra tione quanto quifque gradus altior elt in coclea, tanto debet etiam effe craffior, qux omnia bic diligenter protraxi. Et primùm quìdé coclex fundamentum, fuper quod \& cocleam ipfam, cum omnibus lineis fuis quibus defignata eft:deinde $\&$ coclex lineam nudam tortuofè in altum euectá. Hxc linea potelt arctèfuper feipfam incurrere, vel ftatim in altum extendi prout linea a b.fuerit prolixa,erírque ad multa vtilis opera. Triăgulum etiam a $b$ c.cum arcu fuo b e.ex quo linię a b.partes fuperiores prolongaui,

hic defcripfi,cum reliquis lineis \& numeris neceffariis. Ifte coclearum linex poffunt etiam fieri angulares, $f_{1}$ inter duo puncta fiue numeros femper vnus omitatur:vt fi in coclea eleuata traheres ex puncto $b$. vfque ad 2. lineam rectam $\mathrm{ex}^{2}$, in $4 . \mathrm{ex} 4$ in $6.8 \mathrm{c}$.acita deinceps vfque ad a. 


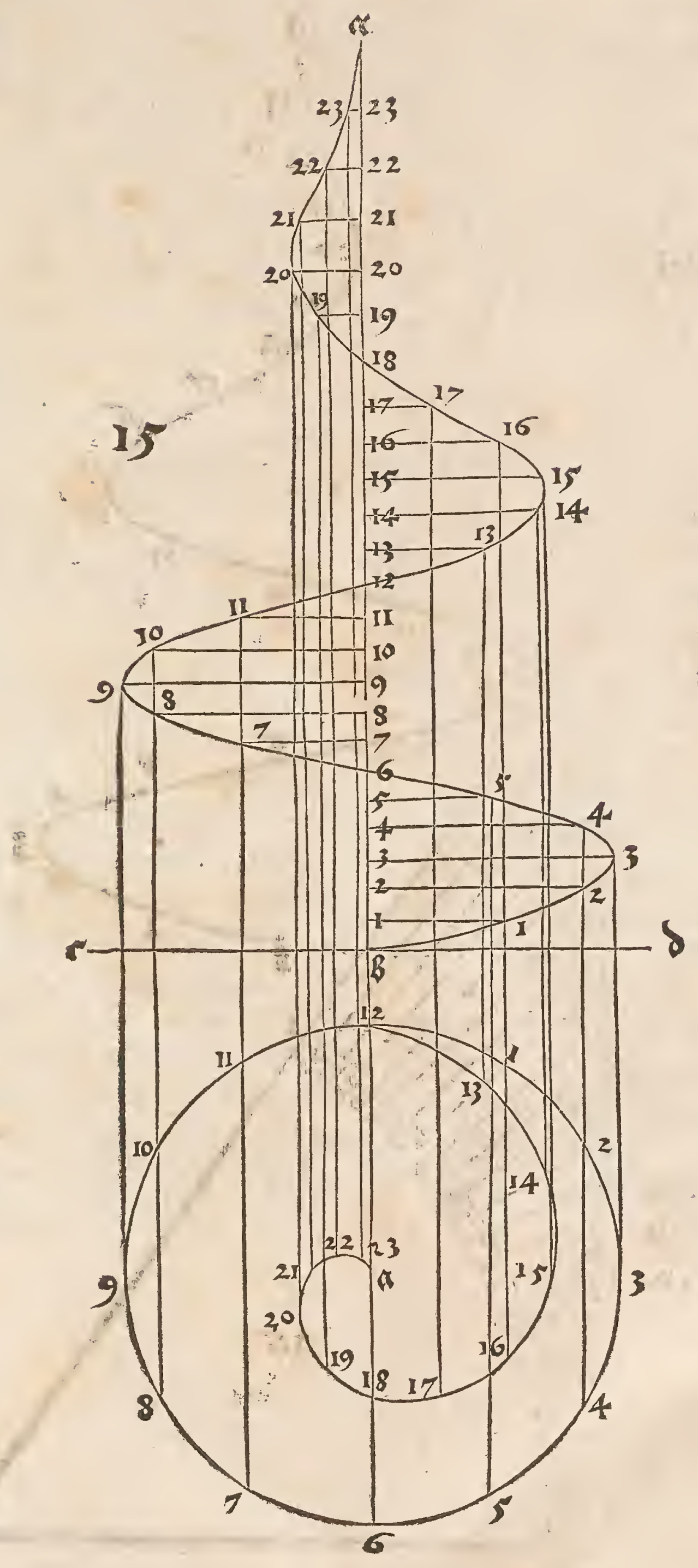

Cochlea ex fúda méto protracta cum omnibus li neamentis necer fariis ex quibus facta eft. 
Hic vides cochleá nudam.

Ex hoc triangulo a b c . diuifa eft linea cochlega $b$.

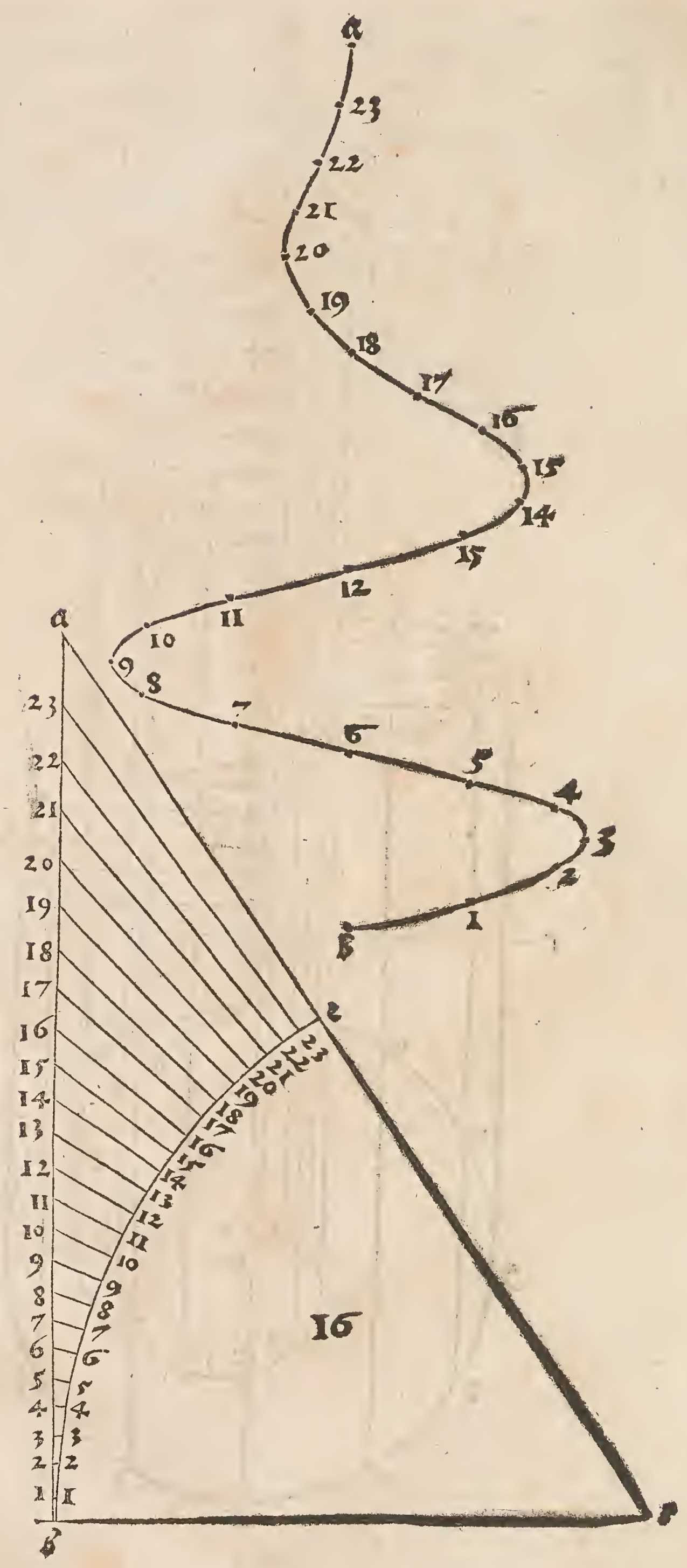




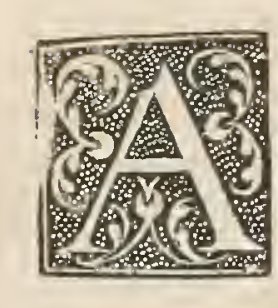

Dhucalia cochlex linea potelt fieri ex fola circuli circúfereútia, quaetiam lapicida in conftituendis cochleis vtuntur, quę tamé com modiùs cocliogramme vocatur, fed vtcunque appelletur, linea hæc eft vtiliffima, quare eam quoque ducere docebo: $\mathbb{X}$ qui inueftigare volet, multa poteft per eam inuenire. P rimùm itaque defcribe circulum, vt in prace. dentibus dictum eft, ex centro a.eum diuide per linea $m$ perpendicularem tranfeuntem per centrum a . in duas partes æquales, \& ad fuperiorem fectionem cir. cumferntię \& perpendicularis linę fcribe ${ }^{12}$. ad inferiorem verò $\sigma$. deinde protrahæ lineam 6.12 in rectum verfus partem fuperiorem quantum opus fuerit, cu ius terminus fupremus fit a. Poft ea abfcinde lineam a a.infernè, prope púctum ${ }^{2}$. per lineam tranfuerfalem $\mathrm{cd}$. ad angulos rectos, fitç feetio illa b.ian diuide circumferentiam circuli in ${ }^{2}$. equales partes, $\&$ adiice punctis diuifionum fuos numeros, incipiendo numerare ${ }^{1.2}{ }^{2} 3.8 \mathrm{c}$. in puncto, qui proximus eft 22 .donec ite rum redeas ad $\mathrm{Iz}_{2}$. Sed deinceps op̀ortet numerum progredi, $\&$ quo vfqg opus fuerit, vnum fuper alium conftituere: venient itague 13 .fuper 1.8 14. fuper $2.8 \mathrm{c}$. fic potelt numerus ter, quater aut quinquies, \& quoties opus poltulat fuper feipfü currere, prout cochlea al ta fuerit cöltruenda. A b folutoiam fundamento, diuide lineam $a$ b.in quot volueris partes, $\&$ appone fingulis fuos numeros incipiendo è puncto b.verfus a. furfum numerare $1.2 .3 .2 \mathrm{c}$. Hoc facto duc lineam à puncto

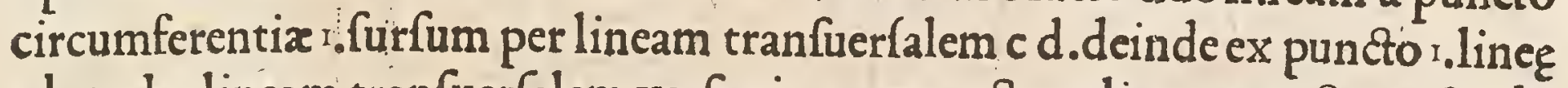
a b.trahe lineam tranfuerfalem verfus iam protractam lineam erectam, \& vbi dux ill $x$ linę faciunt angulum, fcribe r. Sic fac per omnes numeros linę a $b, \&$ fundamenti,atque etiam in numero fupercurrente. Quum iă cochlex linea fic fit per puncta defignata, protrahe eam manu ab vno puncto ad aliud, quemadmodum mefeciffe hic vides. Hęlinę poffunt à puncto ad punctum, angulares protrahi. Talis quog cochlea poteft fieri duplex in circuitu fuo. Primùm qui. défit colúna quę per mediă cochleam erigitur recta $\&$ teres: deinde poteft etiă fieri tortuofa, fic vt à fummo vfque ad imum per eam poffumus videre:id quod lapicidx in fuis protractionibus fcire debent, $\&$ per motionem afferum fundamétalium operi applicare. Ex pradicta linea fiunt cochlę̧ vno, duobus, tribus aut quatuor circuitibus $\& \mathrm{c}$. quibus moles fortes $\&$ graues poffút dimoineri, vt etiam miraculo fimile fit.

B ii 


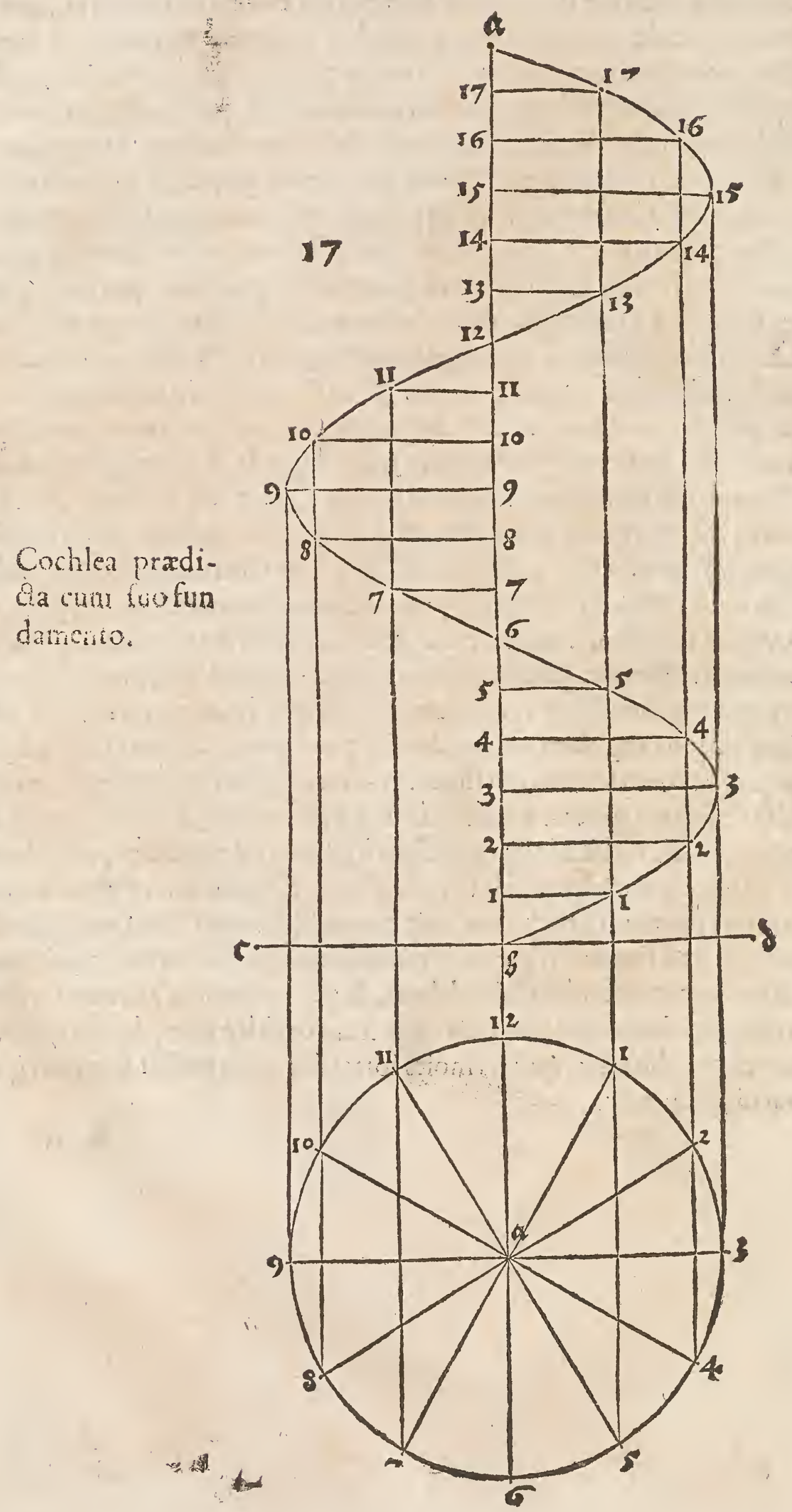


ज政 Vac fimplicem quandam volutá protrahere docebo, \& alia etiam via 61) quàm prius fecimus, in hunc modum. Defcribe quadrantem circuli a 420 b c.\& lit b.cétrum, a.verò angulus fuperior, \& .c.angulus ac latus, deinde diuide quartam illam circumferentix a c.vndecim púctis, in du. odecim partes equales:quas numera à c.verlus a.\&. trahe à fingulis diuifionum punctis lineas parallelas, in lineam tranfuerfam b c.cui etiá adfcribe numeros, quemadmodum in quarta circumferētia, incipiendo à púcto diuifionis c prox imo, \& fic eft linea $c b$.diuifa, ex arcu circuli c a guod primum eft fundamentum. Sub eo nunc defcribe femicirculü, ex centro c.cuius fenidiameter fit æaua lis lateri quadrátis b c.\& fit diameter illa fupernè a.infernè verò b. Deinde par tire femicirculum a b.1n duodecim partes equales, quibus etiam adiice fuos nu meros $a b$ a.verfus $b$.numerando, $\&$ duc lineas rectas à numerorú punctis in cétrú c. Quo facto accipe circinú, $\&$ pone eum vno pede in centrum quadrantis $b$. \&altero in punctum . linex tranfuerfalis $c b . \&$ tranffer interuallum iftud in $f e$ micirculum, in cuius centrum c.pofito vno pede circini, reliquo fub a.in linea a c.à qua vfque ad lineam $\mathrm{c}$.duc arcum, ad cuius finem, fi potes fcribe etiam $\mathrm{r}$. Nunc iterum accipe circinum $\&$ pone eum vno pede in centrum quadrantis $b_{\text {. }}$ \& altero in punctum 2. linex tranfuerfalis $\mathrm{cb}$. \& feruata illa apertura ex centro c. femıcirculi fcribe arcum à linea i c.vfqg ad lineam : c.vbi etıă, fi fpacium ha. bes, pone numerum 2 . Sic operare inter omnes dimidii circul femidiametros. Quum autem omnia pradicta interualla ex quadrantis linea c b. fumis, eáque transfers in femidiametros dimidii circuli, \& ad ́cri bis punctis illis fuos numeros, oftenditur tibi quo pacto ambitum volutx à figno a.circumferentix per fi. gnata puncta ad centrú c.inducere debeas. Potes etiam mobili pede circini femper arcum continuare à linea a c.vfque ad femidiametrú deftinatam, 2 hoc reprafentat aliquid fingulare, $v$ thic vides.
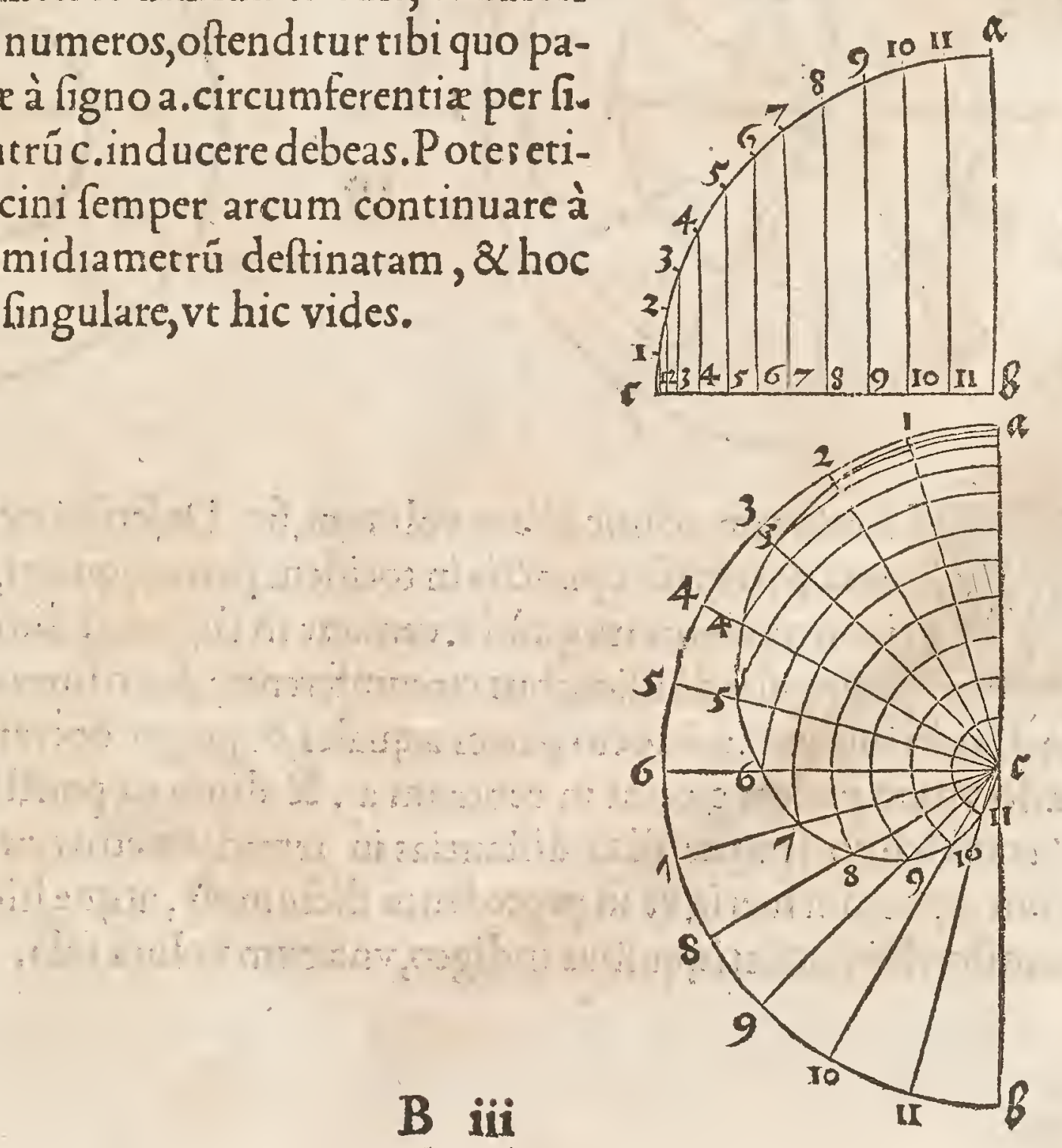
Tem aliter quoq̣ faciá volutá in hune modú, qui fequitur.Primò pono cêtrum a.ex quo defcribo circulum, quem, vt prius, diuido in duodecim equalia interualla, \& à fingulis diuifionibus duco lineas ad cé. trum a.quibus etiam adfribo numeros arithmeticos, ponendo ad fu. premam diuifionem ${ }^{2}$.à quibus numero ${ }^{2}$ 2.3. \& 2 . doneciterum redeo ad 12 .Po Itea diuido lineam 12 .a. trigintaquing punctis in trigintafex partes æquales, 8 incipio numerare fupernè à puncto 12 .defcendédo verlus centrum a. Hoc facto; pono vnum pedem circini in centrú a.\&aliam in lineam 12.a. in punctú 1 .ppe $16 . \&$ duco inde arcum, $v f_{\phi} \phi$ ad femidiametrum 1 .a. Eodem modo relinquo dein

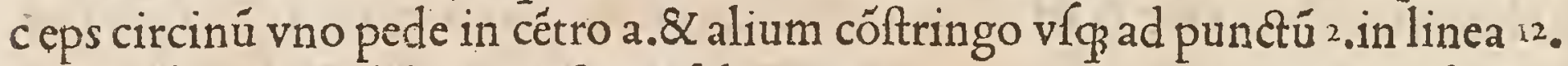
a. \& f cribo arcum à linea .vfque ad lineam $2 . a$.Sic conftringo femper mobilem circini pedem per vnum gradú in linea $"$ "a. \& protraho arcus ordine inter omnes femidiametros donec ter circúiuero . Quú hec om nia circino pfeci,incipio iterum à puncto circúferentix 12 . \& induco volutá $a b$ vno puncto ad aliud, quo $v f_{q}$ in tertia reuolutione peruenero $v f_{q}$ ad centrú a. quod hic defcripfi cú omnibus lineis neceffariis per quas voluta defcribitur; deinde volutam etiă nudá.

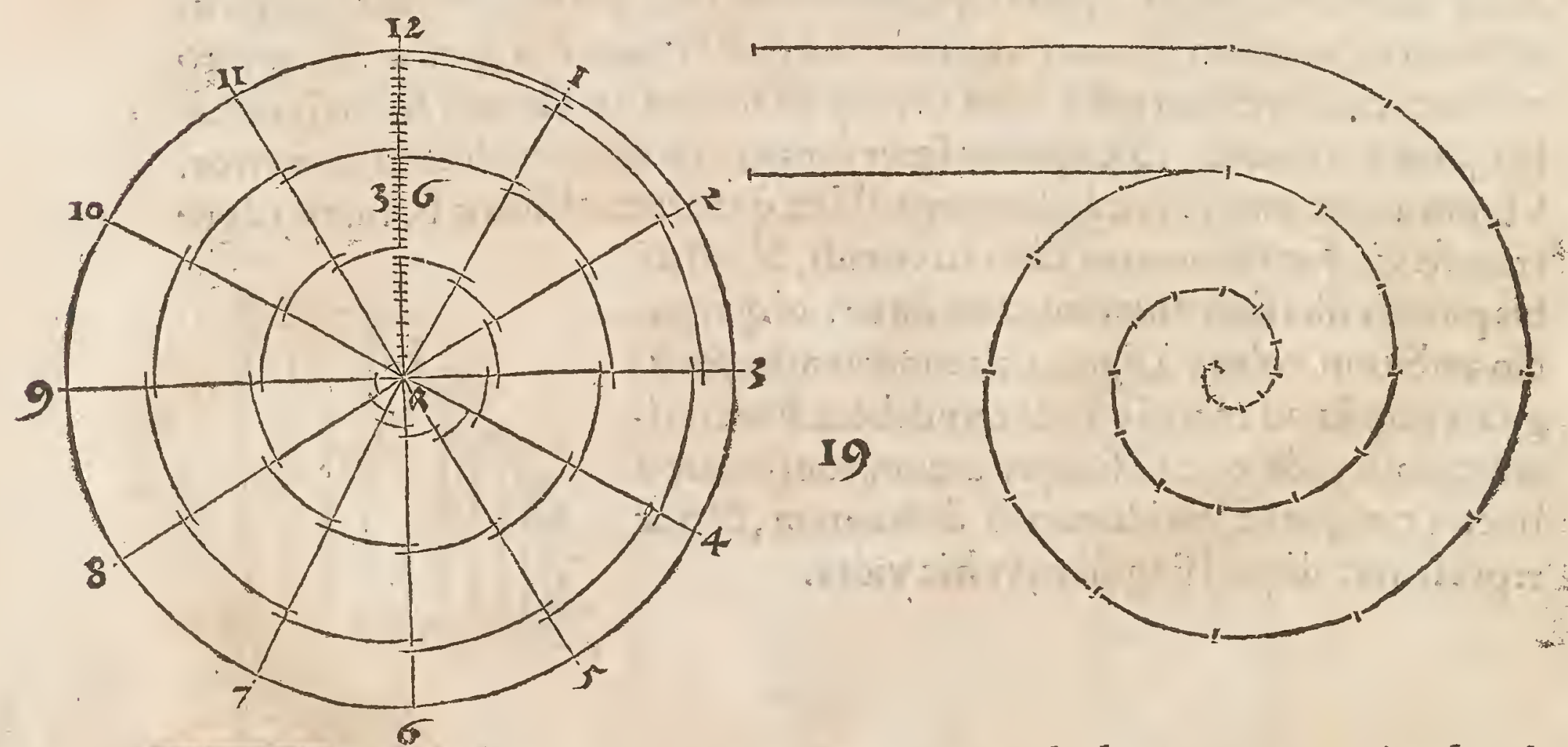

Roducam adhuc aliam volutam, fic. Defcribo ex centro a.circulum, 1. eum partior fex punctis in totidem partes equales, ac punctis illis ad. 1 Cicio numeros ita quòd 6. veniant in fupremú diuifionis punctum, \& à fingulis diuifionibus circumferentį duco lineas ad cétrum a. Dein de diuido lineam 6 .a.in octo partes æquales, \& pergo operari vt prius, conftitu-

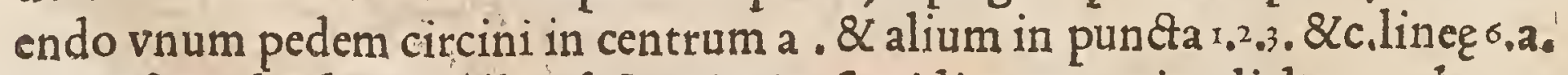
$\&$ trasferendo femper illas diftantias in femidiametros circuli donec ad punctum 7. ventum fuerit, vt in pręcedente dictum eft, atque hic etiam protraxi cú omnibus lineamentis, quibus indigeo, vnà cum voluta fola. 

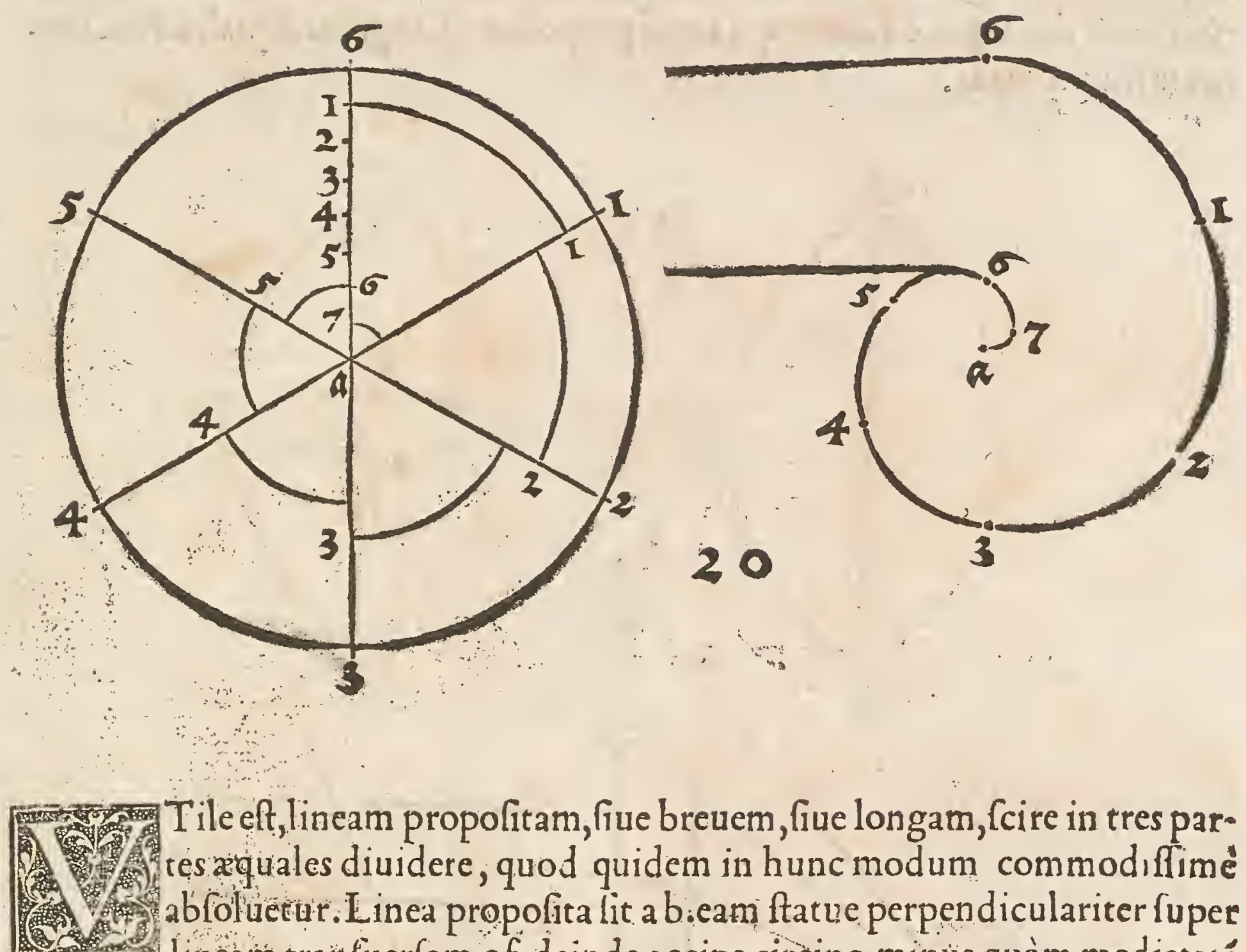

Tile eft, lineam propofitam, fiue breuem, fiue longam, fcire in tres partés aquales diuidere, quod quidem in hunc modum commodiffime abfoluetur. Linea propofita lit a b.eam ftatue perpendiculariter fuper linean tranfuer am c.f.deinde accipe circino, minus quàm nedietaté ex linea a $b$. cuius vnum pedem pone in punctum $f$ a a quo menfura furfum ter aperturam circini, $\&$ per puncta illa duc lineam rectam, qua (it parallela ip. fi a b. fit ç ea linea fupernè e.infernè verò $f . \&$ duobus punctis intermediis adiice numeros arithmeticos 6 . Et protrahe à termino e.per terminum a.lineam obliquam vfque ad lineam $\mathrm{c} f$. quam vbi contingis f cribe literam $g$. ab eo cótactu trahe duas lineas rectas ad puncta $+\& 2 . \&$ fecabitur linea propolita a b. per duas obliquas g. : \& g a in tres partes equales. Si vis adhuc aliter lineă rectá in tres partes equales diuidere, fcribe quatuor lineas tranfuerfas ac parallel as fignatas

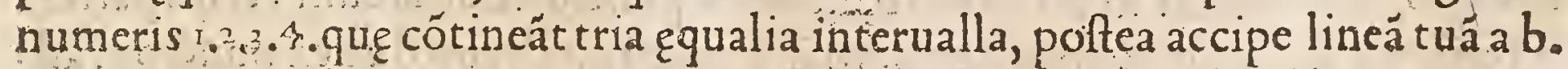
propolıă $\&$ reclina eá extrem itate a.ad linea r.\& extremitate b.ad lineâ 4.8 diuident linex 2.8 .intermedia lineam a b.in tres partes qquales. Iam antequam vlrra progrediar, docebo lineam per medium fecare, in hunc modum. Sit linea propolita tranfuerfa a b accipio igitur circinû, cuius vnú pedé pono in terminum a. \& altero ex termino b.duco arcum in veramque partem ipfius $b$.quantum opus habeo, Iterum immota ci rcini apertura, relinquo vinum pedem in $b$. $\$$ altero ex a duco arcum furfum \& deorfum, qui fecabit arcum priorê in duobus locis, in quibus fcribo literas c.\& d. quas coniungo ducta linea c d.qu $x$ ip. fam a b.propofitam fecat, in puncto e. per medium. Qui verò lineam rectam 
fuper circumferentix portionem volet conftituere, is faciat omnino vt iam dictum eft,ponat'qu a e b.arcum, \& lineam rectam.cc.Item in concauitate arcus, codem modo erigemus rectan lineam. Nam fit arcus datus a $\mathrm{fb}$. in cuius parte cốca!na elt defcripta c f.linea, ipfiarcui propolito ad angulos æaquales. Hxcomnia unfrà protraxi.
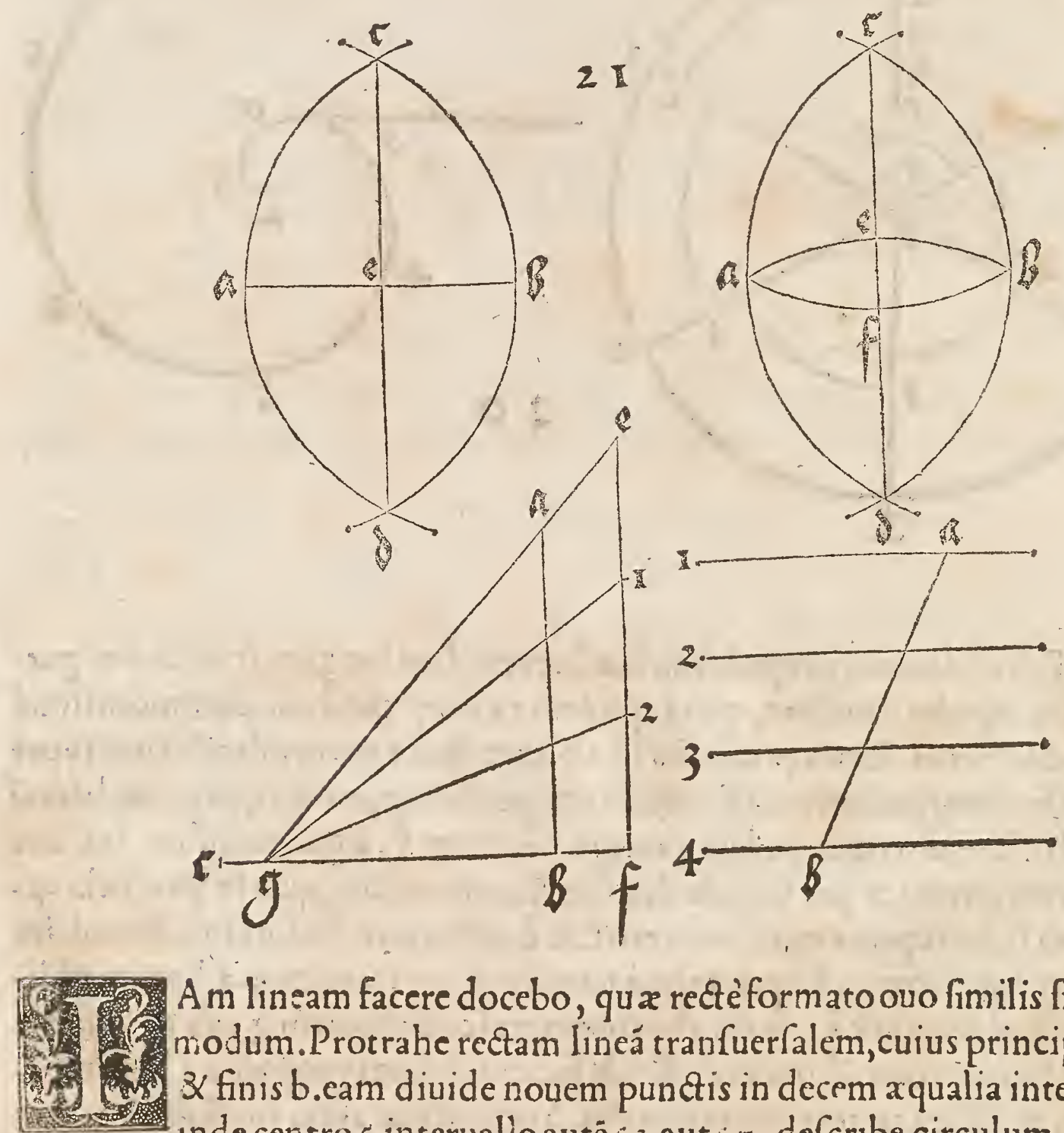

A $m$ lineam facere docebo, qu $x$ recte formato ouo fimilis fit, huns in modum. Protrahe rectam lineá tranfuerfalem, cuius principium fic $a$. $3 x$ fin is b.eam diuide nouem punctis in decem xqualia interualla,de. inde centro s.interualio aute 5.3 . aut $\%$. . defcribe circulum. Poftea pofito vno pede circini in punctum $b$, altero ex puncto $z$ duc arcum verfus partem inferior' $m: \&$ iterum vno pede pofito in puncto a.reliquo duc ex puncto $\% .2 d$ huc alium arcum, verfus prędictam partem inferiorem, \& vbi illi duo arcus fe interfecant, frribe literam e.quofacto, protrahe lineam contingentem circulü in parte inferiore, qua fit parallela ipfi a b.linex, \& vbi illa linea cadit in arcuse. 3.\& e. . Ill ic icribe fub .. literam c. \& fub .d.nunc trahe lineam, ex figno s.ad fe ctionem c. \& vbi linea sefecat lineam $c d$. frrbe ro. Porro diuide quartas circuli $10 . \& 10 . \cdots$ per medium, illam quidem in punctof. hanc verò in punctog. \& fi. to vno circini pede in puncto f.altero ex d. duc arcú verfus e.Iterú ex altera par te, pofito vno pede in punctum $g . \&$ altero in c.duc arcum ex c.deorfum, per li. 


\section{GEOMETRIAE LIB.}

I.

neam $c .10 . \&$ in fectione illorum arcuum in linea.s.c.adiice punctum $h$. hoc ab: foluto partire linea $\mathrm{h} .0$, ad fignum $\mathrm{r}$ in duo fpacia xqualia, \& ibidem polito vno pede circini, alterum extende in arcum $c$ h.in eum punctum, qui proximus eft figno i.\& duc inde arcum per lineam $i h . v f q$ ad $d$ h.erítque oual is linea ab. foluta, quam infrà cum omnibus fuis lineamentis per qua fit, atg etiam nudam protraxi.

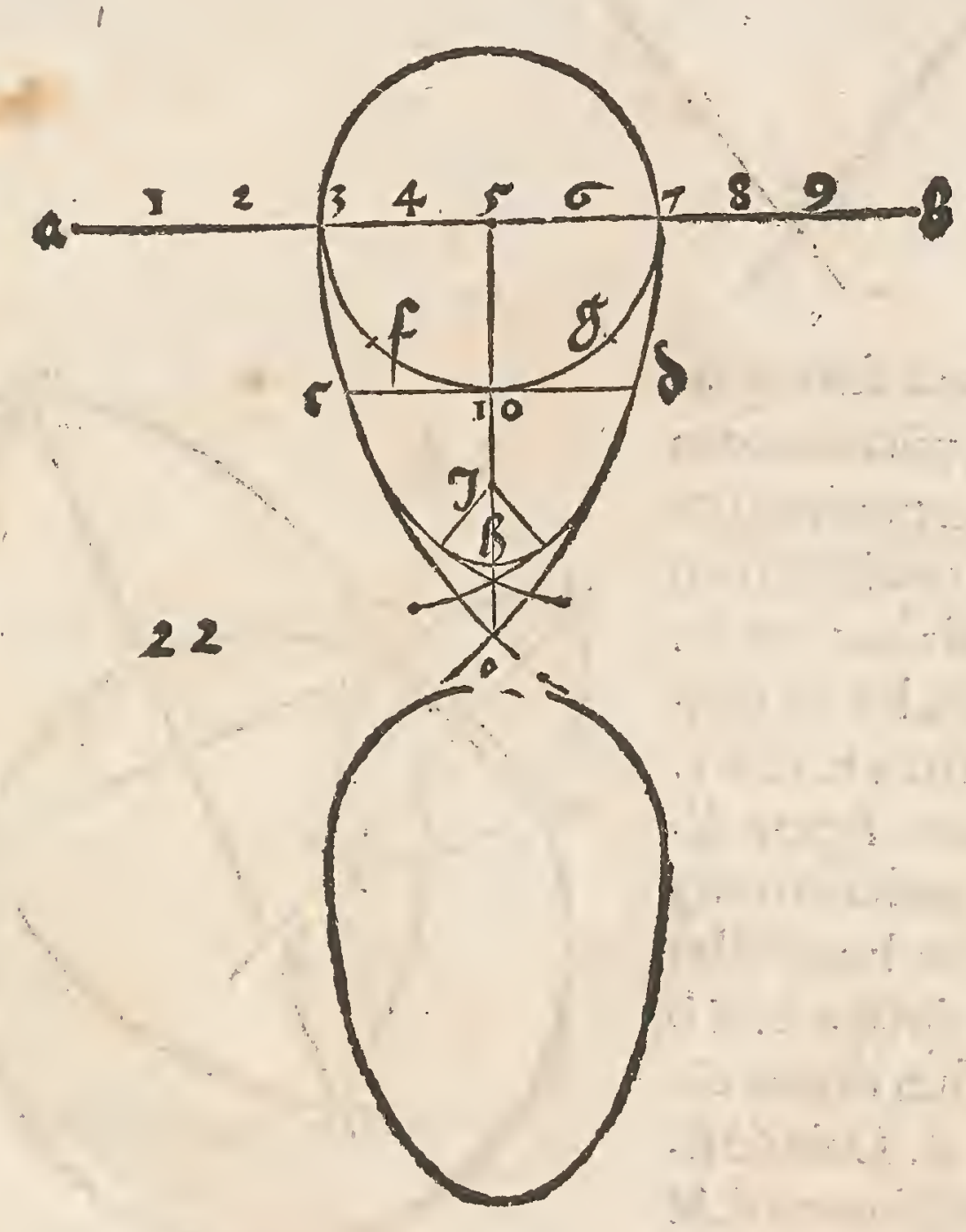

\footnotetext{
Fin Eceffarium erit arcui circuli fuum inueniri centrum, quod quidé co. 1. . dem modo expeditur, quolinea fuper arcum erigitur, id quod etiam

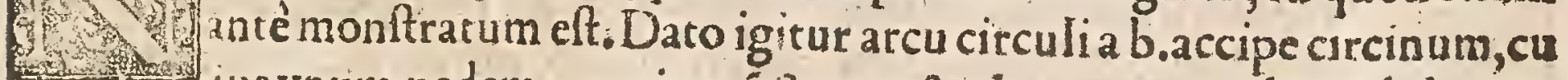
ius vnum pedem pone in púctum a. \& alterum extende paululum in arcum a b.\& duclinean curuam furfum \& dcorfum, quantun opus eft: deinde filte vnum pedem in punctum, gquem linea illa curua fecit in arcu a b. 8 altero ex a.protrahe iterú arcum furfum 2 deorfum, quoad illi duo arcus iam defcripti fecabunt e in duobus locis, per qux lineam trahito rectam deorfum, quăta opus habes:ita facies quoque circa extremitatem b. \& lecabunt fe in parte infe riore binx 1 llę linex recta in puncto vbi centrum elt, ad quod fcribe literam c. Sic igitur centrum b, inuentum ef, quod \& hic videre elt.
} 

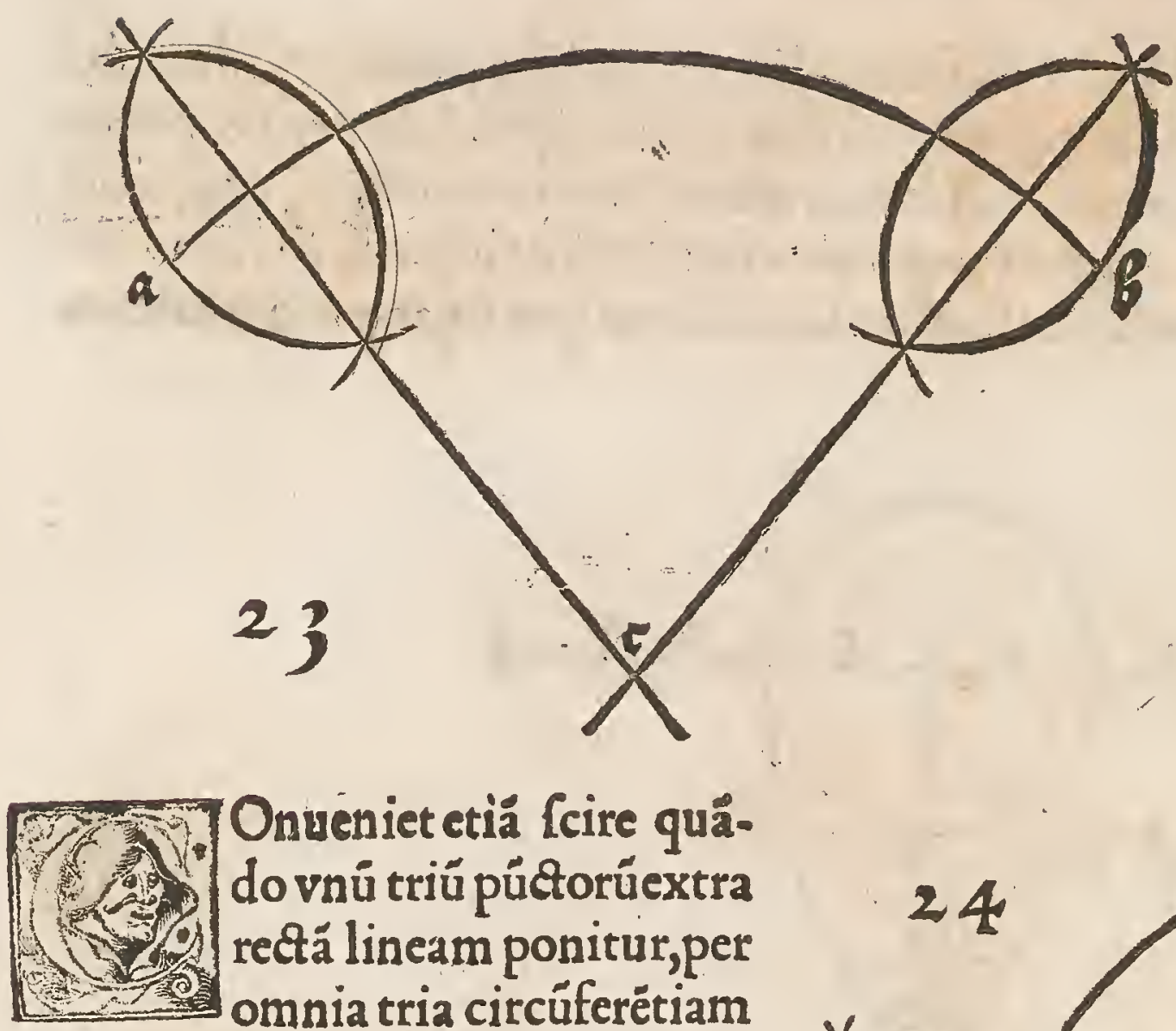

Onveniet etiå fcire quådo vnú triú púctorúextra rectá lineam ponitur,per omnia tria circúferétiam circuli trahere, quod fit hoc modo. Tria puncta fint data a $b c$ c ea coniúge duabus lineis rectis $a b . \& b c$. \& fac quemadmodú in 22 . figura dictú eft : quære púcta media vtriufq̧ linex a b.\& b c . \& duc lineas illas rectas (qux diuidunt ipfasa $b . \& b$ c.per medium) deor(um donec cócurrant ad punctum d. Deinde fige pedem circini in fectionem d. 8 alium in punctum a. \& fcribe circú

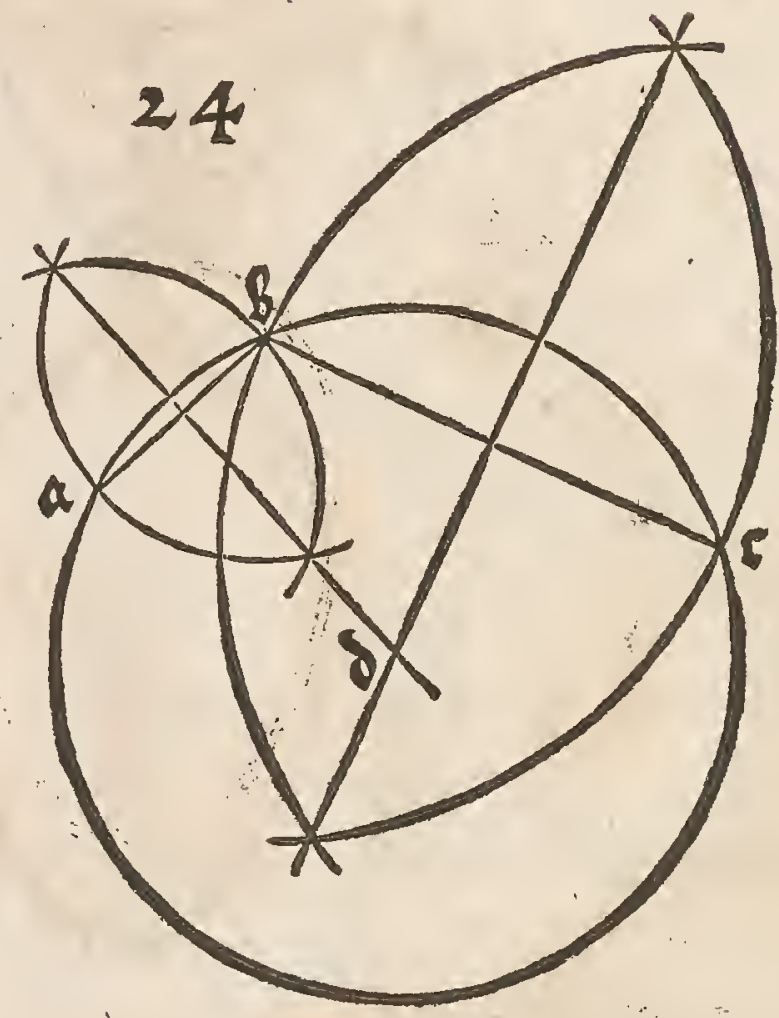
ferentiam, qux tranfibit per ominia tria puncta a b c.vthic vides. Vando magni circuli arcus cốtingit lineam rectă \& prx acie angufta angulorú.vix poffumus locú contingentix percipere, hoc pacto eum 1. inueftigare debemus. Sit arcus circuli a b. qui contingat lineá rect am 12. $\mathrm{c}$ d.iam pone vnum circini pedem in extremitatem c. 8 alium exten de parum verfus $d$. $\&$ duc arcum tranfeuntem per lineam $c$ d.quam vbi fecat fcribe e.rurfus immoto circino fifte pedem wum in e.\& alio arcum liniato per c. fecantem priorem in dwobus punctis, fusernè quidem ad literam f.infernè ve rò ad g ea puncta coniunge, ducta linea $\mathrm{g}$. quęabfcindit $\mathrm{cd}$.ad fignum $h . \propto$ arcum $\mathrm{ab}$. in puncto. à quo protrahe ipfi $\mathrm{cd}$. xquidiftantem, qux vbi interfecat arcum $a b$. prope b.illic feribe K. Nunc transfer diftantiam i K.in lineá $c d$. ponendo terminú i.in punctum $h$.cadétque terminus $K$. verfus $d$.ad fignú 
I. quod coniunge cum K.produsta linea I K. erit'p quad rangul um equilaterum h I $K$ i. Quum autem cupis fcre pun ctum vbi arcus a b.contingit lineame d. fige vnum pedem circini in punctum $l$.ac reliquo arcum circinato ex $h$. furfum \& deorfum, quantum opus eftiterum non variato circino, ex centro h.duc aliú arcum tranfeuntem per angulum I.priorem fecantem in duobus locis (vt monftratum eft in 2 . figura) $\&$ ad fectionem fuperiorem ádiice $m$. ad inferiorem verò $n . \&$ trahe lineam $m$ n. ac vbi ea diuidit $\mathrm{cd}$.adfcribitoliteram o.ad quam ar cus a b.tangit lineam $\mathrm{cd}$. quod in hac figura liquidò apparet.

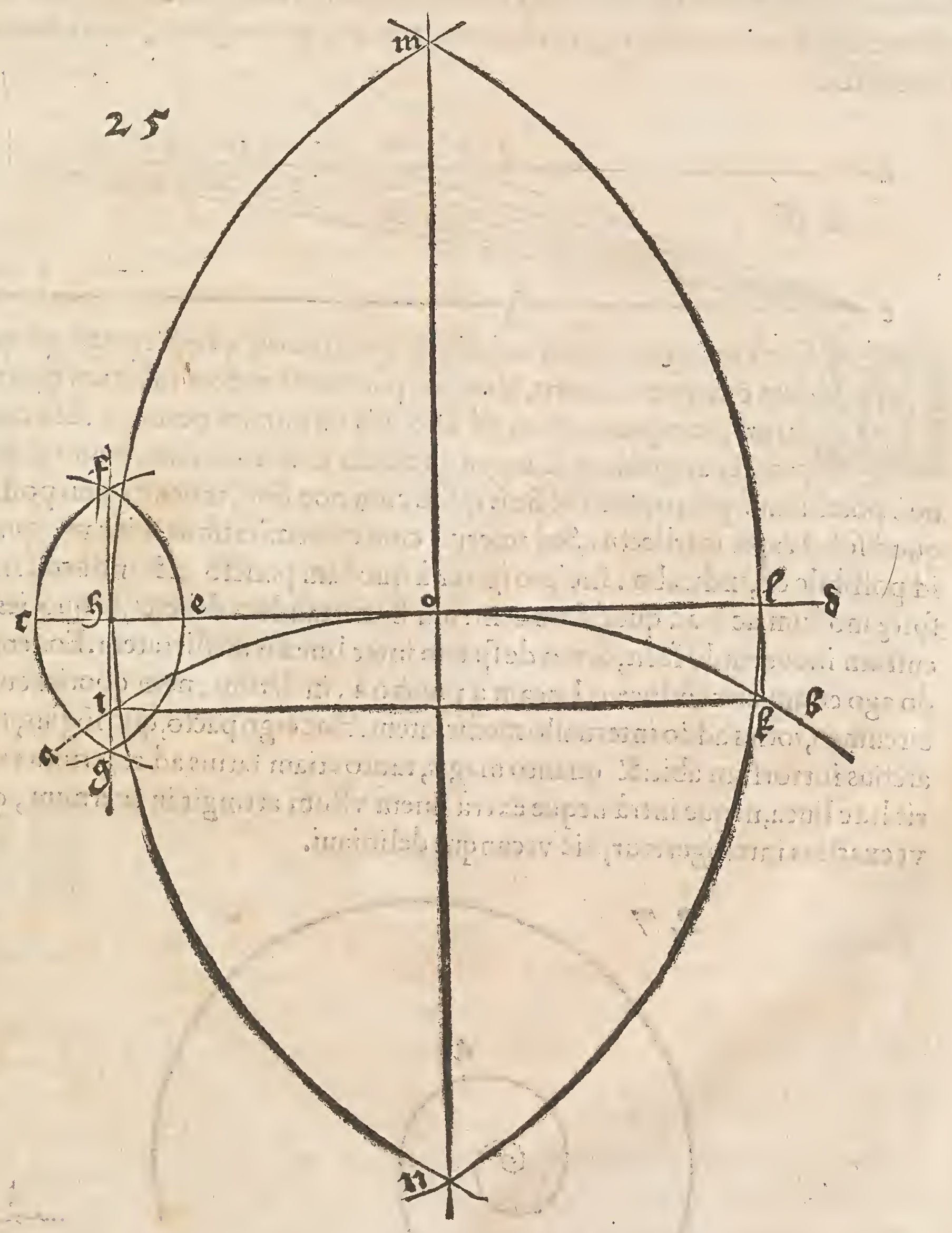




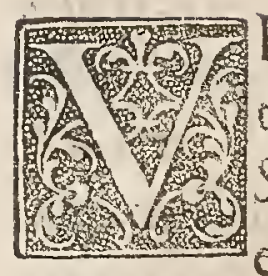

Erum eft, quòd dualinex, qux in aliquo puncto angulú faciunt acucum,perpetiò arctius duci poffunt, nec conuenient tamen vinquam. Sint monftrandi gratia, dux linex a b.\& c d.xquidifantes, quarum extremitates $b \& d$.prolongentur, aut faltem imaginentur prolongari

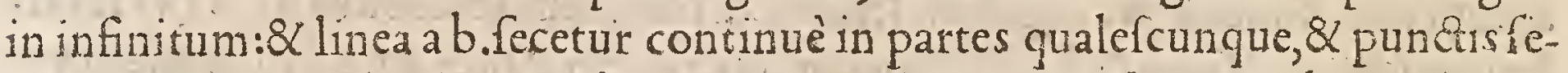
ctionú adiiciantur numeri, ordine fuo naturali, tunc fi pducatur ab extremirate $c$, lineęc d.linea obliqua ad notam .linex a b.facient dux c. \& $\mathrm{c}$ d.angulum acutum. Deinde protrahe femper à figno c.lineas obliquas, ad omnes numeros, linex a $b$. fiét que fubinde poftrema linea obliqua ipfic d.linex propinquior, \& efficiet angulum acutiorem, nec tamen eam continget vnquam, vt hic marifeftè videtur.

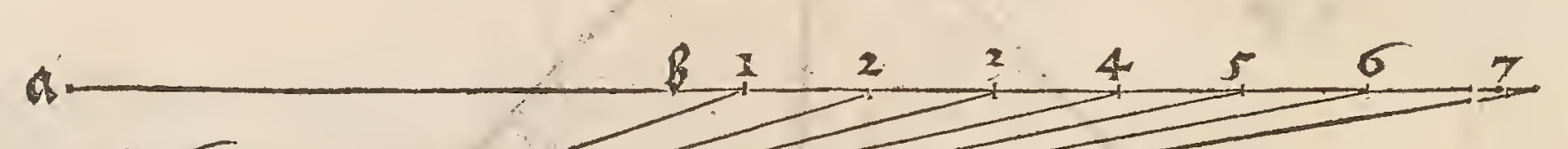

$c$

26

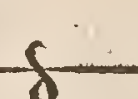

1

Otelt excogitari linea indefinite quantitatis, qux perpetuò ad quo 1. $y$ dam c.ntrum incurrit, \& ex alia parte tantundem in latum extendiC. ditur, nunquam tamen ad aliquem terminum peruenit. Hac linea - 2.0 pter anguftiam \& magnitudinem eius infinitam, manu d'efcribi non poteft:nam principium $\&$ finis ipfius cum non fint, inueniri non poffunt, quod folus capit in tellectus. Sed inferius cum extremitatibus fuis eam, quantú id poffibile eft, indicabo. Incipio igitur à quodam puncto a. $\&$ induco eam ad fpire modum ac fi ad quodd am centrum fluere tandem déberet, $\&$ quoties cir. cuitum incurrendo facit, demo de fpacio inter lineam medietatem. Eodem mo do ago etiam circunducens lineam à puncto $a$. in latum, nam quoties cum ea circumeo, toties addo interuallo medietatem. Hoc ergo pacto, quo lögius, tanto arctius introrfum abit:\& quanto magıs, tanto etiam latius ad exteriora excurrit hæc linea, neque intrà neque extrà finem vllum attingit in aternum, quod vt exactius intelligeretur, hic vtcunque deliniaui.

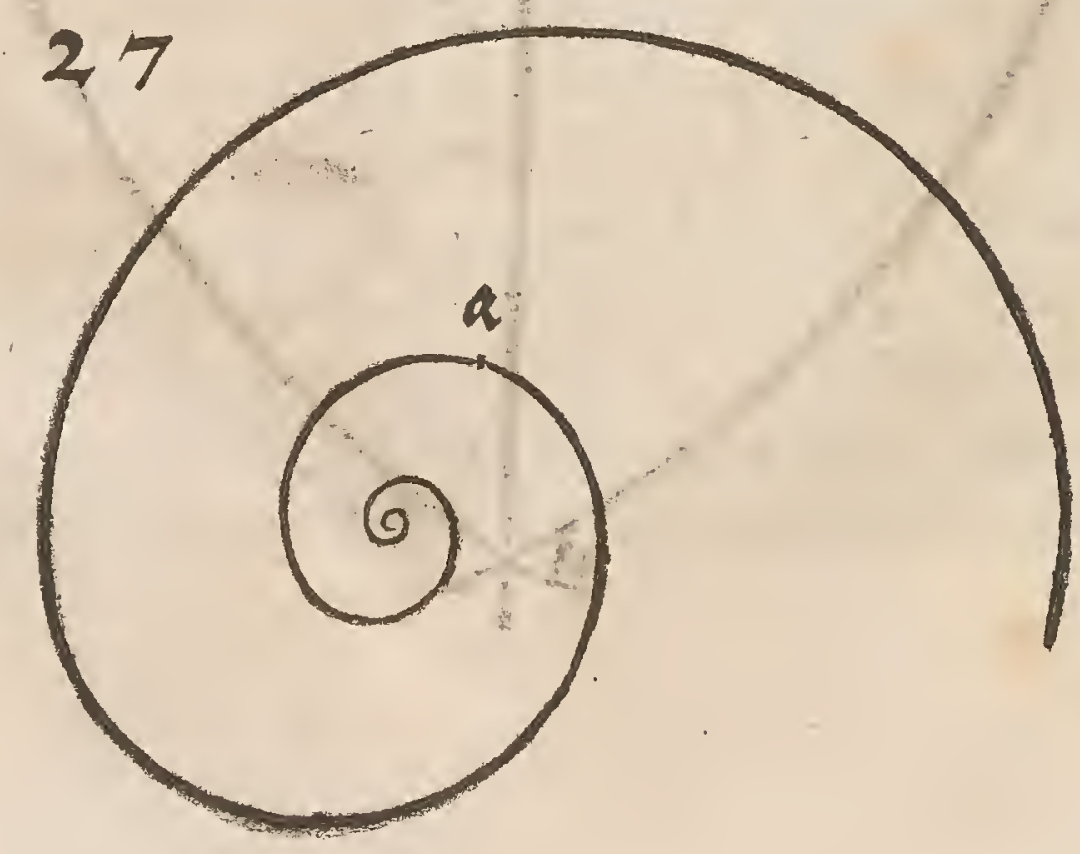


$\mathrm{N}$ fequentibus docebo lineam quandam vilem defignare qua fingulari quodá modo fe flectit.Primò igitur defcribe lineã tráfuerfam $\mathrm{c} d$. eá diuide, púctis in 10 . fpacia æqualia, \& ad púctú intermedium s.erige lineá ad angulos rectos, qux fit fupernèa .infernè autem $b$. hanclineam a b.partire nouendecim punctis in 20 . interualla æqua, $\&$ incipe numerare infernê afcendendo $1_{0}{ }^{2} .3 . \&$ c.deinde accipe regulam $\&$ transfer in eam longitudinem $b$ d.quam etiă figna literis e f. Hac longitudine notátur omnia huius cur uæ linex puncta, per qua ducenda erit. Poftea fume quintă parté ex $\mathrm{b} d . \&$ diui de eam in tres partes equales, \& vna ex illis tertiis prolonga acceptắquintam, \& aperi circinum ad quantitaté prolongatæ quinta, feruatáq̧ ea apertura pone vnum pedem in $\mathrm{d}$.punctum, \& alio duc arcum in parte fuperiore.quo facto applica regu lix terminume, puncto . linę a b.\& terminum $f$ applica arcui, quem nuper pro traxift1, ac figno cótactus adfcribe quoģ notá i. In qua iterum polito vno pede circini, rel iquo arcum producito verfus par tem fuperiorem, $\&$ iunge regulx extremi tatem ead 2 . in linea $a b$. \& punctum $f$. ad iam protractum arcú, \& vbi eum contingit, illic adiice notam 2 . Sic facies per omnes numeros afcen dendo quátum potes. Poftremò protrahe lineam tuam curuè de púcto ad punctú, quemadmodú bifariă nie feciffe hic vides:Primò per lineam $\mathrm{e}$.deinde per e $\mathrm{g}$. breuioré, ex tamen fupernè propius currunt quàm infernè.

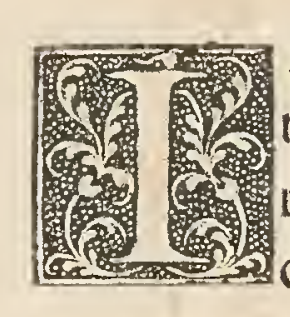

Am protracta linea etiá in húc modú abfoluitur . Primù cốue. nit lineă e $f$. per $\tilde{q}$ futura linea cim cim partes xquales diuidere $\&$ fingulis motionibus ipfi vnam de illis decem $\&$ feptem partibus auferre. Sed hx parteslinex ef.non debent effe xquales:nam qux funt verfus e . fiết maiores, qua verò verfus f.minores, Ad hanc igitur graduú in-

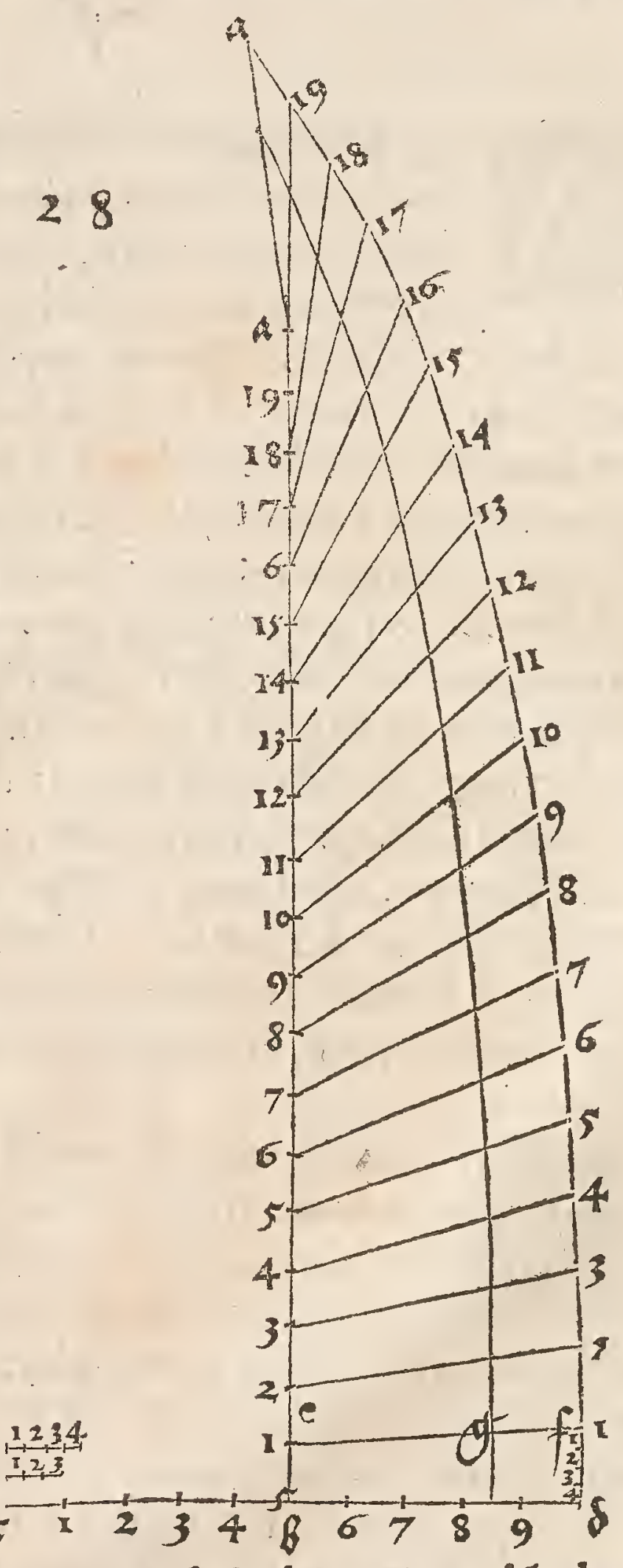
xqualitatem, in regulă tuam transferendam, oportet defcribere triangulú a $b c_{\text {\% }}^{\text {7 }}$ cû arcu b d (vt in octaua figura dictú eft)terminú tamen a.linex a b.declinato maximè $a b$ arcu $b d$.in finiltră. Quúqg arcus $b d$.in feptédecim partes equales diuifus, \& ex puncto c.linex rectę per divifiones arcus $b \mathrm{~d}$. pduct fuerint vfg 
ad lineam $a b$. erunt fpatia in linea $a b$. verfus a.magna. $\&$ verfus $b$.parua, vbi etiam numeri initium fument, quo factotran sfer lineam a $b$.fic diuifam, in regu lam ef.applicando a.ipfi e.\& cadet b fuper f.quemadmodú prafens figura an: te oculos ponit.
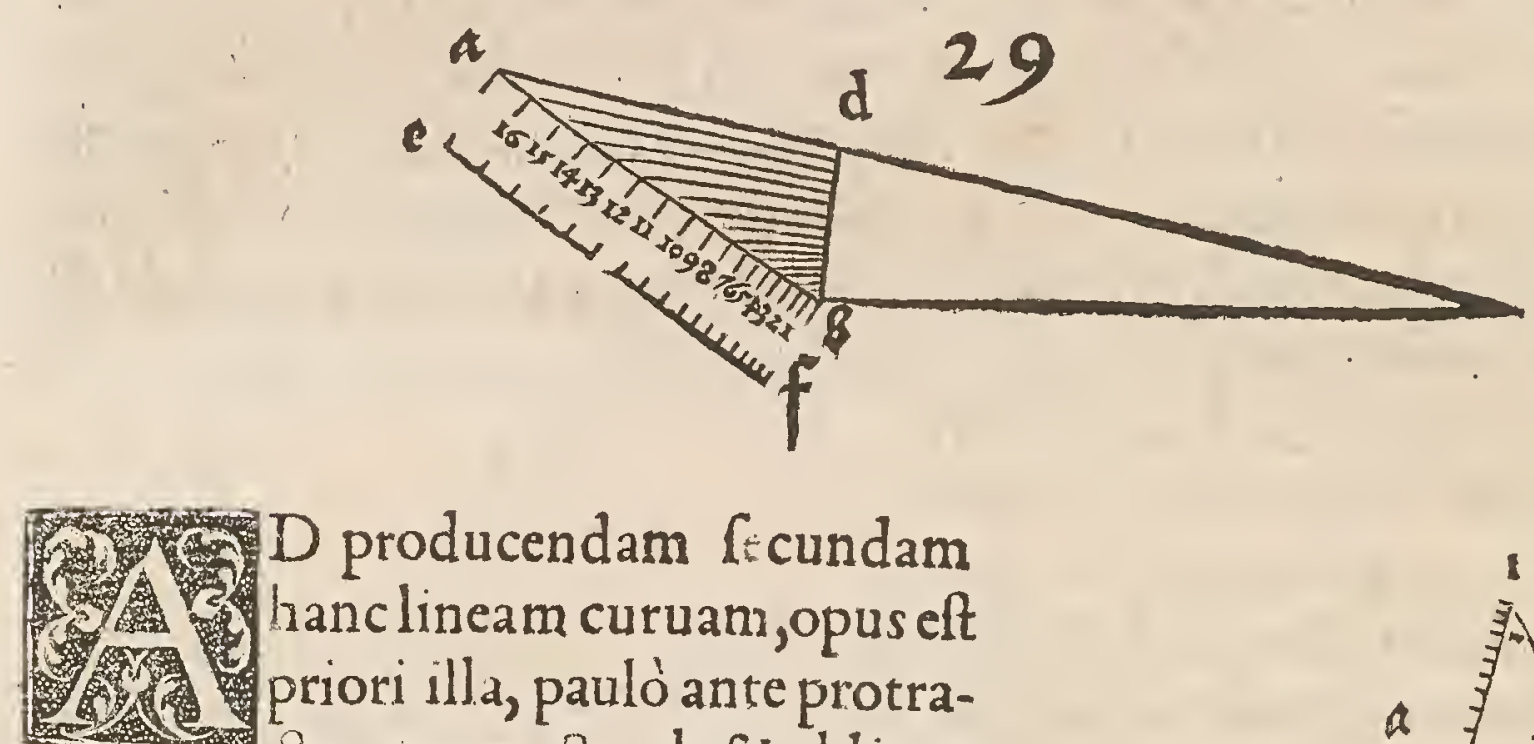

D producendam ficundam hanclineam curuam, opus eft priori illa, pauló ante protracta, cum erecta a b.\& obliqua ef.in hunc modú. Primò vtere perpendiculari a b.vfque ad 17 . punctum, fimiliter quoque curua, \& vt obliqua ef.per numeros prius afcédit fic eam relinquito. Quum verò nuper diuifam lineam e f.ab imo verfus fuperiora, fup prius dictas obliquas afcendere facis, auferipfi femper quoties de gradu in gradú mouetur, vinam parté de extremitate f.donec linę̧e f.ad fupremú numerum recte a b. $\&$ curux 17 . nihil amplius fuperfit. Deinde duc nouá hanc lineá de púcto ad punctũ $\&$ videbis quam habeat diffe rentiam cum priore. Harum rerum hac elt figura.

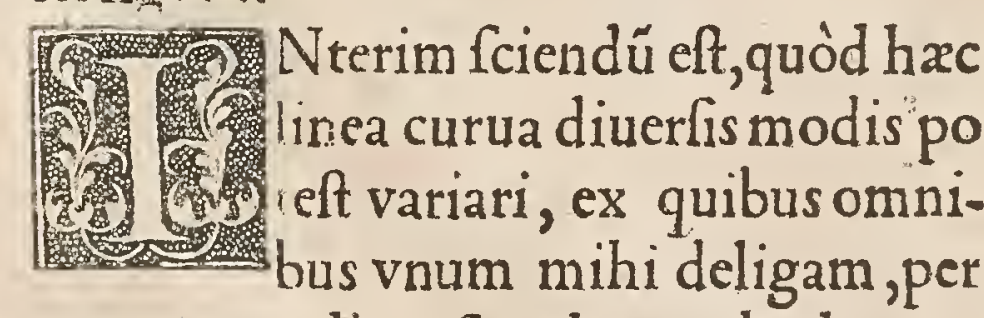
guem eam adhuc femel mutabo hoc pa cto. Lineam perpendicularem $a b$. fac quarta parte breuioré, quàm prius fuit 8 diuide eam rurfus in viginti partes, fed partes ille inter diuifiones debent in

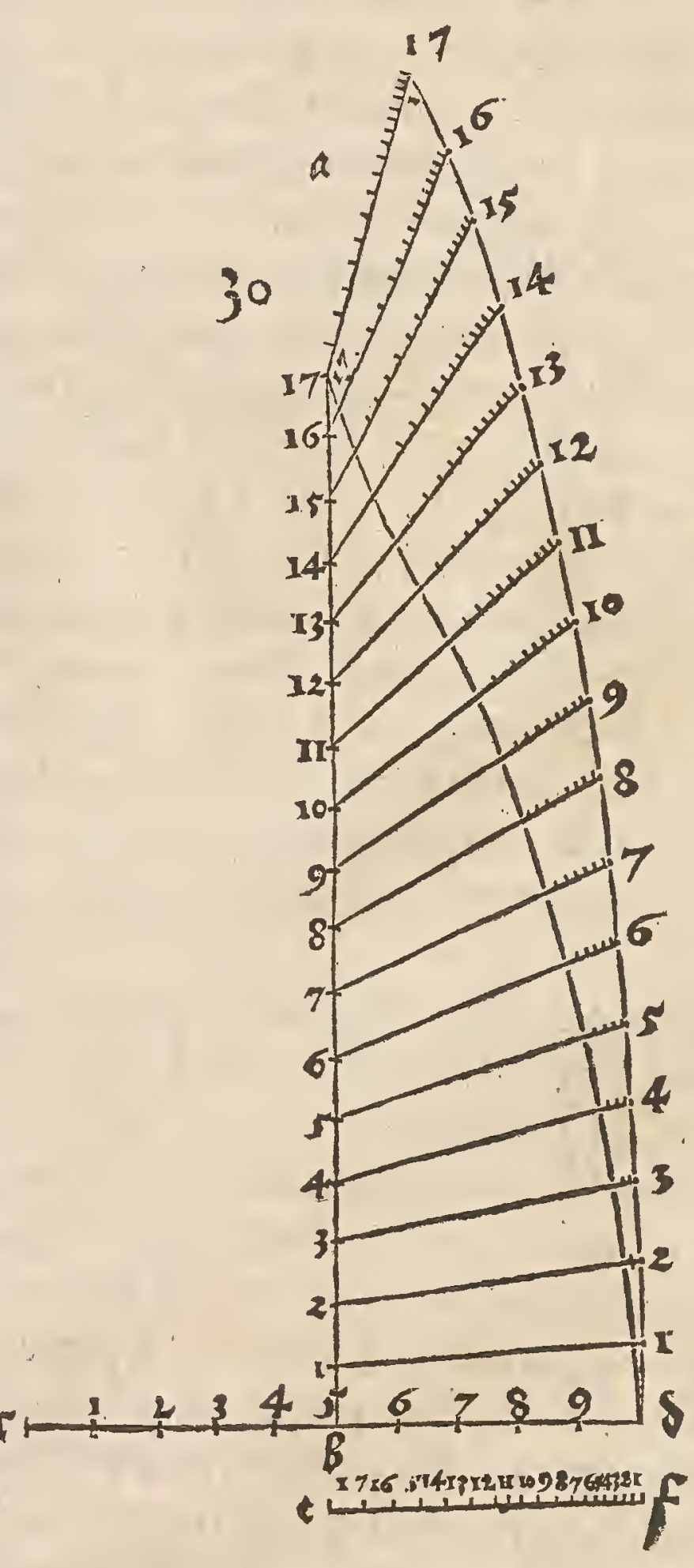
fernè apud minores numeros ampliores effe, \& fupernèapud maiores ftrictiores? certo tamen ordine. Huiufmodi partitionem linex a b.pete ex fupradicto trian gulo a $b$ c.octau $x$ figurę. Pro arcu autem circuli a e. vtemur breui linea recta a b.fic. Accipe iultam longitudiné erectę linę a $b$ diuifxin viginti fpacia æqua- 
lia, ac pone eam ad angulos rectos fuper $b c$. tranfuerfalem, $a c f a c b c$. txanfuerfă vna fexta longiorem quàm erectam $a b$. fic quòd $a b$. quinque fextas habeat ipfius b c.deinde duc obliquam a c. \& applica extremitatem b. tua breusis linex a b.angulob.\& alteram extremitatem a.reclina in lineam a c. Quo facto protra he ex omnibus partitionibus longa linex a b.rectas lineas verfus angulum c. $\&$ vbi obliqux hx linex fecant lineam breuem a b.illic fcribe numeros ipfius lon$\mathrm{ga} \cdot \mathrm{b}$. Sic igitur eft linea a b. breuis diuifa in partes in quales per aquales ipf 1 us a b.longx. Notandum etiam eft, quo quis gradus ipfius a b. infernè vult face re maiores $\&$ fupernê minores, tăto longiores debent effe linex a c.\& b c.\& poterit linea a b.termino a.magis declinare verfus c.angulum. Si tamen opus poItulat, conuenit lineam a b.qux in divifione varianda eft, longiorem aut breui orem facere, vt poffit obliquam a c.contingere. Hunc pradictum triangulum cum variata linea a b. hic defcripfi.

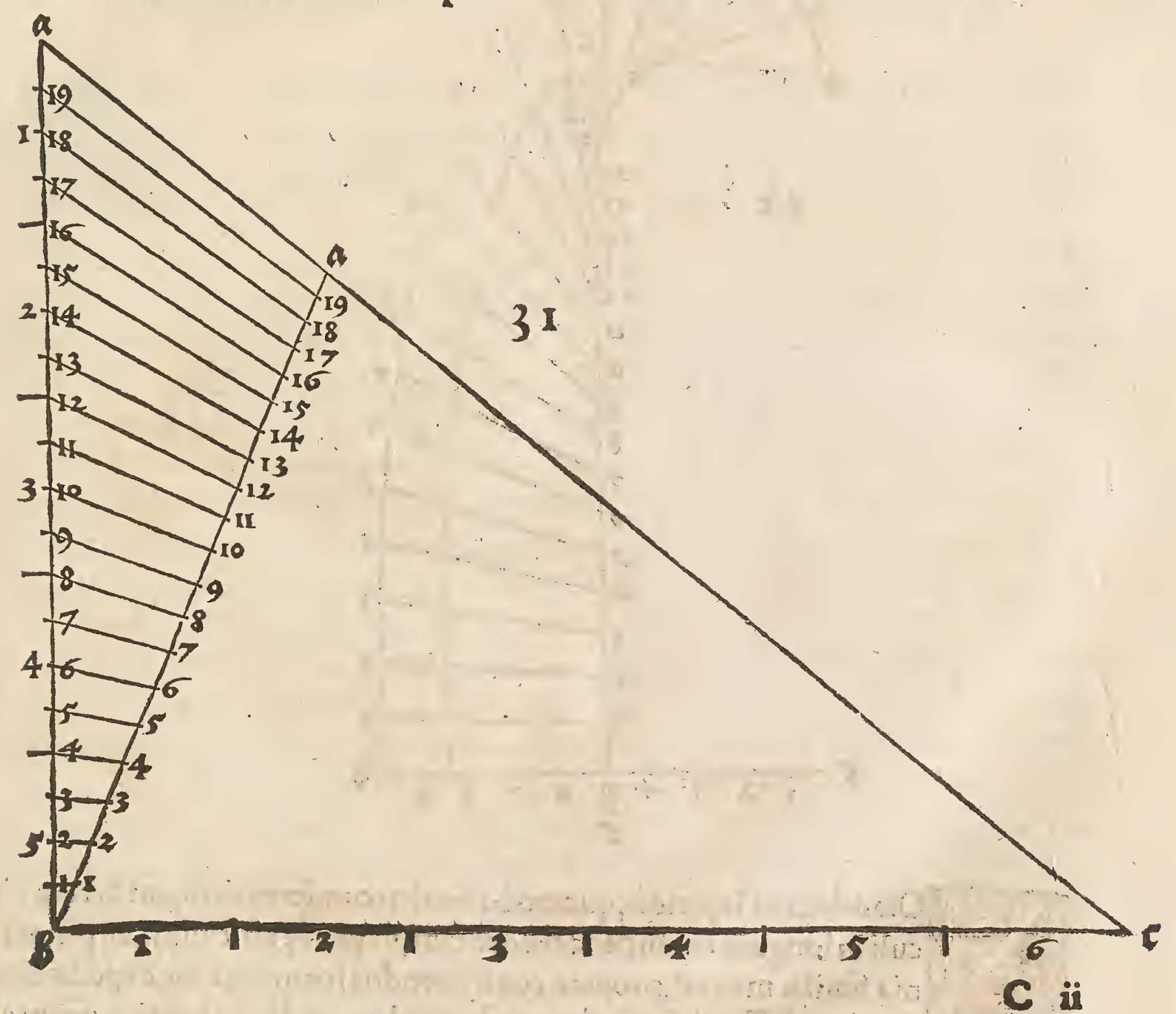




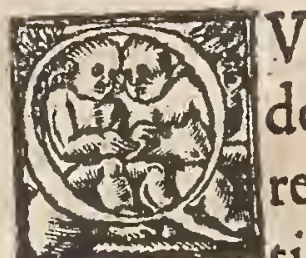

ando nunc breuis hxc linea a b. abfoluta eft, erige eam loco priùs defcripta linex a b.ex qua primam lineam curuam produxifti, $\alpha$ vte reomnibus pręfcriptis menfurationibus in numeris, longitudine, al. citudine, \& latitudine, vt in fequenti figura apparet. Hac linea flut arcuatim longè fupra perpendicularem a b. Vtilis autem eft, tum ad alia opera multa perficienda, tum etiam ad pampinos deliniandos, \& tectum turris, fupernè $2 \mathrm{~d}$ 14.gradum, ad quod hic quoque oftendi craflitudinem muri per interiorem lineam curuam:vti manifeftum fiat quanto murus fupernè debeat effe tenuior quàm infernè, quod hac linea de fcripta declarat,

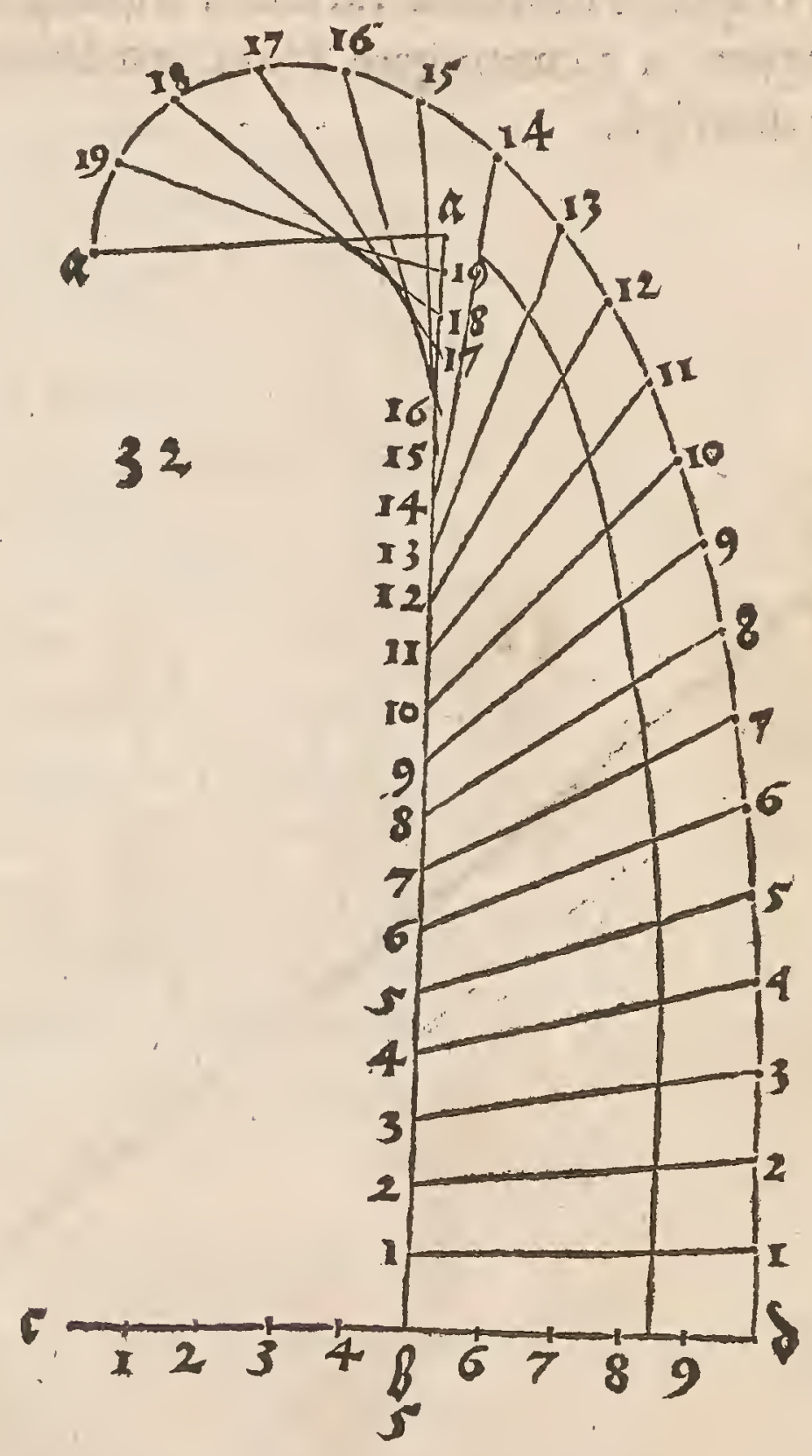

12 (2) Otandum eft lapicidis, quomodo femicircumferentiam, aut arcum cir 1. 1 culi in longum extendendere debeant, vt primęaltitudini,ac per om62. nia fimilis maneat,propter conftituendos fornices. Hoc expediemus fic cómodiffim è. Defrribe quadrangulum in duplolongius quàm al tú, quod fit fupernè a b.Q infernè $\mathrm{c}$ d.cuins latus $\mathrm{c}$ d.diuide in púctoe. per me dium, ac pofito vno pede circini in figno $c$. alio ex c.duc arcum per fuperiorem partem vfq ad d.contingéto hic arcus lineam a b. Deinde partire lineam c d. in i2.partes $x q u a l e s, \&$ ex fingulis diuifionibus ptrahe parallelas furfum, in nuper defcriptum arcum. Iam fac iuxta quadrangulú a $\mathrm{b}$ cd.adhuc aliud quadrä. gulum, xqualis altitudinis omnino, fed longitudinis quantæ volueris, quod fit 
fupernè g.infernè verò $h$ i.\& feca id quoğgn vecim lineis æquidiftantibus, ac erectis in duodecim partes xquales, vt prius:poftea producito ex fingulis interfectionibus prioris arcus, qux per Ir. lineas erectas facta funt, parallelas tranfuerfales per omnes perpendiculares longioris quad ranguli, \& per fectiones illas lógiorem parallelorum arcum, produclineam arcualem de puncto in pun ctum, incipiendo in angulo h.\& finiendo in i:vt hic eft videre.
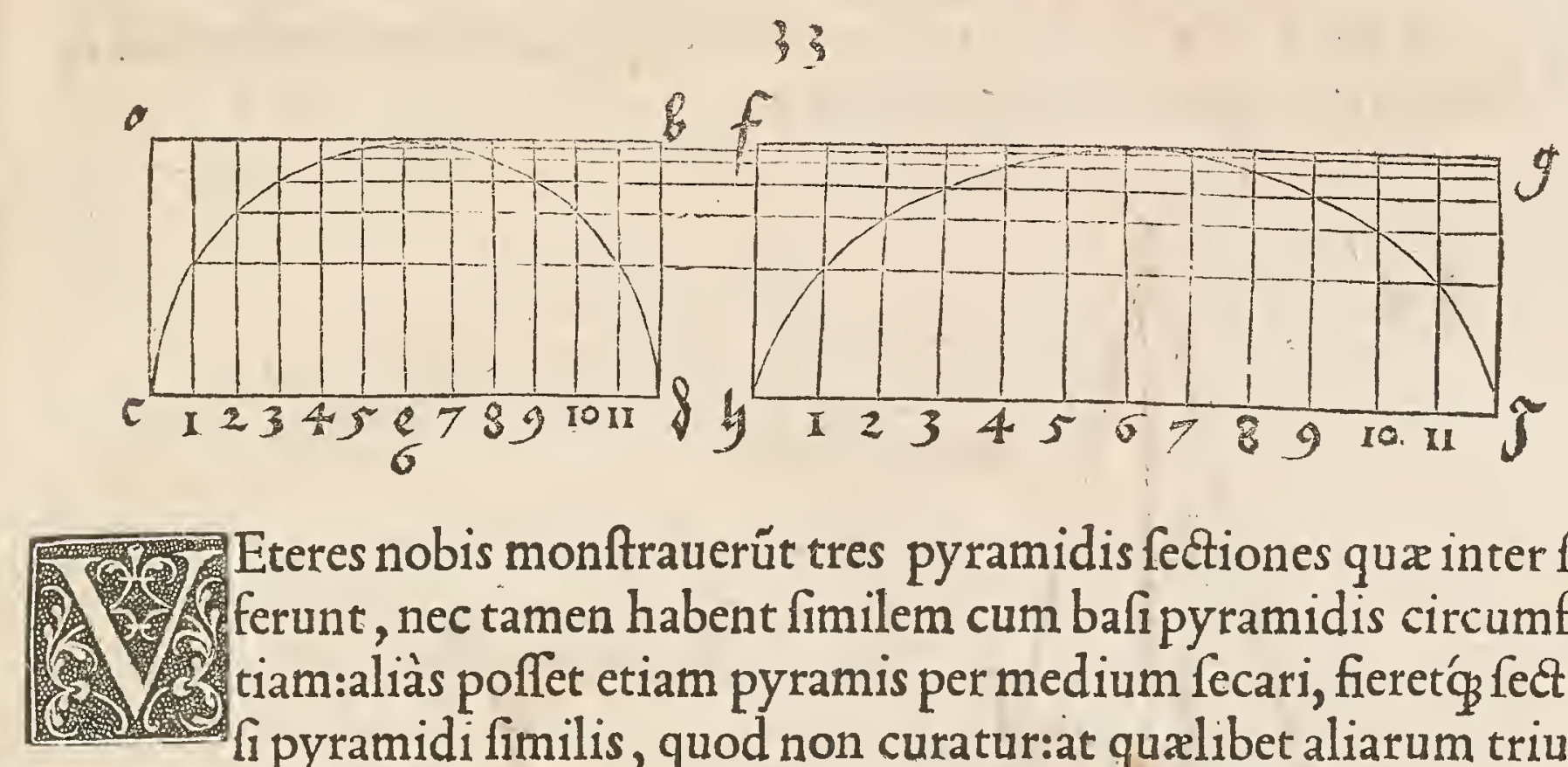

Eteres nobis monftrauerút tres pyramidis fectiones quæ inter fe differunt, nec tamen habent fimilem cum bafi pyramidis circumferentiam:aliàs poffet etiam pyramis per medium fecari, fieretóf fectio ipfi pyramidi fimilis, quod non curatur:at qualibet aliarum trium fectionum, facit fingularem lineam, quas defignare docebo. Prima igitur fectio vocatur Elipfisea fecat pyramidem obliquè, \& bafi nihil aufert, eft tamen ei vna parte propior,altera verò remotior Secúda fectio eft parallela vni lateri pyramidis, \& núcúpatur à doctis Parabola. Tertia eft xquidiftans axi pyramidis, eam appellant Hyperbolen. Iam volens lineă defignare elepfis, primú defcribo pyramidem in qua fectionem oftendo:fimiliter etiam fundamentú fub ipfa pyramide in hunc modum. Pyramidis conus fupernê fit $a . \& b a f i s$ infernè $b c \mathrm{~d}$ e. ex cono autem a. defcendat linea perpendicularis in balim, quax reprafentet axem pyramidis:fectio verò elipfis fit linea obliqua, fupernèf. \& infernèg. hăc fectionem $f$ g.diuido vndecim pundtis in ${ }^{2}$. partes $x$ quales, quas numero $a b f$. verfus $g$. Directè fub hac pyramide delinio fundamentum eius, erítque a.centrum $\&$ b c d e.circumferentia, quod erecta pyramis indicat. Quum iam ex om nibus partitionibus linex $f$ g.cadunt perpendiculares in fundamentum, fiunt fectiones in circulo, eas etiam noto literis \& numeris fuis, quo facto fumo circinum $\&$ pono vnú pedé in axem pyramidis a. in ea altitudine qua eft nota ${ }^{~}$.in linea $\mathrm{fg}$. \& alium peđem pono in latus a d.in xquali altitudine, $\&$ trăsfero eam. diftantiă in fundamentú b $\mathrm{c}$ d e.vbi alterú pedem circini pono in centrum a.\& alterum ad lineam rectam rà qua verfus d.duco arcum vifg ad eandem lineam ex alia parte: deinde figo iterum circini pedem in axem pyramidis a.in altitudine not ${ }^{2}$.in linea $\mathrm{fg} . \&$ alterum in lineam a d.in eadem altitudine, 8 hoc interuallú transfero rurfus in circulú, in quo fito pede vno in centro a.altero à per pédiculari2 . fcribo arcum verfus d.donec iterü venio ad lineá 2 . Sic operari pergovfo ad 4 . Proinde ad altitudinem 5 . verto vnú circini pedem ad latus a b. $\&$ ea dif antia feruata, produco ex centro fundamenti a.arcum à linea 5 ad eandè vlcrad. Ita facio per omnes numeros, träsferendo fpacia ex pyramide in funda- 
mentum eius. Poltea defigno ex hoc fundamento nudam lineam elipfin fie. Ego duco lineam perpendicularem $f \mathrm{~g}$.tanta longitudinis, quanta eft fectio pyramidis $\mathrm{f} g$. hanc lineam $\mathrm{fecO}_{3} \mathrm{vt}$ pritus, in $\mathrm{x}$.partes \&quales, \& per fingulas diuifi ones traho parallelas tranfuerfales in vtranq partem linę erectę $f$. \& latitudi nem accipio ex fundamento, primó quidem in linea r.uám quantú eius excindit intimus arcus circuli, tantum transfero in parallelam r.in vtrangs partem ip hus $f$ g. Non aliter ago in reliquis numeris. Quum autể omnia puncta in equidiftantibus per circuitum funt notata,duco lineam elipfim de púcto in punctú, quemadmodum hęc figura manifeftè declarat.

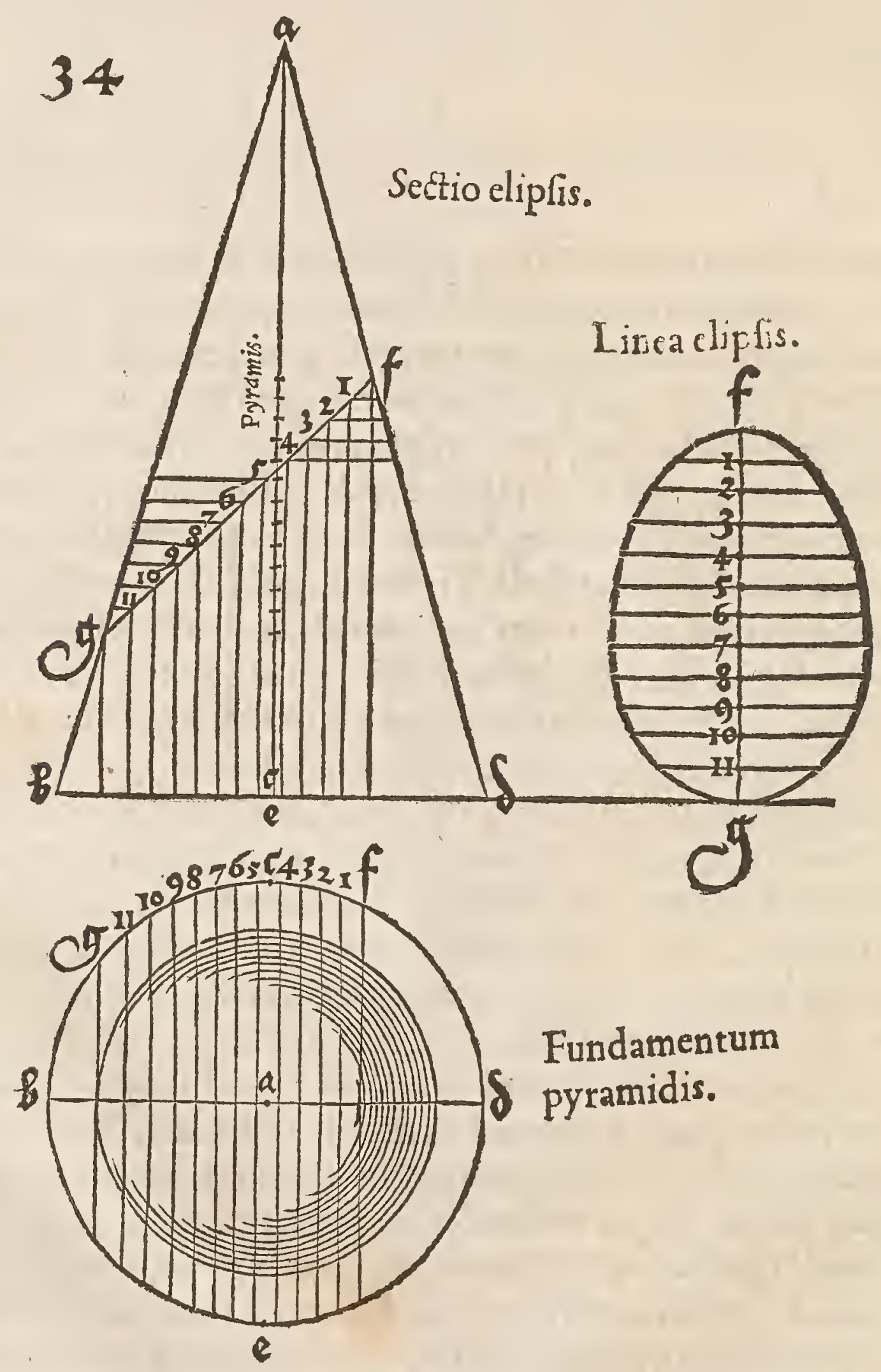


Arabola eo fermè modo quo elipfis fit. Primó protraho pyramidem 1. a a bc de, ato in ea axem a. deinde feco pyramidem à fummo deorfum 19 per batim elus, ita quòd hac fect io fit parallela lateri pyranidis a $b$,

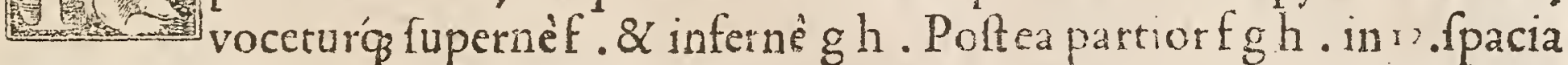
xqualia \& duco lincas tranfuerfas peromnia puncta iplustg h. ac per illa pun ctà qux funt verfis illud latus a d traho tranfuerfas abecedia a vique ad idem latus. Sed perilla qua funt ex altero latere duco trăfuerfas ab axe a.ad latus py ramidis a b:his abcolutis facio fundamentum pyramidis directe fub ea, cuius cé trum fit a.\& circumferentia b c d e.\& fino ex omnibus divifionibus fe etionis $\mathrm{f}$ g h.lineas perpendiculares cadere deorfum per rotundum fundamentú, in quo ipfis etiam adiicio fuos numeros, quemadmodum priùs in fundamento elipfis factum eft. Iam vt etiam in elipfi edocuimus, aperto circino ad quantitatem linex tranfuerfe r.in pyramide, pono pedem in centrum a.fundamenti, $\&$ alium in lineam perpendicularem, \& inde fcribo arcum verfus d. \& vltra ipfum vf'́, ad eandem lineam ${ }^{2}$. Sic procedo per omnes lineas notatas numero donec ad $\mathrm{g}$ h.venio videbitur'́ ilico parabolæ fectio in fundaméto depreffo. Quo facto defignabo parabolam exiam protracto fundamento, hoc pacto.primò duco lineá tranfuerfalé, cui erigo ad angulos rectos lineam quæ fit fupernèf. tante quantitatis quanta eft fectiof $\mathrm{g} h$.in pyramide. Deinceps accipio ex fundamento latitudiné $g$ h.\& traduco eá in lineá tranfuerfam, fic quòd crecta f. ftet in medio, \&

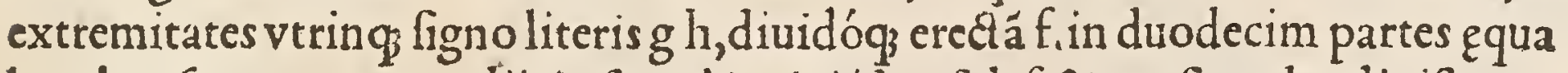
les, qbus fuos numeros adiicio fu pnè incipiédo, fub f. $\&$ per fingulas diuifiones pduco lineas tranfuerfas, tantę longitudinis, quáta opus eft, $\&$ transfero ex fun damento omnia interualla inter fectiones arcuum \& rectam lineam b d.contëta, ad perpendicularem $\mathrm{f}$. ex cuius vtroque latere figno tranfuerfas:Deinde traho lineam parabolx de puneto ad punctum quemadmodú hic etiam deliniaui. 


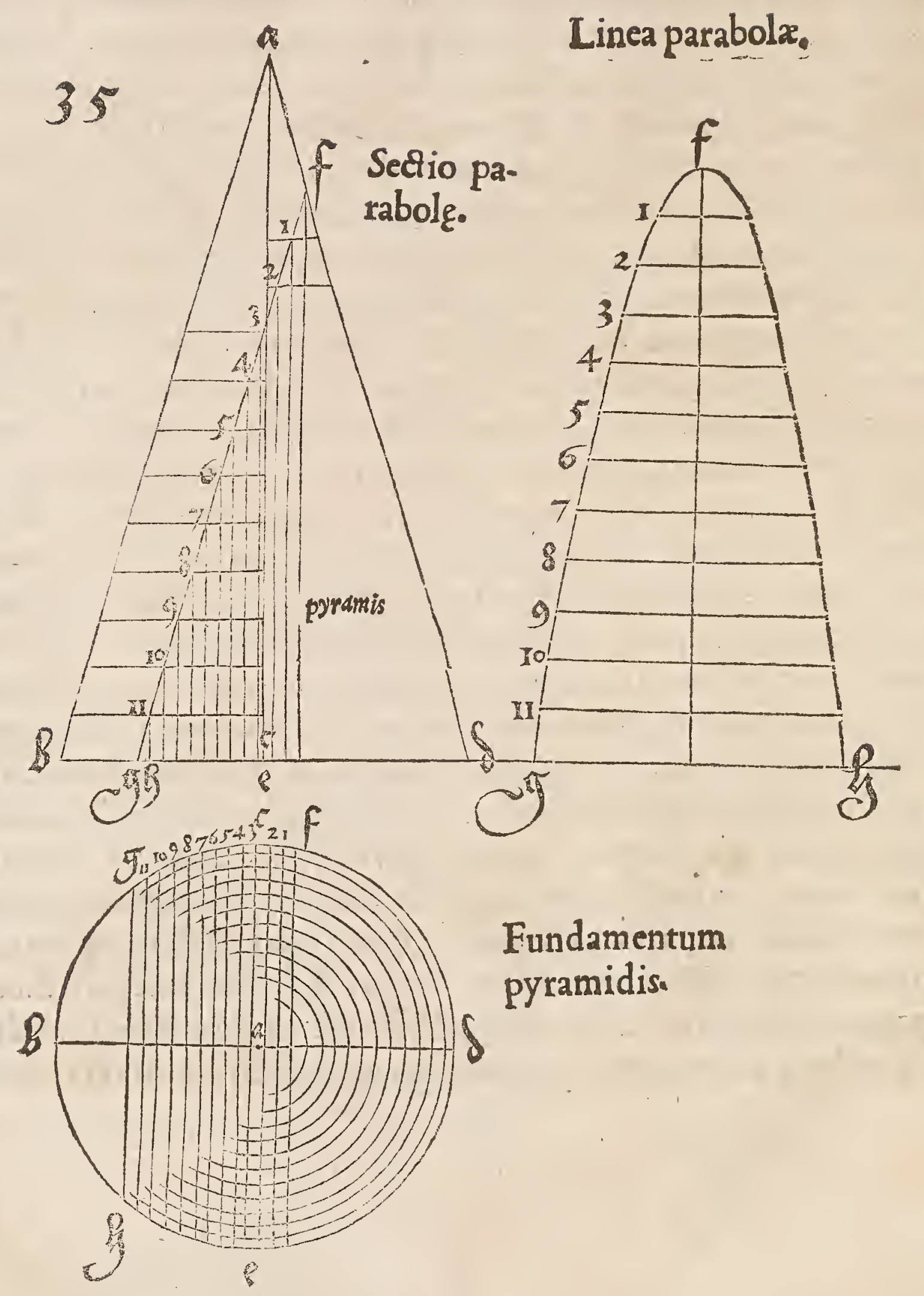

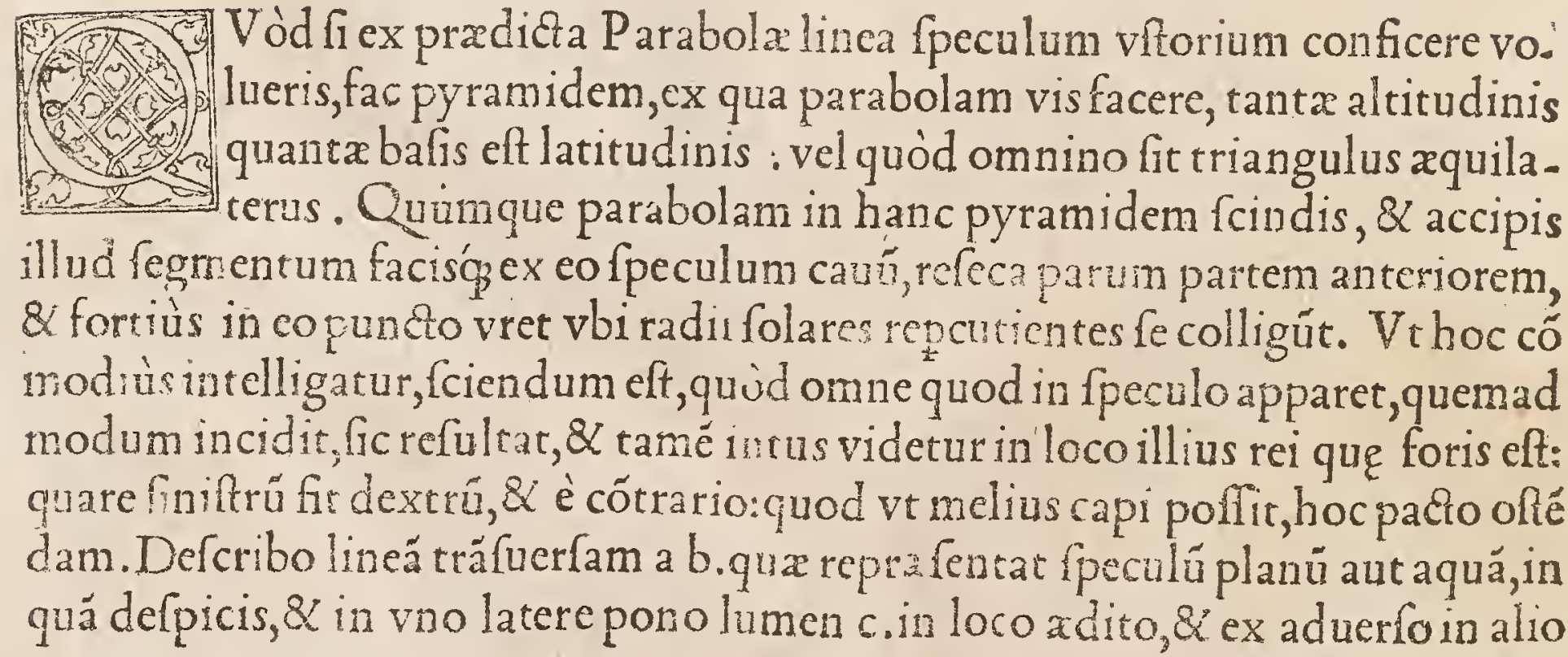
latere 
latere pono homúcionế in fpeculú aut aquam refpicienté, hiuius oculus fit d.cui luméc.non apparebit, donec angulus radii ce. $\&$ aliter linee vifualis de fuerint xquales, $q q^{d}$ fic pcipitur. Quú ex púcto e, in quoreflexio fit'lineă ppédicularé fur fü ducis, \& polito circini pede in figno e. \& altero femicirculü ptrahis à linea a b.furfü, donec iterú eá attingis, ac metiêddo inuenis, quòd radius luminis c e.\&li nea, vifualis d e..qqualiter diftát à linea ppédiculari, túc linea d e.ptracta often. det tibilocũ in quolumé ab oculo rectè videri poteft. Quare cú linea tua vifualis per ́peculú fiue aquá penetrat $\&$ alia à lumine c cadit perpédiculariter, interlecabút fe duę illę linę̧ in loco vbi lumé apparet fitóg illæ f.Cófimiliter reper cutiútur radii folares in fpeculo, quod ex parabolæ linea factú eftexcidút enim omnes, ad vnúq̧ punctú conueniüt, vbi fortiter vrüt. Huius rei rationé monftra. rüt mathematici, $q$ volet, apud eos legat. Quę fuprà dixi prę̧ens figura oftédit:

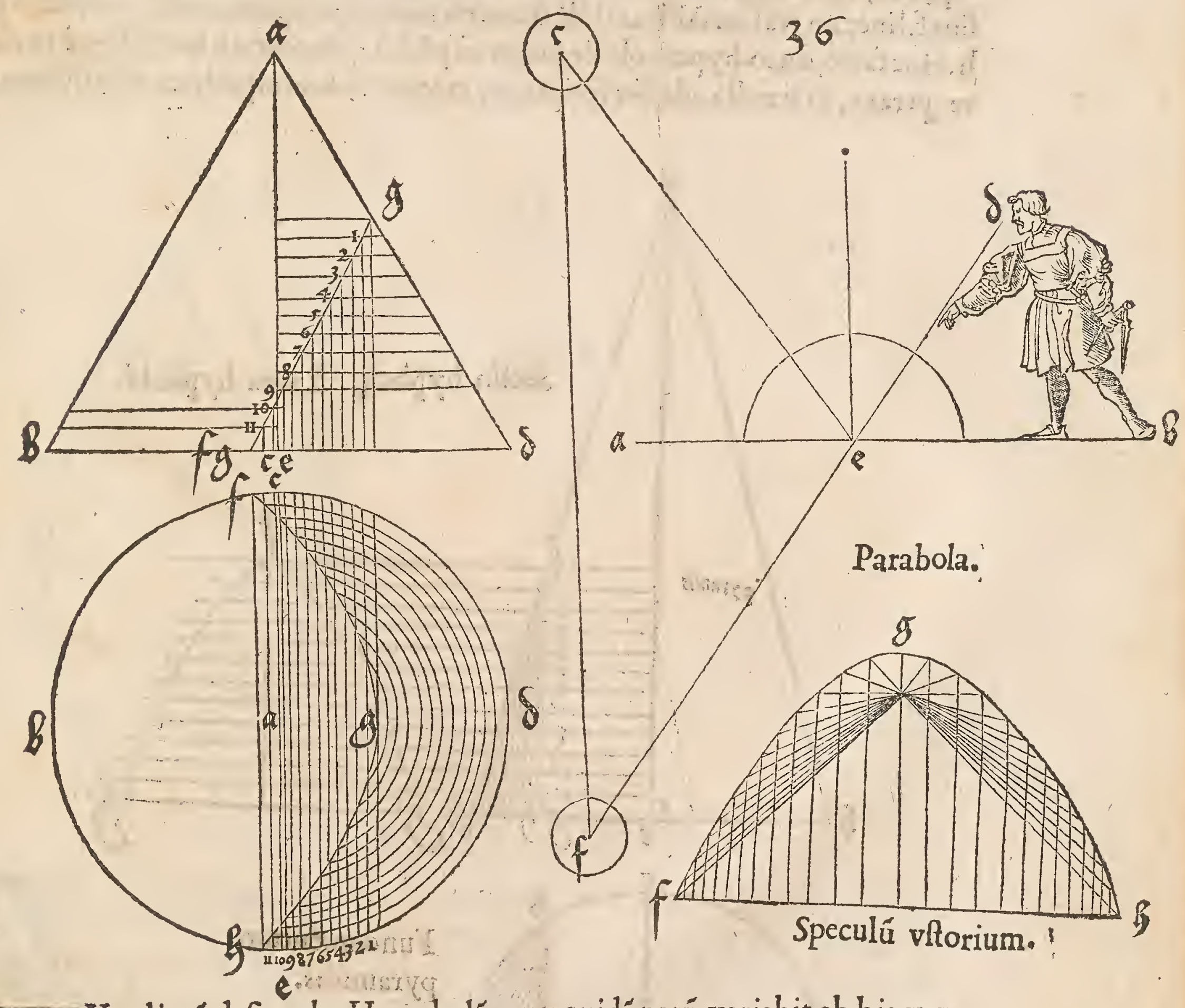

Vnclineá defignabo Hyperbolé, qux quidé parú variabit ab his qua deliniauimus priùs. Defcribá igitur rurfus pyramidē a $\mathrm{b} c \mathrm{~d}$ e.in qua ducá lineájperpendicularem, atq̣ qquidiftar té erectę a.quę frr fupernè f.infernè verò $g h$.per quam abfinditur latus d.eam fectionem $f \mathrm{~g} h$. diuido vndecim punctis in duodecim partes aquales quibus adiicio fuos nume 
ros, \& protraho ex omnibus diuilionibus erectę $f g$ h.lineas tráfuerfas \& equidi ftantes tant $x$ longitudinis, quanta opus fuerit, $\&$ fcribo etıá in latere lineâ perpendicularem, defcendenté per omnes tranfuerfales, fitç ea fupernè f.Poftea facio fundamentum rotundum directè fub pyramide, cuius centrum fit à.\& circircumferentia $b c$ de. $\&$ fino fectionëf $g$ h.etiam hocfundamentum fecare, cui fectioni adfcriboliteras $\mathrm{g} f \mathrm{~h}$.quemadmodum fe ex pyramide in fundamentú tranfponunt :accipióg circinú, cum quo fumo latitudıné femipyramidis in fin gulis lineis tranfuerfis, \& transfero cam in fundamentum rotundum, in quo po fito vno pede circini in centrum a.altero produco verfus d.arcus qui abf́cindú tur per lineá perpendicularé $\mathrm{g} f \mathrm{~h} . \&$ adferibo ipfis fuos numeros:deinde accipi to latitudines ex linea $\mathrm{g} f \mathrm{~h}$. fúdaméti, quę vtrinq $\$$ per arcus circuli diuiditur in x2.partes, trăsferoọ́ eas ad lineă f.erectă \& figno latitudines cx vtrą̧ parte ipfius f.linę.in trăluerfalibus iifdê numeris notatis, à fuprema r.vfọ ad infimá $g$ h. Hoc facto, duco hyperbolế de púcto ad púctú, quéadmodú hoc infernè tá cla re ptraxi, vt fi nulla adeffet fcriptura, crederé ex folo afpectu manifeftü fieri.

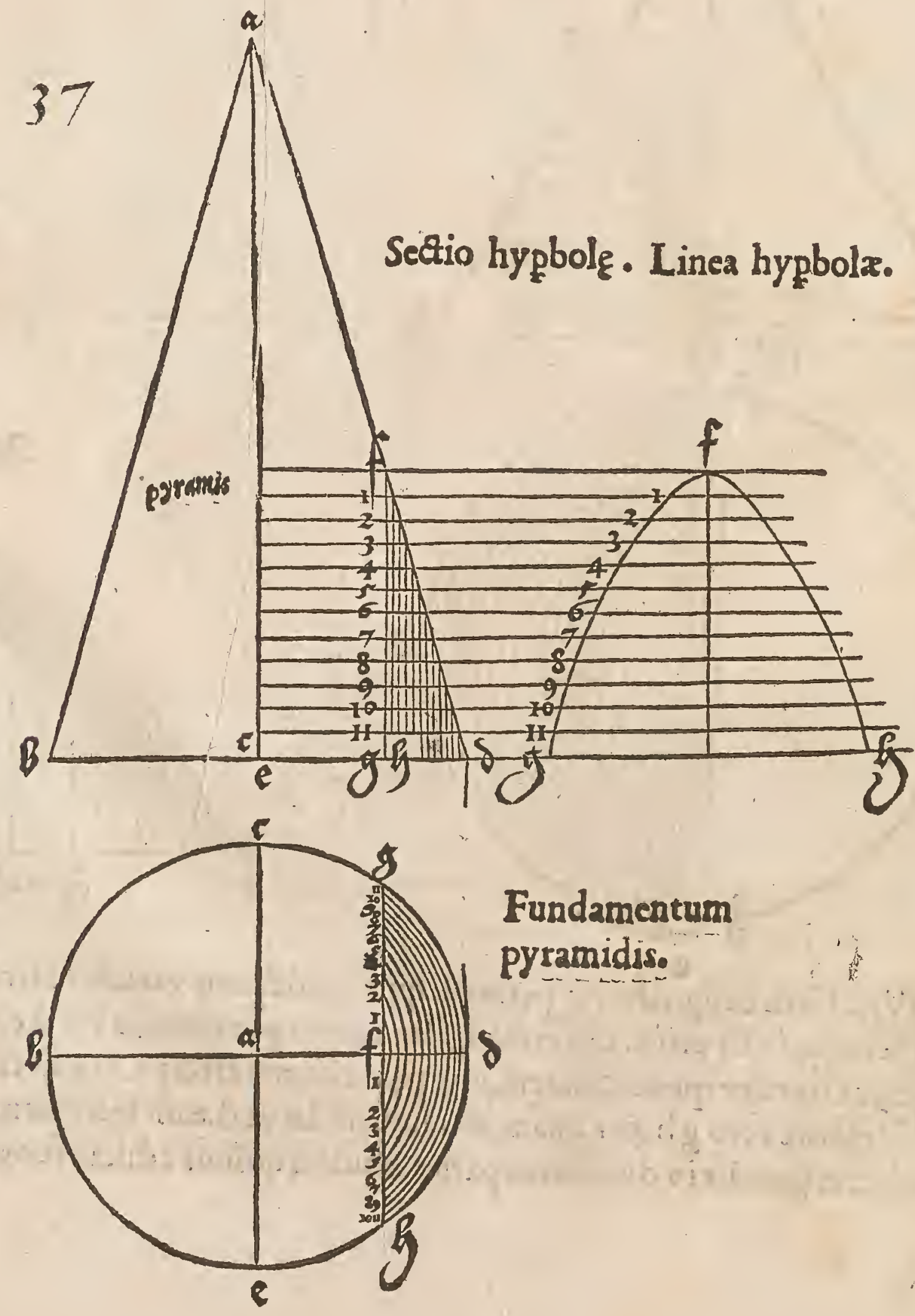


rfus aliam produco lineam, qua in multis rebus vtendú erit,eam fa * ciofic. Ego defcribo lineam tranfuerfam a $b$. in qua pono fedecim puncta, xqualiter diftantia, quibus adiicio fuos numeros, incipiendo ab extremitate a. \& relinquo portionem quandam pro arbitrio inter rerminum $b . \&$ vltimum punctum 6 . quam neque punctis neque numeris noto. Deinde erigo ex puncio iz. lineam perpendicularem, tanta longitudinis, quantá elt ipfa a to . quam etiam diftinguo iifdem fedecim punctis, numeri initium fumendo in parte inferiore. Et accipio regulam in quam transfero longitudinem a b.eam applico vno termino puncto 1 . in linea tranfuerfa, \& alterum cleuo. donec regula contingit punctum tinee perpendicularis, \& vbi alter ille termirus cadit, illic figno etiam notam I. Poftca pono vnam regulæ extremitaten in fignum 2.ipfius a b.tranfuerfalis, \& lubleuo regulam ad punkeú cáfú alterius regula extremitatis noto numero 2 . Sic operor per fingulos numeros trăfuerfęa b. \& ipfius ppendicularis vfọ ad vltimú ro. quo facio duco lineá curuá de pücto ad punctú, quéadmodú infrà vides. Hæclineavarıè duci poteft.

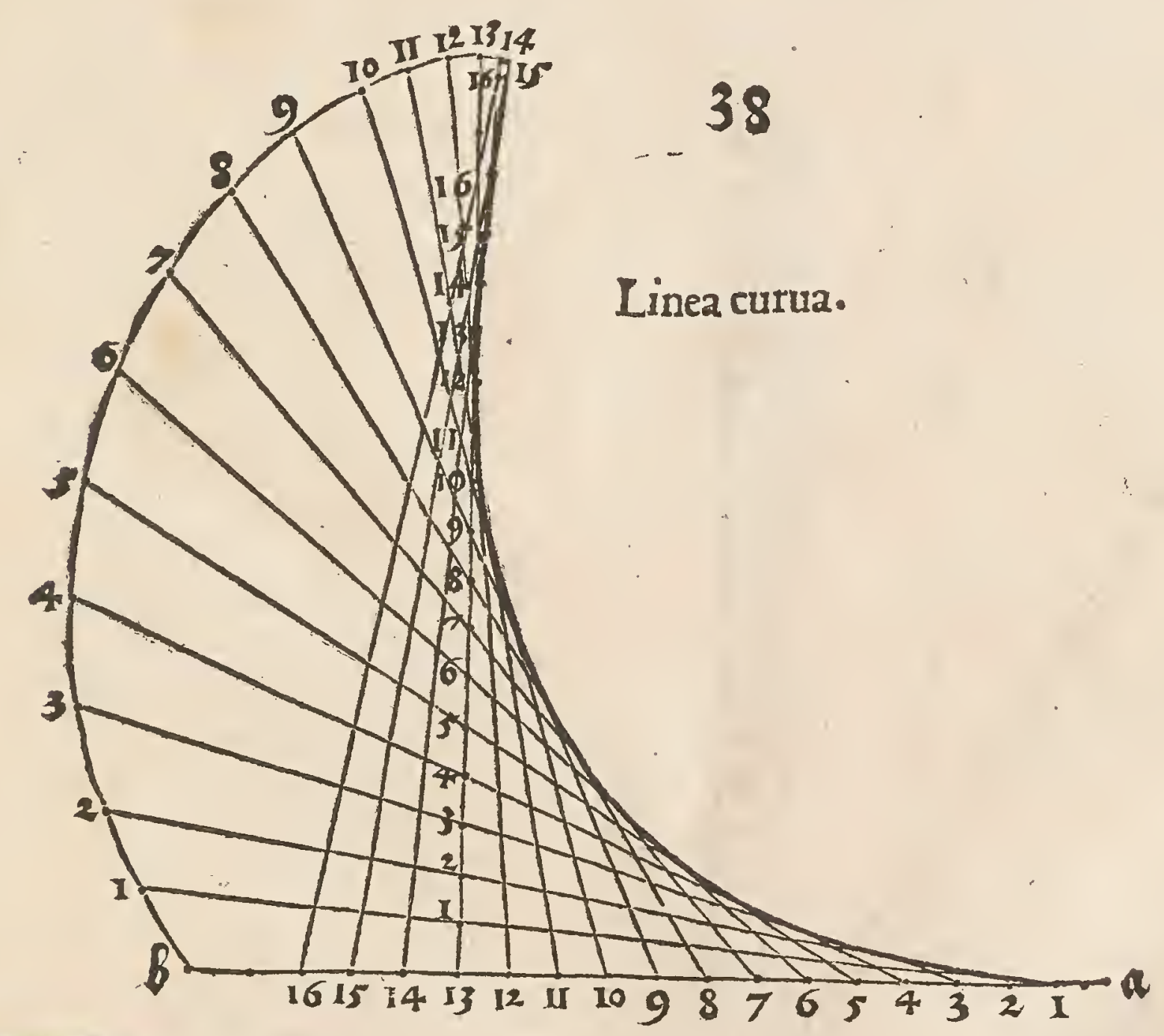

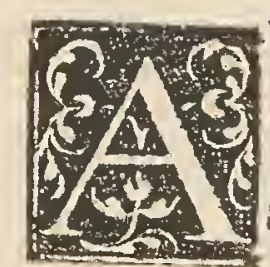

$\mathrm{D}$ nuper defignat am lineam poteft inftrumếtum fieri per quod ea fa. cilè defcribitur, in hunc nempe modum. Praparetur lignú quadran gulum tanta longitudinis, quanta opus erit, per tranfuerfum, cuius principium fit a.\& finis b. In eius fuperficie fuprema fac canalë, tan!profudam vt per eam rotula quædam vltro citróque agi commodè queat, \& diuide lignum illud in tot partes quot volueris, inchoando numerum in extremitate a.Porrò in medioligni huius oblongi erige in vtraque canalis parte regulam modicę fpiffitudinis, longitudinis verò tantx quantx eft lignum vel ca. nalis tranfuerfa, diftent'́g inter fe illæ regulæ, vt lanceola quædam liberè inter
} 
eas eleuari atque deprimi poffit, \& alteram earum nota tot punctis acnumeris quot canalem trafuerfum ab interiore parte verfus fuperiorem afcendendo. Deinde fac haltulam quandam, pro arbitrio lógam, cui in parte polteriore annecte rotulam volubilem, tam craffam, vt facilè per prius factam fcotiam frue canalé volui poffit. Quo facto trăfmitte liberam haftilis extremitatem inter regulas, $8 \times$ age eam verfus $b$.donec rotula centrum applicetur note r.qux eft prope a.Haftulam quoo inter regulas pone ad punctum $1 . \&$ quantum centro rotule appro pinquas extremitati b.tantú eleua lanceolam inter regulas donec tua rotula infernè regulas trắfiueris, atæ̧ ad b.vfọ pueneris:túc enim lăcex cufpis, defcribet tibi lineã hác, quemadmodum ducenda eft. Hanc meá opinioné infrà defcripfi.

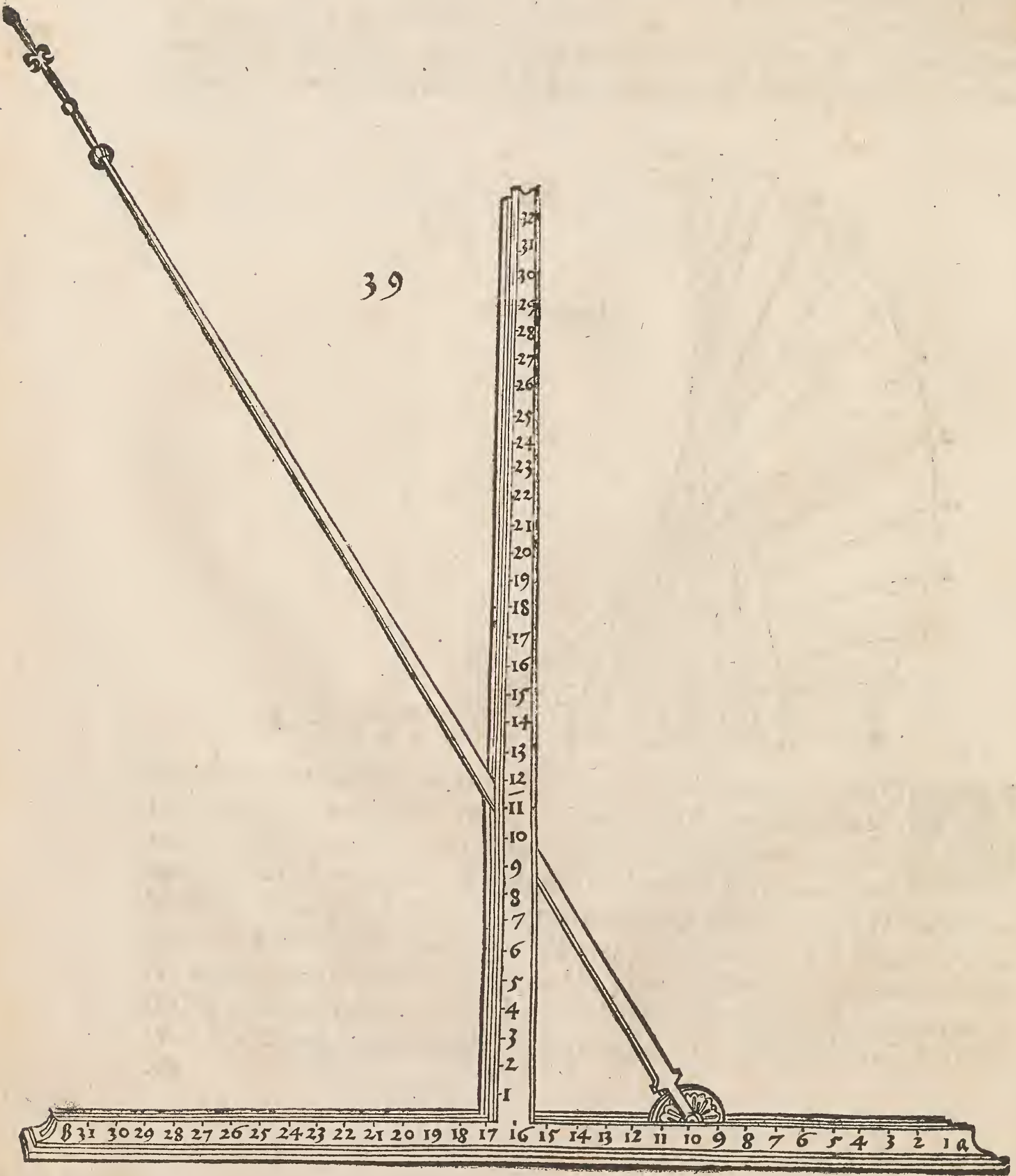


Vnc per lineamenta quxdam, pedibus aranei fimilia, lineam defcribe (1) re volo, quam propterea aranei vocabo : eam per duplicé modum hoc 6. . pacto protraham. Duco lineam perpendicularem, qua fit infernè a, \& lupernèb. huic annecto in extremitate b.aliam quandam b c. Iam extremitatem a.linex $a b$.relinquo inmobilem, extremitatem verò $b$ circumago circulariter, cuietiam per circuitum in omnibus ftationibus literam $b$ adieci. Item linea b.c.debet etiam immobilis manere b. fuo termino, fed terminus c.cir cumducendus eft. Quum igitur linea a b.\& ei adiecta b e.quælibet proprium faciat circuitum, def́cribet terminus c. lineam quandam fingularem. Quò auté hæc linea certè duci poffit, pono vnum pedem circini in punctum a . \& alium extendo aliquantum verfus $b$. ac delinio circulum, quem diuido in aliquot partes, \& punctis diuifionú addo numeros vt linea a b.rectê progrediatur.Con fimiliter facio in puncto b. \& quoties lineam a b.moueo per vnam partem, toties etiam moueo lineam $b c$.per vnam partê in circulob. $Z$ oftendit exeremitas c.púctá per quę linea continuari debet:ea vbiqg notauilitera $c$, vt hic eft videre.

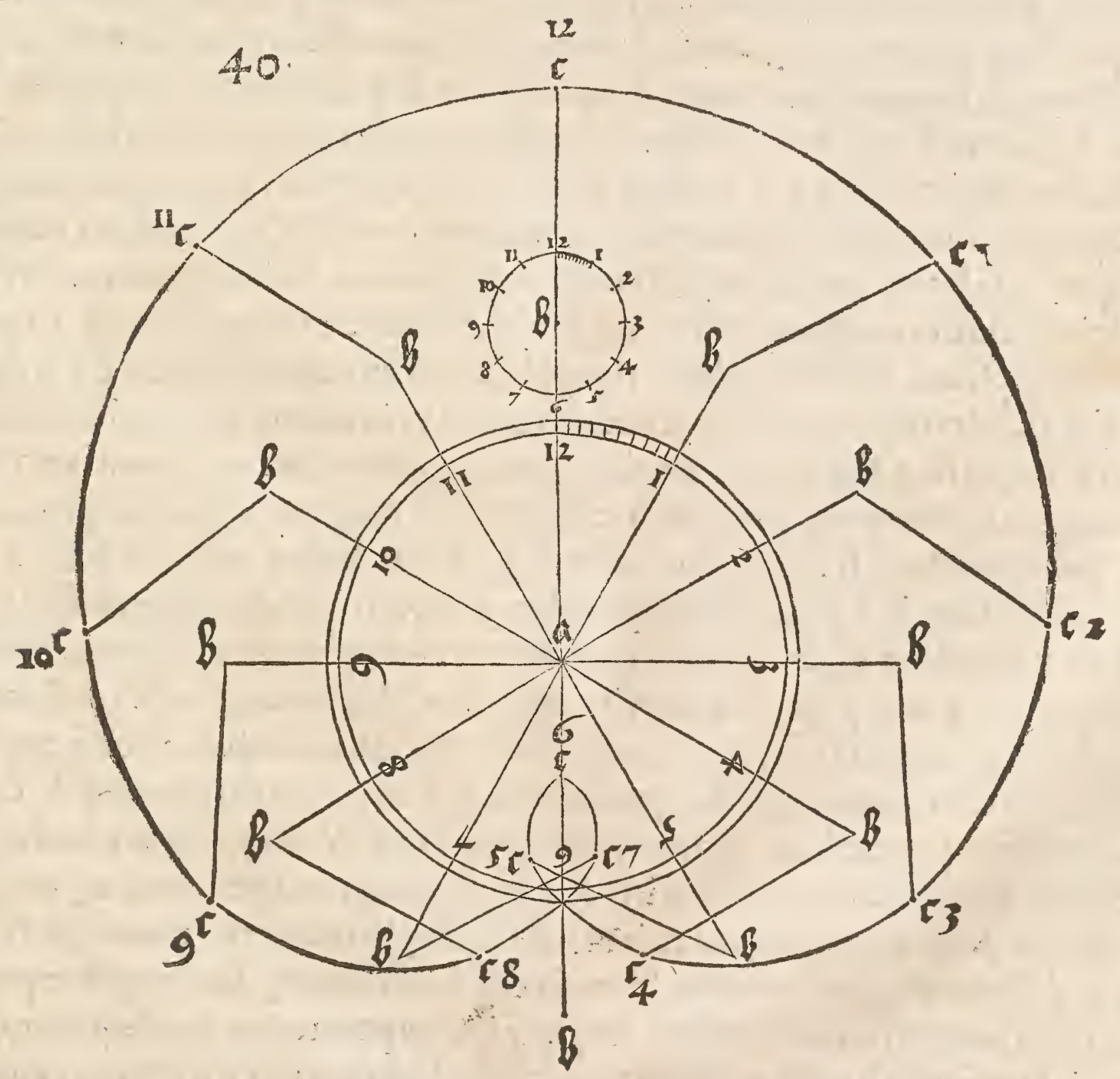

V. Vnc inftrumentum conficiam, quo in diuerfis locis altè, humiliter, 12. Id latera,antè \& póft linea ferpétina notari ac protrahi potelt. Hoc 1. 4 inftrumentum in perticarum extremitatibus flectetur atq circum. agetur, \& in ipfarum iuncturis, rote effe debent, in quarum centris 
flexus fiunt per quos circumagitur inftrumentum: vna pertica poteft in anteriorem, alia in pofteriorem vel in quamcunque partem impelli, aut omnes i $^{-}$mul in vnamquampiam, \& fic conftructas effe conuenit vt quamcunque velis de gradu in gradum queas prolongare aut rurfus contrahere. Item in rota cen tro circumagere, in quamcun $\phi$ partem res poftulet. Licet etiam perticas $\&$ ro tas facere plures vel pauciores, prout vfus inftrumenti exigit. P orrò pertica infima erigenda eft in directum \& parte interiori figenda firmiter. Nam circa eam oportet reliquas omnes volui. Ipfa etiam circa clauum, quo rota affigitur, poteft circumagi ad fingulos rotæ gradus. Verùm vt hæc exactiùs intelligantur, hoc pacto explicabo. Egofacio quatuor perticas, quibus fingul is in fuprema fui parte fingulas fubiungo rotas, in quartum centris ipf $x$ flectuntur : rotz verò debent in circumferentia fua gradibus $\&$ numeris effe diftinctx, atque earum minima acum fiue oftenforem habeat oblongum, iufta tamen craffitudine, qui fuper centrum circumagatur \& linex ductum oftendat. Hic oftenfor etiam ita fit conftructus, vt breuior aut longior, fi opus fit, fieri poffit. Inftrumentum pradiçum poteft fecundú diuerfos vfus multifariam mutari, $\&$ aut magnum aut paruum fieri. In primis tamen curandú eft, guòd perticæ $\&$ rotæ fecundú certá proportionë maiores aut minores fiant $n$ ná poltrema debet maxima effe, \& prima minima, fic quòd hac omnia forma, robore \& pódere apta fint, quod in mo dum fequétem fieri poteft. Primò conftrue rotarum magnitudines tali ordine: Defcribe quadratum b c d e.tantæ quantitatis, quantam rotam maximam fie ri cupis, in cuius medium pone púct um a.fuper quod fcribe circulum, qui omnia quadrati latera contingat, hic circulus reprafentat latitudinem maximè ro tx:deinde duc binaslineas rectas a c.\& a d.\& annectè triangulo a $\mathrm{cd}$. in parte exteriore 1 pfius $\mathrm{c} d$ adhuc talem triangulú,cuius angulus reetus fit $f$. Eft igitur a $\mathrm{f} \mathrm{fd}$ quadratum fecundum, in cuius medio, vt in medio puncto linex diago nalis c d que fit g.fige pedem circini, \& reliquo defcribe circúferentiam tágen tem omnia latera iplius quadrati a $\mathrm{c} f \mathrm{~d}$. Hęc eft magnitudo fecunde rota. Poftea pone punctum $h$. in medio linex b $c . \&$ duc lineas rectas a $h . \&$ a $g \&$ crit quadratum h c g a.cuius diagonalem a c.partire púcto i.per mediú, \& po fito circini pede in figno i.altero protrahe circumferentiam attingentem latera quadrati h c g a.que amplitudo erit tertį̧ rotæ. Poftremò ptrahe lineá rectá i h.habebiśg triangulú h i c . cui adhuc talé adiüge ad latus h c.eritóg angulus rectus additi trianguli $K . \&$ fiet quadratum $\mathrm{h} \mathrm{K}$ ci. cuius diagonalem $\mathrm{h}$ c.feco per mediú in punctol.cui applico pedê circini vnû́, $\&$ alio, circino circulum có tıngenté quatuor latera quadrati h $\mathrm{K} \mathrm{c}$ i.quę magnitudo eft minimę rotę. Ilta rectè funt proportionata:nam prima rota in duplo maior eft fecunda, $\&$ fecunda in duplo maior quàm tertia, \& tertia dupla eft quartę. Iam quęlibet pertica quater debet continere diametrú rotę quę ipfi imponitur, ex quo requitur, quòd omnis longitudo harum quatuor perticarum fumi potelt ex diametris quadra. torû́, qua paulò antè vnum ex alio ded uximus, id quòd fatis amplè oftếlum eft in rotarú quadratis. Item perticarúlatitudines etiă conueniet ex quadratis accipere, hoc modo.Primam \& maximam perticam faclatam, vnam decimamfeptimam 


\section{GEO METRIAE II B. I.}

primam de longitudine fua, à qua fepara fupernè linea tranfuerfa quadratum, à cuius puncto medio duc binas lineas rectas in duos angulos, vni lateri adiacétes, 2 habebis medietatem minoris quadrati, cui adiice $\&$ alteram medietatem erítque quad ratum in duplo minus priore: deinde accipelatitudinem fecund $x$ pertice ex iam facto quadrato, qux etiam continebit viam cecimam feptimam fux longitudinis. Confimiliter age cum tertio $\&$ quarto quadratis, aut fi plura vnum ex alio deducere placuerit, excipe femperex ipfis pertica latitudinem, que quidem fecundum jplius menfuram iufta erit, fic quod vna fimilis fiet alteri. Porrò perticæ ipf $\mathfrak{p}$ variatione quadratorú, fe paulatim leunat at $\not$ erigunt $\&$ extremitates ipfarum denotant lineam volutam. Et fi in illas perticarum am plitudines quippiá ornatus quis addere voluerit, id commodè poterit ex pradietis partirinnam per ea fiant parerga magna $\&$ parua, fecundum proportionể pertice. Qui hoc inftrumentum componere voluerit, diligenter obferuet ne per tica quępiam circuitum impediat alterius:figat quoqg quamlibet perticam vni rotx ad dextram, alteri ad finiftram, \& perticx debent in parte inferiore circumagi per rotx gradus $\&$ in medio extendi atque contrahi, vt longiores $\&$ bre uiores cum negotium poltulet, fiant. Multę funt huiufmodi inftrumentorum vtilitates, nam iis diueris artifices in operibus arduis vtútur. Qua verò arte côftruantur infrà quoque protraxi. V fus autem huius inftrumenti varius eft, pro cuiufque arbitrio qui eo fibi vtendum putabit. 


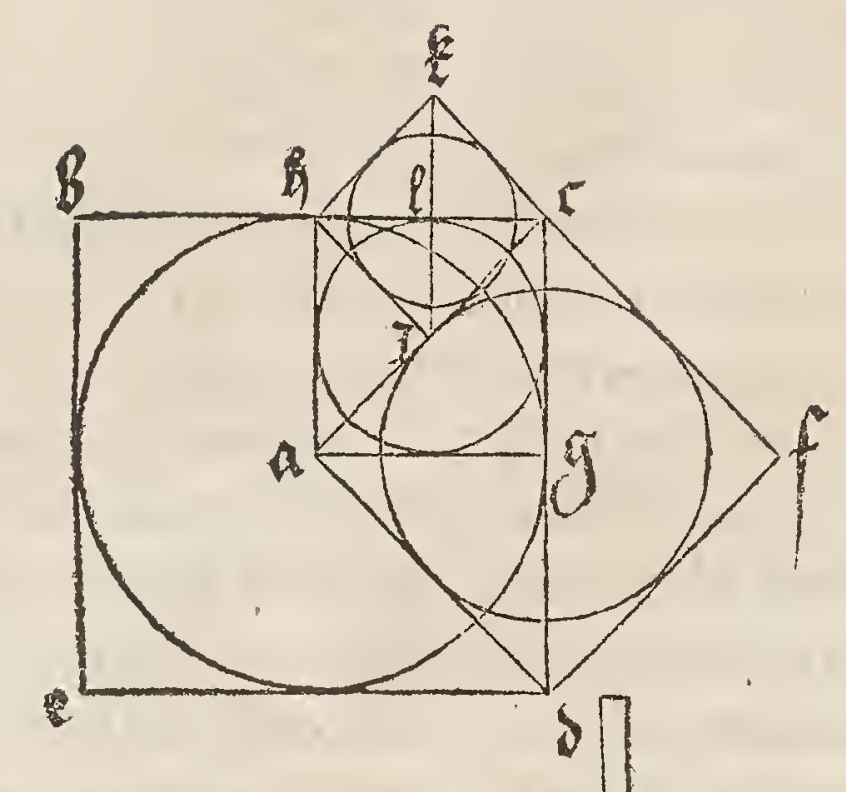

4
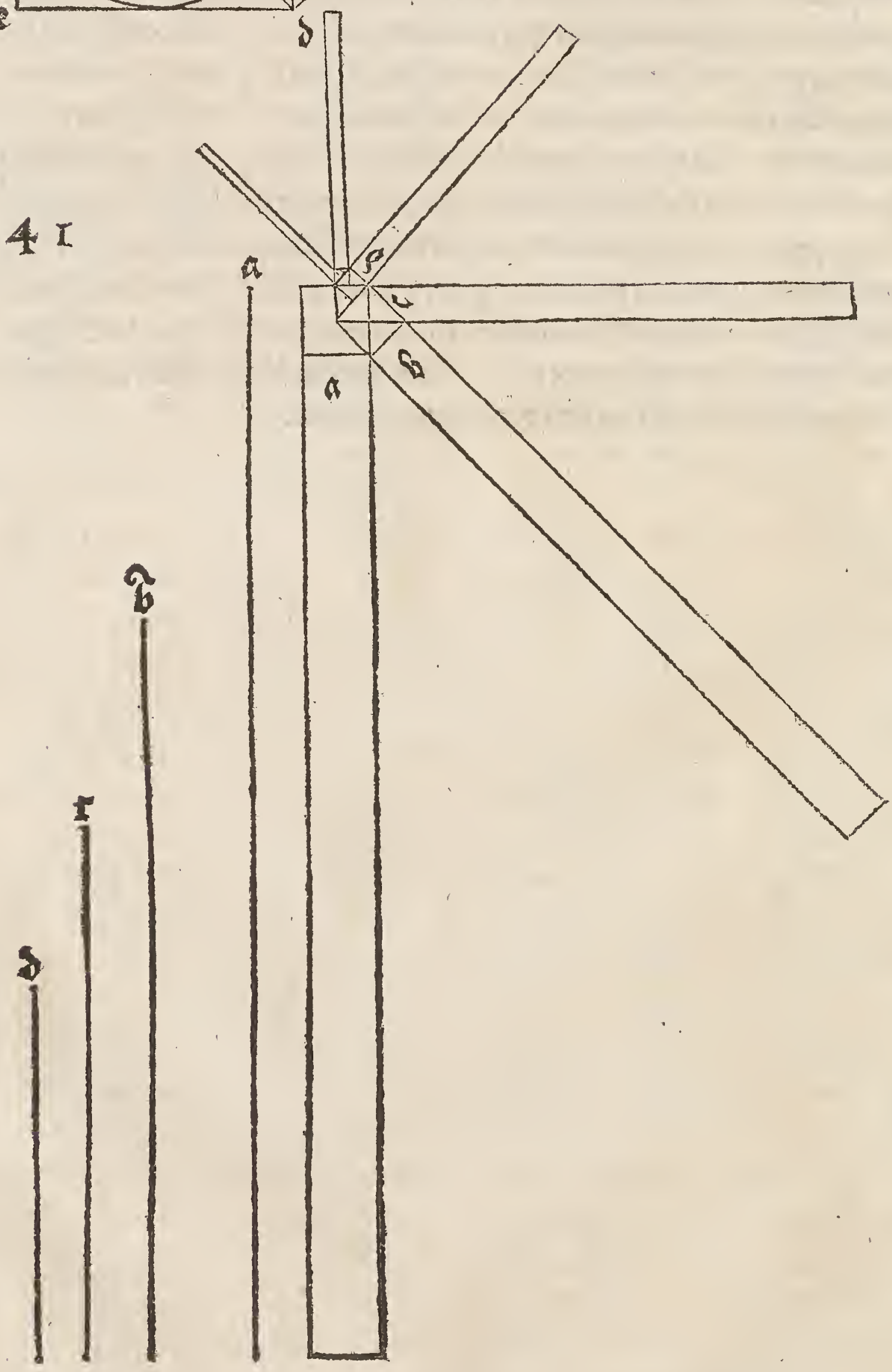

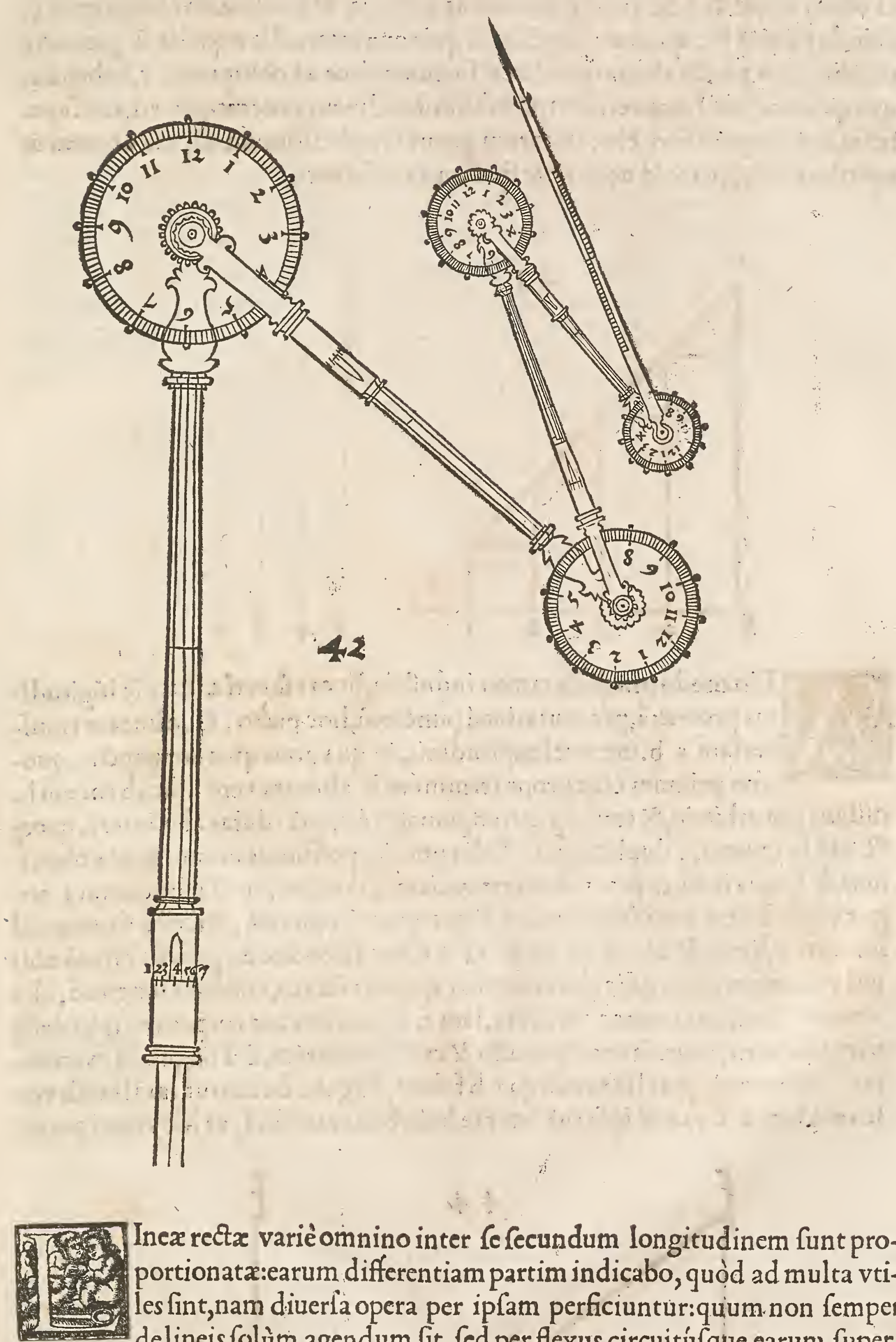

Inex rectx varièomnino inter fe fecundum longitudinem funt proportionata:earum differentiam partim indicabo, quọd ad multa vtiles fint,nam diuerla opera per ipfam perficiuntur:quium non femper delineis folùm agendum fit, fed per flexus circuitúfque earum, fuperficies, atque integra corpora poffumus reprafêtare : id quod operum neceflitas exigit, ex quo res pulchra \& arduę inueniendę. Primo aliquot lineas fecando ordine prolongabo, quod ad hunc modum intelligédum eft. Applica binas lineas

$$
\text { D iii rectas }
$$


as rectas a b. \& $b$ c.fie quòd $b$. fiat rectus angulus, \& duclineam obliquam a $c$. deinde partire bc.quatuor punctis, in quinque interualla aqualia, \& protrahe ex illis $1_{3} 3.3 .4$. punctis lineas parallelas furfum vfque ad obliquam a c, habebúnt que quatuor illa linex certam inter fe fimilitudinem, poterüt'́ ex eis, aut fuper ficies, aut corpora fieri. Hoc linearum genus fimplicifimum elt, mirê tamen in operibus vtile, quare id oculis hic fpectandum fubieci.
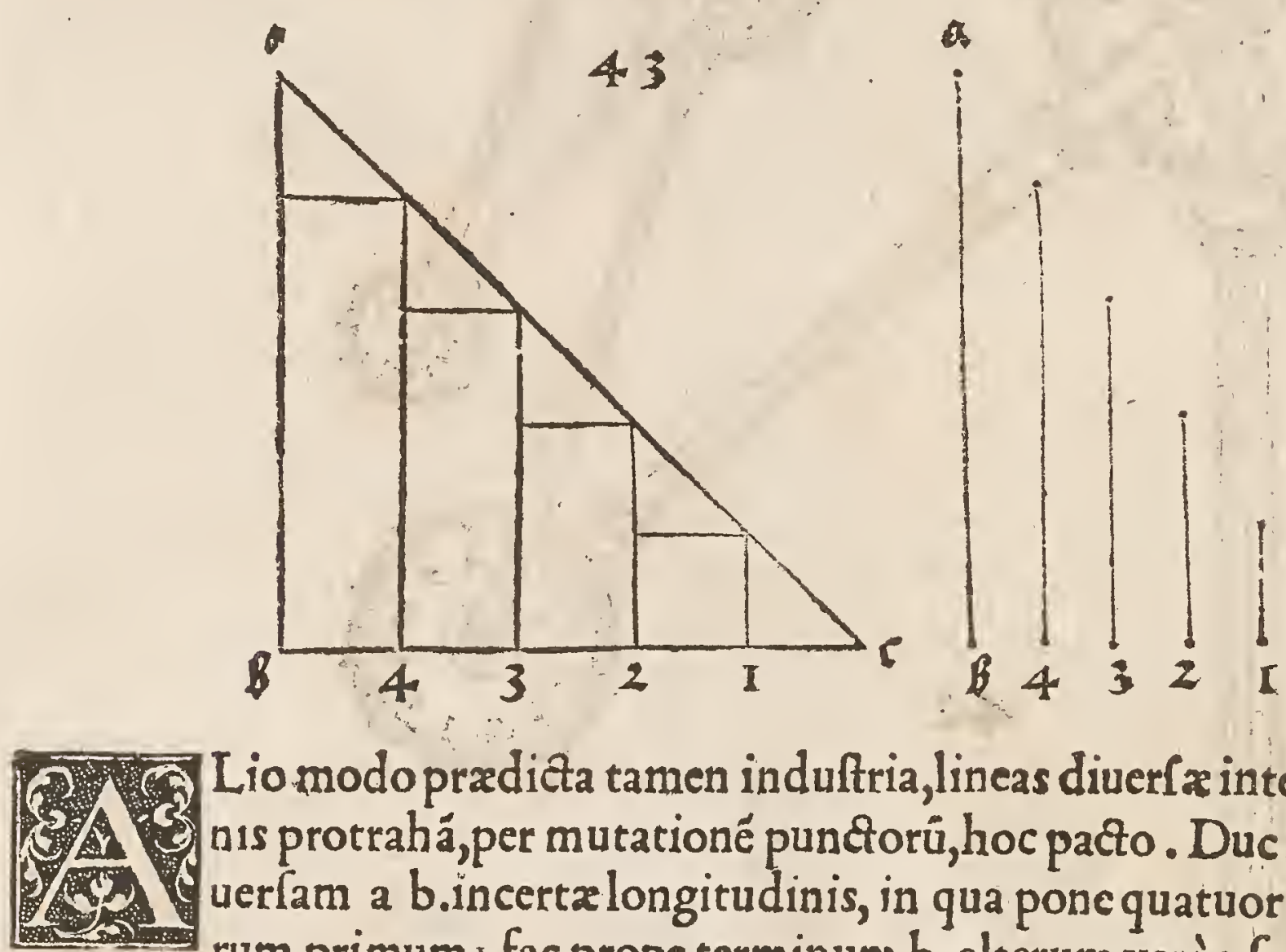

Lio modo prædicta tamen induftria, lineas diverf a inter fe lögitudi: nis protrahá,per mutationé punctorú, hoc pacto . Duclineam tranfuerfam a b.incertalongitudinis, in qua ponequatuor puneta, quo. rum primum . fac prope terminum b. alterum verò 2 .fac ab eodem $b$. diftans pro arbitrio, \& tertium : feiunge magis à $\sigma$.quá : diftat $a b$ s.inter 3 . quoq \&4.fit f pacium 2.3. duplicatum. Talia puncta poffumus notare quot voluerimus, \& fpacia inter ea pro voluntate variare, quúm que puncta funt notata, erige ex iplis lineas xquidiftantes, atğ lógas quantü opus êt, \& fcribe fupernè ad lineam I c.literă, $\&$ ad 2 d.ad ; e. $\&$ ad 4 f.hoc facto duc ex puncto c lineá obli quá per omnes perpendiculares verfus f.qux eas fecans, vnä facit longioré, aliáa verò breuiorë, certa tamen menfura. Iam fi fuperficies aut corpora ex ipfis defig nare placuerit, inuenitur amplitudo $\&$ craffities earum, fi à fingularü extremitatibus fupernè, quas linea obliqua $b$ f.facit, fingula ducuntur tranfuerf $x$ verfus erectam a f.qux \& ipfę fuá inter fe habebunt menfurá, vt hic videri poteft.
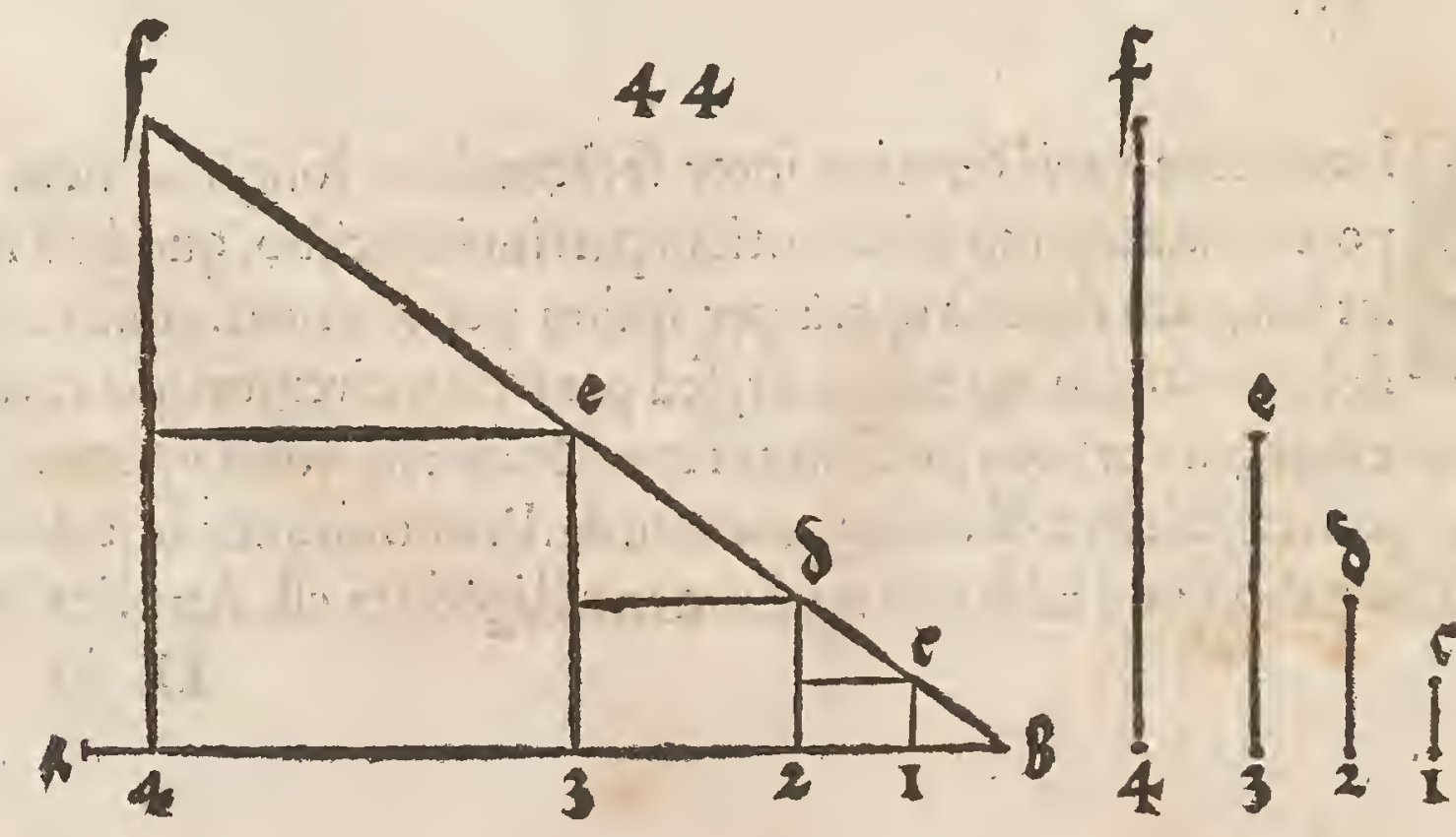
Vrfus aliter per arcum circuli lineas defcribam, certóg ordine vnam circumferentia $b c, \&$ ducam crectá a b. \& aliam tranfuerfam a c.fic quòd a . fiat angulus rectus : deinde partior a $c$. quatuor punctis in quinque fpacia qualia, atque ex illis quatuor punctis producam lineas erectas in arcum circuli $b c, \&$ vbi eum attingit, illic fupra r noto d.fupra 2 , fupra $; f$. $\&$ fupra $4 \mathrm{~g}$. Hequatuor linex fingularě quendam inter fe habent habitü. Nüc quadrantem circuli cum fuis literis ita verto quòd $b$ a.tranfuer $\{a, \&$ a $c$,perpen dicularis fiat, \& duco lineas erectas ex punctis circumferentix $\mathrm{d}$ e $\mathrm{f} g$. deorfum in tranfuerfam a b.habebunt'́s \& iftre linę, nouam inter fe fimilitudinem. No tandú quoq $q$ per illas longitudines, omnes earum latitudines $\&$ fpiffitudines fe offerựt, 8 quemadmodú fe qualibet erga quálibet habeat, velut hic fubieci.
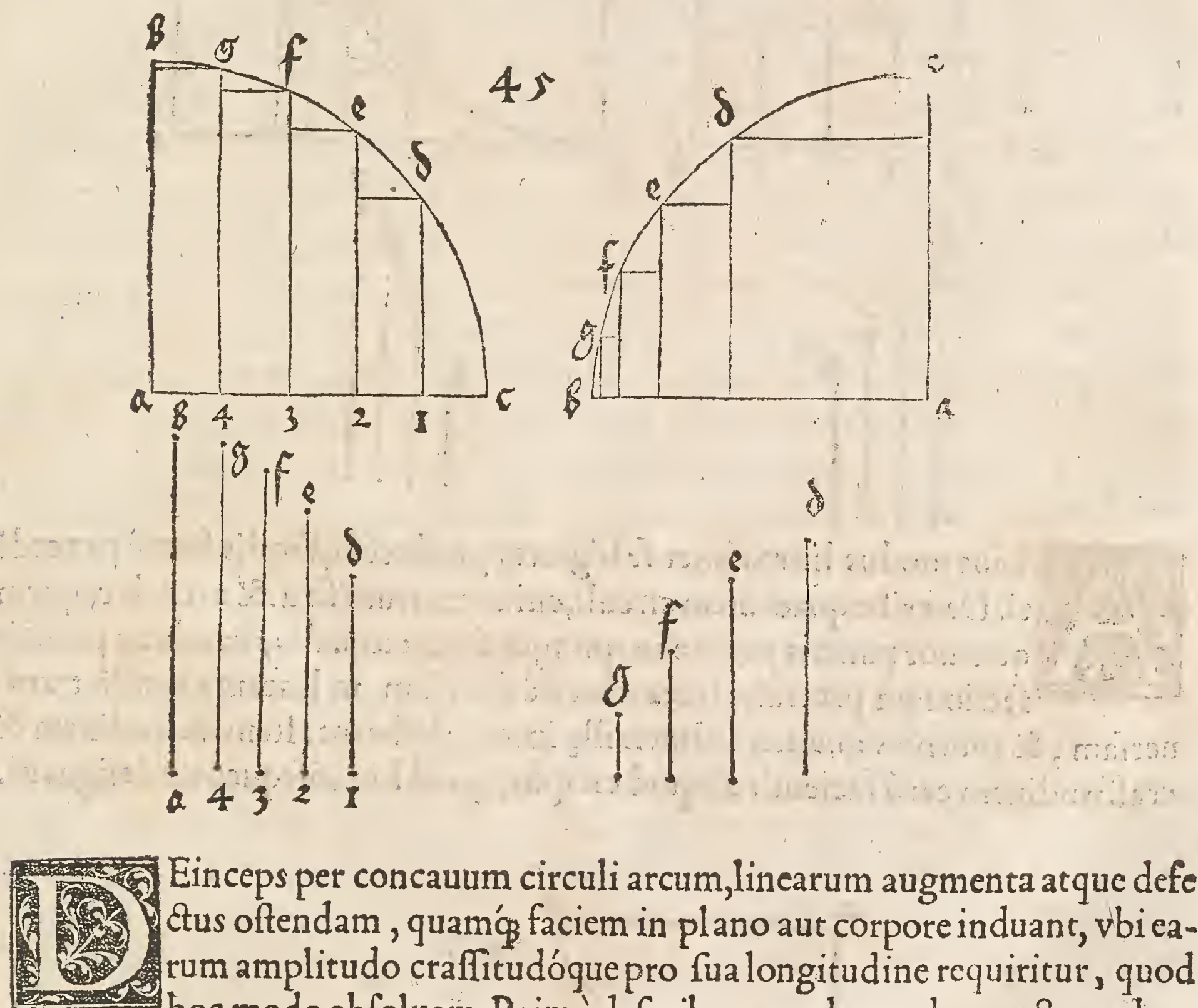

Einceps per concauum circuli arcum, linearum augmenta atque defe ctus oftendam, quamóp faciem in plano aut corpore induant, vbi earum amplitudo craflitudóque pro fua longitudine requiritur, quod hoc modo abfoluam. Primò defcribam quadrangulum rectangulum erectú, quod fit fupernè a b. 8 infernè $c d$.deinceps pono circinú vno pede in pú ctum a.\& reliquo ex b.protraho arcum per quadrangulum vfque ad latus a d. deinde partior latus $\mathrm{c}$ d.in quinģ partes equas, \& à punctis partitionú duco qua tuor lineas perpendiculares furfum vfque ad arcus conuexum, quod vbi attin gunt depingo literas, e fupra r.f fupra $2 . \mathrm{g}$ fupra $3.8 \mathrm{~h}$ fupra 4 . Iam vides quomo do e linęill inter fe habeant, \& fifupernè à qualibetad proximam longioré trahantur tranfuerfx, continuo manifeltnm erit, qualem confequantur latitudinê, qualéóp corpus. Quumetiá quadrăgulü iftud in latus reclino, ita quòd d a. fupernè $\dot{\alpha} \mathrm{bc}$. infernê veniant, \&ex punctis ef $\mathrm{g} \mathrm{h}$. ipfius arcus lineas perpédiculares deduco vfqu ad trăfuerfalem 
verfalem b c.tunc apparebit quomodo inter fe fecundum longitudiné fint pro. por tionatx. Et frex fignis h $\mathrm{g}$ f e $\operatorname{tranfuerf}$ lineas fcribo ad vicinas longiores, continuò videtur quale planum aut corpus habitura fint; qux rotunda aut quá drangula fieri poffunt . Ifta hoc pacto deliniaui.
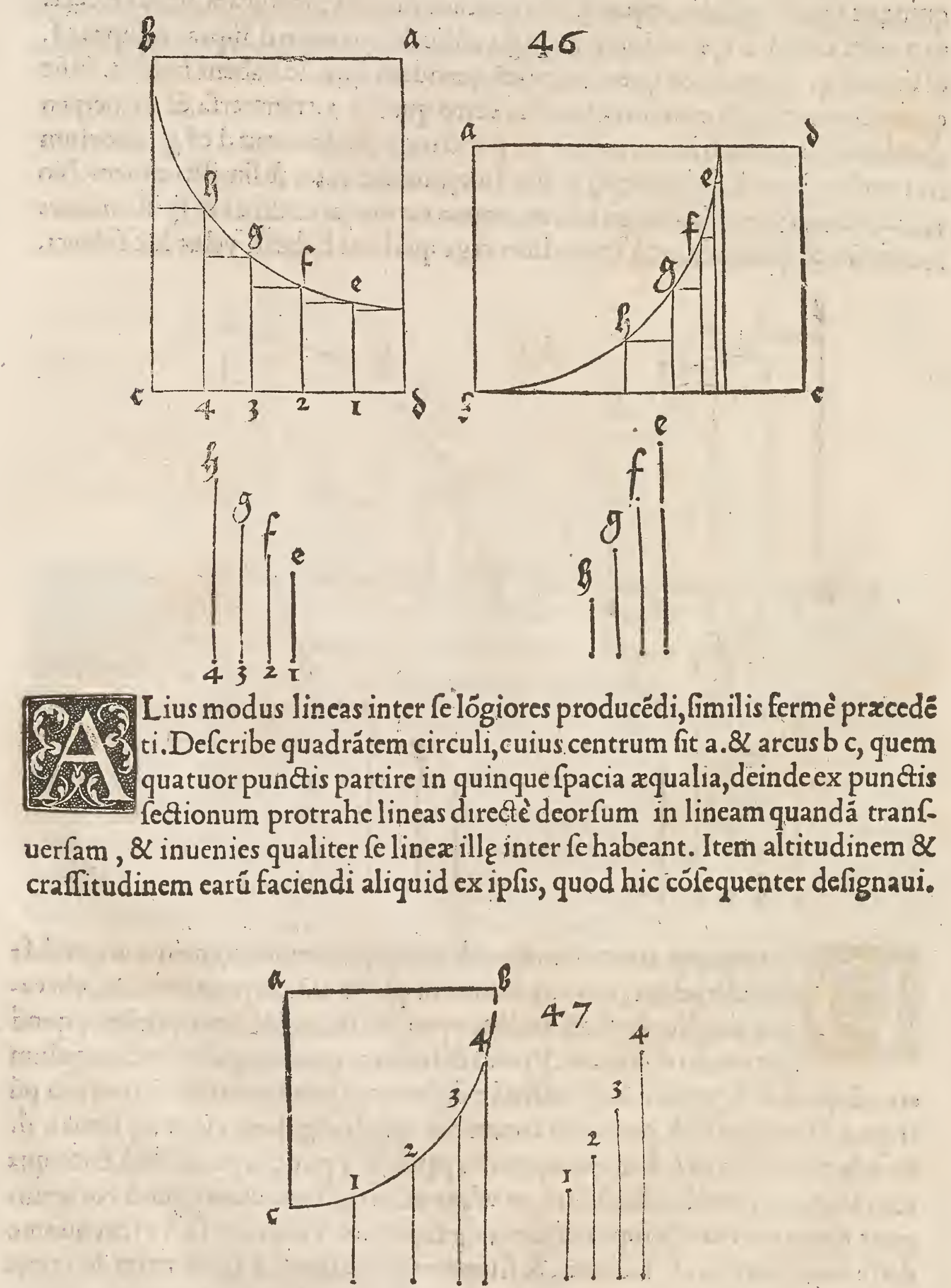
N fumma omnes erecta linex, qux ordine aliquo, xquali fue in $x$ quali diftantia inter $f e$, fuper linea tranfuer $\mathrm{fa}_{\mathrm{ftatuun}} \mathrm{fur}$, triplici mo do abfcindi poffunt, per concauum atque conuexum circuli arcum, \& per lineam obliquam, longă fiue breué:habebítq̧ quodliber fuam peculiarem faciem. Et quanquam hac omnia prius indicauimus, nunc tamen adhuc clarius intelliges. Duc lineam tranfuerfam a $b, \&$ erige fuper eam quatu or lincas rectas, not is numerorú $x_{0} 2.3 .4$. fignatas, $\&$ protrahe ab extremitate a, lineam perpendicularem furfum, in qua vno circini pede polito, reliquo infernè $a b$ eade linea duc arcú per omnes quatuor erétas, qua per eú recto ordine fecátur. Quud fi eafdem lineas breuiores inter fe abfcindere voles, extende circinú adhuc magis \& fige vnum pedem in erecta a. Altius quàm antea, $\&$ alterum in fernè, in locum priorem, ac fcribe arcum per reliquas erectas, quę hoc modo bre uiores erunt.Sic fiunt linex per arcum circuli concauú ing̨uales. Sed per conuexum abfinde eas hoc pacto. Protrahe per extremitates b. lineam perpendicu larem furfum $\&<$ deorfum, in quam pone infernè alterú circini pedem, alterum verò in punctú a.tranfuerfe, à quo fcribe arcú per quatuor erectas, quas abfcindere cupis. Sin auté per fectiones, lógiores inter fefe facere velles, af cëde parü circini pede immobili, in linea perpédiculari, $\&$ alio ex priore puncto a.duc rurfus arcú per prędictas lineas, que abf́ciffe lógiores inter fe erunt. Quòd verò circini pedé femper figo in iifdê rectis lineis a. aut b.hoc fir pppter maiorê cómoditaté. Ná Euclides in tertio fuo Geo.elemen.libro. Theoremate ro. Proporfione ir.do. cet. Si circulus circulú introrfum contingat.erút centra vtriufog remper in linea recta, que ei etiam tranfit per locú vbi circuli fe coniungunt:quod fic intellige. Defcribe ex quopiam ce ntro a .circulum ${ }^{1.203}$ intra cuius circumferentiam pone punctum b.vbi volueris, deinde accipe circinú, qué fifte vno pede in punctủ b. $\&$ alio ex circumferentia 1.2 .3 . fribe circulum minorem quantumcunque : $\sqrt{\text { fi }}$ iam ex centro a.per centrum b.rectam lineam duxeris, ad circumferentiá vfọ 1.2.3.oftendet tibi femper recta illa linea punctum in quo circuli fe inuicem tan gunt. Tali modo vtere in iam monftratis linearum interfectionibus, quanquam circinus aliter etiam figi poffit, fed fic aptius. Porrò linea recta poffunt etiă qua tuor illx propofitx commode fecari, ita quòd plus vel minus inter fe differant: hoc nota fic. Pone fignum ex quo linea procedat, per quam reliquas abfcindere Itatuifti, in extremitate a.à qua produc lineas obliquas per quatuor erectas, altè vel demiffè,prout erectas multum vel parum inter fe differre defideras. Hxc om nia hic defignaui. 

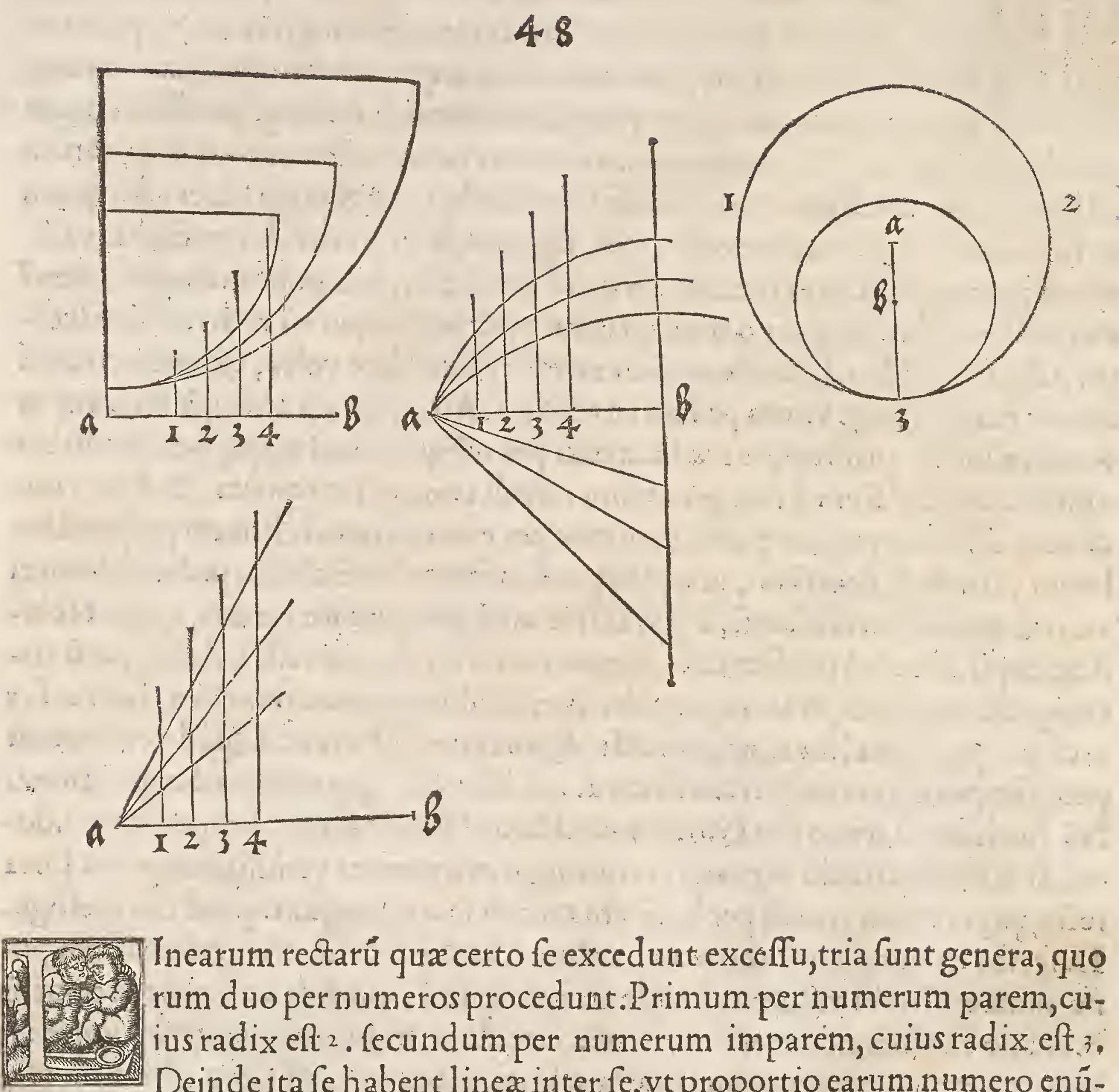

Inearum rectarú qua certo fe excedunt exceffu, tria funt genera, quo rum duo per numeros proced unt. Primum per numerum parem,cuius radix eft 2 . fecundum per numerum imparem, cuius radix eft 3 . Deinde ita fe habent linex inter fe, vt proportio earu mumero enüciari non poffit:hęc fic intelligenda funt: Sratue quatuor lineas ordine, vnam iuxta aliam, \& vtere in multiplicatione numero pari prima igitur continebit 2 . fecunda 4. tertia $8 . \&$ quarta 15. fed quatuor reliquarum imparium linearum prima valet 3. fecunda 9 .tertia 27.8 quarta $8 \mathrm{r}$. Iam numerus par $\&$ impar in vtroq linearum gencre,poteft multiplicari, $\&$ diminui, $\&$ non femper duplum inter fe conftitui aut triplum, id quod facile quiuis qui in numeris vel mediocriter verfatus eft,percipiet. Per numerum quog̣ fimplicế poffumus etiam in lineis afcêdere. At quo pacto linex qux per numeros difcerni non queunt, diftingui debeant, in pracedente figura monftratum eft. Duo autem numerorum genera, quibus in lineis vtimur, infrà ptraxi, qux etiă lapicidis, in productionibus fuis vtiles erunt. Licet pręterea huiufmodi lineas infernèlongius protrahere in quá dam lin eam tranfuerfam, \& rurfus alia erit inter eas comparatio. 


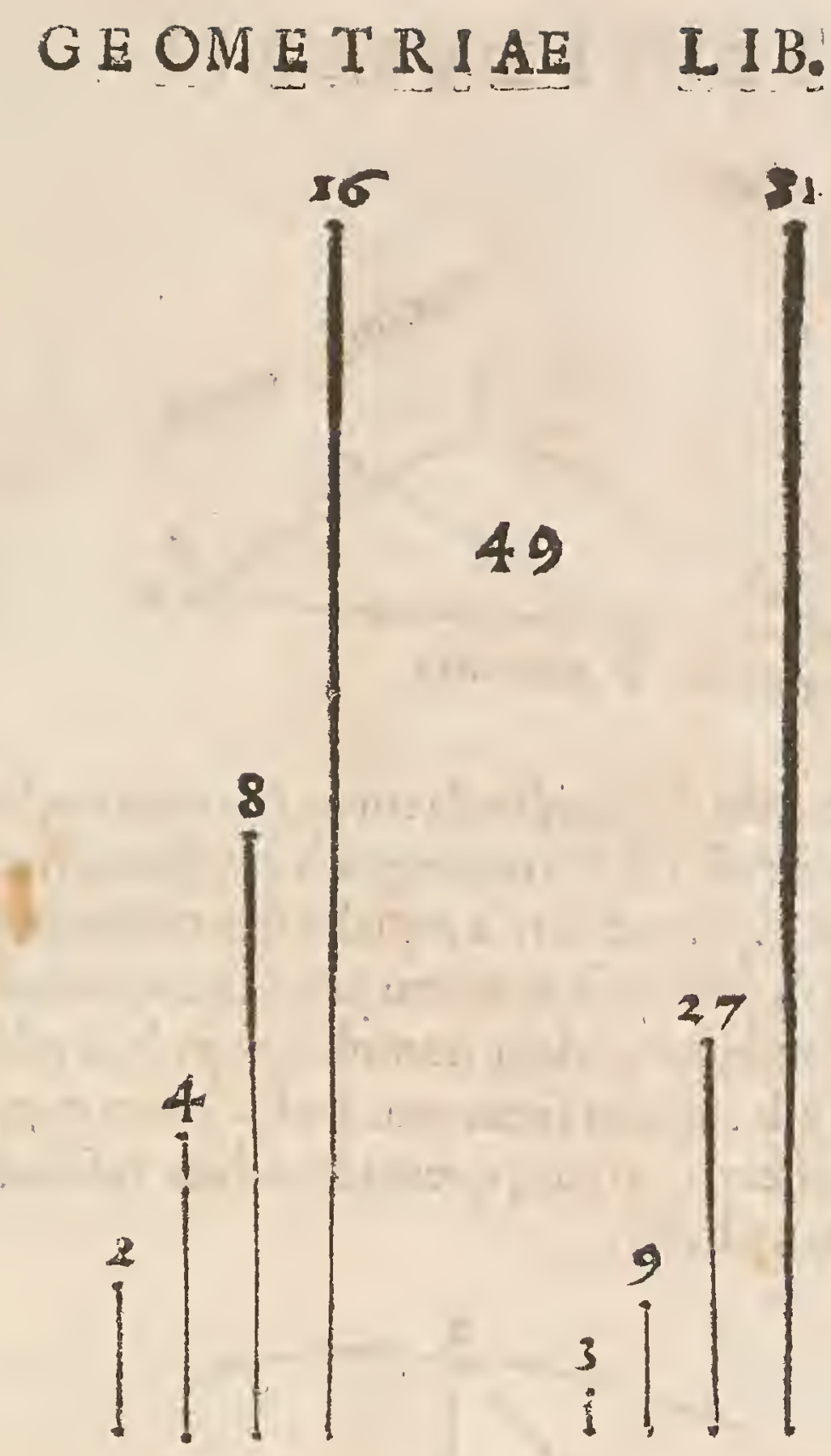

Tể quădo duas lineas, alteră longam, alteră verô breué habes, $\&$ defị: deras vnam tertiam illis breuiorem, proprortionalem tamen, ita quòd quemadmodum fe illæ dux inter fe habent, fic fe habeat illa tertia inuenta atque minima ad mediocrem, tum adhunc modum operare.Coniunge duas illas fecundum longitudinem, ita quòd ex eis vna fiat tranfuer₹a, quam figna literis a $\mathrm{b} c . \&$ pone a.in extremitate maioris, b.verò in loco iuncture, \&, c.in fine minoris : deinde accipe lógitudinem breuiorem b c.\& applica eam termino fuo b. extremitati a. \& termino c. declina eam plurimum verfus tranfuerfam a b c, \& duc ab extremitate $c$. declinatx lineam obli-

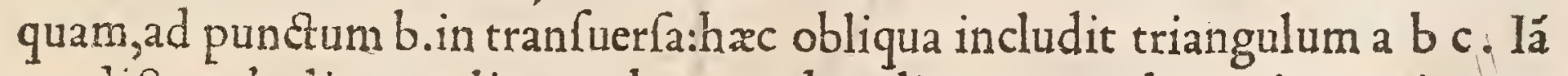
predictam declinatam lineam $b c$. produc aliquantum vltra $c$. in continuum \& rectum atque ex puncto c.tranfuerf $x$ duc parallelam ipfi b cobliqux, $\&$ vbi ea fecat prolögatá,illic fcribe literă d. Erit igitur linea breuis c d.proportionalis ipfa a b.\& b c.longioribus, \& fic fe habebit ad b c . mediocrem vt fe habet ipfa b c.ad a b.maximam:nam xquidiftantes $c$ d. 8 b c.ablcindunt has lineas proportionaliter. Heg funt digna cognitu, $\&$ ad multa vtilia. 


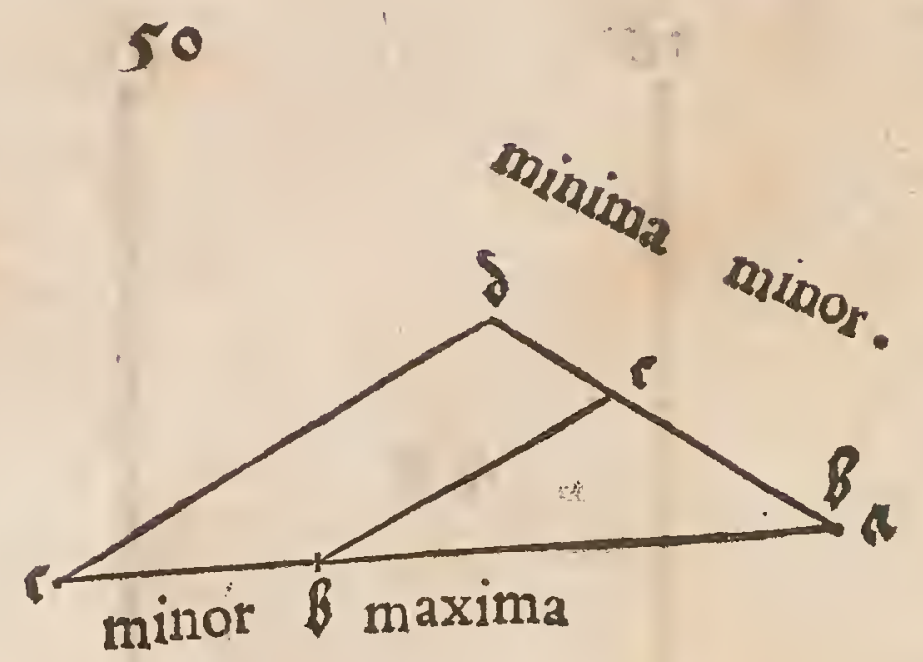

(2) Dhuc ex linea recta eft diuidenda curua, per curuam, hoc modo. De5. 3 fcribe arcum circulia b.\& coniunge a b.per lineam rectam, quam per 1. 6 duo puncta $1 . \$ 2$.partire in tria æqualia interualla:quo facto, fige vnú circini pedem in puncto b.\& altero per a.duc arcum:deinde conftrin ge circinum parum, $\&$ relinque pedem immobilem in $b . \&$ reliquo ex figno. protrahe arcum,ipfum a b arcum fecantem. Ita fac etiam cum nota 2 .manebitóg b. centrum ad omnes tres arcus, quemadmodum infernè protraxi . Ifta quoque divifio ad multa vtilis eft.
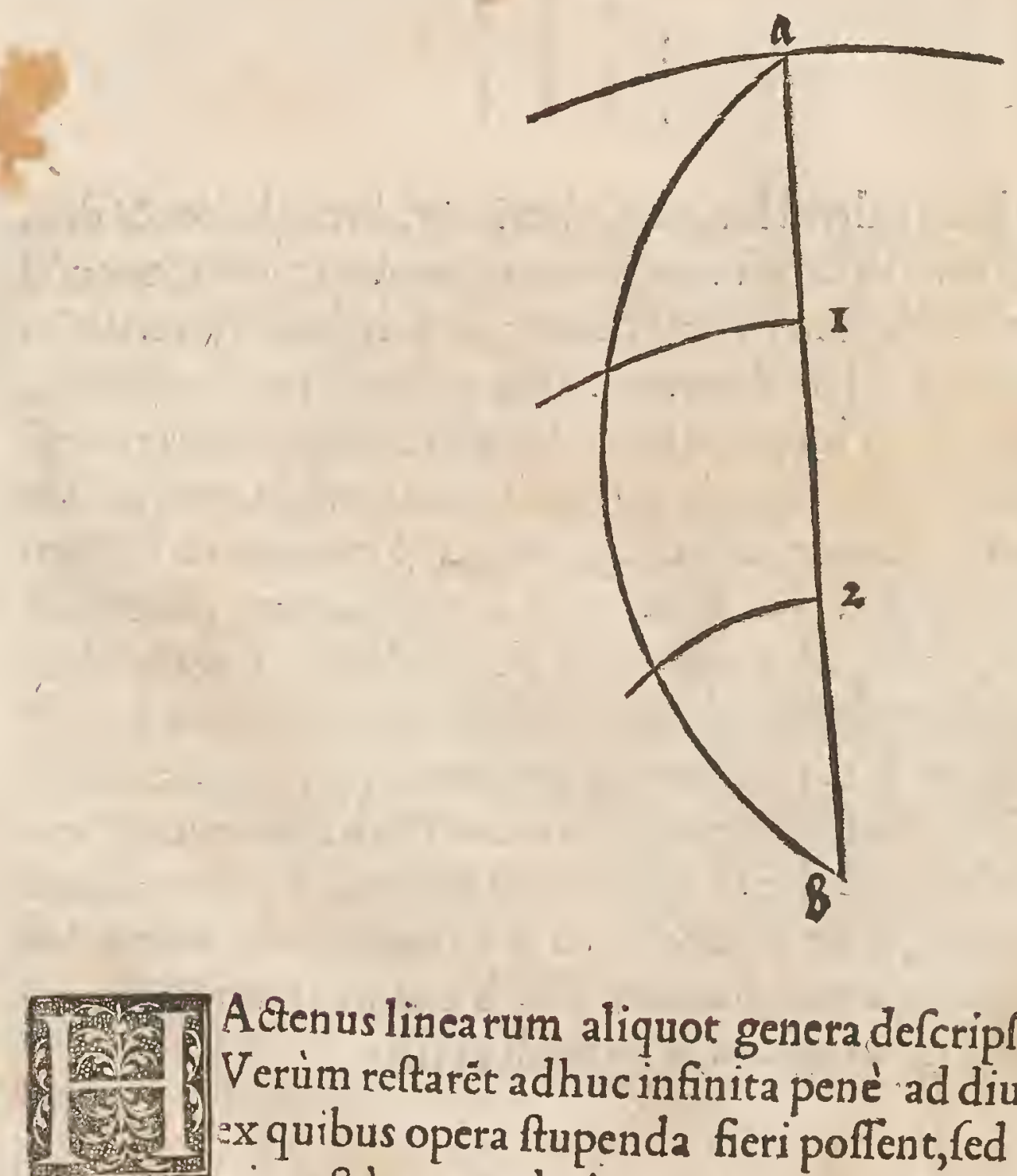

Actenus linea rum aliquot genera defcripfi, atọ etiă oculis fubieci. Verùm reftarét adhuc infinita pené ad diuerfos humang vitę vfus, ui, rectè perpenderit, atque in manus fump qui hęc, que ego monftra fructum, $\propto$ longèaltiora inueltigabit. 


\section{ALBERTI DVRERI}

PICTORIS EXCELLENTISSIMI INSTITVtionum Geometricarú Liber Secundus qui elt de fuperficiebus.

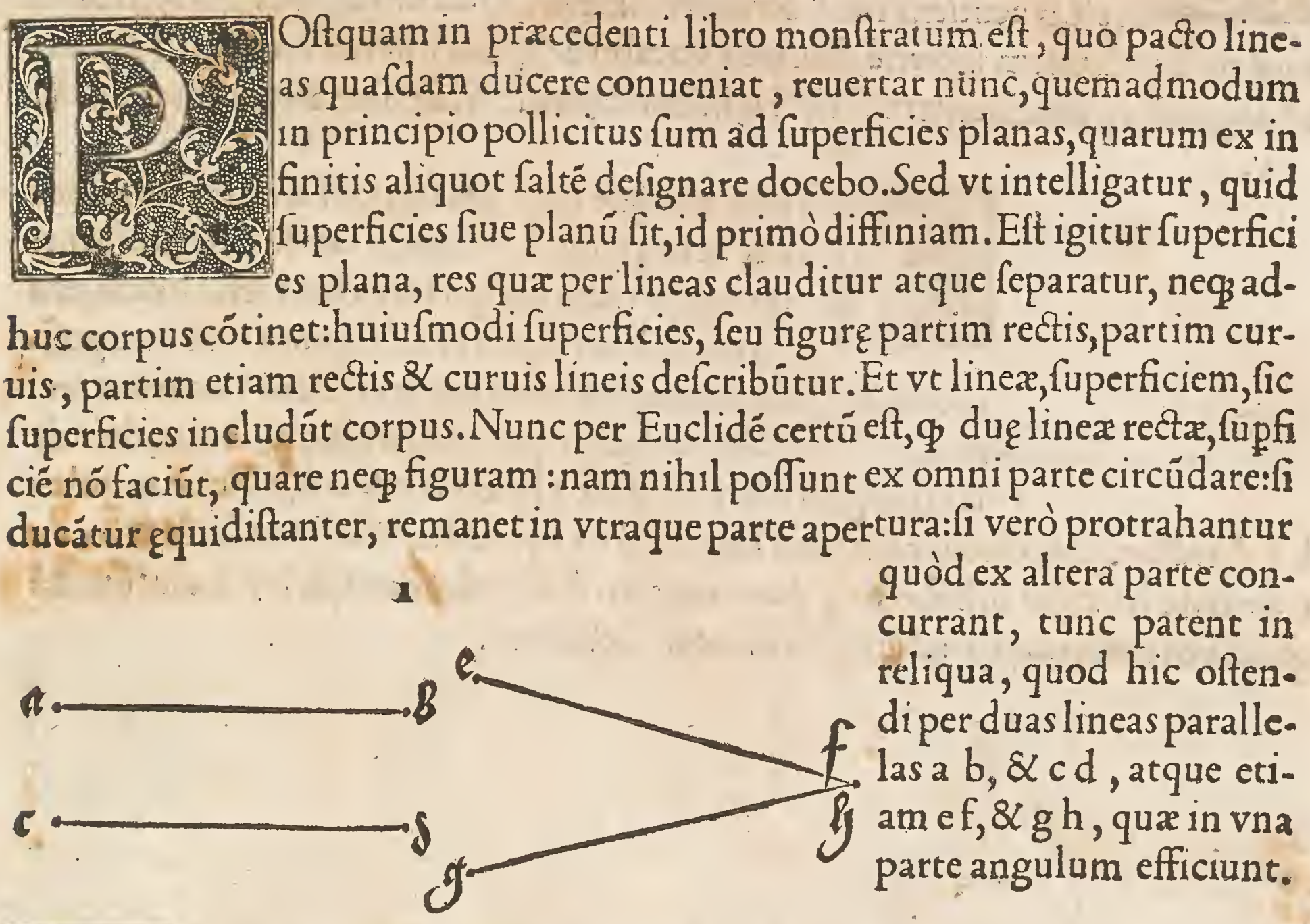

Vum verò dua linex curux a \& b, fe inuicem refpiciunt con cauita2. 1 tibus fuis, ex protractę claudét figuram:fed ę̧dem linex figuram eti am efficient fi conuexitas vnius applicetur concauitati alterius, ato hac figura fimilis erit nouę lunx. Pari ratione, fi curua d, protrahatur fuper rectam c.comprehêdet fuperficiem. Item linea curua, qux fupperficiem intra fe continet, non habebit angulos, \& fi-altera parte longior ducatur. Rur-

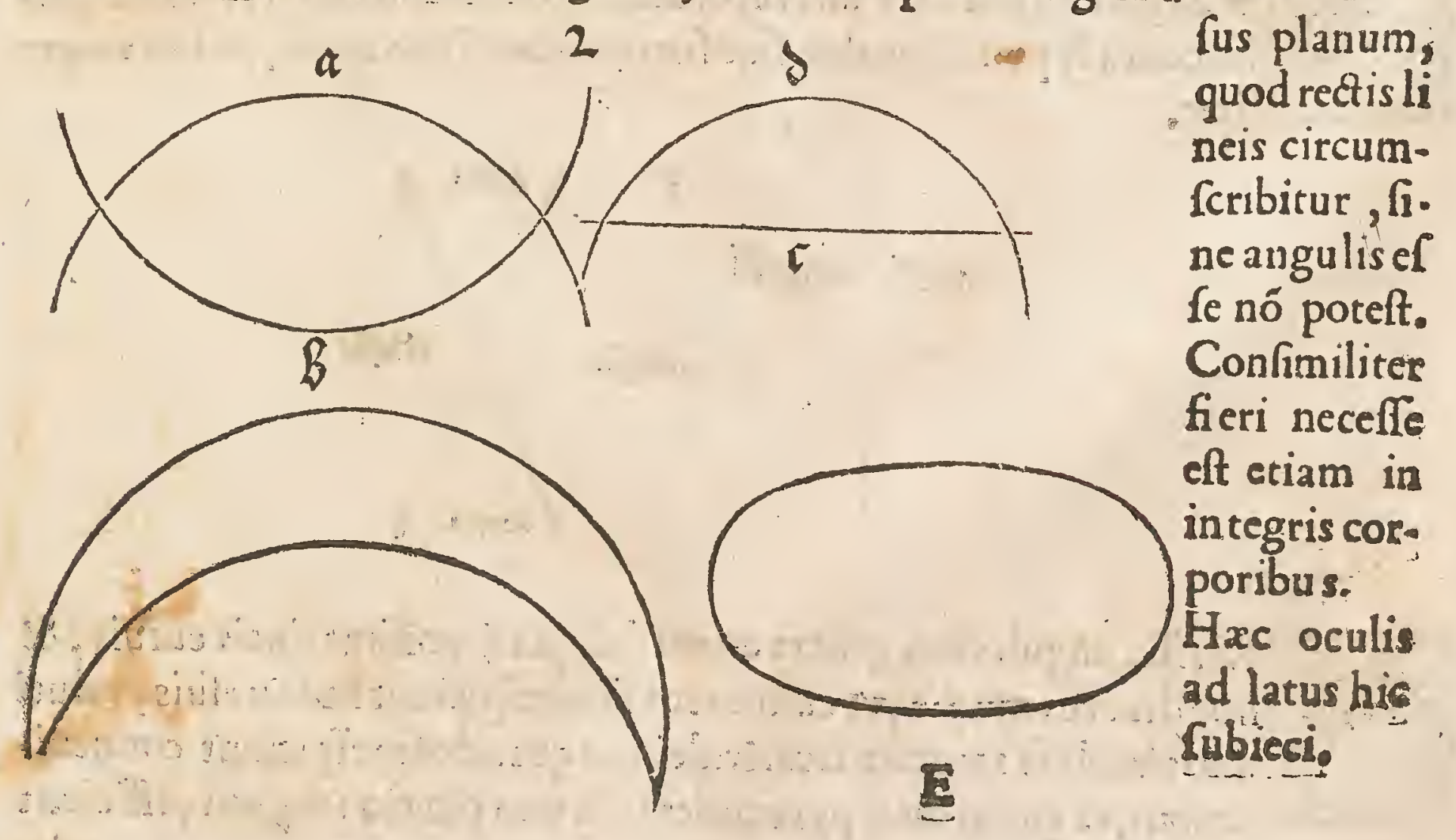


Orrò priufquam figuras defignare incipiam, de angulis nonnulla prefabor.Primò igitur notandum eft quòd angulus prominens \& finuacus $x$ quales habent lineas, difcrimen tamen eorum in opere huiufmo di eft,fi acutiem confideraueris externè, dicetur angulus porrectior : fi verò profunditatem internè,finuatus vocabitur:quod infernè hoc pacto ptraxi ad angulum prominentem fcripfis a, in concauum au tem fiue finuatum pofui $b$.
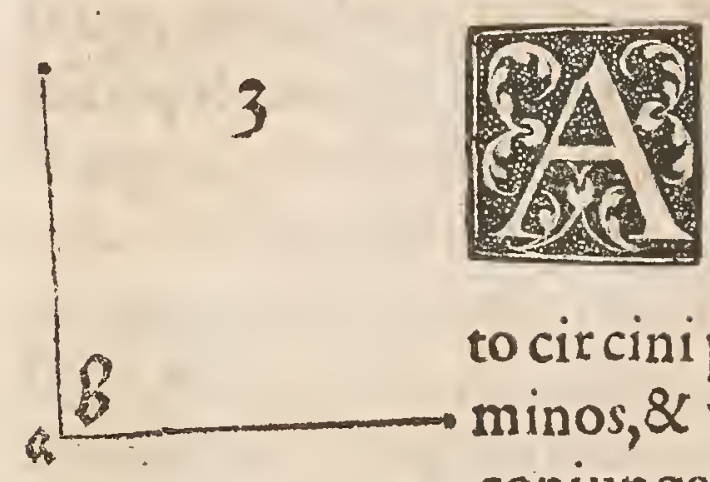

Nguli quoque triplices funt, eft enim rectus, obtu fus \& acutus, qui hoc modo facilè defcribuntur: pro angulo recto defignádo, duc binas lineas pro arbitrio, fe interfecantes ad fignum a, in quo pofito cir cini pede protrahe arcum per tres illarú linearum ter. minos, \& vbi abfcinduntur, illic adiice $b c d$, literas, deinde coniunge $b c \& c d$, ductis lineis $b c \& c d$,erítque angulus $b$ cd,rectus. Quo facto continua lineam d c vique ad $c, \&$ inclina lineam $b$ cex tremitate $b$ verfus d, habebisón duos angulos b c e obtufum, \& bcd acutum:nả quod vni aufertur, alii adiungitur:hoc lic deliniaui.
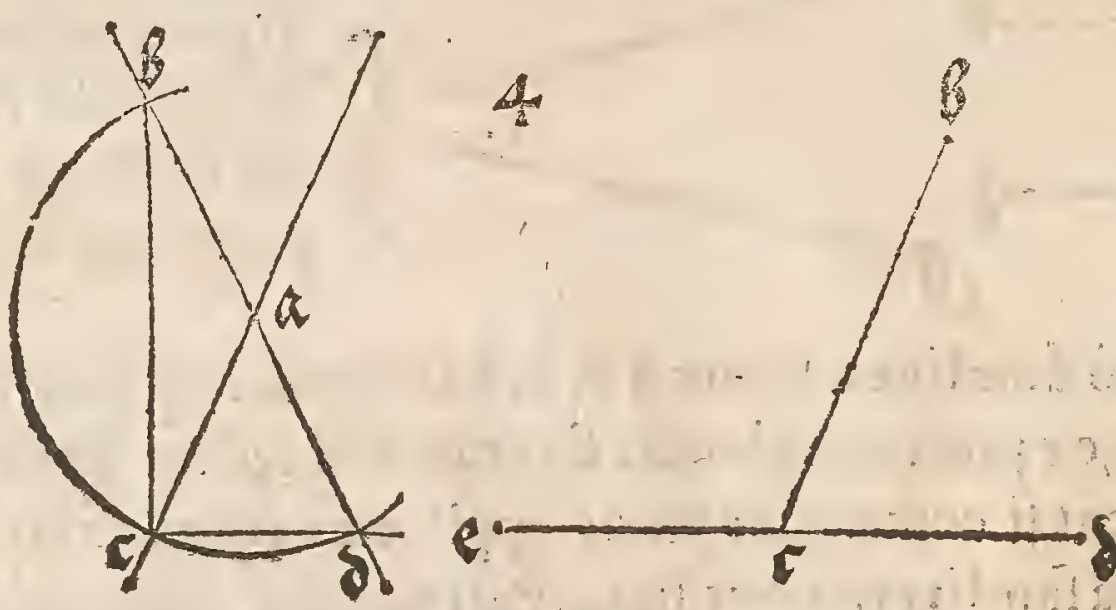

Vando linea fuper lineam cadit perpendiculariter, tune fiunt quatu or anguli recti:fi verò vna fuper aliam ceciderit obliquê, erunt angu. li contra fe politi qquales, fiue fuerint obtufi fiue acuti, vt hic videre licet.

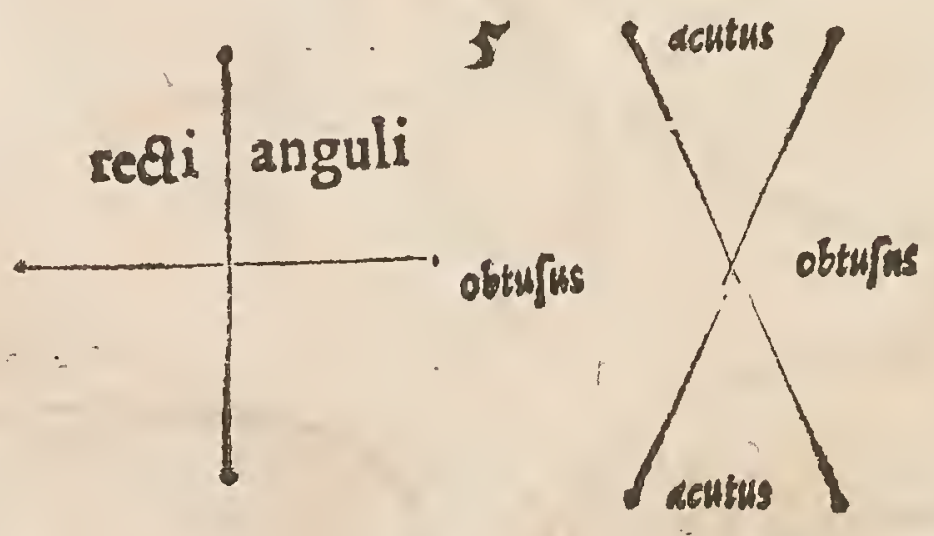

Ria illa angulorum genera etiam defignari poffunt lineis curuis, \& hoc diverfis modis, vt cum arcus fefe relpiciunt fnibus fuis, veluti duo circuli fe in uicem fecareat:deinde quando fe refpiciunt conuexitatibus, vt quum dux pyramides fe in vno pundo tangunt, efficiunt 
tales angulos duos. Variatio etiam exiftit in magnis $\&$ paruis circulorum arcu bus, quum ex eis anguli fiunt. Anguli quoque defcribútur arcubus circuli,quorum conuexitas vnius afpicit concauitatem alterius qui formam inducunt détis apri, qui etiam poflunt æqualibus aut inæqualibus arcubus fignari. Tădem angulos claudunt, curu $\&$ rectx linex, \& poteft curua linea rectę opponi conuexitate vel concauitate. Huiufmodi angulos hic aliquot fubieci.

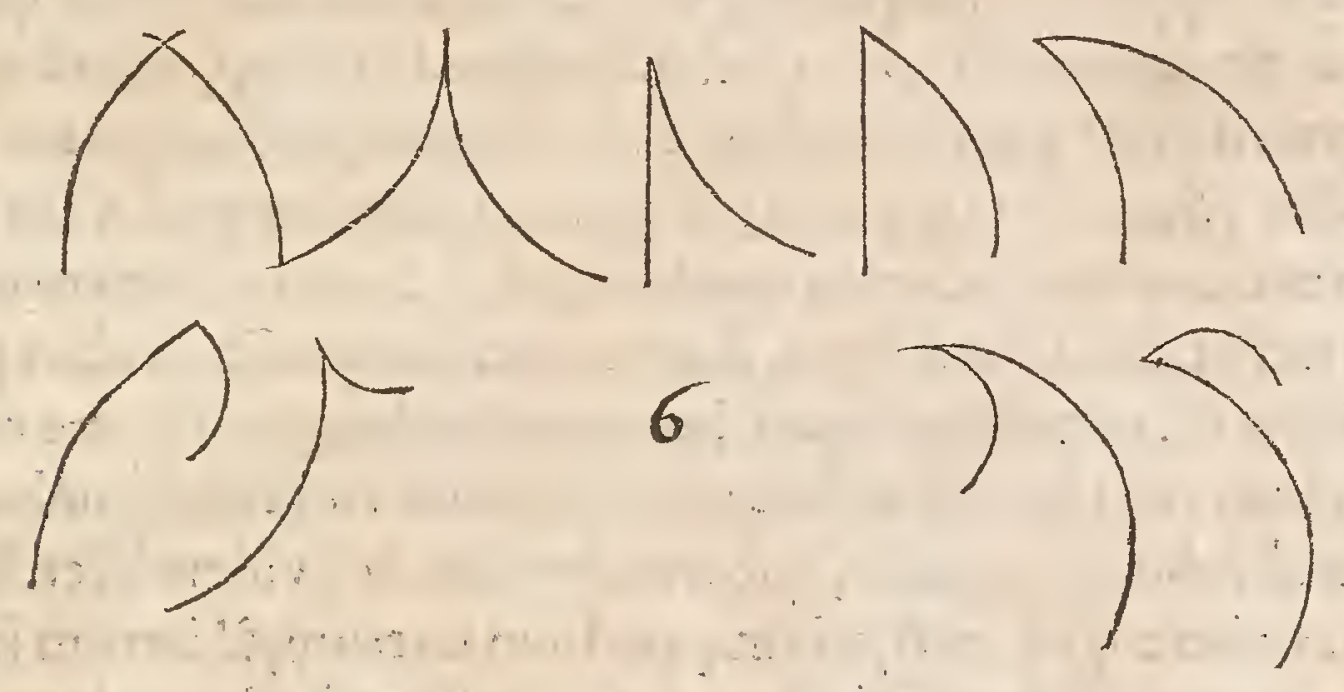

Orrò clarum ett quôd nulla fuperficies recti linea paucioribus quàm 10 Iribus rectis lineis circumfcribi poteft : nam ad minimum claudunt 4 tres linex recta figuram triangularem. Triangulorum verò rectiline - orum tria Cunt genera. Primo eft triangulus qui tria æqualia habet la tera, atọ tres angulos inuicem æquales. Deinde elt triangulus duorum æqualiü laterum, $\&$ vnius inæ qualis illis duobus, qui etiam continet duos angulos xqua les, $\&$ tertium inæqualé. In poltremo triangulo funt tria inqqualia latera, $\&$ tres inæquales anguli. Triplices illi trianguli poffunt etiam curuis defignari lineis, verfus interiorem vel exteriorem partem flexis. Item lineis tortuolis. Fiunt pre terea figuræ, qux nullum habent acutum angulum, ex circulorum arcubus aut lineis tortuofis fignătur, quemadmodum infernè protraxi.

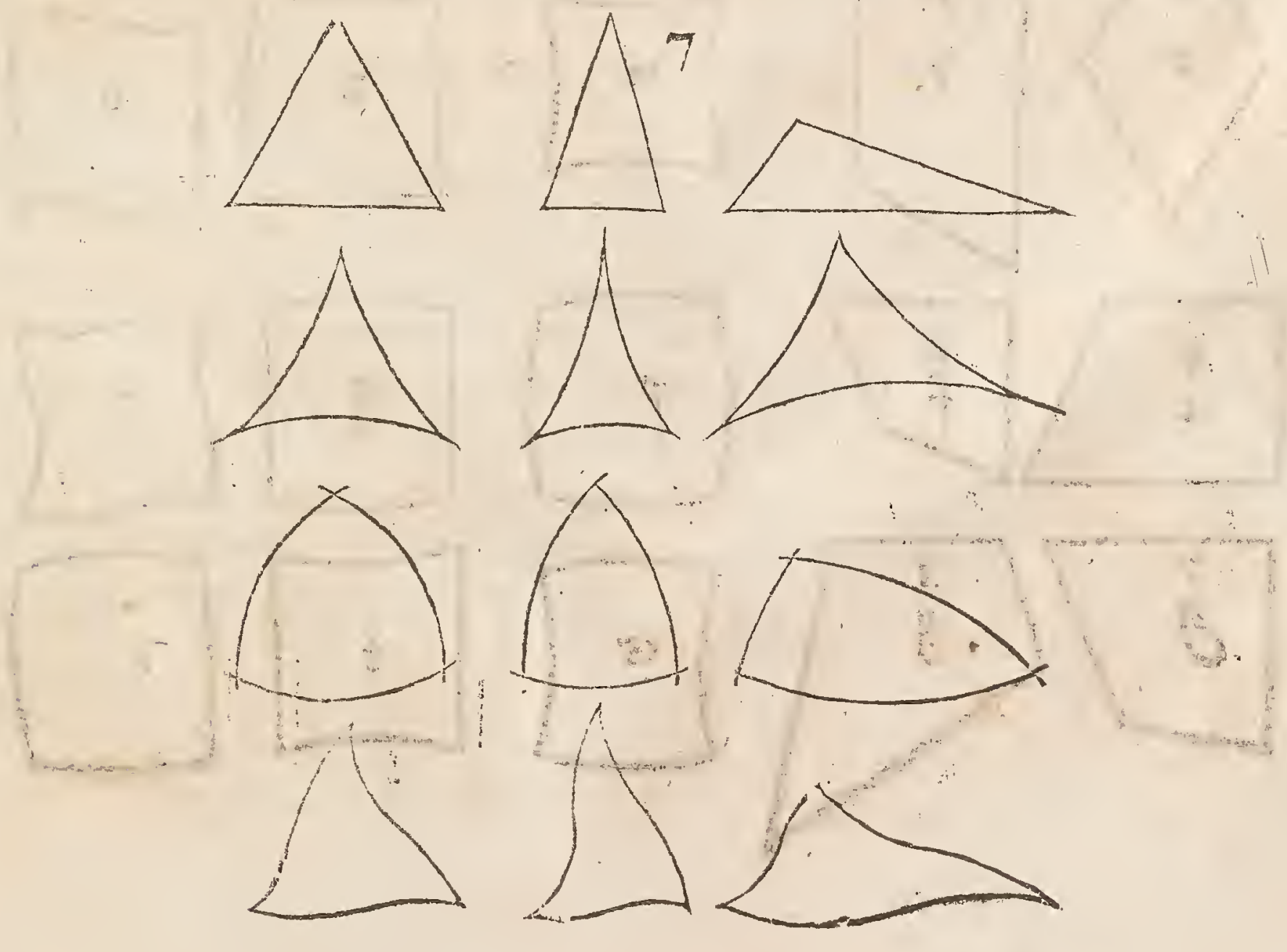


Am fciendum eft, quando quatuor linex reex, qua omnes habent eandem longitudinem, applicantur ad angulos rectos, efficiunt quadratum quod fit a. Deinde funt et1am aliæ figuræ quadrăgulæ, at nó xqualium la terum: vna fcilicet eft quatuor æqualium angulorü, $f e d$ duo latera oppofita funt longiora reliquis duobus, hxc efto b. Tertium quadră gulua habet quatuor latera equalia, \& duos angulos oppofitos, equales inter fe: duobus veró reliquis oppofitis in $x$ quales, qualis eft figura c. Quartum quadrágulum eft fimile ei de quo iam diximus, fed habet duo latera oppofita longiora aliis dnobus oppofitis vt eft quadrangulum $\mathrm{d}$. Quintum quadrangulum habet duos angulos rectos, duo latera xqualia, \& duos angulos inequales ac reliqua duo latera vnum maius altero, veluti in quadranguloe, apparet. In fexto quadrá gulo eft angulus reetus, duo latera xqua, duo reliqua inæqualia duobus prioribus, fed equalia inter fe, ea poffumus aut breuiora aut longiora facere duobus aliis rectum angulum continentibus: hoc quadrăgulum vno recto, duobus acutis, quarto obtufo abfoluitur angulis, quemadmodum in f, viderelicet. Rurfus eft quadrá gulum vnius anguli recti, trium qqualium laterum, $\&$ iterum in ६qua lium angulorum vt manifeftum fit in figura $g$. Poftremò eft quadrangulum quatuor inæequalium laterum, \& angulorum, fitóp illud h. Huiufmodi quadran gula multifariam fieri queunt vtpote lineis curuis, in interiorem vel exteriore partem flexis, quemadmodum infernè diuerfis modis deliniaui fecundú alpha: betiordinem.
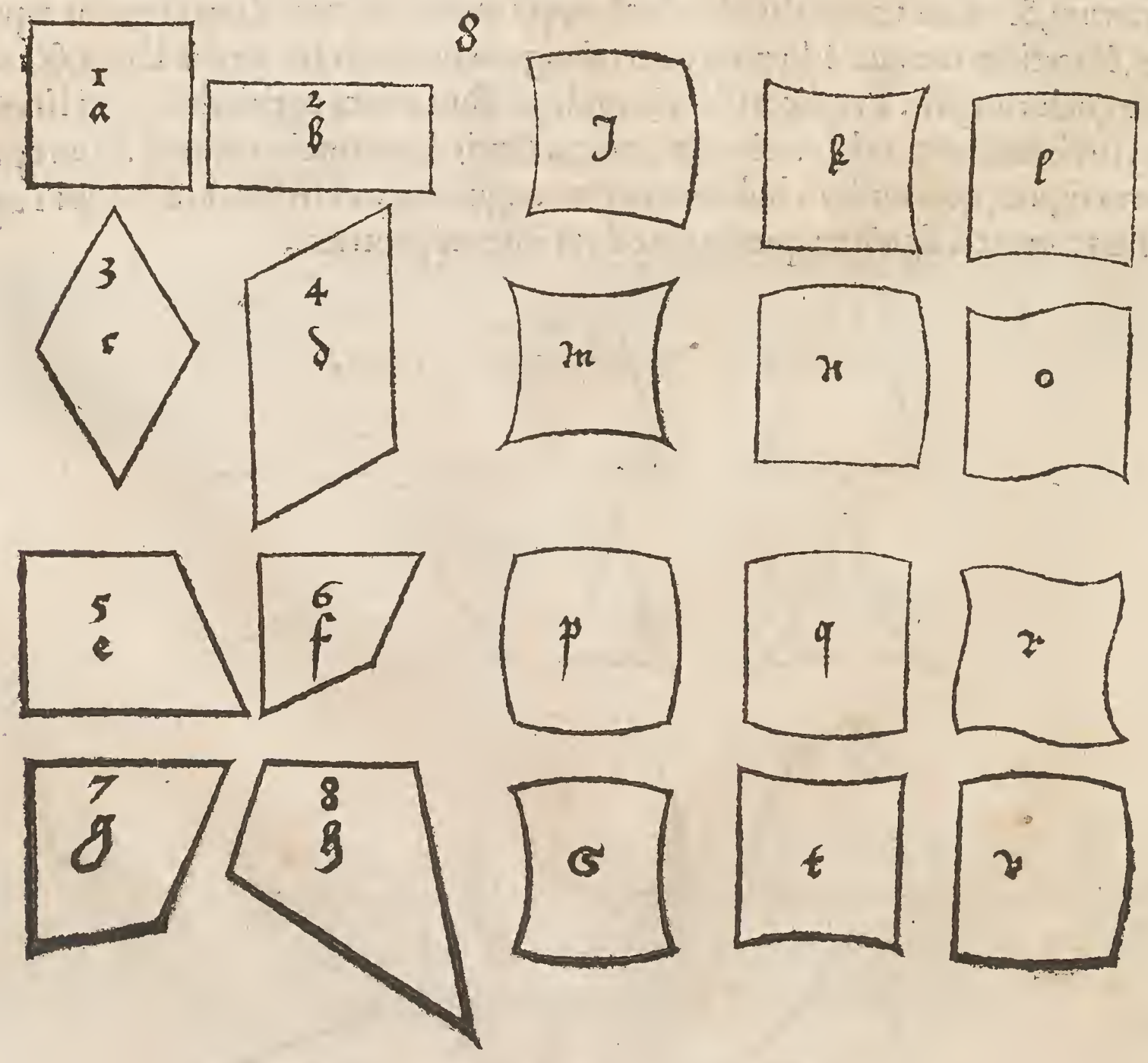


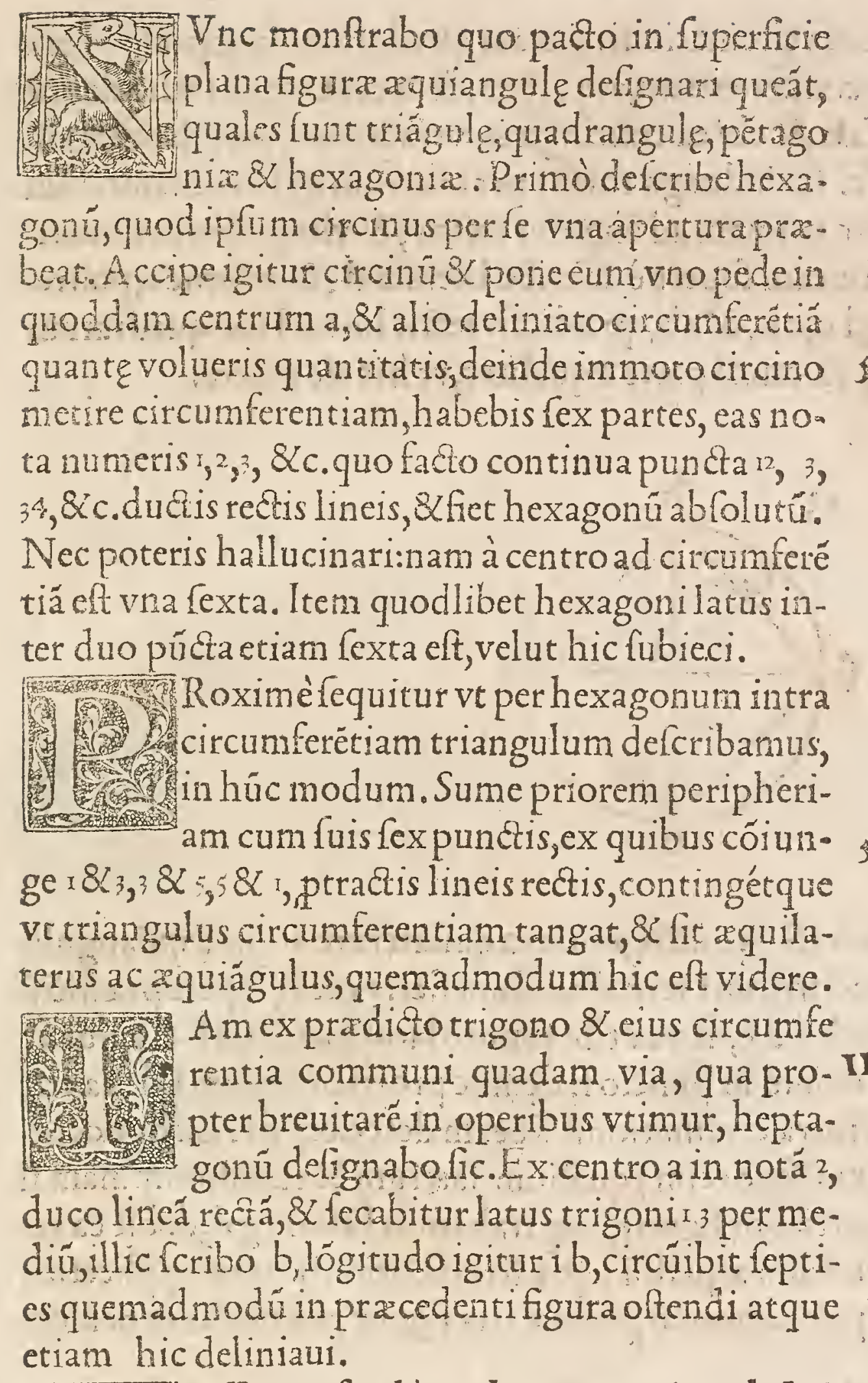

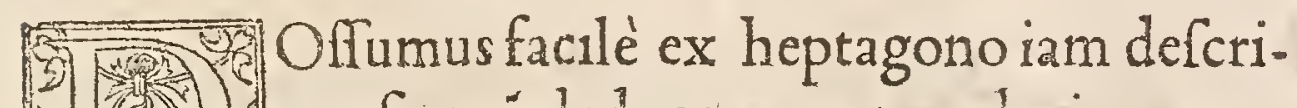
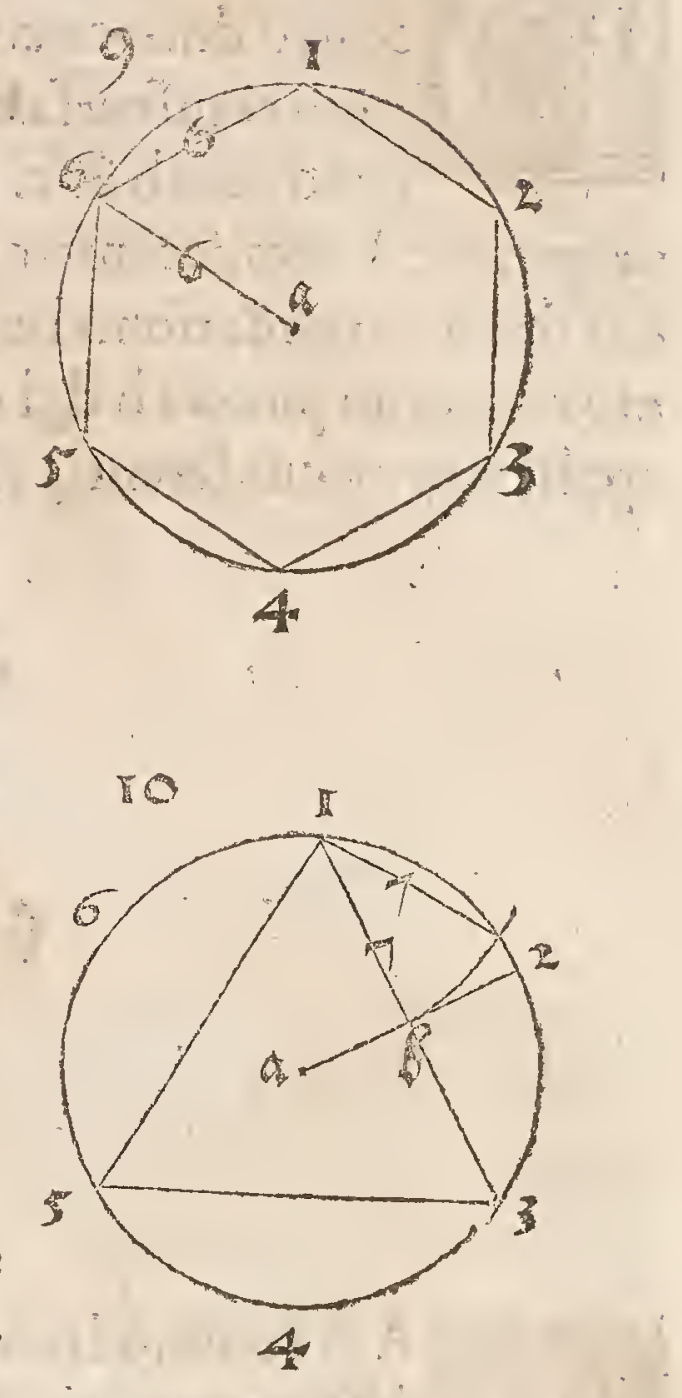

1. ${ }^{-}$pto figură deducere quatuordecim \&qua-

(3) (3) lium laterum, atque angulorum, in hunc modum. Accipe arcum circuli heptago - I2 ni inter $\&$ \& ac diuide eum bipartitò, cum hac longitudine metire circumferentiam, fiéntque in ea quacuordecim puncta, qux per lineas rectas coniun-12 ge, 2 habebis figuram quatuordecim xqualium laterum, \& angulorum, vt hic manifeltè apparet . Ex hac fuperficie per predictam artem potelt fieri alia 8 laterum atque angulorum xqualium.

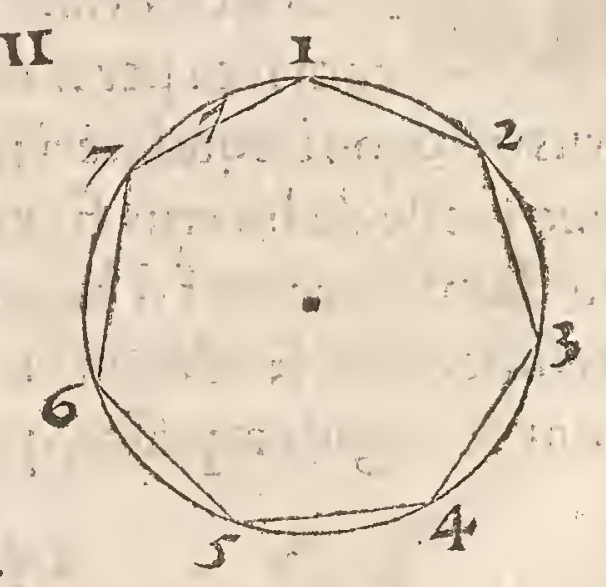

\section{E $i i i$}


Efcribo nunc intra circinationis lineam quadratum hoc pacto . Ex a 7. 16. centro duco circumferentiam, atque per idem centrum a, traho diame 2 trum tranfuerfam, $\&$ vbi ea contingit in vtraq parte peripheriam, illicadiicio $b \& c$. Deinde ago rurfus per centrum a aliam diametrum perpendicularem, fecantem priorem ad angulos rectos, $8 \mathrm{vbi}$ pofterior dimetien s fcindit rotüdationis lineam fupernè, afcribo literam d, \& infernè e, poftremò con tinuo puncta $b \mathrm{~d}, \mathrm{~d} c, \mathrm{ce}, \& \mathrm{e} b$, predictis rectis lineis. Hoc quadratum applicatur circunferentie ex omni parte aqualiter, vt hic eft videre.
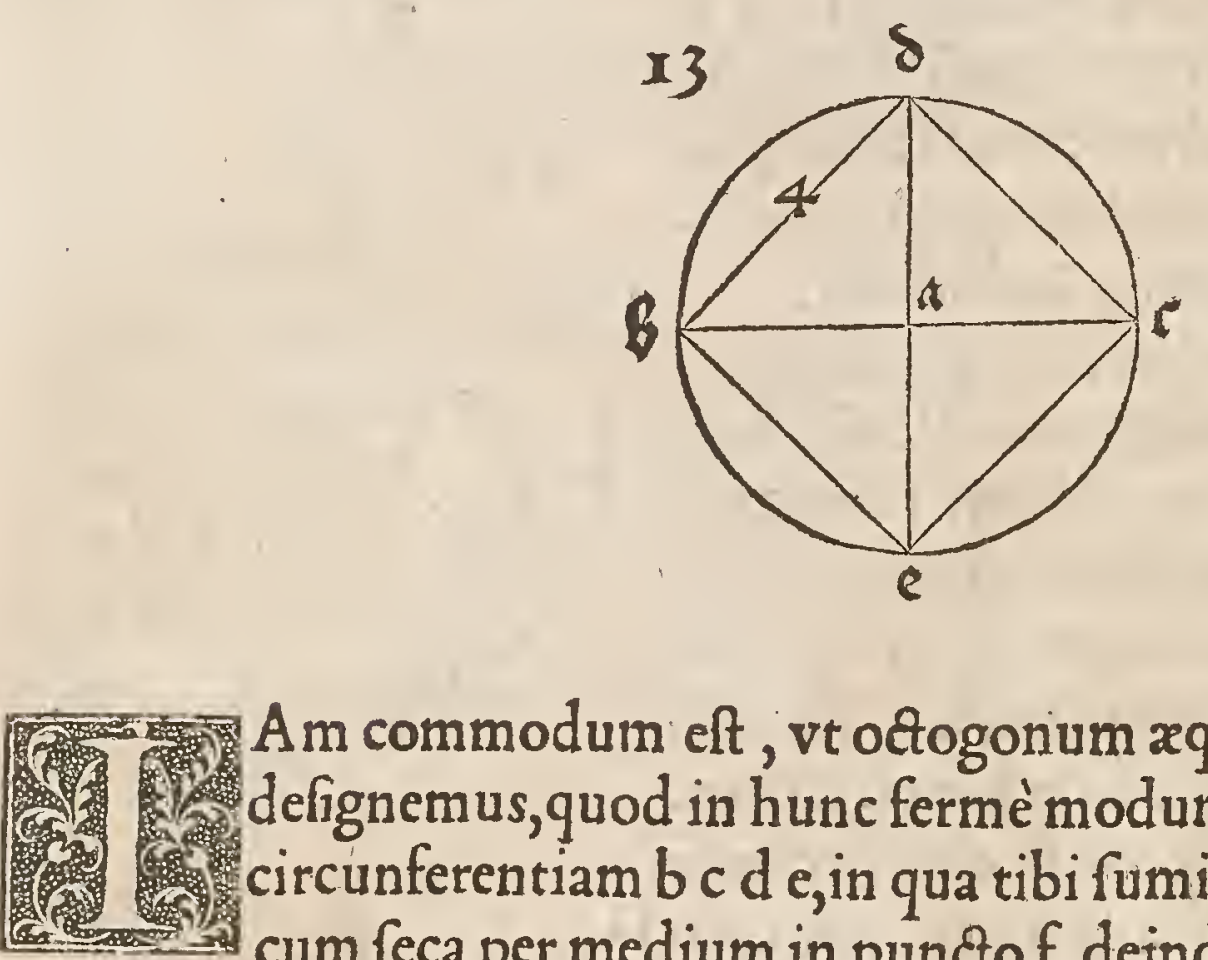

Am commodum eft, vt octogonum xquilaterum atque æquiangulú defignemus, quod in hunc fermè modum fieri foteft, repete predictá circunferentiam $b c d$ e, in qua tibi fumitolatus quadrati b d, cuius ar cum feca per medium in puncto $f$, deinde protrahe lineam $f d$, ea erit latus oetogoni $x$ quilateri in tra circinationis lineam def cribendi . Ex hoc octogono facile deducetur figura fexdecim equalium laterum $\&$ angulorum:nam fi diuidamus arcum $\mathrm{fd}$ bipartitò in figno $\mathrm{g}$, at $_{3}$ trahamus lineam $\mathrm{g}$, dabit ea la. tus fuperficiei fexdecim laterum $x q u a l i u ̈$, qux fi rotúdationis linex applicetur, erunt $\mathcal{Q}$ anguli $æ$ quales, quemadmodú pxim $x$ tres figuræ ponunt ante oculos.
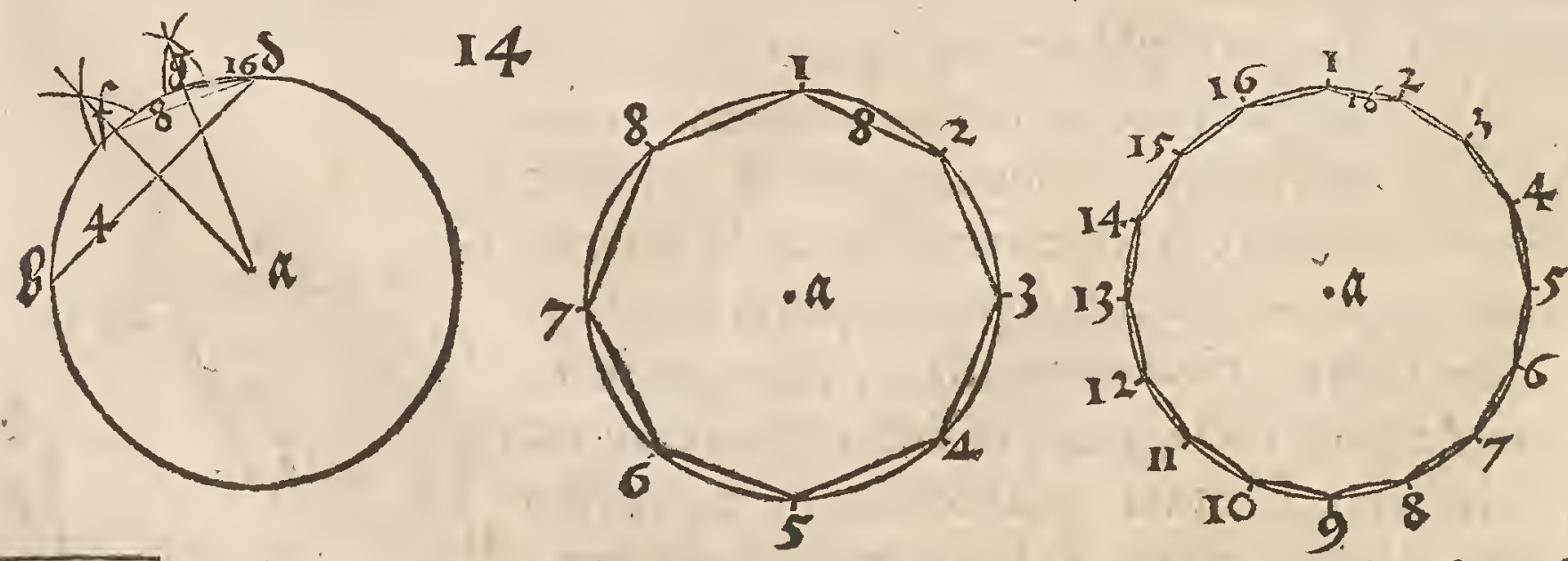

Equitur nunc monftrare quo pacto pentagonus intra circulum defi-
gnari poffit, quod fic expediemus. Ex quodam centroa, defcribito cir
cumferentiam, in qua diametrum ductranfuerfam, que vbi fecat in
vtraque parte peripheriam, adiicito b $\&$ c.Deinde trahito quoque di
metientem perpendicularé ipfit tranfuerfo ad angulos rectos, \& fectioné illius fu
pernè cú linea rotǘdationis,nota litera d.Poftea partire a cfemidiametrú p me
dium 
diú, fitóg púctus partitionis e.Iá centro e, interuallo verò e đ, liniato arcủ à figno $\mathrm{d}$, vique ad lineam a b, quam vbi abfcindit, fac notam $\mathrm{f}$, eam continuatocum $\mathrm{d}$, producta linea $\mathrm{fd}$. Hac longitudof $\mathrm{d}$, exit latus pentagoni intra circination is $\mathrm{li}$ neam ftatuendi. Item $\mathrm{f}$ a, latus dabit decagoni equilateri 8 : $x$ quiangul. Si nunc ex figno e, linea excitaretur vfo ma circumferentix pars mechanicè, vt hic videri poteft.
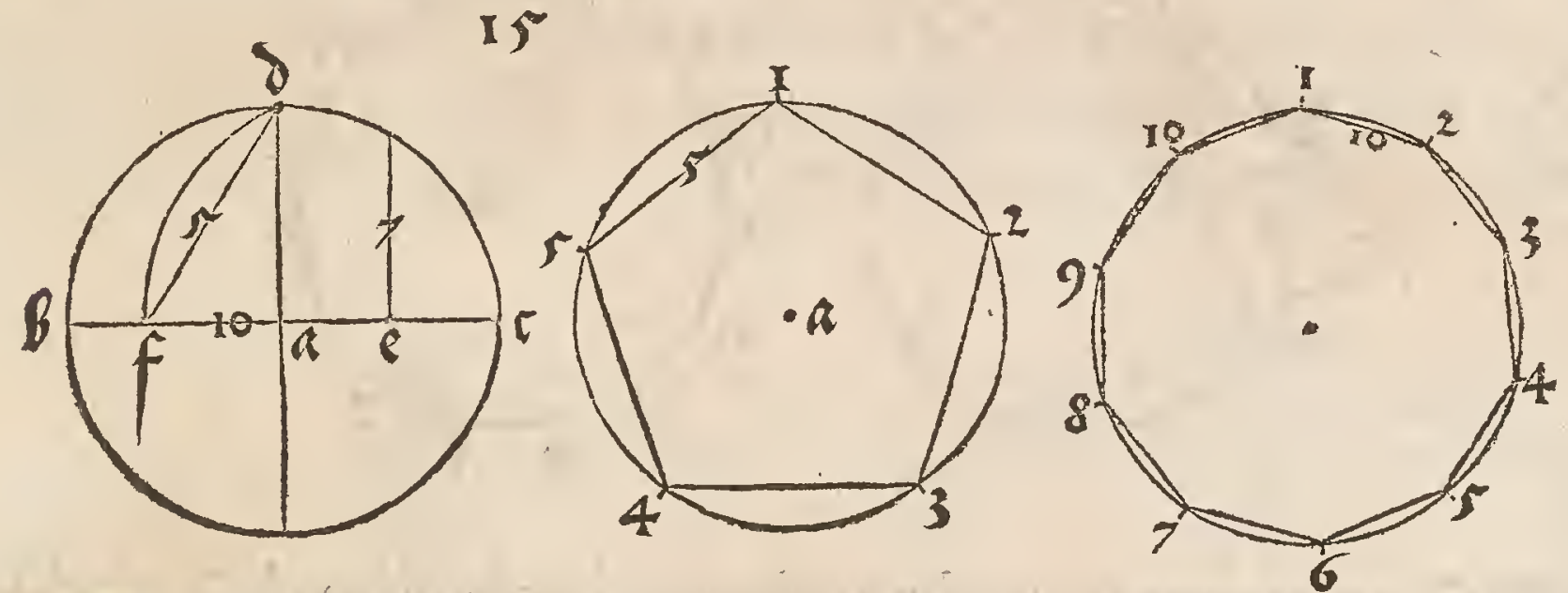

(1) Am pentagonum conftruere docebo vna circini apertura, hoc qui fequitur modo. Efto linea a $b$, vnum pentagonilatus, cuius extremitatë a, facio centrum $\&$ ad interuallum a b, defcribo circulum:rurfus cen tro $b$, fpacio verò $b a$, delinio alium circulum fecantem priorem, fupernè quidem ad $c$, infernè verò ad $d$, qua duo puncta linea recta coniungo. Nunc fuper centro d, protendo arcum, per vtriufque circuli centra $\&$ circunferé tias, quas vbi abfcindit noto per ef, item fectioni ipfius $\alpha$ linex $\mathrm{cd}$, adiicio $\mathrm{g}$ literam. His perfectis cótinuolineam e $g$, verfus $g$, vf́que in peripheriam a $\mathrm{g} f \mathrm{~d}, \alpha$ vbi eam contingit, illic fribo h. Confimiliter etiam produco lineam $\mathrm{fg}$, donec cadat in circinationis lineam b c ed, \& locum contactús figno litera i. Deinde duco lineas a i, \& b h, habeóǵ tria pentagoni latera, reliqua duo applico perpen dicularid c prolongate, $\&$ terminis i $\&$ h, quo facto erit pentagonus abfolutus, velut hic defignaui.
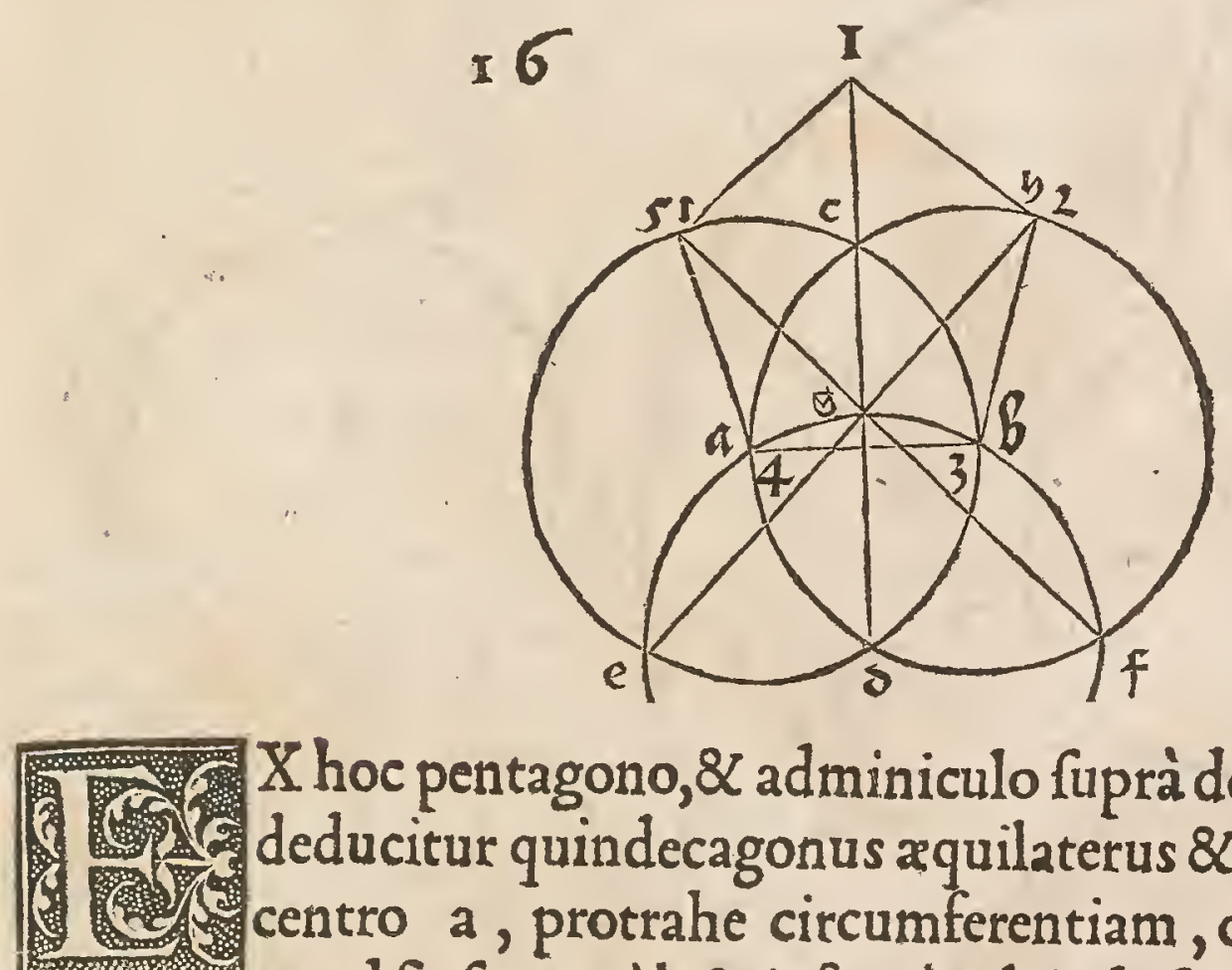

$X$ hoc pentagono, $\&$ adminiculo fuprà defcripti trianguli,commodè deducitur quindecagonus æquilaterus \& æquiangulus, hoc pacto. Ex centro a, protrahe circumferentiam, cui applica latus trianguli, quod fit fupernè $b, \&$ infernè $c$, deinde fumelongitudinem lateris pen tagoni, quam pone vna extremitate fuper $b$ punctum, \&aliam iunge periphe- 
riz, inter $b \& c$, ac locum contactus fignatolitera $d$, quo facto remanet inter $c$ $\& \mathrm{~d}$, arcus quidam, quem diuide per equalia in puncto e. Iam $\mathrm{fl} \mathrm{ed}$, puncta cốt nuaueris per lineam rectá, habebis latus quindecagoni intra circulum defignandi,quemadmodum hic deliniaui.
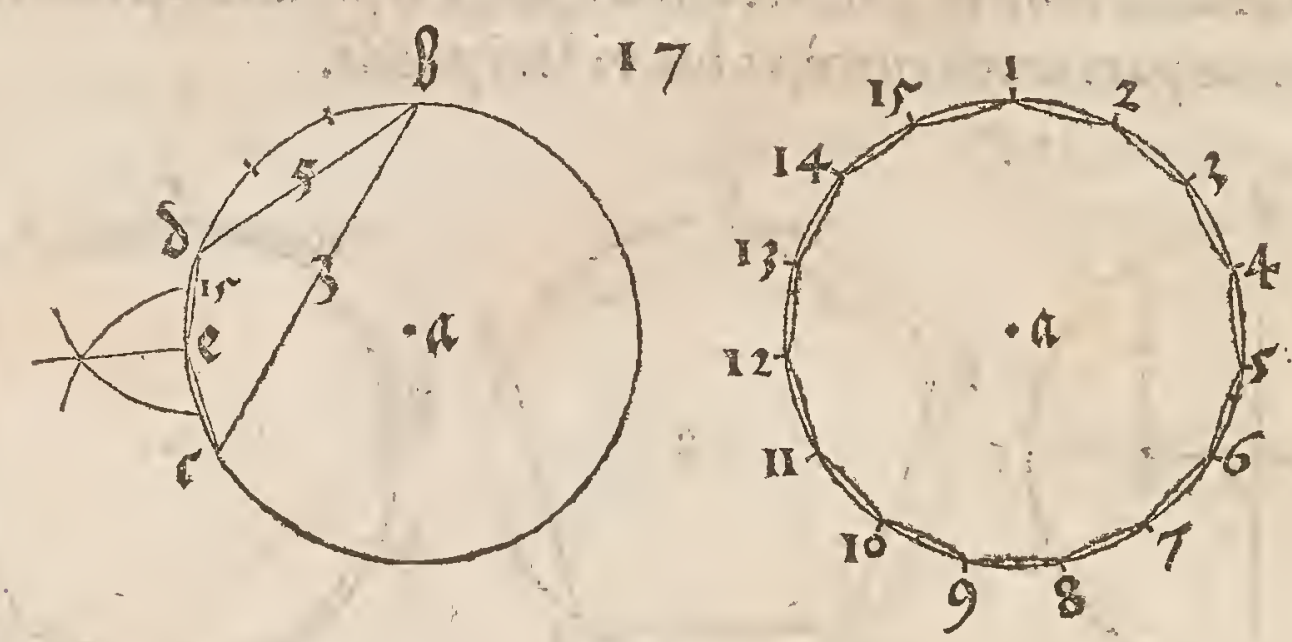

\begin{abstract}
5. 1. U. centro a, circulu fatis amplum, in quo defigna circino inuariato tres 5. pifcium veficas, quarum fuprem $x$ terminus in circumferentia efto b; 1. reliquarum ad latera fint c.d. Hoc facto protrahelineam rectam a $b$, eam duideper puncta \& 2 , in tria fpacia zqualia, fic quòd 2 fit propiusipfia:per hoc fignum , age lineam tranfuerfam ipfi a $\mathrm{b}$ perpendiculari ad angulos rectos, \& vbi hac tranfuerfa linea vefica in vtraque parte abfindit, illic nota e \& $\mathrm{f}$. Iá fume circinum, cuius locato pede vno in cetro a, alio ex frgnoe,per ipfum $\mathrm{f}$ duc rotund ationis linea m, circuibitque longitudo ef, nouies intra hanc peripheria quod hic etiam fubieci.
\end{abstract}

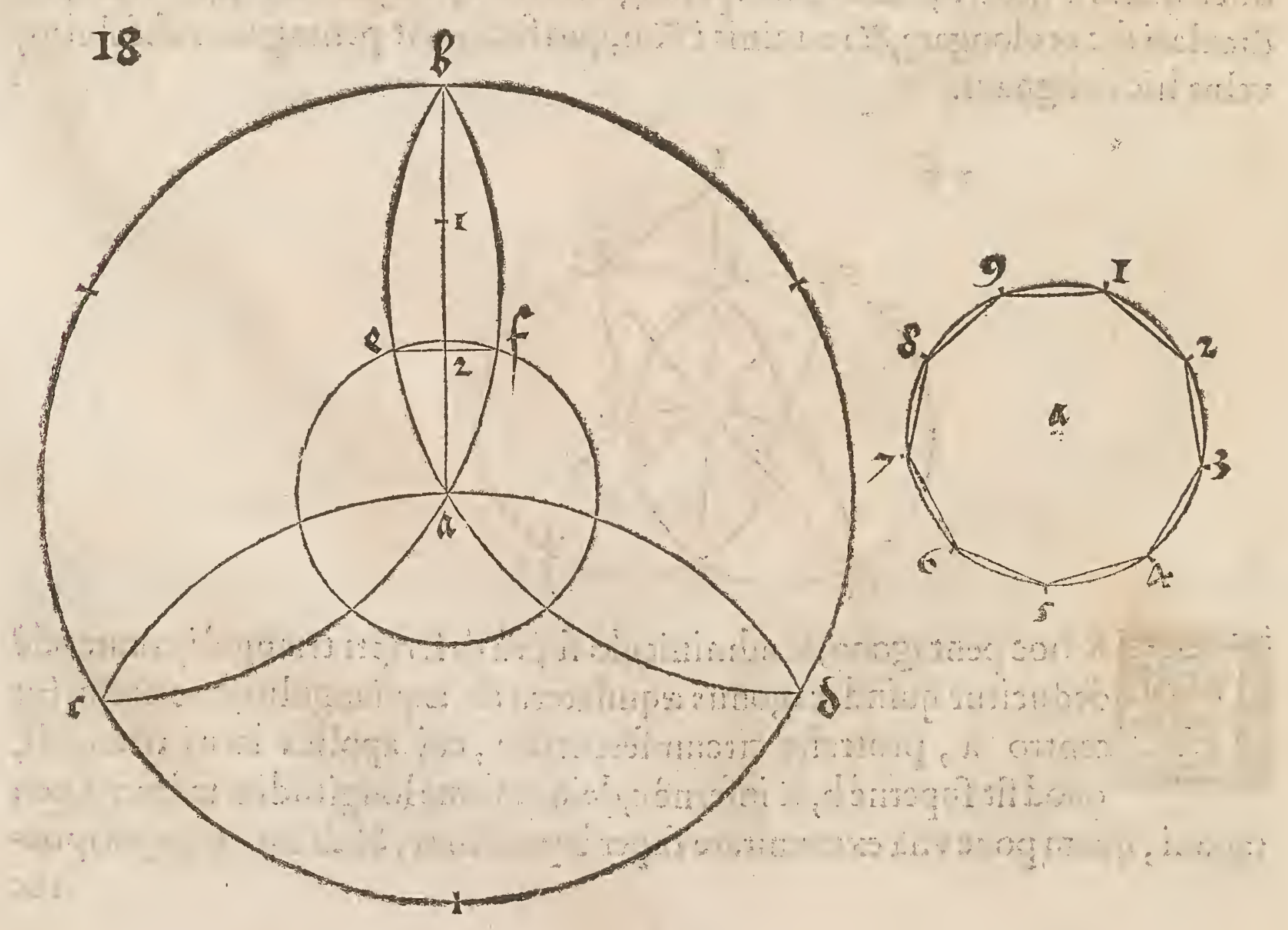


Vum promptè hendecagonum intra circulum defignarecupio, fumo (1. 4 quartam diametri partem cui adiungo octauam eiufdem quart $\&$ (1) cum hac longitudinecircumeo intra circumferentiam, quam fic pra1. cifèadmodum metior, ita quòd mechanicè non autem demóftratiuè id quod propofitum erat, inueniatur. Quòd fi circulo figura tredecim laterum atque angulorum $x$ qualium infcribenda fuerit, tunc ex quodam centro $a$, circu lum fcribo in quo excito femidiametrum a b, quam per medium feco in puncto $\mathrm{d}$, quo facto vtor longitudine d c, qua tredecies intra peripheriam circumeo, fed hoc quoque mechanicè, $8 \mathrm{k}$ non demonftratiue verum effe credendum eft.

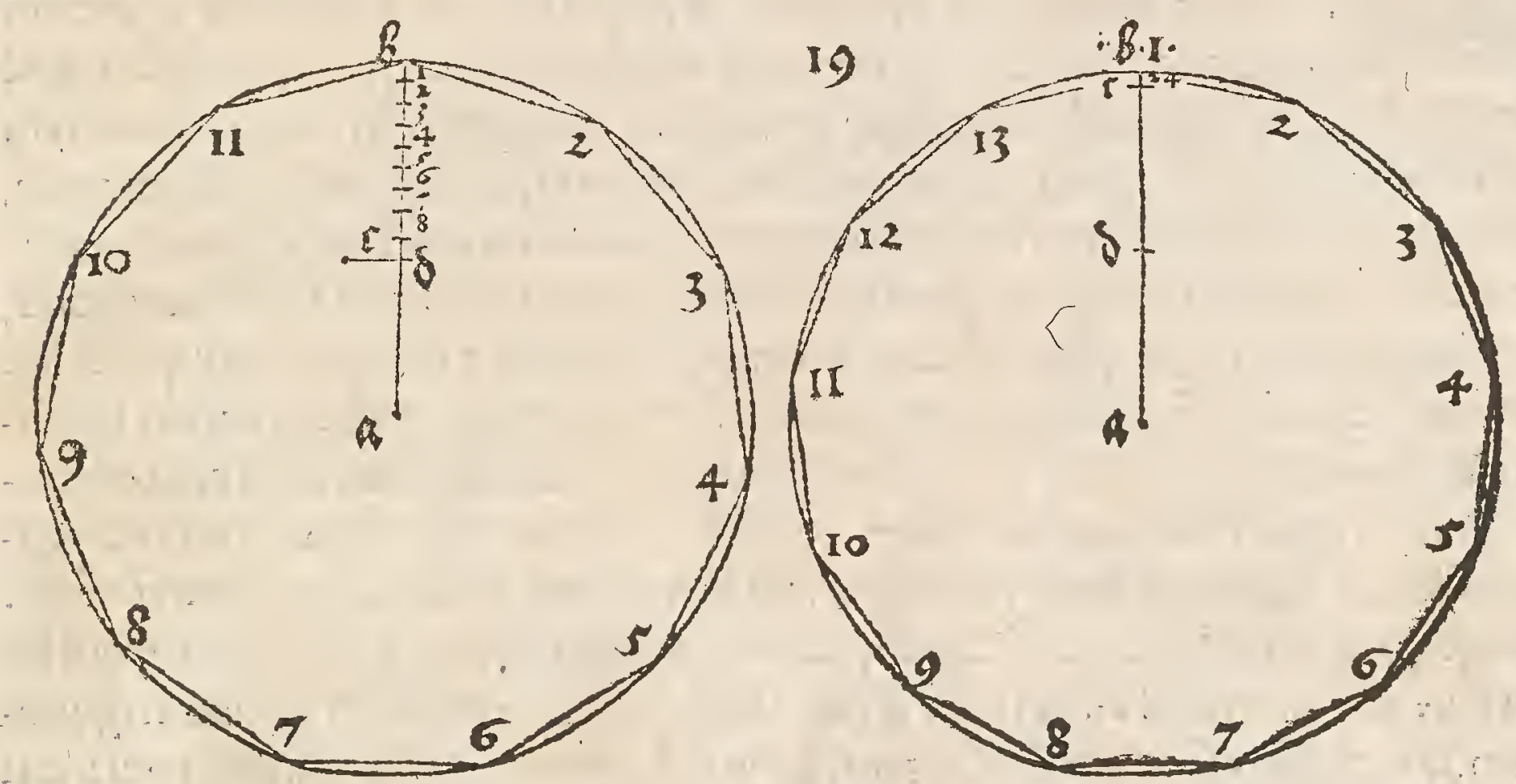

Vemlibet circuli arcum, qui mihi proponitur, diuido in tres partes:
hoc pacto. Arcus ille efto a $b$, cuius extremitates fint coniunct a recta
linea, quă vt fuprà docui, feco duobus punctis $\mathrm{c} \&$ d, in tria interualla
qqualia, deinde pono circini pedem in fignum a, $\&$ alio protendo ex $c$, lineam curuam, vfque in arcum $a b$, quem vbi abfcindo, llic fcribo literam $e$. Poftea centro $b$, interuallo veró $b d$, arcum delinio vqque in ipfum a $\mathrm{eb}$.arcú, $8 \mathrm{vbi}$ is fecatur, adiiciof. Hoc facto erigo duas lineas perpendiculares, ex púctis $c \& d$, qux vbi cadunt in arcum e f.noto $g \& h$. Sic erunt longitudines a $e, g h$, $\& \mathrm{fb}$, in dato arcu a b, xquales, $\mathbb{Q}$ remanent duo fegméra eg $\& \mathrm{~h} f$. Iam circini pede fixo in a, alio duco arcum ex $g$, in lineam $\mathrm{cd}, \&$ vbi ea fecatur, pingo i lite ram. Rurfus ex centro b, excito arcum perh $\&$ rectam i d, \& ei fectioni in linea $\mathrm{i} d$, adfcribo $K$. His perfectis partior $c i, \& K d$, in tria fpacia \&qualia, \& pede circi nifito in a, reliquo ex puncto, qui propior eft ipfi $i$, arcum circino vfque ad fegmentum e g, vbi figno fectionem litera l. Poltremò facio b centrum, ac ex diui fione, que eft proximior ipfi $K$ arcum delinio verfus $h$, inter qua ad terminum eius arcus frribo $\mathrm{m}$. Hac arte datus arcus a b, partitus elt per púctal $1 \& \mathrm{~m}$, in tria zqua in terualla, quemadmodum hic oculis fubieci. Sed qui hoc exactiùs expe. dire vo!et, quęrat demonftationem. 


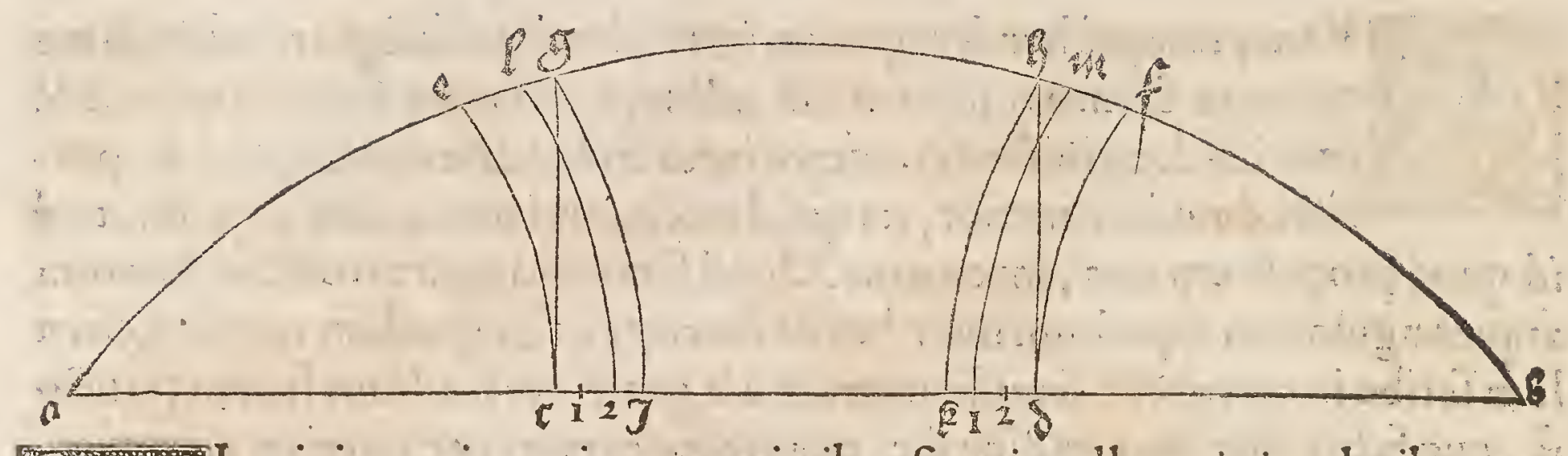

7. I quis in pauimentis a ut parietibus figuris vellet vti circularibus, eas 12 duobus modis inter fe poterit applicare : Primò quidem per quadra16. 1 ta, deind e etiam per rhombos. Perquadratum hocfermè agendum erit modo. Defcribe quadratum æquilaterum atque equiangulum, $\&$ feca id duobus perpendicularibus, ac totidem tranfuerfis in nouem quadrata xqualia, in quibus fingulis quære punctum medium, in quo polito circini pede, alterum extende tantum, vt omnia omnium quadratorum latera queat contin:gere, ac fic delinia in fingulis quadratis cirumferétias, ipforum latera tangentes, atop ita vnus circulus quatuor alios attinget. Remanent quoque inter quoflibet quatuor circulos figura quædam quadrangulares, quarum latera verfus interiorem partem funt flexa. Porrò circuli quoque in rhomborum modum coniunguntur, $\&$ tunc refiduæ erunt inter quoflibet tres circulos fuperficies triangula res, latera habentes flexa verfus, partem inferiorem. Hoc ita expedire cóuenit. Erige quadrangulum 1.2 .3 .4 . altum, quatuor triangulorum æquilaterorú, quilateribus $\&$ conis rectè vnus fuper alium ftent: latum verò trium talium trigonorum, qui angulis fe inuicem tangant, fic quòd totum quad rangulum contineat vigintiquatuor triangulos integros, ex integris $\&$ dimidiis collectos:at integrorum quidem angulos nota literis Alphabeti vfque ad $r$, quo facto, pone circinum vno pede in puncta literis fignata, $\&$ alium diftende per dimidium triăgu li latus \& fribe circulos; \& femicirculos quotquot poteris, habebifque feptem integros $\&$ to. dimidiatos, qui omnes faciunt duodecim integros. Et vbi huiufmodi circuli multi applicantur, vnus alii, coniungút femper fex feptimum. Pof fumus etiam circinationes multifariam inter fe $Q$ diuerfa opera ex eis cóficere: harum aliquot fed eiufdem form e defignabo, ex quibus multa alia deduci queant. Ex centro a defcribo peripheriă, ea diftribuo in duodecim partes equales, $\&$ ex fingulis partitionum punctis inuariato circino circumferentiam linio tranfeuntóg duodecim illi circuli per decimitertii centrú a, ex quo rurfus extra primú circulum per fectiones (qua funt $\mathrm{c} d \mathrm{e}$ ) duodecim aliorú ptraho adhuc quatuor circulos maiores. Qui in circulo ftella fex radiorum voluerit defignare, is immoto circino id hoc pacto expedire poterit. Ex centro a, excitetur circü ferentia in qua ad fignum $b$, figatur circin us vno pede, $\&$ reliquo protendatur arcus per centrum a in vtranque partem peripherie, quam vbi fecuerit, fcribatur $\mathrm{h} \& \mathrm{c}$. Deinde facto centro g, ducatur ex $\mathrm{b}$ arcus per a centrú,vfque in alteram circumferentix partem, vbi noteturf. Poftea locato circini pede in $c$, alio protrahito ex $b$ arcum per ip fum $a$, ad rotundationis lineam in qua fignetur d.Con- 
d. Confequenter ex centro d, fcribaturper $\mathrm{c} \&$ a, arcus vfo ad circinationis $l_{\text {- }}$ neam, vibi ponature. Nunc ex e, ducatur arcusf a d.Poftrenò ex centro f, fribendus eft arcus g a e, \& erit ftella hac abfoluta. Potes tamen filibet, circinú pa rum conftringere, \& paruos circulorú arcus inter literas defignare. Item aliter: ex centro a defcribe circulum, eum diuide quatuor puntis b $\mathrm{d} d \mathrm{e}$, in totidem xqua interualla, ita quòd $b$ fupernè, $\& \mathrm{c}$ mnfernè, $d$ e verò ad latera veniant. Deinde diuide quartam $b d$, per punctum $0, \&$ b e per $q, \&$ e c per punctum $p$, item cd per $r$, quamliber bipartitò. Quo facto fume circinum quem pone pede vno in $b, \&$ alio duc arcum $f$ ag, iterum fige circini pedem in $c, \&$ reliquo protrahe arcum $h$ a i.Poltea ex centro e, arcun deliniato $m$ a $n$. Tandem fixo altero circini pede in d,altero excita arcú $\mathrm{K}$ al. Iam locandus eft circini pes in ofignum, \& altero producendus eft arcus per a centrum, in vtranque circunferentia partem, in quibus inter b $\& \mathrm{~m}$, fcribêda aft litera $s . \&$ inter d $\& \mathrm{~h}$, thitera:facito núc punctum p. centrum, $\&$ trahe per a, circinationis arcum, in ambas peripherix partes, quas nota inter $1 \&$ c.litera $v, \&$ inter $z \&$ e, litera $x$. Pone porró circini pedem in $r \&$ reliquo per a centrum protendito arcum per totam circuli fuperficiem, \& fectiones in rocundationis linea fignato, eam qux eft inter $f \& d$, characterey : aliá verò qua elt inter $\mathrm{c} \& \mathrm{n}$ litera $z$. Nunc ex centro q, producito arcum per a, in circumferentie partem, \& vbi eas contigent, illic vtrin , inter $^{K} \& 2$ $b$, fcribe a ${ }^{2}, \&$ inter e $\alpha^{\prime} i^{2}{ }^{2}$. His perfectis excitato in fingulis foliis acutis, qux percirculorum arcus facta funt, binos arculos hac arte. Sifte circini pedem in púctum $g$ \& alteroliniato arculü à centro a, per foliun quod eft fub Kai, deinde pone vnum circini pedem in fignum $x, \&$ reliquo duc ex centro $a$, fecundum arculum in eodem folio. Sic operare per fingula octo folia acuta, \& quare horum arculorum centra in extremitatibus arcuum qui folia claudunt. Sunt quoque duo ftellarum genera delinianda, quorum alterum ex pentagono, alterum ex heptagono procedit in modum qui fequitur. Defcribe circulum circa pentagonum atque etiam heptagonum, deinde applica pedem circini vni angu lorú qui in circumferentia funt, \& alium extéde in proximos angulos qui funt in vtraq parte, quos per arcum continuato fic circumi per omnes angulos pentagoni \& heptagoni, et videbis quales ftellas he protractiones defignent. Ea qux fupra dicta funt, proxim̨ figurę ponunt ante oculos, anfam q $_{\text {p }}$ præbent inueftigandialtiora:nam $\mathrm{ex}$ circulis $\&$ arcubus earum, rerum diuerfarum inueni id untur proportiones. 

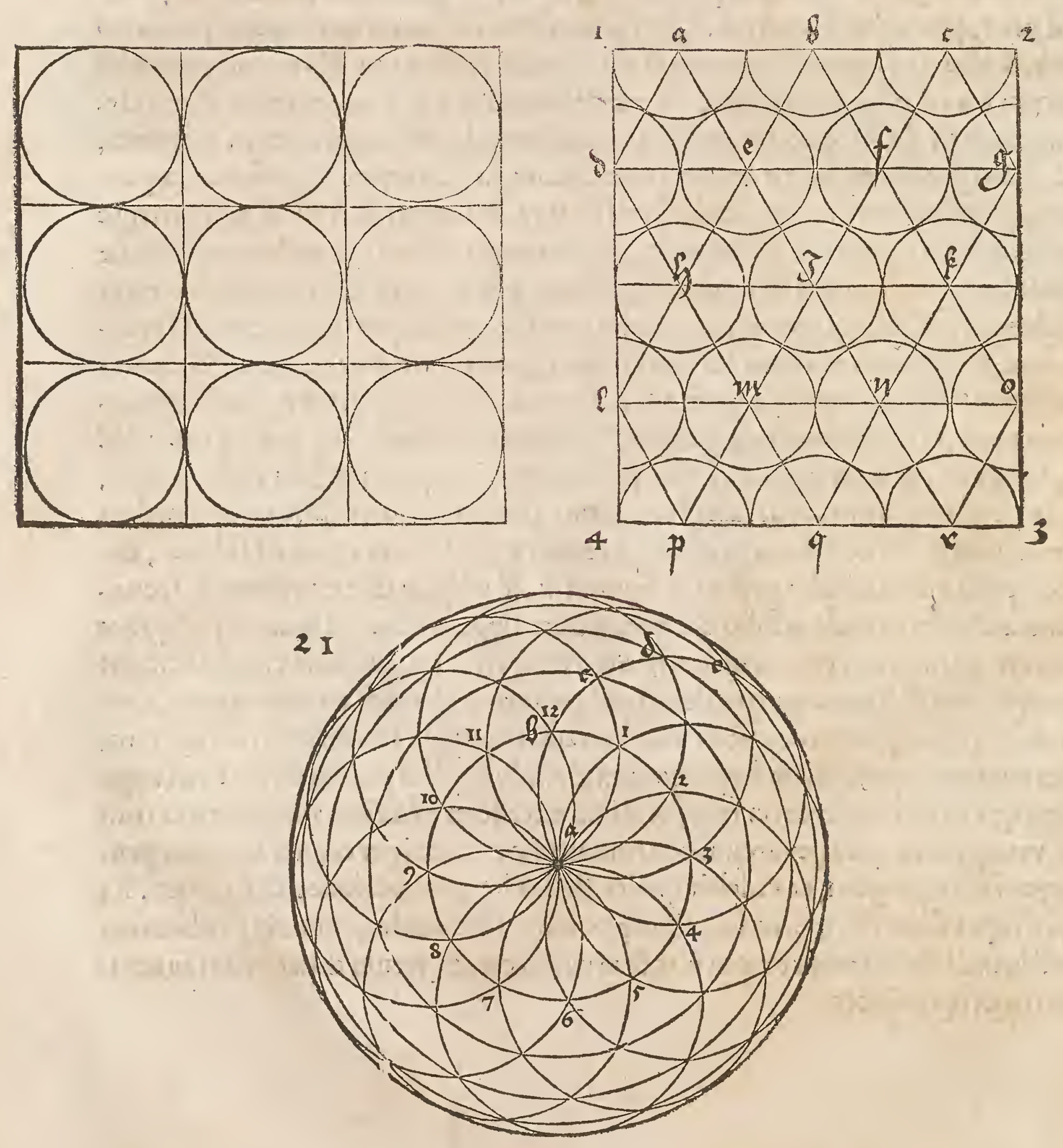

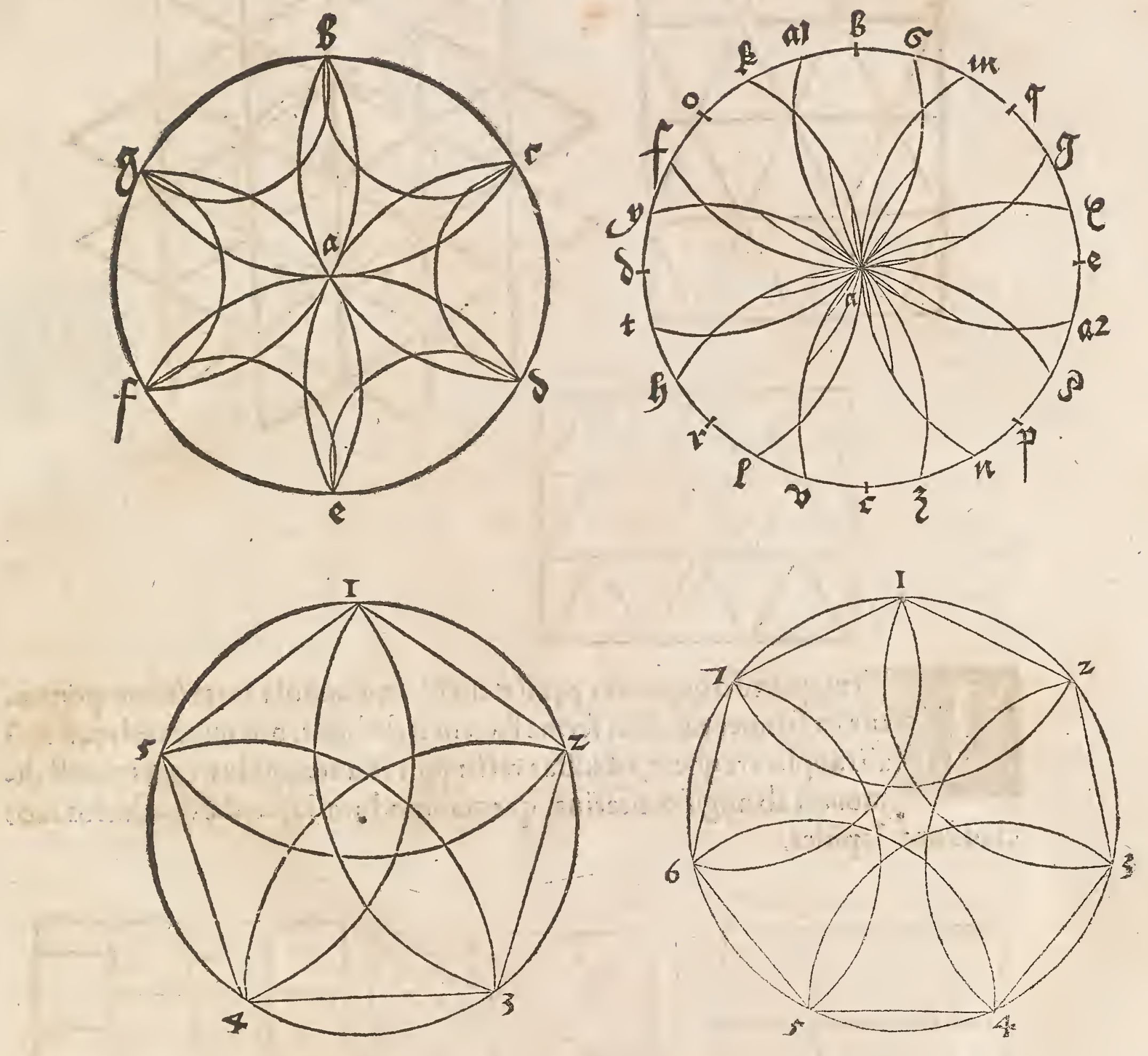

\begin{abstract}
7. Vnc figurarum aliquot angularium in pauimentis docebo dirpoliti onem, \& quanquam in præcedente de triangulis inter circulos fuerit pertractatú, tamen in fequentibus cos extra circumferentias defigina bo, $\&$ alio modo inter fe coniungam, nempe hoc. Ego applico fex tri angulos fuis conis cuidam centro a, deinde necto ad latera exteriora, fingulis fex triangulis adhuc talem trigonum, quibusiuterpono rurfus alios duos, \& lic deinceps producendo triangulorum latera, \& erunt nou trianguli. Aliter poffunt adhuc trianguli inter fe difponi, ita quod nullum fpatium inter eos relinquatur, quum fcilicet angulus vnius trianguli medio applicatur lateri alterius trianguli.Quando fex trigoni fuis angulis coniunguntur,tunc efficiüt hexagonum, cui poffumus addere, fil lubet, adhuc alios triangulos.
\end{abstract}



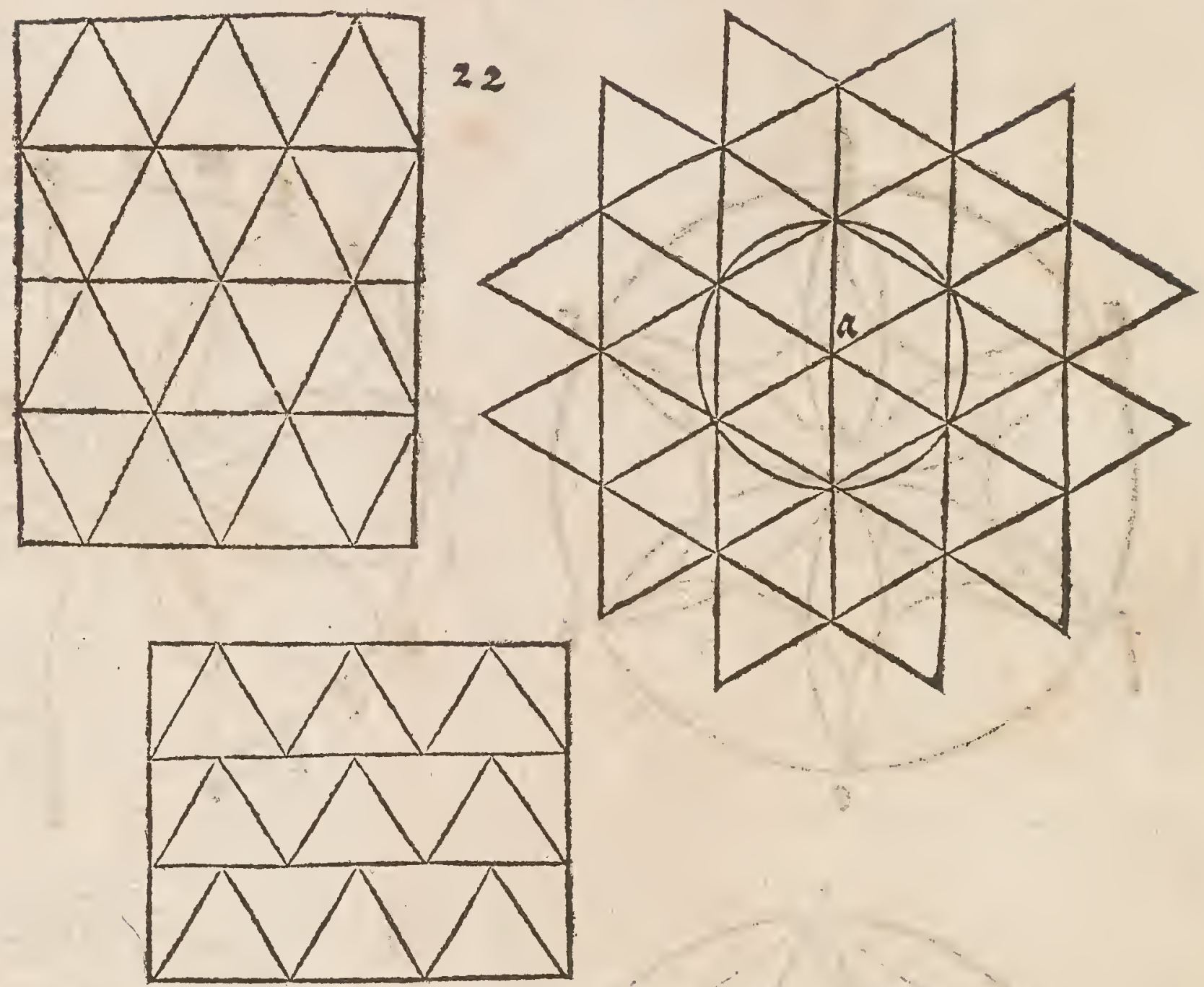

17. Tem quando quadrata qquilatera \& a quiangula inter fe componun: tur,ea femper eandem fermè faciem habebunt, nifi quod obliquê vnú alii applicari queat, ad inftar retis:quin etiam angulus vnius poteft dimoueri ab angulo alterius, quemadmodum lapicidæ quadratos fuos clạudunt lapides.

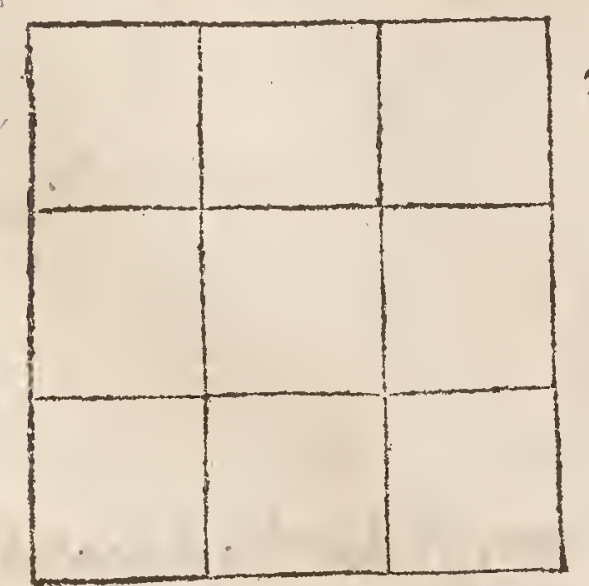

23
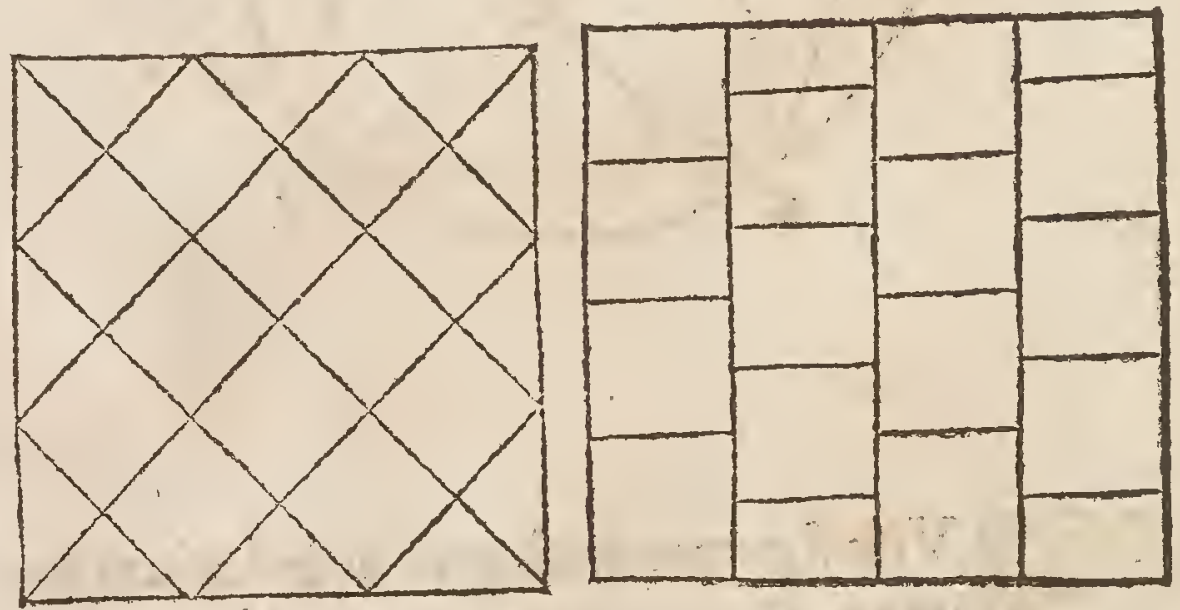

Hombi verò qui duos angulos oppofitos acutos, reliquos duos obtu19 los habent, duplici modo inter fe coniunguntur. Primò feparantur 1 omnes per lineas obliquas:deinde applicantur duo lateribuis fuis, \& tertius ipfis tranfuerféimponitur, habebunt'́g figură depię tefferæ: hoc pacto plures licet difponere. Quando octo rhombi angulis fuis acutis iunguntur, tunc efficient ftellæ formam, qux per fex quoque \& quing fieri poteft, velut hic eft videre. 

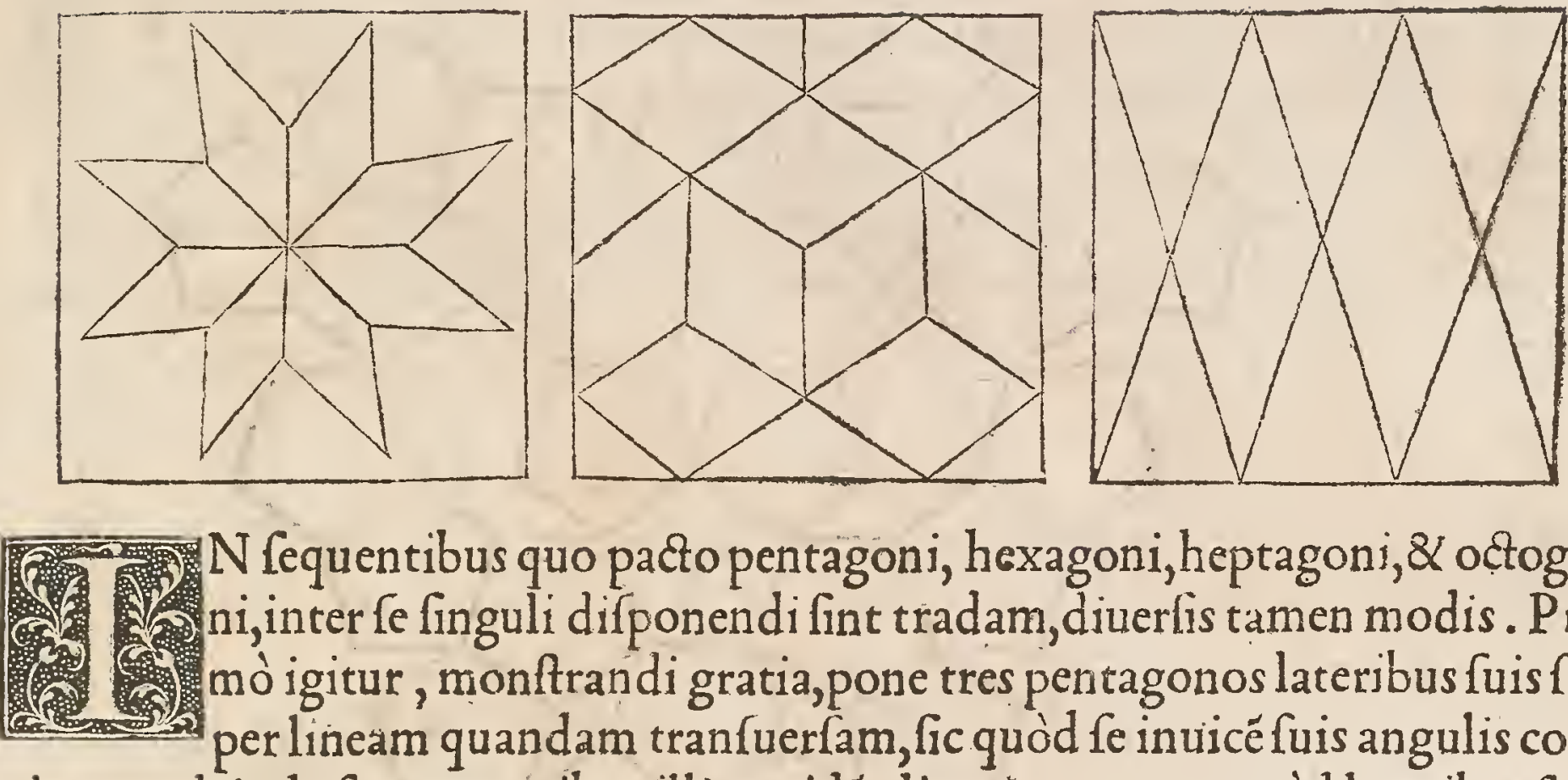

N fequentibus quo pacto pentagoni, hexagoni, heptagoni, 8 octogo ni,inter fe finguli difponendi fint tradam, diuerlis tamen modis. Primò igitur, monftrandi gratia, pone tres pentagonos lateribus fuis fuper lineam quandam tranfuerfam, fic quòd fe inuicéfuis angulis contingant, deinde fuppone tribus illis totidé alios, ita quoque quòd lateribus fuis applicentur lateribus priorum, fintóg omnes in vna linea tráfuerfa locati, quare $\&$ hi fe angulis fuis inuicem tangent, quò fit $v t$ inter pentagonos illos rhombi relinquantur oblongi. Huiufmodi pentagonorum ordinem compone adhuc vnum cuius vertices finbus immitte prioris:hic eft primus modus, atque arctiffima pentagonorú compolitio. Iam compone tales ordines duos quemadmodú priùs factum eft, $\&$ coniunge eos angulis fuis, ita quòd quilibet pentagonus, vno latere $\&$ tribus angulis quatuor alios tangat pentagonos, remanebúntque inter pentagonos 1 llos, duplices Rhombi,erecti admodü longi, $\&$ trăfuerfi breuiores, fed latiores. Hęc cópofitio extendi etiam poteft quátum voluerinuss. Porrò pentagonos in hunc quog modú componere licebit. Primò fifte pentagonum vnum, cui iunge ad fingula latera alium pentagonum æquálem, deinde applica quing illis pentagonis, cuilibet ad duo latera extrema adhuc duos pentagonos, \& fient inter pentagonos illos quinqu rhóbi fatis tenues. Poftea impone pentagonos finibus, qui facti funt in circuitu, fic quòd verticibus fuis tangant rhombos illos tenues, qui priùs formati füt:fic facere perge quoad opus fuerit. Potes etiam quinq rofas ex pentagonis conficere, quę omnes cohæreant, deinde alias quinq inuertere, $\&$ prioribus adiúgere, atq $\beta$ ita deinceps cótinuare $\&$ fpacia in ter eas parergis quffúq libuerit implere ifta omnia hicfunt defignata.
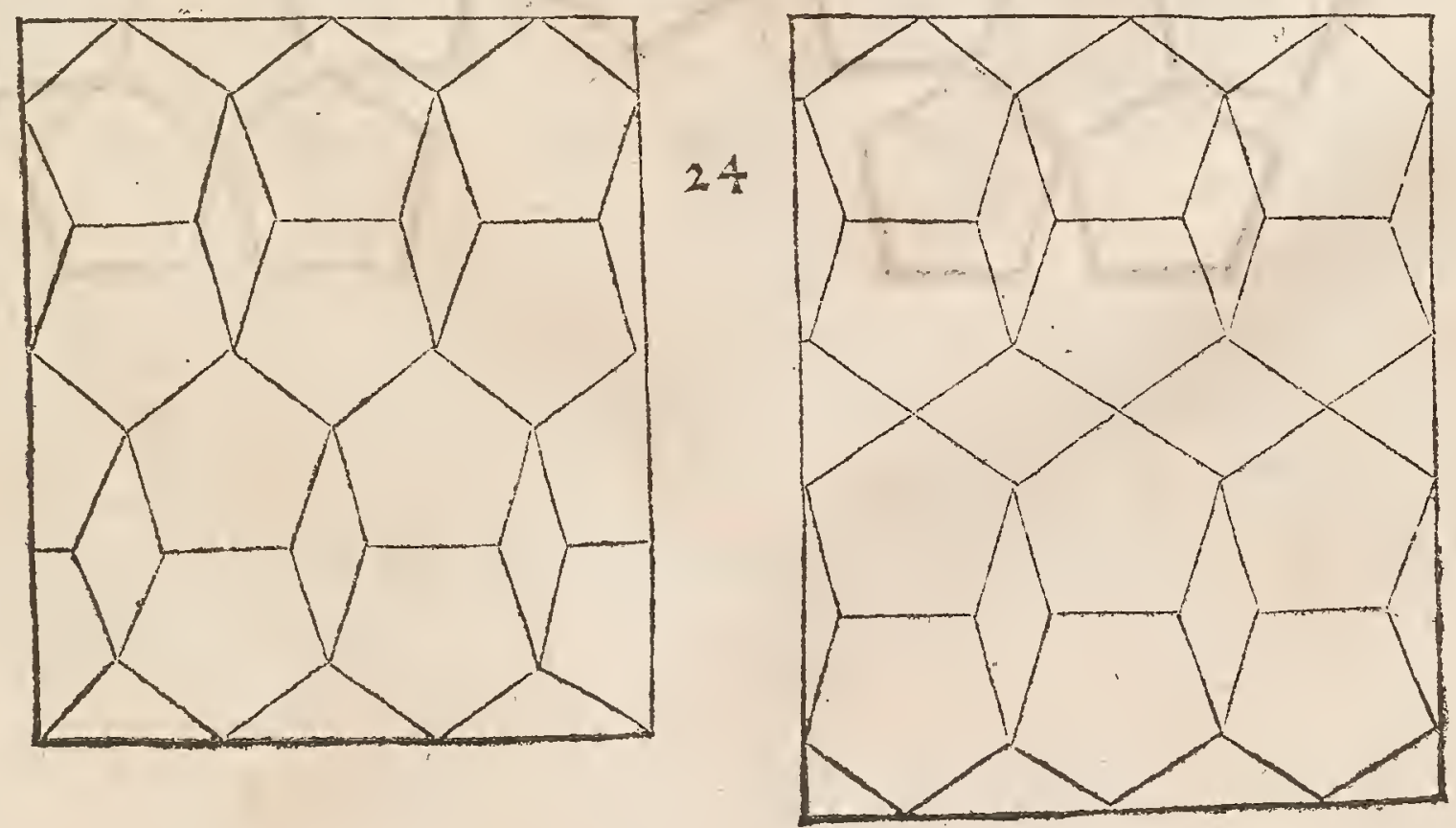

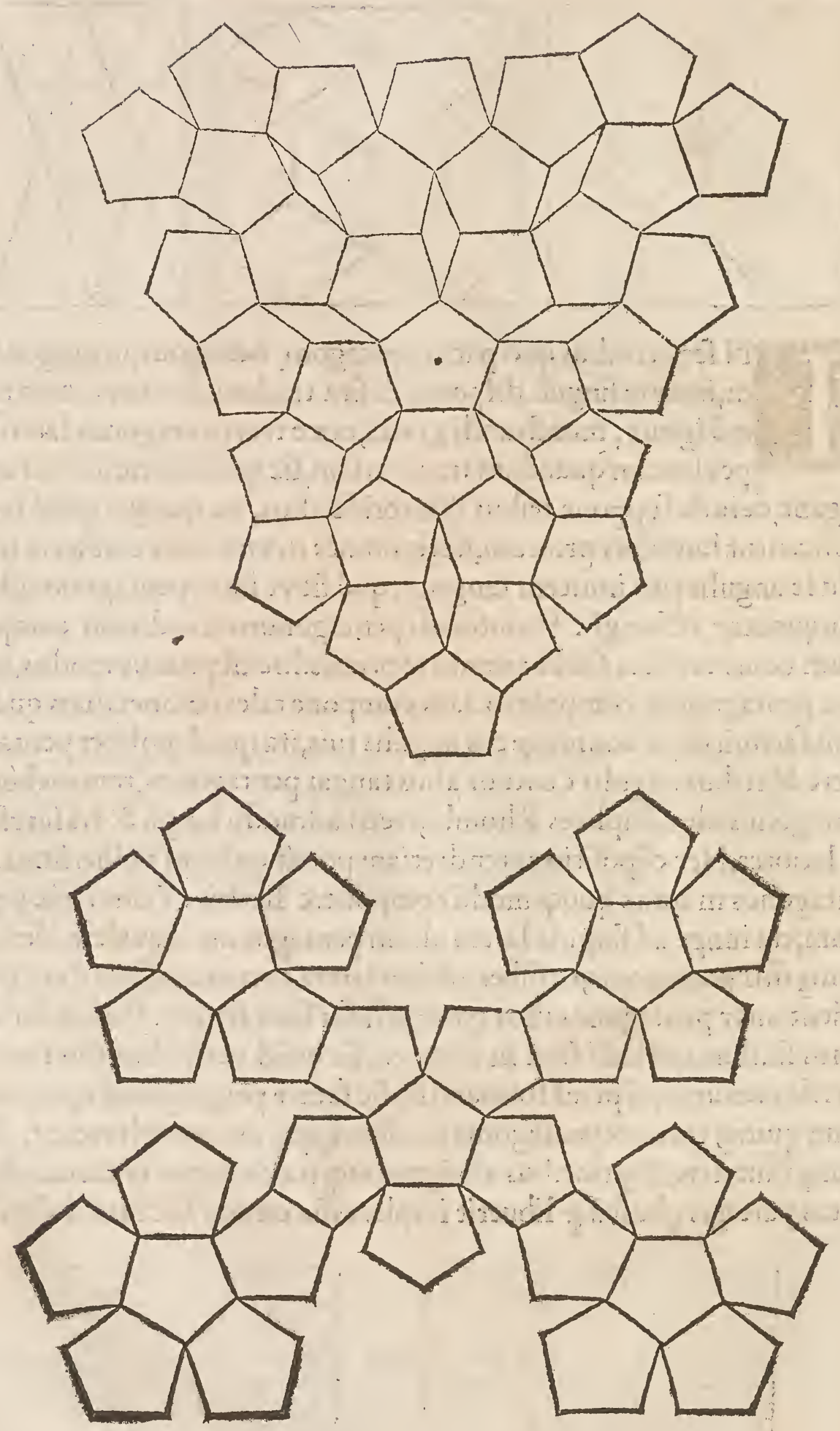
Tem hexagonos tribus modis componere docebo. Primò eos fic im. plico,vt omnia latera, atque etiam anguli fe inuicem tangant \& $\mathrm{ni}$ hil fpatii inter eos remaneat. Deinde eos compono, vt folùm anguli fe inuicem contingant, \& fic relinquentur triäguli inter hexagonos. Tandem compingo cos ve quilibet duobus lateribus, ac totidem angulis, circinos quatuor contingat, \& tunc rhombi inter eos erunt vacui : ifta fic deliniaui.
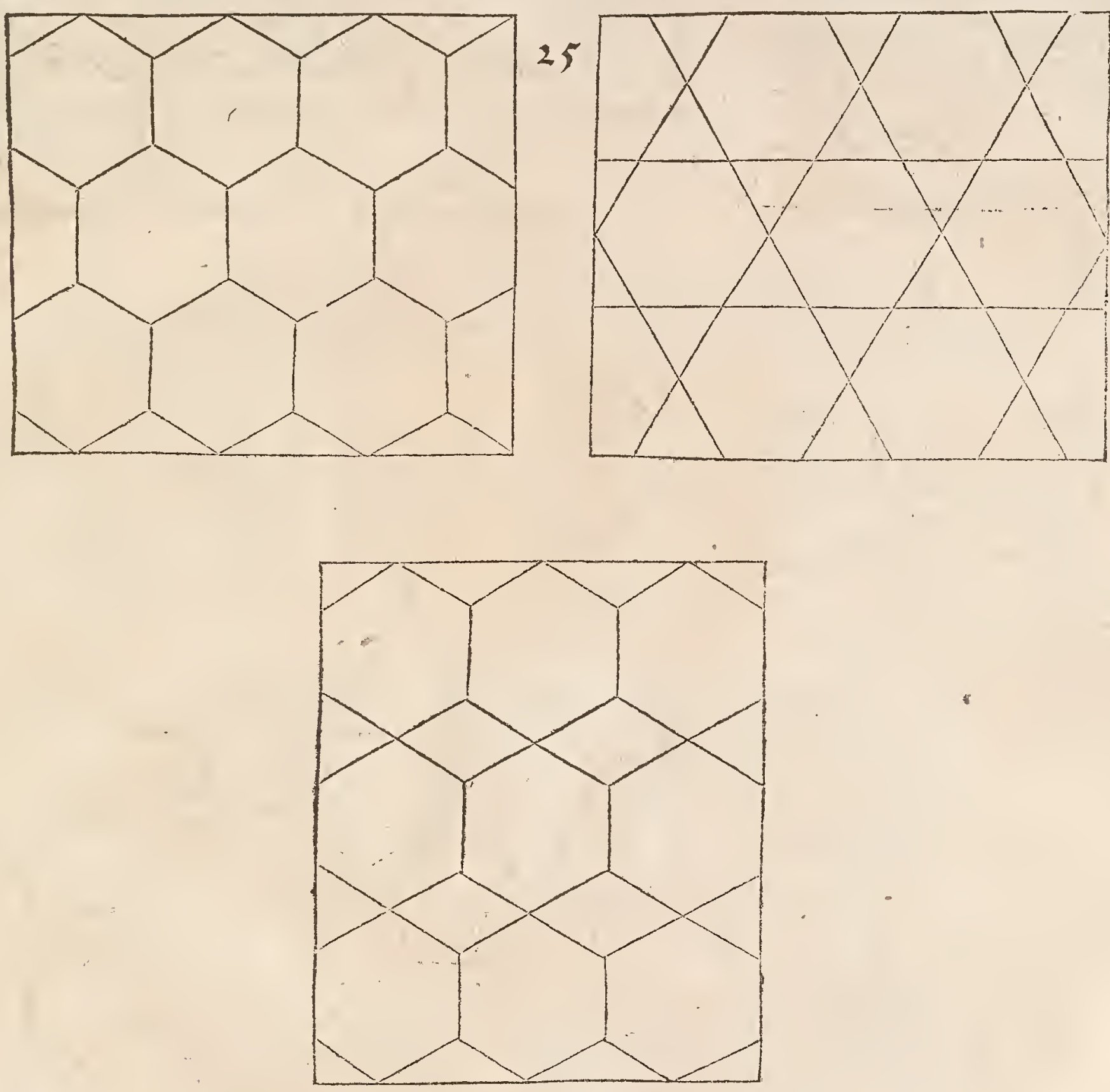

Eptagonos duobus modis inter fe compingam : primò vt angulis fo (1. 1 Lù inuicem fe tangant, $\&$ fic relinquentur trianguli, \& \& fella quatu or radiorum inter splos in eis ftellis folent fieri quadrangula, qux fuis angulis attingunt angulos heptagonorum:vel linex ducuntur obli qux qux fe in medio ftellx interlecant. Poftea applico duo latera, deinde duos angulos, drrectè vnum fuper alıum, \& quando tales ordines vnum iuxta alium pono, tunc fino angulos, qui ad latera funt, fe inuicem tangere, manebúntque quadrangula \& priores ftell inter heptagonos. Item quando heptagoni fuis lateribus iunguntur, ita quòd femper duo anguli extra $\&$ vnus intra promineat, tunc circuitum conficiunt in modum circuli, fed non complent eum, fic faciunt etiam pentagoni. 

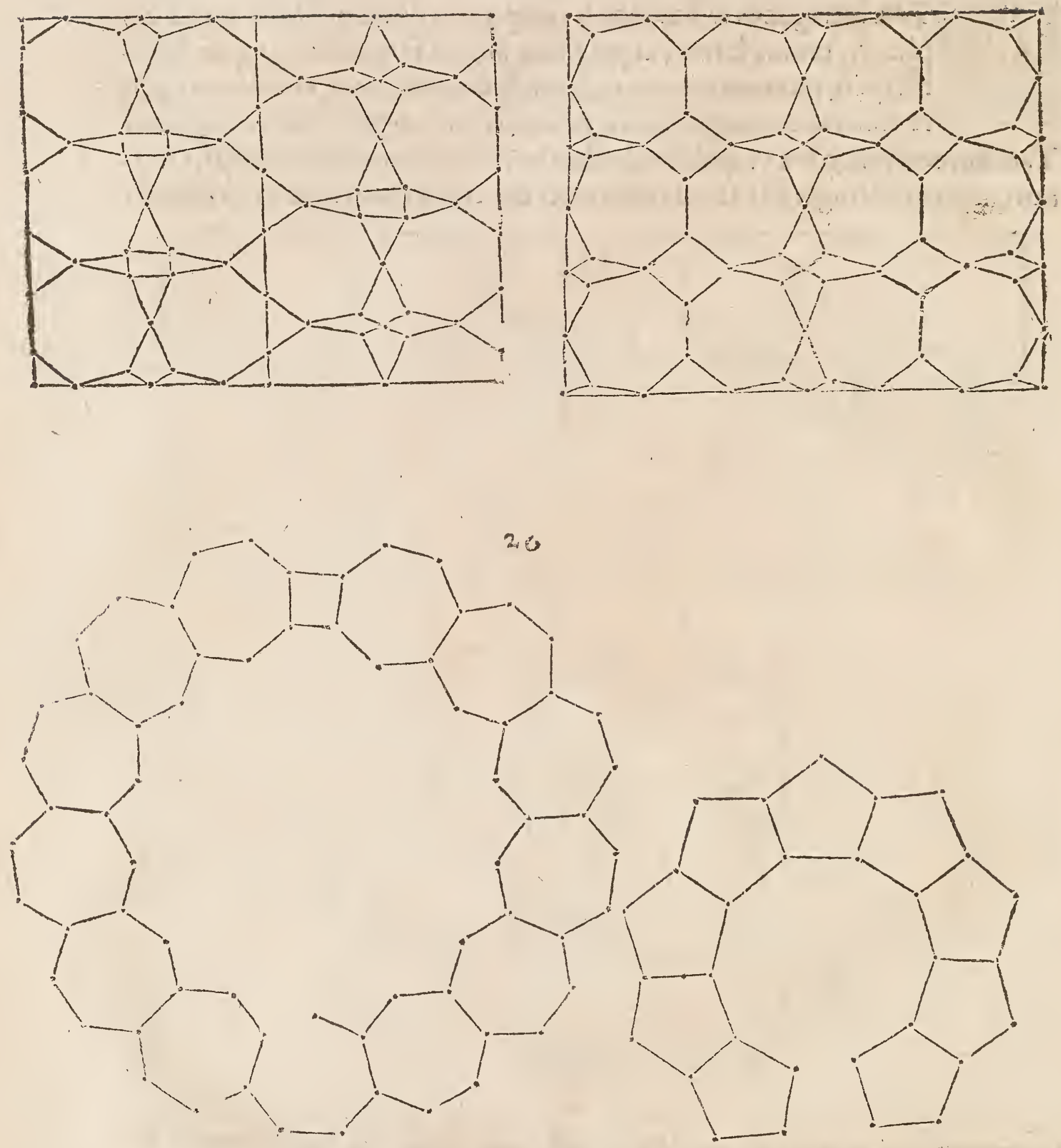

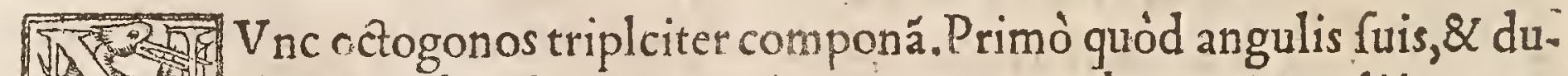
obus lateribus fe inuicem cótingant, \& manebunt trianguli inter eos vacui. Secundo cos fic coniungo vt quilibet quatuor lateribus fuis quatuor alios tangat:ac directê fupra 8 iuxta alios, fecundum du: as lineas, quę fe ad angulos rectos fecant, confiftat:tunc relinquútur inter eos quadrata ftantia obliquè. Tertiò vnum alii applico obliquè, \& reftát inter eos figurx quadratę perpendiculariter erect x:ifta omnia infernè deliniaui.

Huiufmodi figuris licebit vti in ędificiorú imo cęlo, \& pauimétis. Item nos poffumus diuerfa predictarum figurarum genera certo in fe ordine difponere, $\&$ areas qua inter eas remanét, vacux egregiis quibufdam lineamétis exornare. 
Sunt etiam qui irregulares quaflam figuras qua inæqualia habét latera pulchrè inrer fe diftrbuant, ex quo onamenta multa fumuntur. Sape vfu venit, vt triangulos, guadrata,pentagonos, at $\phi$ alias iftiufmodi figuras per le ipfas di ducamus, \& angulos vnius per latera alterius pminere finamus, cuius rei infer. nè fex fchemata fubieci. Aliquando figura circa, aut intra aliam locatur, quòd commodiffimè fit quum figura parium angulorum aliis etiam parium angulo rum applicantur vel e contrario . Poltremò figurx paruorum angulorum plures poffunt circulo infcribi, quàm polygonia, quòd ha feipfas impediant. Que fupra dicta funt in hunc modum protraxi.
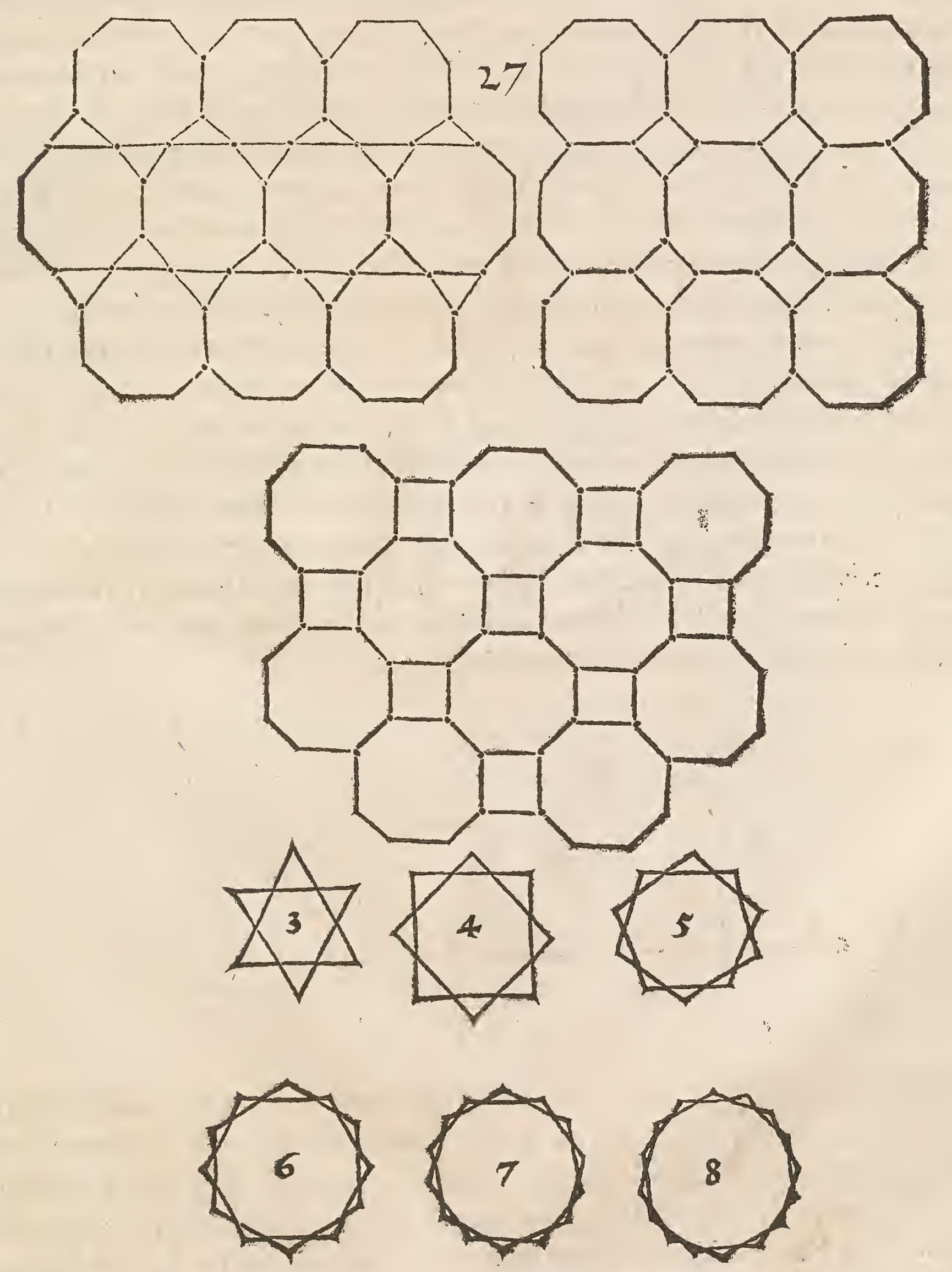
Offumus innumeras figuras inæqualium laterum defcribere, quæ fu1. 2. Is angulis circumferentiam attingere nequeû́t; ex quibus egregia có1. Pofitiones ficri folent . In plano quoque varix figura coniunguntur, 15elut trianguli, quadrata, pentagoni, hexagoni, heptagoni 8 octogoni,ex quibus ardua opera multa in pauimentis $\&$ coelis imis perficiuntur, vt dictum eft priùs. Item regulares $\&$ irregulares figurę fimul etiam compinguntur, qux rurfus pulchram conftituunt conftructionem, vnde varix $\&$ excellentes proueniunt deductiones. Si ifta omnia indicare conarer, fieret liber nimis prolixus:quocirca fibi quilibet de his rebus fumat cogitationem.

Iam dignum cognitu eft fcire, quo pacto diuerfa figuræ in capacitate, fibi inuicem frant equales, vt fi triágulus aliquis proponatur, cui æquale quadratum fit conftituendum, fic quòd quadratum tantum contineat, quantum datus trigonus, quod $8 \mathrm{X}$ de aliis figuris regularis cenfendum eft, hoc in hunc modum ex peditur:efto triangulus a $b c$, cuius latus a $c$ diuide bipartitò in puncto $d, \&$ duc lineam $b d$, qux triangulum $a b c$ fecat per medium, tunc fimedietas $a b d$, invertatur atq applicetur alteri medietati $b c d$, erit a b cd quadratú altera parte longius, quod tantum comprehendit, quantum a b c datus triangulus. Nunc potes ex parallelogrammo illo rectangulo quadratum conficere, quod fit $x$ quale triangulo primitus propofito:id fic abfolue. Accipe de quadrangulo a $\mathrm{bcd}$ duo latera, maius $\&$ minus, ea applica directè ac noca tria eius puncta e $f g$, deinde fone in medio linex ef $g$, punctú $h$, in quo fito circini pede alio protrahe arcum ab e vfq ad g, quo facto erige ex functo flineam perpendicularem, qux circumferentiam tangat ad fignum $i$. Si nunc quatuor lineas æquales $i p f i f i$ ad angulos rectos coniungeres, fieret ex eis quadratum xquale tetragono longo a $\mathrm{b} d \mathrm{c}$, atģ ctiam trrgono a b c. Triangulus etiam hoc modo breuitatis caufa qua drato comparatur: diftribue latus quadrati in duas partes, qualium tres fume pro latere trigoni.Hęc omnia hic oculis fubieci.
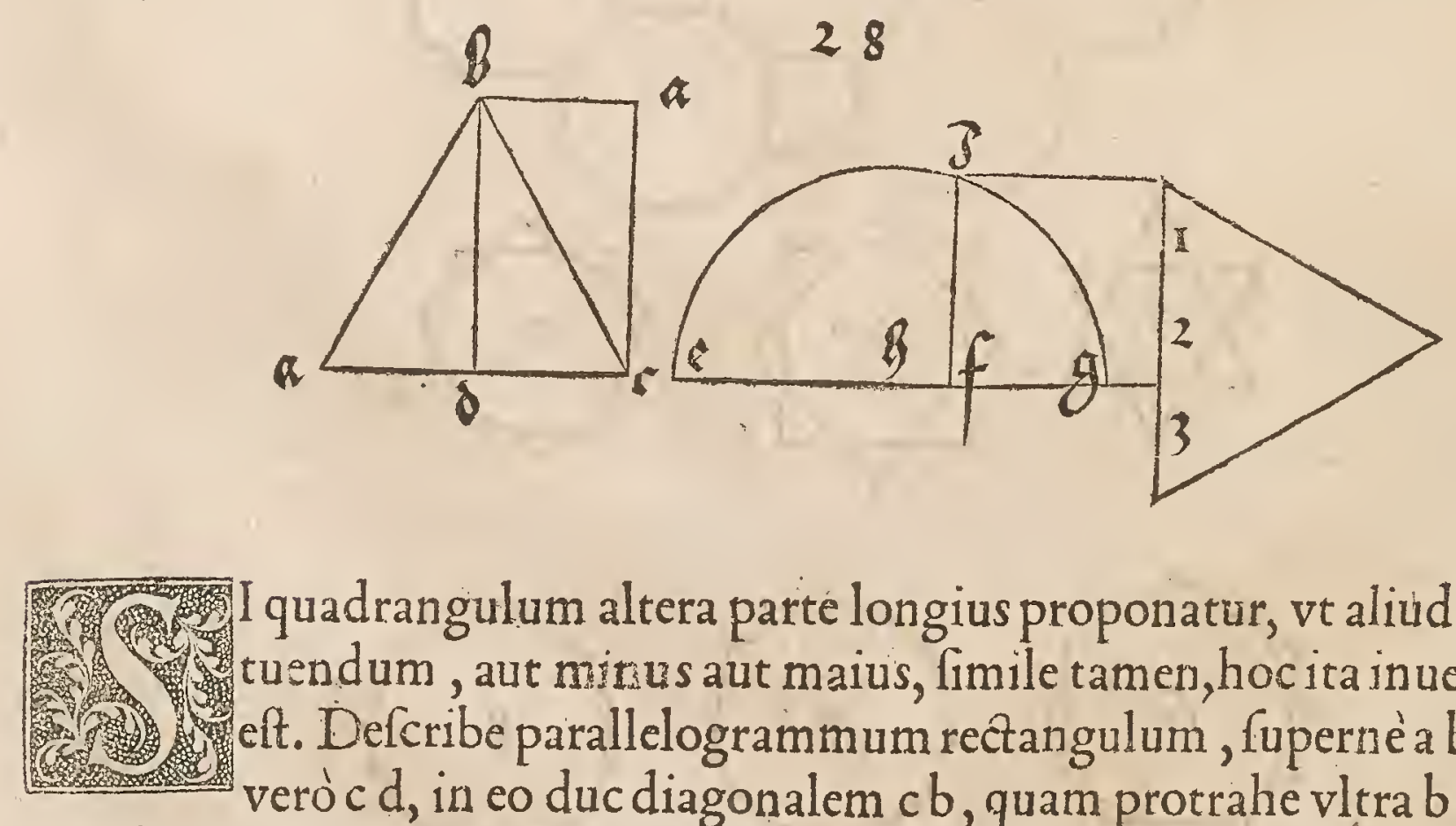

I quadrangulum altera parte longius proponatur, vt aliud fit conftituendum, aut minus aut maius, fimile tamen, hocita inueniendum eft. Defcribe parallelogrammum rectangulum, fupernè a b, infernè verò $\mathrm{c}$, in eo duc diagonalem $\mathrm{cb}$, quam protrahe vltra $\mathrm{b}$ quantum opusfuerit: $\propto$ bafin $\mathrm{cd}$, continuato etiam vltra d quoad fatis videbitur. Quum iam quadrangulum vis tacere minus, pone in tranfuerfa $c d$ fignum $e, a b$ ipfo $d$ diftans pro arbitrio, \& erige ab hoc puncto e,perpendicularé vf $q$ ad c $\mathrm{c}$ diametrum vbif notato, à quo ipli a b trahe parallelam ad a c vfque, quem locum fi- 
gnato litera g habebitóg tetragonus longus $f g$ ce, fimilem menfură ipfi a b cd. Quood fi parallelogrammum maius conttruere velles quàm fir a b cd, id eo modo extra datum quadrangulum abfolues quo priùs abfoluittintra, velut hic quoque defignaui.

\section{9}

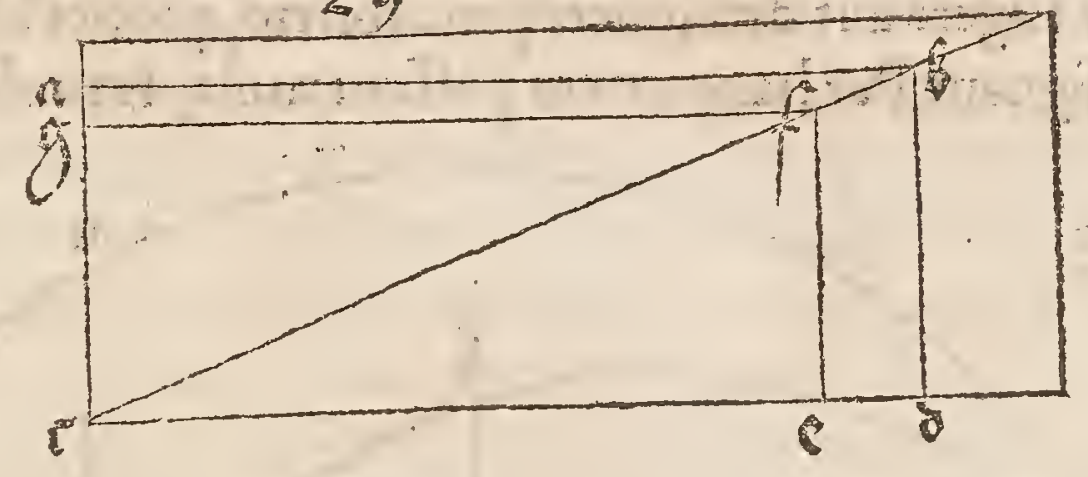

Vòd fipradieta quadrangula qua femper manent ciufdem fimilitu. dinis velles augere, ve in duplo, triplo aut quadruplo plus caperent quàm antea, hoc modo pro e endum effer. Lefignato quadrangulü oblongum a b c d, velut in precedente dictum eft, id pone fecundum longitudinem tranfuerfé. Dende fac duos ad buc tales tetragonos longos, ac applica eosvtrinque ad latera primi atque angulos iftius longi parallegrămi notato fupernè e $f$, limliter etiam inferiè. Quo facto diuide fuperius latus ef, in punctogper medium, \& fige circinum vno pede in pundum g,acaltero ex anguloe,producito lemicirculum per partem fuperiorem vfque in $\mathrm{f}$. Poftea protrahe lineam $c b$ furfum, donec contingat femicircumferentiam ad fignum $h$. Hac linea b h longicudo eft quadranguli, quod duplum erit ad quad rangulum $a b c d$. Sed a t inuentend m huc longitudinidebitam latitudinem, vt quadrägulumfimile fiat priori, fac iendum erit quemadmodu in pracedente iuffimus. Sctibe in tetrago o a bc d diagonale d $b$, quam vltra b prolongato quantú placuerit. His pfecti accipe lineá b $h, \&$ applica eá in vno remino pücto d, \& vbi ahus cadit inter $\& \mathrm{f}$, in ip fa linea d fillic adiceliteram inde trahe lineá perpendicularem furfum, vfque ad diametrum d b, quam viblecat notak, auo duc pli $\mathrm{f}$, aguidiftantem vfque ad tinean da prolongatam; $\&$ vbi eam abfin dit illic adfcribito literamt. Er continebir quadrangulum $1 \mathrm{~K}$ id in duplo pliss, quá quadrangulum a $b$ d, \& fune fimilia inter fe:Sin auten quadrangulum $a$ $\mathrm{bc}$ d triplandum fuerir, tunc adiunge adhuc tale quadrangulum lecundum ló gitudinem tribus prioribus, 8 erunt quatuor anguli iftius oblongi parallelo. gramm e m e m:iam fifte crrcini pedem in punetum blinęe $m$ fuperioris, $\&$ al tera liniato arcú ex ligno e per partem fuperiorê $v f_{g}$ in $m, a c$ ptrahıto lineam $f$ f furfü donec cadar in nuper defriptü ar cú,cui loco adde literă niifa lineaf $\mathrm{n}$ erit lógitudo quadrăguli triplicati, q pone fup lineá e f applicădo vná extremitatẻ figno d, \& vbi relıqua ceciderit vltra i, illic pinge oliterä. Ex hoc ligno o age lineá directè furfú ad prędictădiagonalé, ac fectionis locú lignato ch sractere $\mathrm{p}$, 
à quo protrahe lineam tranfuerfalem atque parallelam ipfi o d lateri, víque ad lineam erectam quę ex $\mathrm{d} b$ furfum producitur, $\&$ angulum, quem du $x$ ill $x$ linę efficiunt, notato litera $q$. Quadratum igitur oblongum $q$ p o d ter capit quadrangulum a b cd, \& habet fimilem quoque proportionem, propter obliquam diametrum. Hoc ergo pacto, quo monftratum eft,poffumus huiufmodi paralle logramma aut augere aut diminuere pro arbitrio, quoties libuerit, quemadmodum infrà defignaui.Per hanc artem poffunt multę res arduaexpediri.

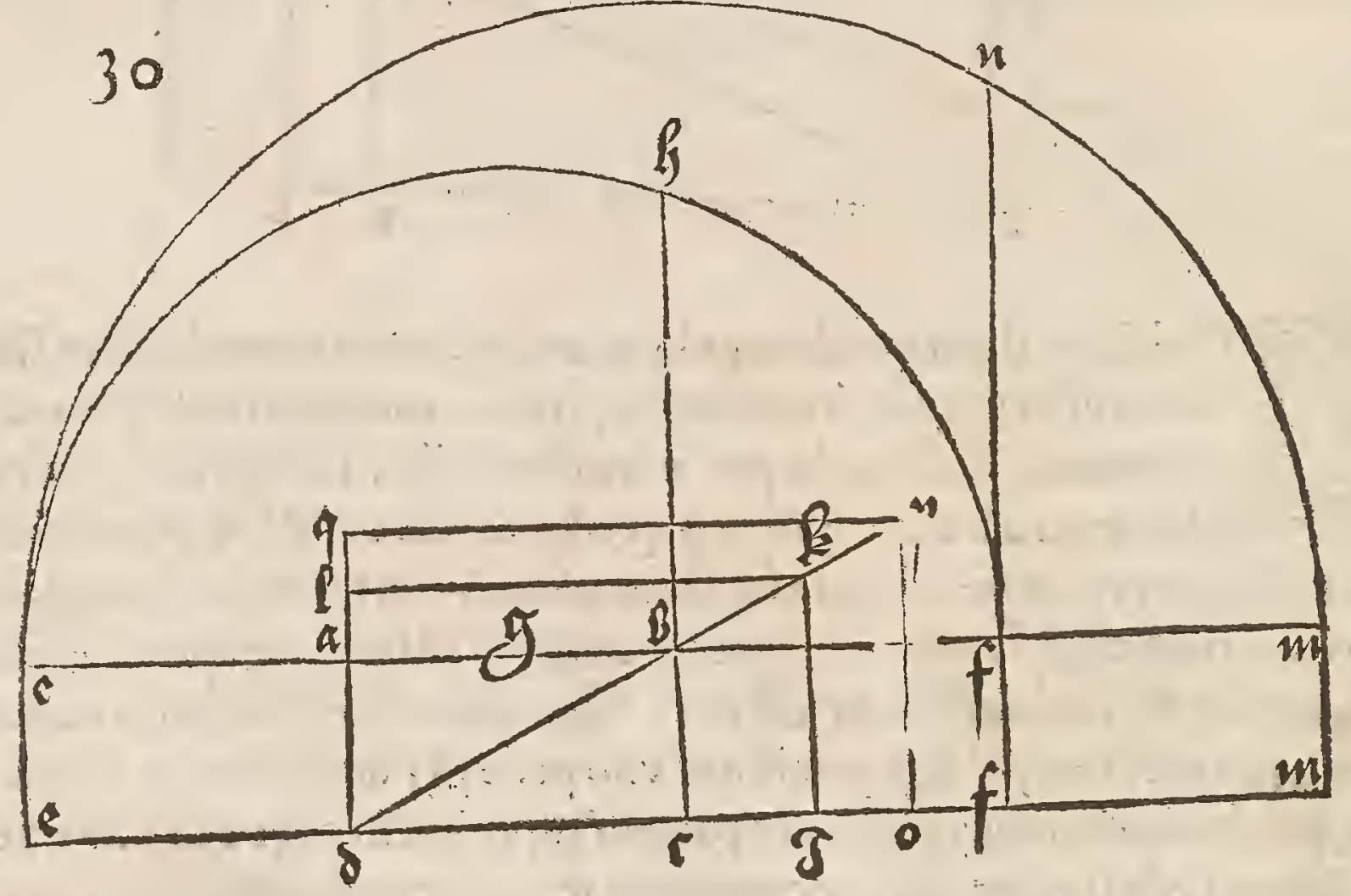

] 5 F. $T$ exactiù intelligantur ea qux hactenus dicta funt, propter ingente corum commoditatem multiplicabo adhuc quadratum rectágulum, 2. 5 . 5 atque xquilaterum fepties, quod hoc pacto abfoluam. Ego ftatuo qua . 2.20 dratú a b c d.cuius vnum latus octies in recta linea repeto, eius aggregati principium fit $\mathrm{d}, \&$ finis e, deinde diuido d.e. per punctum fbipartito , \& pono circini pedem in $f, \&$ alio duco femicirculum d e, atque latusc $b$ quadrati $a b c d$ produco vltra $b$, in continuú $\&$ rectum $v$ fque ad arcum de, $\&$ vbi eum contingit illic fcribo g literam:fi iam ex quatuor lateribus, quorum quodlibet fit aquale ipfi $\mathrm{cg}$, quadratum conftruo, continebit ipfum fepties tantum quan: tum quadratum a bcd, velut hic deliniaui.

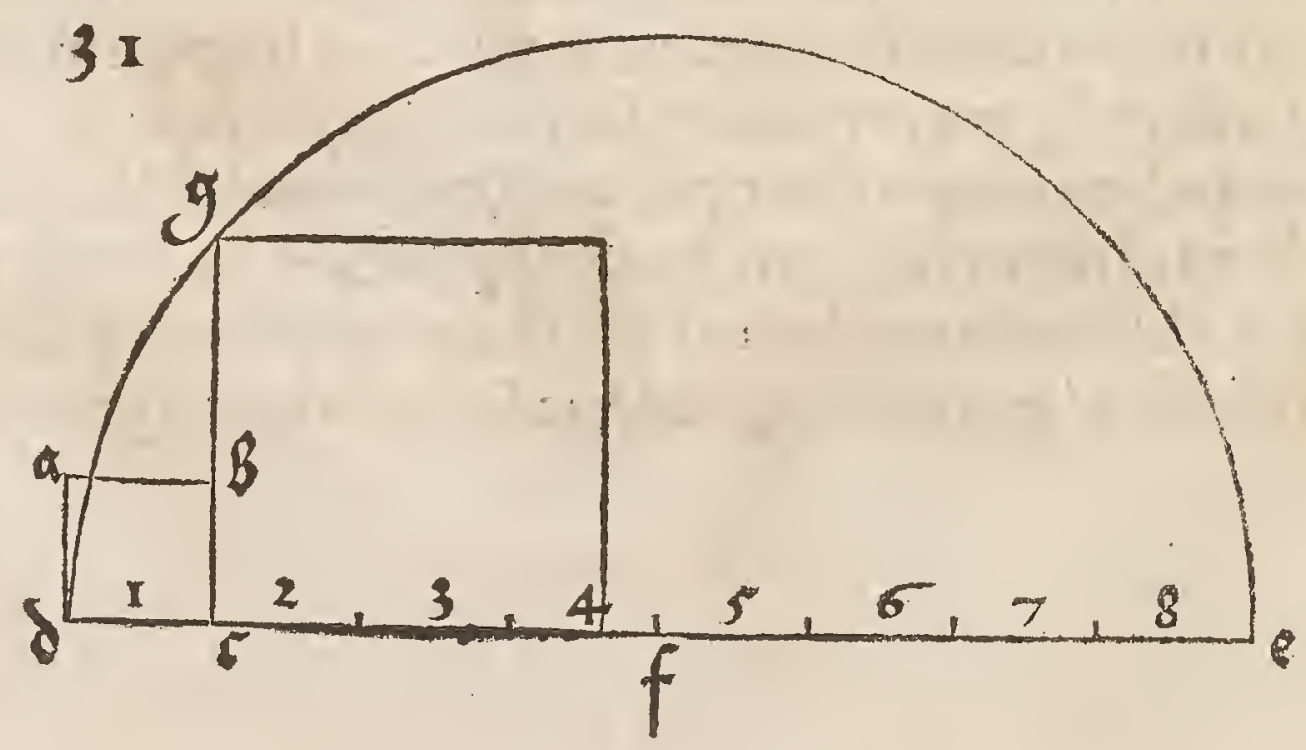


I triảgulum inæqualium laterú ad quadrat um reducere velles, quod 1 Iatus a c lit tranfuerfum ac longiffimum, $\&$ angulus b fit eredus, ex quo in tranfuerfum a c, duc lineam perpendicularem, \& vbi ea fecat $a$ c, illic fcribe d literam. Deinde partire $b d$, per medium in puncto $e, \&$ a $b$ in $f$, $\$ \mathrm{cb}$ ing, \& trahe perfeg glineam rectam, tantæ longitudinis, quantx eft a $c$, atgex punctis a \& c erige binas perpendiculares, qux cadant in lineam $\mathrm{f} g$ trăf uer $\int a m, \&$ loca fectionum fignato literis, 11 lum quidem qui exiftit fupra a litera $j$, eum verò qui eft fupra $\mathrm{c}$ nota $\mathrm{h}, \&$ continebit quadrangulum i h $\mathrm{c}$ a, tantum quantum triangulus a bc. Sed fi huic parallelogrammo æquale quadratum cố. ftruendum effet, id per circulum velut antè monftratum eft expediri poffit.

Item aliter poffum us etiam quod fuprà dictum eft efficere:fac triangulum inasqualium laterum a bc, 8 include cum in quadrägulum rectangulum a $\mathrm{b} c \mathrm{~d} e$, ita quòd trianguli latus maximum $a b$ etiam latus longum fit ipfius parallelo. grammi circumfcripti, $\&$ latus quadranguli e $d$ fupernè tangat trigoni angulü $c$ ampliffimum : fic circumdatur triangulus a $b$ comnino à quadrangulo $a b$ d c, $\&$ capit trigonus parallelogrammi medietatem præcifè:quocirca quadrangus lum $a b \mathrm{~d}$ e, altera parte longius, fcindito per lineam $f g$ in duo xqua parallelo. gramma, erítque quadrangulum a $\mathrm{g}$ e aquale trigono a $\mathrm{b}$ c.Poftremò conuenit huic tetragono longo xquale quadratum conftituere per iam repetitam ato tem, hax omnia hic defignaui.

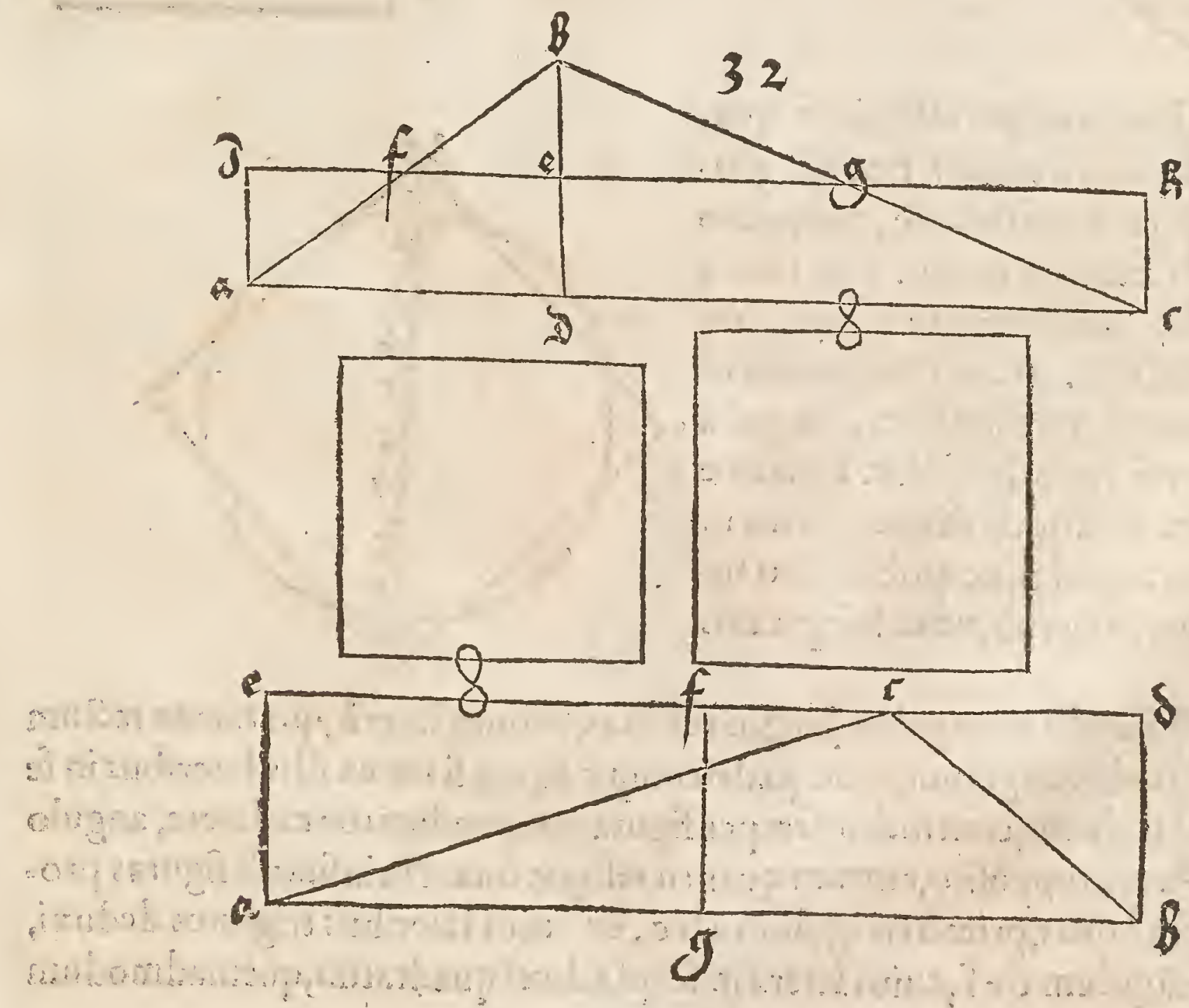


Is Ic animaduerte quomodo quadrangulum fit faciendum, quod tan. 2. 1. cum capiat quantum hexagonus:operare hoc modo. Defcribe hexa16. gonum xquilaterum \& aquiangulum, intra circuli circúferentiam:

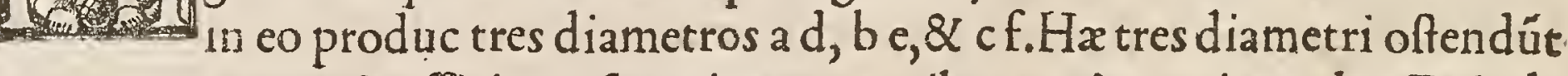
vnum centrum $g$, \& efficiunt fex trigonos qquilateros $\&$ xquiangulos.Deinde extende lineam tranfuerfam $\&$ pone fuper eam ex hexagono tres triangulos, $q$ feinuicem angulis fuis contingant, \& fuper vertices eorum protrahe ctiam lineam rectam, \& fient quinque trianguli aquilateri, qui fe inuicem includunt; cui aggregato applica ad latera duos dimidiatos trigonos erit' q $_{\text {e }}$ ex illo hexago no quadratú al tera parte lógius, æquale tamen ipfi hexagono:hunc tetragonú longum reducito poftea ad quadratum, quemad modum priùs edoct us es:quod tantum continebit quantum hexagonus, vt in fequenti figura videre eft. Ita po. tes trahere omnes regulares figuras quotquot angulos habuerint.

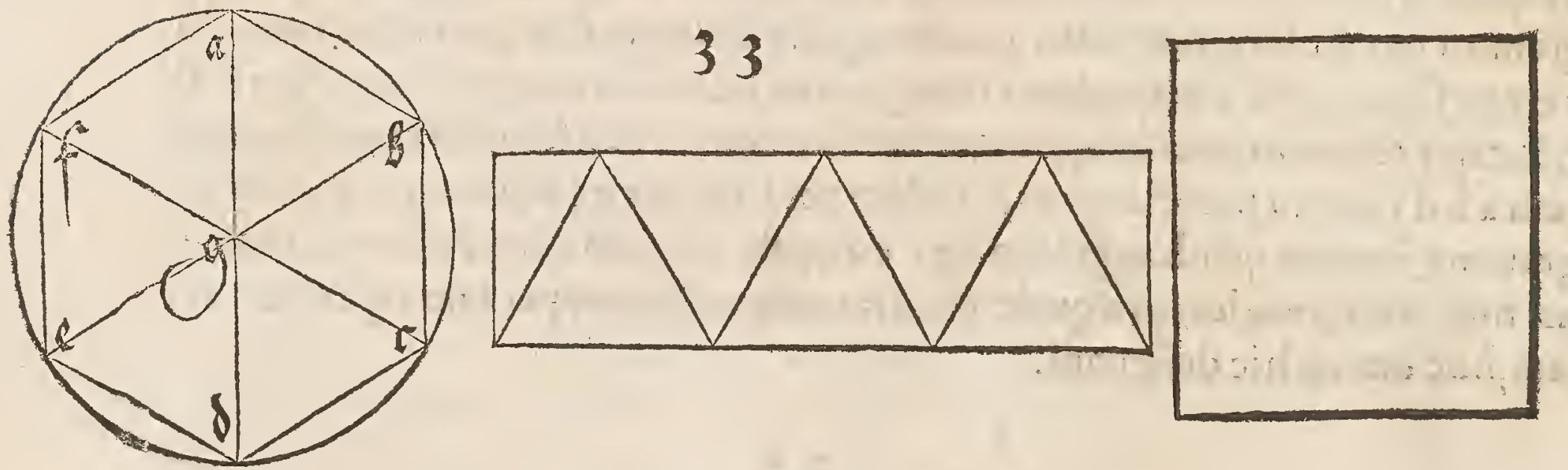

Perequecium effet fcire qua-
draturá circuli, hoc eft, qua-
dratú conftituere, propofito
circulo xquale. Sed hoc à philofophis mathematicè adhuc non demonftratú eft. At in hunc modú id expediri poteft verifimiliter, ita quòd in opere parú aut nuhift fallat. Defcribe quadratum \& diuide diametrú eius in decé partes aquales, ex quibus octo fu. me pdiametro circuli, velut hic ptraxi.
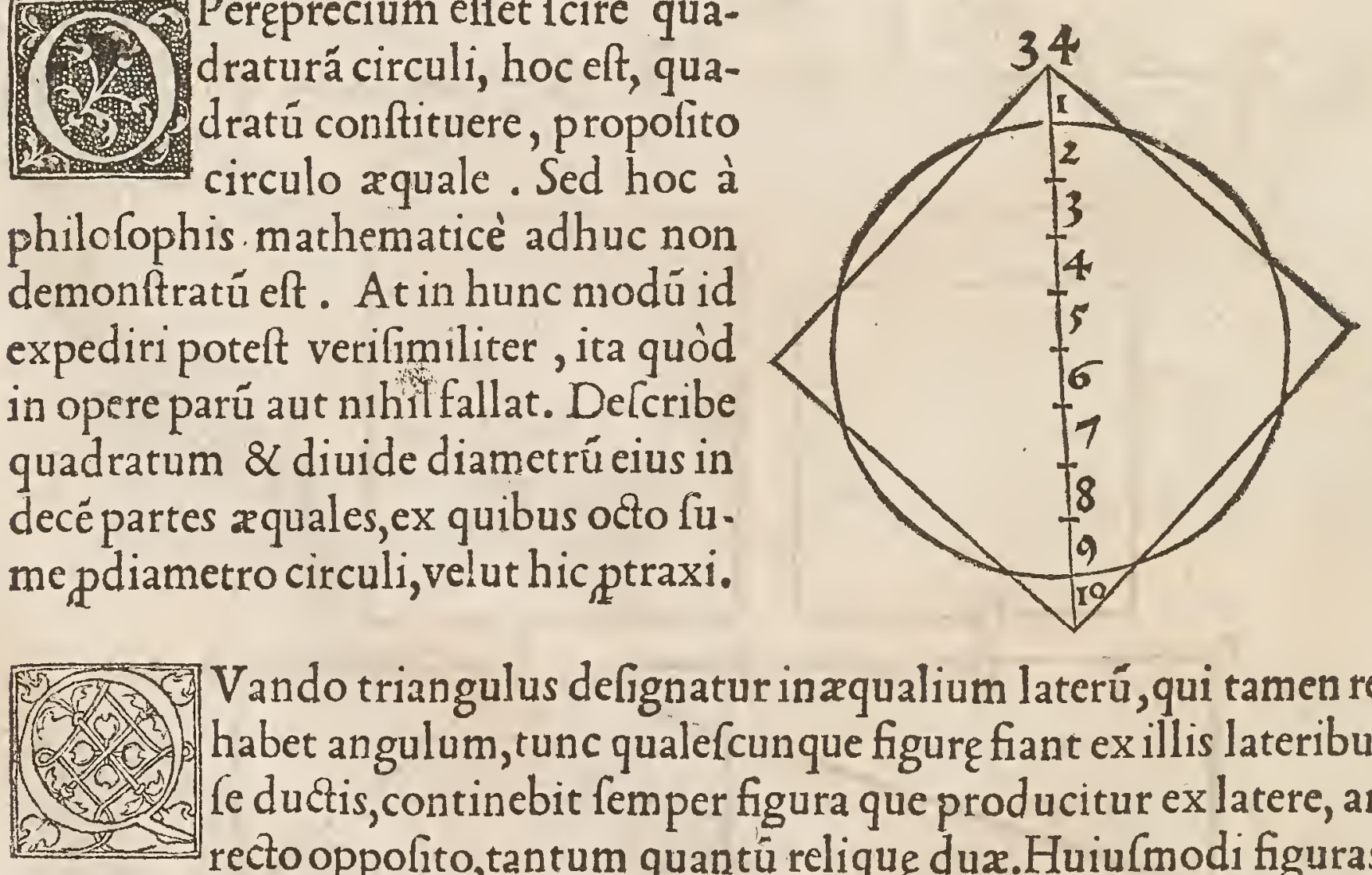

Vando triangulus defignatur inæqualium laterú, qui tamen rectum habet angulum, tunc qualefcunque figure fiant ex illis lateribus in $f e$ fe ductis, continebit femper figura que producitur ex latere, angulo recto oppolito, tantum quantü reliquę dux. Huiulmodi figuras pro. traxi infernè duas, primò triăgulum a b c, ex cuius lateribus trigonos deduxi, deinde triăgulum def, cuius latera in fe ipfa duxi quadratim, quemadmodum hic apparet. 


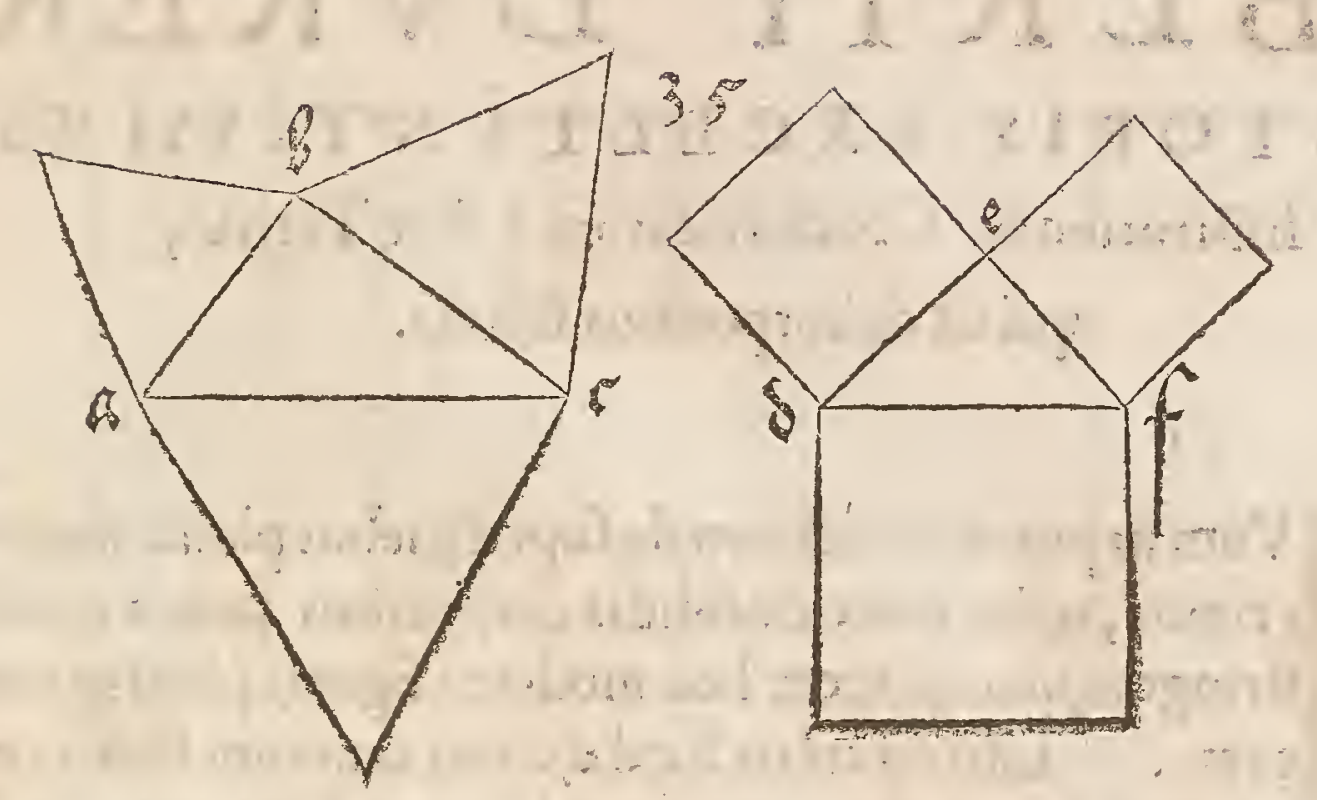

2. Vum duo circuli arcus concauitatibus fuis fe inuicé refpiciút, atque (2) (1) claudút, ita vt fpatium inter cos relinquatur longü \& a redum, tunc fi 2. 0.0 id fpatium partirı commodè voluerimus per lineas tranfuerias, hoc 12-2 pacto operandú erit. Defcribelineá perpendicularế fupernè a, $\&$ infernè b, cui duc aliá tranfuerfam, qux ipfam a b fecet ad angulos rectos, \& pone in linea tranfuerfa verfus finiftră punctum $c$, diftans à perpendicularia $b, p$ arbitrio:in eadem diftantia, locato etiá verfus dextră punctú d, in linea tranfuer fa:quo facto fige circini pedem in fignú $c, \&$ alio ex a,produc arcú verfis $b$, confimiliter fac etiá ex puncto d. Deinde liniato duos circulos paruos, prinún fupra tranfuer $\{a m, \&$ fecundü infra, ita tamé, quòd vterģ contingat lineă tráfuerfam $\&$ ambos circuli arcus. Poltea duc duas lineas tranfuerfales, vná contingentem fupremam partem fuperioris circuli,alteră verò in fimá inferioris:iterum produ ciro duos minores circulos, alterum fupra $\&$ alterú infra eos quos iam defignauimus, quorü veerq̧ attingat circulü maiorem, $\&$ concauitaté amborum arcuú: item his minoribus circulis adde etiam. fuas tranfuerfas, qux eos contingăt, fic deinceps progredere, quătum potes, \& recto fe ordine omia habebút, veluti infer nè oculis fubieci. Ex huiufmodi partitionibus multa egregia opera deducútur.

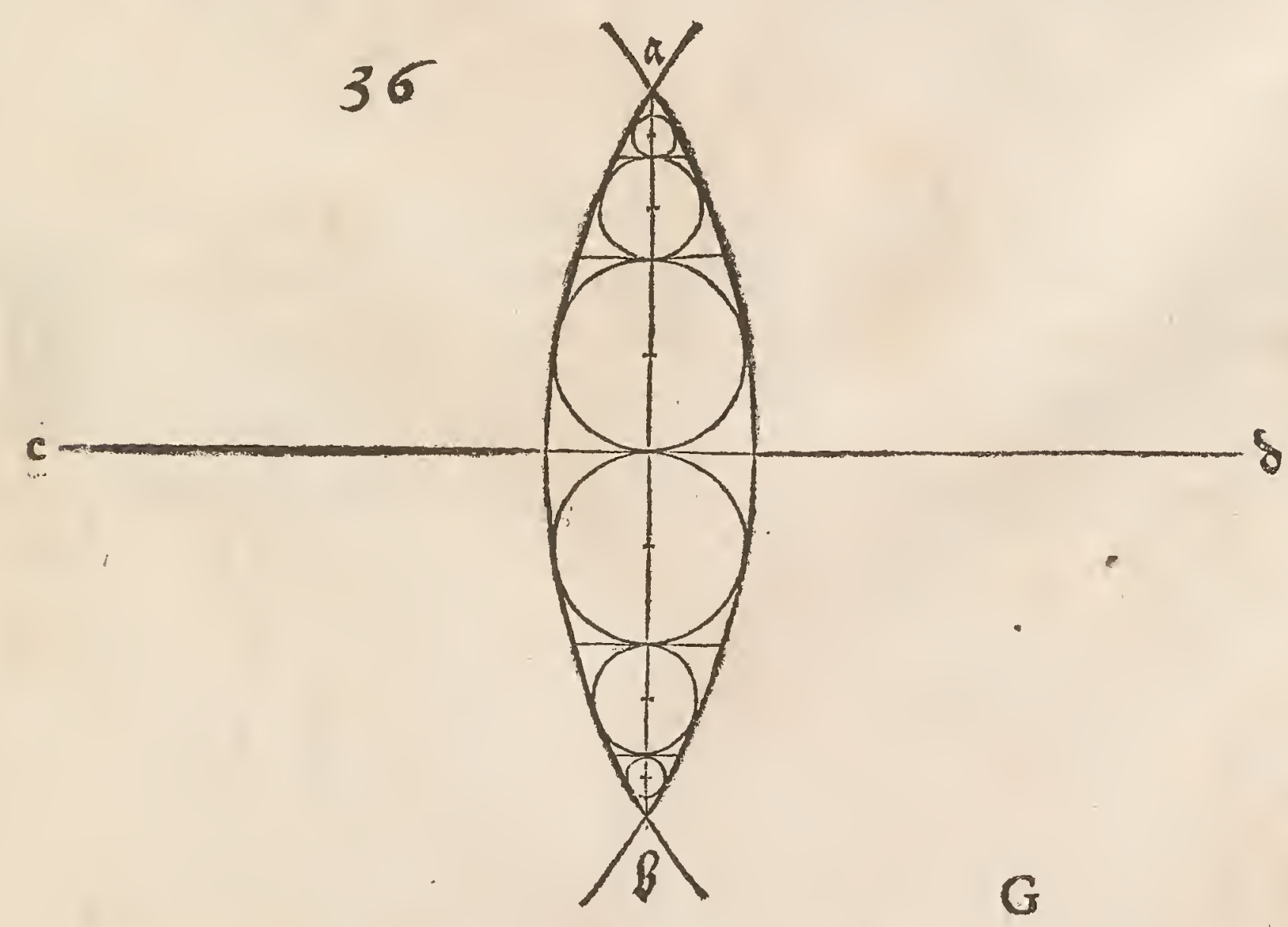


74

\section{ALBERTI DVRERI}

PICTORIS EXCELLENTISSIMI

Inftitutionum Geometricarum Liber Tertius,

qui eft de corporibus folidis.

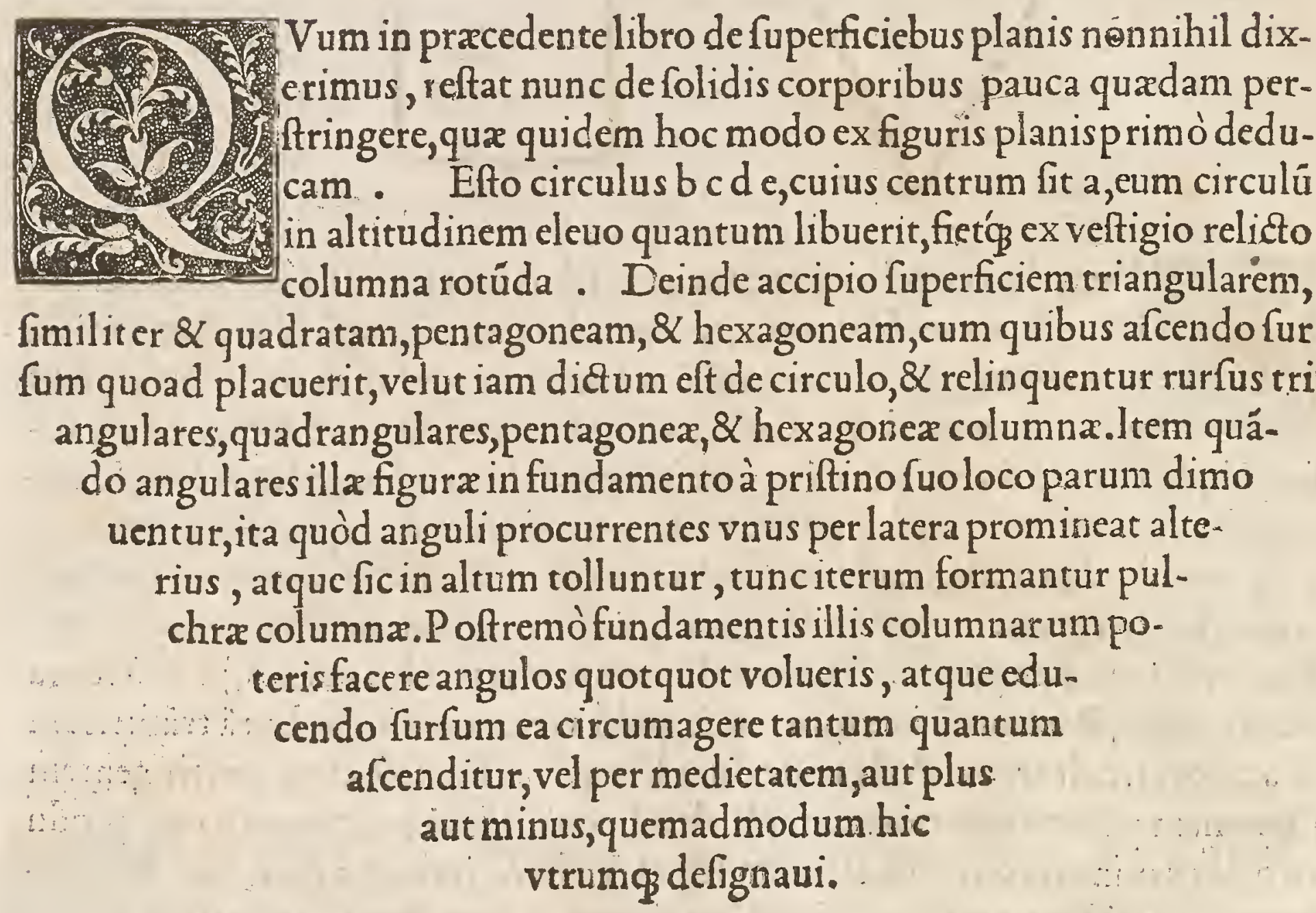




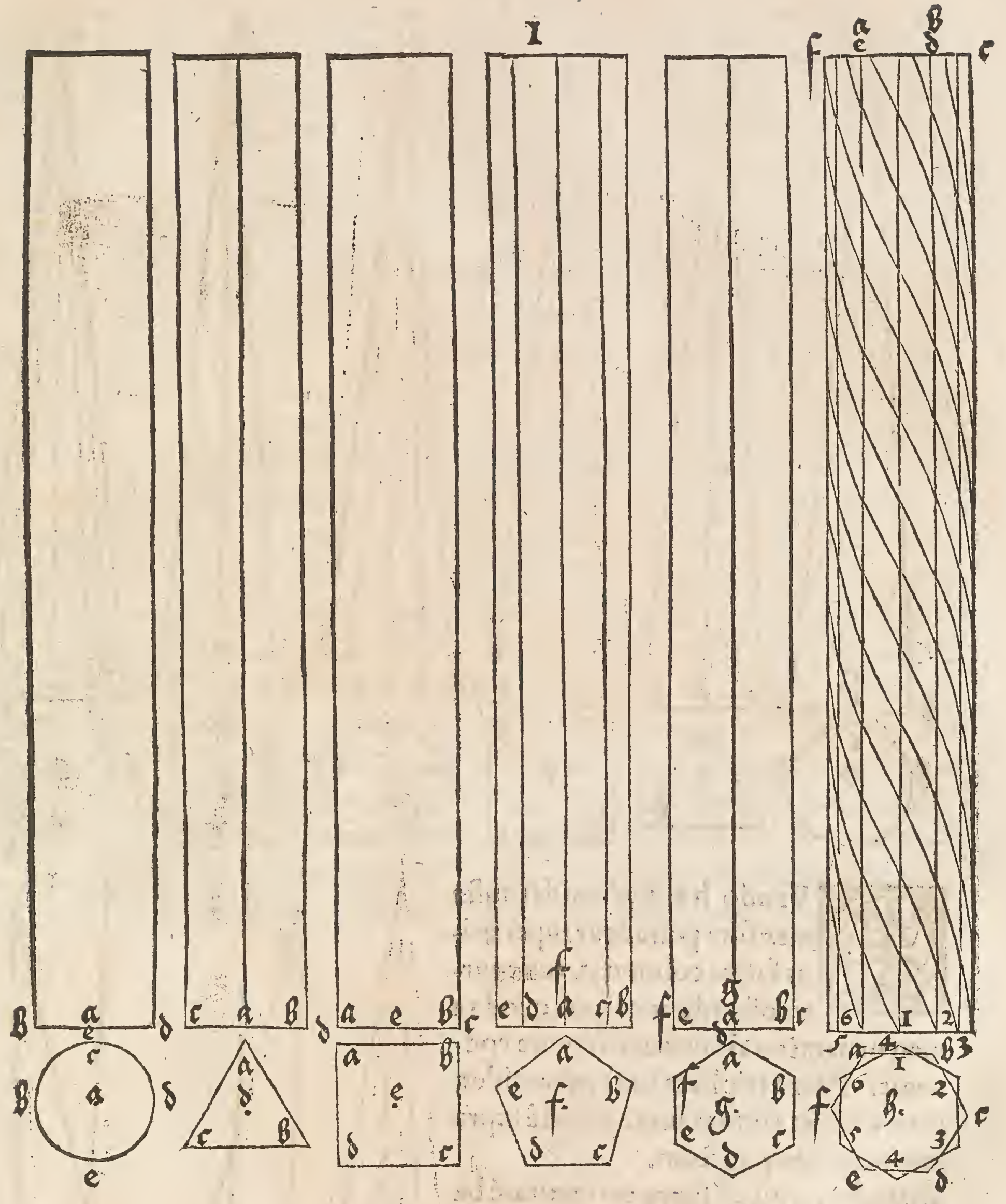

MODVS PYRAMIDES CONFICIENDI. Am ex pradictis fundamentis furfum afcendo quantum placuerit ad
quendam verticem acutum, \& fient pyramides triangulares, quad ran
gulares, pétagonex $\&$ hexagonex, quas rectas aut in modum coclex
inuolutasfacere poflumus, atque angulis vti quot voluerimus, quem-
admodum in precedenti de columnis dictum eft, \& hic quoque protraxi. $G$ ii 


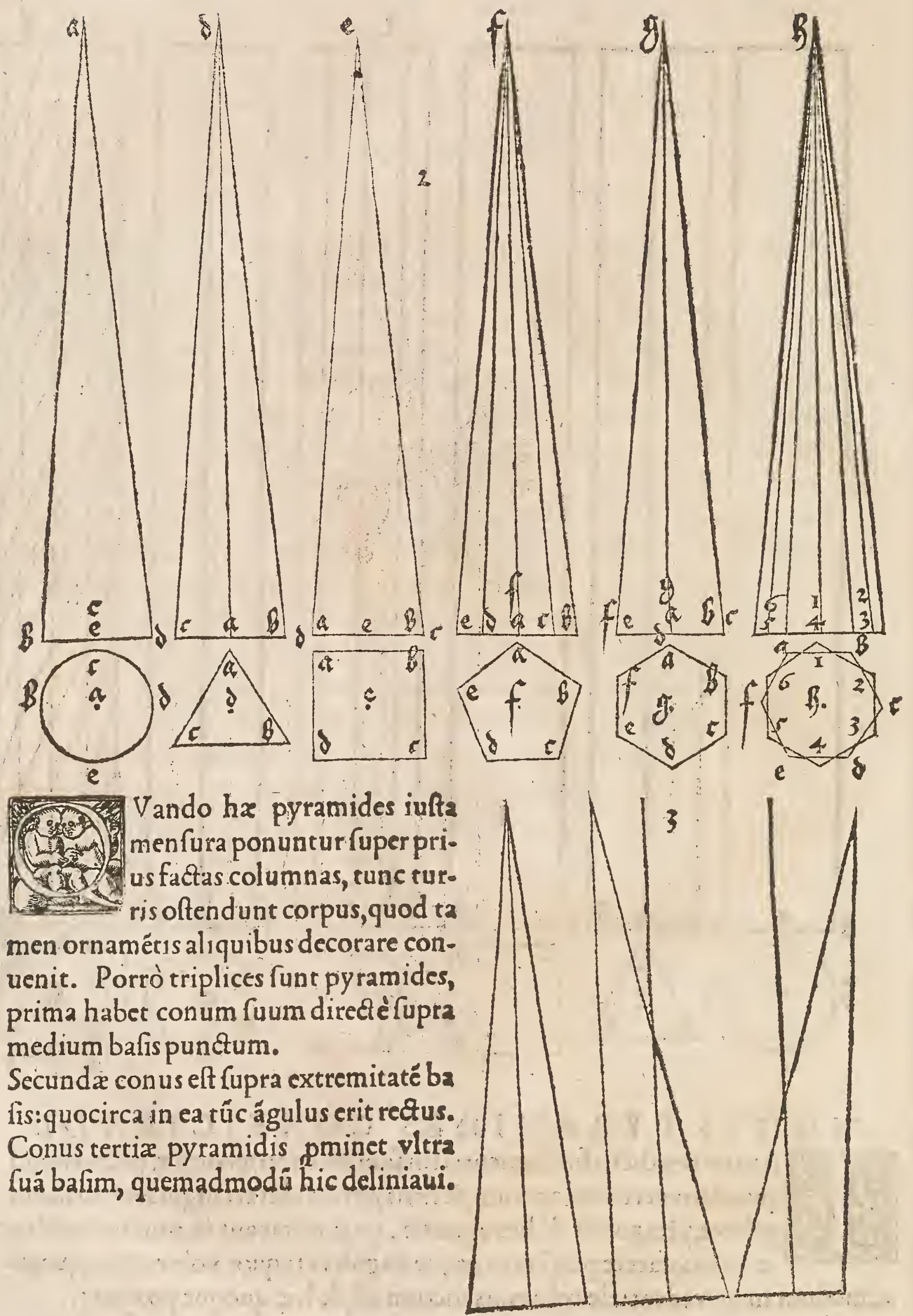


Vum in pracedentibus à columnis librü aufpicati fimus, iam eas pro 1. Lixiùs paulo pertractabimus. Nam multifariam omnino colún $x$ con 1. . . F trui folent, $\&$ fecundum onus, quod iplis imponetur, earum fumëda elt proportio. Sunt qui columnis ipiras \& capitula faciăt, alii verù folùm fcapos ex fundamento erigunt:in fummitate autê propter fornicum arcus diducuntur in partes, aut fcapi in altú continuantur, vt fingularia quædam arcuum ornamenta in ipfis fieri queant, velut fi ftria aliqua per canalem tran feat obliquè, vel contrarium quiddam per alterum producatur:quando ifta certo fiunt ordine, tunc redd unt opus infolens at que venuftum valde, quod ingeniofi architecti obferuare folét diligenter. In huiufmodi fcapis licet diverfis v ti mutat1onibus in Ariis, trigibus, fafciis, angul is \& trochilis.Sed hæc omnia in füda mento proportionaliter defignare conuenit, atque deinceps in opus deducere. Cæterùm fị quatuor fcapi eiufdem magn itudis vnus iuxta alium ponătur, quorum quilibet in fundamento proprios habeat ornatus, quando hi diducantur in arcus,vt fornicế cốttituant,erit conftructio mirabilis atģ elegans. Qui hifce rebus delectetur,pro arbitrio ipfis vti poterit. Porrò cum plures fint, $q$ magnopere ament peregrinas quafdam arcuum coniunctiones in claudéd is fornicibus propter carum venuftatem, infernè exemplar oculis fubiiciam, item aliquot fca porum fundaméta, quibus vti licebir, fi cui placent, interim etiam cy matia quedam addam prominentia, quæ ad fcaporum bafes pertinent humiliter fiue altè à pauimento. In iftis proportionem quilibet facilè animaduertet, nam fi omnia fcribi deberent, fieret liber nimis prolixus. Tandem fundamenta qua fimplicibus lineis defignata funt nihilominus iuftam craffitiem atque amplitudinem exigunt, quemadmodum protracta cymatia deinceps monftrabunt. Poffumus etiam columnas facere diuerfis angulis atque ornamentis quibufcunque,

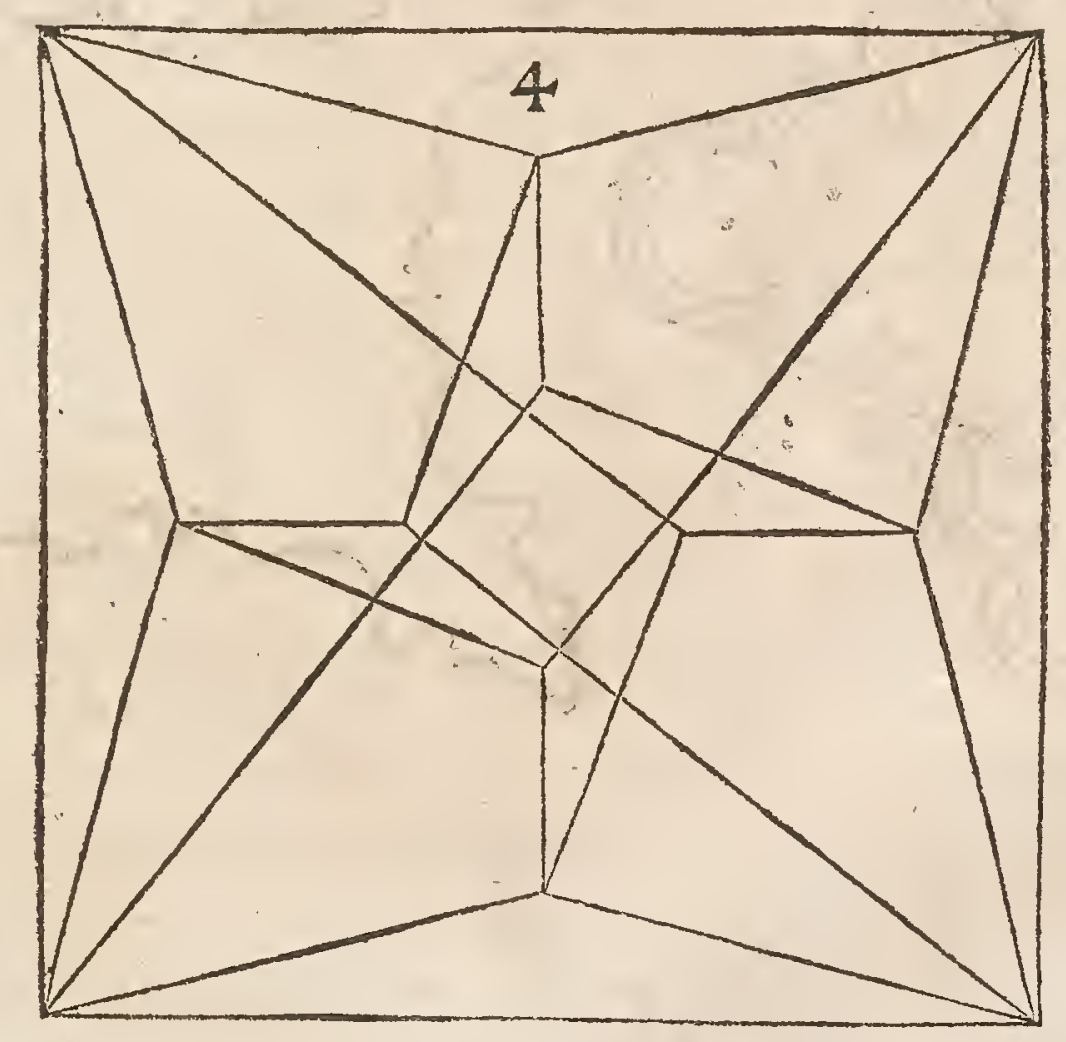




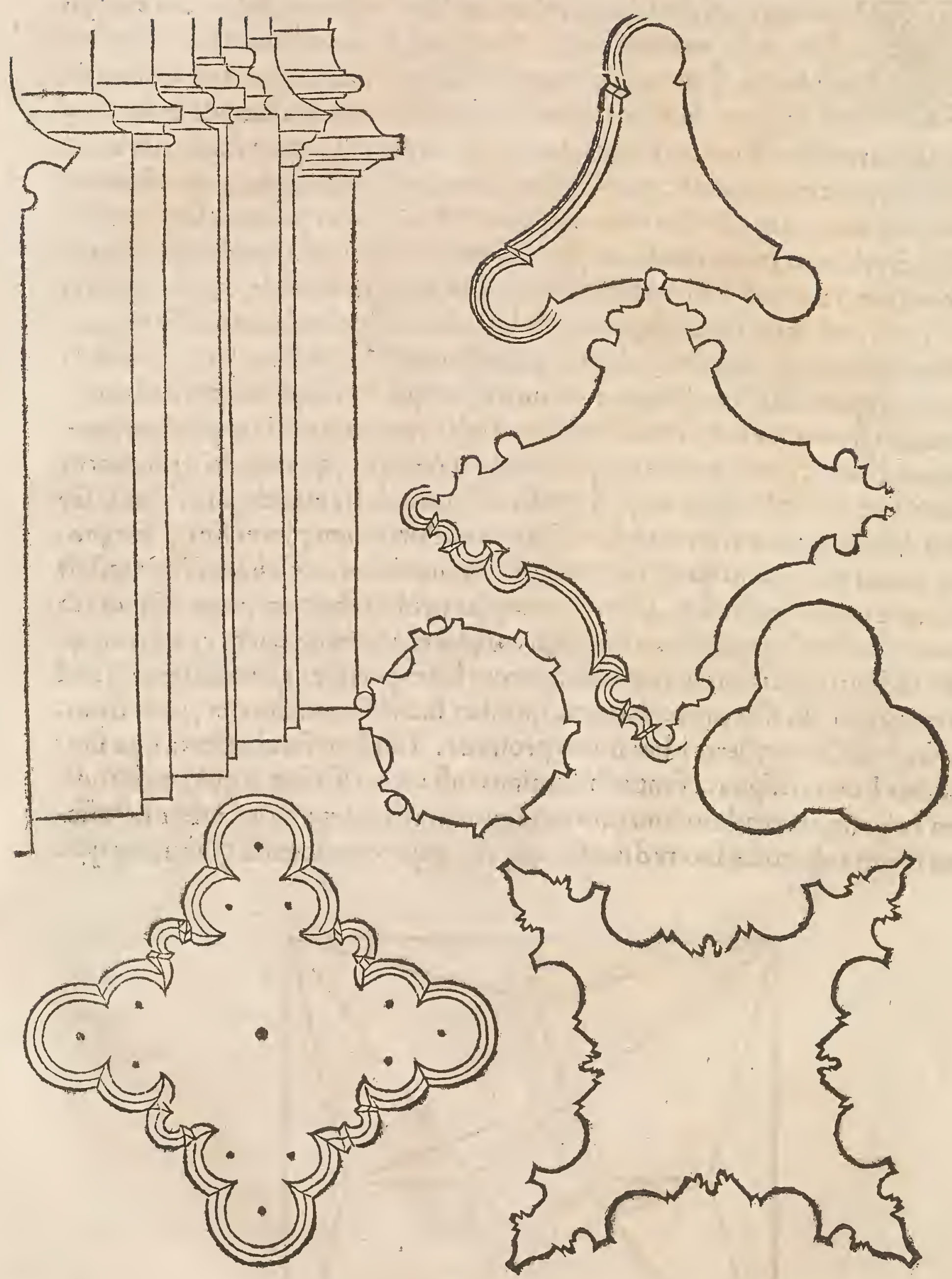




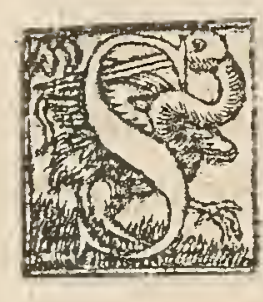

I de tota architectura aut partibus eius differendú fuerit, neminế ex.: cellétem architectum latere exiffimo, quàm ingeniofè artificioféq an tiquus ille Romanus Vitruuius in libris fuis, de frmitate, vtilitate at que ornamentis adificiorum confcriplerit : quapropter iplus in primis doctrinam fequendam effe cenfeo.

Quum verò pro exercenda iuuentute columnam vnam aut alteram conftruere conor, Germanorum mihi fubit animus, qui quum noui aliquid ædificare volunt, nouum etiam ædificii genus habere cupiūt, quòd antè vifum non fit:quo. circa peregrinum quiddam facere docebo, ex quo quilibet quod fibi placet, fu. mat atque pro arbitrio applicet. Interim etiam monftrabo qua ornamenta, dolobra, \& torno fieri queant, rationem q $_{\text {sed }}$ rem vbi ea maiora atq̧ minora effe conueniat. Ornatus ifti ad rectas teretesóg res pertinent. Primò igitur columná parato, cuius altitudo craffitudinem imu fcapi fepties continear, cum dimidia eius parte, fed fafciam octaua parte craffiorem conftrue ip fa columna, arque latam vnam octauam eiufdem columnæ fpiffitudinis, \& fupernè contrahe colúnam ad feptem octauas : fafcia tamen $\&$ annulus tantum pronineant, quantus eft imus fcapus, fitóplata ad proičura quantitatem : columna fic defignata $\&$ fundamento eius rotundo ex centro a protrado, inuolutis quibufdam parergis eam exornato, ad quod quidem vti poteris coclez linea primi libri, ac primò quidem circuitibus verfus alteră folùm partem productis, vel ctiam in vtran ${ }_{3}$; vt féfe obliquê interfecent:fed in col umna licet eiuf́nodi circuitionibus ad mi nus octo verfus eandem partem protrahere:earum principia fumuntur ex cir. cumferentia depreffi fundamenti, quæ in $x$ quales diuiditur partes, à quibus linea rectę ducútur in colüna furfum. Quòd fi tortuof illa reuolutiones in partes diuerfas productę fuerint, tunc ab octo fundamenti punetis fexdecim excút linex flectuof $x$. Tales fpiras poffumus per totam columnam protrahere, vel infernè in tertia eius parté finire:verùm reuolutiones iffę multis modis variătur, \& plures excogitari queunt, at que arctè vna fuper aliam fl eftatim in altum dù cuntur, item in imo arctiores, \& verfus fummú diftantio s quog, ptrahuntur, per triangulum a b c decimafexta figuræ primi libri. ftiufmodi ptractionibus vti poteris in columnis qualibufcunque ficefuerin in medio ampliores quàm in ino 2 fupernè fiue vbique equales, aut infernè habuerint adiectionem in fummo verò contract uram, modo pro dimenfione columnx lineamentorum principia diuidantur. Porrò prędicta coclę linea ad col mná diuidendá hoc pacto vteris. Diltribue primò fundamëtum rotundum in quot volueris partes, quibus adiice fuos numeros, incipiendo à diametro tranfuerfa, ex his numeris educere f́piras in columná $\&$ in ea xqualiter protrahere oportet, hoc qui fequitur nodo. Afcede cum lineis rectis ex fingulis partitionibus depreffi fundaméti, furfum vfog ad imum fcapi, ea puncta illic notato quemadmodum in fundamento fuerunt rotundo : codem ingenio, divide etiam columnam fupernè, vbi ftrictiffima eft in partes cófimiles, quas numera vt inferius factú eft. Deinde lineis rectis continuato puncta fuperiora \& inferiora in ipfo fcapo, cuius longitu diné deinceps partirequatuordecim lineis tranfuerfis in quindecim interualla equalia 
¿qualia \& incipe numerare à bafi verfus eapitulum $2.3 . \& c$. atog.fic totus fcapus erit reticulatus. Ex illo multa fieri poffunt vtiliffima, led huc propterea pofui, vt reuolutiones circa columnam commodius duci queant. Nunc inchoato infernè, fupra fafciam à perpendiculari linea $r, \&$ duclineam tortuofam in colum na obliquè vfo ad angulum perpédicularis $2 . \&$ tranfuerf $x$. Deinde ex aduerfo protrahe furfum aliam tortuofam obliquè à puncto linex perpendicularis : vfque ad angulúm tranfuerfe \& perpendicularis s. Hoc modo operare cum fingulis numeris omnium quadrangulorum qua funt in columna. Aut delinia fpiras illas verfus alteram folùm partem, vt fe non interfecent $\&$ hoc in infima tertia duntaxat, \& producito lineas perpendiculares furfum per totum fcapum: aut ne hoc quidem, nifi parum vltra infimam tertiam. In fumma his fingulis po teris vti feparatim, fiue aliquot eorŭ a aut omnibus fimul . Itế fiue ducantur tranf uerfr, frue non, poffumus tamé multas res pulchras ex huiufmodi protractioni bus facere, quod rectè intelliget qui periculum fecerit.

Pro hac columna capitulum fex modis parato quod centies, fi opus fuerit, variar1 poffit. Fac igitur quadratum quoddam tantę craffirudinis, quătus eft $f$ capus fupernè fub fuo annulo, altitudinis verò dimidia craffitudinis parte, fuper id quadratum pone plinthum, qux habeat tertiam partem fpiffitudinis capituli, fitóg rectágula atq̧ quatuor axqualium laterú, lata vt poffit fuis latéribus capitu li fupremam fafciam prominentem contingere, quanta verò capituli proiectura effe debeat, ftatim dicetur. Plinthus hac octogona fieri poteft, quemadmodum paulò an tè dictum eft, at fí quadrata fuerit, latera eius ad circinú fic excauari po terunt, producantur in ea dux linex fe fecantes ad angulos rectos in puncto $a$, atque diuidentes plinthum in quatuor quadrata $x$ qualia, $\&$ continuato quamlibet linearum in vtranque partem quantum opus fuerit, ac terminis earum adfcribito b c d e, quofacto, aperi circinum ad quantitatem lateris plinthi, ac fifte pedem in quatuor illas literas, \& reliquo arcus defigna in plinthi lateribus, \& quoflibet duos arcus, vbi ex plinthi lateribus procurrunt, linea tranfuerfa extra plinthi angulos abfcinde.Caterú in, plinthi craffitudine res diuerfę ful pi poffunt vt fafcia, ftrix, canaliculi, $\&$ alia huiufmodionamenta. At qui ea qua fupra fcripta funt variare volet, is faciat femper fuperioribus conuerfü in hunc modum. Primò fculpe plinthum fic:diuide craffitudinem eius bipartitò \& fuperiorem medietatem rurfus in duas partes, quod dupliciter inuerte, ex fuprema parte fac vnam fafciam, ex fecunda verò fcotiam,tam profundam quàm ef alta. Deinde ex inferiore medietate facito fafciam profundam fux altitudinis, vel pro fcotia fiat quadrans tori, quorum vtrunque fi inuertatur, vt inferior pars veniat fuperné, alia erit cóftitutio. Aliud:diftribuatur plinthus per mediñ $\&$ dabit fuperior medietas tori quartă partê, inferior verò fafciă,aut pone quod inferiùs eft fupernè, $\alpha$ habebit iterú aliă faciê. Aliud:fiat ex fuperiore medietate quarta pars tori, $\&$ inferior pars excauetur, fecundü altitudinis q̉titaté. Aliud:ptirecraflfitudiné in tres partes, fuprema maneat vna fafcia, inferiores duęad circinu concauettur $p$ f piffitudinis qutitate:inuertatur hoc, \& rurfus fe aliter habebit, aut partiatur altitudo in duas partes, ex inferiore fiat fcotia, que abfcedat 
fecundum altitudinéfuam, fuperior pars iterum diuidatur bipartitò, fiátque ex inferiore medietate fafcia, ex fuprema autem abfceffus. Aliud:feca crafficudiné in fex partes, ex fuprema fac fafciam, ex duabus fequentibus fcotiam, tam profundam, quàm eft alta:ex infimis duabus trochilum, quod inuerfum iterum ali: ter erit. Item fub fafcia licet cymatium conficere, vel è contrario:aut fupernè facere paruam fcotiam, in medio eorum, $\&$ infernè magnam fcotiam, fed quồd. fupra $\&$ infra torum duętæniç maneant. Huiufnodi variationes infinta funt. Quare hac nó propterea huc fcribo, quòd neceffario fic fieri oporteat, fed quòd aliquid ex eis fumi poffit, \& quilibet admoneatur, quid noui adhuc inueniendú reftet:nam in talibus partitionibus nô fatis eft vti vna quapiam dimenfione, fed diuerfis, fi quis nouerit:quapropter femper al iquid inueftigandum, quemadmodum Vitruuius ille excellentiffimus, $\&$ alii inueftigarunt;atque res vtiles inuenerunt:fed non idcirco nihil aliud, quod etiam fit bonum, excogitaripoterit, $\&$ pracipuè in rebus quas probare nemo poteft eas effe factas optimè.

His perfectis capitulum fimpliciffimè defcribam, atqg quadrato precipuè vtar abfque omni ornatu, poftea tamen oportet id exornare parergis aliquibus maxi mè cum opus eft magnum : poffumus etiam fafciaś, trochilos, ftrias, \& reliquas res omnes fingulariter ornare, a liquid egregii imponendo, a ut infculpendo ad húc ferc'modü. Diuide altitudinêcapituli, quin qg notis in fex partes equales, ex fuprema fexta facito fafciam planá, eam producito vna fexta de altitudiñe capituli:deinde punctum notato fub fafcia, abfcedếs ab extrenitate eius, medieta te craflicudinis:ex hoc püeto cum linea perpédicuları defcende per tres fextas, hos magna erit fafcia planä:fed ex infimis duabus fextis, fac ex fuperiore fcotiam, tam profundam quàm alta eft, fic remanet fupra inferioren fa ciam produ ctio quxdam, \& capitulum paruam habebit contracturan. Secundo fic facito: Supremam fafciam, codem modo vt fuprà ditum eft telin que, \& diuifa capituli altitudine in fex partes, notam poine in medio quarta fext rex qua lineá per capitulum ducito tranfuerfam, $\mathbb{Q}$ fpacium illud arêu, quod fupra infinas duas fextas remanet tęniam dabit; prominétem fecundum cruffitudinem fuam:dux infima fextr ita maneant velut collum quoddam planum, fed grod fupernè re linquitur, ad circuli arcum rotúdetur, atque inclinetur fupremęfafciz, fic quòd fafcia per medietatem fux amplitudinis promineat. Tertiò fiat fafcix p roiectura vt prius, de qua medietas folùm detur craffitudini:nihil aliud in hoc capitu. lo faciendū nili quòd reliquum eius excauetur ad circuli arcum, vfọ ad pròduetronem fuperné faficia, fic quòd fub ea cymatium proveniat. Deinde conficito in reliquis tribus capitulis fafcias fimiles prioribus, \& fecta altitudine capituli vti fuprà fcriprum eft in fextas, producito fub tertia fexra per capitulú lineam tranfuerfam, inter quă $\&$ fafciă facit fcotiá magná, qux tamé amplitudini capi tuli concauitate fua nihil auferat:ex hoc inuenitur prominentia acutiei in iam protracta linea tranluerfa.Poltea trahe per medium quarti ́patii lineá tranfuer fam, ex quo interuallo fiac parua fcotia, ita quòd profunditas eius, capitulitangat foifitudinem, tü circumferentia inferiori tranfuerfæx fuam dabit menfură quantú extare debeat. Deinceps infima fexta partita duobus punctis in tres par 
tes æ̇quas, ex quibus infimam linea tranfuerfa å reliquis fepara, atq̧ ex ea tæniam facito prominétem quanta altitudo eius eft, ex reliquo'torú effice cuiuș ambè decliuitates capituli latus tangant, rotundatio verò nó magis emineat quàm acuties scotix, quæ ipfi incumbit. Quinta fuprema fcotia magna faciêda eft vt prius, \& inferior capituli pars duabus tráfuerfis diuidenda eft in tres partes, ex fuperioribus duabus fiant dux fcotix, \& infima maneat fafcia. Sexto partiatur capitulú à fuprema fafcia vf́g in imum tripartitò, fiantóg dux partes fupremæ trochilus, in fima itidé trochilo relinquatur, fic tamen quòd vtriufque trochili pfunditas de capituli craffitudine ni hil demat. Quú iam hæc capitula perfecta fuerint, licet ea exornare diuerfis modis, pro arbitrio cuiuflibet, id quod vtrúq̧ monftrabo, Sume plinthum de qua fnprà dictum eft, \& primo octogonam,ac impone eam primo capitulo, \& fub quolibet angulo fac dêticulum quadratum, ea tamen parte, qua capitulo iungitur, craffiorem:huius proiectura fit quarta pars craffitudinis capituli. Déticulus autê eam habeat fpiffitudinē, quam plinthus octogona. Item quando capitulú aliquod ornare volueris, tunc adiiçe craf fitudini tantum quantum pro conficiendis ornamentis fatis videbitur. Secundo capitulo impone plinthum quadratum, $\&$ ex fafcia capituli, in duobus lateri bus conficito volutas, parergis tamen aliquibus ornatas. Tertio capitulo:impo. natur plinthus quadrangula excau ata, cui fub angulis pro ornamentis faciende funt proiecture quadratx, qux fint quarta pars craffitudinis capituli atgue craffiores plin tho, vna quarta eiufdem. Deinde conftituenda funt ornamenta totúda in excauatis plinthi lateribus, tam craffa quàm plinthus eft, atque prominen tia,vt latera plinthi, fi adhuc quadrata effet, tangant. Poftremò quum quadrangula pro ornamentis parata funt, tunc aliquid pulchri in ipfis fculpitur $\mathrm{yt}$ ra: malia aut folia quędam fingularia, vel animalium capita, aut aliquid de auibus, aut rebus aliis quibufcun que, pro orbitrio eorum, qui talia operantur. Cęterùm capitula abfoluta imponuntur fcapis, $\&$ dabit vnum alteri decorem.

Nunc ad columnæ bafim accedamus, qux quidem in hunc, qui fequitur, modum expeditur:quàm craffus imus eft fcapus, ex dimidio eius fiat bafis fiue $f$ pi $r \mathfrak{x}$ altitudo, atque in vtraque parte à fafcia deducantur linex perpendiculares $v f q$ ad finé bafis, quę diuidatur duabus lineis tranfuerlis in tres partes, ex quarum infima fiat plinthus quadrangula, ea proiectură habeat quanta eius craffitu do eft.Sed proiectura fiue productio reijalicuius prominentia eft à proprio corpore cui adhęret:deinde fecáda eft media tertia in tres partes, quarum fuprema fa fcia maneat plana, at ex reliquis duabus pars fiat tori, fic:fifte cırcini pedé in fuperiorem tranfuerfam plinthi, atque perpendicularem lateris, \& reliquum in angulum fafcix iam formate, ac fcribe circination is arcum à bafis latere in partem exteriorem, vfque ad plinthi fuperiorem tranfuerfam. Poftea diuidaturfuprema tertia in tres partes, quarum infima detur fafcix, que excedat eam cui incumbit per medietatem altitudinis fux, reliqux dux relinquantur toro qui vltrafafciam, cui fuperiacet producatur quátum dimidius rotundationis arcus excurrit, fi principium eius fumatur in angulo pradicta fafcie.

Iam locanda eft hęc tota columna cum capitulo \& fpira fuper fty lobatam qui 
hoc pacto faciendus eft. Primò quadrangulum paretur altitudinis quadrantis totius columnæ cum capitulo \& bafi, amplitudinis verò quanta eft infima plin thus bafis: hic ftylobata in fundamentum ponendus eft quadrágulus, deinde ab eo fupernè fecanda eft vna decimaquarta, per lineam tranfuerfam, ea tantundé à perpendiculari lateris producenda erit, atque fegmétum iftud rurfum per medium diuidendum eft linea tranfuerfali, ac fuperior pars fafcia maneat, inferior autem quadrans fiat tori, qui in angulo hareat, $\&$ ad dimidiam proiecturam $f a-$ fcix procurrat, hoc facto abfoinde infernè ab hoc ftylobata feptimam partem quam tantundem vltra latera eius producito, atque ab eadem feparato fupernè vnam quintam ex qua fafcia fiat, producta pro altitidinis quantitate, $8 \mathrm{x}$ angulus ipfius fupernè ad circinum rotundetur: iam quod reliquum elt fub hac fafcia diuidatur bipartito, per lin eam tran fuerfam, atque ex inferiore fegmento fafcia fiat,cuius proiectura vfque ad perpendicularem lateris fecetur lineis erectis in partes tres:atque à termino fuperioris fafcix vique in fafciam inferiorem circuli arcus ducatur quidimidium faciet torum, ita tamen, quòd prominentia infim $x$ fafcix, torum illum excedat vna tertia. Stylobata hic diuerfis modis exornari poteft : led ea qux fuprà fcripta funt, infernè cum fuis fundamentis defignaui.

Verùm vt intelligatur, qux fint ornamenta, qux dolabra \& torno fieri queant, notandum eft fex effe res prxcipuas, quibus hæc ornamenta conftent. Prima igi tur elt fafcia plana, fecunda fcotia fiue trochilus, tertia torus, quarta angulus p. minens, quinta angulus cócauus, fexta cymatium eft, tortuofum pro cuiuflibet arbitrio: his rebus vti licet omnib us fimul; vel aliquibus folum, atq fieri poffü cóuexę fiue concaux, planę vel pminentes, amplax aut ftrictx, acutę fiue obtufe, magnæ aut paruæ inter $\mathrm{fe}$, late vel arctę, aut quocúg tandem modo placuerit: fed fymmetria debita quilibet operarius vti debet, vt operibus magnis ornaméta magna, $\&$ paruis parua adiiciat $:$ hęc infrà duabus fácciis lóga $\&$ breue iuxta fefe politis monftrabo:nam cuiuflibet hominis intellectus facilè percipit, quòd res longa merito craffior effe debeat, quàm breuis fui generis:quocirca fiarchiteetus cymatia aut proth y rides, aut aliud quiddam fimile conficere velit, debet exteriora, qux longius currunt ampliora facere, quàm interiora que breuiora fune. Si huic conuerfum fiat erit deformè, quod hoc pacto accipito:fafcia efto erecta, inter duas lineas contenta, eam abfcinde fupernè per lineam tranfue: ato angulos illos nota literis a $\&$ b, deinceps pone forte fortuna iuxta medium fafcix punctum $\mathrm{c}, \&$ duc duas lineas obliquas a $\mathrm{c} \& \mathrm{~b} \mathrm{c}$. Poltea notato interioré faf ciam quę breuior fieri debet linea recta iuxta a $b$, tantum $a b$ ipfa a $b$ diftante, quàm latum tympanum effe velis, \& vbi ea linea obliquam a c tangit, illic fcribe $d$, à quo duc tranfuerfa $m$ vfq ad aliam obliquam $b c, \&$ punctum contactus nota charactere e,ex eo trahe perpendicularem deorfum, $\&$ habebit $d$ e, rectam proportionem ad a $b$, quemadmodum hoc vnà cum fuprà dictis fex rebus, qux ad ornaméta pertinent, atø colúnis, capitulis, bafibus, \& Atylobatis, infernè om nia ocul is fubieci. A c veluti cum fafciis iam poftremò actum eft, fic agendum erit cum reliquis rebus omnibus. 


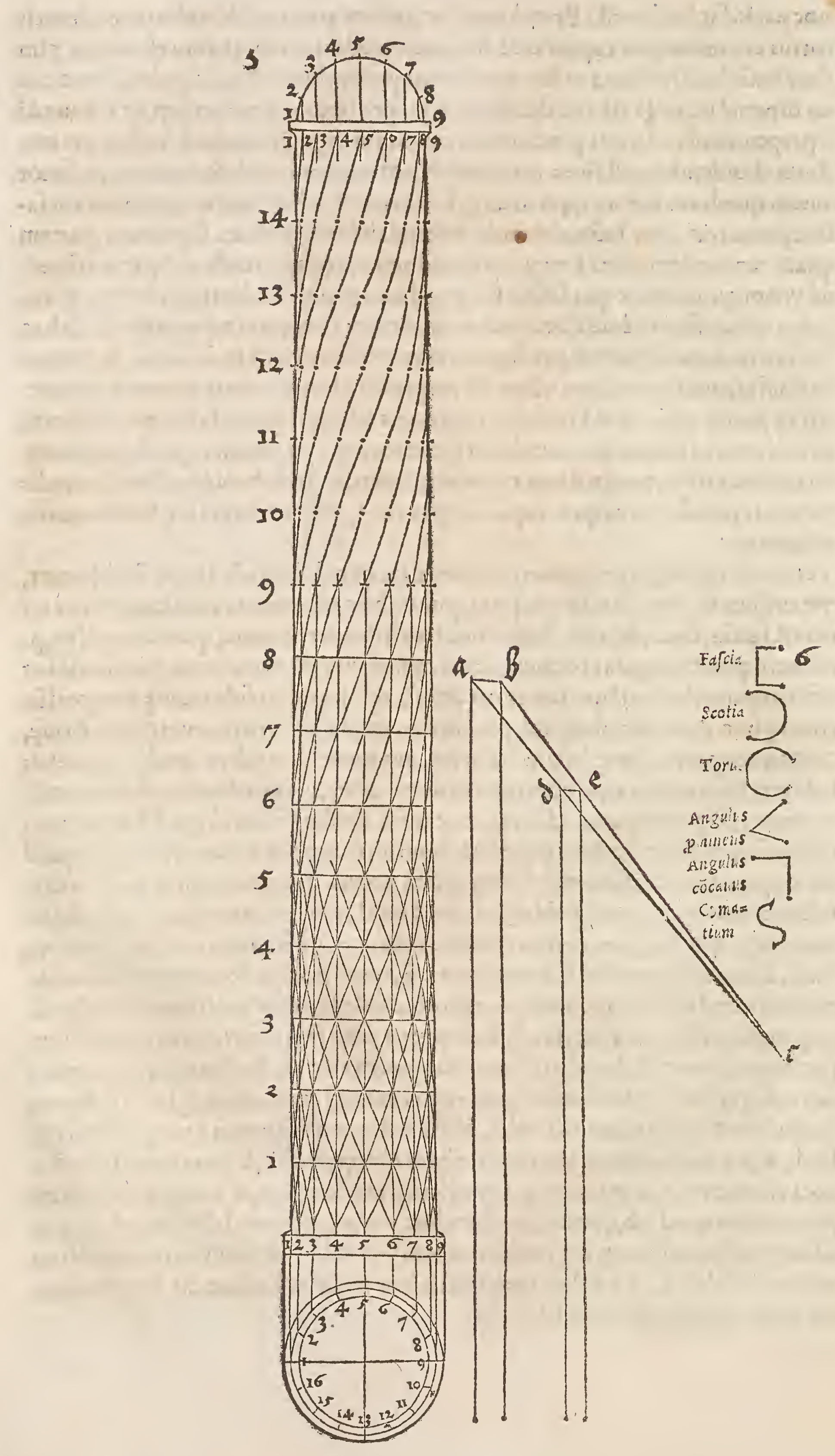


GE OMETRIAE LIB. III. 85

6

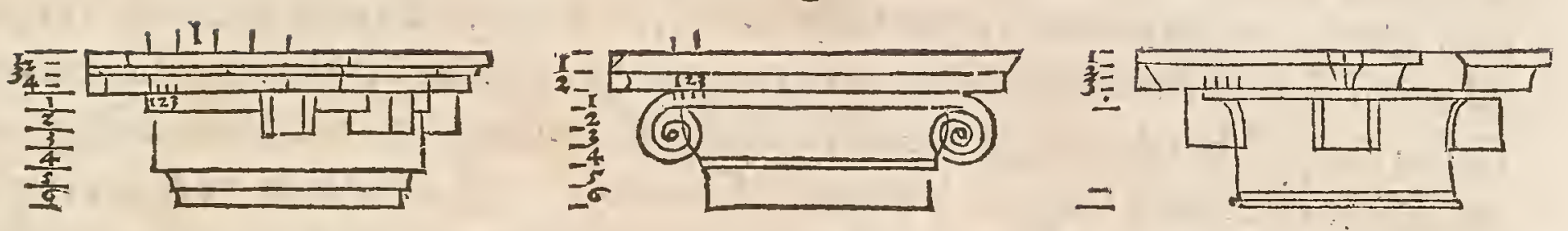
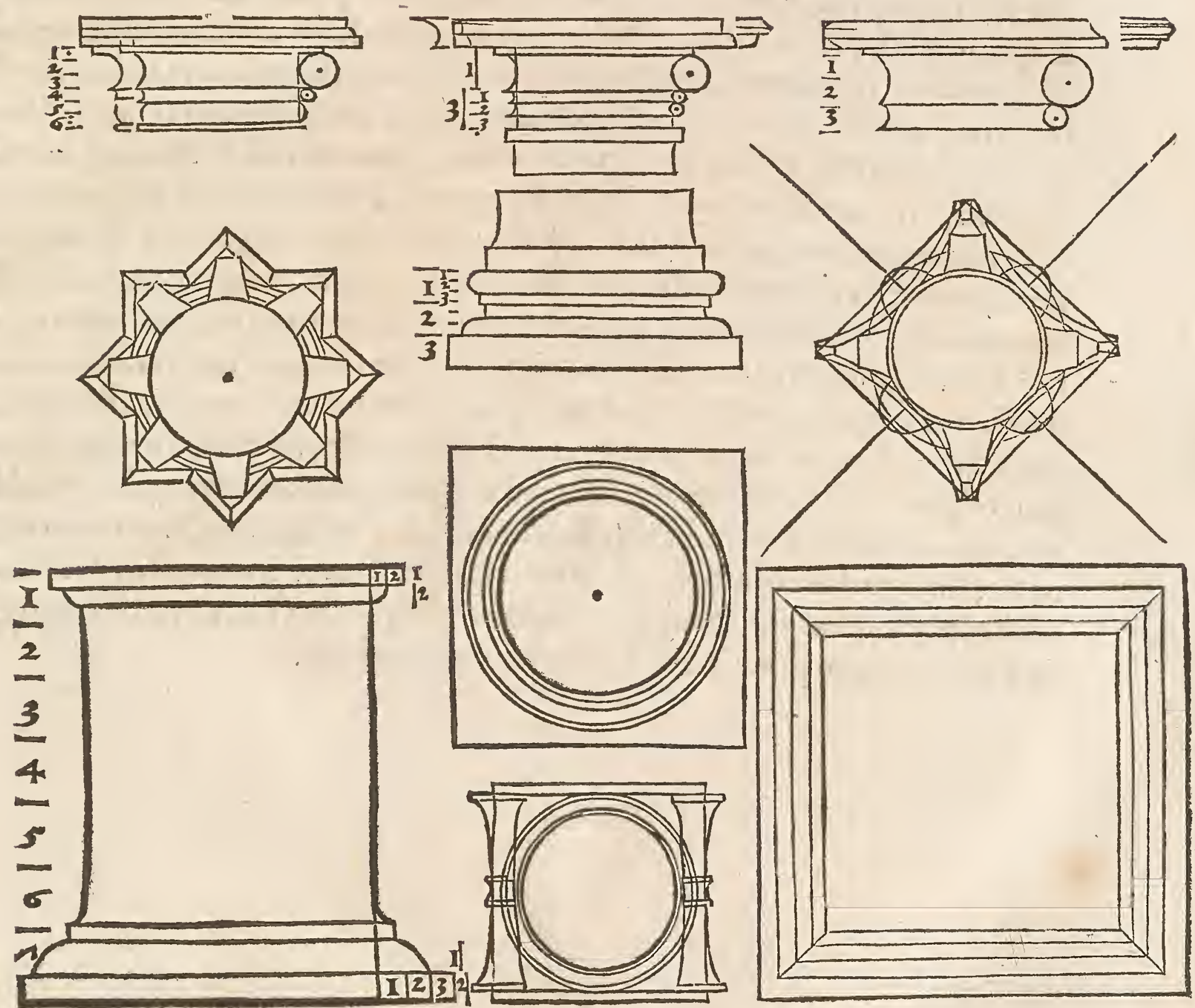
Vi fuprà defcripte colünar ventrem fuve adiectionéfacere velit, id du? obus modis expedire poterit. Primó fecetur columna longitudo in tres partes qua rum infimam linea tranfuerfa cốnenit à reliquis diftin guere, ac in eodem loco fcopus tam craflus fiar, quàm fa fcia elt inferi or, deinceps notêtur tria puncta in columna lateribus. Supremum quidem fub fafcia fuperiori,infimum fupra inferiorem, \& tertium ad eum lozú vbi fcapus eft craffiffmus. Per hac puncta arcus circuli ptendatur veluti vigelima quarta figura primi libridocuit, ex co nancifcetur columnacirca medium additioné venuft am:Secundò fcapi adiectionem fic abfolusto, diuide eum, vt prius tripar tito $\&$ tranfuerfam, qux infimam tertiam abfcindit prioris ftatux lógitudinis, ac fpacii ffud, quod extra perpendiculare columne larus adiicitur in vtrog, latere, partiatur in quartas. Demde diftribue duas tertias fuperiores, tribus tranfuerlís in quatuor parres:confimiliter etiam infimă tertiá in quartas fecato.Porrò maxima addurio colünę fiat in tertia infima, à qua furfum $\&$ deorfum recedẻ do, aufer à traluerlis, vnam ex illis paruis quartis, deinceps duas, poltea tres, tan dem omnes quatuor, atque ita ad:ectio formam habebit elegantem. Si iam columnam ornare placuerit, id expedire licebit lineis rectis perpendicularibus, $\mathrm{f}_{-}$ue in modum cochlex tortuof 1 , guarum rerum principia ex fundamento fumu tur depreffo:quo circa hoc in promis conftituêdum erit pro fcapi craffitudine, quo facto fundamétum diuidarur in triginta duas partes ex quibus alternatim nunc friges fiant, ad femicirculum excauara: nunc ftrig, in medio harum bacu. lum erigito craffum ex dinsidua fria parte. lam fic columna per ambitum obli què ltriandofuerit circuiribus fimplicibus, vel duplicibus inter fefe concurrentibus, tune partes furfum vbi circuitiones fe interfecant prolongato, deorfum verò côtrahe, quod aptiffimè Geri poteft per triágulum a $c b$, ex arcus centro $c$, qua arte antè quoque vif fumus, at que hic deliniauimus. 
GE OMETRIAE LIB? III.

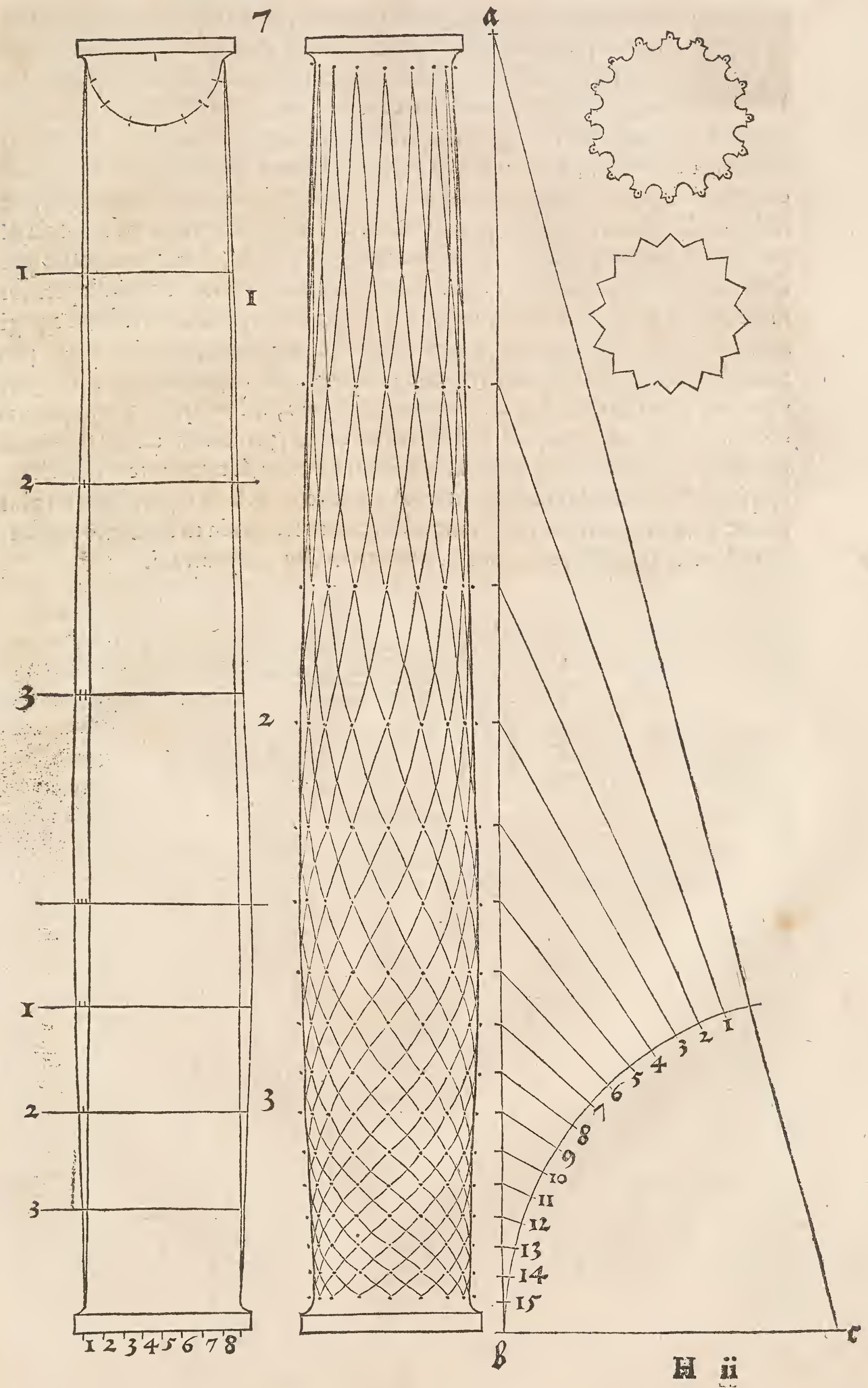




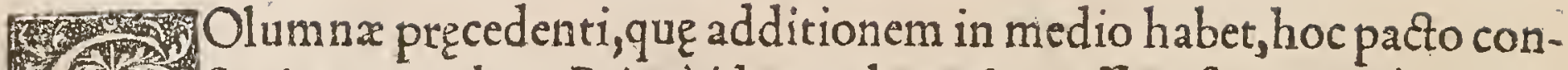
- 1. Itruito capitulum. Primo id tam altum \& craffum facito, quàm eft fu prema fcapi craffitudo, ab hac capituli,altitudine fepara fupernè plin thum, qua fit fexta elufdem capituli pars, ea proiiciatur ex dimidia amplitudinis capituli, reliquum capituli in fex partes diftribuatur, ex quibus fuprema deturfafcix cum cymatio, ea fafcia habeat fpiffitudinem ex quarta crafitudinis plinthi, atque producatur ad dimidiam proiecturam eiufdem. De inde fac fub cymatio tertiam graciliorem vna tertia, quàm fuperior fit fafcia, 8 proiectura eius ex quinta fiat parte prominentiq plinthi, ab hac tęnia,caua qux dam rotundatio procedat, ad capituli latus, \& habebit inferior capituli pars for mam cyathi ampli, qui cum lance, qux ipfi incumbit, venuftatem referret egregi am. Iam poteris capitulum exornare foribus, animalibus, aut aliis parergis pro tuo arbitrio. Et fi quid ornamëti, fub quatuor plinthi angulis adiicere placeret, id per volutas fub cyathi tęnia com mode abfolues, \& hoc quadratum poteris coltituere tam magnum, quàm plinthus eft craffa, prominens ad duas quartas pro iecture plinthi, licet quoque lancis illius formam, 8 eam partem qux eft in. ter plinthi angulos, $\&$ volutas, ornatu aliquo decorare. Item fi plinthus tibi plus extare videtur quam par effer, tunc ad circinum fcotias in ea excauato profun: dius, \& refeca angulos eius quoad fatis fuerit.velut hic protraxi.

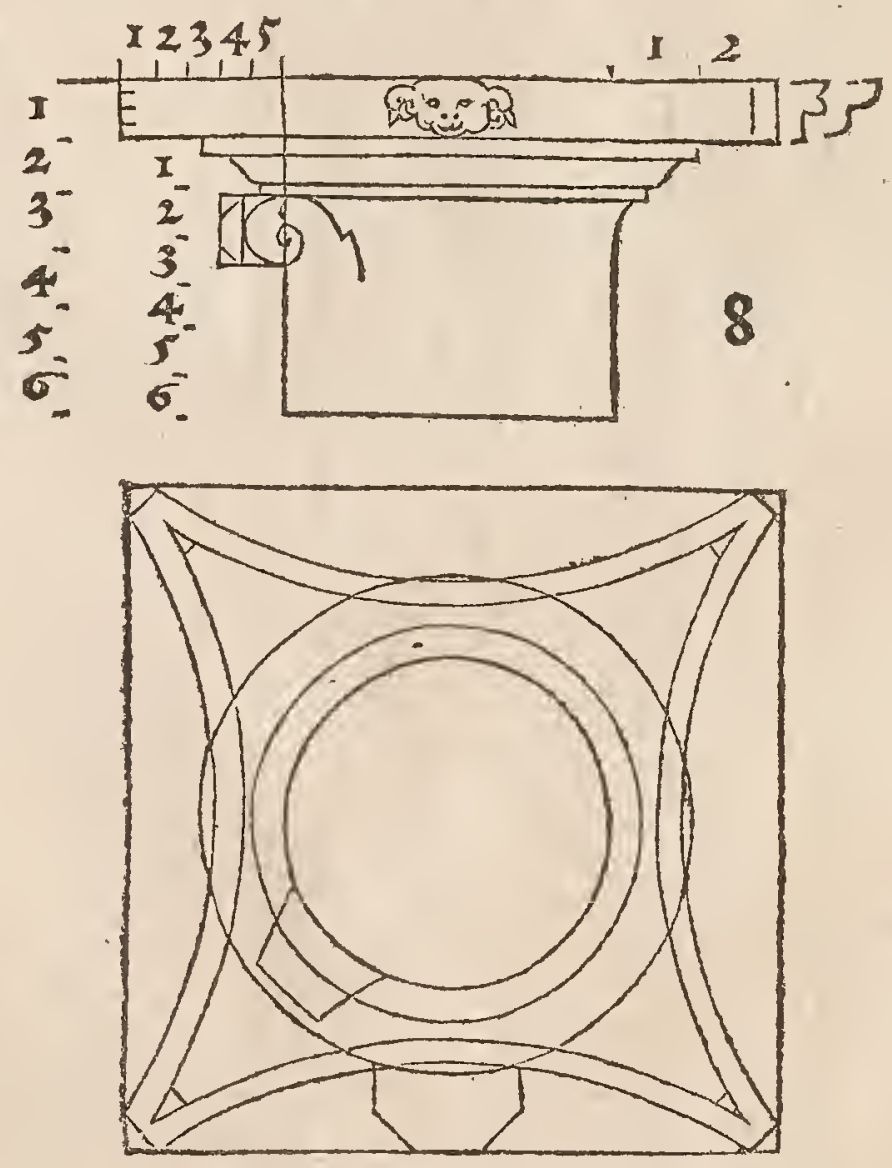




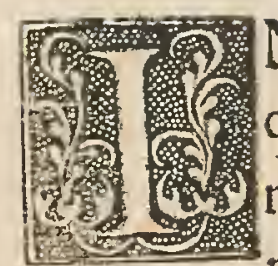

$\mathrm{N}$ fequentibus quandam aliam teretem columnam conficere docebo, qua fingulari quodam modo inflectitur atque diftorquetur, ea pro or namento v ti poffumus, ac ftatuam ipfi ad memoriam rei alicuius impoinere, qua hoc modo abfoluitur. Quam craflitudinem imus colum næ fcapus habet, ea pro totius fcapi altitudine nouies repetatü, contrahatúrg fupernè ex fexta fcapi imi craffitudinis parte, $\mathcal{Q}$ annulus ibidem tantum proiiciatur, quanta infernè épiffitudo eft columna, \& fiat craffus ad proiectura men furam:ea ornatus altitudo duabus tranfuerfis diuidatur in tres partes zquales, in quarum fuprema annulus maneat, in mediofafcix, cuius prominentia terminatur inter annulum \& fupremum fapum, infima tertia cymatio detur, quod in fupremo finitur fcapo. At fafcia qua fcapo fupponitur, eius craffitudinem fu perabit ex vna feptima,de hac craffitudine fexta pars tribuatur altitudini, quá in tres partes fecato, earumg fupremam linea tranfuerfa a $b$ inferioribus diftin. gue, ac facito ex ea cymatium, quod à fcapi extremitate procedat ad fafciá qu $x$ ex reliquis duabus tertiis perficiatur. Sed priufquă ftilobata \& capitulum conftruantur, debet repeti columna recta, ac primùm quidem fundamêtum locetur, ex quo fcapus nofter flectend us erit. I tag columna recta defignata fecundum li neas extremas, ducatur per medium eius linea perpendicularis, qux fit infernè $a, \&$ fupernè $b$, hrc linea a $b$, in cochlę modum diftorquenda erit ex fundamé to quodam, quod hoc pacto expeditur.Pone centrum a, ex quo circulum defcri be tantæ quantitatis, quanta craffitudo imi fcapi, in eo circulo diametrum ducito perpendicularem, cuius fuperiorem medietatem puncto $c$ partire per medi um:hoc facto ponatur in inferiori femidiametro centrú $\mathrm{d}$, ex quo ducatur circi nationis linea qux fupernè tranfeat per punctum $c$, infernè verò tangat circúferentiá priùs def́criptam, deinde diuide portionem diametri a $c$, bipartitò in figno e, eo centro ad interuallum e $c$, fiue e a, protrahatur peripheria. His perfect is diuidentur tres illi circuli in partes fexaginta, quibus adiciantur fui numeri qui exordium fumant internè ad punctum a, à quo in circulo intimo numera $a b$ vno vfque ad fex, qux locum habebunt in figno $c$, deinceps $7.8 .9 .8 \mathrm{c} . v f_{q \beta}$ ad is fcribio media in circumferentia, qux eius medietatem occupabunt. Cum I, poftea exi in maximam peripheriam, quam circui totam, fic vt 42 veniant ad. punctum 18 , hoc eft ad circulorum diametrum . Deinde introito rurfus cum 4 ; in mediocrem rotundationis lineam donec cum 54 veneris ad fignum $c$. Porrò 55,8 qui eum fequátur numeri fcribito in circulum minimum, quoad cum 60 attigeris punctum a. Hos numeros licebit ducere in quam partem volueris, per eos autem oportebit axem erecte columnç torquere. Igitur abfoluto ifthoc fundamento diltribuatur columna erecta in fexaginta partes, fed eo quo iam dicetur ingenio. Protrahe lineam, quę fcapum infernè à cymatio feparet, in continu um \& rectum tranfuerfè in duplolongioré quàm columnæ ibidem craffitudo exiftat, cuius finem nota flitera, à qua ducatur linea obliqua, $v \int_{q}$ ad finem $f_{\text {capi }}$ fupremi, ac fifte circinum vno pede in punctum $f, \&$ alium prope fcapum ad fignum $h$, in linea tranfuerfa, à quo ad obliquă arcum liniato, quam vbi attingit fcribe characterem $g$. Hunc arcum $g h$, feca in fexaginta partes aquales $\&$ tra$\mathrm{H}$ iii he ex 
he ex puncto $f$,per omnes eas partes lineas rectas ad fcapum vfque, in quo puncta illa notato manifeftè, $\mathcal{Q}$ ab eis lineas producito tranfuerfales, per columnam erectam, quibus adiice numeros depreffi fundamen ri, qui in columna $\&$ arcu $h$ g, debent infernè incipere. Ex hoc apparet quomodo portiones in columna ere cta alcendendo fiant longiores.

Ruxfus ducito lineă perpendicularem a $b$, qux columne axem repręfentet, cum omnibus fuis tranfuerfis ac numeris, \& fumpto circino perge ad fundamétum rotundum, per quod puncta axis dimoueri flectióp debent, \& pone remper pedé in diametrum perpendicularem rotundi fundamentique circulos per medium dinidit, 8 quemad modum puncta numerorum femper inter fefuccedút, ita pede reliquo minima meorum à pradicta diametro diftantian fume tranfuerfè ac defer can in columne axema b, locando circini pedem in iplum axem, in linea tranfuerfa qua fignata eft eo numero, cuius diftantiam in fundamento accepirti, \& altero pede mprime notam in eadem linea tranfuerfa, vbi variatus pü ctus tortuofi axis ftare debet:Sic operare perfingulos numeros in vtroquelatere rotundi fundamenti $\&$ axis ereeti a $b$, atque lineis tranfuerfis, $\&$ defiginantur puncta flexuofi axis retortæ colúnx, qux longior ipfa recta propter flexus quos facit, nec tamen al tior fiet. Poftea defer circino ab axe $\dot{\alpha}$ tranfuerfis er êt $x$ colú na craffitudinem finuof $x$ ad axem eiufdem, vbicirculos defcribito ex quibus tui fcapi inuenies fpiffitudinem:nam meminiffe oportet quòd ex rotunda colú na in omnibus lineis tranfuerfis, medióg puncto globus excindi poteft. Item quòcun globú locaueris mouerisóg in partes diuerfas, nihilomin us femper ver fus te poterit plano aliquo per medium fecari. Quum igitur colúna rotunda vl tro citróque flectatur, manebunt femper in ea huiufmodi globi inaaninandi, qui per pradictas fectiones fcind untur in omnibus punctis medis, quotquot volue rimus. Porrò cogita omnem punctum in axe rortuof columna effe elufinodi globi centrum, quare ex eo in eadem linea tranfuerfa, in qua punctum axis acci pitur, circulum defignato, tam magnum quàm crafla in codem loco ereeta colü na eft:at $\$$ hoc faciendü eft per omnia axis puncta columna flexę:\& fic habebitur eius craffitudoper fingulos flexus: def criptis his circulisominibus linea cótinuato conuexitates ipforum, \& apparebunt columnx flexus.

Si verò per omnes circumferentias, qux fectiones in globo reprefentant ac centrum fiue axis punctum lineas duxeris tranfuerfas veluti prius duct $x$ funt, $\&$ in curua columna peripheriam fignificant, ac per earum extremitates in vtraque parte per totam curuam columnam in ominibus circumferentis fecundum lon gitudinem lineas traxeris, videbis quomodolinea in flexibus propter circuitus columnx contrahuntur atque abbreuiantur. At cum in colúna recta tranfuerfales linex omnes rotunda plana defignêt, ac directê vnum fuper aliun ftet, tamen linex hę in circuitu flexę colún̨ non amplius manebút vna recte fuper ali am, fed declinant, pendent $\&$ vertunt fe vltro citróg, furfum $\&$ deorfum ac in omnia latera, \& fient obliquę, oblongx, rotundx linex ex eis. Hxc omnia infer nèvtcunque deliniaui. 
GEOMETRIAE II B

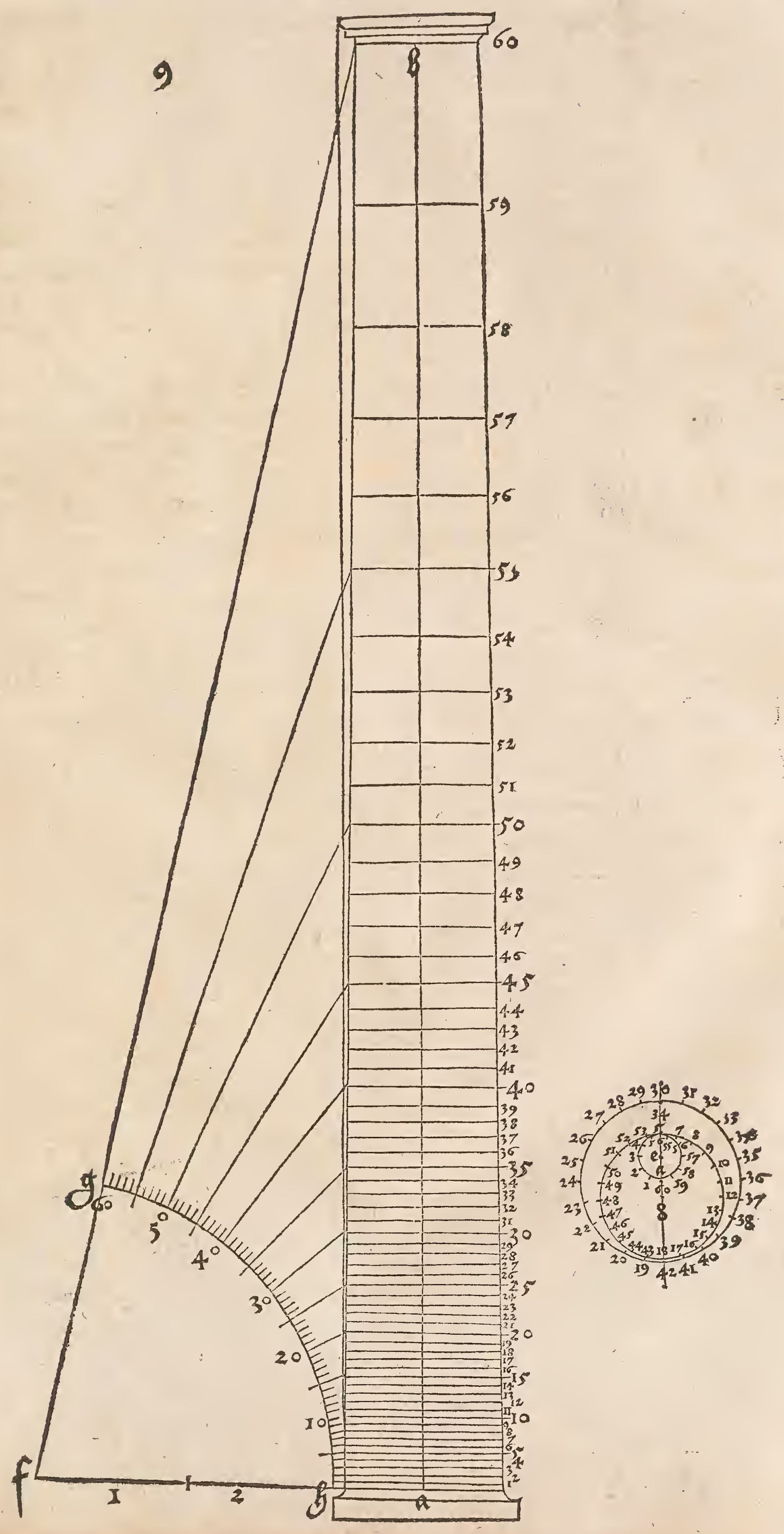




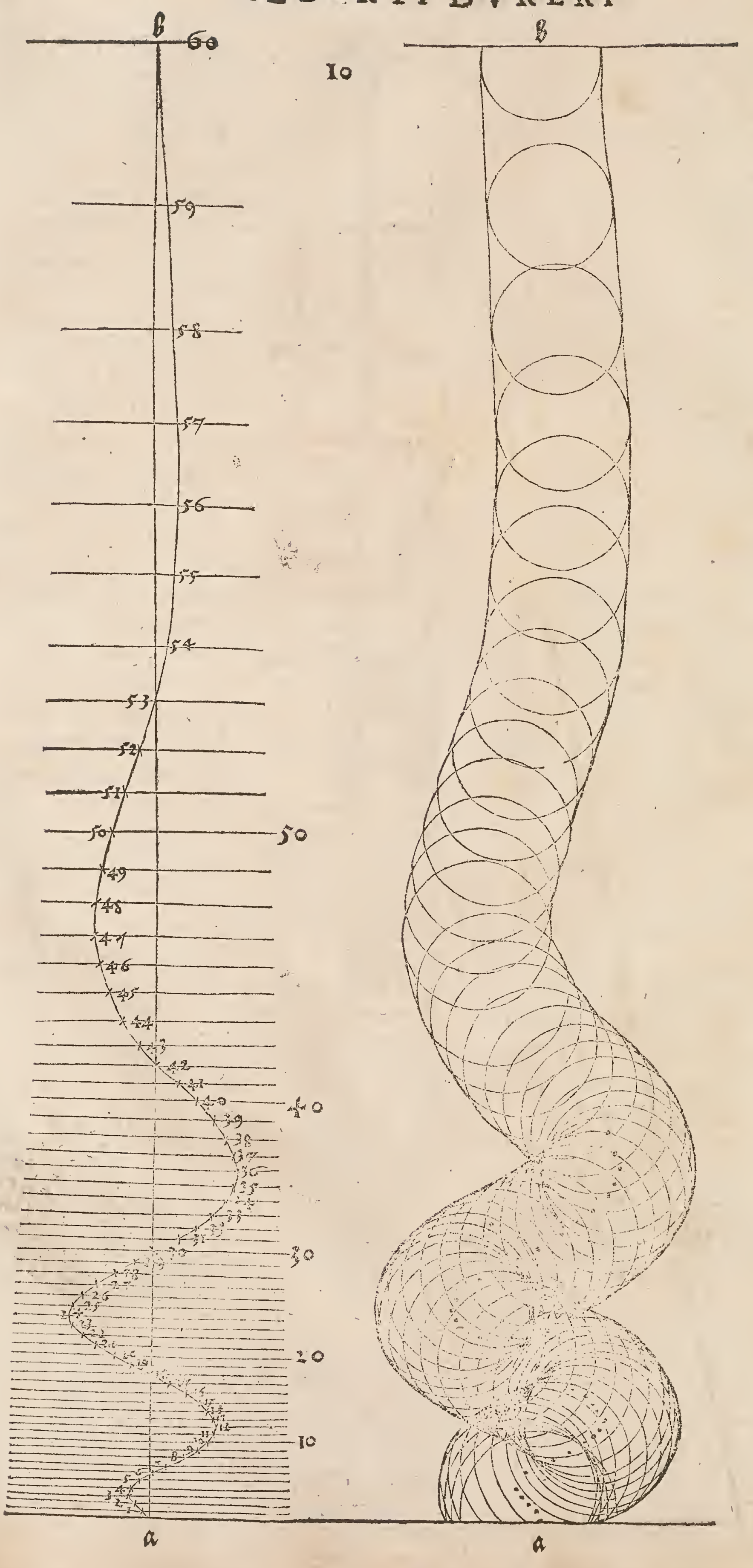


Ortuof huic columnx huiúmodi facito bafim. Primò quadrangu7. 15. tripla fiz ad altitudinê fafcię, qux eft fub imo fcapo:id quadrăgulum (2) - (e) lineis tranfuerns per literas alphabeti fignatis hoc pacto diftribue.Su periùs quadranguli latus tranfuerfum fit a $\&$ inferius $b$. Deinde partire a $b, d u$ abus tranfueris $c$, \& d in tria aqualia interualla, \& a c tranfuerfa e per medium. Foltea fecato a e guatuor punctis in quinq partes, quarum fupremá ab alis feparato linea $f, \&$ e c tribus pútis difpefce in fpacia quatuor, ex quibus infimú à reliquis diftingue linea $g$. Cecterum $e \mathrm{~g}$ partire in quartas, ac fupremam tranfuerfa $h$ ab aliis relecato. Porro $d b$ in fex partes diuidito, quarum infimas duas linea i, à fuperioribus abfeinde:fupremam verò ab inferioribus linea K. Quum iam linez tranfuerfè pro ornamentis, que inter eas facienda erunt, def criptę fint omnes, pergito ad perpendiculares in quibus ornamentorum amplitudines fini ri debent, atg eas fir vn votantum latere produxeris, feruiết tibi $\&$ in reliquo. Latus ergo perpendiculare quadráguli efto $1, \&$ linea quę à fcapifafcia per qua drangulum defendit fit m:nuncl $m$ per lineam $n$ fecetur in medio, hęc linea inter $\mathrm{c} d$ magnam terminat fafciam, in medio balis conftitutam, quo facto diftribue fpacium m n per linearn o in duas partes, finiet'́p hac linea torú, qui eft inter $\mathrm{f} f$. Sed o $\mathrm{m}$ feca in duo interualla per lineam $p$, in qua terminan tur dux tęnig, altera inter af fupra torum, altera autem inter h h fub toro. Item fpacium no per lineam q fecandú eft bipartitò, vt ad eá proiiciatur tania inter $c g$, at fco tia qu $x$ inter $g h$ in ppendiculari $m$ finitur. His perfectis pducatur fafcia inter $\mathrm{d} K$.vltra n tătum, quáta fpiffitudo eius eft, fed fafcia infima porrigitur $\mathrm{vf} q \mathrm{~g}$ ad lineam 1 , trochilus autem inter fafcias illas tangit lineam 11 . A tqui ex fuperiori fafcia $\&$ trochilo licet fcotiam vnam conficere $\&$ fic deperditur predicta fuperi or fafcia, quemadmodum hic omnia pręter poftremum illud defignaui.
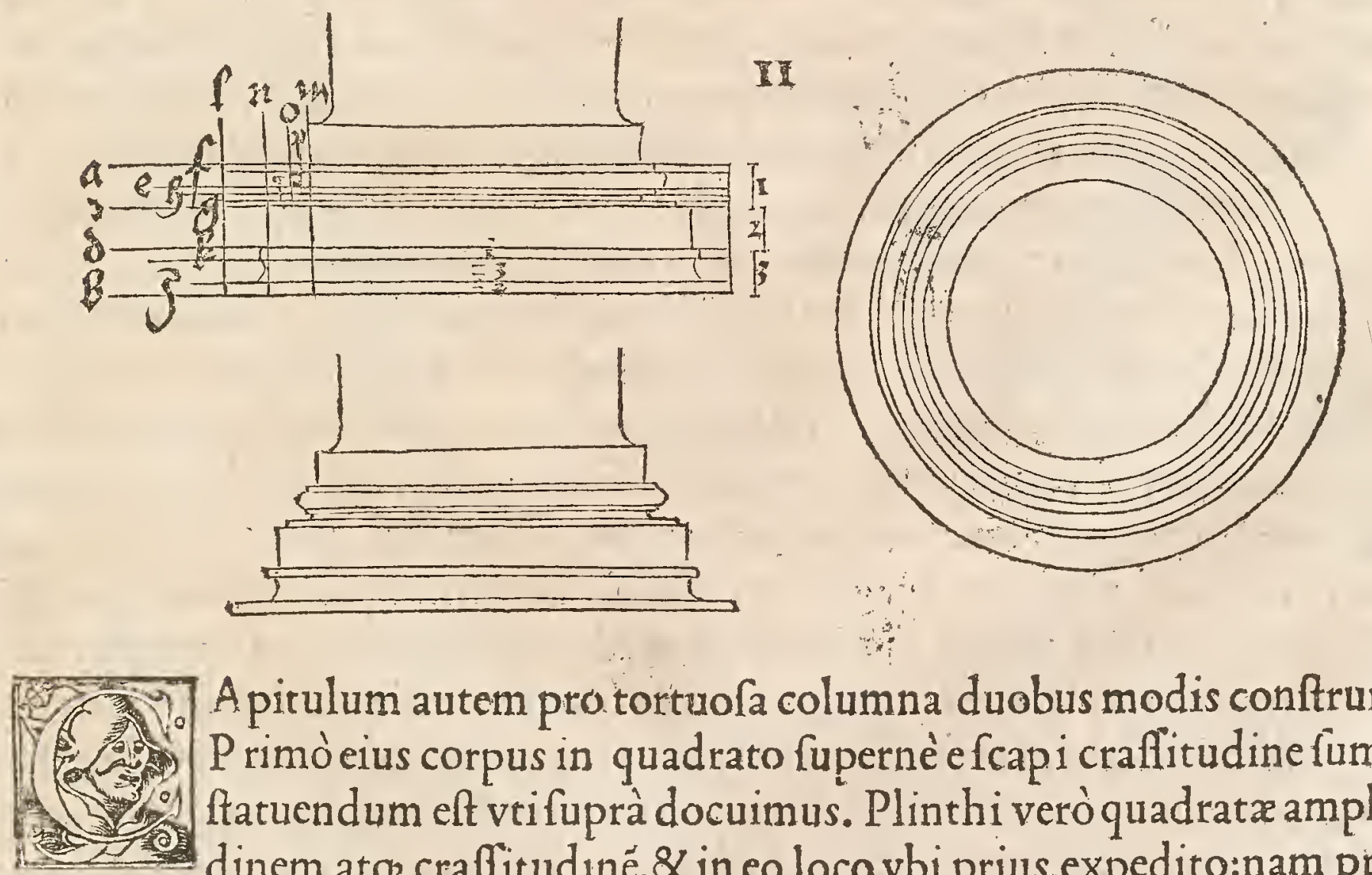

A pirulum autem pro tortuofa columna duobus modis conftruitur, P rimò eius corpus in quadrato fupernè e fcapi craflitudine fumpto ftatuendum eft vti fuprà docuimus. Plinthi verò quadratæ amplitudinem atog craffitudiné, $Q$ in eo loco vbi prius, expedito:nam proieetura eius plurimùm prominet propter columnę flexus, vt capitulo conferat ve 
nuftatem. Item fupremam fafciam fub plintho, cum latitudine $\&$ piectura fua conficito, velut de pracedente capitulo dictum eft:in medio inter hanc fafciam $\&$ imum capitulum lineá ducito tranfuerfam, atọ ab eo fpacio quod inter eam \& fafciam efl feparato rurfus alia tranfuerfa tertiam infimam:eo facto trahe line am obliquam à termino fuprem $\mathfrak{f a} f \mathrm{cix}$ ad imum capituli, in qua fifte circini pedem, \& alıo à fafcix extremitate in fpacio 1 llo ampliffimo circinationis arcum defcribe verfus capituli latus fic quòd Cemicirculus infernè proximá tranfverfam tăgat : \& fecabic hic arcus ibidem trochili acutié, quantü extare debeat, fietọ́ fcotia magna, deinde inter anguftiores duas tranfuerfas circulum delinia to, qui vtram q̧ earum tágat, vnà cum capituli latere ac linea obliqua, ex eo proueniet fcotia parua, qua fuam acutiem ipfamet prominetem indicat, à qua víg ad capituli latus iterum paruam defignato fcotiam. Quum iam capitulum exornare ftatuifi, fac fub plinthi angulis ornatus in fuis quadratis eius craffitudinis, cuius magna eft fcotia, \& proiice eos paulò amplius quàm ex media capituli craffitudine:in minori fcotia potes ramú ftatuere, à quo fe folia furfum erigant, at in cauo plinthi latere rolam aut aliud quiddá egregii facere licet. Secú dò capitulü hoc modo variatur. Plint hus cú fafcia fub ea vt priùs relinquitur, fed paoxima fexta fub eademplintholinea tranfuerfa feparatur, ac diuiditur in duas partes, quarú fuperior fotį detur profunde, quę capituli latus tágat:po. ftea diftribuitur \& inferior medietas bipartitù per lineam tranfuerfam, at lis fegmentis binę fiunt tenia, quarum inferior proiecturam habeat fubduplam ad proiecturam fafcie, qux fub plintho elt:at fuperior tantùm retrahatur quàm craffa eft.Porrò fexta, qua tertiò iam fequitur in duas partes fecanda eft per li. neam tranfuerfam, ac in fuperiore quidem parte cymatium conftruetur pro ornaméto, quod oriatur fub infima fafcia, in ea prominétia qua elt fuperior tęnia: id cymatiú fupernè flectatur verfus partem exteriorem, \& infernè verfus interi orem, atque definat in medio inter exordium fuum \& capituli latus. Ex angulo autem qui iam factus eft protendito arcum deorfum vfọ ad capituli finem, cuius medium non amplius extet quàm proxima acuties \{́upernè. Caterú ornatỉ fub quatuor plinthi angulis in fuis quadratis craffum conftitue ex vna fexta $\&$ dimidia, \& proiice eum ex media capituli craffitudine. Item in concauis plinthi lateribus quadrata quoque perfice pro ornamentis magna aut parua. Hoc iubeo in quadratis fieri propter eos qui in lapidibus aut lignis operantur. Iam plinthi craffitudo in hunc modum exornanda eft. Altitudo eius in tres partes fecetur, quarum fuprema, fafcia fiat, reliquæ duæ cymatio rel inquătur, cuius concauitas infernè verfus partế exteriorê definat, ac fupernè quidem id cymatium tantùm poft extremitatem fafcix incipiat, quàm fafcia eft alta. Tanden plinthi latera ad circinum fic folent excauari. Ducantur per plinthi fuperficié binę linex qua $\mathrm{fe}$ in medio eius interfecent ad angulos rectos, ita vt ex plintho quatuor fiant quadrata, eas lineas protrahe vltra plinthi latera, quantú fatis eft. His perfectis defignentur fundamenta pro vtroque capitulo in fuis quadraturis ac fumatur circino prater vnam maxima circulorum diameter:ea diftantia inuariato circino trásferatur ad lineas plongatas vltra quadraturam, fic : figatur 
circinipes vnus in circumferentia, cuius dimetientem accepifti in eo loco vbi linea tranfit tranfuerfa, 8 alius pes extendatur verfus fidem eiufdem linee, atg punctus is fignetur litera a, ita quęrantur etiă reliqua tria puncta qua notentur $\mathrm{b} c \mathrm{~d}$ characteribus, deinceps feruato priore interuallo fifte circinu in ligna a $b c$ $\mathrm{d}$, ac delinia arcus per plınthi latera, poftea defigna etiam circino ex iifdè cêtris $a b c d$ quantū parerga abfcedere debeát in plinthi craffitudine. Poltremò acro terium quod capitulo imponitur, eiufdem capituli habeat craffitudinem, cuius quarta pars detur altitudini, id fcabellum aftragalis quibufdam decoretur.Statua autem, qux fuper columnam locatur fit in duplo altior quàm capitulú cum fuo acroterıo. Hęc capitula cum Luis fundamentis inferius oculis fubieci.

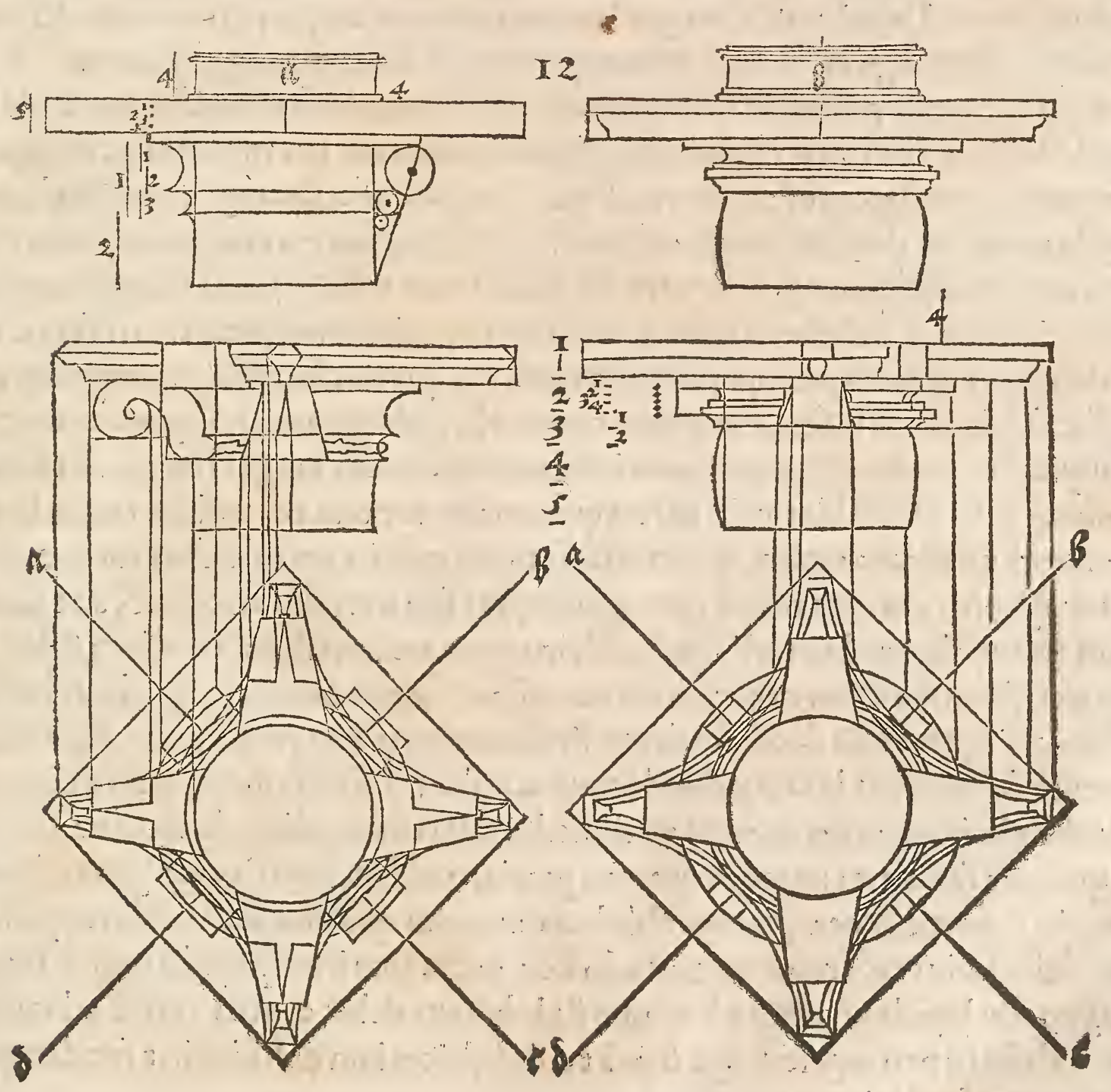




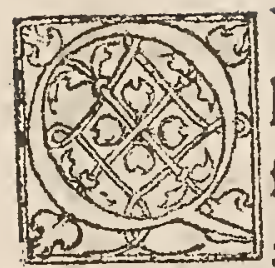

Vum iam abroluta fuerit columna cum bafe $\&$ capitulo fuo, tune fty lobatam ipfi parato, ac primò quidem quadrangulum oblongum cóficito, 1 dque erectum, tantæ latitudinis quanta elt in fima bafis fafcia, fus lapidem itidem quadrangulum, qui fit altus ex quarta erecti altitudine quadranguli, quíque ex omni parte promineat ad quantitaté craffitudinis erectiqua dranguli , ac ab eodem erecto quadrangulo, infernè diftingue linea tranfuerfa vnam quartam à cuius altitudine linea defcendat obliqua ad mediam quadrati lapidis proiecturam, atọ fuper obliquâ illam fcalæ conftruantur quatuor gradibus, qui fupernè promineant ex fux diametri quantitate, habeánt que ibidem fafciam latam ex quarta gradus altitudine, fub qua contractura ad fcotix fiat fimilitudinem. Deinde refecato per lineam tranfuerfam, fupernè vnam octauă aberecto quad rangulo, id fegmentum produc ad altitudinis quantitatem, \& à fuprema fegmenti proiectura, ad infimam fuam tranfuerfam trahito lineă obliquam. Poftea diuide hoc fegmentum linea tranfuerfali per medium, ac ex fuperiore medietate fupernè fecetur vna fexta, quafafcia tribuatur, cuius inferior angulus excedat defcendentem obliquá. Ex reliquo fiat toti medietas, cuius có. uexitas vltra obliquam producatur, fic quòd arcus in ea fupernè incipiat, atque definat infernè in eadem ad lineam tranfuerfam. Caterum medietas inferior bi parcitò diuidenda eft, ac fuperior pars rurfus in quartas fecanda, quarum fuprema fiat $\mathrm{fafcia}$, cuius inferior angulus currat vltra obliquam, reliqux tres fcotix relinquantur profund $x$, qux acutie inferiore obliquam tangat lineam. Refidua medietas infernè diuidatur in partes quatuor, ex fuprema $\&$ infima tania fiant extantes à quadrato erecto, quàm alta funt:ex mediis verò duabus torus expediatur, qui vltra tænias proiiciatur, paulò plus fuo dimidio circulo. Nunc dimi dium torum fupernè ramulis quibufdam exornare licet. Item inferiora ftiloba. tx parergis quibufdam pro arbitrio cuiuflibet. Quatuor autem quadrati latera hifforiis \& epitaphiis decorare decet.Priùs tamen in eis tympana conftituătur, quorum fafcix fiant latx, ex duodecina amplitudinis ftilobatx, fed fafciarum abfceffio in interiorem partem ex dimedia fafcix latitudine abfoluatur. Porrò ad quatuor fcalarum angulos ornatus gratia, totidem viros locato pro cuftodibus. At fupernè fuper quatuor ftslobatx angulos quatuor ftatuito cercopithecos. Iufta huius columnæ magnitudo hoc pacto fumitur:quando homo fuper magno illo lapide quadrato ftat ante ftylobatam, debet capitis vertex ad medium ftylobatx pertingere. Qux fuprà tradidi, hic etiam deliniaui:at fundamentum quum facile fit,omifi. 
GE OME TR I AE L IB. III.

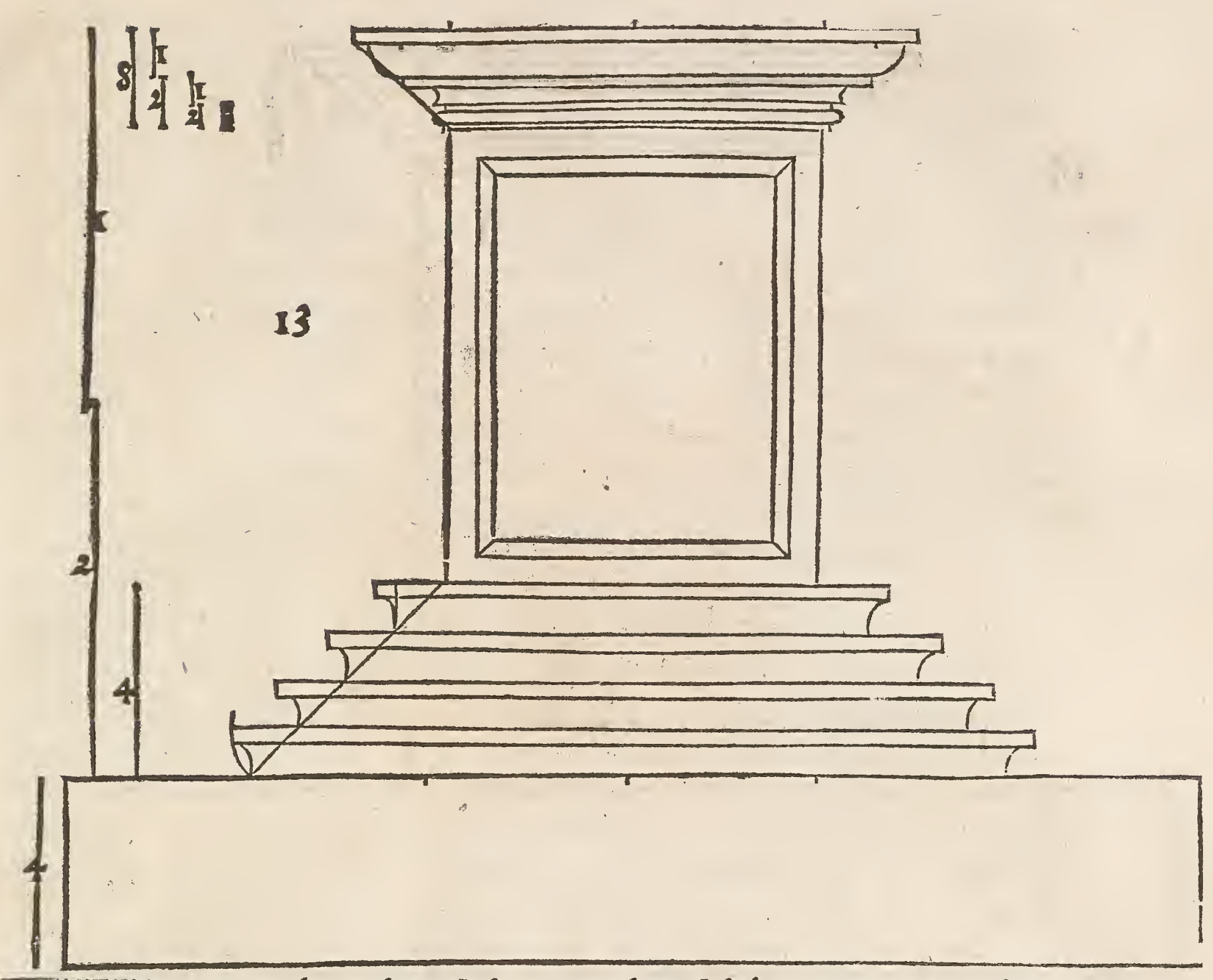

6. In autem volueris, licet fcalas in prædicta tilobata omittere, \& aliter 2. exornare quàm prius, in hunc modum : conftituc altitudinem erecti quadranguli duplam ad fuam amplitudinem : fpira verò alta fiat ex. dimidia quadranguli craffitudine, éque tantundem piiciatur,à quo proiecturatermino inferiore, ad angulum ei oppofitum in quadranguli latere perpendiculari linea ducatur obliqua, qux menfuram dabit productionibus. Hxc fpiræaltitudo in tres diuidatur partes, ex quarum infima plinthus fiat qua drangula, medix infima, tertia fafcix detur, reliqux duę trochilo relinguantur: fed fuprema tertia per medium fecetur, at $\$$ inferior medietas rurfus in tres $p a r$. tes, quarum infima maneat fafcia, cui fcotia fuperponatur, velut prius:in fuperiore medietate dux tęnix conftituantur, in quarum medio totus efto tantum occupans, quátú tęnięambę. Porrù altitudo capituli ex dabus afoluatu r fpiræx tertiis, qux tantundem etiam producatur, éáque altitudo diuida da eft in tres partes æqualiter, ex quarum fuprema fiant dux tęnį̧, fed fuperiorpaulò amplior, inter quas mediet cymatium quod craffitudinem vtriufque habeat tanix, quòdque fupernè in interiorem partem, infernè verò in exteriorem flectatur. Poltremò ex inferioribus duabus tertiis totidem fcotix perficiantur, atque infe riori fafcia incumbat admodum gracilis, quemadmodum hic protraxi. 
14
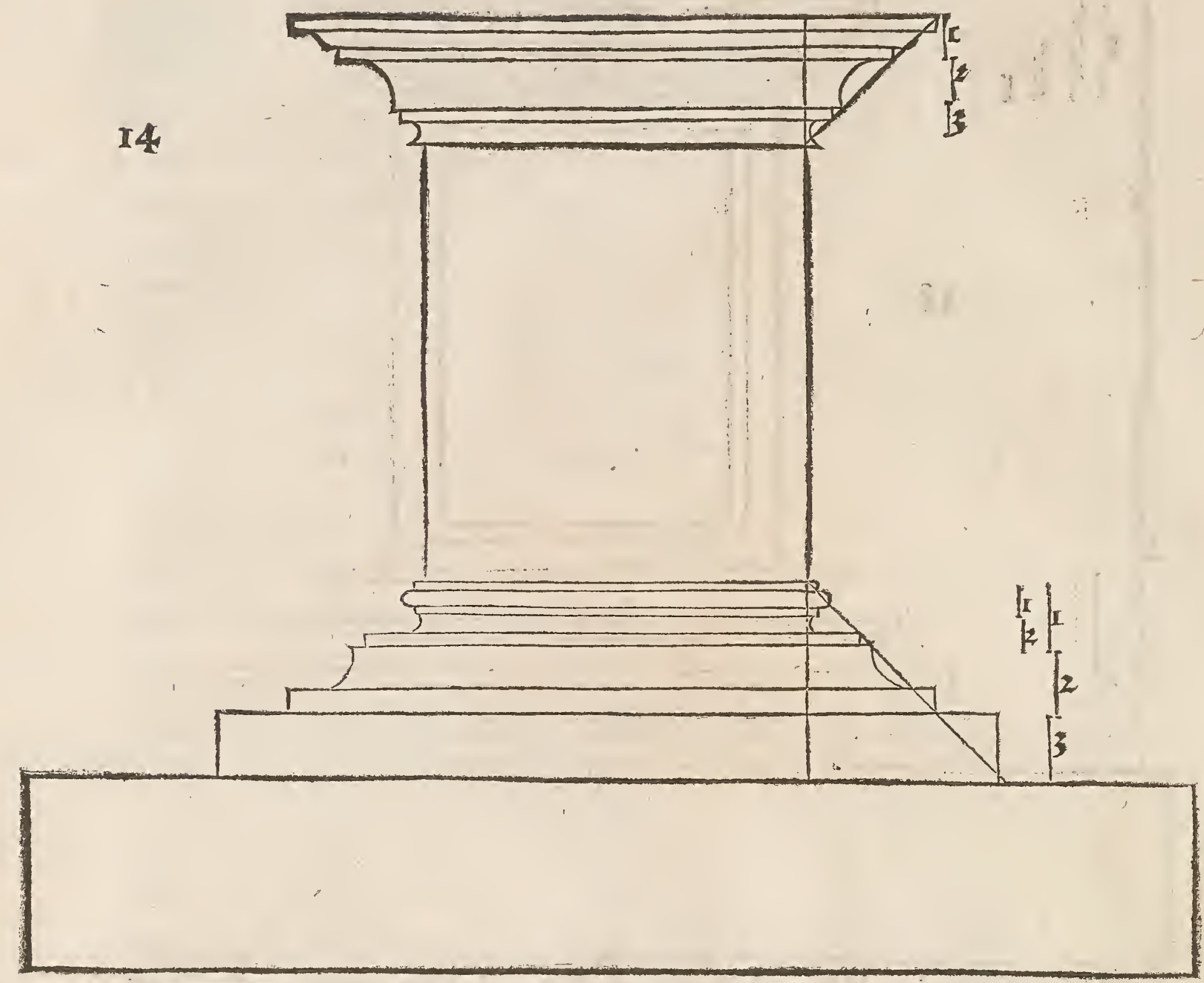

m

Aepe accidit quádo in bello exercitus vincitur,vt in eo loco ad memoriam columna erıgatur, fignificans qui fuerint, qui fuperati extiterunt:quòd fi potentes, poteft de apparatu corum bellico, huiufmo. di colúna extrui . Primò locetur ftrues lapidü quadrata fuper aliquo colle, qux omnı, quæ ei imponuntur fuftinere queat, cuius quodliber quatuor laterum viginti $\&$ octo pedes in amplitudine contineat, extétque nouem fuper terram, illis quatuor angulis fuperpone pilas bombardicas, al tas fefquipedem, \& in media hac cógerie quatuor gradus facito, tribus pedibus al tos, corum infimus habeat altitudinem vnius pedis cú quadräte, reliqui tres certo ordine diftribuantur, fic vt fupremus fiat humilimus, ac medii duo proportionales ertremis velut in fine primilibri de lineis móftratum eft. Gradus infimus longus efto vigintiquatuor pedes, at fupremus viginti 82 vnum, fuper hos quatuor angulos doliola locato pulueri apta bóbardico, altas duos pedes cum dimidio:diameter verò media fit duorú tantú. Porrò graduú angulos linea abfcindito obli qua. His perfectis erige fuper mediam fcalam lapidem quadrangulum oblógum duodecim pedes altum, 8 octo latum. Cuius productio fuperior per tres quartas pedis promineat, eamq ${ }^{\prime}$ habeat craffitudinem, in cuius quarta fuprema 
fiat fafcia, fimiliter 8 in infima, fed hæc minor $\&$ tantum extans quàm alta eft, in harum medio cymatiú conftituatur, quod fupernè in inferiorem, infernè verò in exteriorem flectatur parcem. Verùm ornatus in lapide hoc infernè proiiciatur in quolibet latere ad duos pedes, ac infima fafcia fiat alta pede vno. Deinde in lapide erecto altè ad vnum pedem fupra fafciam lıneam ducito trắ uerfan:atqueex eius fegmenti in fima tertia quadram facito, qux ad vnum pedem extra quadranguli latera promineat, $\&$ ex reliquis duabus tertiis trochilus fiat, cuius fuperior acuties per tertiam pedis partem a quadranguli latere extet. Caterùm fuper erectü hunc lapidế totum tormétum fiue machina ponatur, qua cum globi in altum torquentur, qua cum fuo apparatu latitudinem habet octo pedum, ea cum apparatu cui incumbit alta fiat decem pedum, ac circa os propter firmitatem \& decorem amplitudo efto quinque,at corporis eius quatuor cú quadrante:annuli autem \& reliqna ornamenta extare à corpore parumper debent.Item in eoloco vbi foramen ef incendii, diameter habeat tres pedes, cum quárta eiufdem. Super hac machina bombardam erigito magnam $\&$ fortem, cu ius longitudo fit viginti $\&$ vnius pedis, amplitudo autem pofterior trium, anterior verò duorum, globi, quem emittit, diameter efto vnius pedis. Os auté bom bard $x$ fit firmiffimun: nam ornatus in anteriore $\&$ pofteriore parte debet bombardx corpus proportione certa excedere, id quod ingeniofi bombardarum có flatores exactè folent obferua re. Poftremò huic bombard $x$ fuperponatur tinti nabulum , fic vt anfxeius, bombardx ori inferantur, fit $\&$ altum duos: fuper eo locentur duo afferes inter fefe tranfuerfi, fuper quibus erigantur quatuor virorum arma que dorfa inter fe cóiungát, ita vt in quatuor locis fuper afferes tibiarum dependeant arma:id totú habeat altitudinem quatuor pedum. Sed galearum criftas potes in altum erigere ac diducere in latum quantum placuerit:at afferem vnum fac longum fex pedum, quemadmodum hic defignaui. Verùm quando ifta omnia magno fiunt in opere, tunc fingula exornare decet. 


\section{5}

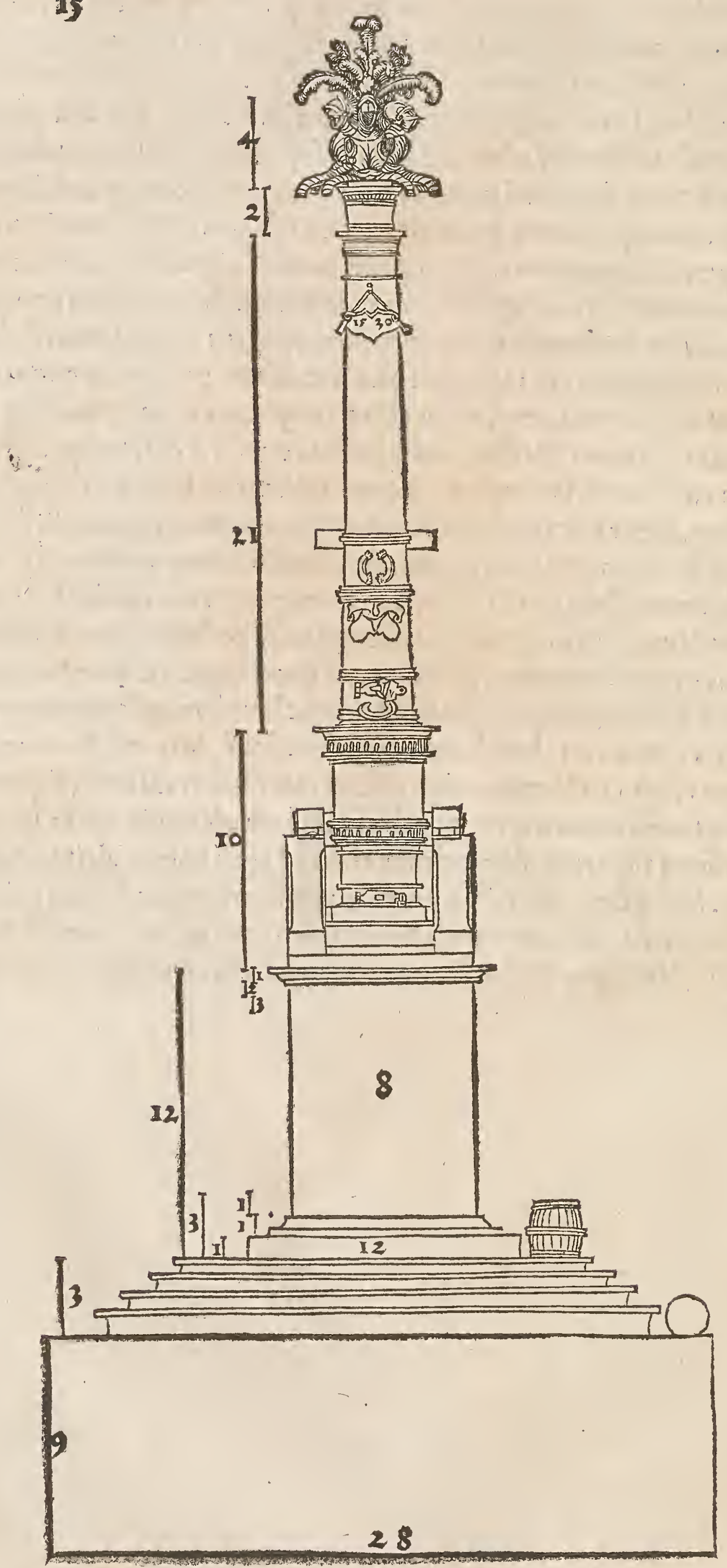


I quis victoria erigere vellet, quòd feditiofos agriculas deuicerit, is $\mathrm{hu}$ II 5 iufcemodi rebus vti poterit, quas deinceps dicani. Prımò locetur la(2) pis quadrangulus, cuius quodlibet quatuor laterum fit longum decé pedes, \& altum quatuor:is plintho cuidam incumbat, qua latera habeat viginti pedes longa, alta autem vnum, fit qu $_{\text {fita }}$ in colle aliquo: fuper quatuor eius angulos ponantur vaccę, oues, porci, atọ́ alia pecora ligara, fed fuper an. gulos quatuor lapidis quadranguli, totidé ponantur corbes, pleni cafeo, butyro, ouis, cępis, herbis, aut quicquid tibi in menté venerit.Super húc lapidê, alius adhuc cốtituatur itidê quadrágulus, $q$ latera lóga habeat feptếpedes, $\&$ alta vnú, in eius medio locetur capra auenaria, cuius altitudo fit quatuor pedes, ac infernè vnú latus longú fex cú dimidio, at fupernè vbi fera eft folú fex, fuper oper culum autélögitudo fiat quatuor, cui impone ahenú inuerfum, tress cú dimidio pedes amplum:fed in fundo fupernè folú tres, huic rurfus gabata in qua cafeus formari folet imponatur, alta dimidio pedis, fupnèduobus pedibusá pla, at in fú do folú vno cú dimidio, eă orbiculo cooperiadmodú craflo, quị́̂p fatis pmineat:in medio huius orbiculi vas locato in quo butyrú recondi cófueuit altitudinis trium pedü, $\&$ in fundo vnius $\&$ dimidii, fupernèverò vnius amplum, ro It rum tamé per quod effundimus atq̣ anfa aliquantulú proiiciătur. Deinde vrceus bene formatus ftatuatur, in quo lac reponi folet, cuius altitudo fit duorú pe dú cú dimidio, in vếtre auté amplitudo vnius, \& fupernè dimidii, at bafim eius infernê largiore facito:in eo vrceo erige quatuor raftros, qbus carbones corradú tur, quilongi fint quinq pedes $\&$ dimidiú, circú quos ligatofruméti fafciculum altú pedes quinqu, fic $q$ raftri fupernè extết per dimidiú:illi rufticorum appéde inftruméta, ligones, palas, furcas, flagella, \& a lia huiufmodi . Tădê pminentibus raftris fupponatur gallinarú cauea, huic rurfus fidelia butyri inuerfa, cui rufticus triftis atq gladio perfoffus infideat:quéadmodú infernè defignaui. Homi ni ebriofo poffet aliquis hoc pacto fup monumen tú memoriá cóftruere.Primò fepulchrú eig Itatuatur cú epitaphio, quod voluptatê ironicêlaudet. Deinde fu per fepulchrum erigatur dolium vinarium, quod alea fupertegatur. Poftea locë tur dux patin $x$ quarum vna fuper alteram fit inuerfa, ė gulam contin ebunt:iá fuperiori patinx imponatur cantharus humilis, fed admodnm capax, duabus anfis, qui orbe coopiatur, iup quế inuertatur poculú vitreú ampliffimú:cuius fü do infideat corbis plen, pane, cafeo, butyro, $\&$ aliis efculétis. Cófimiliter ex aliis rebus poffemus diuerfis modis fecundú vitá cuiflibet, fepulchrú eius exornare. Hæc infolétix caufa indicare placuit, ac vnà cum cxteris columnis deliniare.

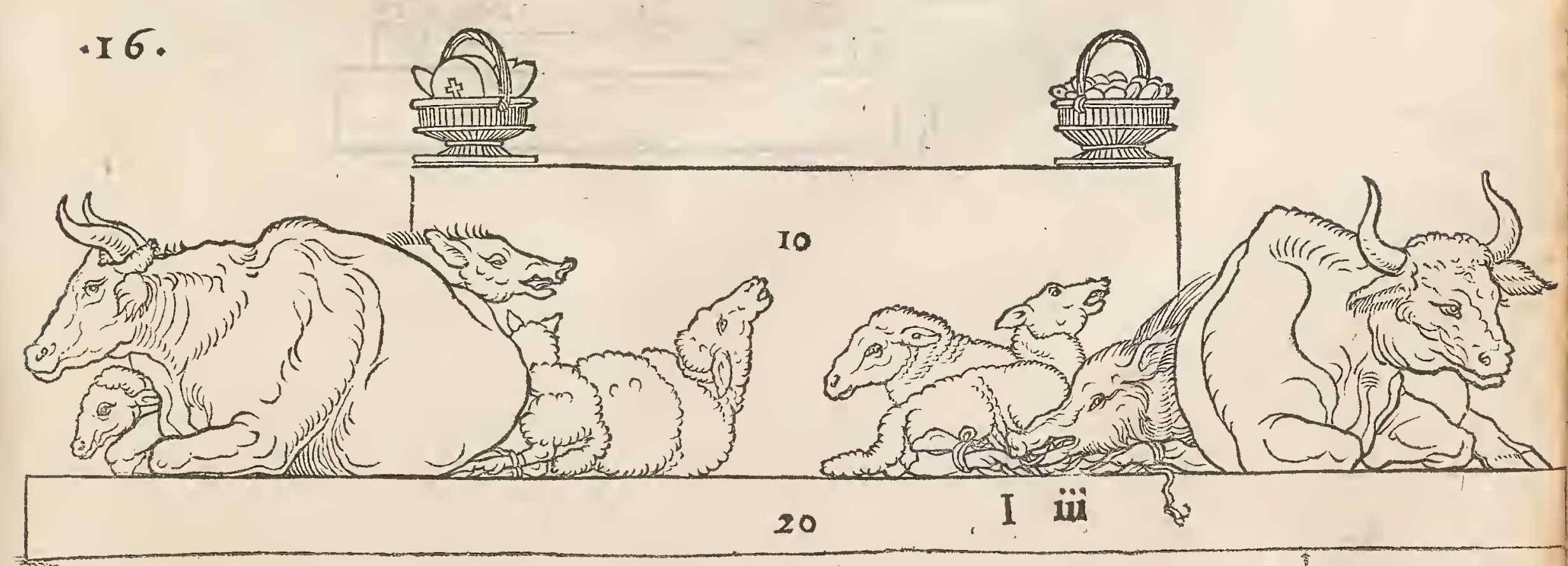




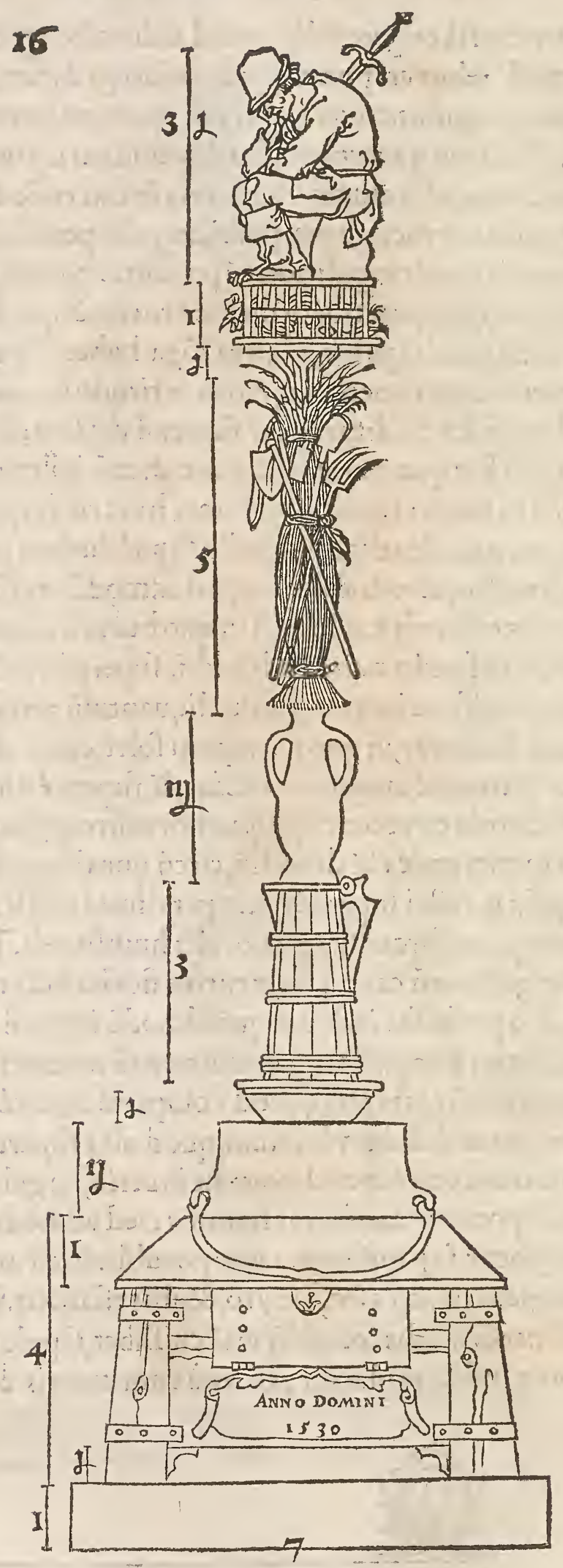


GEO METRIAE I I B. IT ?
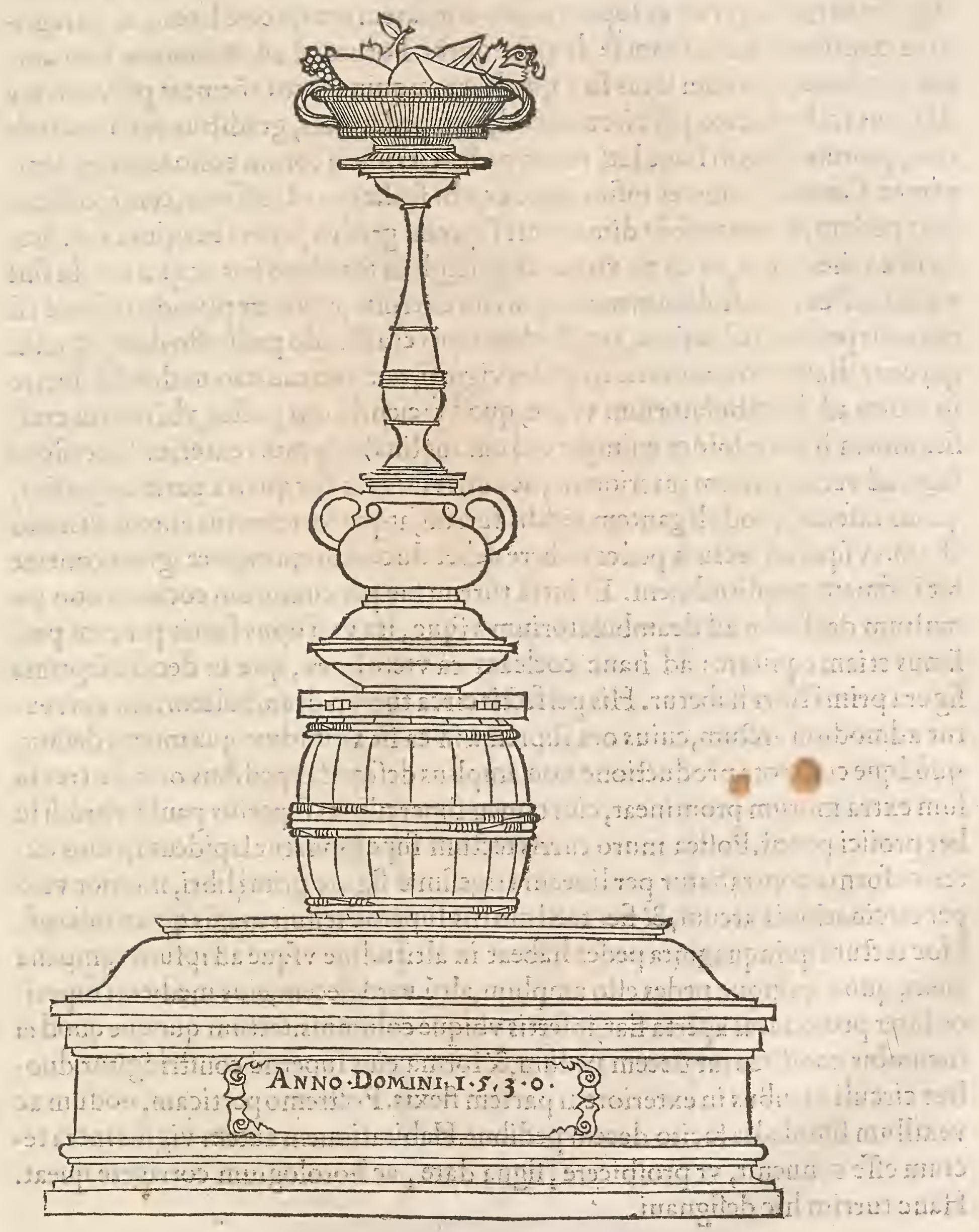


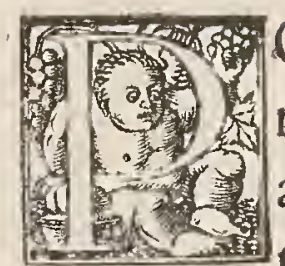
Oftquam in pracedentibus de corporibus longis nonnihil pracepimus, iam turrim rotundam, firmam'g facere docebo, at fol um corpus abfque omni ornatu, qualem fi quis conftruere volet, is eam pro arbitrio exornet. Hac turris in ciuitatis loco xdificetur valde oportuno, atque foro medio, vt ex ea fuper totam ciuitatem profpicere liceat, ac peregri$n i$ in omnibus vicis ad eam fe dirigere queát. Forum id ad minimun tam am. plü effe debet, vt vnum latus fux quadratu ræ quingentos cótineat pedes: in me dio eius fcalas locato per circuitum decem pedes altas, gradibus autem octode cim, quorum finguli fiant lati vnum pedem, eruntóg eorum collocationes tem. peratæ.Cæterú diameter infimi gradus, vbi fcalæ funt latiffimæ, centü cóftituatur pedum, \& remanebút dimetienti fupremi gradus pedes fexaginta fex. Sca$1 x$ ift vtiles erunt, vt ab eis videatur quicgd in toto foro fiat ac qua res ibi fint vanales. Porrò in fcalarum medio turrim extruito, cuius amplitudo infernè cú muro fit pedú quadraginta, atọ̧ ibidem muri craffitudo pedû efto decé, $\&$ relin quentur diametro concauitatis pedes viginti, eam concauitatem directè ducito in al tum ad deambulatorium vfque, quod faciendú erit poltea, vbi murus craffitudinem habeat folùm quinque pedum:inclinabit'́g muri exterior fuperficies fupernè verfus partem interiorem, ac turris ibidem fiet quarta parte anguftior, quàm infernè, quod elegantem reddit turrim, atque fortem:eius autem altitudo $a b$ imo vfque ad tectum ped es habere debet ducentos:quinquies igitur contine bit infimam amplitudinem. Et intra turrim fac per circuitum cocleam non ita multum decliuem ad deambulatorium vfque, ita vt fi opus fuerit per eam porfimus etiam equitare: ad hanc cocleam ea vtere linea, qux in decimafeptima figura primi libri habetur. His perfectis circa turrim deambulatorium extruatur admodum aretum, cuius ora fuprema in ea fit altitudine qua murus definit, quód que cum totaproductione non amplius defcendat pedibus octo, ac tres fo lùm extra murum promineat, eius tamen fupercilium fuperius paulò vltrà, fil lu bet proiici poteft. Poltea muro turris tectum fuperponatur lapideum,cuius exterior forma con trahatur per lineam trigefima figure primi libri, interior verò per circinationis arcum, \& fiet tecti mu rus fupernè tenuis magis quàm infernè. Hoc tectum quin quaginta pedes habeat in altitudine vfque ad ipfum campana rium, quod quinque pedes efto amplum, altá verò decem, eius medietas fuperior inter proiecturas aperta fiat, infertis vbique columnis: tectum quoque quod ei incumbit conftituatur decem pedum, \& forma eius fupernè conftringitur duobus circuli' arcubus in exteriorem partem flexis. Poltremò perticam, nodum ac vexillum fimul al ta facito decem pedibus. Habitationem autem vigilis intra tectum effe conuenit, vt profpicere, figna dare, ac horologium corrigere queat. Hanc turrim hic defignaui. 
GEOMETRAE LIB. III.

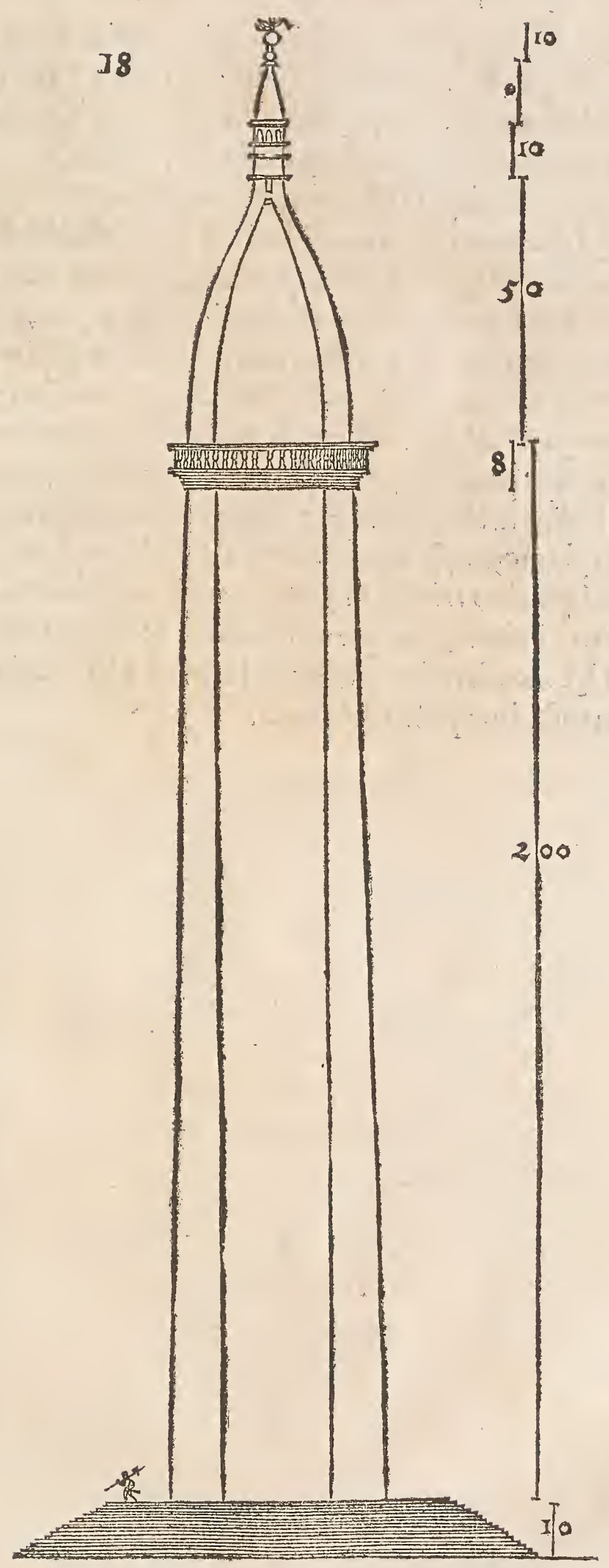


Ontingit aliquando vt quifpiam fcire cupiat alicuius turris altitu. dinem, ad quod quidem expediendum multa funt inftrumenta, ve. lut aftrolabium, \& eius quadrans, baculus Iacob, \& alia nonnulla, ied hic facilem quendam modum indicabo. Quando turris altitudinen capere vis, tunc figna eam fupernèb, infernè verò $c \&$ oculus tuus fit a , quem ab ea fige quàm longè placuerit,at in ea altitudine qua c eft:deinde fumito normá iuftam cui in vna parte affigatur regula quadam, it avt in clauo quo affixa eft, circumagi, atque in alia parte eleuari ac deprimi queat : eam normam locato in lineam a $c$, fic vt angulus eius rectus turris bafim refpiciat, \& oculus tuus fit in parte polteriore vbi mobilis regula circa clauum verfatur:quo facto erige regulam anreriore parte ad altitu dinem $b$, quam vbi recte notaueris, facito fignum in norma ad eum punctum quem anterior regulx pars oftendit, atque fic firmetur regula.Poltea inclinanda eft in codem loco norma cum regula inuariata in horizontis planiciem, $\&$ veniet turris altitudo in planum, quam fignato charactere $d$, fián tque duo trianguli equales, erectus ad turris altitudinem a b c $\& \mathrm{acd}$, qui in planicie iacet, funtque altitudo b c ac longitudo $c d$ in plano inuicem xquales. Hecc menfuratio certa eft,fed tibi eft adhibenda diligentia,ne er rorem committas:ifta hoc pacto deliniaui. 


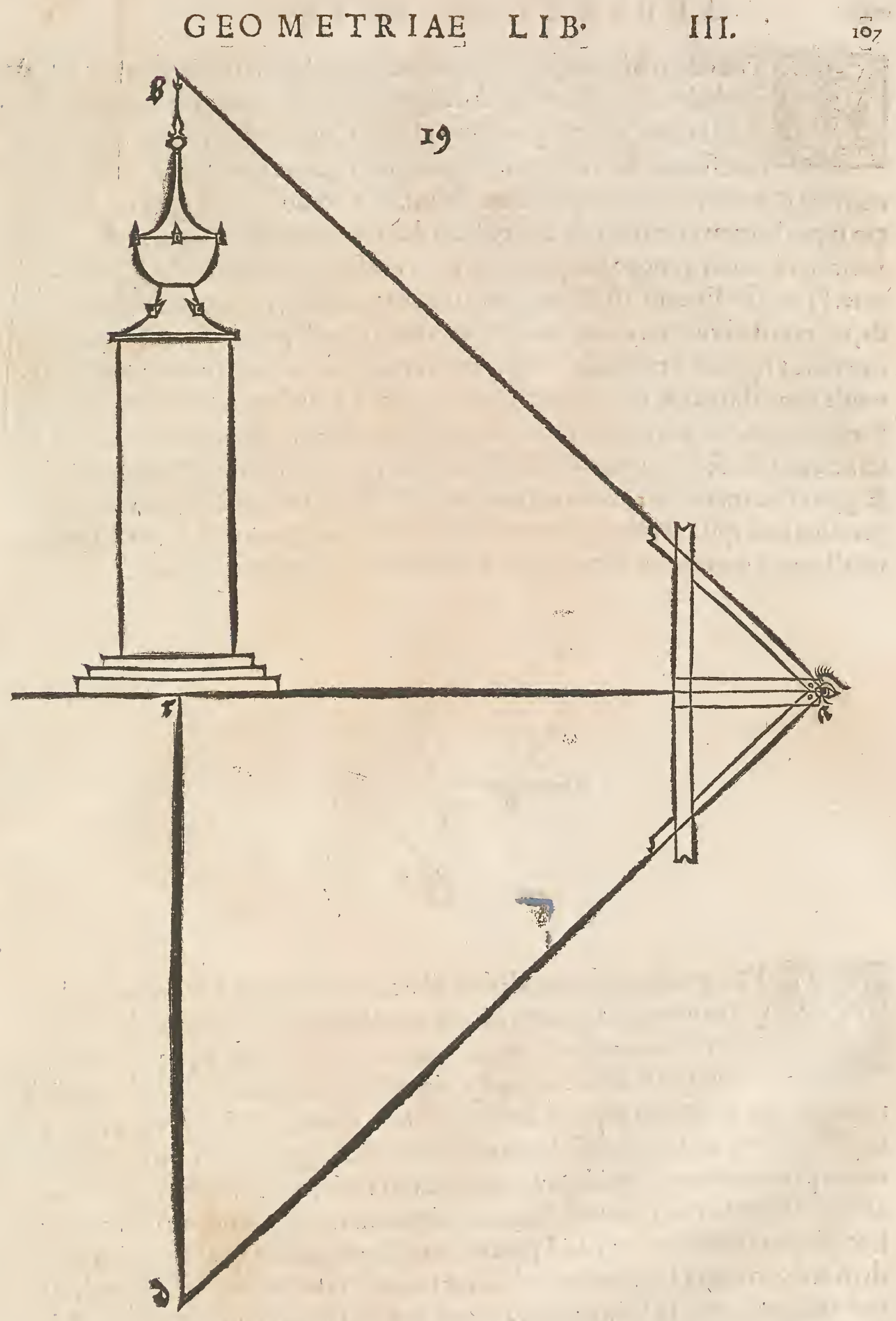




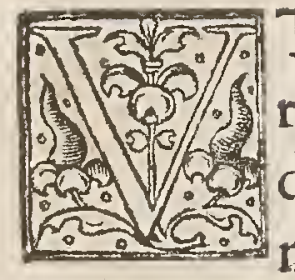

Tile eft architectis, lapicidis ac pictoribus, vt in turribus, $m$ unris $\& p_{i}$. rietibus vulgare horologiú defignare queant: quocirca in liequêtibus de his rebus pauca quadam tractabo, qux ingeniofo cuique magis neceffaria effe videntur: horologium itaque duodecim horarum, à meridie $\&$ media nocte incipientium deliniare docebo. Primò igitur à dato pú cto fupra lineam tranfuerfam difce aliam ducere perpendicularem, velut prius in libro de lineis quoque fcriptum eft: fed vt rectius intelligatur, hoc pato ope rare.Protrahe lineam $a b$, fupra quam notato punctú $c$, in quo polito circini pel de, reliquo defcribe circinationis arcum tranfeuntem per lineam a $b$, quam vbi interfecat fignato d e:deinde locato circini vno pede in punctum d, al terum extende pro arbitrio, ac ducito arcum per lineam a b, furfum $\&$ deorfum:confimi liter etiam fac ex puncto e; circino inuariato:at vbi duo illi arcus fe invicem $a b$ fcindunt, illic fcribe fupernè $f, \alpha$ infernè g: his perfectis cốtinuentur puncta c f $f$ \& g,per lineam rectam, ac vbi a $b$ fecatur, 1 lic adiiciatur h, erítq, linea $c h$ g,perpendicularis ipfi a b. His igitur pro conficiědis horologiis in primis opus eft, vt. intelligatur quid linea fit perpendicularis, velut hic quoque protraxi.

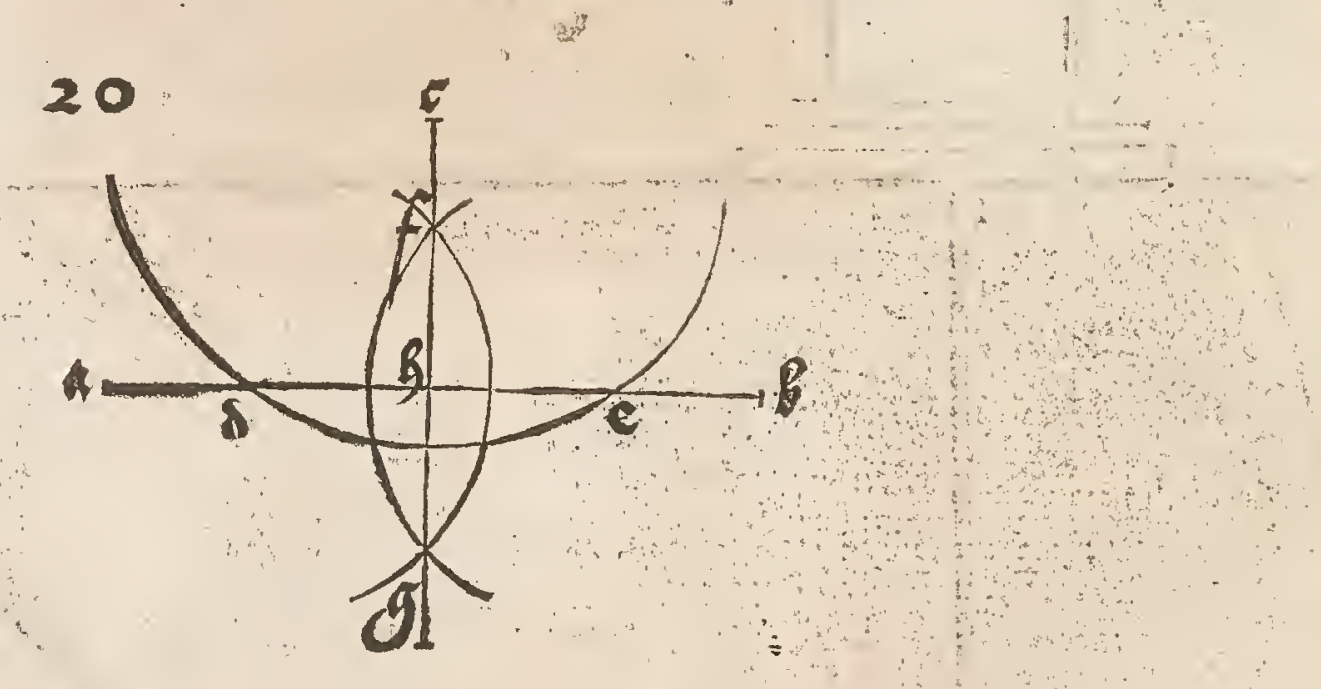

Vnc quadrantem circuli defcribe cuius cétrum fita a $\&$ arcus eius b c:
qué in nonaginta partes diuide æquales, ac primo qdé in tres, \& quá-
libet tertiam rurfus in tres, \& fient nouem fegmenta, qux fingula fe-
centur per mediú,eruntóg fpacia octodecim, quorú quodlibet diuida tur in quinq interualla æqua, $\&$ habétur in tota quarta circufferentix nonaginta partes fiue gradus. Deinde latus quadrantis a c erigatur, a $b$ verò in horizóte iaceat plano; ac gradus quidem à $b$ numerandi funt fur fum verfus $c$. Porrò $f i$ in aliqua ciuitate horologium folare conficiendum fuerit,primò per inftrumentû́ frue tabulas aftronomicas ad id paratas inueftigandum eft, quátum polus mundi in eo loco fupra horizontem eleuetur : velut Nuremberga arctici polialtitudo eft quadraginta \& nouem graduum, cos à b fupputa furfum vfque ad finế quadragefiminoni gradus vbi d locandum eft, quod cum a coniungetur perlineă rectam a d,qux axế repræfentat mundi:nă circa eum verfari mundum ima ginamur. His perfectis ducatur ex puncto d linea perpendicularis ( $v t$ in precedente 


\section{GEOMETRIAE IIB. : III. Iog}

dente figura monftratum eft ( ad lineam a b cui incidit in puncto e ad angulos rectos:hęc linea d e muralis à quibufdam núcupatur, $q$ ex ea horologia folaria in muris erectis fieri confueuerint. Cæterum ex figno e, linea trahatur recka jpf a dad angulos xquales, per doctrinam pracedentis, ac vbi ea fecat a d axem, illic fcribe fliteram:linea igitur ef aquinoctialem denotat, qui mundi axem per medium diuidit orthogonaliter. Hæc liniamenta pro parallelo tranfeunte per Nurembergam folùm conftituta funt, ex quibus ad prædicú clima folares qua drantes fieri queunt. At circuli quarta iam defcripta eam debet habere quantitatem vt horologium commodè ex ea deduci poffit, nam vt plurimú ipfa vtendum erit. Triangulus quoq a e d ex metallo aut ligno fieri debet vt eius adminiculo ftilus feu gnomon infigi poffit:in parietibus itaque infigitur extremitas d $\&$ a polum relpicit antareticum fub terra:in plano autem figitur terminus a $\&$ d erigitur verfus polum arcticum. Poftremò quando e $\mathrm{d}$ plano applicatur ho rizontis, tunc oftendit d a altitudinem xquatoris. Hæc omnia hic defignaui.
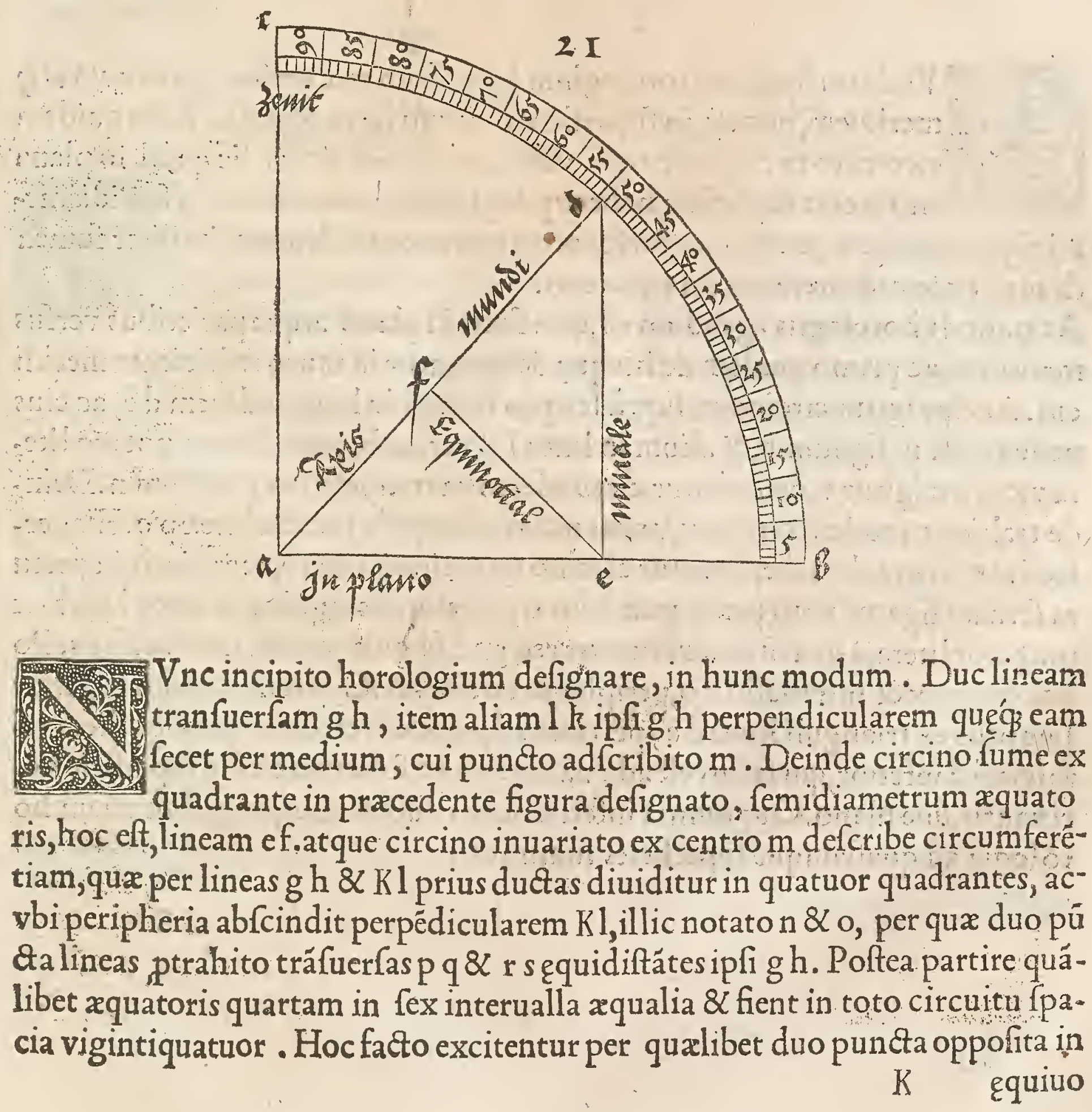
xquinoctiali linex rectę tranfeuntes per centrum m, \& cótinuentur in vtram partem vfque ad lineasp q \& r s. Hac omnia infernè deliniaui. Porrò quandocunque tibi folare horologium eft conficiếdum numera diligenter in quarta cir culi altitudinem poli eius regionis pro qua folarium paras.

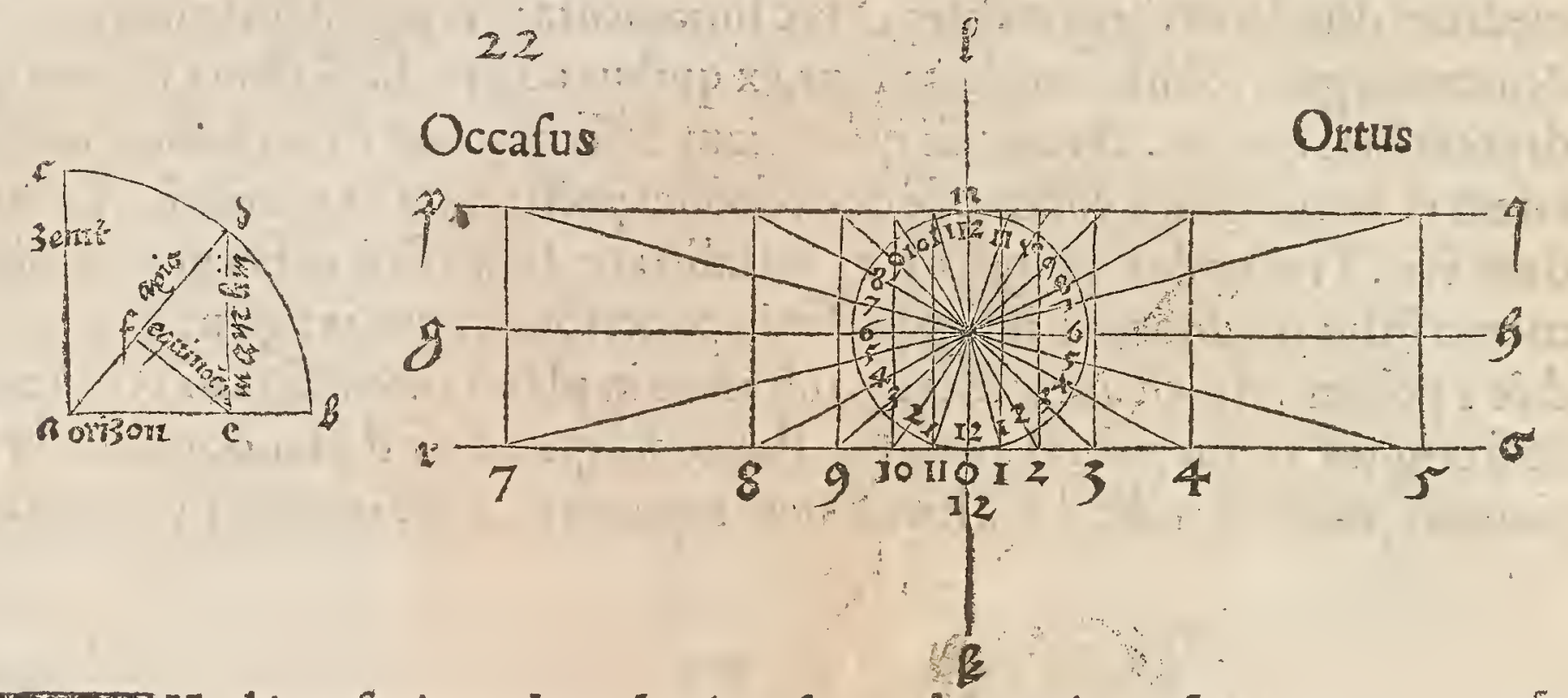

Vod iam fecimus horologium locandum erit verfus æquatorem at (1) 1 meridiem, hoc eft, in fuperficiem circuli horx fextx, ac ftilus quidem N. ex centro $m$ erigatur rectè verfus æquinoctialem, fic quòd in plana eius ia ceat fuperficie, habeat'í lógitudinem $m$ n fiue $m$ o femidiame. tri æquatoris. Item gnomones verfus orfentem aut occidentem fimiliter fumen difunt ex femidimetiente fui \&quatoris.

At nunc de horologits agend um eft, qux funt in plano atque parietibus verfus meridiem, ac primo quidem de his que defignantur in muro refpiciente meridi em. Accipe igitur circino ex fuprà fcripto triangulo longitudinem de, ac eius pedem fifte in fignum $n, \&$ alium in linea $l K$, verfus $b$, quem locum not ato litera v:centro igitur $v$, defcribatur circumferentia tranfiens per punctum $n$. Deinde trahito ex eodem centro $v$, lineas reetas ad fingula puncta linexp $q$. His perfectis ex triangulo a d e, fumatur circino quantitas linę̨ a e, ac ea circini apertu ra feruata figatur vnus pes in punctum o, \& reliquus applicetur linex $1 \mathrm{~K}$, vbi $\mathrm{x}$ pingatur litera, à qua linex excitentur rectę ad fingula puncta tranfuerfę $r s$. Po ftea centro $\mathrm{x} \&$ interuallo $\times$ o, peripheria circinetur. Cetterum circulus fuperior fumptus ex trianguli linea d e, pertinet ad parietem erectum qui meridiem refpicit:fed inferior, qui factus eft ad quátitatem a e, folarium erit in fuperficie ho: rizontis, frue plano. Quęomnia infernè oculis fubiecimus, ex quibus etiam ho rologia ad quafcunque fuperficies fumuntur. 


\section{GEOMETRIAE LIB. III.}

$\mathbf{x} \boldsymbol{x}$

23

Hoic congruit erecto parieti

Anite meridiem

Horizonti aut plano hocaccommodatur.

\section{Ante meridiem. $\quad \& \quad$ Polt meridiem.}

\section{Poft Meridiem.}

Verfus

orientem

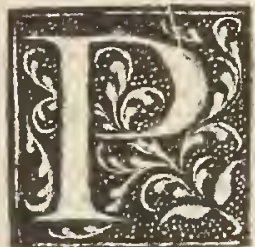

Rohuiufcemodi horologiis poffunt fieri cubi diuerforum angulo. rum, in quorum lateribus varia quoque defignantur folertia, fed hic vulgarem quendam modum per cubú indicabo, cui anguli quidam refecantur, atque iis fuperficiebus horologia infcribuntur, quas fol $\mathrm{fu}$ is radiis contingit, quod hoc pacto expeditur. Vnum cubi latus fignato in quatuor ang ulis characteribus $\mathrm{K} / \mathrm{m} \mathrm{n}$ ac in medio lateris ponito fignum c ex quo circulum liniato, quatuor contingentem cubi latera, is duabus diametris inter fefe tranfuerfis diuidatur in quatuor quadrantes, ita quòd trafuerfa a $\mathrm{cb}$ horizontem nobis reprafentet $\& \mathrm{~d}$ e perpendicularis fupernèfit zenith capitis, infernê verò púctus pedum: deinde feca quamlibet quartan in nonaginta partes xquales, velut prius dictú eft, \& numera à b furfum verfus $\mathrm{d}$, gradus quibus in regione eleuatur polus (at eius altitudo hic Nuréberga, vt antea nominauimus eft quadraginta $\&$ noué partium ) ac vbi gradus illi finiútur illic fignato literá f. His perfectis protrahelineam $\mathrm{f}$, quam continuato vltra $\mathrm{cad}$ circumferentiă vfque, cui gadfcribito:hæc linea axem mundi fignificat, circa quem firmamentum verfatur, atque fecundum eum debent gnomones folariorum parietibus infigi, quanquam etiam verfus equatorem aut punctum verticis, pręfertim $\mathrm{K}$ ii in muris 
in muris declinantibus aut fuperficiebus planis dirigi queant. Stili auteri qua tuor horologiorum in æquatore circulo debent finguli in fuo campo erigi ad an gulos equales. Cęterùm per centrum cezcitetur dimeriensh $\mathrm{i}$ iph f $\mathrm{f}$ axi curtho gonalis, qui equinoctialis indicat fuperficiem. Quum itą̧ linea aquatoris mú. di axem fecet ad angulos rectos, quantum polus feleuatur tantum deprim itur xquator hatque alius polus $g$, conlimiliter facit puncus equatoris i opp ofitus ipfi h:nam quod polo ad ditur hoc aufertur qquinodiali : eruntergo in thingulis quatris quadraginta nouem gradus, hoc eft, altitudo poli Nuremberge; $8 x$ quadraginta \& vnus gradus refidiui vfque ad punceum noftri verticis $x q u a l e s$ funt maxima diftantia equatoris ab horizonte. Eo modo cófequitur cubi qua dratum latus $\mathrm{K} I \mathrm{~m} \mathrm{n}$ octo angulos, quòd fic commodiffimè abroluitur. P roducantur linea $\mathrm{f} g \mathrm{~h}$ i in vtramque partem vfque ad extremitatem lateris, atque arte iam fepe repetita trahantur expunctis $f \&$ glinea parallele ipfi $h$ i qquatori. Item ex punctis $\mathrm{h}$ i ducantur qquidiftantes ayi ifg:fecundum has parallelas refcinde quatuor cubi angulos, 8 fient in eo odto fuperficies quadrangula: oblonge,per quarum omnium medium linea fcribitur meridiana. Reliqua duo cubi latera octo habebunt angulos, fed in zquales. Porrò corpus hoc angulare opponitur latere ita defignato ortui folis, $\&$ a meridiei, $b$ verò mediæ nocki:fta. tuuntúrque folaria in omnibus lateribus, que fol afpicere poteft, quemadmodum infernè quoque deliniaui.

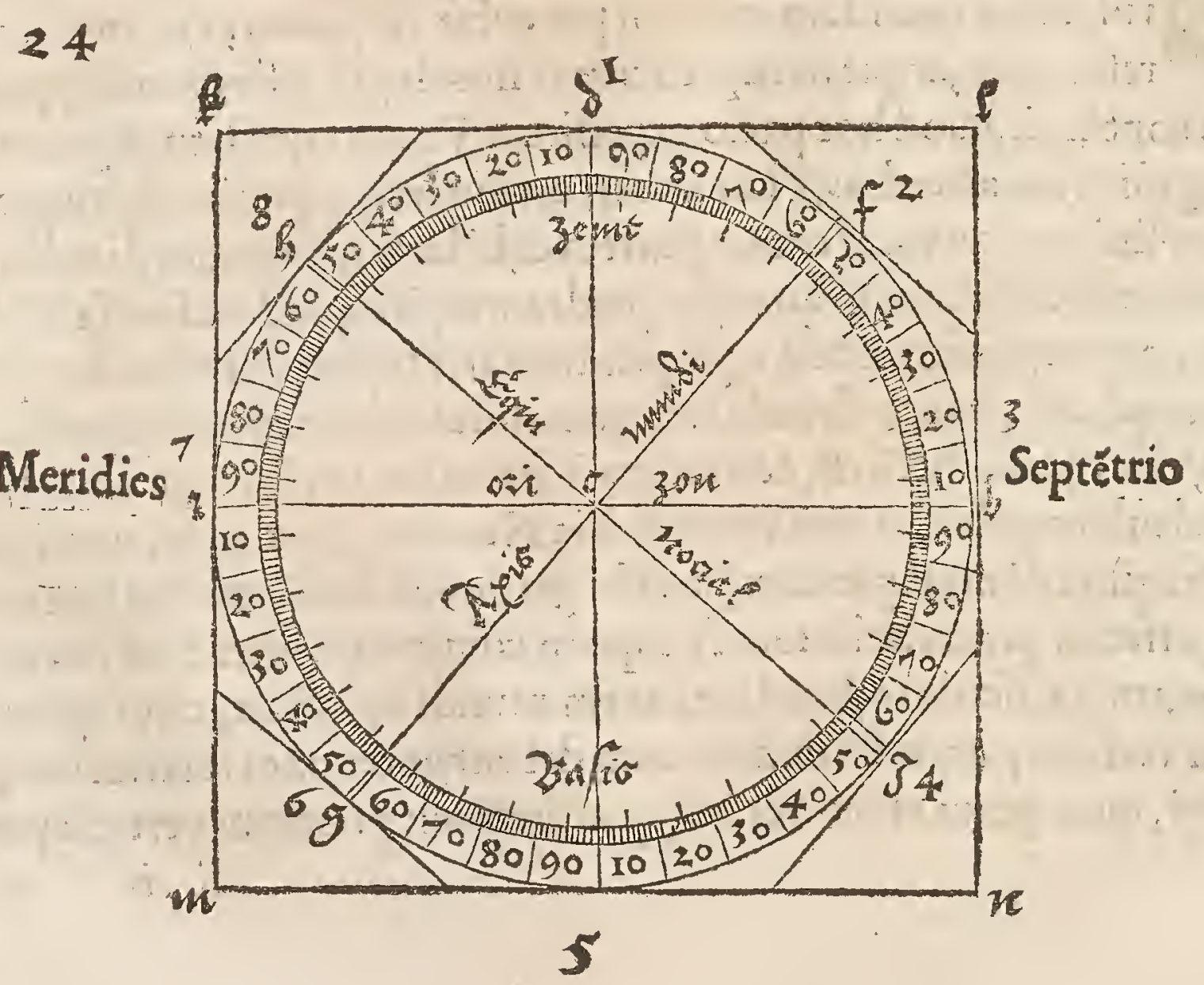



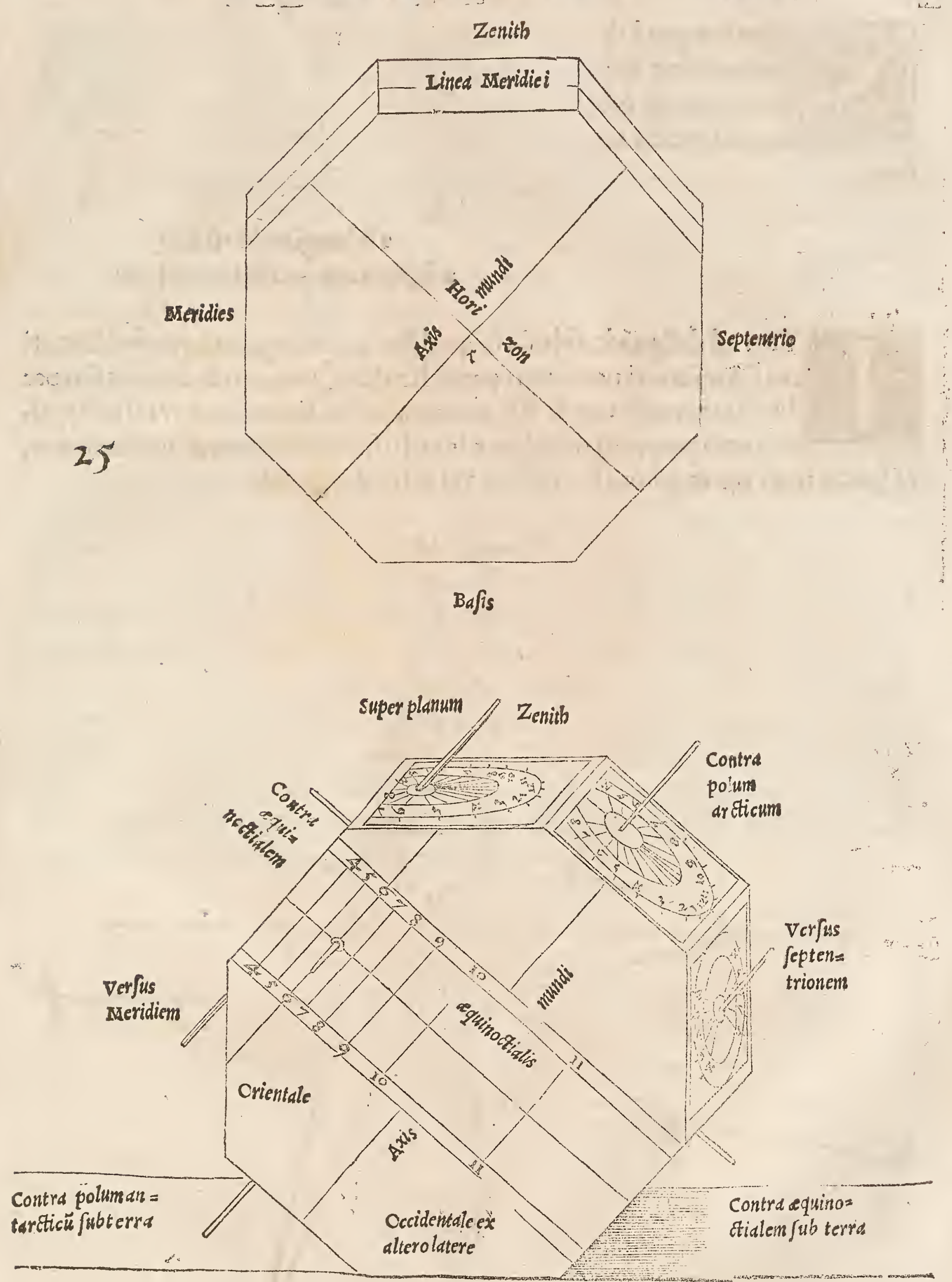

Bdfis

R iii 
Ond
quitur ftatue ver-
fus aquatoré fub atem.
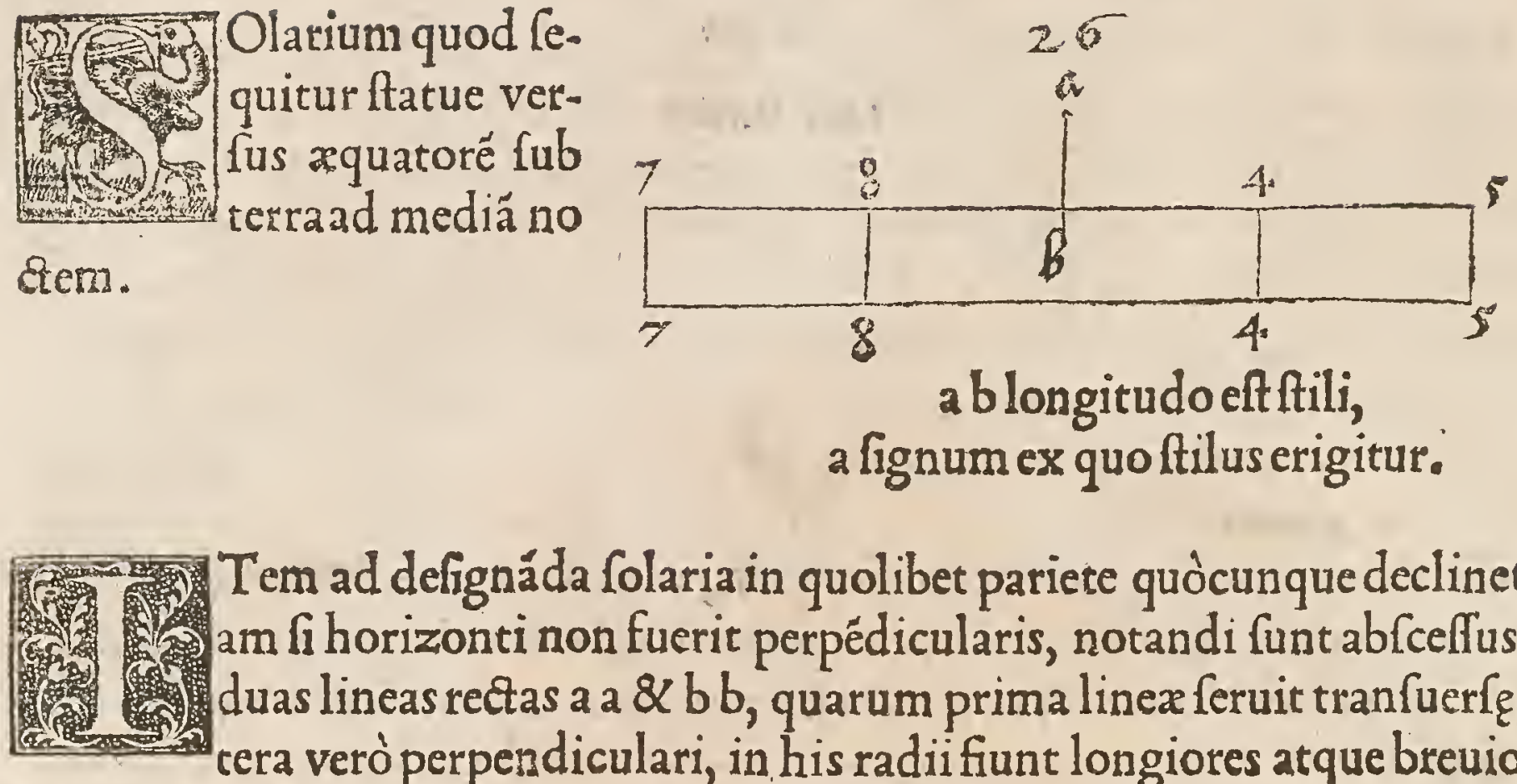

Tem ad defignáda folaria in quolibet pariete quòcunque declinet,eti am fi horizonti non fuerit perpédicularis, notandi funt abfceflus per duas lineas rectas a a $\& b$ b, quarum prima linex feruit tranfuerfe, alcera verò perpendiculari, in his radii fiunt longiores atque breuiores, aclpacia inter cos ampliora \& aretiora, velut hic defignaui.

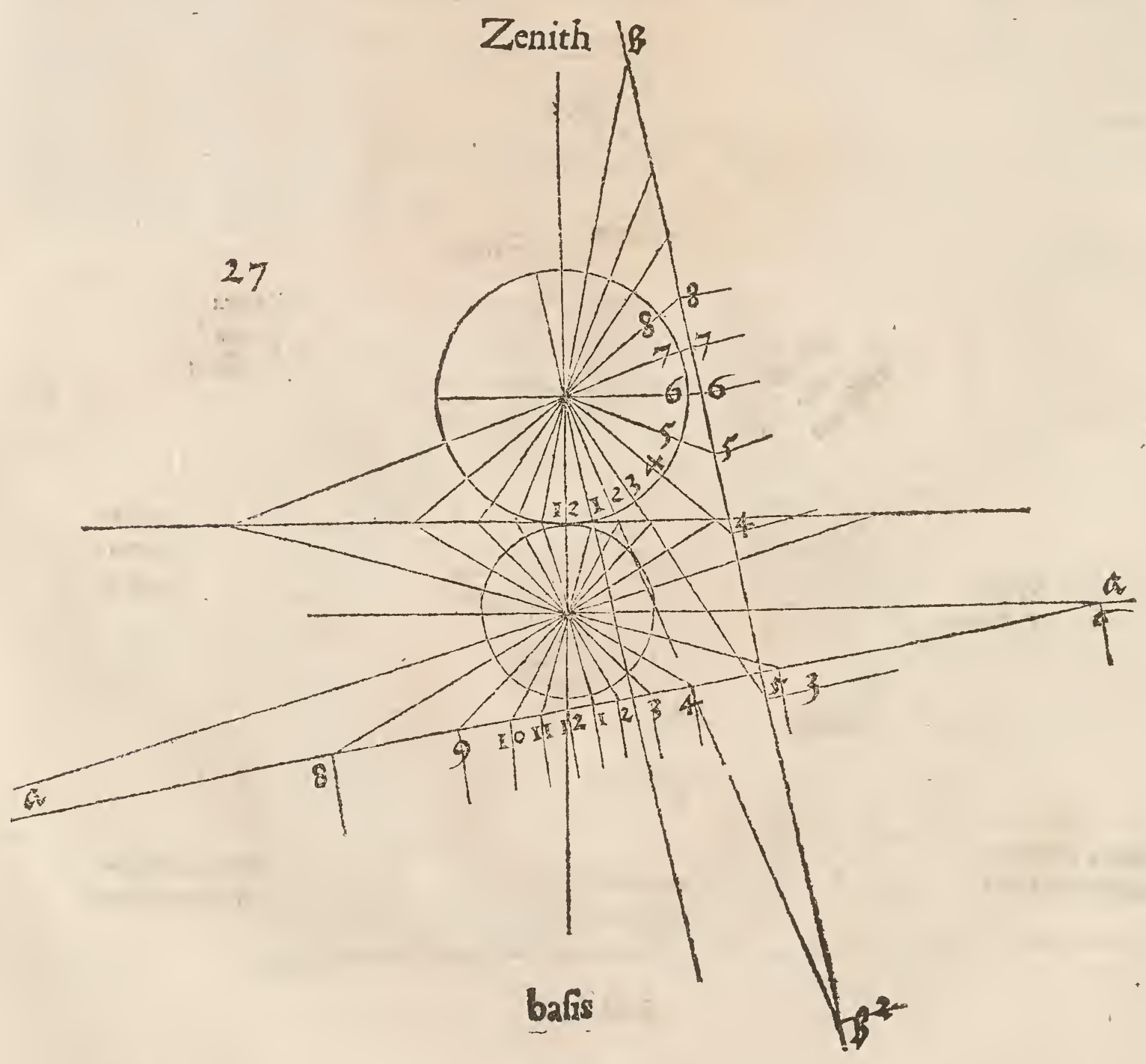




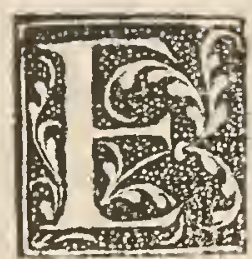

T quanquam varia adhuc fint horologia, in quibus fingulis horis vi. dere eft fignum afcendens, dierum augmentum atque decrementurn, locum folis, horam planetarum, \& alia huiufmodi, tamen volui dun. taxat indicare horas vulgares, \& hoc breuiffime, quum hac horarum traditio non fit inftituti noflri, fed potiùs digreffio quadam. Sape fcriptura in columnis, turribus, aut parietibus altis fingenda eft, quocirca qui altè aliquid cófcribere velit, ita quod literę fupremi verfus tam magnx appareant quàm in fimi, is 〔uperiores inferioribus ampliores faciat, co qui lequitur modo:lifte vifü tuum in altitudine atg; diftantia à turri quanta volueris, fitóp ille punsius $\mathrm{c}$ : deinde fume triangulum decimafext figurx primilibri, \& imaginare a b efie altitudinem turris, aut parietem, in quo frrbere ftatuifti, arcum veró $b$ e in xqua verfuum $\&$ fpaciorum diuide interualla, per que ex cpuneto vifus ducito lineas rectas ad turrim fiue parietem a b, ex his notis in pariete trahito lineas tranfuerfas atque inter fefe aquidiftantes inter quas locanda erit feriptura. Exeo. manifeftè videtur quantum verfus fuperiores inferioribus debeant effe maiores. Cæxterùm fi lineam breuem fecundum longam a $b$ fecare placeret, tunc diuifio nes $a b$ continuand $x$ effent vfque ad cper lineas rectas, que perpendiculari $\mathrm{f} g$ abfcindédę erunt ppe c:diuiditúrọ $\mathrm{f} g$ ad fimilitudinể $\mathrm{a} b$ cui parall ela exiftit. His vti poffumus in parietibus, decliuibus, aut prominentibus, ad res maiores aut minores faciendas. Sic omnes linę fecundum alias diuid $\mathrm{i}$ queunt in partes xquales, aut inæquales, \& eas quas nominare non poffumus . Porrò partitiones huiufmodi non folùm feruiunt pro defignădis literis, fed aliis etiam rebus quibufcunque, \& pracipuè quando turris alta opere ftatuario eft exornanda, ita quòd ftatu $x$ fuperiores inferioribus appareant æquales: id hac arte abfoluitur, quemadmodum infernè deliniauimus. 

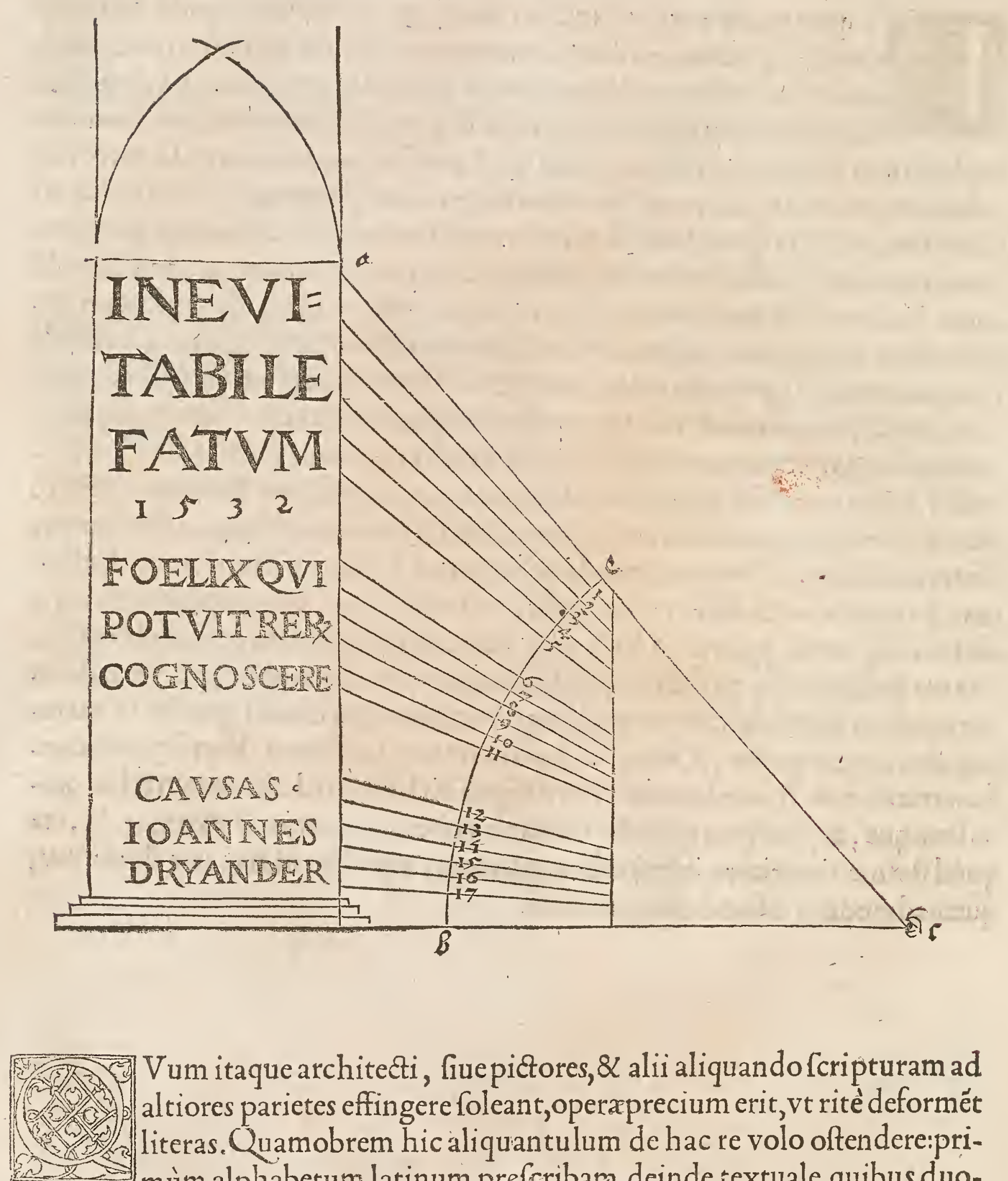

Vum itaque architecti, frue pictores, \& alii aliquando fcripturam ad altiores parietes effingere foleant,operaprecium erit, vt ritè deformét literas. Quamobrem hic aliquantulum de hac re volo oftendere:primùm alphabetum latinum pręifribam, deinde textuale, quibus duobus generibus literarum maximè in talibus rebus vti confueuimus. In primis ad literas romanas fingulas fac quadratum æquum in quo contineatur vnaquag litera. At quando in eo ducis litera tractum maiorem, hunc fac latum parte decima lateris quadrati: \& minorem tertia parte latioris, idq $q_{\beta}$ obfer ua per omnes literas al phabeti.

Primò fac $A$, hoc pocto. Deligna eius quadrati angulos literis a $b c d$, idé fac in omnibus reliquis literis, \& diuide quadratum perduas lineas ad angulos rectos fefefecantes:erectam e $\mathrm{f}$, trañfuerfam $\mathrm{g} h$, deinde pone duo puncta $i K$ infernè iuxta $\mathrm{c}$, decima parte dilt antia introrfum ab c \& d, \& ducito tractum tenuiorem furfum $a b i_{8}$ ad quadratum:inde deducito latiorem tractú deorfum, ita vt amborum 
amborum latitudines exteriores cótingant puncta $\mathrm{i} \& \mathrm{k}$, tunc relinguar ur in me dio triangulus, $\&$ punctus e cadet fuperiùs in mediam literam, deince co iiunge ambos tractus fub linea tranfuerfa $\&$ hic tractus terciam partem latitudinis ha beat maioris tract us. Deinceps permitte arcum circuli a d fuperiorem $\&$ exteriorem partem maioris traetus fupra quadratum egredi, ac amputa liceram fuperùs per lineam ferpentinam feu curuam, ita vt finus cauatus ad fubtiliorem tra ctum declinet, $\&$ acue vtrunque literg traetum infersùs vtrin que, ita ve quadrati angulos $\mathrm{c} d$ contingát:hoc facies arcu circuli, cuius femidiameter feptimam partem lateris quad rati continet. Sed inferiores finus fibi mutuò oppofitos finito egredi tantum quanta fuerint dua tertia maioris tractus, id fficies per arcum circuli, cuius diameter fit a qualis latitudini tractus maioris.

Item ipram literam A poteris etiam fupernè per quad rati latus recum amputare, $\&$ literam acuere vtraqgex parte, ficut inferiores pedes, ita tamen vt longior egreffio lit antè. Sed tunc oportet k tractus fuperius aliquantulo propius coniungere.Irem ipfum A poteris \& alio modo defcribere, nempe fuperius acutum, \& tunc inclinant fe tractus fupernè ad fe inuicem propius:deinde deprime pau lulum tractum tranfuerfum, $\&$ duplica laticudinem fuam. Poffes $8 x$ tractum fupernèobrutè abfindere, vel antè exacuere. His tribus formis te affuefacias oporter, aut ei quę maximè placuerit. Et obferua fimiliter, quo pacto hac litera fupernè,ac infernè exacuitur, codem modo reliquas, quarum obliquè tractus du cti funt, excauendas effe, ve funt $v \times y$, quanquam paululum immutantur, vt in frà audies. Literę forman exaratam dedi hic fubiunctam.
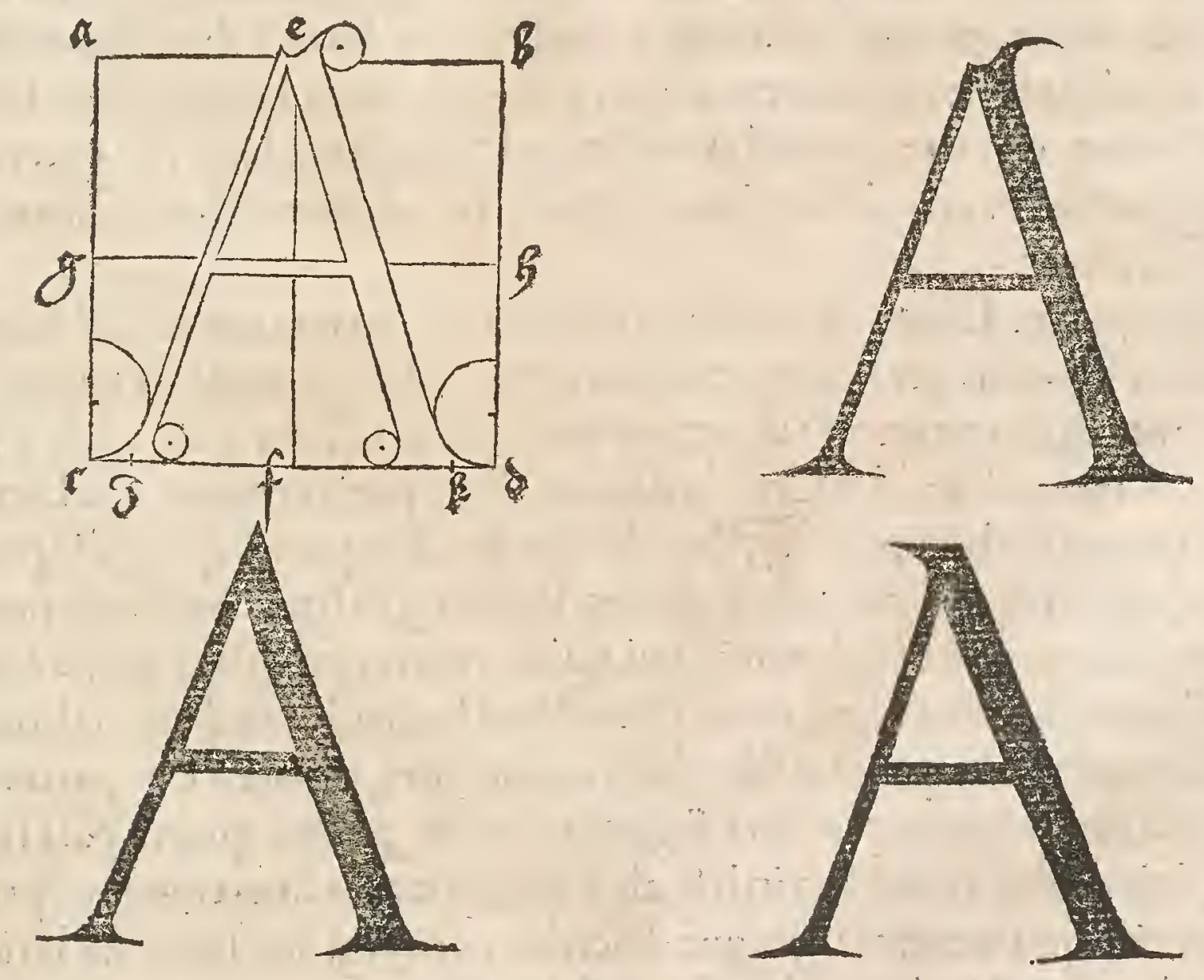


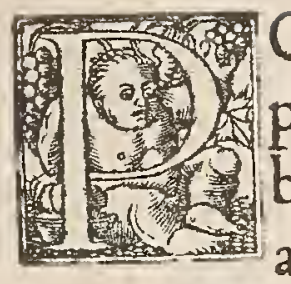
Orrò $\& b$ in fuo guadrato exarato fic . Imprimis diuide quadratum per lineam tranfuerfam ef in duas partes, deinde diuide lineas a e $\&$ bf per lineam $g h$ item in duas partes. Poftea locabis primum erectú ac latum tramitem literx,tantum ab latere a $\mathrm{c}$ quadrati diftantế, quá ta eius fuerit latitudo, deinde erige lineam $i K$ polt hunc tractum litera, verfus medium intus decima partelongitudinis lateris quadrati,\& vbi fecat lineam $g$ $h$ ponatur 1.Deinceps duc tramites anguftiores ac tranfuerfos à tramite erecto liter $a$, quibus ducuntur ventres rotundi ad lineam erectam vique, quęeft $i K$, fupernè quidem fublinea a $b$ fupra lineam $\mathrm{e} f$, atque infernè fupra lineam $c d$. Ponatur poftea pes circini in puncto $1 \&$ defcribefemicirculum intus à tramitibus tranfuerfis, ita vt ambę extremitates lineę circularis in linea ik erecta fub a b, \& fuper ef, breues illos tractus tran fuerfos contingant. Deinde partire latitudinem ductus angulti, atque tranfuerfi, qui eft fuper lineam e fin linea $\mathrm{i} K$ per punctum medium $\mathrm{m}$. Deinde pone latitudinem literx fuper lineam $\mathrm{g} h$ in pun cto $n$ polt lineam circularem, $\&$ poftea duc ex puncto $m$ fupra eflineam tranfuerfam breuem verfusf quantula fuerit opus. Deinde duc femicirculum, qui hanc lineam, \& punctum $n \&$ fupernè latus a b contingat, \& per $n$ tranfeat ere cta linea. Hxc omnia contingunt infernê concauum ventris, quemadmodú fupernè conuexum ventris contingunt. Deinde produc tractum tranfuerfum fu. pra $c d$ intantum verfus $d$ quanto erit opus, illic ponatur opus q:deinde diuide $\mathrm{m} g$ per tranfuerfam o $\mathrm{p}$ in duas partes, $\&$ vbi fecuerit lineam $n$ ponatur $r$, dein . de duc femicirculum contingentem tranfuerfam ef,punctum $r \&$ g:deinde po ne latitudinem literx tractus per punctum $s$ poft $r$ fupra lineam o $p, \&$ defcribe femicirculum contingentem lineam $m$, punctum $s, \&$ latus $c d$ dita remaneát in litera tres anguli,infimus autem excauetur per circularem lineam cuius femidi ameter contin eat duas tertias delatitudine tramitus litera lati. Sed exteriores egreffiones exacue per circularem lineam, cuius femidiameter latitudinem illius tractus contineat.

Aut fac b hoc pacto. Diuidatur quadrati latus a $c$ in partes nouem, \& amputa partes quatuor fuperius per lineam tranfuerfam $e f$, deinde erige tramitem erectum, ficut ante defcriptum eft : ac fuperiorem ventré efficies inter $a b \&$ ef, inferiorem verò inter ef $\& \mathrm{~cd}$. Deinde diuide $a b$ in partes nouem, $\& a b$ fcinde quatuor partes verfus $b$ in púcoog, deinde diuide $c$ d in partes quinóg \& proxi. má verfus $d$, abfcinde in puncto $h, \&$ ducito lineam $g h$ : hac contingat neceffe eft foris ventré fuperioré \& inferioré : qui quidế ventres fingulari quadă forma funt defcribendi: $\&$ circinus ad ductus circulares faciendos in diagonalibus $1 i-$ neis tranfponendus. Diagonales illas duas fic inuenies, diuide a $\mathrm{e}$ in partes qua tuor, proximá fupra efigna puncto i. Signetur etiam pxima quinta pars fupra cpuncto , deinde duc rectas $a b i$ in $b \& a b K$ in $f$, fuper his lineis moue ato $\beta$ tranf pone circinum, quo ventres vtrofque defcribis, eruntóg ita fuperiùs latiores quảm inferiùs, ficut $\&$ calamus id efficere folet, ideóque ventres non erunt circulariter rotundi:quia oportet te circinum fuper diagonalibus tranfponcie, \& nihilominus manus ductu adiuuare, quemadmodum feci in proxima pi. 

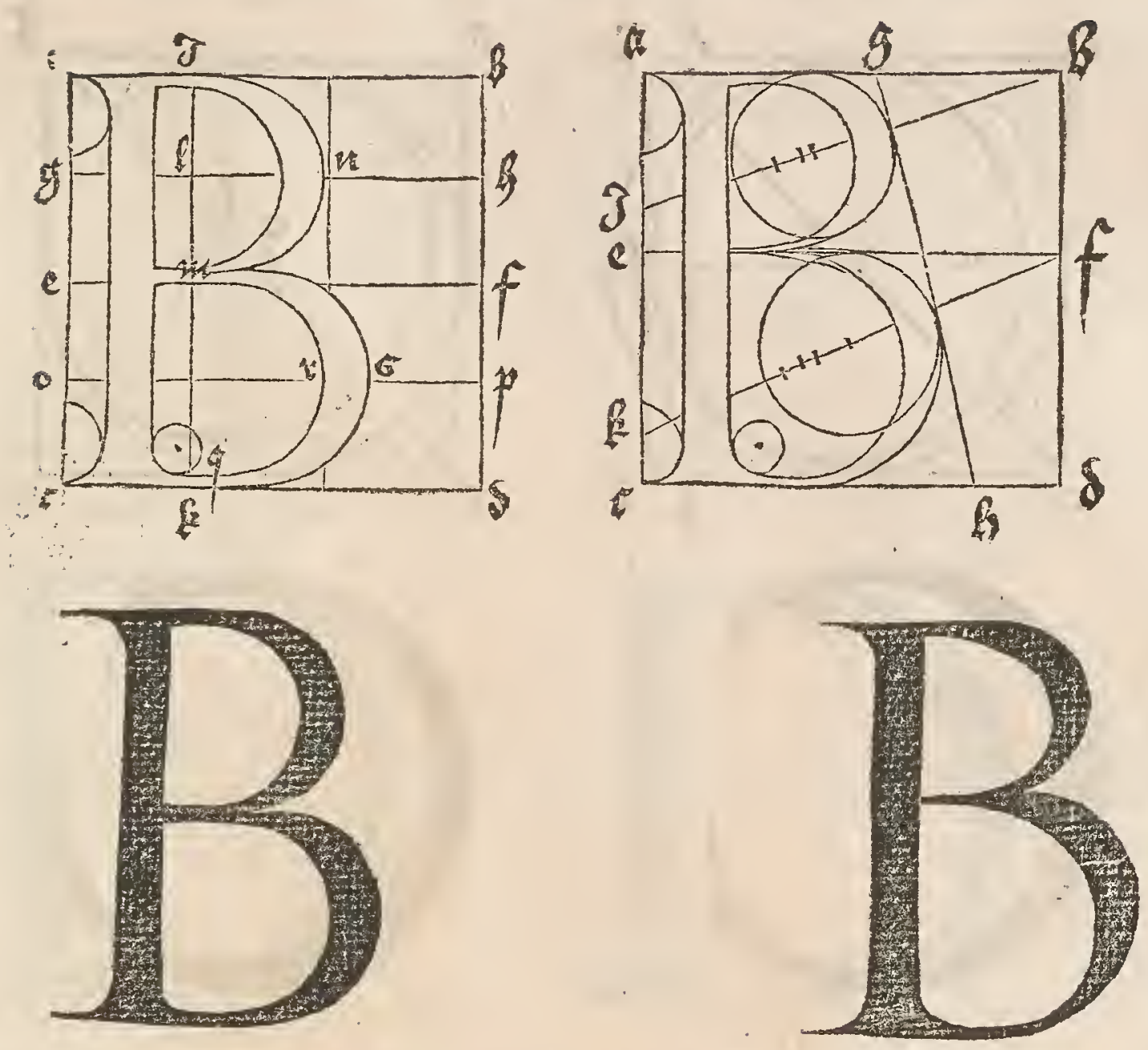

(16) Ofea fac literam $C$ ita in fuo quadrato. Ductranfuerfam ef,per me1. dium quadrati, \& pone punctum $i$ in medio linea ef,ex quo defcribe 1. circulum, qui quadrati a b c d latera quatuor cótingat. Deinde poltpone pedem circin inuariati fupra lineam e $f$, tantum polt $i$, quanta fuerit latitudo literx tractus latioris in punctum $k, \&$ circumfcribe quoque circulum, is tranfibit lineam $b d, \bar{\alpha}$ dabit a fronte in rotunditate litera fuam iuftam latitudinem. Deinde erige lineam $g h$, decima parte diftantem ab d b internè, hac abfcindet literam c infernè $\&$ fupernè, eo modo quo antiqui funt vî. Sed volo inferiorem tractum abfeindere in medio loco inter $\mathrm{gh} \& \mathrm{~b}$ d. Deinde ducito tractus fubtiliores atģ rotúdiores intra literam fupernè atọ infernè vbi circuli fefe fecăt:ad pfectionê quog liter ducito rotunditates, fupernè atộ in. fernè ad quadratilatera a b \& c d. Porròinferius, vbi litera pede vno trälit lineá $\mathrm{g} \mathrm{h}$, illic fub circulari linea paulò incuruatiorem efficies formam, ita tamen vt cufpide fui termini iterum circularé contingat. Similiter \& fuperius redde pe. dem interius magis cauatum, quàm circulus fecerat, ita due circulares linę̧ ferè totam literx formam obfoluunt. Secundò ita poteris literam c formare. Due in quadrato diametrum $\mathrm{cb}, \&$ pone pedem circini in medio puncto $i, \&$ altero pede defrribe exteriorem circulum ficut priùs, fupernèterminatum in diametro $c b$, fed inferné finito circularem pauló vlteriùs tranfire quàm priús. Deinde pone ped em circini inuariati tantum fuprà i, quanta eft latitudo tramitis literæ fuper diametrum, \& fcribe interiorem circulum, \& fiet tractu s fecundum calamum infernè latior quàm fupernè:reliqua manu protrahito, \& abfifiones terminorum literæ fiant fupernè furfum, infernè verò deorfum quemadmodum fubrcripli formas. 

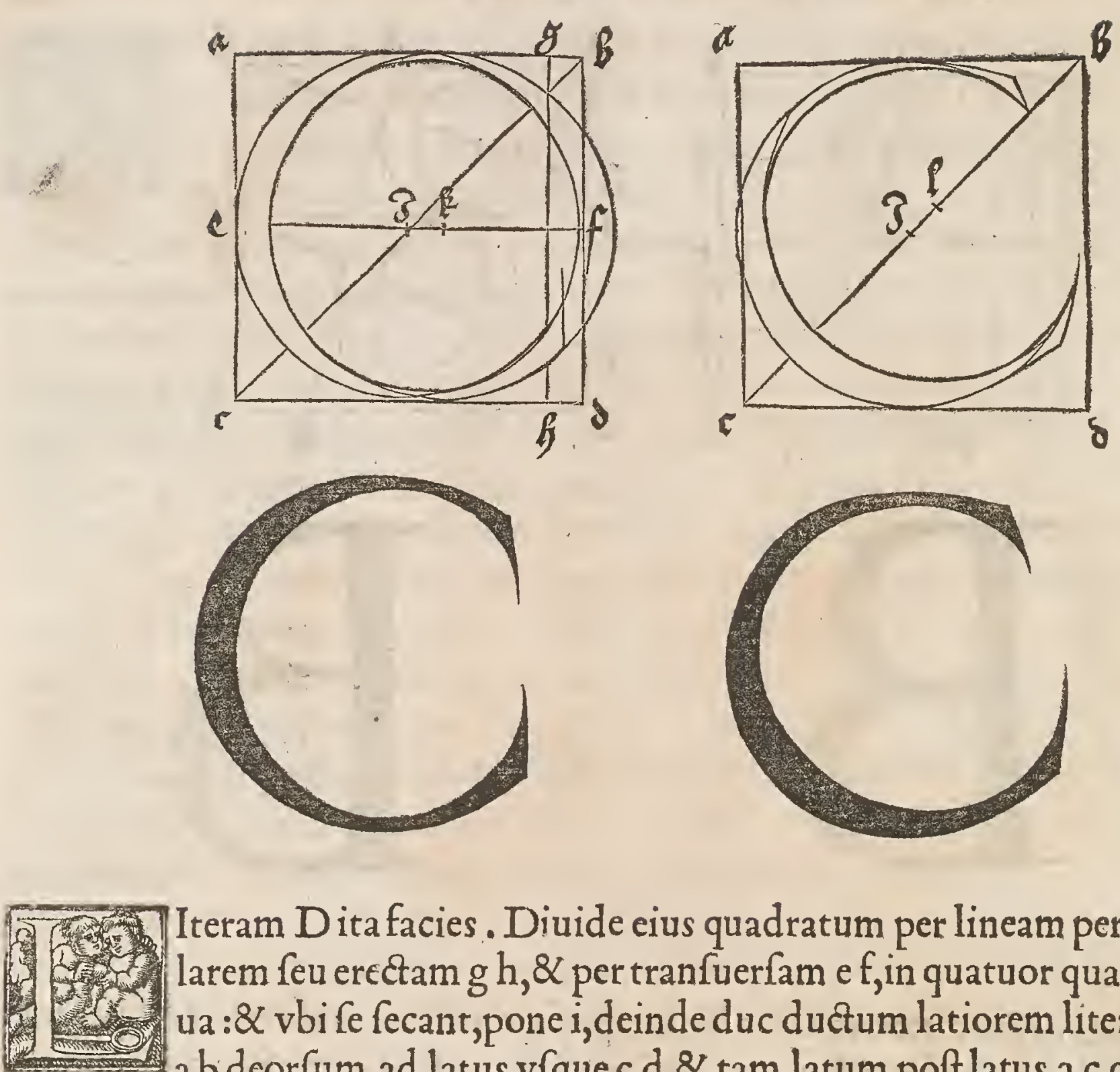

Iteram $\mathrm{D}$ ita facies. Diuide eius quadratum per lineam perpendicularem feu ercetam $g h, \&$ per tranfuerfam ef, in quatuor quadrata par ua : $\&$ vbi fe fecant,pone i, deinde duc ductum latiorem litere à latere a b dcorfum, ad latus vfque $c d, \&$ tam latum poftlatus a c quanta eft eiufdem ductus latitudo: \& exacue ductum fupernè $\&$ infernè vfque in angulos a $\&$ c,vt prius in $B$ fcriptum eft,eodem modo vtere in omnibus rectis ductibus fequentium literarum. Deinde producito $a b$ hoc ductu duos tractus fubtiliores tranfuerfos, à quibus cicumducatur arcus litera circularis fubtus lineam $a b, \&$ fupra lineam cd, vfque ad erectam $g$ h.poltea circino cóiunge $g f h$. Deinde pone latitudinem litere tractus maioris fuper lineam ef, per punctum $k$ ante f:deinde comprime circinum tantum quanta fuerit literæ dicta latitudo, \& pone eius alterum pedem in $k$, alterum verò in linea $\mathrm{e}$, atgi illic pone punctú $l$, in hoc cófítat pes circini immobilis:altero verò ex $k$ circúfcribe internè ad fub tiliores tractus tranfuerfos, arcum contingentem vtrofque: \& fuperiorem angu lum finito acutum, inferiorem verò excauato per circularem arculum eadem quantitate qua tractus antè exactus fuit. . Rotundum ductum ipfius D,eti am aliter facies quàm prius, nempè vt calamum imitetur, fupernè latior quàm infernè. Ad hoc duc diametrú c b, \& f cribe exteriorem arcú vt prius. Sed ad internum defcribendum pone punctum $m$ infra i,tantú diftans quanta eft latitudo tractus maioris, fuper diametrum $\mathrm{cb}, \&$ circumfcribe inuariato circino internam lineam . At vbi tractus fieri debet fubtilior, ibi manu erit ducendụs in: fernè $\&$ fupernè:quenadmodum fubfcriptum eft. 


\section{GEOMETRIAE LIB. III.}
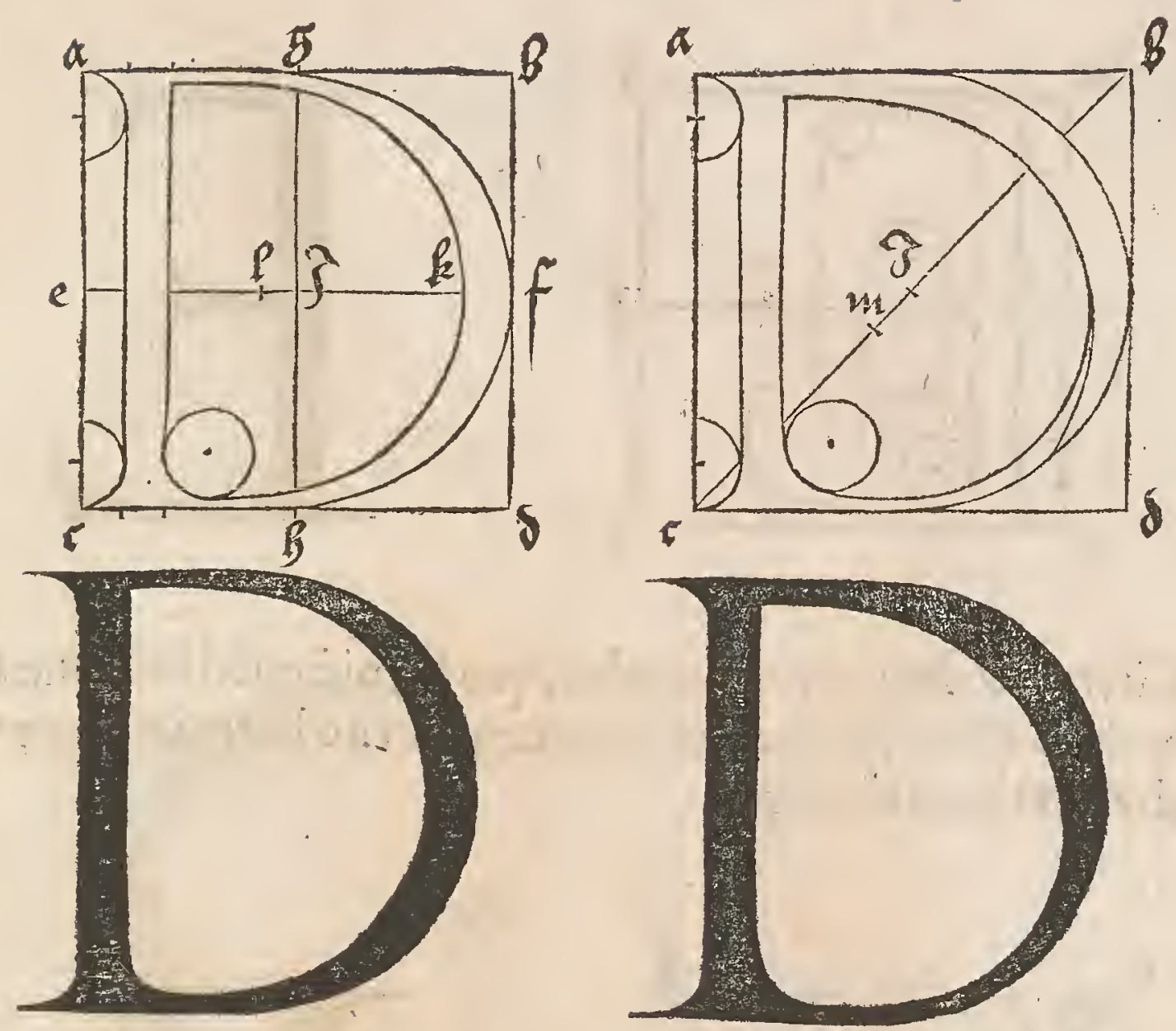

Literam in fuo quadrato ita formabis. Duc lineam tranfuerfam ef per medium a b c d, deinde duc literæ tractum magnum \& erectum à fronte, eo modo quo in $\mathrm{d}$ friptum eft. Deinde duc etiam fupremum trafuerfum fubtiliorem ductú fub linea a b ita vt terminus eius contineat fex decimas minus tamen vna tertia vnius decima partis, $\&$ egreffionem huius fac deorfum defcendere, in fine continentem decimam partem a b:tanta etiam fit diameter circuli, quo egreffio illa excauatur interné:deinde duc medium tractum fubtilem tranfuerfum fuper mediam lineam ef, ita vt breuior fiat fupremo tractu parte decima a b:ad finem tamen in duplo illo latior, \& excauetur per circularem arcum cuius diameter contineat fextam ef. Sed infimum ductum fupra lineam $\mathrm{c}$ d fac, vt infimo angulo excedat tractum fupremum longitudine decima partis $c d$, cufpidem tamen vltra hunc locum tranfire facies per duas tertias vnius decime partis, erigeśg furfum eum per vnam fe $x$ tam longitudinis $c d, \&$ excauabis eundem per circulum cuius femidiameter fit fexta quoque $\mathrm{c}$ d. Item infimum angulum in liter a excauabis per arcum eiuf dem circuli, quo medium tractú tranfuerfum excauabas, reliquos angulos permitte acutos vt hic fubf criptum eft. 

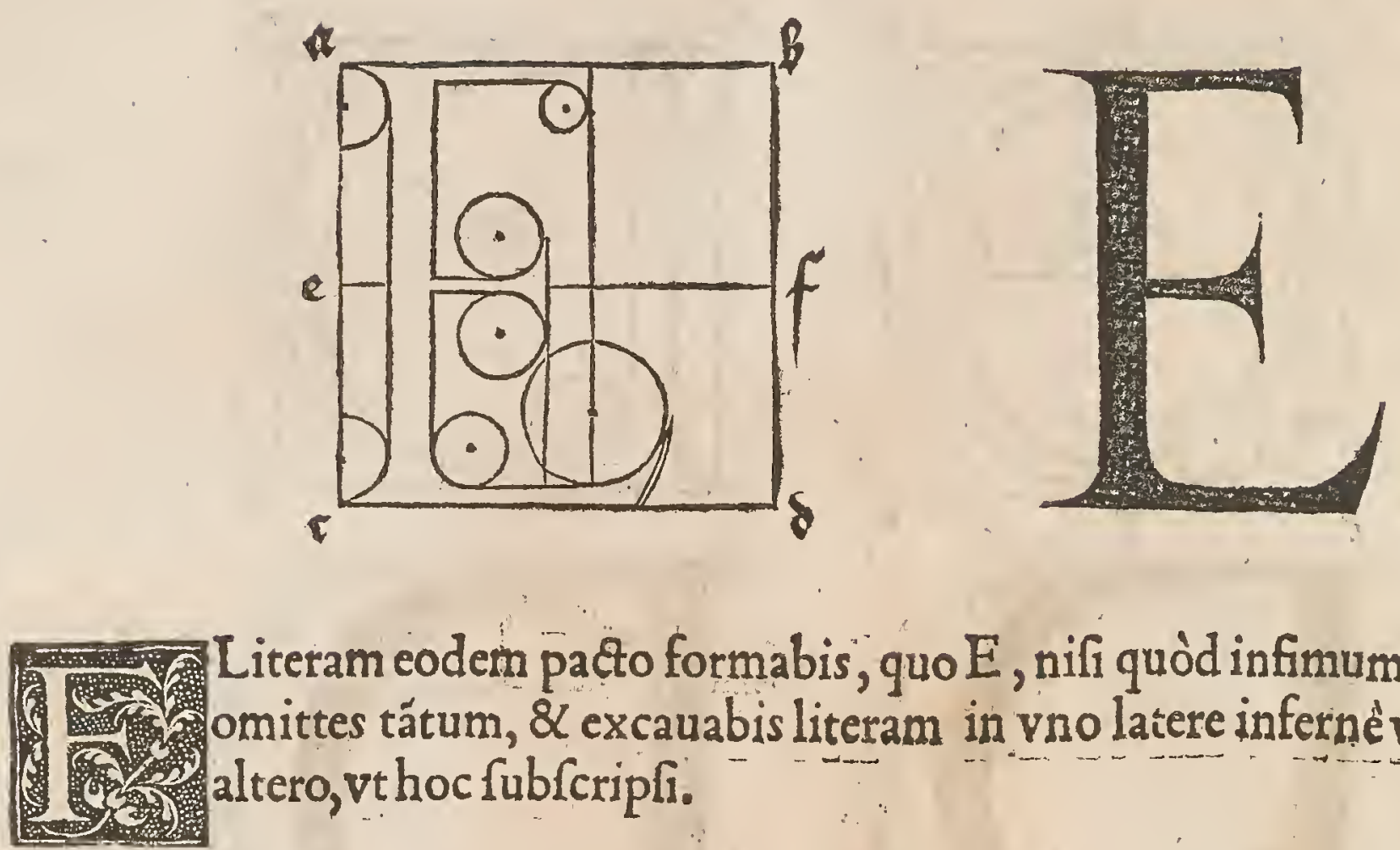

Literam eodem pacto formabis, quo $E$, nifi quòd infimum tractum omittes tătum, \& excauabis literam in vno latere infernèvt antè in altero, vt hoc fubfcripfi.
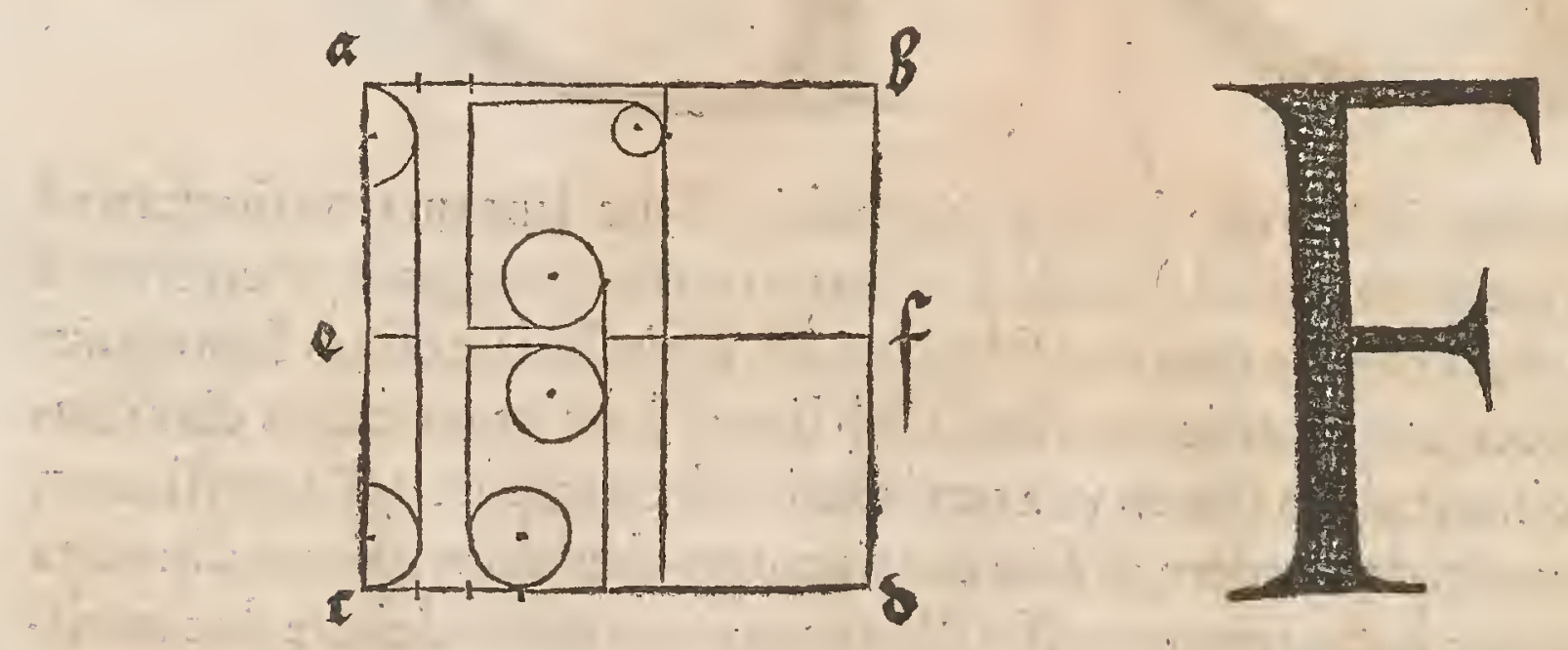

Tem literam $G$ facies fimiliter atquê $C$ priùs defcriptum eft, duntax at hoc excepto, $q$ ante lineá $g$ h erigitur ductus latus literę à rotundi tate furfum ad lineam ef $\&$ fupernè exacuitur ficut pri s dictum eft, fed infernè remanent anguli ambo. Vel Ghoc pacto formabis in dicto quadrato preqdiuifo, duces diametrum $\mathrm{c} b, \propto$ pones circinum pede altero in púcto i \& altero pede duces arcú ex $\mathrm{e} v \mathfrak{\text { q }}$ ad mediú locú $\mathrm{c} d$, ibi pones punctú 1 ,ita quoque duces arcum furfum ad lineam $a b$ vfque in erectam $g h$, atque ibi dem pones 3. Deinde locabis fuper lineam $\mathrm{g} h$ punctum $m$ in parte decima longitudinis $g h, \&$ coniunge $1 \& \mathrm{~m}$ manu circulariter mota. Deinde educito ex 3 li neam furfum, quanta eft tractus latitudo liter $x$, fed obliquam ac inclinatam in mediointer circularem $\&$ eręctam $g h$, ab extremitate huius manu ducitolineam rotundam vfque ad lineam a $b$, vbi circularis eandem tangit. Poltea $a b$. fcinde $g$ infernè in parte tertia, id'̧ in puncto $n$, atque tantum afcendat tractus latus ab m erectus furfum : fiant'́g, eius egreffiones fupernèin duplolatiores ipfo ductu. Poft hoc pone pedcm circini fuper diametrü $\mathrm{c} b$.tantum fupra $i$, quanta fuerit tractus literglatitudo $\&$ circumfcribe diftátia e i fumpta arcum, qui exteriorem fupernê tangat:infernê verò finitur fupral, hinc manu duces lineam ad tractum rectum iuxta altitudinem m.Idem fac fuperne ducendo tra ctum fubtilioremlitere, vt fubfequitur, formatum. 


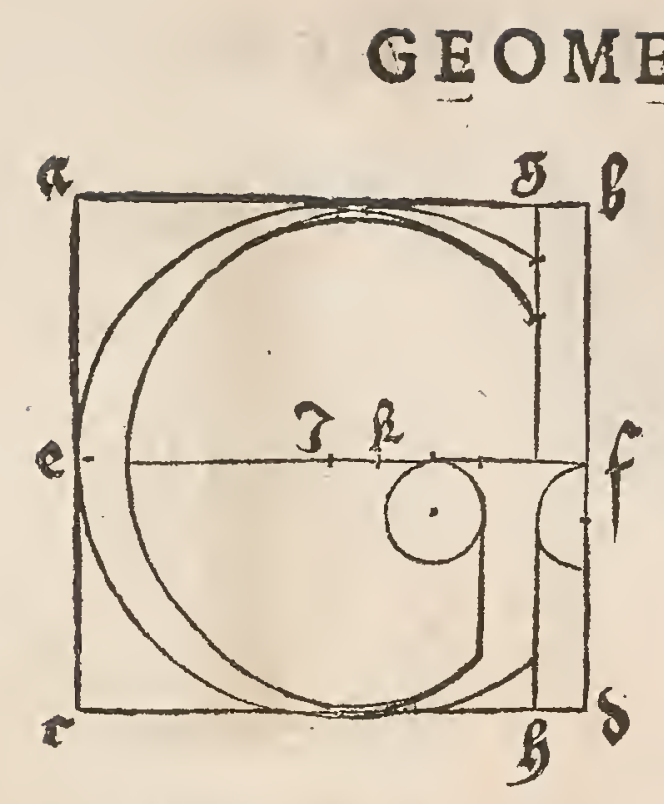

IIB. III,

129
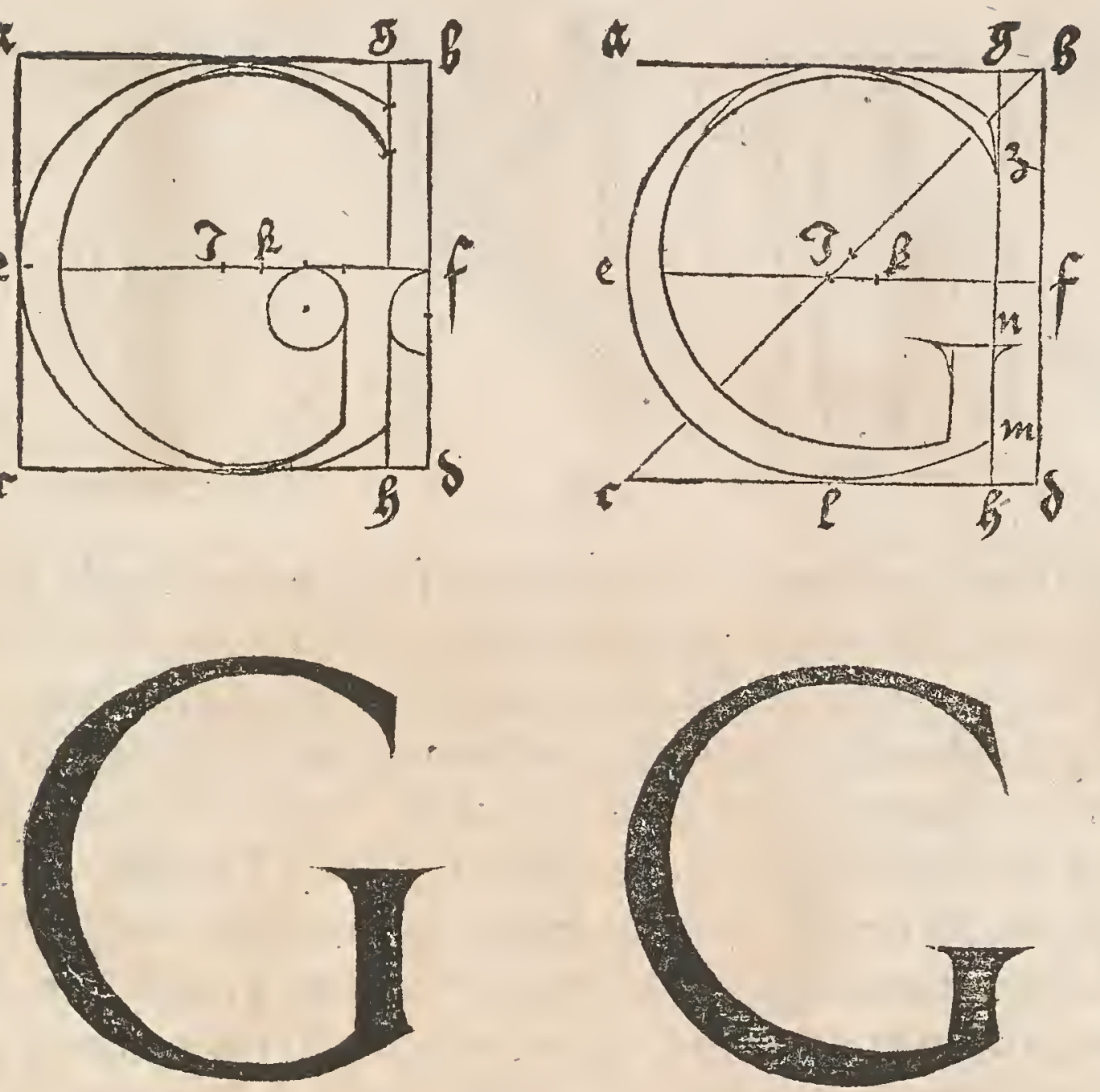

Litera forma bitur ex duobus latis magnis at‡ erectis ductibus, quan ta fuerit altitudo quadrati, ita vt corum egreffiones exteriores angulos quatuor quadrati a $c \& b$ d contingát. Et quonam pacto latiorem literarum dụctuum exacuentur infernè ac fupernè vtrinque proiectus rx, fuperiùs edoctus es. Nam qualibet litera in quolibet ductu latiore $\&$ erecto fuperné $\&$ infernè in fuis piecturis triplo latior eft, quàm circa mediü:modo nó iungatur tractus lubtilior . Cum ifta fuerit peracta, tunc duc tractum tranfuerfum fubtiliorem inter erectos in medio fuper lineam ef, quemadmodum fub. fcriptum eft.
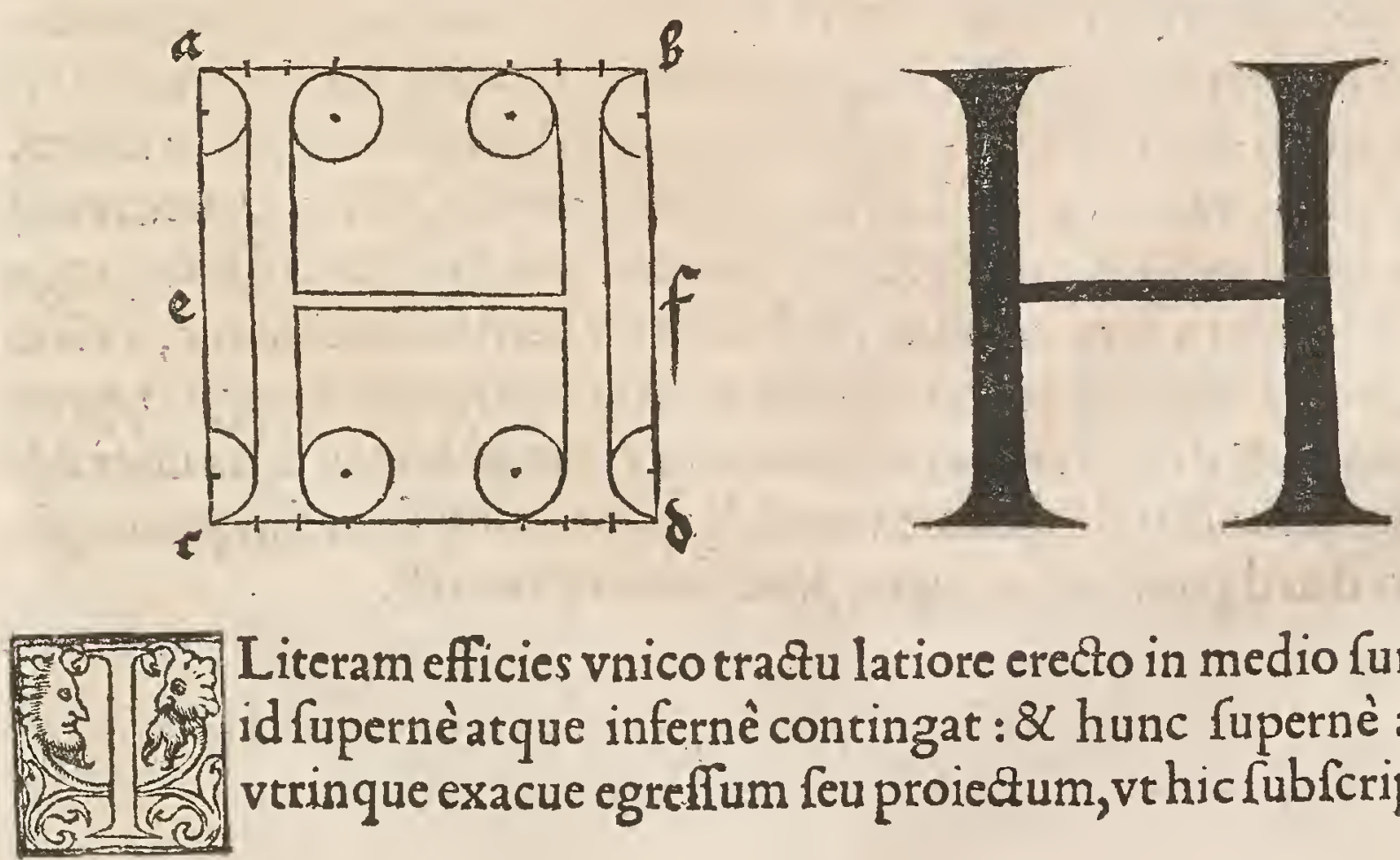

Literam efficies vnico tractu latiore erecto in medio fui quadrati, vt id fupernèatque infernè contingat : $\&$ hunc fupernè atque infernè vtrinque exacue egreffum feu proiectum, vt hic fubfcriptum eft. 

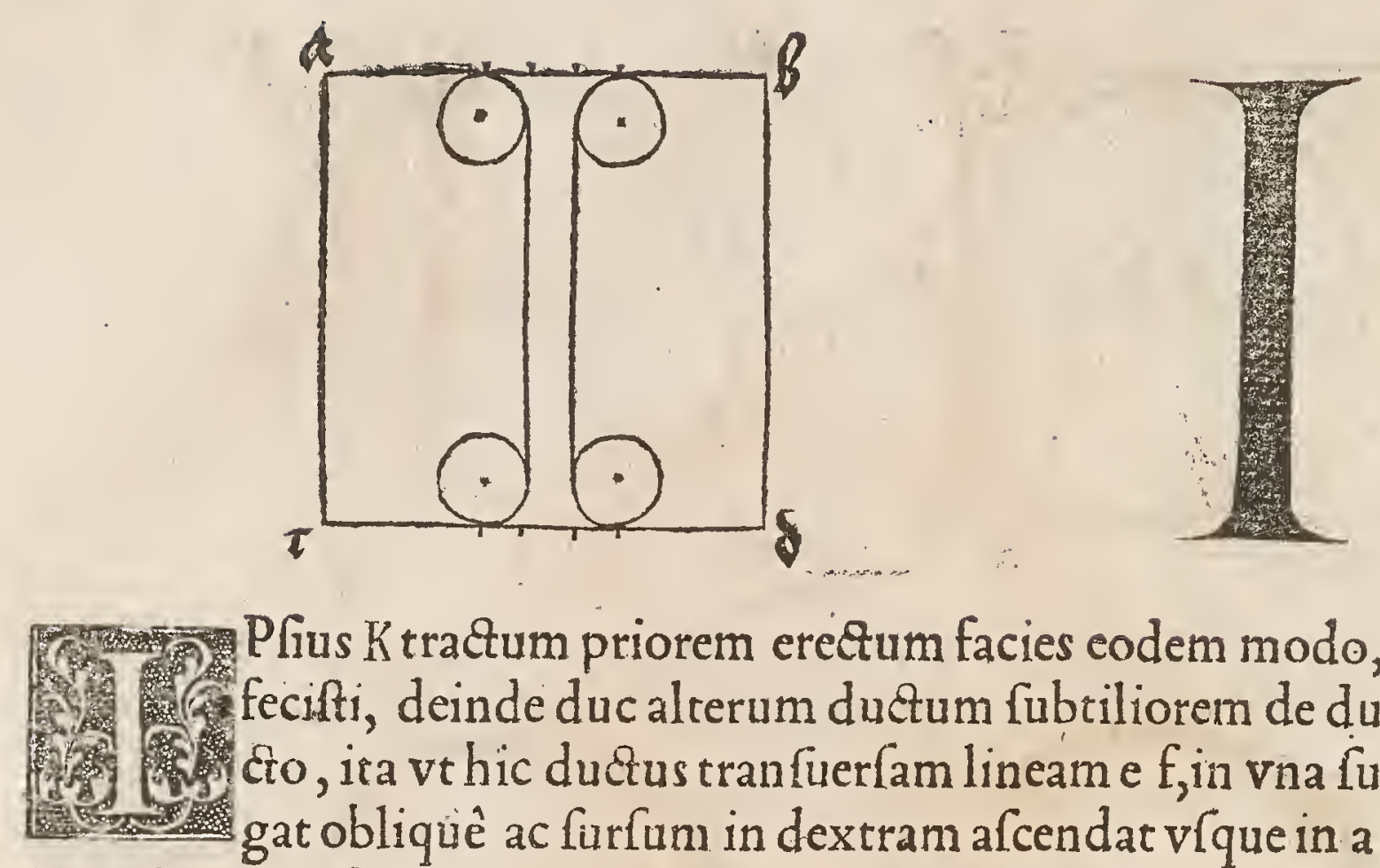

Pfius K tractum priorem erectum facies eodem modo, quo in $\mathrm{H}$ prius ecifti, deinde duc alterum ductum fubtiliorem de ductu latiore erecto, ita vt hic ductus tranfuerfam lineam e $f$, in vna fui parte contingat obliquè ac furfum in dextram afcendat vfque in a $b$, atque paral lelusfiat rectę dia metro. Sed huius proiecturas lupernê fac vtrinque ad lineam a $b$,partem decimam linea a $b$ continentes fingulas. Proiecturam anterioré excauabis per arcum circuli, cuius diameter non excedat latitudinem tractus exilioris, fed alterius arcus diametrum quo pofteriorem proiecturá excauas fac duplo maiorem diametro arcuum, quibus pracedentes proiecturas ductuum ere ctorum atque latorum excauare cófueuifti, deinde de ifto ductu exiliore duc alterum latum deorlum, vt etiam parallelus fiat diametro quadrati:atque huius initium fumatur ex angulo acuto, quem facit ductus exilior cum ductu erecto \&lato:ducarúrque hic cum proiectura in angulú d, ita tamen, pone puncta duo ante $d$, hoc pacto, vt prius punctum parte decima linex $c d$ diftet ab d, tantundê $\&$ alterum à priore; ducatur itaque dictus ille tractus in fpacium; quod eft in ter puncta duo, lineis cœecis \& occultis. Sed poltea addes proiecturan quianfacies hoc modo. Pone antef in linea ef punctum g, non plus abf diftans, quàm quanta fuerit latitudo tractus exilioris. In hoc puncto ponatur pes circini vnus ac alter extédatur in angulum d, ex quo reducatur per latum ductum ocultum $\&$ cœcum:tunc exibit rotunditas inferior iplius caudx. Sed fuperiorem concauationem quare hoc pacto:partire $f d$, per punctum $h$ mediu, in quo ponatur cir cini pes vnus, altero defcribe arcum egredientem ex $d$, vfque ad ductum latum. Aut hoc modo efficies $k$. In primis finito ductum erectum, \& fuperiorem exilé permanere, quemad modum iam defcripti funt:nifi quòd exilioris ductus, angu lus interior ad lineam a b relinquitur, fed exterior verfus b excauatur, vt iam dictum eft. Deinde ducatur ductus inferior \& Iatior obliquus ex angulo, quem concludit linea ef, $\&$ ductus erectus: defcendatq $\mathbf{v}$ fque ad latus $\mathrm{c}$, ita inter $\mathrm{d} \&$ ductum ductus latitudo relin quatur vacua, $\&$ anterior angulus relinquatur, po. fterior verò verfus d, paulùm excauetur, ficut fubfcriptum eft. 


\section{GEOMETRIAE LIB?}
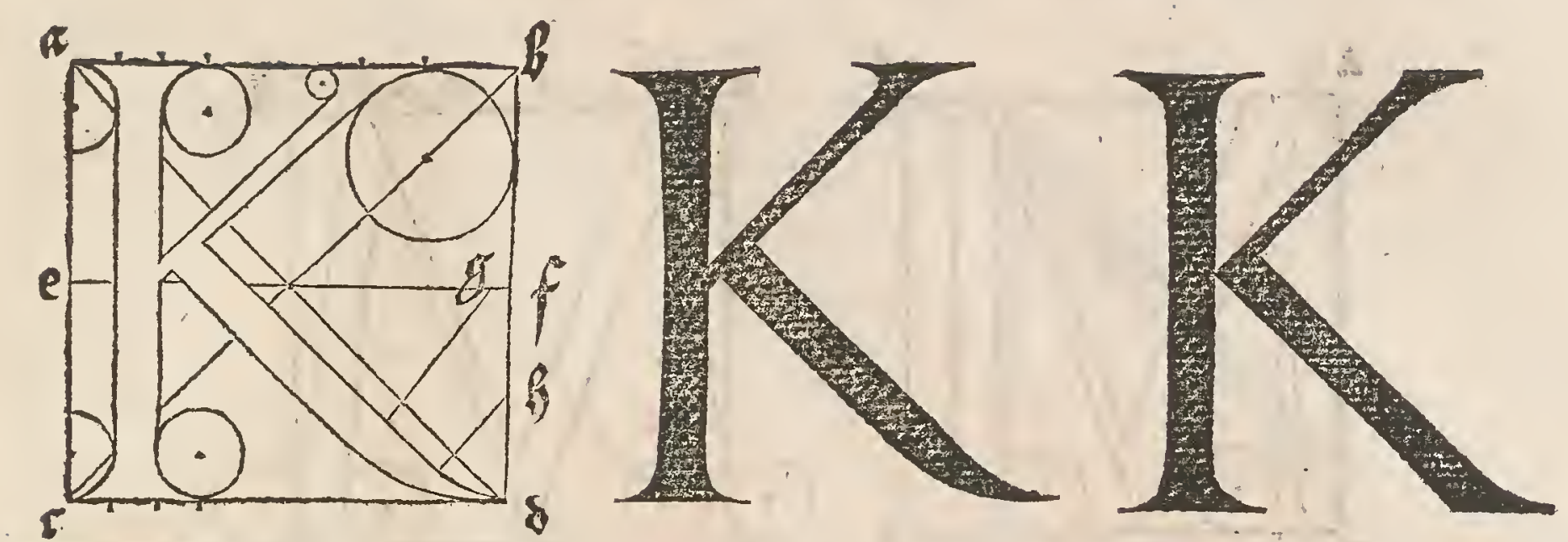

Aeterùm ipfü L efficies ex duabus literis pręcedentibus fimul iüctis, nempe facies priorem tractum erectú, \& latum, ficut prius in i. Ccriptú eft. Ad hunc iunge pedem infernè priùs formatx literx $E$ : ita defor: matum L fubfcriptum eft.
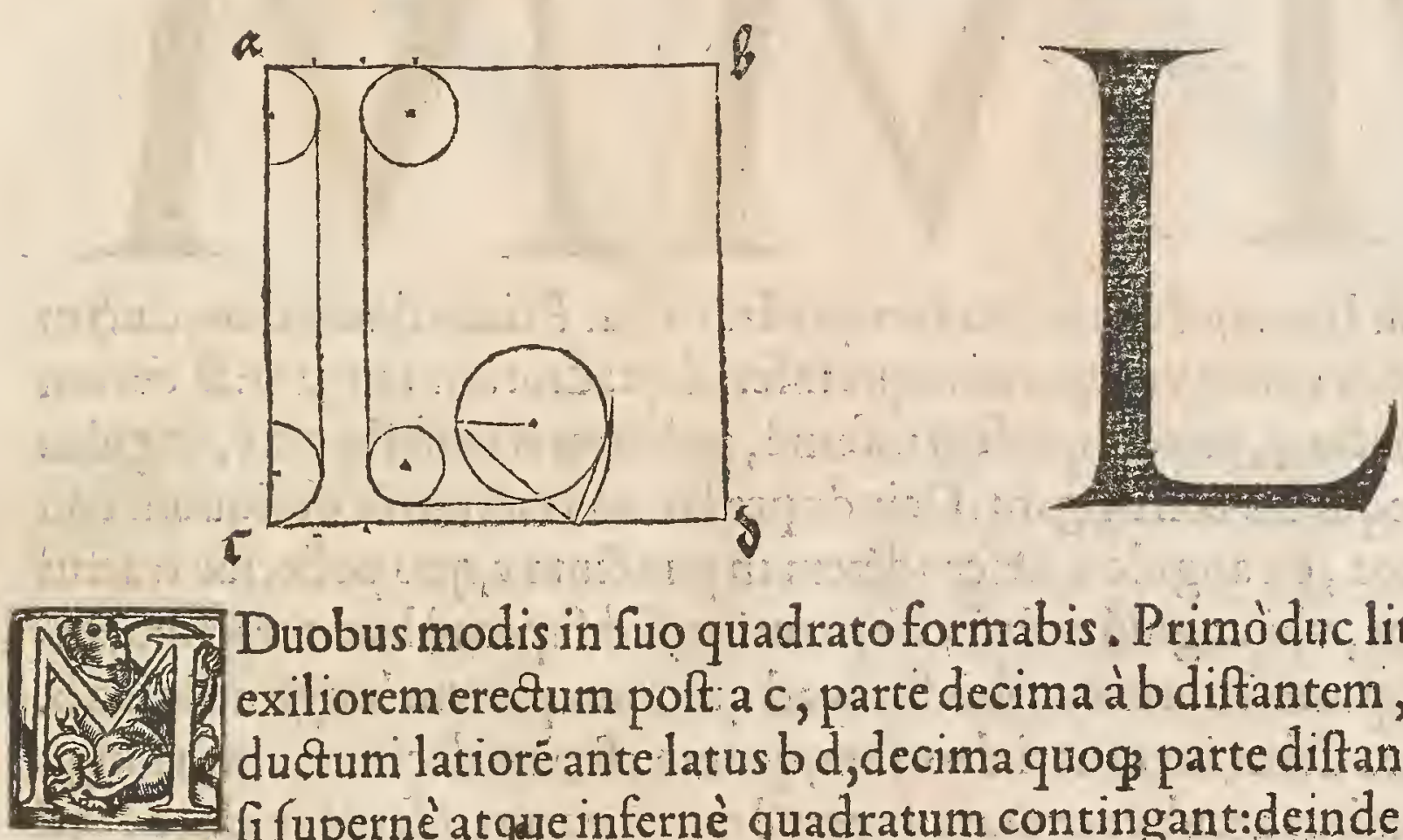

Duobus modis in fuo quadrato formabis. Primòdnc litera tractum exiliorem erectum poft a $c$, parte decima à b diftantem, fac alterum ductum latiore ante latus b d, decima quog parte diftantem, ita vt ip fí fupernè atgare infernè quadratum contingant:deinde partire in ter duos hos duetus lineam c d per xquahia in puncto e, \& ductractum latum ab angulo an teriore exilioris tractus deorfum in punctume, deinde ductum exile furfum abe, in angulum etiam anteriorem tractus latioris erecti, angulośćptractuum fup eriores $\&$ interiores non cauabis, fed acutos fines:fed exterior es, fimiliter amborrum ductuum erectorum inferiores confuetis proiecturis, vt in prace dentibus literis fecifti, ornabis vtrinq. Notabis quoque, quando hr literx calamodefign andę eflent, vnico ductu forent perfcribendx. At in tuam gratiă quò teinftituerem, elt litera hęc ita vt pradictum elt, fubfcripta.

Altero modo fic. Diuidelatus quadrati a $b$ in fex equa fpacia, $\&$ abfcinde duo fpacia ext1:ema vtrinque per duo puncta $f g$, duc interiorem tractum latiorem cum cufpi de fuo e, ve fuprà:\& huic furfum exiliorem ita vt inter $f \mathrm{~h}$ relinquatur vnum fpacium vacuum, \& fic amplius litera fefe proclinat. Deinderelinqui to tractus rirectos ad latera, priorem exilem, \& polteriorem craffum, fupernè qui dem vt pri us, fed infernè extende eordem in duos angulos $\mathrm{c}$ d:tandem adde $\mathrm{p}$ iectu:as v1: in priore $M$ es edoctus. Sed proiectura excedit infernè quadratum iu $x$ ta $\mathrm{d} \& \mathrm{C}$ : :aut facito $\mathrm{N}$ fupernè cum angulis acutis, tunc magis fe acclinant tractus later: les:aut amputa eofdem obtufê, atque eo modo, qui placebit maximê, vtaris, qui amadmodum \& hic fub criptum vides. 

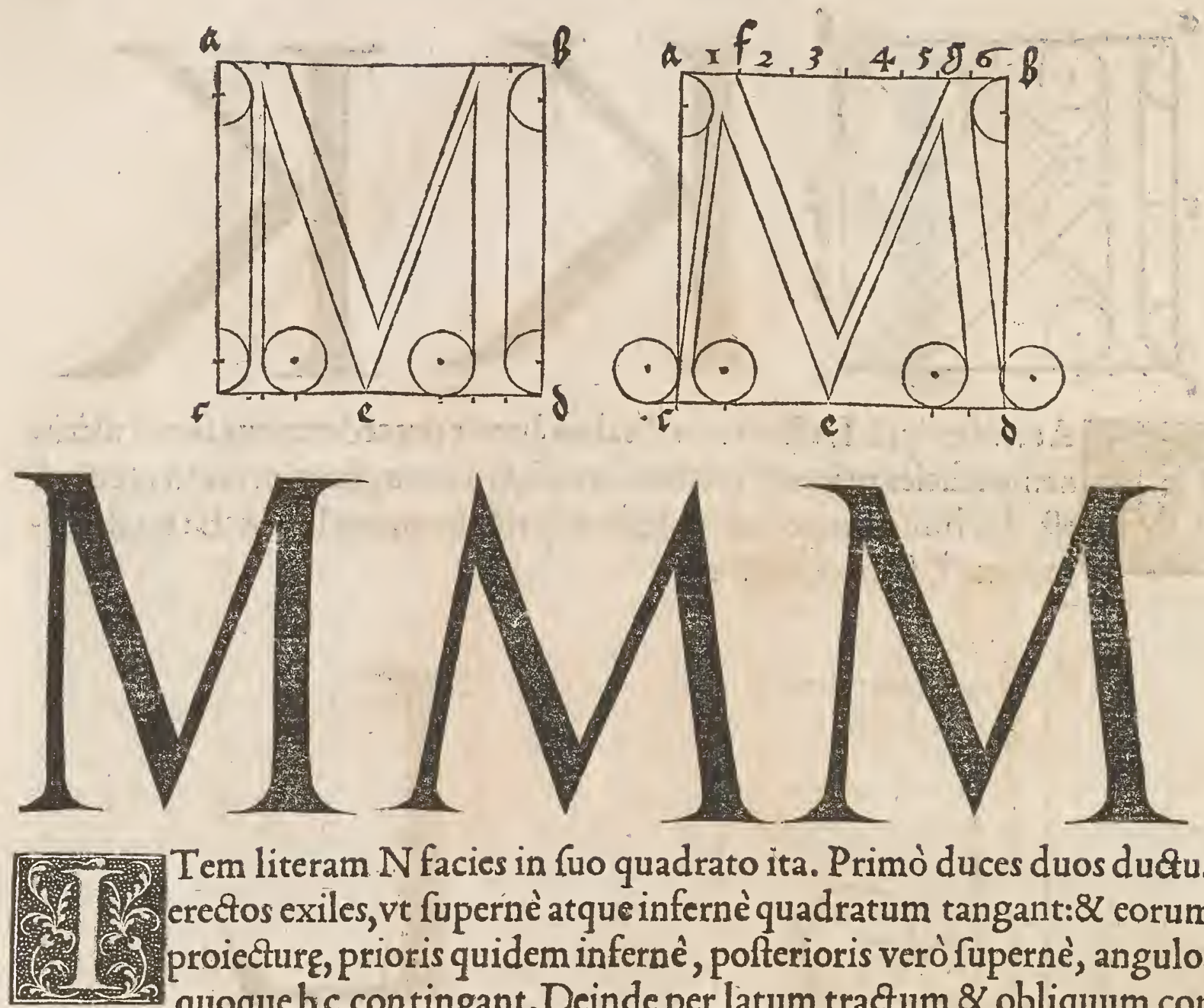

Tem literam $\mathrm{N}$ facies in fuo quadrato ita. Primò duces duos ductus erectos exiles, vt fupernè atque infernè quadratum tangant: \& corum proiecture, prioris quidem infernè, pofterioris verò fupernè, angulos quoque b c contingant. Deinde per latum tractum \& obliquum con iunge illos duos, ex angulo a defcendétem in punctum $e$, quo pofterior tractus à terigo notatur, vbi angulú acutú effe permitte, fed fupernè hunc tractum, ante angulum a porrectum excauabis foras ad quintam partem longitudinis $a b$. Hæc proiectura debet incuruari deorfum, quanta eft pars decimaquinta longi.tudinis a $b$, duobus arcubus exarata:fupernè quidé paruo, infernè maiore. Ad arcum igitur minorem fumatur pro diametro circuli pars quinta longritudinis $a b, \&$ ponatur centrum extra quadratum, ita vt pes circini, contingat finem $p$ iecturę, \& angulum a:deinde aperi paululum pedes circini, \& mutato cétro do nec arcus tangat finem quoque proiectura, $\&$ obliquum latúm que duć tum literæ in puncto medio, inter latus a c $\&$ ductum exiliorem erectum atquie prioré.

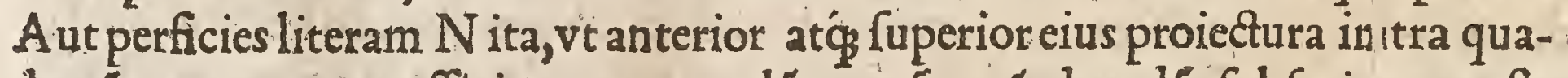
dratú remaneat:aut efficies ex co angulú acutú, quéadmodú fubfcrip ıtum eft. 
GEO METRIAE LIB! III:
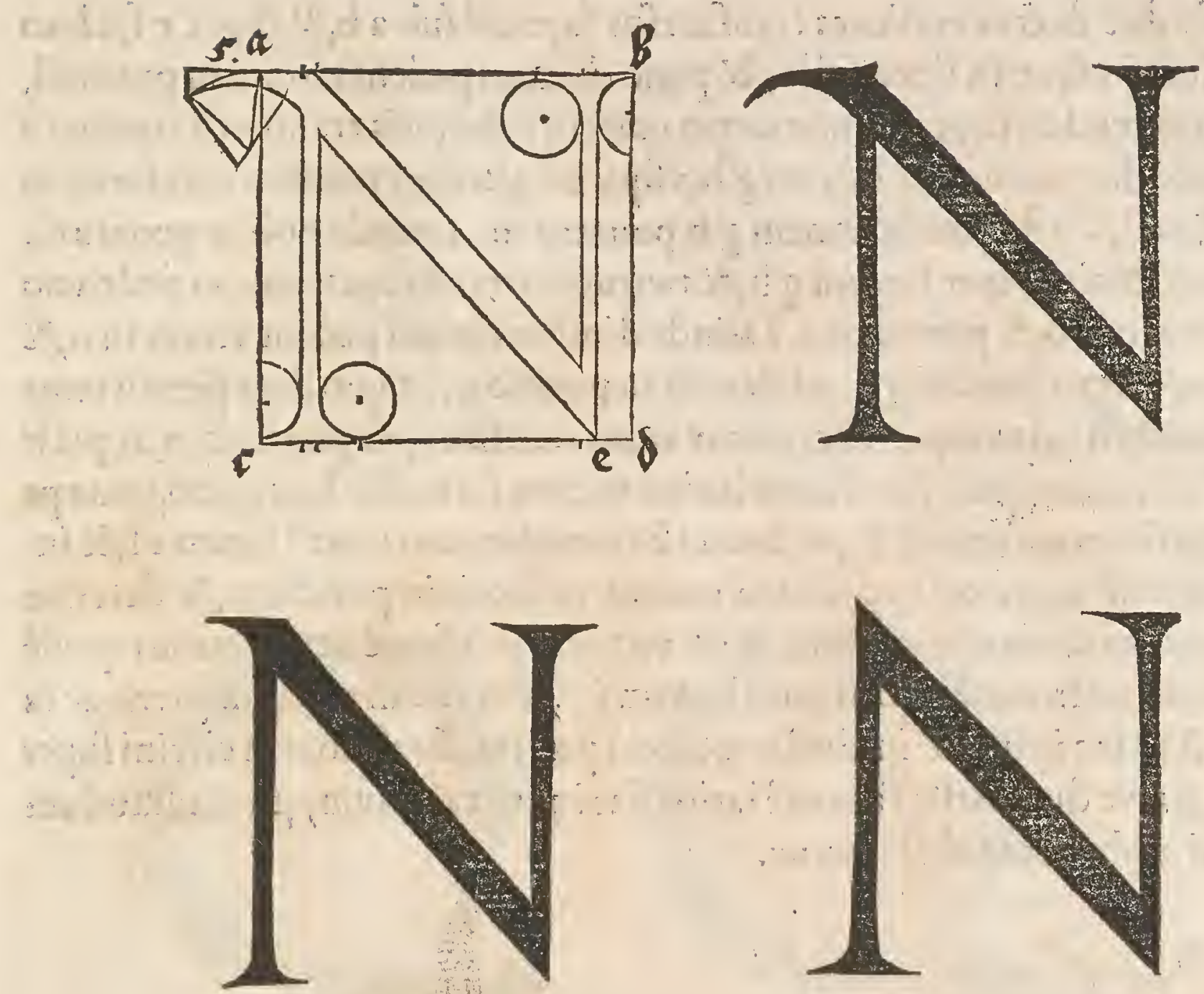

Verù perficies ita in fuo quadrato. Decito in quadrato diametrum $\mathbf{c}$ $b, \&$ diuide illam in medio per púctum e, \& pone liter $x$ maiorem craf fitudinem circa $e$, ita vt $e$ fit in medio duorum punctorú $f g$ :qua fint duo centra, \& ex vtroque ducatur circulus qui bina quadrati latera tangat, $\&$ vbi circuli fe fecant, illic manu ducas latitudinem exiliorem literalis tractus iufta forma, ficut fubfcriptum eft.
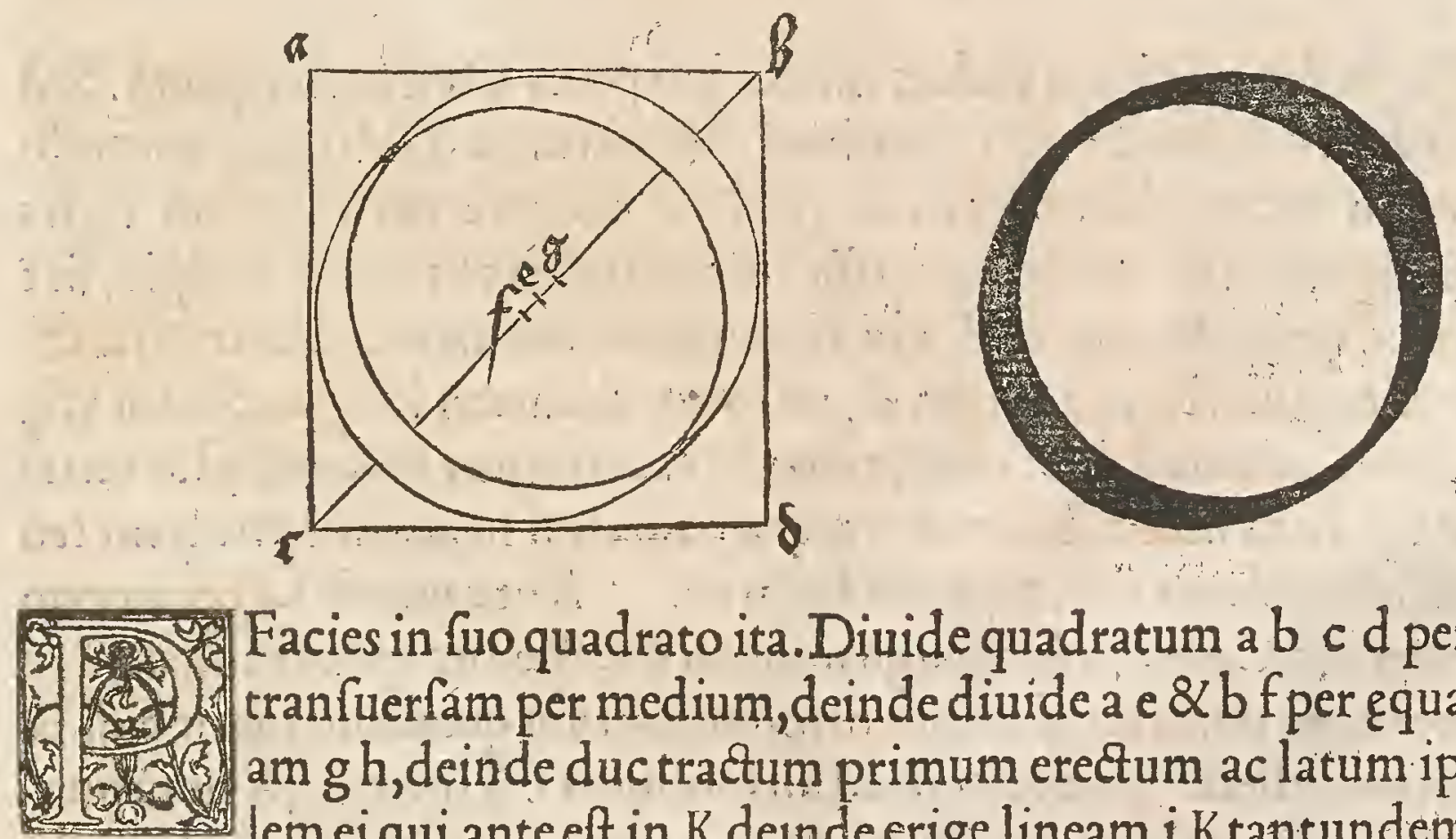

Facies in fuo quadrato ita. Diuide quadratum a $b$ c d per lineam $e f$ tranfuerfam per medium, deinde diuide a e $\& \mathrm{~b}$ f per equalia per line am $g$ h,deinde duc tractum primum erectum ac latum ipfius $P$ fimilem ei qui ante eft in $K_{2}$ deinde erige lineam $i \mathrm{~K}$ tantundem poft hunc tractum erectum, quantam idem habet craffitudinem (hoc hic obleruandum eft femper, quòd in quadrato, in quo fteterit litera, vocamus angulum a antè feu ad finiftrá, $\&$ b pòft feu ad dextram). Porrò vbilinea i $k$ fecat $g h$, ibi pona- 
tur $l$, deinde duc ductus exiliores tranfuerfos fupernèfub a $b, \&$ fupra ef, à lato $\&$ erecto ductu vfque in lineam ik, \& pone alterum pedem circini in punctol, \& alterum extende vfque ad inferiorem partem inferioris tranfuerfi tractusiu $x t a k, \&$ inde duc arcum per lineam $g h$, vfque ad alterum tractum tranfuerfum exilem ipfius $P, \&$ ad interfectionem $g h$ ponatur $m$. Deinde poft $m$ ponaturli ter latitudo maior fuper lineam $g h, \&$ tantum circinus aperiatur vt pede vno cotingat lineam a $b, \&$ punctum $n$. Deinde dimitte circini pedem vnum in $n, \&$ alterum pone fuper lineam $\mathrm{g} h$, ad dextră in puncto 0 , in quoftare permittatur pes ifte immobilis, alteróque defcribatur arcus tranfiens per punctum $n$, atque li neas a $b \&$ e f contingens. A ut liter $x$ huius vncum formabis hoc pacto, pone pe dem circini fub tranfuerfa $g h$, in linea ik in medio loco inter lineam e $f, \&$ inferiorem partem fuperioris tran [uerfi tractus exilioris in puncto $p, \&$ defcribe arcum vt priùs tranfeuntem per $m, \&$ fic vncus erit infernè acutus:cuius quidé acies terminetur in medio loco inter lineam i $K, \&$ tractum erectum liter $x$ ac la tum Aut fac ipfum $P$ in ductu rotundo per tranfpofitionem circini fuper diametro, ita vt ductus ille fupernèlatior fiat iuxta calamum, quemadmodum in fequenti alphabeto fubfcribetur.
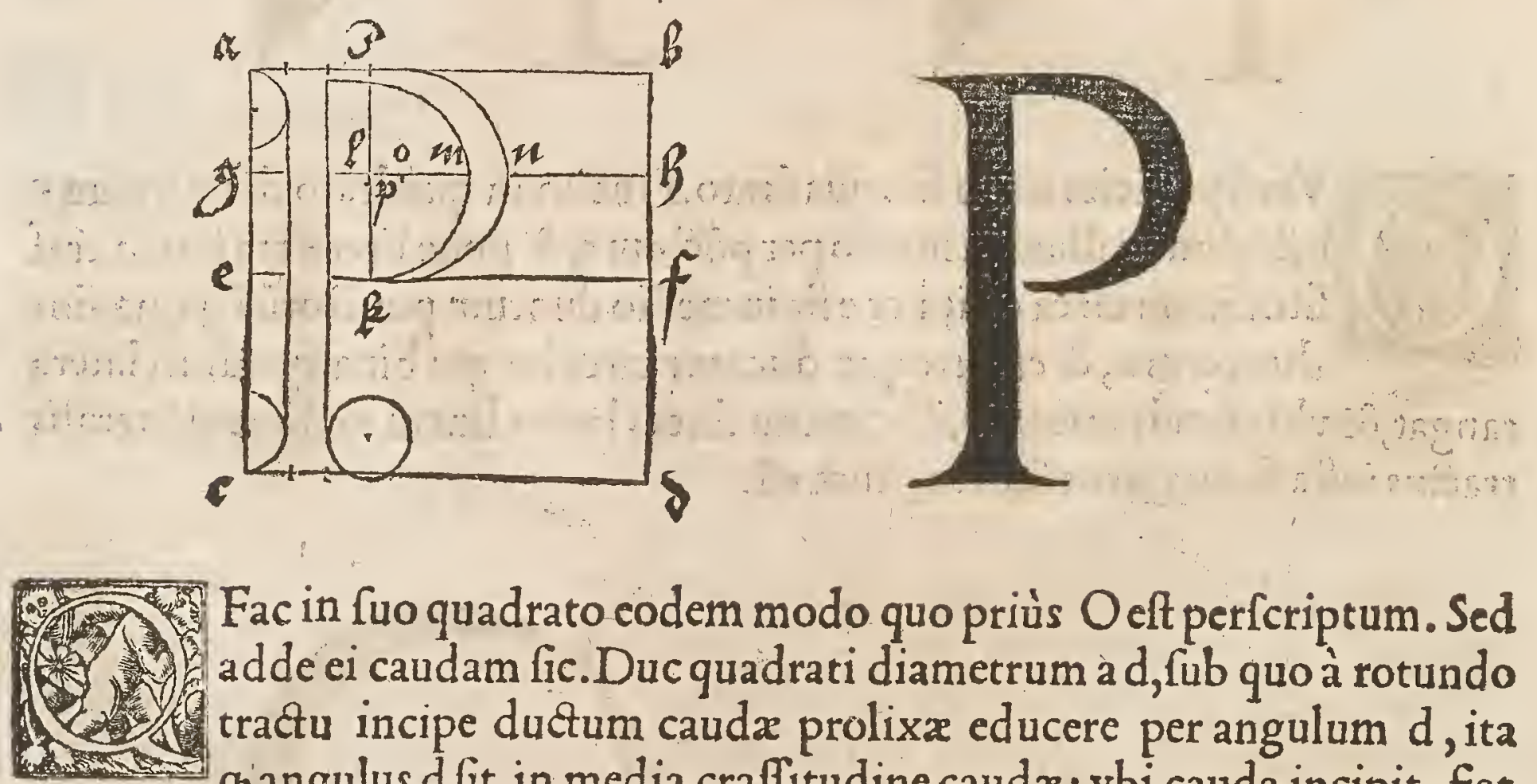

Fac in fuo quadrato codem modo quo priùs Oeft perfcriptum. Sed adde ei caudam fic. Duc quadrati diametrum à d, fub quo à rotundo tractu incipe ductum caudx prolixa educere per angulum $d$, ita qangulus d fit in media craffitudine caudx: vbi cauda incipit, fiat paulò angultior, quàm in angulo $d$, vbi veram fuam obtin ere debet craffitudinem:deinde educatur vltra angulum $d$, ad totius diametri longitudinem vf $q$ $\&$ deorfum, ita vt curuetur dum obliquatur, $\&$ vt terminus eius non plus tertia lateris parte fublatus imum defcendat atque paulatim in acutiorem aciem ten dat faftigiata, ac tandem exilis multum finiatur. Aut caudam $Q$ breuiorem reddes, fic videlicet. Cape circino longitudinem $\mathrm{cd}, 8$ educ ipfius caudam à ro tunditate literx, per punctum d arcum priorem tantum quantum fuerit $\mathrm{cd}, \&$ vt cauda furfum feflectat, donec iterú ad altitudinem $\mathrm{c} d$ furrexit, $\&$ nota punctum per h, deinde tranfpone circinum, \& altero pede iterum à rotunditate literæ educito arcum fubtus $d$, donec iterum redeat in $h$, ita cauda in fuo initio maximam habebit craflitudinem, ficut id in fequenti figura dupliciter elt fub. criptum. 


GEOMETRIAE IJ B: III. I
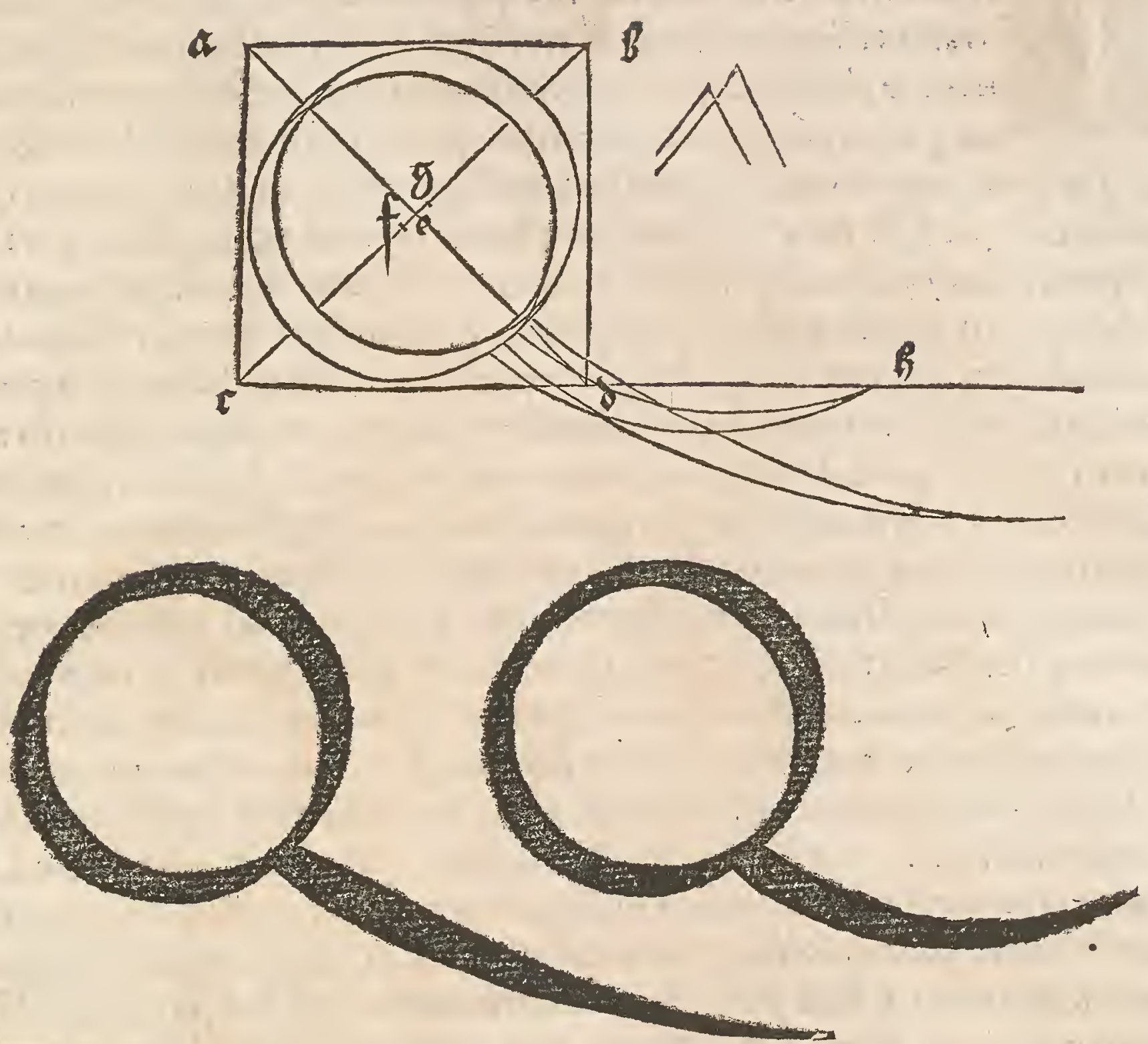

2. Raterea $R$ fac ita in fuo quadrato vt $P$ defcriptum eft,deinde erige $r$ 28 ctam lineam q r per medium quadratum, \& vbifecat exteriorem arC. Cum tractus rotundi ponatur s, à quo deorfum verrfus angulú d. duca tur tractus latus, xqualis ferè illi qui eú fupra in literam $k$, hic tamen introrfum aliquantulum elt flectendus ideóg manu ducendus eft, $\&$ acumen eius bene formatum vf $\mathrm{g}_{3}$ in angulú d ducendữm. Aut ita fac $\mathrm{R}$, vt ductus eius rotundus iuxca calamum fupernèlatior, inferne exilior fiat. Ad hoc faciendum erit tranfponendus circinus fuper diametro $q \mathrm{e}$, neque rotundus erectum tratum continget, quemadmodum in $\mathrm{P}$ defcriptum eft. Praterea obliquus tractus à rotundo paululo incuruatior eff ducendus, ficut id fubfcripfi.
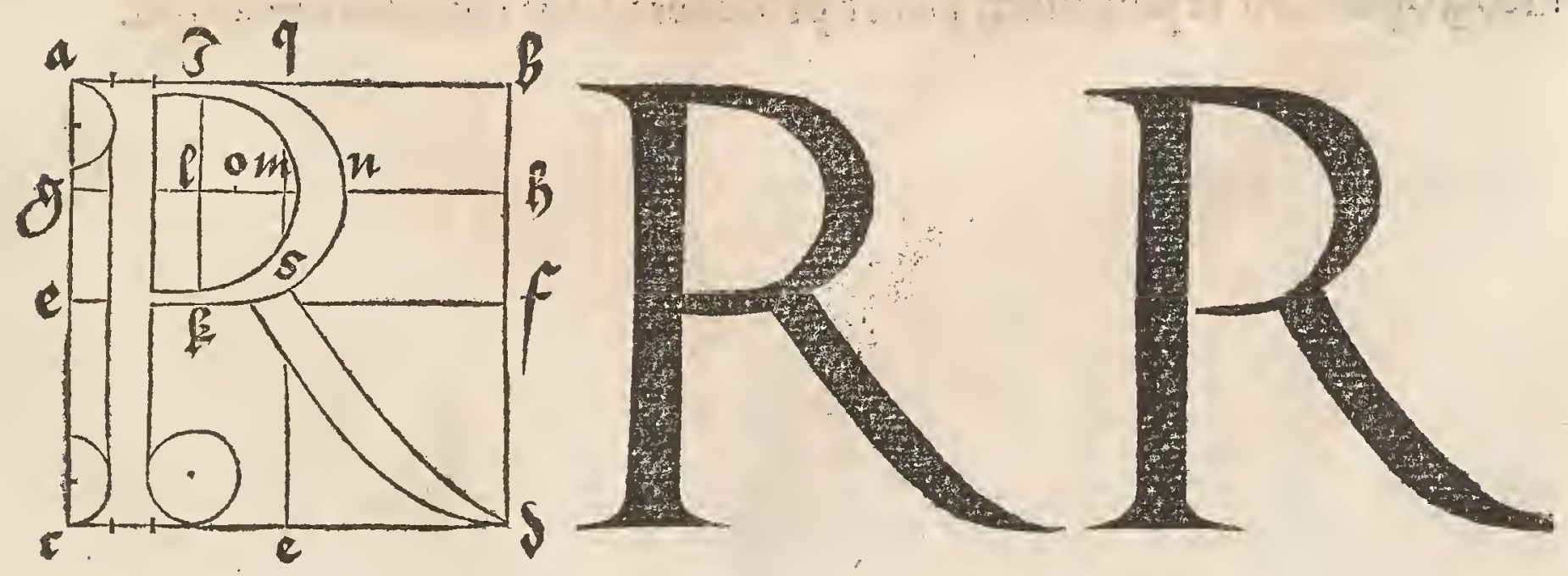


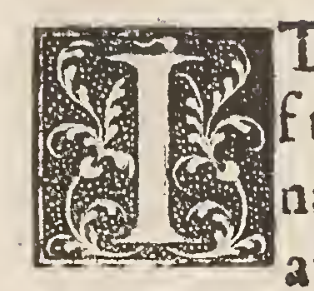

Tem literam $S$ fic facies in fuo quadrato a $b \mathrm{~cd}$. Primò duc lineam e $f$ tranfuerfam, mediam's \& erectá $g h, \&$ vbi hx fe in medio fecant,po natur $m$, deinde cape literæ craflitudiné maiore $\&$ eá pone fuper lineam $g h$,ita vt punctum $m$ tertiam parté craffitudinis fub fe abfindar, deinde pone litera craffitudinem minoré fupernè fubtus g,per púctum $i$,infernèfupra h,per $\mathrm{K} ; \&$ nota craffitudinem literæ fupernè per $n$,infernè per 1 . Dein de pone pedem circini fuper lineam $\mathrm{g} h$ in medio inter $\mathrm{n}$, ac altero pededuc cir culum per in,deinde quoque fuper lineam $g$ h pone circinum in medio inter $g$ 1,8 defcribe circulum per $\mathrm{g} l$, deinde iterum fuper eandem lineam $\mathrm{g} h$ pone circinum in medio inter $n h \&$ circumfcribc circulum per $n h$, ac tádem in medio inter $1 \mathrm{~K}$ polito pede, def cribatur altero circulus per eadem púcta $1 \mathrm{~K}$, deinde $\mathrm{ab}$. fcinde huius liter $x$ fuperiorem tractum erectè, ita vt hęc amputa tio in extremi tate contineat maximam craffitudinem litere, \& infuper tertiam partem: $\& \mathrm{vt}$ acumen tantum defcendat, vi cétro circuli i n æqualiter ad latus ftet, propterea acumen illud ad dextrum à circulo in recedit vfque in primá tertiam inter minorem \& maiorem circulum. Deinde prafcinde literam à finiftris infernè per li neam erectam,per medium duorú circulorum, $\&$ vt hæc amputatio quarta partelatior fit fuperiore ac pofteriore, $\&$ vt acumen eius afcendat ad xqualitatem cétri circuli in h. Aliter facies $S$ literă.Pone in medio quadrati a $b \mathrm{~cd}$, fuper linea $m$ tranfuerfam efpunctum $m$, deinde pone circini pedem vnum in medio $\mathrm{g} \mathrm{m}, \&$ altero pede defcribe lineá circularem verfus a e, per $\mathrm{m} g$,deinde pone cir cinum in medio $m h, \&$ defcribe circularem lineam per $m h$, verfus $f d$. Hiduo arcus contingúr fupernè antè, infernè pòft, curuaturas ipfus $S$ literę exteriores, deinde duc diametrum $c b$ per $m$, in medio eius pone craflitudinem maiorem literę duobus punctis $\mathrm{p} q$, ex quibus ducantur dux rectę linex furfum ac deorfü ad duos arcus illos $v f_{\mathrm{p}}$, deinde duc duas parallelas ex duobus punetis $\mathrm{p} q$, lineis circularibus, intra eas tamen, $v$ fque ad altitudinem $\&$ depreflionem amborum centrorum earundem circularium. Deinde figna fubtus $g, \&$ fupra $h$ minorem craffitudinem litere, hinc manu dabis formam liter $x$ introrfum fupernè $a c$ in fernè, $\&$ produci to tractum $S$ fuperné verfus $b, \&$ prafcinde eum vt acumen in ferius circularè tangat, $\&$ quòd pręcifio furfum decimá contineat partem a $b, \&$ quòd circularis acumen amputatum excedat, deinde pone erectam lineá r s poft e c parten quintam $\mathrm{cd}$ :vbi hæe diametrum fecat, illic pone $t$, at que in illú angu lum duc extremitatem liter $¥, \&$ fac præcifioné tertia parte latiorem fuperiore: ideóg oportebit te paululum vltrat procedere, ficut continenter fcripfi. 

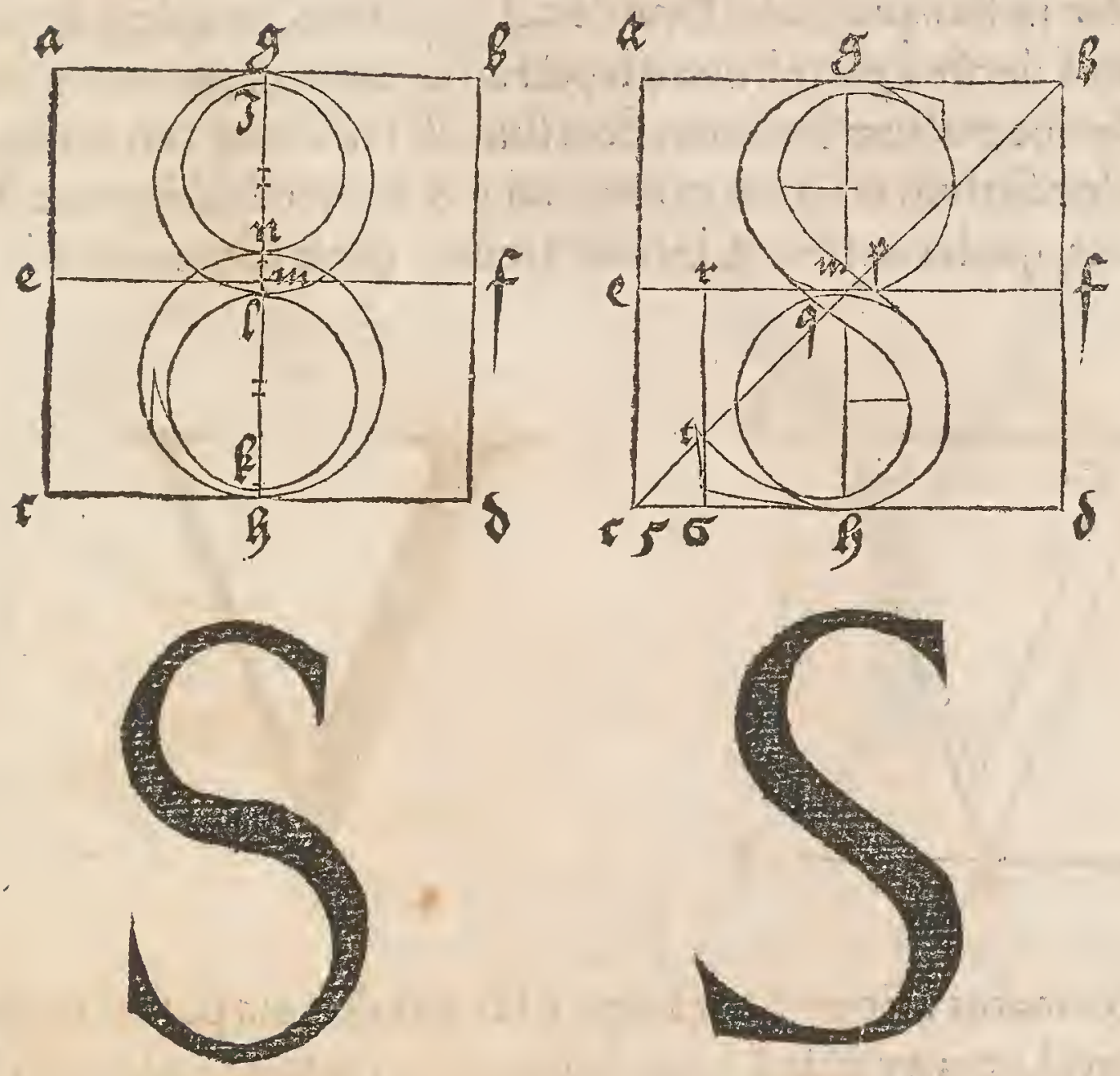

Literam ftatue in medio fui quadrati erectam, exacuéq infernè vtrá que proiecturam, quemadmodum priùs literam I fecifti,deinde pone duo puncta, fingula per partem decimam longitudinis a $b$ poft a $e$;an te $b \mathrm{f}$, tantæ longitudinis fiat ductus literæ trăfuerfus fublineam $a b$, extremitates autem huius tranfuerfi ductus piecturas habebunt, 8 oblique ab: fcindentur, \& fupernèlineá a bexcedent proiecturarum acumina verfuśg finiAtrá dependentia, \& lineas has obliquas proiecturaru fac longas parte guinta a $b$, deinde proicturas excauabis per duos diuerfos circulos, in minore angulo vte re diametro duas tertias latitudinis maioris cótinente. Sed in angulo maiore fu me diametrú, quantum fuerit latus quadrati inter tractú latú $\&$ erectú $\&$ angulú a vel $b$. Aut fac ita $T$ in fuo quadrato. P one punctú e vt priùs a, $\&$ pręcinde tranfuerfum tractum per diametrum vt priùs. Sed vt piectura dimidiata fit ad prioré, $\&$ vt fupernè fimplex angulus maneat, idem quoop in al tera extremitate. Sed punctum $\mathrm{t}$ in dupla propiùs ponatur $\mathrm{ad} \mathrm{b}, \& \mathrm{ab}$ fiffio paulò erectior $\&$ lati or fiat anteriore, alioqui omnia relinquantur vt priùs, quemadmodú fubfcripfi.

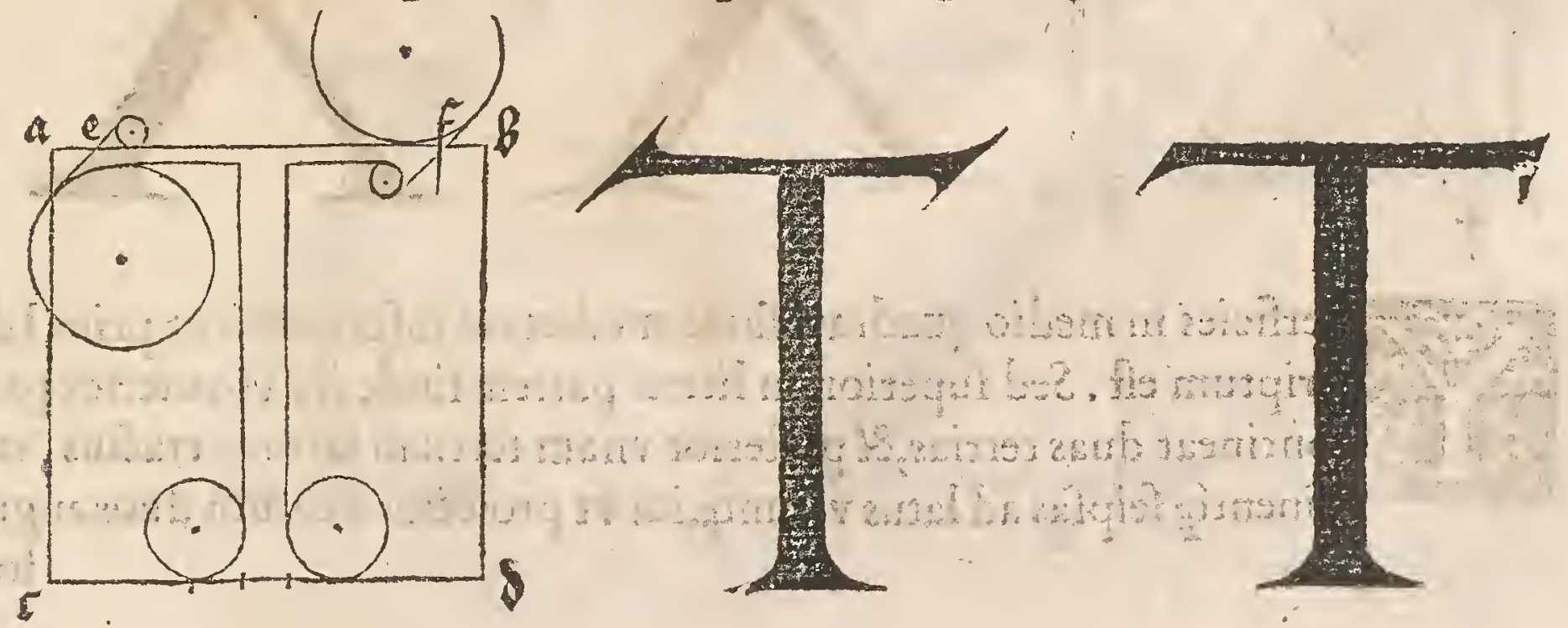




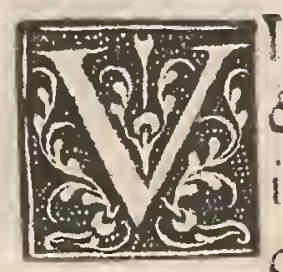

Tta fac in fuo quadrato. Diuide $\mathrm{c} d$ púcto medio e,deinde pone punctumf decima partelinex a $b$ poft a,tantundem ponatur $g$ ante $b$, deinde duc tractum latiorem deorfum ab fin e cum fuo acumine, $\&$ inde duc furfum tractum exilioré ad $\mathrm{g} \&$ fupernè adde proiecturas ductibus ambobus, quales antè in $\mathrm{A}$ infernè fecifti, quemadmodum hoc fubfcri. ptum eft.
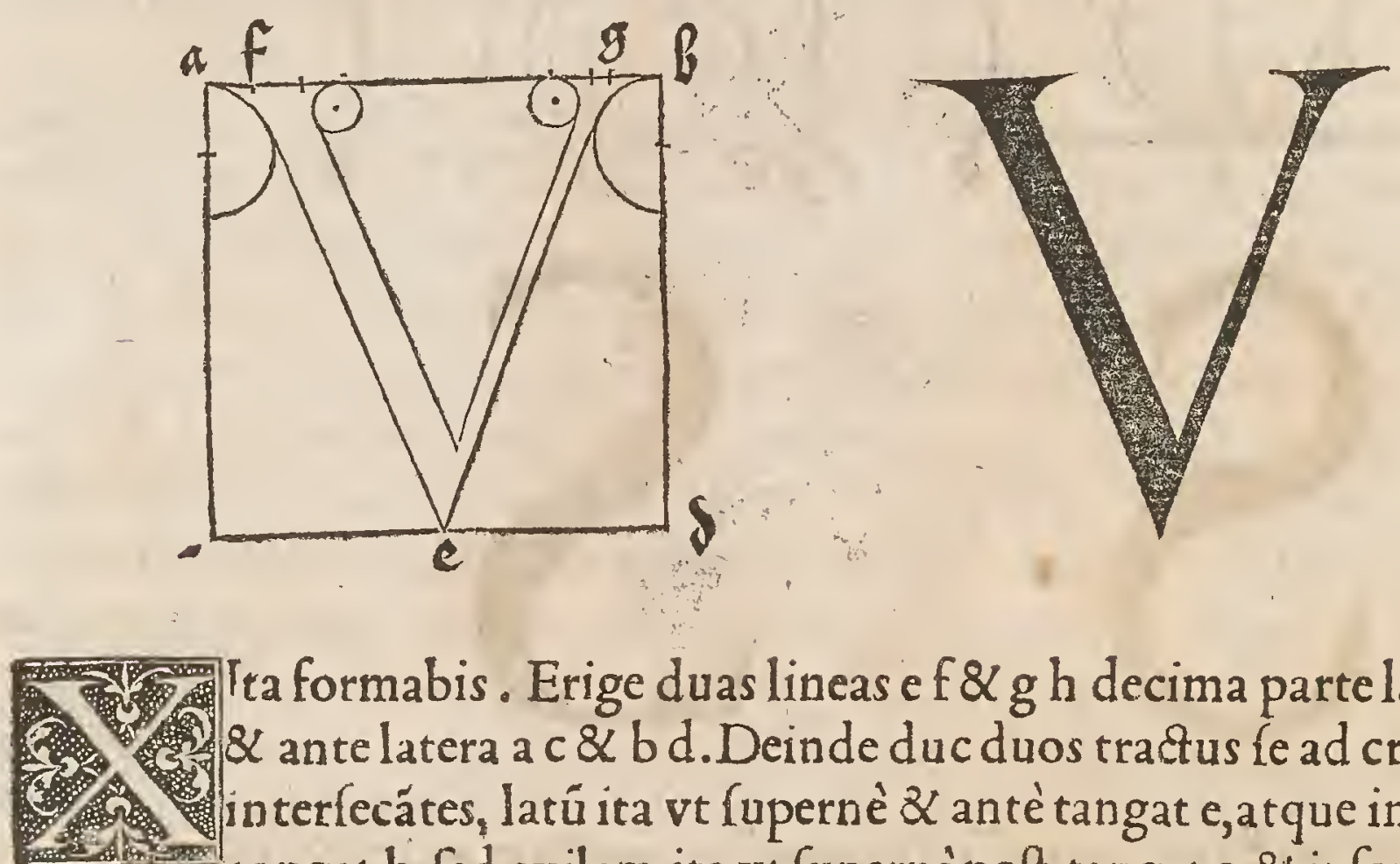

Ita formabis . Erige duas lineas $\mathrm{e} \& \mathrm{~g} \mathrm{~h}$ decima parte lateris a $\mathrm{b}$ polt $\&$ ante latera a c \& bd.Deinde duc duos tractus íe ad crucis formam in terfecátes, latú ita vt fupernè $Z$ antè tangat e, atque infernè, $\&$ poft tangat $h$, fed exilem, ita vt fupernè poft tangat $g$, $\&$ inferne ante $f$. De inde adde proiecturas, quatuor angulos a b c d tacturas fupernè atọ infernè, \& fac femidiametrum maioris circuli latam quintam partem a b, atque eo exca uabis quatuor maiores angulos: fed minoris circuli diametrum fac longum duas tertias latitudinis latioris ductus.

Aut variabis $X$ ita. Omnia relinquantur ve prius prater tractum fubtiliorem, guem fupernè per medietatem lati tractus erectiorem facies, \& fic fuperior pars literx erit minor atque anguftior quàm inferior ac aliter apparebit, ficut fụb. fcriptum elt.
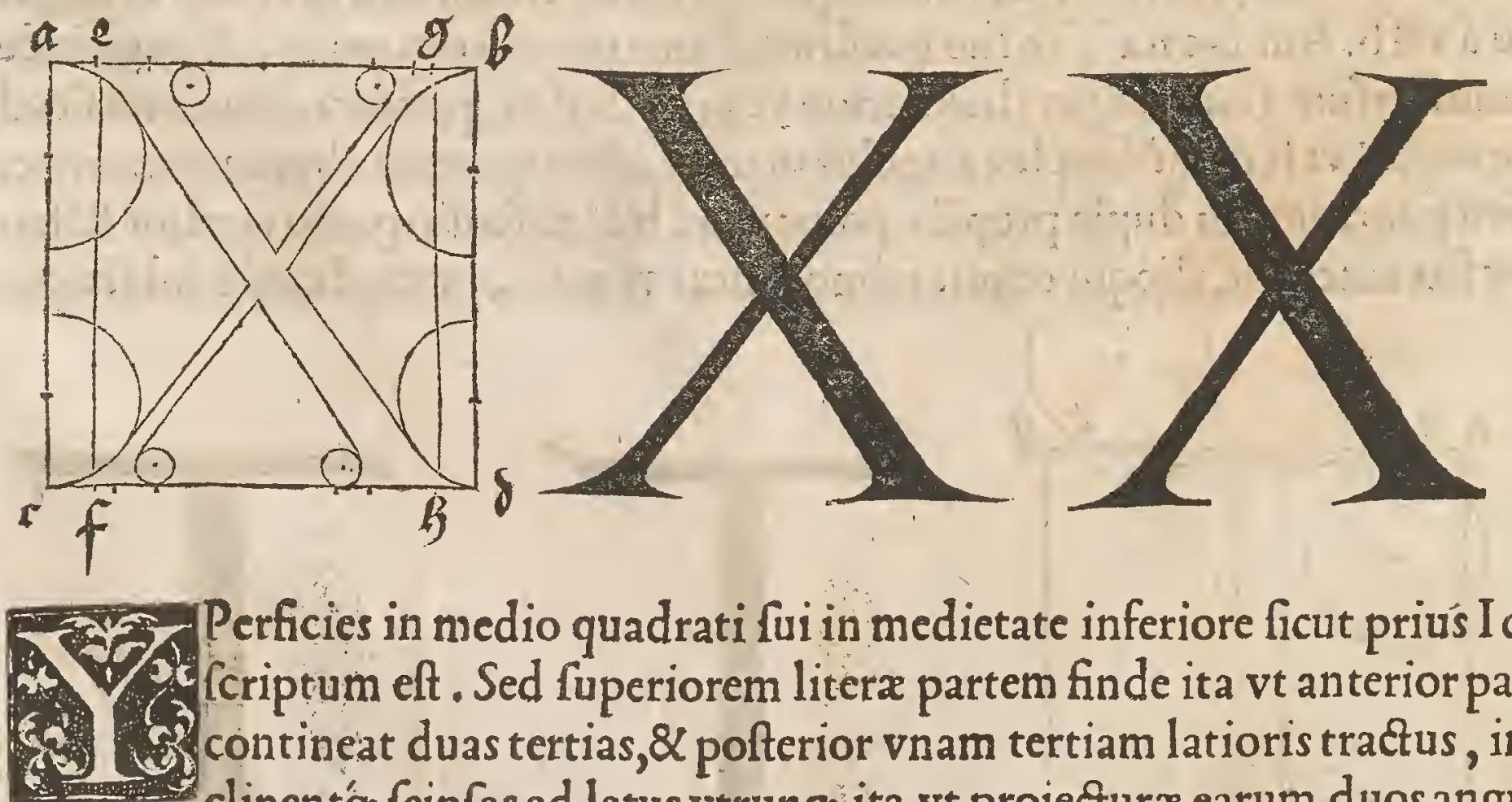

Perficies in medio quadrati fui in medietate inferiore ficut priuśs I de fcriptum eft . Sed fuperiorem literæ partem finde ita vt anterior pars contineat duas tertias, \& pofterior vnam tertiam latioris tractus, inclinentóg feipfas ad latus vtrung, ita vt proiecturæ earum duos angu- 
los a \& b contingant, \& maiores circuli, quo obtufos feu maioris angulos exca uabis; fac deametrum quantum fuerit fpacium quadrati iuxta literă, ficut in $T$ dictú eft. Diametros verò circulorú ad angulos acutos $\&$ minores fac vtrafque Iongiores quàm ductus latitudo fit ex eadem parte,ficut fubfcriptum eft.
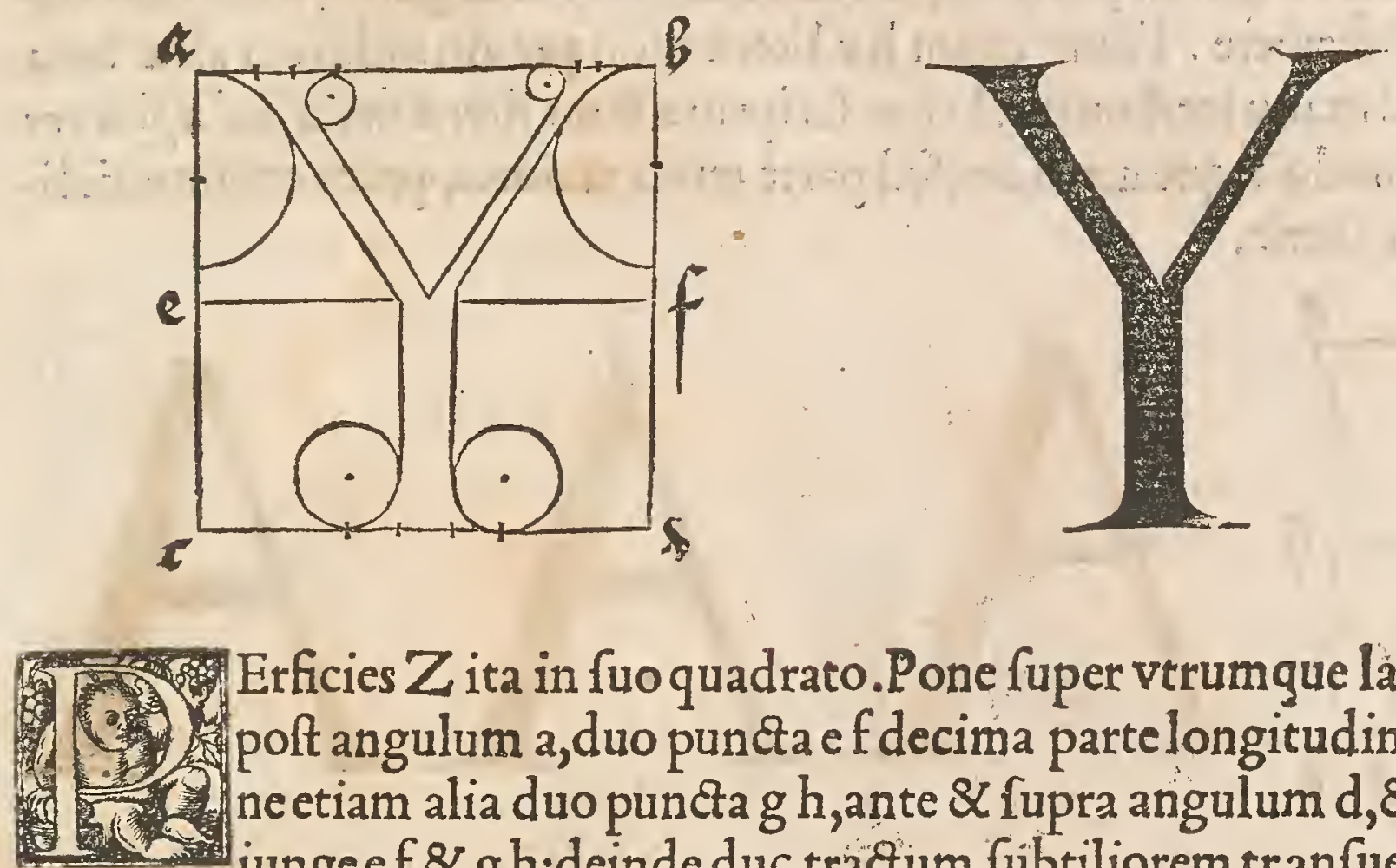

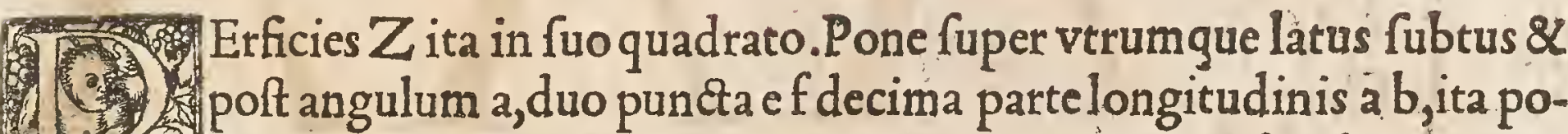
(1) ne etiam alia duo puncta $g h$,ante $\&$ fupra angulum $d, \&$ lineis rectis - - iunge ef $\& \mathrm{gh}$ : deinde duc tractum fübtiliorem tranfuerfum fub $\mathrm{a} b$ $\mathrm{ab}$ retrorfum in angulum $\mathrm{b}$ vf $q_{\mathrm{s}}$. Inde duc latum ductum obliquum vfque in $c$, deinde duc exiliorem ex c in g,deinde manu excauabis duo acumina $\mathrm{e} h$. Aut ita facies $Z$. Ab cinde quadratum a $b c$ d per lineam erectam $e f, \&$ ducli teram $Z$ iterum in hanc, vt prius, fed ita vt duo ductus tranfuerfî fupernè ante $\alpha$ infernè pòft per erectas a c \& ef ab cindantur, ficut fubfcriptum eft.
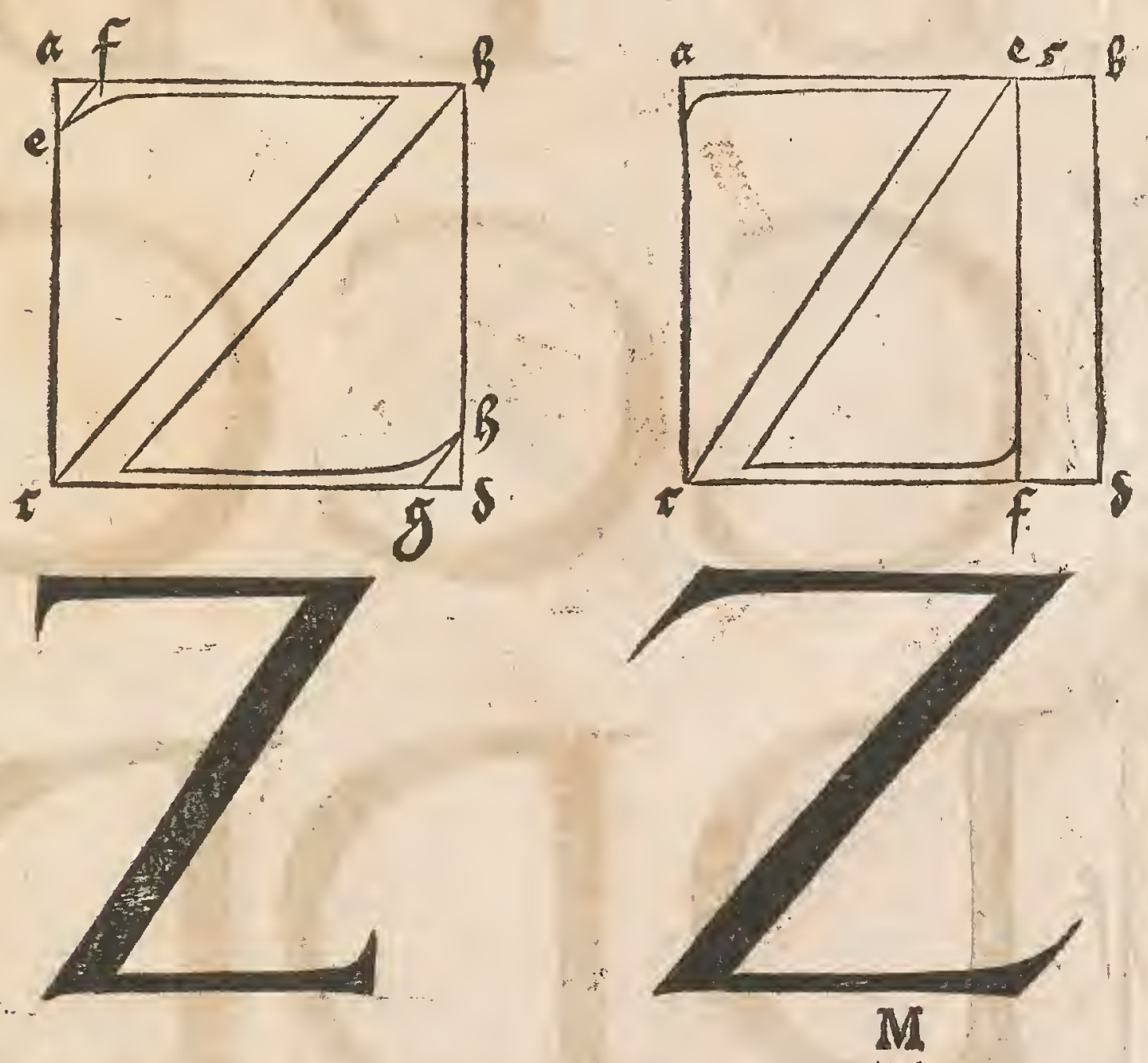
Téomnes alias priùs formatas literas poffumus ex noué latitudini: 1. 3 bus altas efficere, quemadmodum $\&$ iam defcript $x$ liter $x$ decem lati15. tudinibus longx funt ac alt $x, \& 2$ codem modo iuxta fuam proportio (G) (.) nem ac menfuram ex nouem partibus in fuis quadratis a $\mathrm{b} c \mathrm{~d}$ diui détur,vt prioris ex decem partibus, \& vt hoc meliùs intelligatur, volui tales etiam literas fubfcribere. Fiunt etiam hx liter $x$ quinque altitudinum alt $x$ cum minufcula, \& manu fcribuntur. In hac fcriptura fiunt litera maiufculx, feu ver fales eodem modo atque men fura, fed parte tertia maiores, quàm communis linea literarum fuerit.
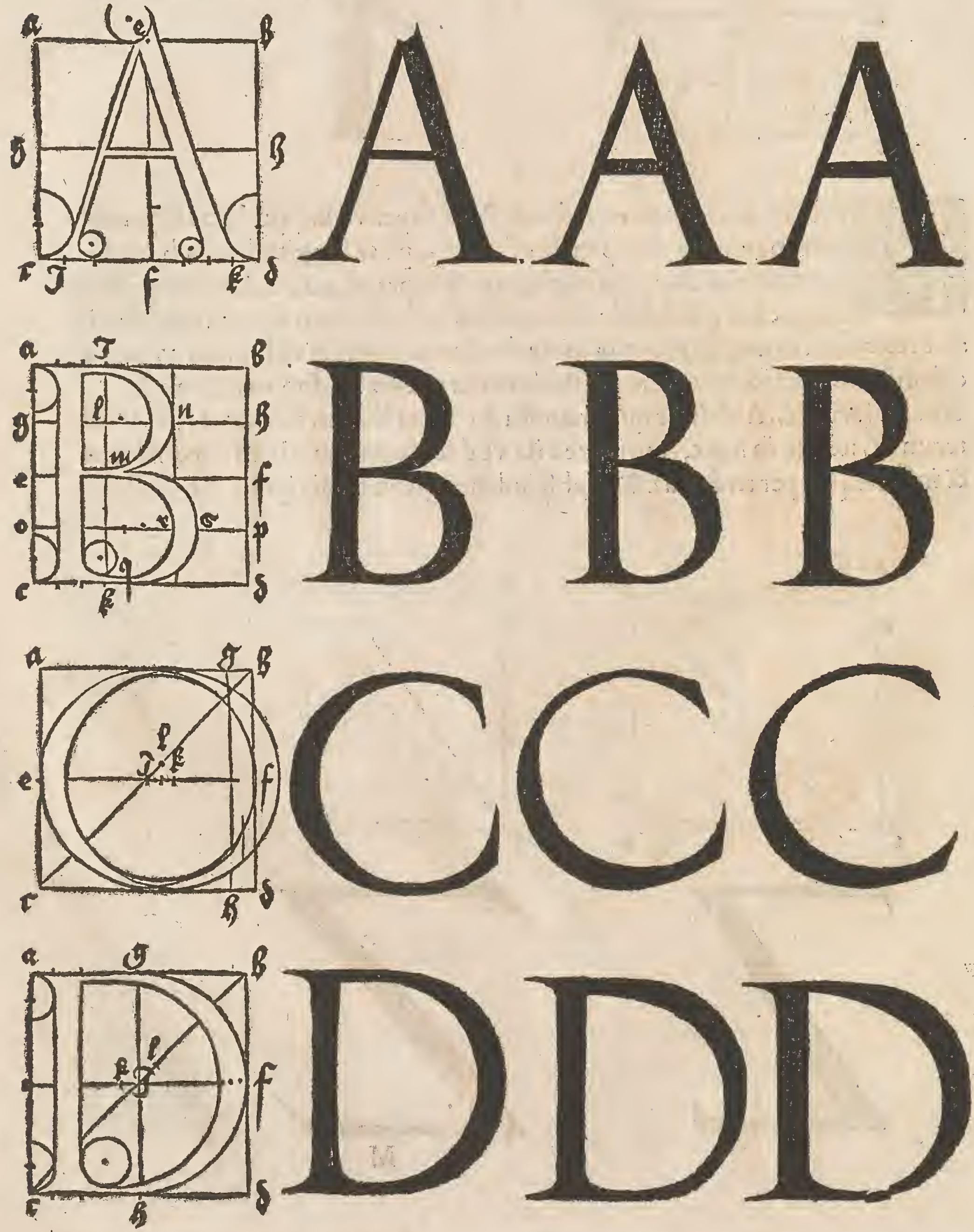
GEOMETRIAE. LIB. III. 335
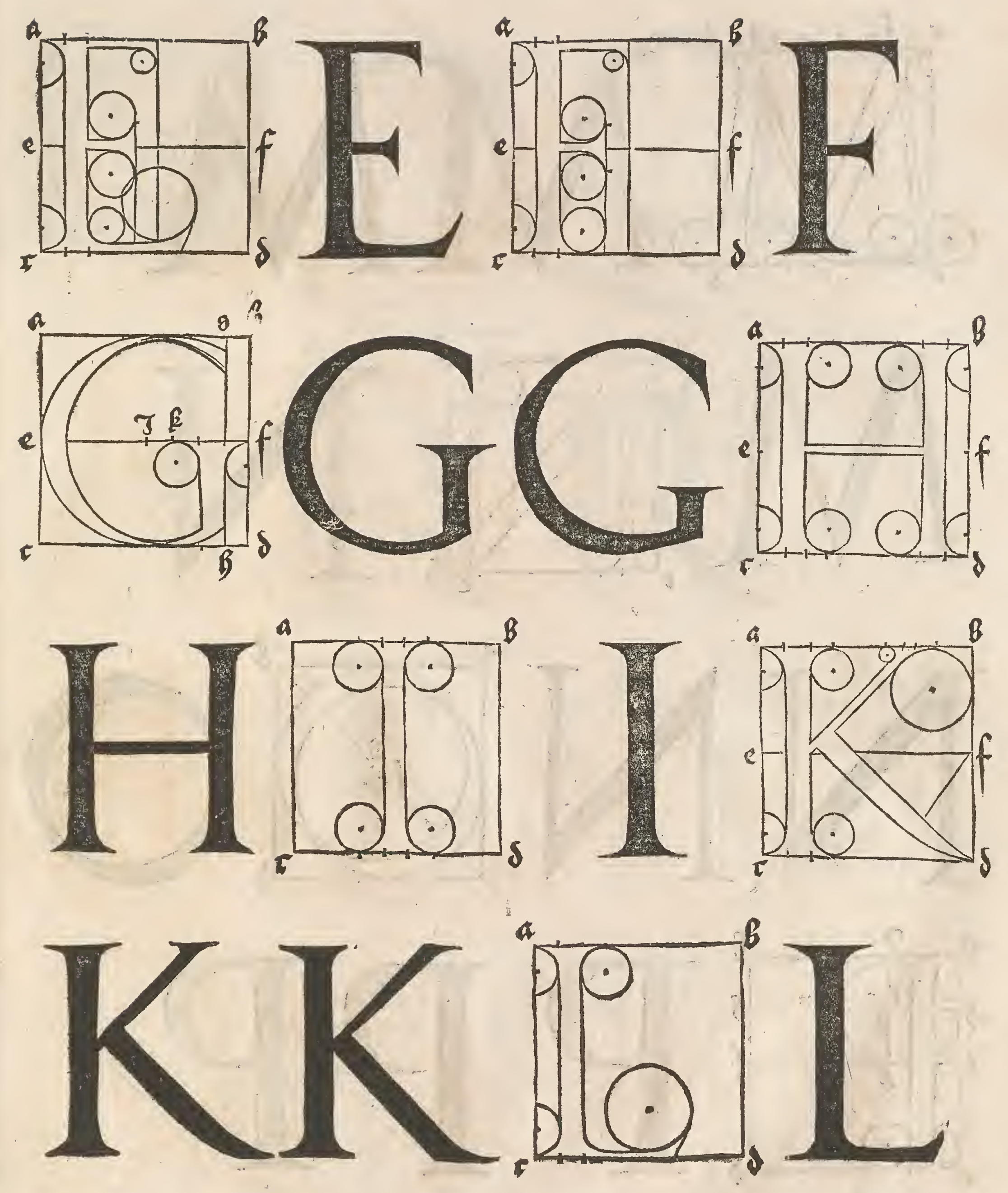

$M$ ii 

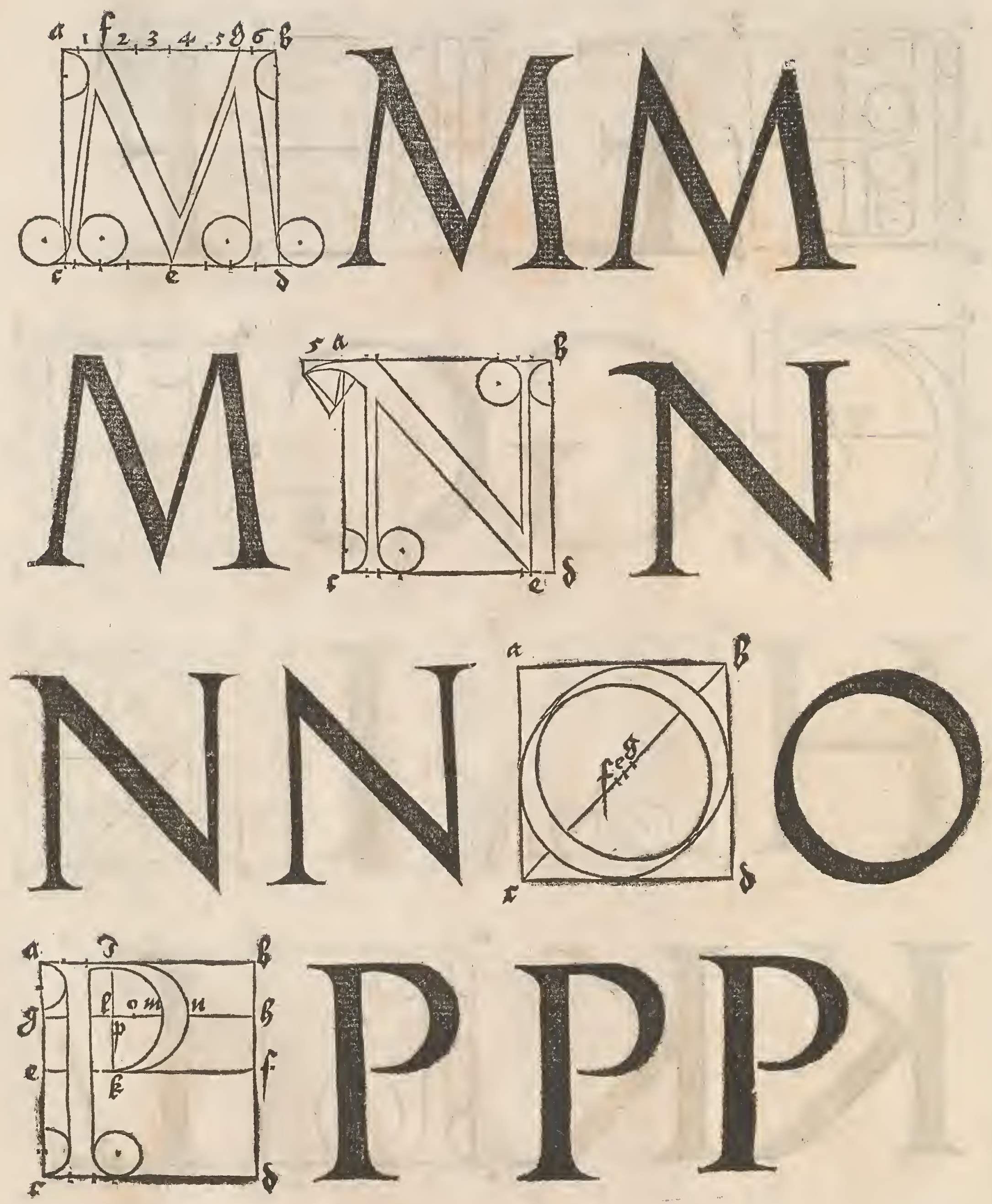
GEOMETRIAE IIB. III.
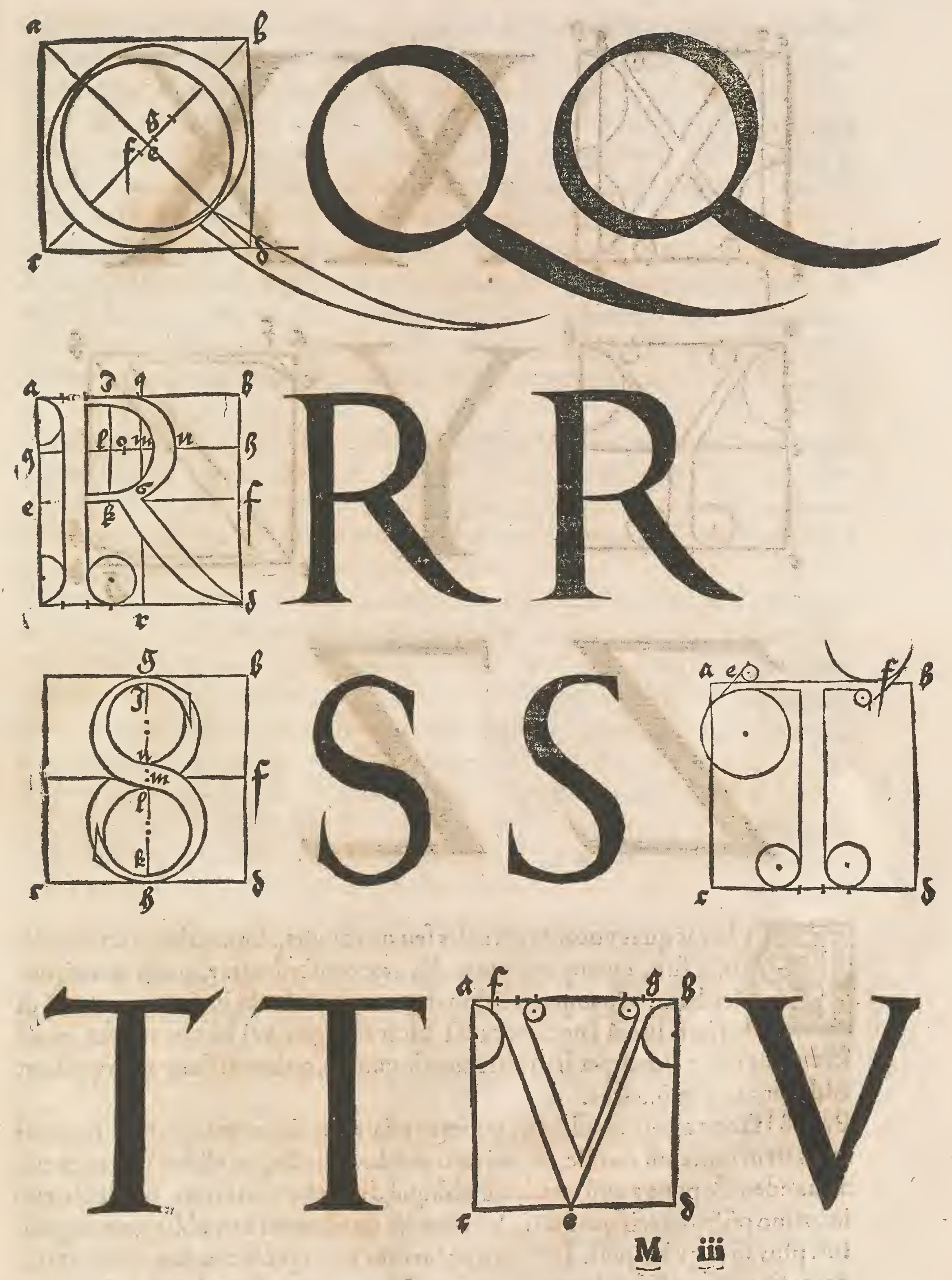

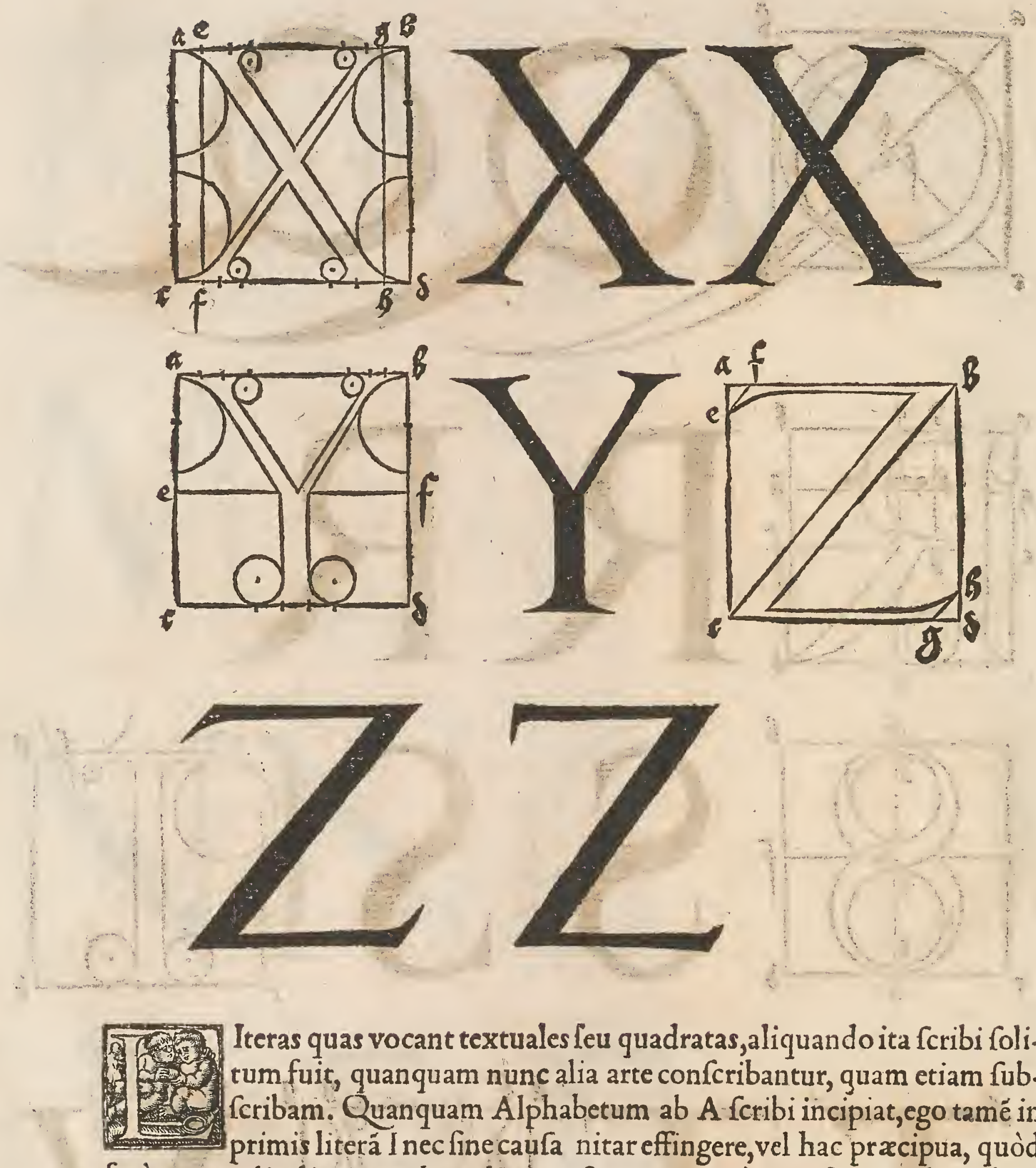

Iteras quas vocant textuales feu quadratas, aliquando ita fcribi foli. tum fuit, quanquam nunc alia arte confcribantur, quam etiam fubfcribam. Quanquam Alphabetum ab A fcribi incipiat,ego tame in primis literá I nec finecaufa nitar effingere, vel hac præcipua, quòd ferè omnes alia litera per hanc literam finguntur, quàmuis femper aliquid aut addatur, aut diminuatur.

Primó l fac ex xquis quadratis, quorum tria recte fuperponantur, \& fupremi latus item fupremú diuide, $\&$ imi imú per duo puncta, quodlibet in partes tres xquas:deinde pone aquú quadratú obliquè, diametro eius erecta, \& angulo eius in primo púctolateris quadrati. Sic excedet quadratum hoc obliquum angulis fuis plus ante, quàm pòft. Deinde duc furfum vtrinque fecundum quadrata fuperpofita rectas vf $q$ ad latera obliquè politi quadrati. Deinde infernè fimiliter facies ato̧ \{upernè, nifi quòd angulü obliqui quadrati ponis ad fecundum pü- 
Etum, aut polterius inferioris lateris quadrati, \& demitte lineas vtrin que ad qua dratum tranfpofitum, ita perfectum erit 1 , fupra quod defcribe exili calamo minutulam ac dimidiatam lunulam.

Item $\mathrm{N}$ facies ex duobus ductibus ipfius I, ita ve eorum fummi atque imi angu li fe inuicem contingant. Sicfpacium inter duos ductus erit anguftius, quàm $\mathrm{la}_{2}$ titudo vnius ductus: fed non amplius facies lunulas fupra literas; $8 \%$ breues literasomnes fac eiufdem lougitudinis per totum Alphabetum.

Item $M$ fac ex tribus ductibus, ficut $\mathrm{N}$ ex duobusfecifti.

R fac vt I, folummodo fupernè pone quadratú xquum à dextris, \& obliquè, vt angulofuo angulum tangat. Retiam hoc modo facies:pedem relinque in fernè vt prius, fed fupernèfuperpone duo quadrata tranfpolita, qux angulis fuis, fefe tangant in medio erecti ductus, $\&$ duc vtrum $q$ latus plenè furfum $v f_{\beta}$ ad quadracum.

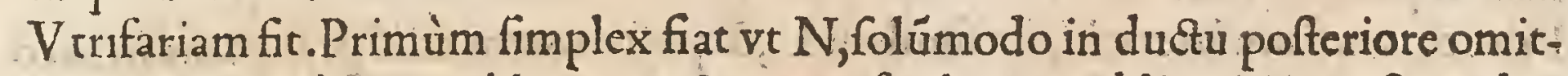
te fupernè quadratum obliquum, \& pro eo fac lineam obliquä,ita vt fiant duo angulisn hoc duetu, quorum pofterior fit in ea altitudine qua eft angulus fupre mus quadrati obliqui in tractu priore, fed anterior angulus fit in eadé altitudine cum angulo fibi proximo in priore tractu.

$V$ fecundum, quo vimur in initio dictionum . facita, Ductum priorem facies $\mathrm{vt} \mathrm{I}$, fol ummodo infernè quadratum obliquium trude paulo plus ad dextram, ita vt angulus anterior non exced at latus erecti tractus, fed fit in linea eadé defcendète. Deinde pone fecundum ductum polt hunc, $\&$ infernè eum abfcinde per lineam obliquam qua ducatur ab ino angulo ad dextram $v f_{q}$ ad altitudinem mediam im quadrati ex tribus fuperpolitis.

Deinde $f a c$ duplex, ficut V fimplex, modo praponas ei ductum liter $I$. $B$ fac vt fecundum $\&$ fimplex $V$. Sed in priore ductu omitte fupremum quadratum obliquum, $\&$ fuperpone ad huc alia tria quadrata tribis inferioribus, fed feptimum per diametrum antè prafcinde.

Item quando inuertitur hoc $B$,vt fummum eius fiat imum; tunc erit $Q$ $X$ facies ex I. A ppende à dextris fuperne aquum obliquium quadratum, vt in priore $R, \&$ infernè ducito acutam caudam in finiftram ab obliquo quadrato; \& in medio duc tractum tranfuerfum per erectum, ita vt is antè $\&$ pòft per diame. trum abfcindatur:angulus anterior atque inferior terminetur tantum ante erectum, quanta fuerit femidiameter ante abfcindens, fupernè tamen tractum erectum tangat diameter illa, fed à dextris egrediatur vfo ad eum locum qui eft fubtus angulum obliqui quadrati fupernè quidem, hinc amputetur per lineam obliquam anteriori obliqux parallelam.

C ficfacies ex I. Remoue fupremum obliquum quadratum, \& erigátur linea la terales vique ad iuftam literx altitudinem, \& prafcinde angulum anteriorem per diametrum, deinde duc tractum latum tranfuerfum fupernè tantum poft erectum, quanta fuerit erecti craffitudo, $\&$ abfcinde hunc per diametrum, ita ve inferior proiectura fit dimidia ad fuperiorem.

Ductú erectú ad E facies, vt in C, fed defuper defcendat in dextră tractus latus 
ex anteriore diametro ad angulos rectos vnius quadrati \& eius tertiă partế lógi tudinis, ducaturó linea parua $\&$ obliqua ab angulo inferiore ad ductú erectum. $T$ fiet frmiliter ac $\mathrm{C}$, nifi quòd huic in diametro fupernè aliquid adiicitur , per quod acquirit in fummo aciem, at q̧ fimilé antè ad finiftram ad latú ductü, ficut in fummo:ideóg elegantius eft $T$ fupernè, $q \mathrm{C}$, nég ita incuruum videtur.

I fac infernè vt I, fed quadrata fex fuperponătur: $\&$ feptimum antè prǽ́cinde per diametrum, fic remanebit literæ acies fupernè ad dex tram.

Literam $S$ fac ficut $\mathrm{L}$, nifi quòd fupernè ad dexteram ducendus eft tractus latus in lóngitudinem diametri, quem poft abfcindes per lineă parallelă anteriori.

F facies vt $S$, tantummodo adde ei ductum tranfuerfum in altitudine breuiorú literarum,duplo longiorem. q latiorem, ita vt acies antè' $Q$ infrà excedat tantú quanta fuerit eiufdem media latitudo, ve duæ abfciffiones obliqux fibi inuicem fiant xquidiftantes.

Priorem tractum liter $\mathrm{H}$, fac fimilem $\mathrm{L}, \&$ polteriorem adiüge fupernè in loco fuo parem ipfi I.Sed infernê proobliquo quadrato perfice ereétum quadratum quartum, \& quintum imun abfcinde pòt per diametrum.

K priorem tractum fac vt $L$,ad dextram appende quadratum obliquum ad tràn ctum, $\& \mathrm{ab}$ angulo infimo procedat linea obliqua vfque ad tractum illum ereCtum, à qua deinceps obliquè educatur tractus latus: $\&$ hunc infernè amputabis per diametrum, ita vt infernè fpacium inter duo acumina non amplius fit quadrati vnius diametro.

$D$ in medietate fua inferiore fac $v t B$, fed anterior tractus fupernè afcendat fur fum ad literarum altitudinem, \& angulum anteriorem per diametrú refeca, deinde fuperpone adhuc dimidium quadrati fuper alia tria quadrata polterioris tractus, $\&$ iterum fac hic eodem modo, ficut infernè, \& tractus hic fractus incúbat fuper angulum tractus anterioris, at $\phi$ hunc excedat vfo ad finem erecti anterioris ductus:ita paulo minus q̄ tria quadrata couiúcta habebit, nam iuxta anteriorem tractum erectum, fractus ille ad angulos rectos eft abfcindendus.

$O$ infernè vt $D$, fimiliter hoc fupernéfac vt idem infernè, ac fi inuerfum fit . Anteriorem tractum $P$ fac fimilem $L$ inuerfo, fed pofteriorem fimilem erecto $I$, infernê tamen non addes obliquum quadratum, fed amputa tractun per diametrum, $\&$ duc inferne tractú tranfuerfú latú, qui item per diametrum abfin datur, vt inferne acies excedat in finiftram, quanta fuerit media tractus latitudo. Item $A$ in medietate inferiore fac fimile $N$, fed anterioris tractus erecti in medio quadrato per diametrü angulum prioré abfcinde, polterioris verò fine tria quadratà confiftere fuperpofita, $\&$ inclina fuperiore parté quadrati magisfiniftrorfum, ita vt fi adhuc iungatur illi dimidium quadrati, tunc altitudıné literæ con tingat:\& quadratum oblique pręcinde, vt tamen inferior acies latiùs excedat quàm fuperior. Inde circumfcribe circulum in finiftră atọ deorfum, ita vt cius quantitas diftantiam anterioris tractus contingat.

$Z$ fit trifariam, Primò pone quadratum obliquú quod altitudinem literæ con tingat:deinde adde alterum fimile ad dextram iunctis lateribus, ex quibus fiet quadrangulum defuper in dextram dependés:deinde quadratum obliquum po 
ne rectè fubtus quadratum fupremum, tantum ab inferiore diftans, quanta fue. rit diameter eius:ab hoc duclineam obliquam ab angulo fcilicet huius ad angulum alterius, aut fac tractum rotundum ad quadratum vfque inferius. Sed à iam dicto quadrato infimo deorfum atq dextrorfum educito rotunda proieEturam per diuer fos circulos, quæ infernể longitudiné literæ contingat, \& aciê, acuta ac tenuis finiftrorfum vergat:aut hanceffice per tres lineas obliquas vnă fupra alteram $8 x$ duc diametrum per eas qua in dextram declinet.

Alterú $Z$ fac ita, ve tria obliqua quadrata fibi inuicé fuperponantur, \& imum quadratum habeat proicturam rotundam ficut prius.

Priorem tractum $G$, fac infernè limilế $I, \&$ fubiunge infernè alterum quadratús obliquum iunctis angulis. Supernê verò huius tractus polteriorem aciem exalta furfum vfque ad altitudinen litere, ex hac acie duc diametrum deorfum ad finiftram, víque ad priorem angulum primi quadrati erecti ex fuper fe pofitis tri.bus. Deinde, duc pofteriorem ductum erectum totum tanta longitudinis quan tafuerit prior ductus, \& duc infernè diametrum ab angulo imi quadrati obliqui vfque ad finem polterioris tractus anguli, $\&$ demitte interius latus in aciem deorfum vfque ad diametrum:ad hanc quoque per vnam Iineam iunge quadra tum imum $\&$ anterius, deinde duc tractum fupernè tranfuerfum, latúmque $a b$ prioris tractus erecti dorfo per pofteriorem erectum \& vltra hunc tantum, quata eft eiuidem latitudo, quem tandem ab cindes pòft per obliquam lineam parallelam priori.

Y fac vt N, folummodo omittatur infernè pofterius quadratum obliquum, pro quo fiat quadratum erectu $m$ fub aliis tribus fuperpolitis: $\$$ quintum quadratú diffeca per diametrum, vt acies antè frat,à qua diameter egredia tur in lơngitudinem vnius lateris quadrati.

$S$ rotundum fiue breue ita facito. In medioaltitudinis litera iuxta fe ponátur iunctis angulis duo quadrata obliqua, à priorè quadrato erige ductum erectum 8 latum vfọa ad altitudinēlitera, ita â polteriore demitte deorfum, pariter at tọ I fupernè atque infernè formatum eft:hos ambos ductus abfinde fupernè $\mathcal{Z}$ in fernè per diametros; ita vt ambę acies relinquantur verfus medium. Deinde du cantur duo tractus lati à fuperiore quidem acie dextrorfum, atque deorfum, inmiliter $a b$ inferiore acie finiftrorfum, atgue furfum:in latitudinem ductus fur: fum $\&$ deorfum, fed non latius vtrinqge educantur, quàm quantum lati ductus fe inuicem dift ant:deinde duc diametrum defuper à dextris in finiftram, \& per eam a breindes ambos tractus obliquos. Ad eandem etiam adducitolatera quadratorum in medio politorú. Talia ego in albis per lineas, $\&$ in nigro, ordine recto fubfcripfr. Hęc vt prędictú eft an tiqua literarú forma eft. At hoc têpore elegantior fit textura, \& obliquú quadratü ponitur in medio loco lateris quadratì erecti, ita vt linę literarú non tantú curuétur: \& fiút quidá duçus adiuncétiatğ diffiff: fuperponútur etiam quadrata tria $\&$ femis, fiunt $\oint^{\prime}$ Spacia inter duos du ceus tắta quantafuerit ipfius ductus latitudo. Tales etiä literasfubfcripfi, vnà củ literis maiufculis, quas v ocăt verfales, $q$ ab initio verfus poni cófueuerint: qux eriá tertia parte altiores fieri debết reliqgs in fcriptura breuioribus literis. 


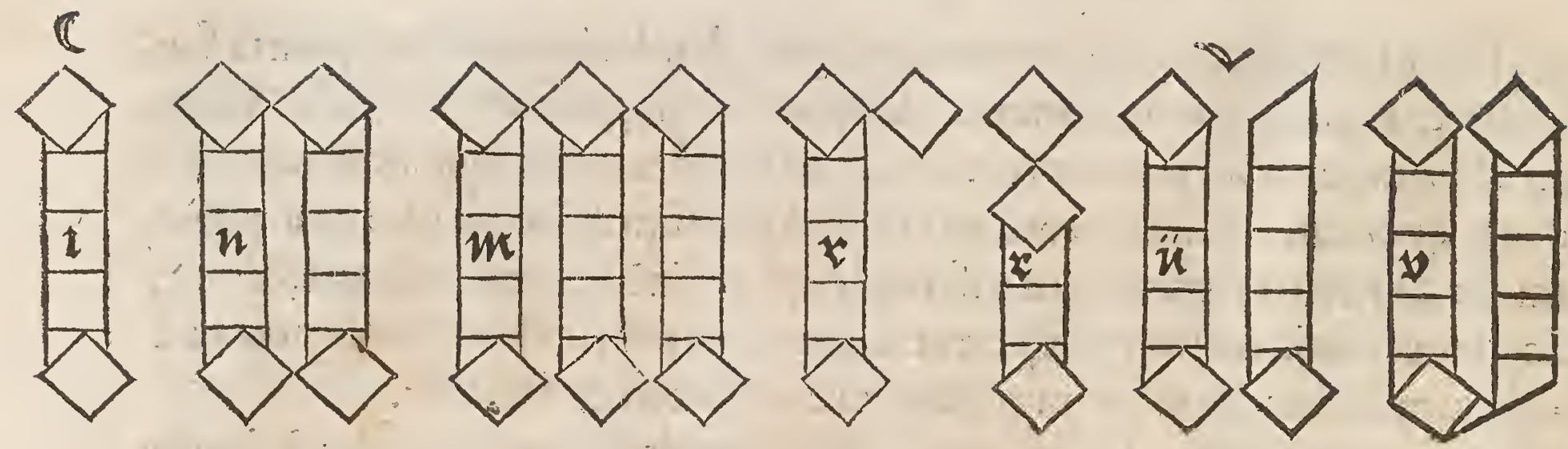

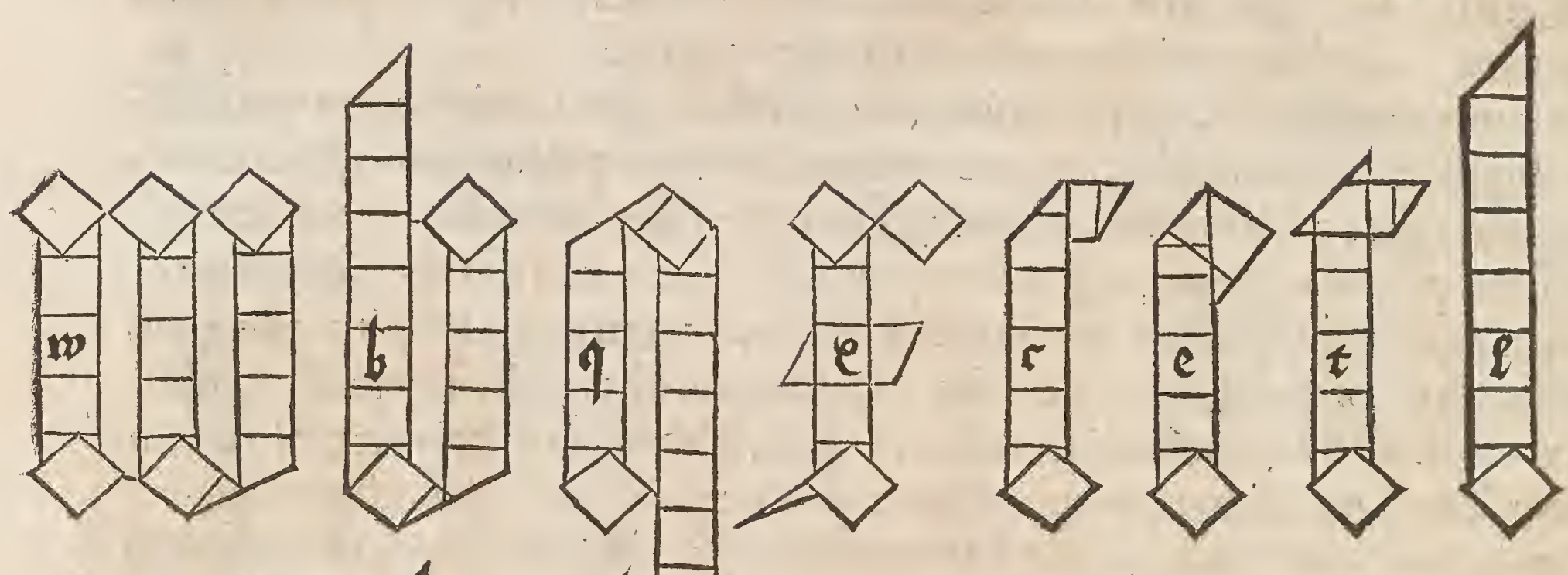
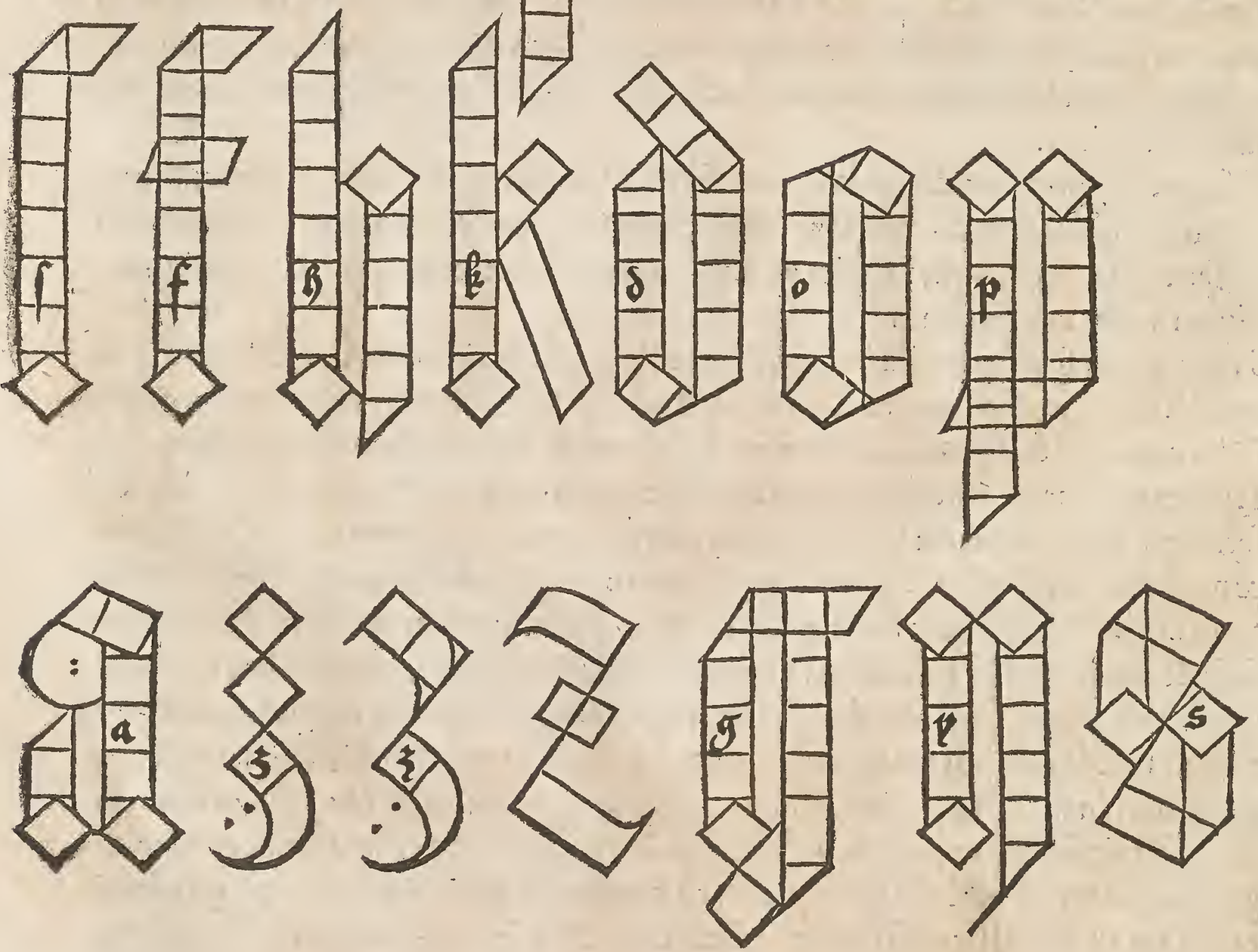

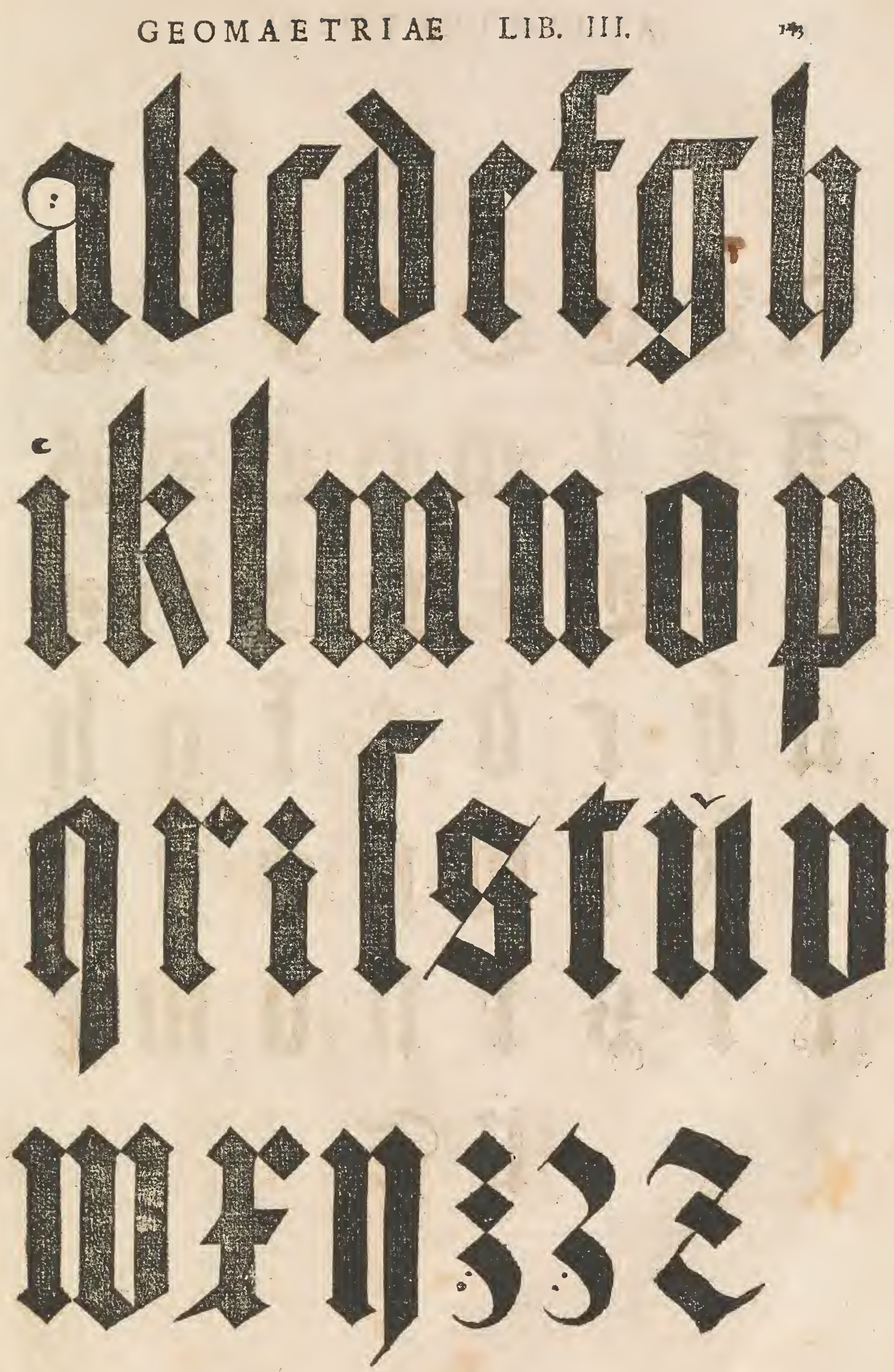


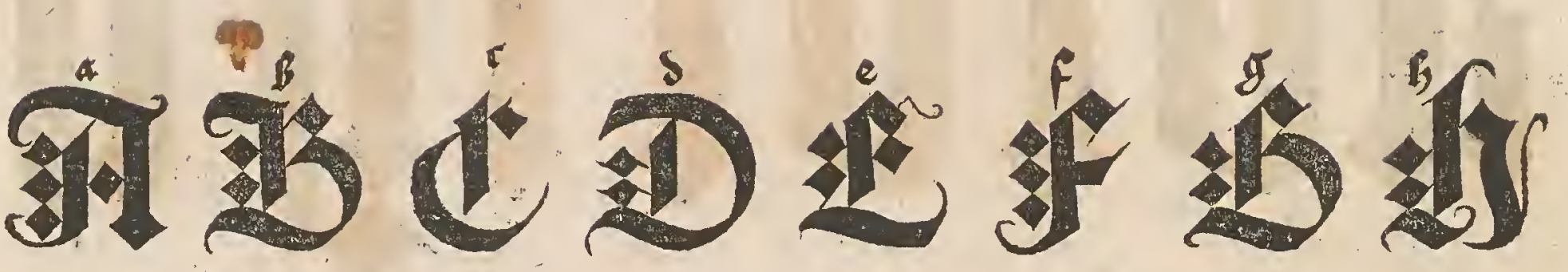

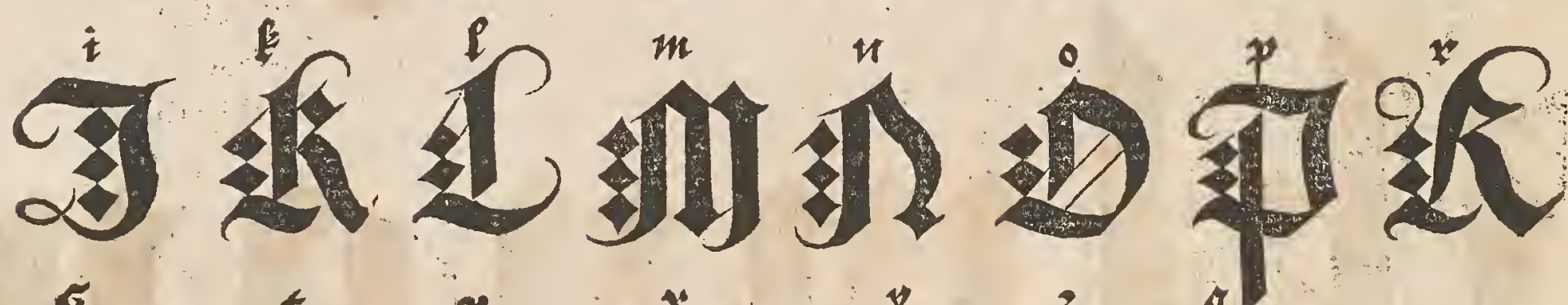
5 1)

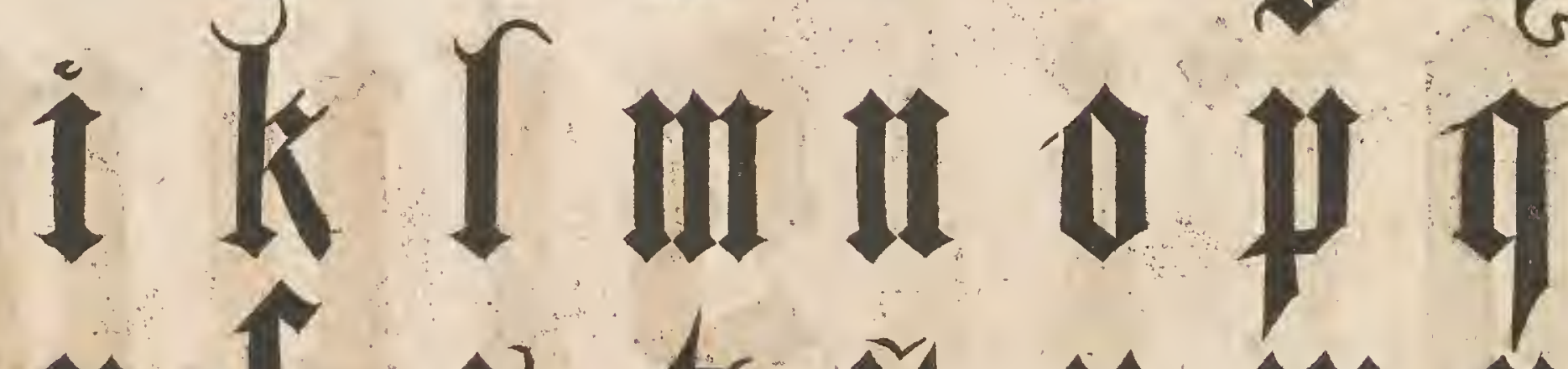
II 11 


\section{A LBERTI DVRERI}

\section{PICTORIS EXCELLEN TISSIMI}

Inftitutionum Geometricarum

Liber Quartus.

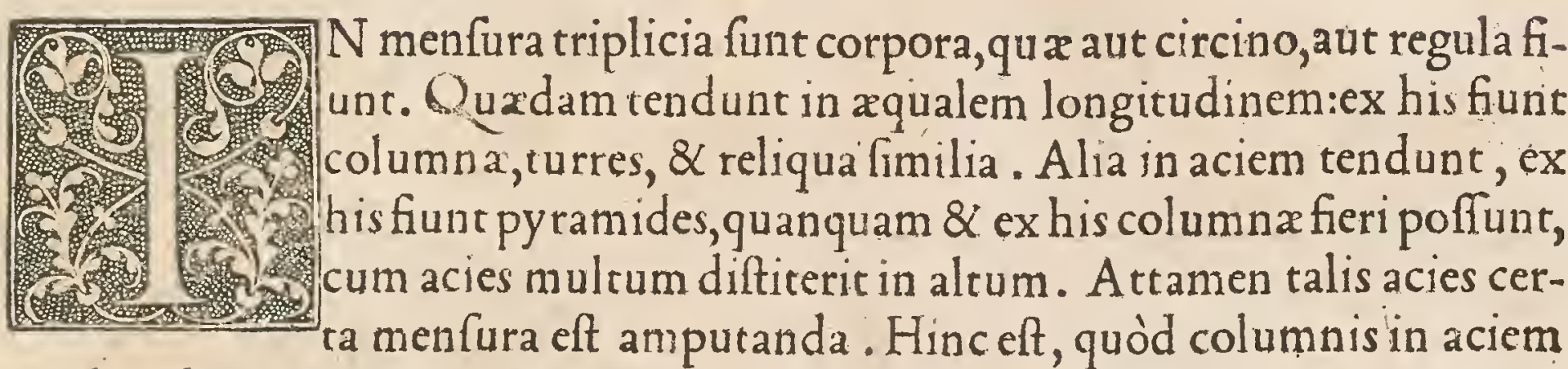
tendentibus non altius onus imponitur ferendum, quàm acies earum trianguI contingit. Tertio funt corpora, qua vndiquaq $x$ qua funt, fuperficiebus, angu lis, \& lateribus, qux Euclides vocat regularia corpora, quorum quin $q_{3}$ defcribit,
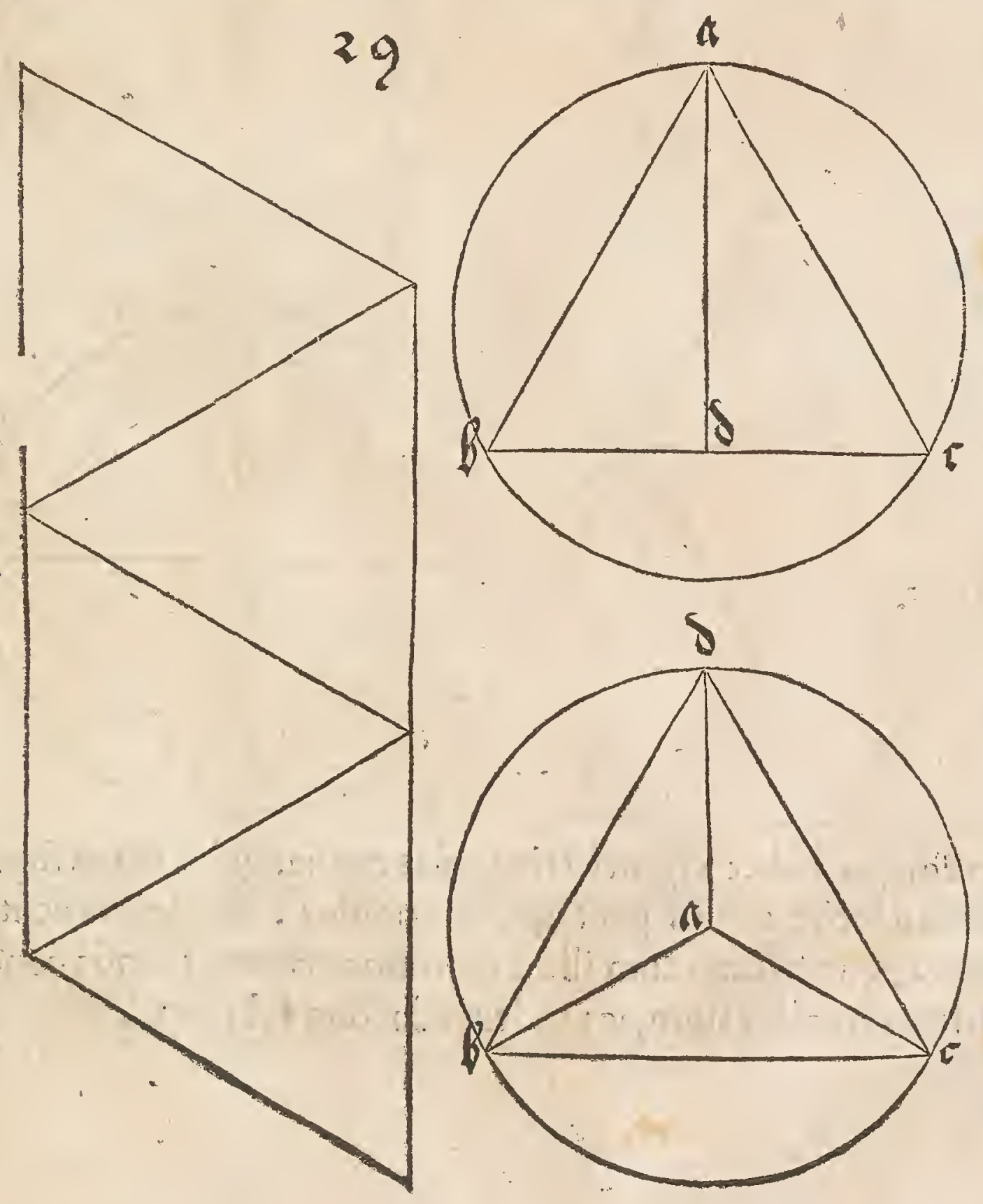

quon plura effe poffint:que in ca uo globo contéra vndio tangant. Hac quia ad plu rima cótingunt, hic fublignabo. Inprimis eft corpus triangulare, cótinet hoctrian gulares fupficies quatuor equas equiangulasós, habet quoq quatuor xquos; trigonos'́; angulos, 8 fex equa latera acuta, quemadmo dum hoc patefa. ctum in folo poli tum: deindéque iterum eductum. cubleripfi. 
Fin. Ecundum corpus eft fimile cufpidi feu puncto adamantis, equiangu= lum , fex xquorum quadrangulorum angulorum, $\&$ duodecin acucorum laterum, quemadmodum $\mathcal{Q}$ illud omnino patefactum, deinde claufum, ad folum depofitum, \& deinde eductum, reuolutum, ad alte rum latus collocatum fubfcripfi.

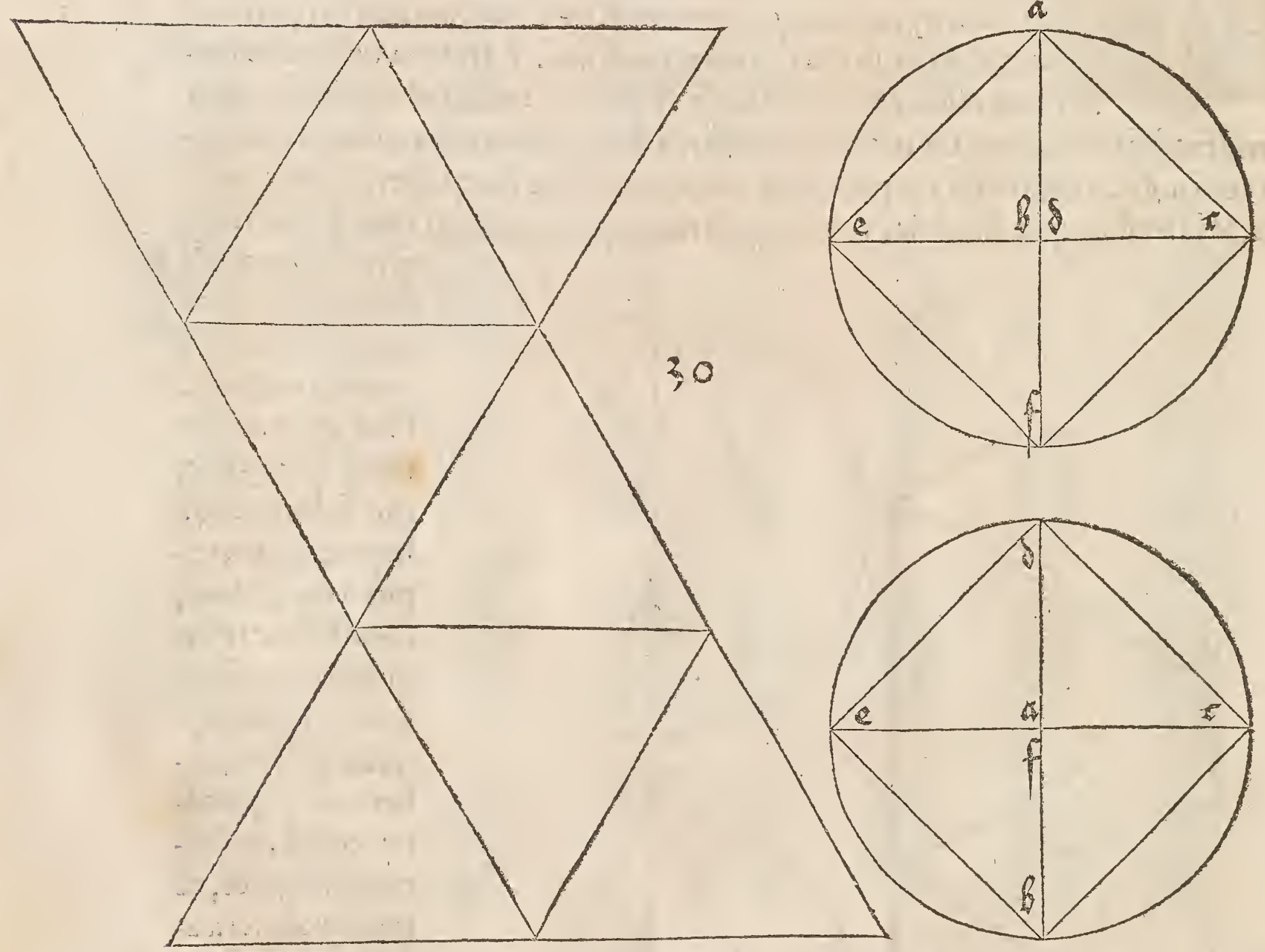

Ertium corpus habet viginti triangulas æquiangulas equas fuperfi: cies, \& duodecim $x$ quos pentagonos angulos, \& triginta acuta \&
xquilatera, quemadmodum illud omnino apertum, deinde conclu: fuim, in terram collocatum, ac tandem eductum ́ubfcripfi. 

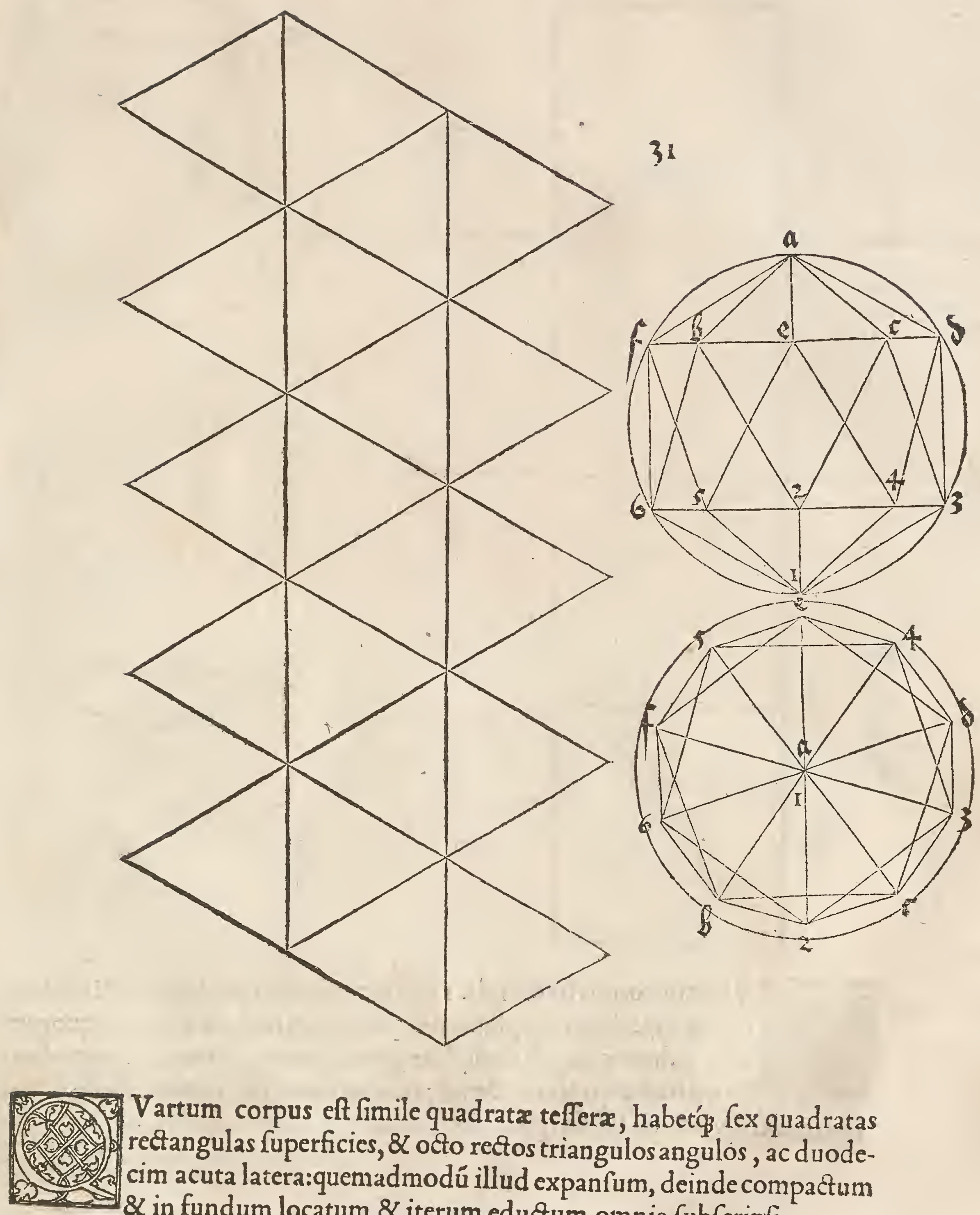
cim acuta latera:quemadmodú illud expanfum, deinde compactum $\&$ in fundum locatum, $\&$ iterum eductum, omnia fubfripi. 


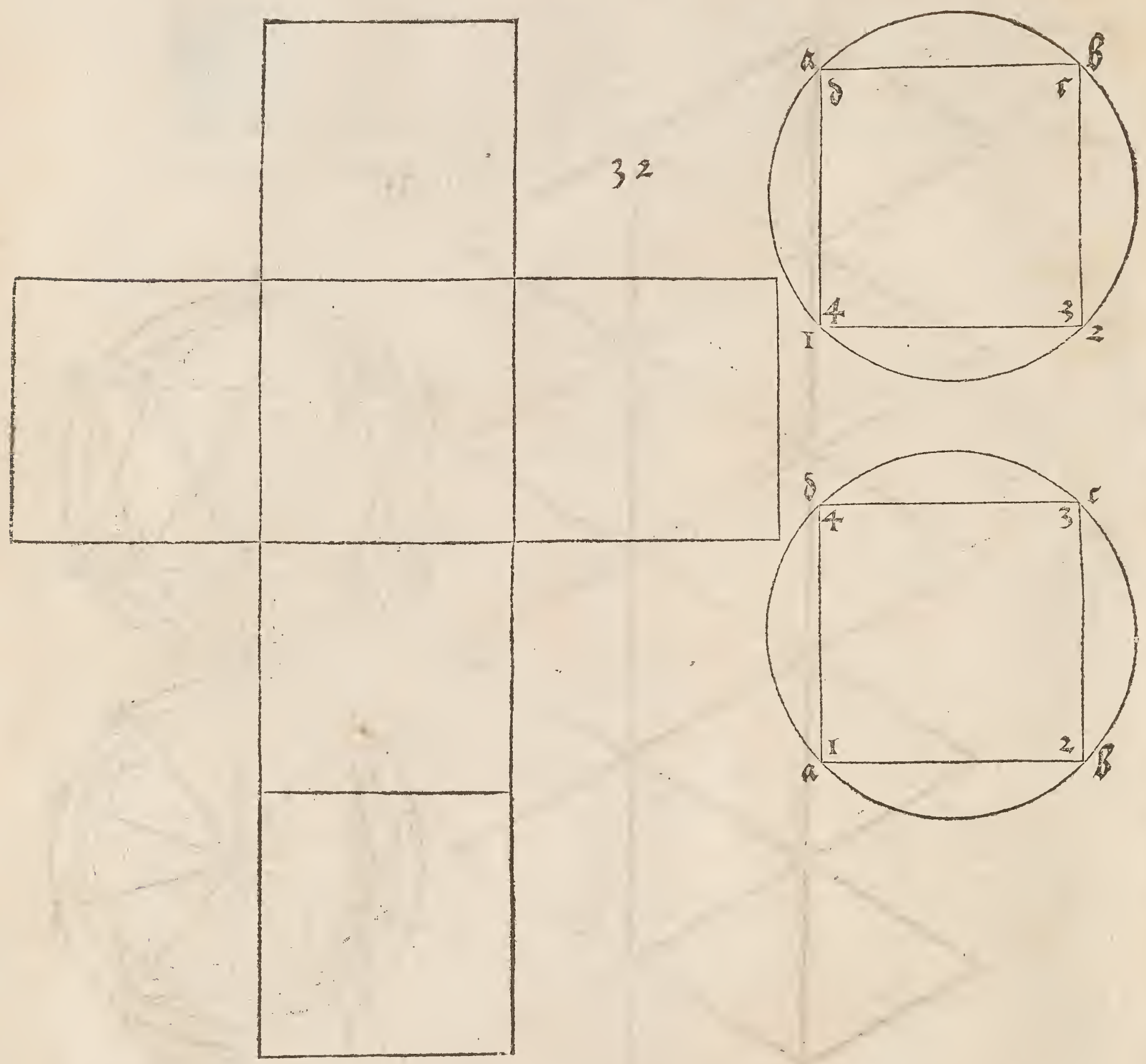

Vintum corpus fit omnibus fuis fuperficiebus pentagonis, \& eft duodecim fuperficierum planarum, pentagonarum, \& viginti æquorum triangulorum angulorum, \& triginta acutorum laterum, quemad mo dum illud expantum, deinde compactum, in bafim locatum, \& iterum eductum,omnia fubfcripfi. 
GE OMETRIAE LIB? IIII:
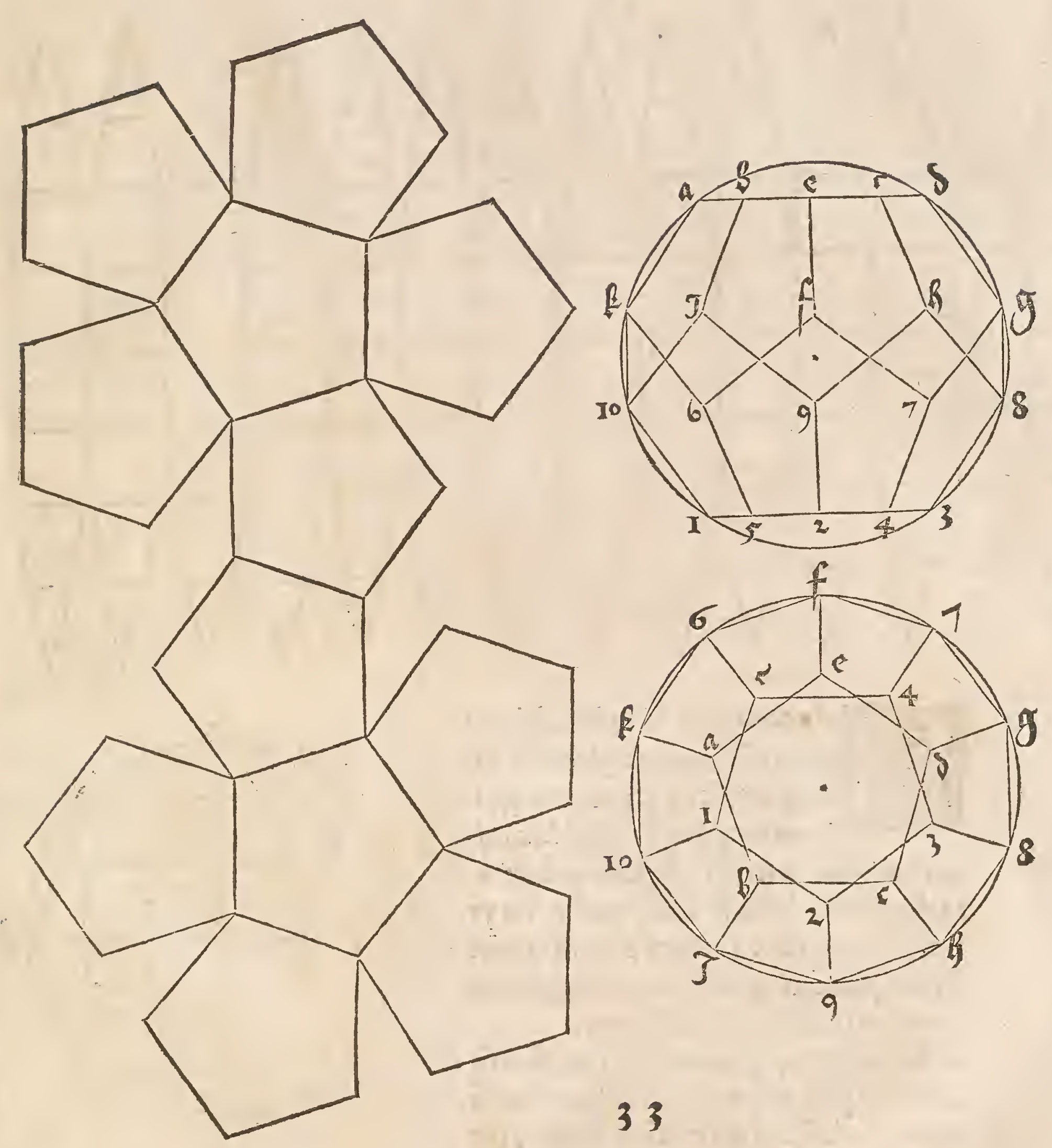

33

(9.) Phra aut globus quando per meridianas lineas diffecatur, 8 in plano collocatur,formam pectinis accigin, quemadmodum id fpharicè 8 apercùm fubicripli. 


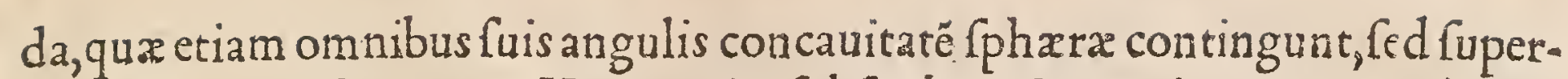
ficies eorum nó funt pares. Hæc partim fubfcribam, \& omnino aperta, quò quifque ea claudere poffit. Si quis igitur ea componere velit, is accipiat duo folı pa pyri, bitumine, aut pafta cohærentia, \& corpora illa fuper eadem ita defcribat, vt acuto cultello alterum foliorum fecundum lineas ductas fcindatur, 8 cum omnia reliqua ex refiduo papyri fuerint foluta, tunc facilè complicabirur in du ctibus \& fciffuris. Ideóg aduertendum elt ad fequentem ductionem,nam talia ad plurima conducunt.

Rimum corpus, quod non xqualibus planis fuperficiebus conftat, eft 1.21 quod continet quatuor fexangulas, \& tres triangulas fuperficies. Sed 13 C. latera acuta omnia funt aqualia inter $f e, \&$ cú hoc corpus, vt apertú 1. eft cópingitur, acq rit angulos duodecin, \& latera acuta octodecim.

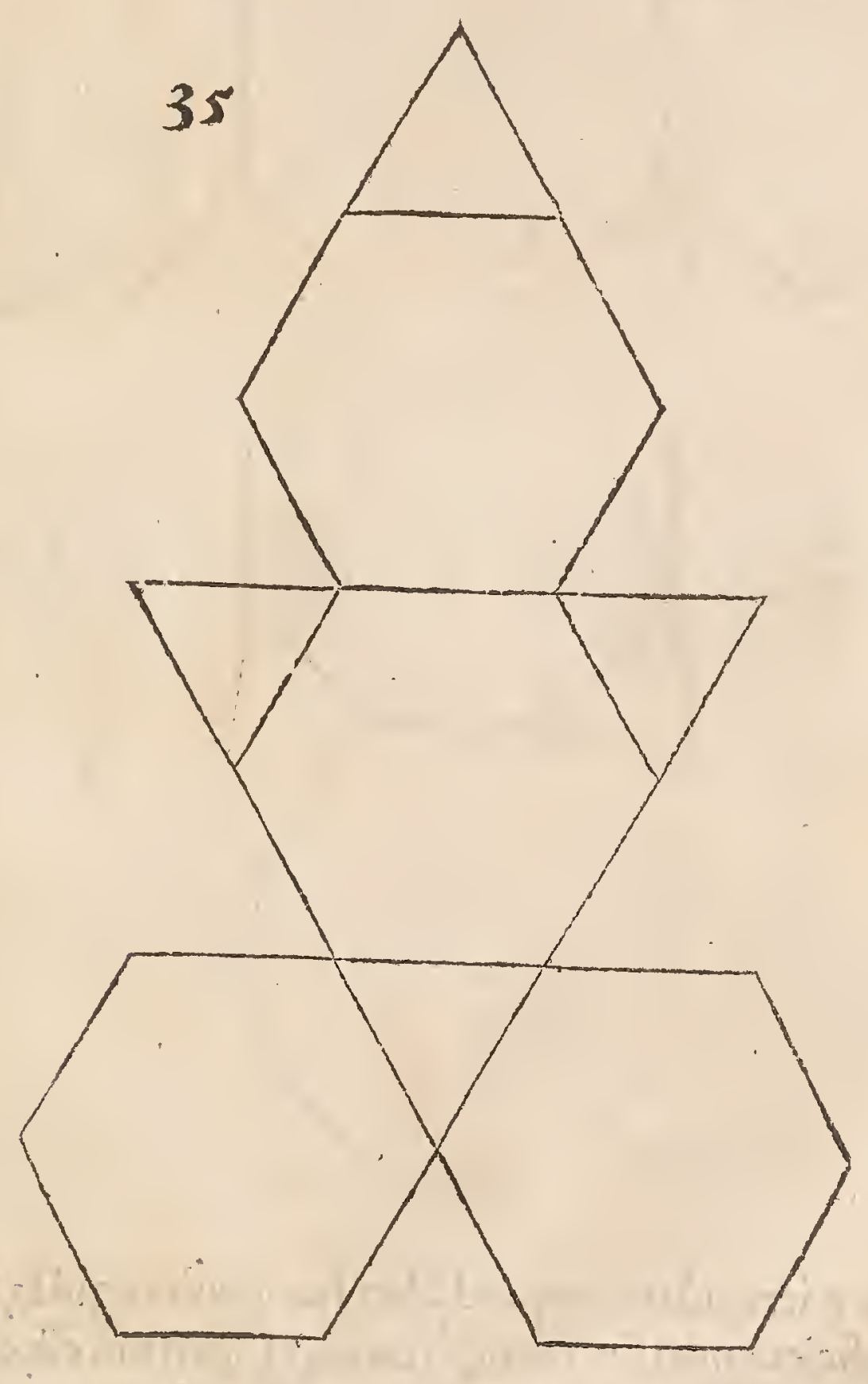

Ecundum corpus irregulare habet fex octangulas; 82 octo triangulas [upficies. Cum hoc corpus vt apertum defcriptum eft, coniungitur, habebit angulos quatuor \& viginti, \& latera acuta fex \& triginta 

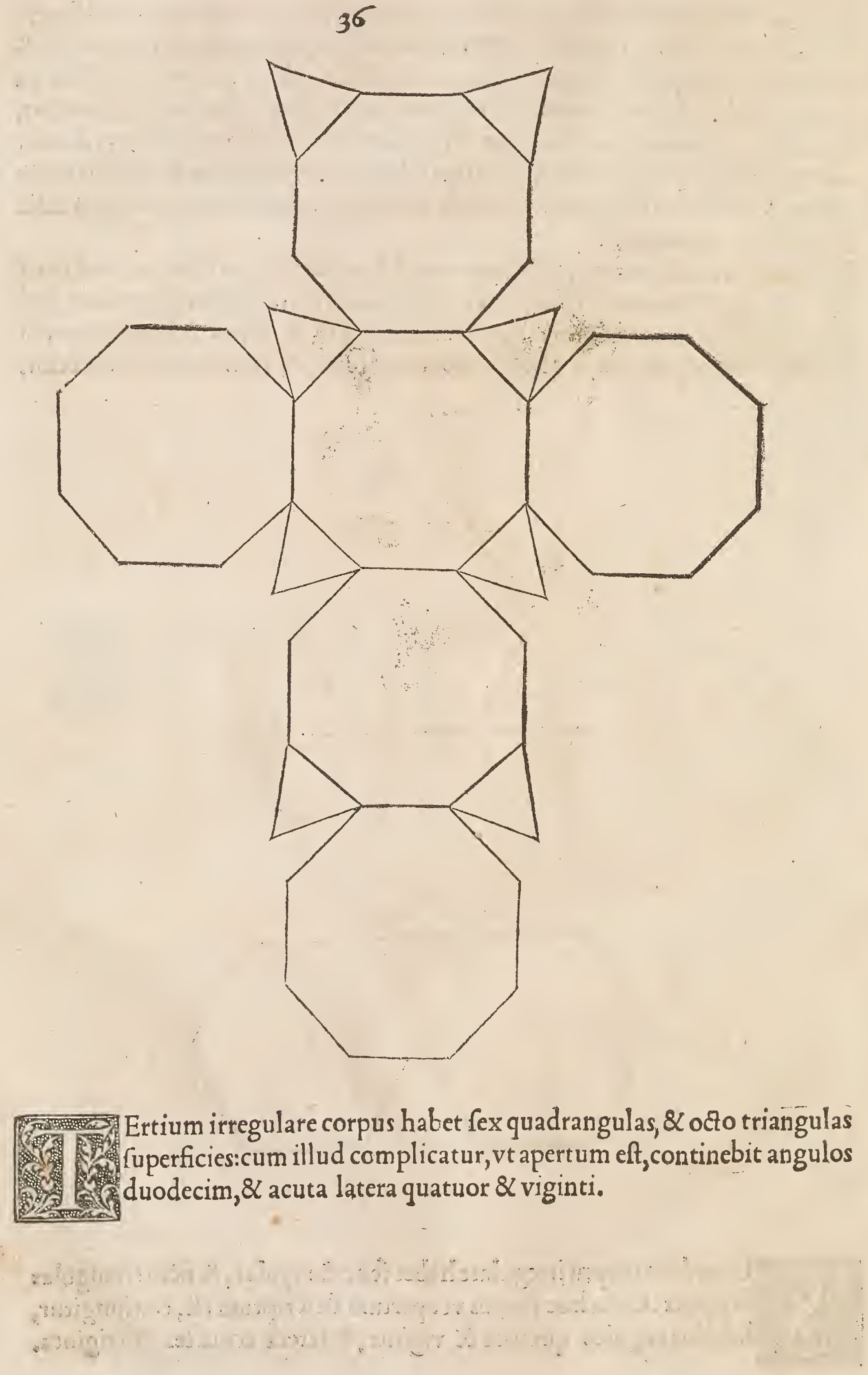


\section{GEOMETRIAE IIB: IIII :}

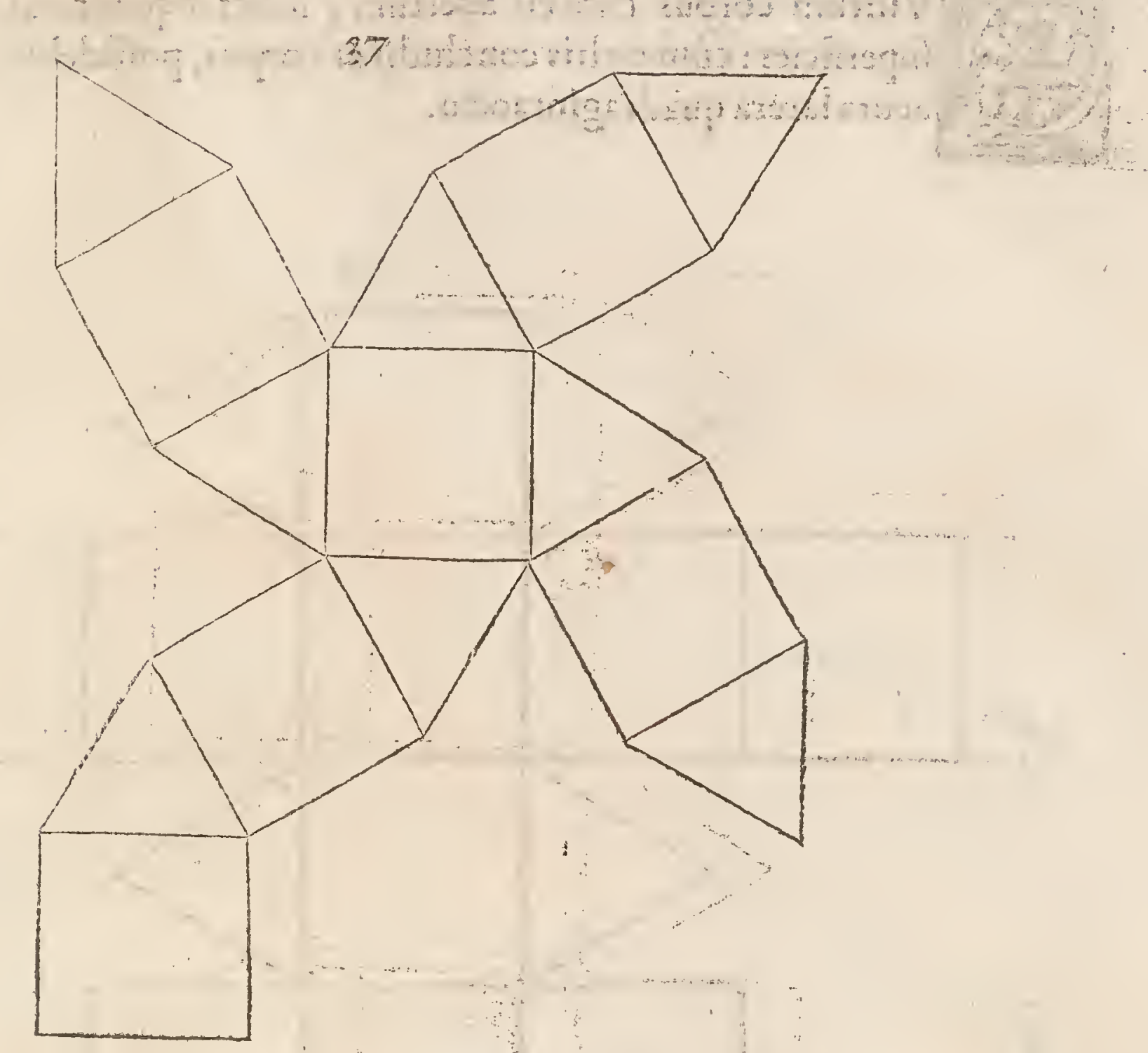

Vartú corpus apertú, habet octo fexágulas, \& quatuor quadrágulas fup ficies, củ côpingitur cóprehendit corpus hoc angulos 24; \& Iatera acuta 38

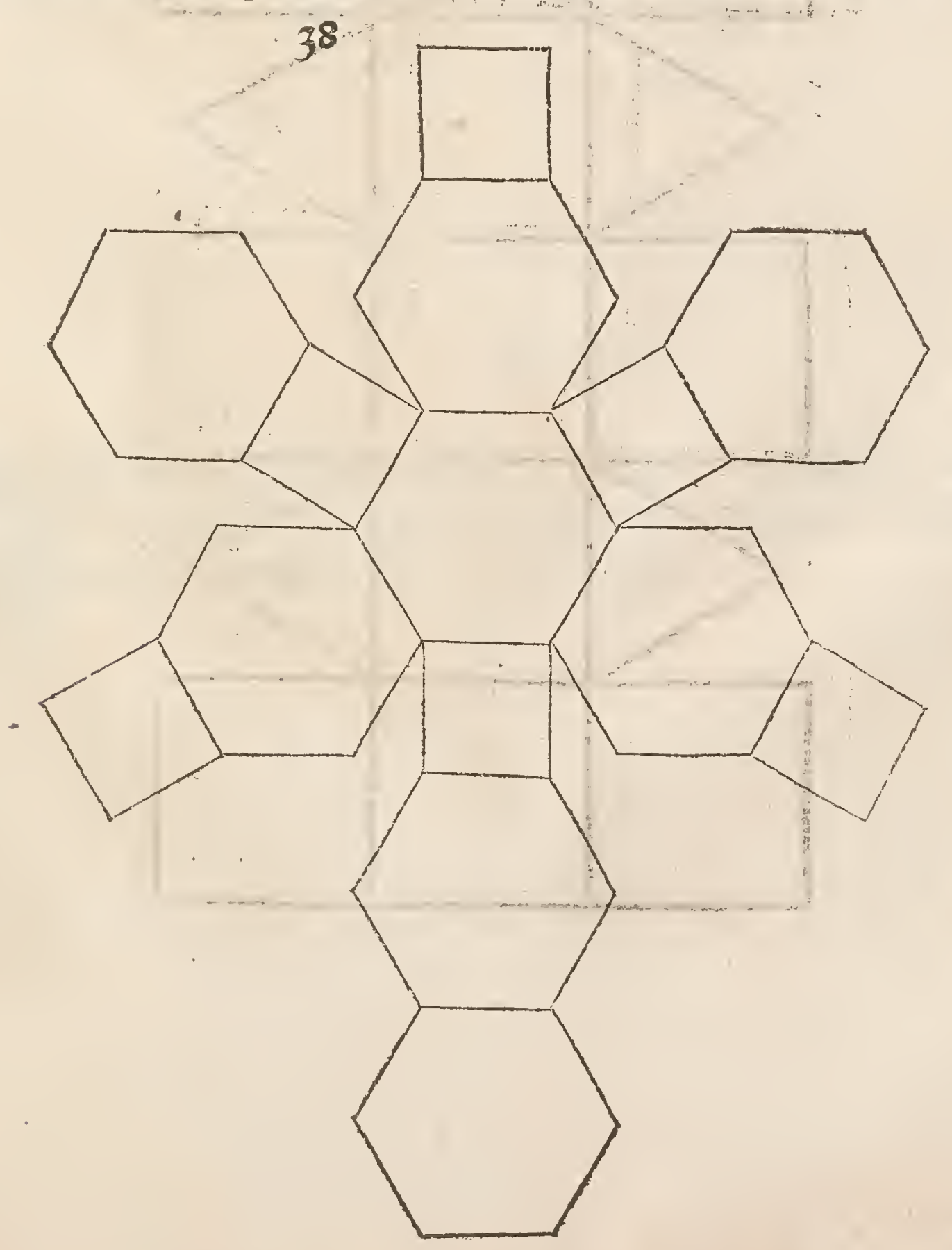



fuperficies : cùm ex his concluditur corpus, poffidebit angulos if \& acuta latera quadragintaocto.

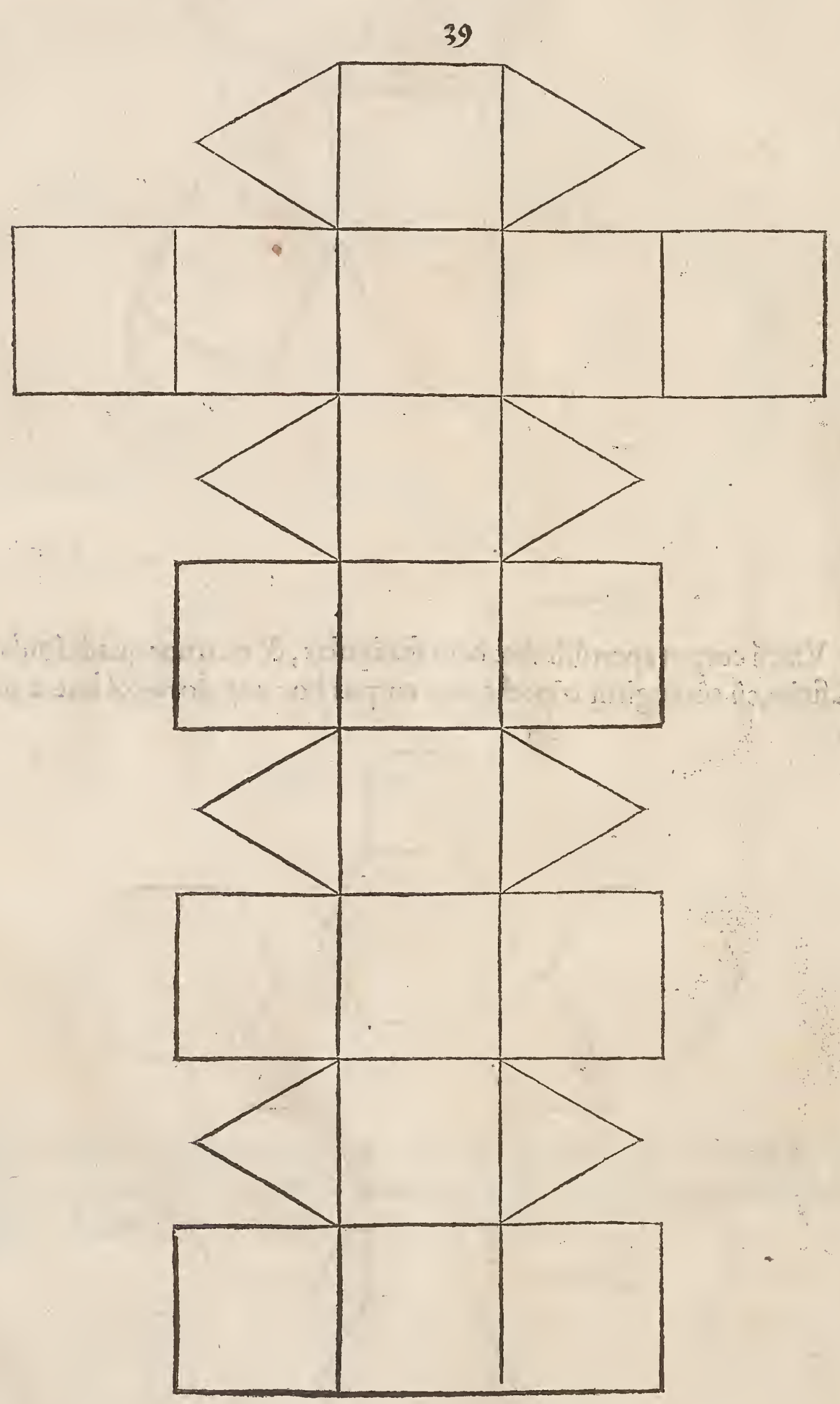




\section{GEOMETRIAE LIB. III.}

Extum corpus cùm ef apertum, habet fex quadratas \& 32 triangulas fuperficies: cum verò plicatum eft, habet 24 angulos, \&6: latera acuta.

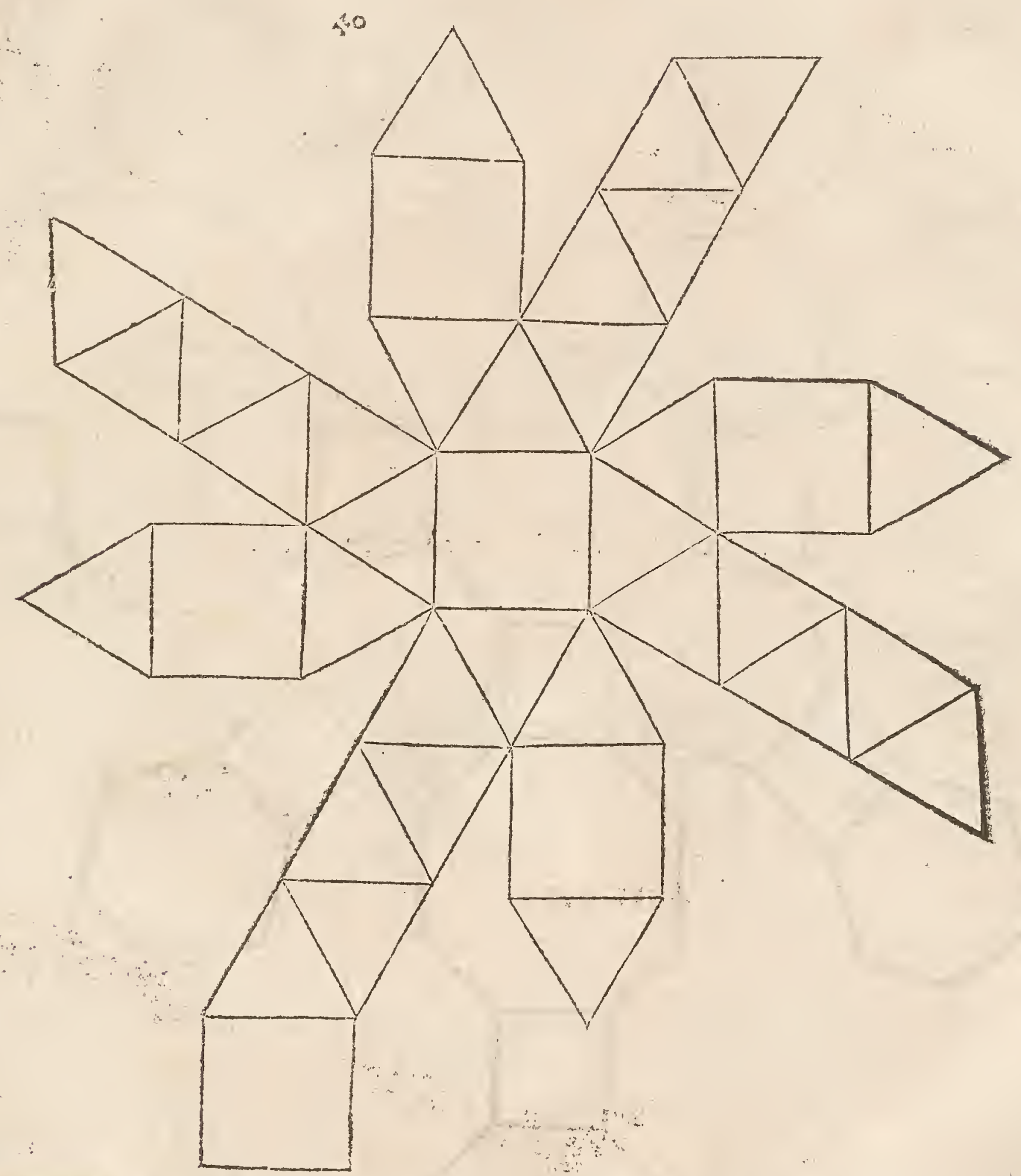

Eptimum \& fequens corpus, cùm aperitur habet lex octangulas, \& \& fexangulas, \& duodecim quadrangulas fuperficies, \& cum plicatur. continet $4 \delta$ angulos, $\&>2$ acuta latera. 


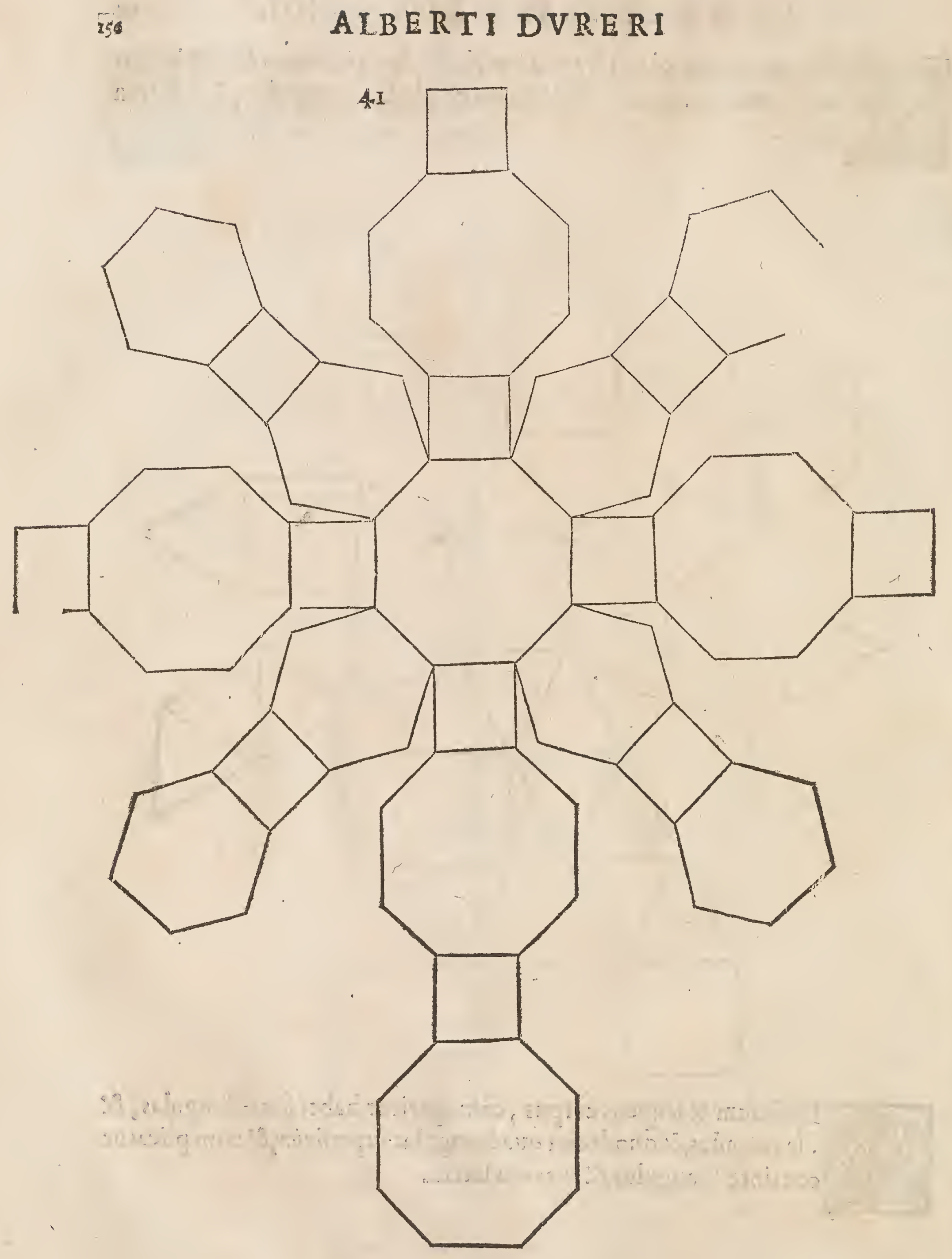




\section{GEOMETRIAE : LIB. III. II. 157}

tac

Ctauum corpus fac ex fex duodecágulis fuperficiebus, inter quas fac 32 duos triangulos, qui non habent omnia latera æqualia, quemadmo dum id fubfcriptum eft apertum.

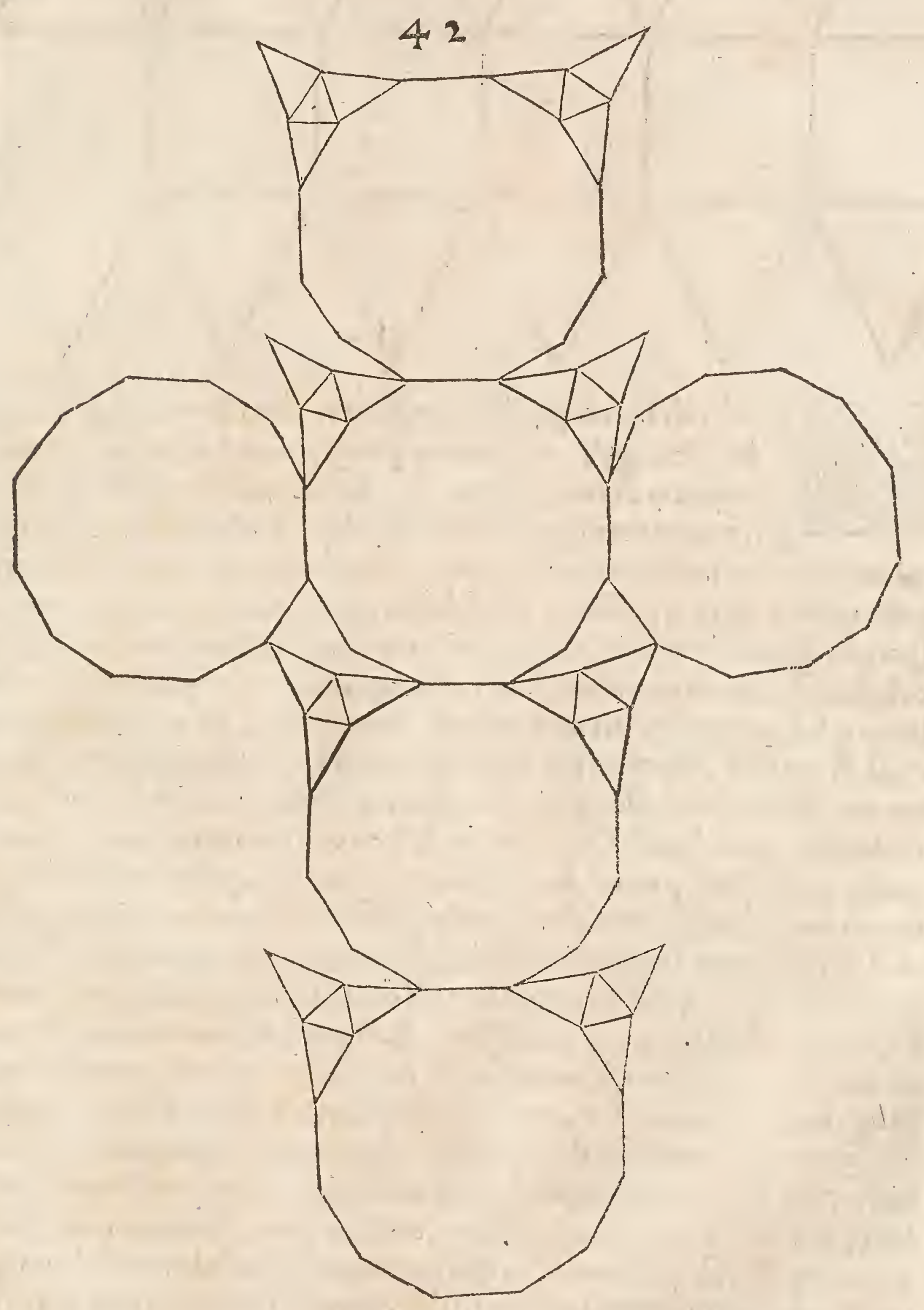

Vando fex recta quadrata ad fe compofueris, \& duodecim triangulos, quorum quilibet tantam habeat altitudinem, quantum fuerit latus quadrati, \& hec omnia compofueris, fiet corpus quale hic apertum elt fubfcriptum. 


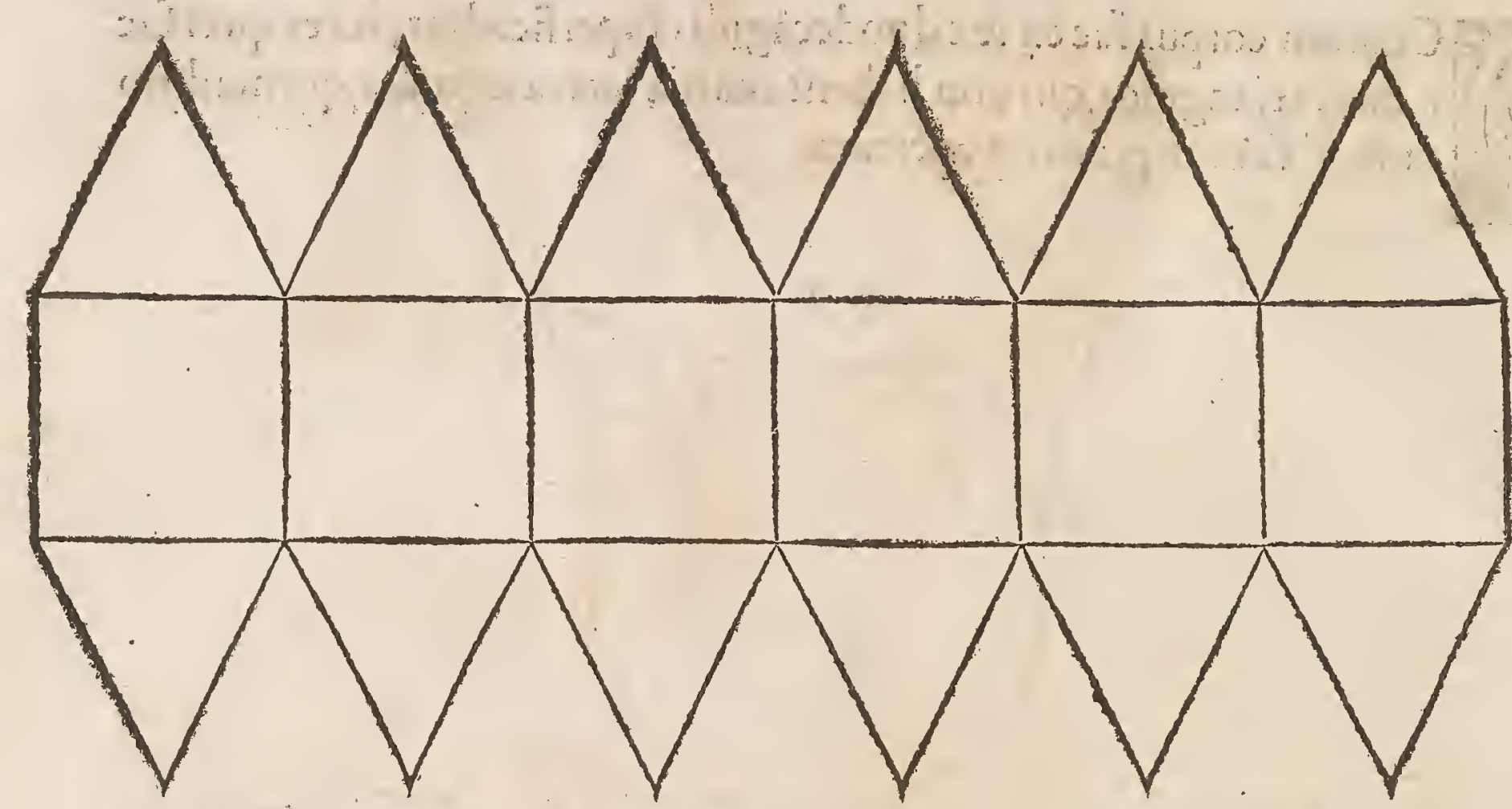

Vando ab his corporibus per planas abfiffiones anguli amputătur (2. 2 ) \& deinde anguli remanentes quog abfinduntur, fic poterunt fieri multiplicia ex his cotpora. Ex his rebus varia fieri poffunt, cùm pars earum tranfponitur inter $\mathrm{fe}$, id quod ad excifionem ftatuarum $\&$ columnarum earumóg ornatú conducit. Quemadmodú vrbis Athenarü femel pefte infectx ciues A pollinem confulebant, quo pacto morboliberarentur. Refpondit A pollo liberatú iri, qua ndo altare eius decuffarent.Itą̧ accepto ora. culo,lapidem para nt eiufdem cum altari quantitatis, atø̧ eum fuper altare collocant. Quum verò peftis non ceffaret, denuo Apollinem confulunt, rogantes quid in caufa fit, cur minus ceffit peftis, quum ipfi dicto paruiffent . Refpondit cos non ita executos effe, quemadmodum ipfe praceperat, fed al tare multo maius feciffe quàm duplú. Cum verò architecti eorú inuenire non poffent, quoná pacto id efficerent, confulebăt de hac re doctos, $\&$ pręcipuè philofophú Platonem:hic eos docebat, qua arte inuenire poffent ad duas lineas impares datas, du as aliaslineas qux fe pportionaliter haberét. Nam per talế arté poffent cubum (quod eft corpus quadratú, vt teffera) \& omnia alia duplicare, triplicare, $8 \mathrm{C}$ con

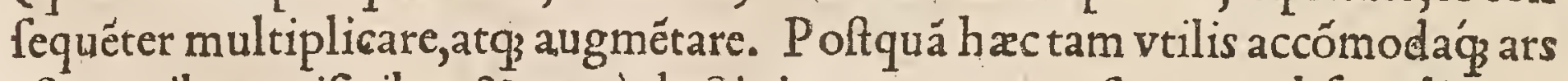
eft omnibus opificibus, \&\& qux à doetis inter arcana conferuatur abfcondita, vo lo ego hanc in lucem ędere, ac docere:quoniă per hanc artě etiam bombardarü, campanarum'́ conflatio difcitur, quæ augentur aut duplicantur pro fuforis ar bitrio, ac nihilominus femper fuum pondus, fuam q́p proportionem feruăt. Simi liter per eandem dolia, arce, menfurx, rota, carpenta, ftatux, \& quicquid lubeat augentur $8 \mathrm{l}$ multíplicátur. Ideo quilibet artifex animaduertat ad eam qux ( $v e$ reor) hactenus nun quam in lingua, (alté vernacula defcripta fuit. Primò iungeduos cubos a $\mathrm{b} c$, hanclongitudinem a ceetam pone fuper tranfuerfam lineam $\mathrm{d}$ ead angulos rectos, $\mathbb{Q}$ circumfcribe femicirculum d a ex centro c, deinde ex e duc rectam per $b$ vfop ad circumferentiam vbi ponatur $f_{\text {, deinde accipe }}$ regulá, feu baculú anguftú, \& in medio eius figna punctú ab hoc verfus vtramóg extre- 
extremitatem diuide, $\&$ cifras adpone in vna parte, ficut $\&$ in altera vt punctus me dius principiú vnitatis fit verfus vtramq partem, quoniam per motú regulę quęres primam lineam, per quă fecunda inuenitur ad duplicatú cubú, deinde pone iam factam regulă cum vna parte fuper punctum d, in quo femper hareat, fiue regula furfum, frue deorfum moueatur. Sed cum alteram regulæ partẽ moues, maneat fem per púctus medius fuper lineam a b c, \& tătifper moue regulă, donec inuenias medium locú inter lineá e $\mathrm{\&} \&$ circumferétiá, $\&$ vbi mobilis regula fecat lineá e $f$, illic poneg, $\&$ vbi fecat lineam $a b c$, ibi pone $h, \&$ vbi circumferentia fecatur, ibi locetur i. Ita erunt $\mathrm{g} h \mathrm{Qh}$ i duę lógitudines xquale $s$, tunc erit h c prima inuéta linea per quam inueniendum eft latus duplica ti cubi,deinde compone lineam h c, \& la tus fimplicis cubi a $b$ trăfuerfè ad fe inui cem, ex quibus fiet linea a $\mathrm{h} c, \&$ pone pe-

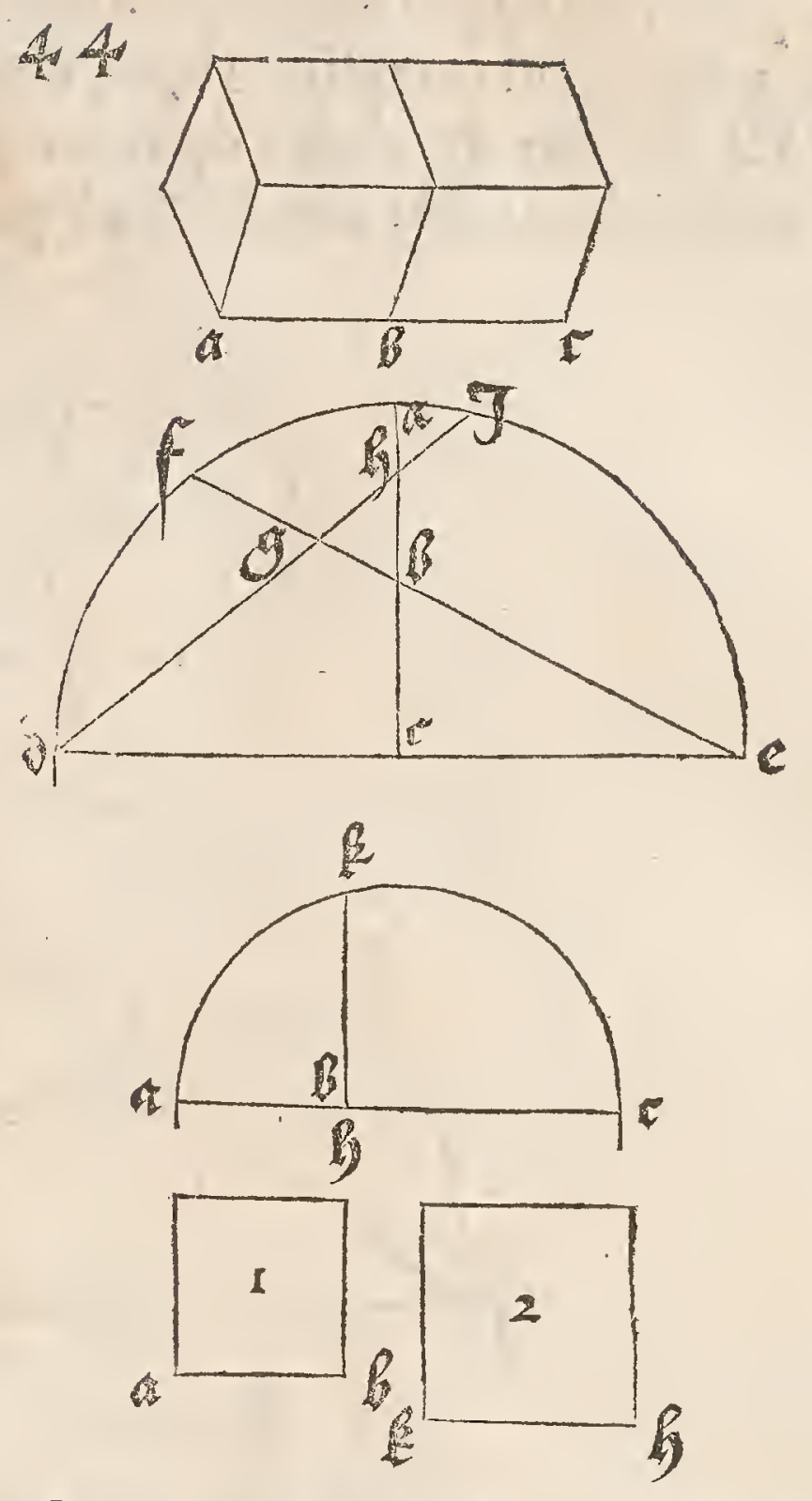
dem circini in medio linex a $c, \&$ defcribe femicirculum a c, deinde erige perpendicularé ex $h$ vqque ad circumferentiam, ibierit $k$ : hæc linea $K h$ dabit tibi latus ad duplicatum cubum quemadmodum hoc fubfcripfi.

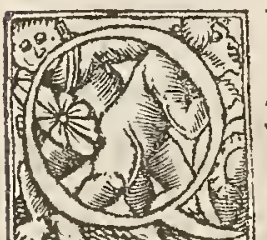

Vòd fi voles cubum triplicare, aut quadruplicare, aut quouis modo multiplicare, id efficies per viam prędictam. Sed vt fequitur. P rimò capelongitudines trium cuborum a $\mathrm{b} \mathrm{cd}$, aciunge eafd em: $\&$ pręd $\mathrm{u}-$ ctam lineam a d,erige fuper tranfuerfam $f$ e ad angulos rectos $\&$ duc femicirculum $f$ a e ex centro d, deinde duc obliquélineam rectam ex eper lineá a d, \& per eam abfcinde infimam tertiam parté, hoc eft longitudinem vnius fim plicis cubi, illic pone b. Sed vbilinea obliqua ex e ducta ad circumferentiam tá git, hic pone g,deinde colloca regulam per gradus diuifam cum altera fua parte fuperpunctum fin quo permaneat :cum altera veró parte moue regulam, \& quare in linea erecta a d locum medium interlineam e $g$ \& peripheriam e af, \& quádo regula ita fuerit fita, $\&$ linea fecundum eam ducta fecuerit lineam e $g$ ibi ponatur h, vbi verò erectam a d fecat, illic $i, \&$ vbi peripheriá, iftick locetur. Ita erunt h i \&iK eiufdem longitudinis, \& per hoc eriti $d$ prima longitudo per quam inuenienda eft fecunda longitudolateris triplicati cubi. Confequenter 
cape lineam i d pro longitudine linex tranfuer $\{\&$ adiunge eidem longitudi nem lateris cubi fimplicis a $b, \&$ pone circinum in mediolinee coniunctara $b$ \& i d $\&$ duc femicirculum a d, deinde erige perpendicula rem bl, hac linea bl eri latus triplicati cubi ad cubum a b quemadmodum fubfcriptum eft.

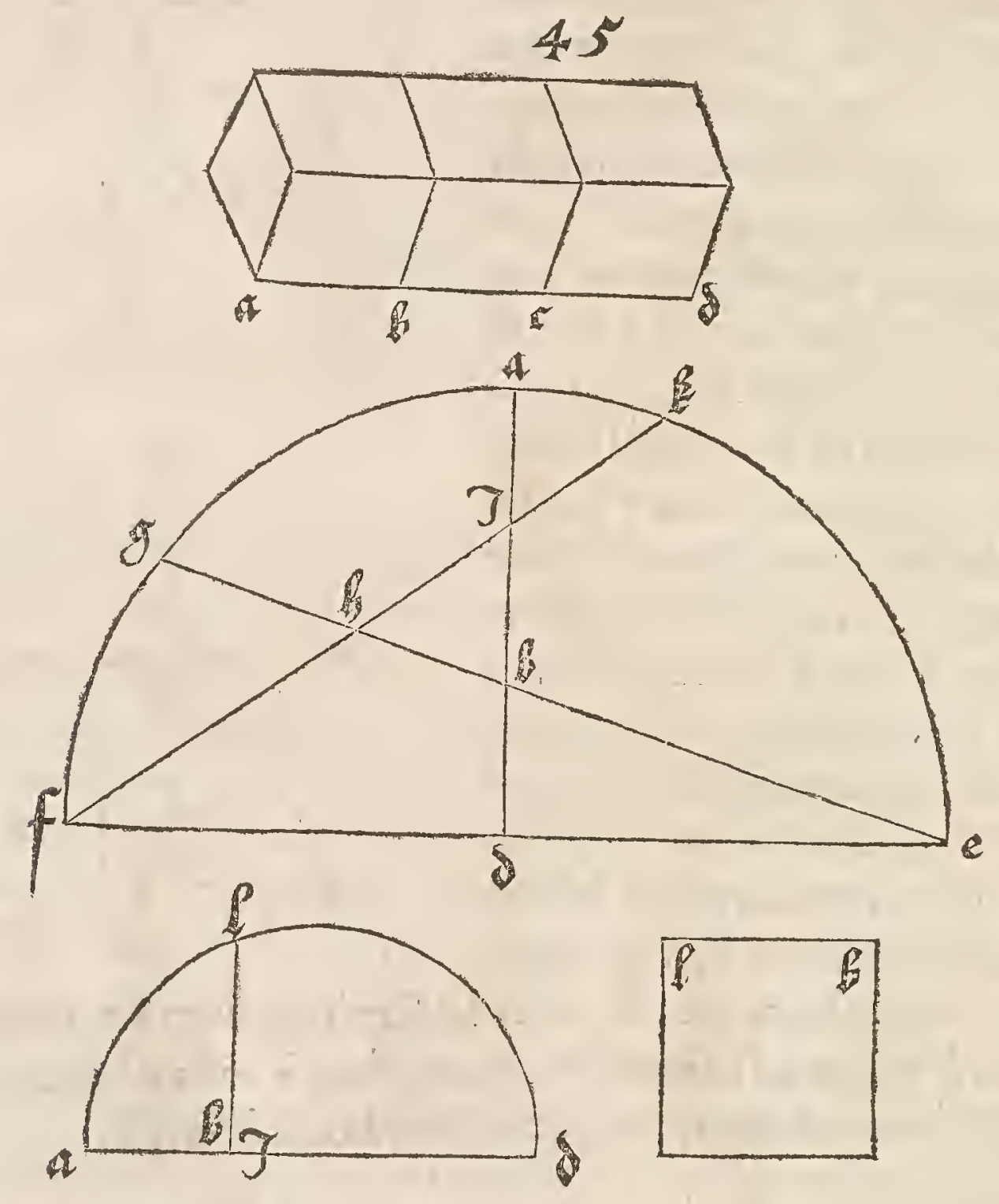

Of ea coniunge quation longitudines fimplicis cubi a b \& fiet a b cd 1. 2 , hanc lineam a e erige perpendiculariter fuper tranfuerfam $g \mathrm{f}$,dein. 1. Te duc lineam ex $\mathrm{g} \& \mathrm{a}$ abfinde cum ea quartá partem inferioréip. fius a e, \& vbi hæc linea peripheriam fupra $g$ tangit, ibilocetur $\mathrm{i}$ : deinde cape regulam tuam graduatam , \& punctum eius medium pone fuper erectam a e, \& alteram extremitatem fuper $g, \&$ quere medium in a e inter lineami f \& peripheria , \& vbififecabitur,ponek:\& vbi a e, ibi ponel: \& vbi circularis inter af tangitur, illic m pone, fic erunt $K 18 \mathrm{I} \mathrm{m}$ xqualium longitudinum. $\mathrm{Ex}_{\mathrm{X}}$ hoc inuenitur quod le prima eft longitudo, per quam inuenitur latus quadru. plicis cubi, hoc quere vt iam dictum eft. Coniunge longitudineml e \& lateris ló gitudinem fimplicis cubia $b, \&$ pone pedem circini in medio inter a,$\&$ altero pede ducfemicirculum a e. Dein de erige perpendicularem ex l in peripheriam vque tangentem, $i b$ íg pone $n$. Sic erit $n 1$ longitudo lateris quadruplicati cubi, quemadmodum id fubfcripfi. 

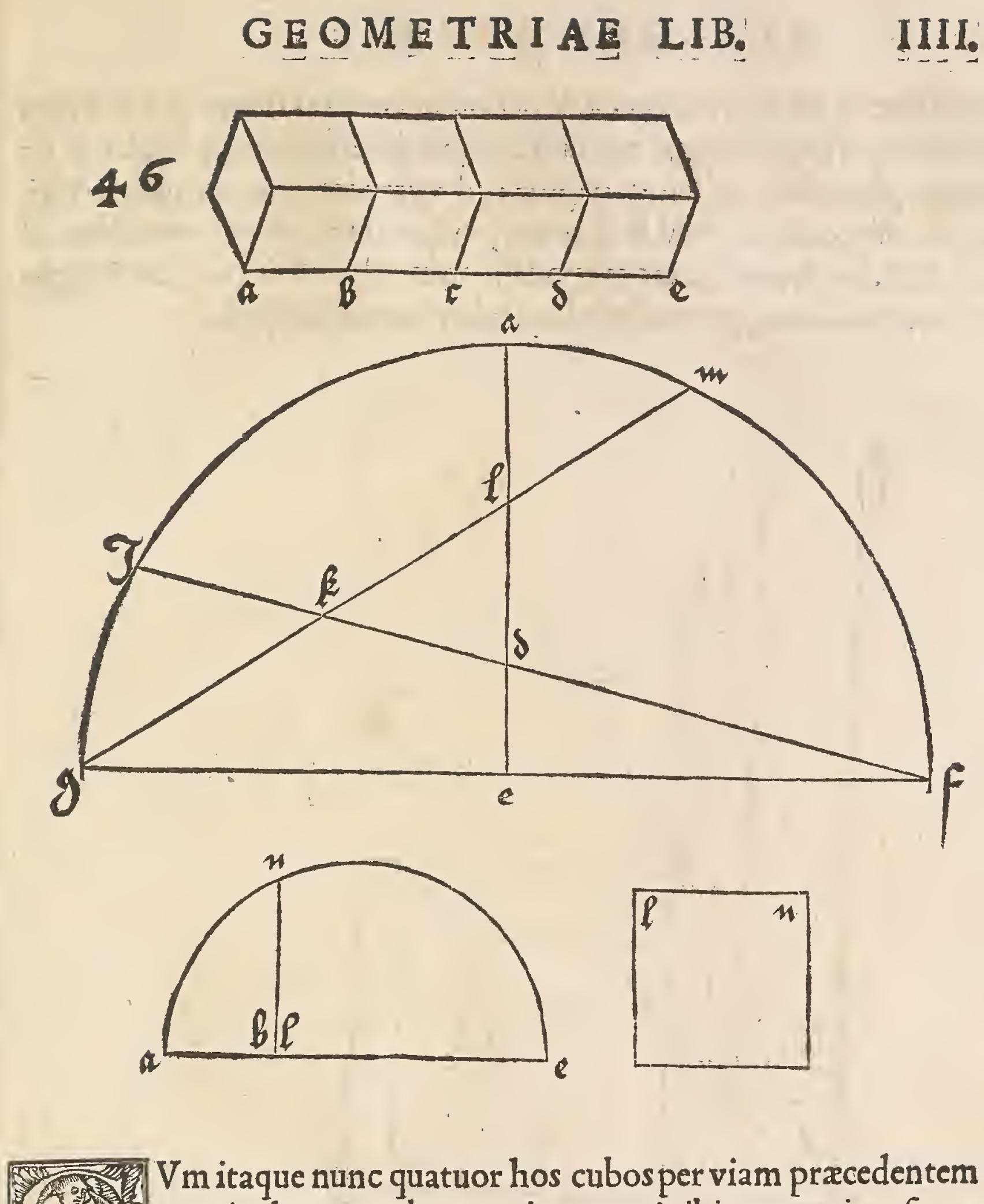

Vm itaque nunc quatuor hos cubos per viam pracedentem effeceris? poteris alterum cubum maiorem qui tibi proponitur fecundum primos pari modo multiplicare, dupliciter, tripliciter, aut quadrupliciter.

Augulum rectum conftitue, cuius angulus fit e, linea erecta $f \&$ linea i tranfuer$f a, \&$ pone cubum fimplicem, duplicem, triplicem, $\&$ quadruplicem in defcripti one omnes ad locum vnum, ita vt cuiuflibet cubi $\&$ angulus in angulo e, $\&$ duo latera fuper duas lineas fi permaneant: $\&$ defigna quatuor cuborum angulos in linea i fecúdum cuiuflibet cubi capacitatem $\mathrm{e}, \mathrm{e}^{2}, \mathrm{e} ;, \mathrm{e} 4, \&$ duc diametrum, feu lineam obliquam e $\mathrm{h}$ per quatuor angulos quatuor cuborum, deinde furfil meducito quatuor cuborum latera, qux fignata funt per $\mathrm{e}^{\mathrm{i}}, \mathrm{e} 2, \mathrm{e} 3, \mathrm{e} 4$, fupra obliquam eh æquidiftanter prolongata, quantum fuerit opus. Cùm tibi nunc propofita fit longitudo maior alicuius cubi, quem iuxta priores cubos vis mul tiplicare, tunc colloca propofitam longitudinem erectam fuper lineam i fimplicis cubi,vt infernè tangat trafuerfame $i, \&$ vbi fupernè finit, lllic pone $a$, de-

$$
\mathrm{O} \text { iii inde }
$$


inde duc obliquam lineam ex e per a, \& per omnes erectas lineas $2,3,4$ \& huius oblique extremitas fit g, vbi tunc erectx linex $, 3,4$ interfecantur, pone $b \mathrm{~cd}$ : fic erit a latus fimplicis, $b_{2}$ duplicis, $c_{3}$ triplicis, $d$ 4 verò latus quadruplicis cubi, $\&$ funt in eadem proportione ad fe inuicem, in qua funt cubi minores. Hoc ad plurima eft vtile. Nam per hęc omnia poffunt multiplicari ritè, vt fuam femper obtineant proportionem, quemadmodum tale vides fubfcriptum.

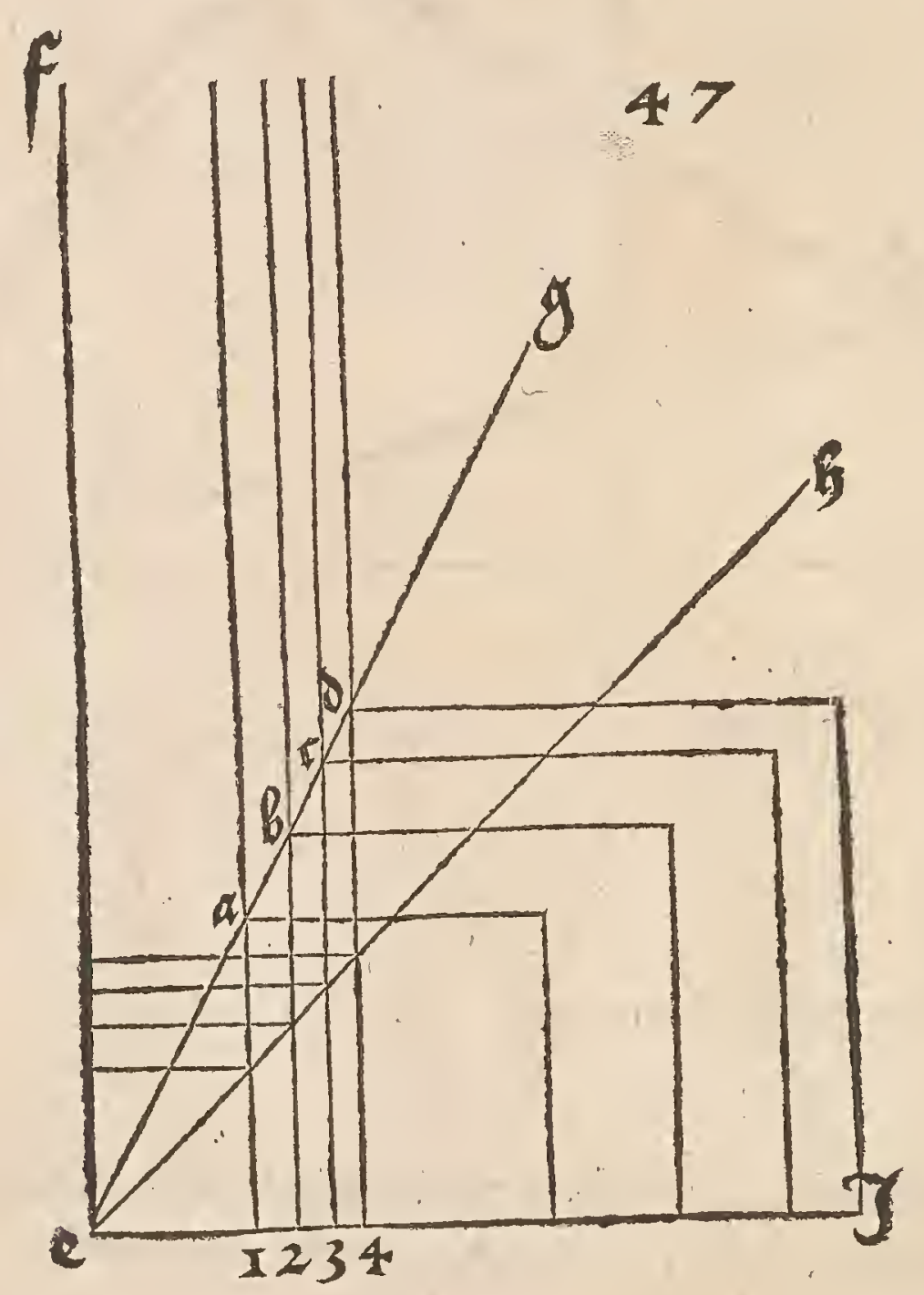

Tem alia forma, qua cubos continuo in duplovel maiores, vel minores facere poteris, vbi prius habueris duos cubos, quorum vnus alteum in duplo contineat, fac ita.

Ponelineam erectam a $b$, in qua ponantur dictorum cuborum latera, $v t$ fe contingant, \& vt maior fupra minorem collocetur cubum, fuperiores anguli duo anteriores ad lineam a b ftantes fint $c e$,pofteriores verò fint $d \mathrm{f}$.Inferioris verò cubi minoris angulos duos ad lineam $a b$ anteriores figna $g . j, \&$ pofte riores $h K$, tunc coniunge duos angulos $\mathrm{f} K$ per rectam lineam, deinde ean dé duc furfum quantum volueris, atque illic pone $r$, deinde deorfum vfque ad lineam $a \mathrm{~b}$, ibi pone 3 .Cùm itaq, exangulo d ducas lineam erectam in punctum 3 , ea feca bit inferiora duo latere cuborum. Cùm verò cum eadem obliqua furfum tendas vique ad finem $x$, ex hac fumitur cubi maioratio, fed infernè deorfum minoratio. Id facies ad hunc modum. Primò educito latus tranfuerfum cubi $\mathrm{c} d$ fupernè vique ad lineam obliquam $3 \mathrm{r}$, ibi ponitol, deinde afcendito cum linea erecta 
crecta ex l, furfum ad lineam $3 \mathrm{x}, \mathrm{ibi}$ que ponito $\mathrm{m}$, deinde concludito cubú $\mathrm{cl} \mathrm{m}$ $\mathrm{n}$, is continebit in duplo cubum $\mathrm{cd}$ e f.Idem toties in duplo alcédit quoties for mare poteris, hócque certum atọ rectum reperies. Sed deorfum cubus femper in duplo redditur minor, idquefit fermè vfque in cufpidem 3 $\&$ hoc eodem mo do defcendendo efficitur, quo alterum afcendendo, hoćq ita facies. Vbi linea $x$ 3 interfecat infimi cubilatus $i \mathrm{~K}$ ibi pones $\mathrm{o}$, ex quo duc deorfum lineam erectá ad obliquam 3, , ibi pone . Inde duc rrăfuerfam ad angulos rectos, vfque ad erectá a b, hic fige q. Sic continebit hic cubus i op $q$ in duplo minus, quàm cubus fuperior $\mathrm{g} \mathrm{h}$ i $\mathrm{K}$, codem pacto vlterius vti poteris ferè ad punctü vfque, tale fub. fcripfi inferiùs.
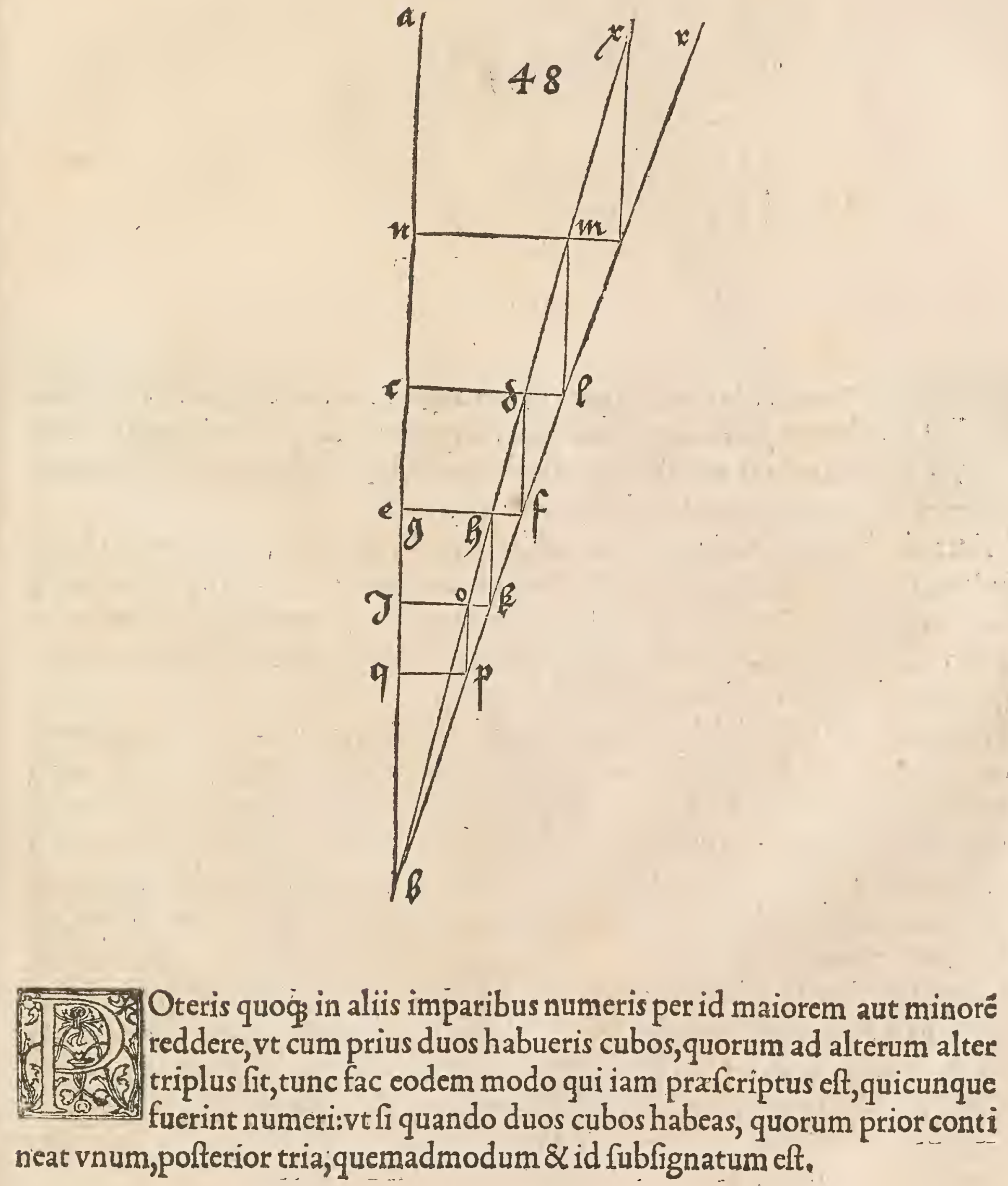

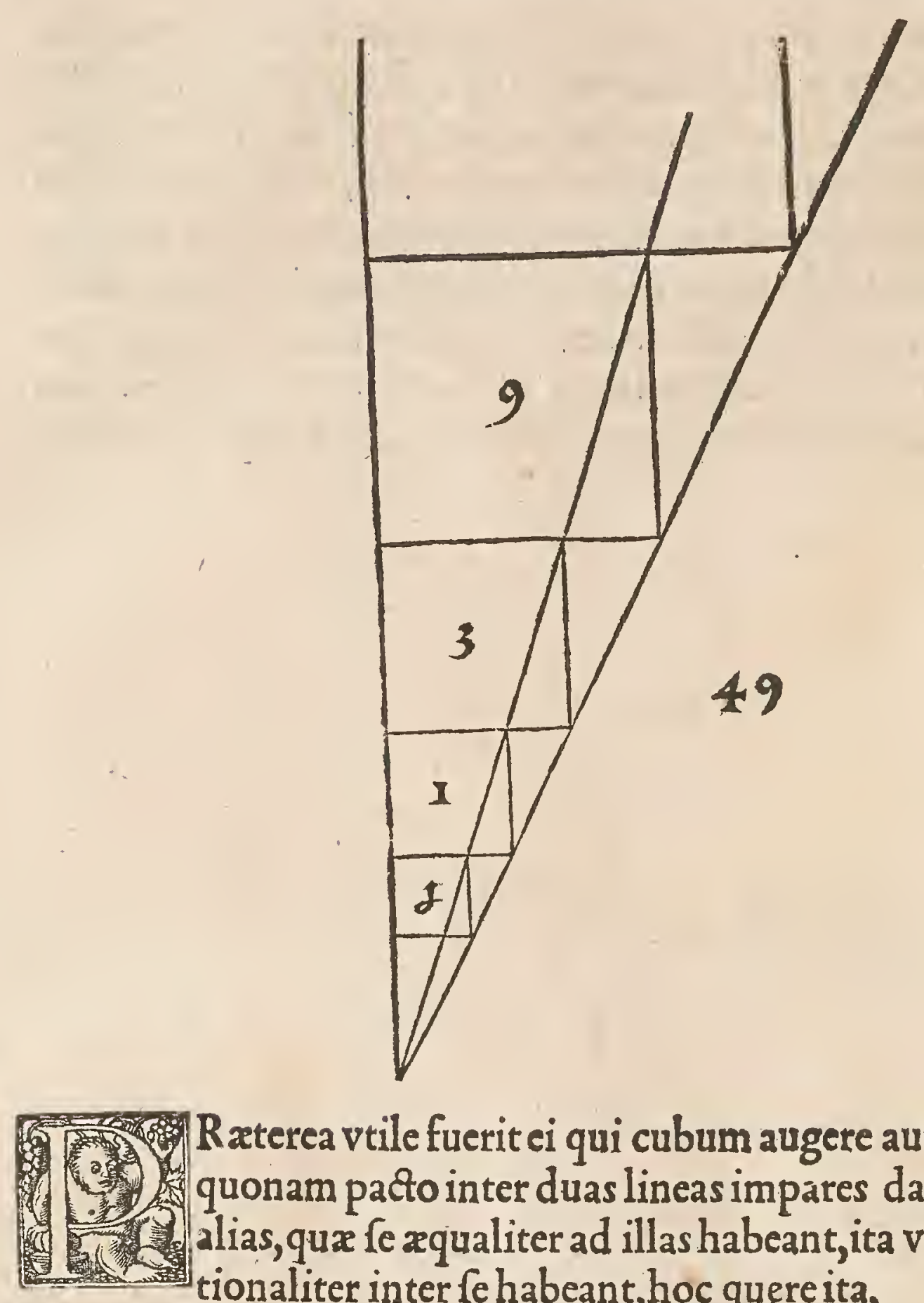

Raterea vtile fuerit ei qui cubum augere aut multiplicare volet, fcire quonam pacto inter duas lineas impares datas, inuenire poffit duas alias, qux fe xqualiter ad illas habeant, ita vt omnes quatuor proportionaliter inter fe habeant, hoc quęre ita,

Prim $x$ dux linex dat $x$ impares fint $\mathrm{eb}, \& \mathrm{~b} \mathrm{~g}$, has duas iunge in puncto $b$ ad an gulum rectum, deinde duc ambas lineas vltra angulum $b$ quantum fuerit necef fariú vfque ad d $8 \mathrm{c}$, deinde cóftitue duos angulos orthogonos $\mathrm{g} c \mathrm{~d}$ cuius angulus rectus fit $c$, alterum verò triangulum $\mathrm{cd} e$, cuius rectus fit $d$, per quod inftrumentum id fieri debeat, poftea fcribetur.

Nunc probat Euclidis libro fexto, propofitione octaua, lineá c b effe proportionalem inter $d \mathrm{~b} \& \mathrm{~b} g, \&$ lineam d b effe proportionalëinter $c b, \& b$ e, ideo $g b$ ad cb fe habet,ficut $c b$ ad $d b, \&$ ita quoog $d b$ ad eb, \& per confequens inuentr funt due linex proportionales $b c \& b d$ ad duas datas impares fcilicet $b \mathrm{~g} \& b$ e.Pradictos verò triăgulos $\mathrm{g} \mathrm{cd} \& \mathrm{~cd}$ e fac ita.Cape regulam rectangulam $\mathrm{r} p$ $q$, cuius rectus angulus fit $p, \&$ regul $x$ latus $p$ q excauabis, \& in medio rimulac $c$ locabis regulam rectam $t s$, qux cum latere $\mathrm{q}$ femper faciet angulum rectum vt cunque furfum fiue deorfum moueatur, \& vt femper $t s$ verè parallela maneat ad latus p r regulę rectangulęr pq . Quando hęc omnia fuerint ita abfoluta, túc fuperpone latus p riplius regule, ita vt tangat punctum $g, \&$ vt angulus reEtus $\mathrm{p}$ fitus fit fuper lineam e $c$, \& vt alterum regule latus p q iaceat fuper line- 
am $\mathrm{d} b$, hic moue regulam $\mathrm{t} s$ donec angulus $s$ fit fuper lineam $\mathrm{d} b$ \& regula $\mathrm{s}$ tangat punctum e, $\mathcal{X}$ cum totum hoc ita fuerit perfectum, \& defignatum, tunc erit $\mathrm{p}$ r ficut $\mathrm{c} g: \& \mathrm{p}$ s ficut $\mathrm{c} d, \&$ s ticut d e, $\&$ ex hoc pateई quòd duo triangu lig cd $\& \mathrm{~cd}$ e defreripti funt ficut ab initio fuerant propofiti, quemadmodum id in fequéti figura fupfcribtum vides
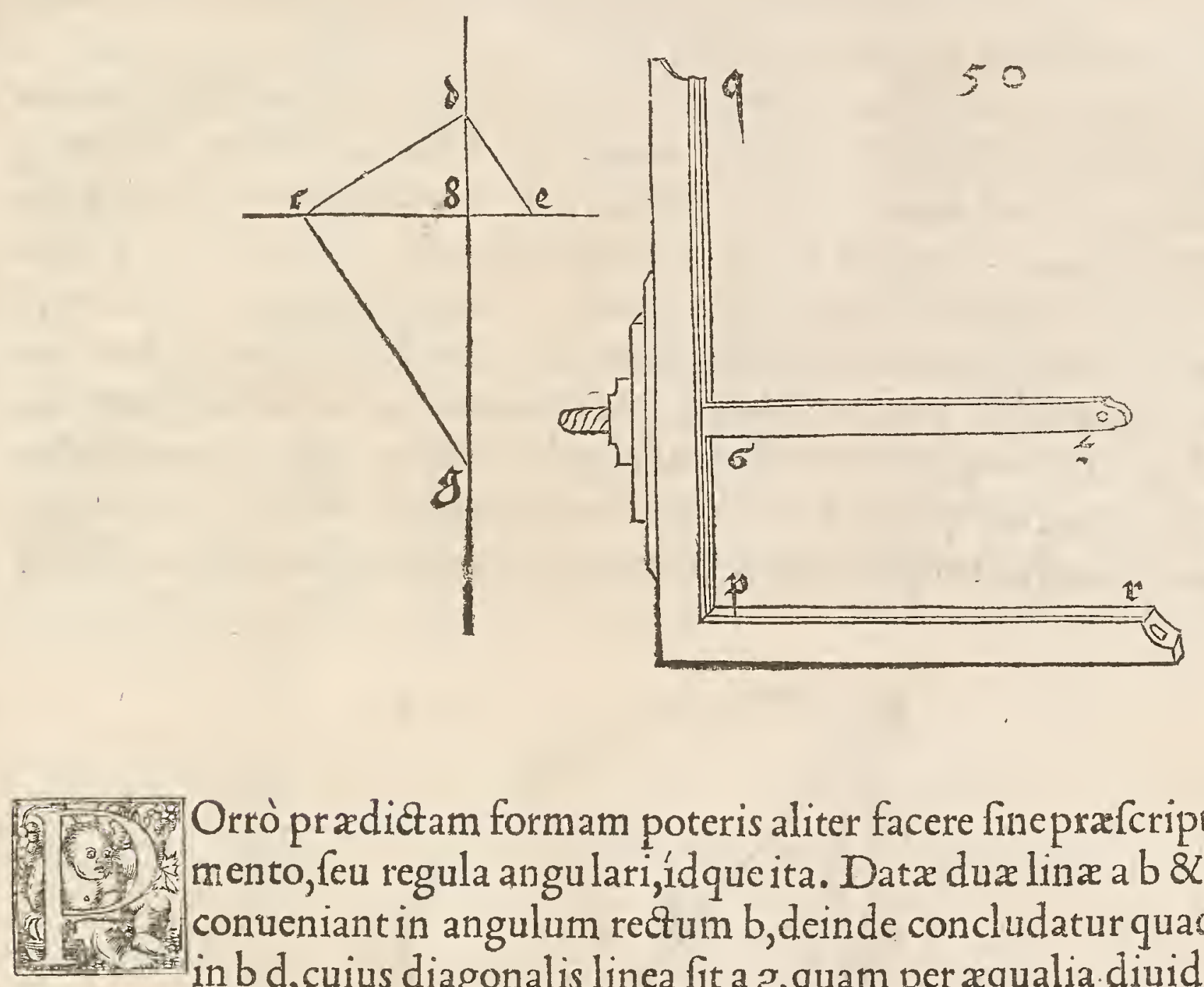

Orrò prædictam formam poteris aliter facere fineprafcripto inftrumento, feu regula angu lari,ídque ita. Datæ du linæ a b \& b g iterú conueniant in angulum rectum $b$, deinde concludatur quadrangulü in $b \mathrm{~d}$, cuius diagonalis linea fit a g, quam per æqualia diuide in puncto e, atque duo latera d a $8 \mathrm{~d}$ g prolonga quantum fuerit opus, deinde pone regulam fuper punctum $b$, ita ve moueri poffit huc atque illuc donec $a b f c i n d a t$ d h $\& \mathrm{~d}_{3}$,ea quidem menfura, qua lineqe $3 \&$ e h fiant pares, \& vt regula femper fuper b iaceat dum vtramque abcindit, id đq̣ oftendit circularis linea. Cófequen ter duc perpendicularée t fup lineá $\mathrm{d} g$, ita diuidetur d $g$ per equalia per fecundam propofitionem libri fexti Euclidis. Hinc fequitur quod figura rectangula quadrágula qua fit ex $t 3 . \& 3 \mathrm{~g}$ cú quadrato linę $\mathrm{g}$ t equa eft quadrato, quod fic ex $t_{3}$. Vtriq etiá addatur quadratü, quod fit ex t e. Pręcerea quadrágulúquodfit ex $d_{3} \&_{3}$ g cú quadrato e g,equú eft quadrato quod fit ex e 3 . Similiter quadran gulú quod fir lineis $d \mathrm{~h} \& \mathrm{~h}$ a cú quadrato ex a efacto, $x$ quú ef quadrato quod. fit ex linea eh. Cùm núc, vt iă oftếfú eft, due linę e h $\&$ e 3 equę füt, frmiliter etiă dux linex e a $\&$ e g xqux. Ex hoc fequitur quòd figura quatuor rectorum angulorum (quam quadrangulam vocamus) qux fit ext $3, \&$, $\mathrm{g}$ æqua eft in arex 
fpacio rectangula figure, quęfit ex lineis $d \mathrm{~h}, 8 \mathrm{~h}$. a. Id ita eft intelligendum. Linea $d \mathrm{~h}$ pro lateribus quadranguli longioribus, \& h a pro breuioribus lateribus fumuntur; pariter $t 3$ linea ad longiora $\&$ 3 $g$ ad breuiora latera alterius quadranguli fumuntur. Sic tantundem in fe continebit quadrangulum $d \mathrm{~h}$ a atquè quadrangulum $\mathrm{d}: \mathrm{g}$.

Ad hoc amplius probandum, fac hæc quadrangula ad vera quadrata, ficut antê in libello planorum in figura 3 oftenfum eft.

Sed quòd primum ita fe habeat demonftratur per decimamquintam propofitio nem fexti libri Euclidis, quòd fcilicet linea $\mathrm{d}$ ad lineă $\mathrm{d}$ h fe habet, vt linea $\mathrm{g}$ $\mathrm{h}$ a ad lineam $\mathrm{g}_{3:}: \&$ vt linea $\mathrm{d}_{3}$ ad $\mathrm{d} \mathrm{h}$ habet, ita fe quoggh habet linea $\mathrm{g}_{3}$ ad linea h:eodem etiam modo linea $a b$ ad ha, deinceps per quartam libri fexti Euclidis linea $a b$ fe habet ad lineam $a h$, ficut linea $a h$ ad $g ;, \&$ ita quoque linea $g$; ad line am $g b$. Et fic pa tet quòd inter duas lineas $a b, \&$ b g inuentę funt due mediaproportionales, que funt a $\mathrm{h} \& \mathrm{~g} 3$ quemadmodum id fut friptum eft. Item differentia inter quadratum \& quadrăgulum eft, quòd quadratum habet quatuor rectos angulos, \& qqua latera, quadrangulú verò habet duo latera lógi ora, \& duo breuiora, attamé quatuor quoq̧a angulos rectos, ficut intè dictú eft.

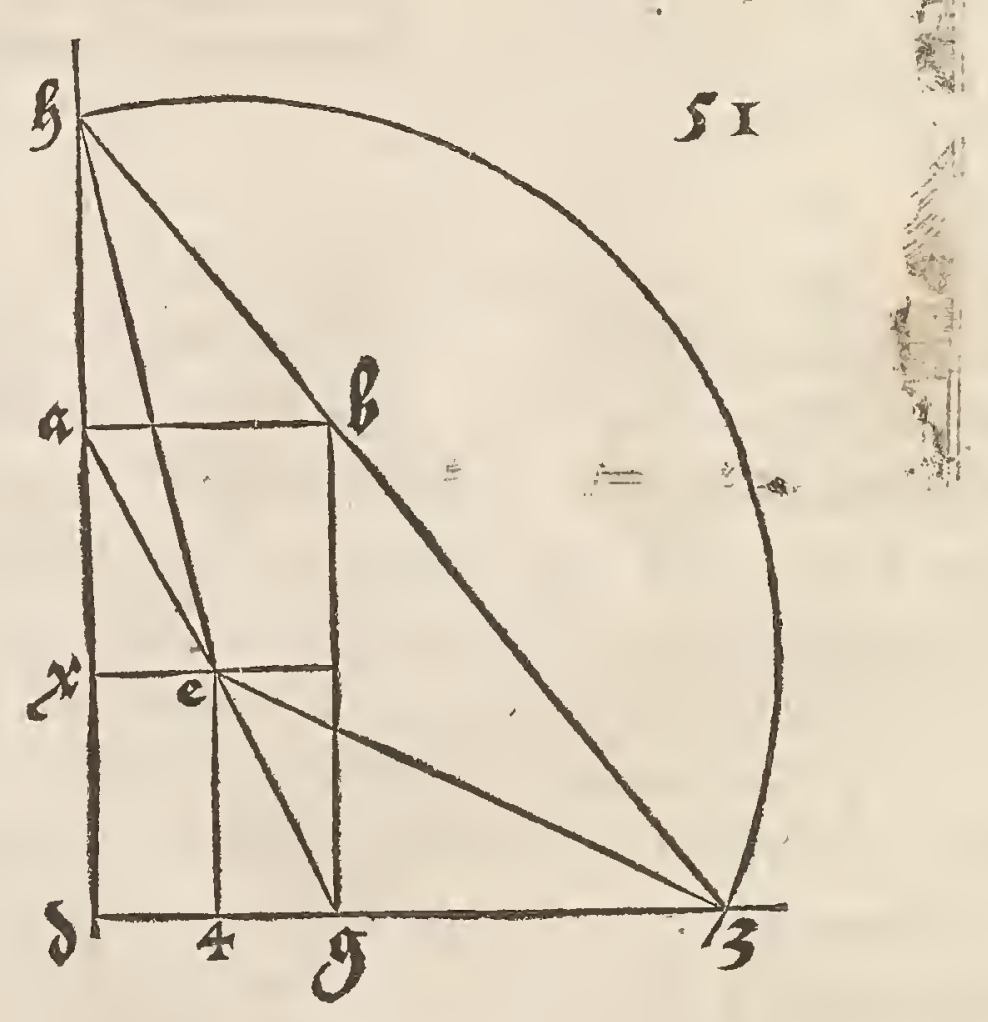


Vum igitur per doctrinam iam defcriptam \& demonftratan, cubum (1) datum voles duplicare, tunc latus ppofiti cubi duplabis, \& inter hoc 1. 1atus duplicatum, \& inter prius fimplex cubi per dictam artem con1. Iuncta debes inuenire duas veras medias proportionales lineas: $\& \mathrm{fr}$ ex minore inuentarum linea rum cubum erexeris, is in duplo fe habebit ad cubum datum.

Sic etiam datum cubum triplicare licebit. Nam quotiés inter dati cubi latus, $\&$ inter latus in iriploprolongatum duas medias inueneris, \& iterum ex minore cubum feceris, is in triplo fe habebit ad datum cubum. Ita toties cubus multipli cari poterit, quoties latus auxeris prolongando. Exemplum his fubiungam ad ponderaita.

Quando habueris globum æreum bombardæ, vnius libræ grauem, poteris eúndem cótinuò per prædictam viam grauiơre reddere delibra ad libram. Nam proportio in fimili metallo, fimilia pódera prodit.Cúmq̧ deinde globum in cu. bo fignaueris, \& deinceps cubú in duplo, triplo, quadruplo maiorem feceris, ac tandem iterum globos in his omnibus fignaueris, tunc pondera quoque qux fe cundum hos fuerint fufa, ad fe inuicem in duplo, triplo, quadruplo habebunt quemadmodum id fubfripar. Idem etiamad centum libras perducere potes. 

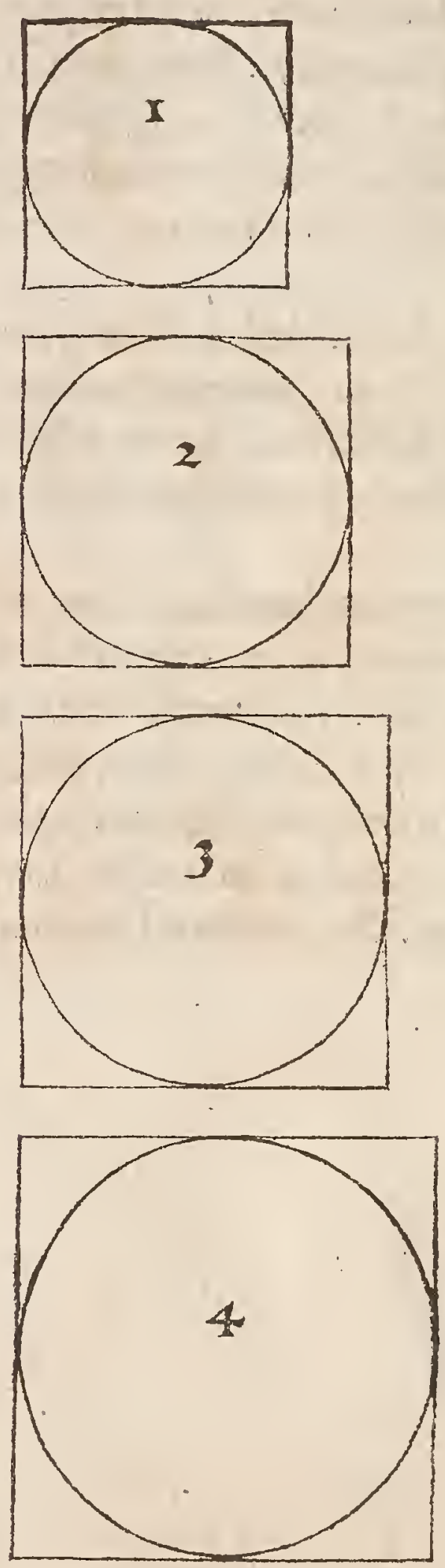

V. Vm iam antècorpora varia, quo pacto fierent, declarauerim, nunc quoque docebo quonam pocto facta eadem $\&$ vifa in picturam veniant, atque ad id fumam fimplicifímú corpus, vt elt teflera,pro exem plo, fimul oftenfurus cum reliquis omnibus corporibus pariter operádum effe.Caterum $\&$ de lumine $\&$ vmbra quippiam commemorabo, ac vno cum altero vtendum efle. Nam quod in confpectum venire debet, id ante ftet necelle elt, $\&$ oculo videbitur. Ad hoc etiam lumine opus erit, cùm tenebra om nia occulrent:praterea \& mediam quandam intercapedinem effe opus eft inter oculum, \& rem viram, vt fubfequitur 
Lumen omne rectas extendit lineas, quantum radii eius fefe extendere poflut: quando verò res opaca lumini obltiterit, tunc radii in eam rem impingeitst, $\&$ vmbra cadet in tantum, quantum linex radiorü ipfius luminis detinêtur, quod in fignaturis fubfcribam. Propterea in primis parato frontem planam, quadrangulam, æquiangulamóg ef $g$ h. In hac planicie locetur teffera, hac dabit in fundo quadratam formam:nam quatuor anguli fuperiores decidét ad quatuor inferiores, ideóq quilibet angulus dupliciter fignabitur. Inferiores quatuor anguli funt $a b c d$. Sed fuperiores quatuor funt $,{ }^{2}, 3,4$. Igitur vniếtur $a, b, c, d$ t fimul. Sic abfoluta erit hęc frons plana.

Deinde fuperficiem hanc quadratam $\&$ tefferam quęin ea eft,conuenit eleuare quemadmodum architectus fundamentum fuum è plano ducit in altum: quod fic abfoluitur. Trahe fupra quadratum ef $g$ h, lineam parallelam ipfi quadrato, atque eiufdem longitudinis cum quadrati latere, cuius principium fit $\mathrm{e} \mathrm{h}, 8 \mathrm{fi}$ nis $\mathrm{f} g$. Hęc linea repræfentat planum inferius ef $\mathrm{g} h, \&$ ideo extremitates eius $b$ is funt fignatax. Iam ex fundamento plano tefferæa $\mathrm{a}, \mathrm{b} 2, \mathrm{c}_{3}, \mathrm{~d} s$, afcende furfum cum lineis rectis per trăfuerfam e $\mathrm{h} f \mathrm{~g}$, in eam altitudinem, qua teffera ere. cta finire debet,inueniésqg locum tefferæ fiue cubi, qui fupra pradictá tranfuerfam ftatuendus elt : ita erit inferius teffera latus, quod tranfuerfę $\mathrm{e} h \mathrm{f} g$ incumbit ad b c, fed fuperius latus fignabitur $1_{4} \&{ }_{23}$, fic quatuor anguli erecti cubi manifeftè funt fupra fundamentum defignati. His perfectis lumen locandū eft: verùm vt duo funt facta fundaméta, alterum planum, al terum erectum, ita pro fingulis fundamentis fingula erunt figenda lumina: ad fundamentum erectum luminis obferuo altitudinem vel humilitatem, ad depreffum verò , partem declination is à fundamento. At hic pono ad fundamentum erectum lumen $o$, in ea qua placuerit altitudine atog diftantia, \& ad fundamentum in plano figo alterum lumen $p$, ad quod latus voluero, modò vtrunq lumé æqualiter à füdamento in plano \& teffera erecea diftent fecundum latitudinem. Nuncex punctoo luminis protrahe binos radios rectos per tefferá erectę füperiores angulos 14,$82 ;$ ac vbi hicadunt in tranfuerfam e $\mathrm{h} f \mathrm{~g}$, illic fcribe literas $\mathrm{i} \& \mathrm{~K}$, tantum protenditur vmbra. Poftea ex fignop tuminis inferioris, excitato lineas per angulos fü daméti plani, qui fignati funt $b_{3}, c, 8 \mathrm{~d} 4$, atq̣ eas lineas cốtinuato quantú opus

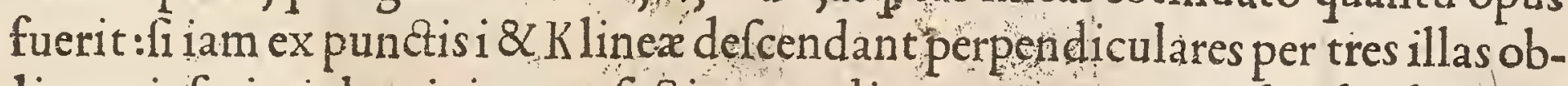
liquas inferioris luminis, tunc fectiones radiorum cum perpendicularibus terminant vmbræ longitudinem atglatitudinem: quare notand $x$ funt literis $1 \mathrm{~m}$ $\mathrm{n}$ quas rectis lineis coniunge. Confimiliter etiam continuato $\mathrm{b}=1,2 \mathrm{~d} 4 \mathrm{n}$, habe bísg integrum vmbrę circuitum. Poftremò fciédum eft, duo illa lumina vnicú folum fignificare: item tranfuerfam e hfg idem effe cum quadrata fuperfcie ef $g \mathrm{~h}, \mathrm{vt}$ dictum eft priùs: pari ratione cubum in plano $\&$ erectum eundem effe. In fumma, vtrum q̧ fundamentum, $\&$ vtrúq lumen idem funt, fed propter faciliorem defignationem feparata, vt infrà deliniauı. 


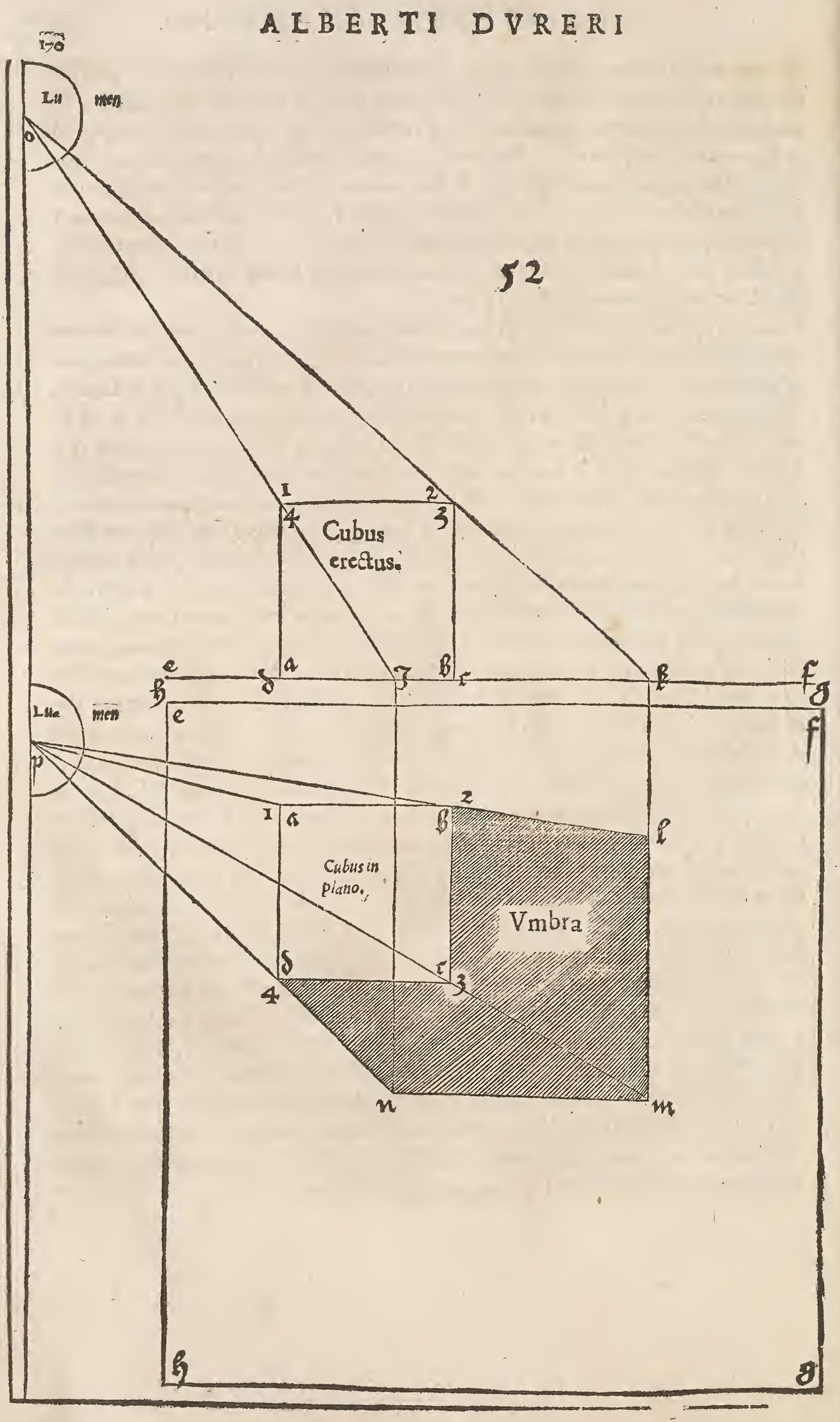




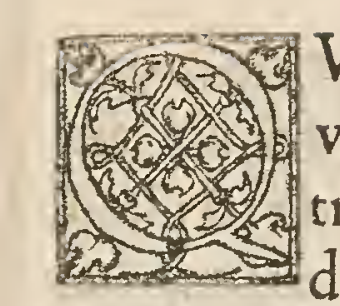

Vum iam prædictü cubum in fuperficie fua quadra vnà cum lumine $\&$ vmbra pictura vis reprafentare, tria prius notanda erunt:primo punctus oculi, deinde res qua videnda elt ex aduerfo, fiue in altera parte, poltremò lumen fine quo nihil videri poteft:velut fuprà dictum eft. Oculus folùm per lineas rectas, non autem per curuas videt eas res, qux ante eum funt: quocirca quando dux res opacx atq; xquales in linea ponuntur recta qua ab oculo procedit, tunc an terior folum $\&$ non pofterior ab eodem perfpicitur : quarefi res multa vidend $x$ fuerint, debent exdem inter fefe difiunctx effe, vt oculorum radii eas contingere gueant. Item mediocrem opor tet effe intercapedinem inter oculum \& rem qua videnda eft: nam fi ea oculo propius admoueatur, tum obtegit eum $\&$ vifus impeditur. Neque res ab oculo nimis fit remota, ne vifui difperdatur:quando enim res ab oculolonge diftat, tunc radii vifuales prope oculum tam arctè conftringuntur, vt oculus hoc paruum interuallú, quod eft in ter eos, percipere nố poffit:ifta infernè hoc modo demonftrantur. Punctus a efto oculus, prope eum trahatur linea b c,à cuius vtraque extremitate ducátur linex ad oculum a, qui fic omnino erit coopertus : hoc facto aufer lineam b c \& trahe aliam d e mediocriter aboculo a remotam, qui cum de extremitatibus continuetur per lineas rectas, hanclineam d e oculus commode videre poteft. Iam depone $\alpha$ lineam d e, ac fribe tertiam $f g$ admodum longé aboculo a diftantem, à quo itidé ad terminos $f$ protrahantur radii vifuales, a $\&$ a g, eritó prope a oculum fpacium tam arctum, vt id vifus vix amplius cố pręhendere queat:vnde fi homolongius côfpiciatur, vifus propter debilitatem eum cognofcere non poteft:ideo res qux tam accuratê contemplandx erunt, in debita fint ab oculo diftantia oportet. Porrò ad pingendas regiones, per quas fex aut feptế videmus paftuum milia, rutfus propria eft fymmetria.

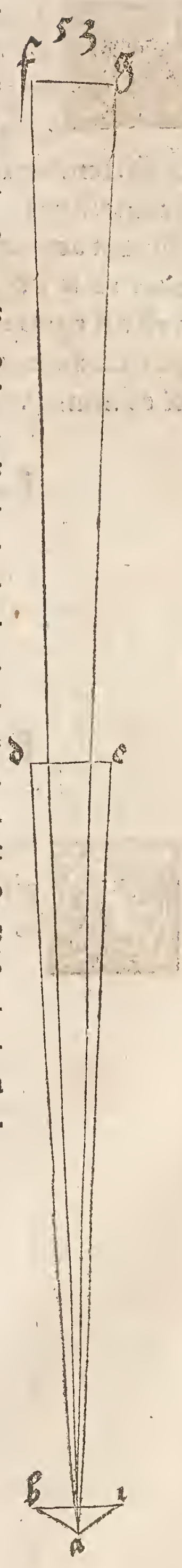


Otato nunc quod inter oculú $\&$ rem vifan radii, qui ex oculo in eam prorumpunt,per planum guoddá abCcindi poffunt prope oculú aut 2. Longe ab co,ac proximè ren videndam:quòd fi prope vifum id planú Itatuatur, tunc pictura in id cadet areta:fi verò planum hoc longè ab oculo remoueatur, ac prope rem vifam, tunc pietura in id incidet amplior, quod fic intelligito . Applica binas lineas fuis extremitatibus ita vt angulum conftituant acutum, qui fit a, \& earum reliqui duo termini fint $b c$, inter eas duas lineas $a b \& a c$, protrahentur $d u x$ alia linex erecte, quarum qu $x$ magis diftat $a b$ a eftod e,propior vero f $\mathrm{g}$ e, ex his duabus quanta inter $\mathrm{c}$ a $\&$ b a obliquas comprahenduntur, duo efficito quadrata, fietóg $d$ e quadratum $a b$ a remotius maius \& ef minus và̀ cum omnibus lineamétis, qux in id cadút, velut hic deliniaui.

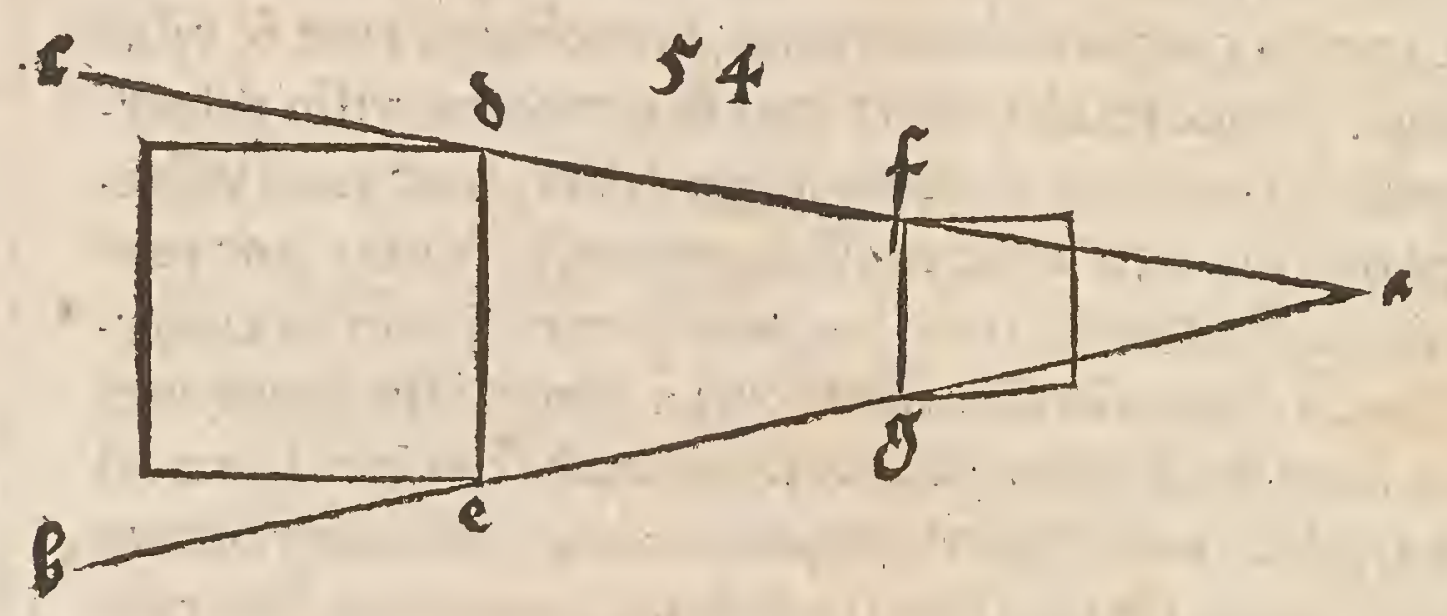

Vod inter duaslineas tranfuerfas a b \& a c cadit, ató eas vt ing tan git,fiue hoc fit propè, frue longè, erectum, obliquum aut curuum,oculo a in eadem apparebit quantitate, quemadmodum hic defignaui.

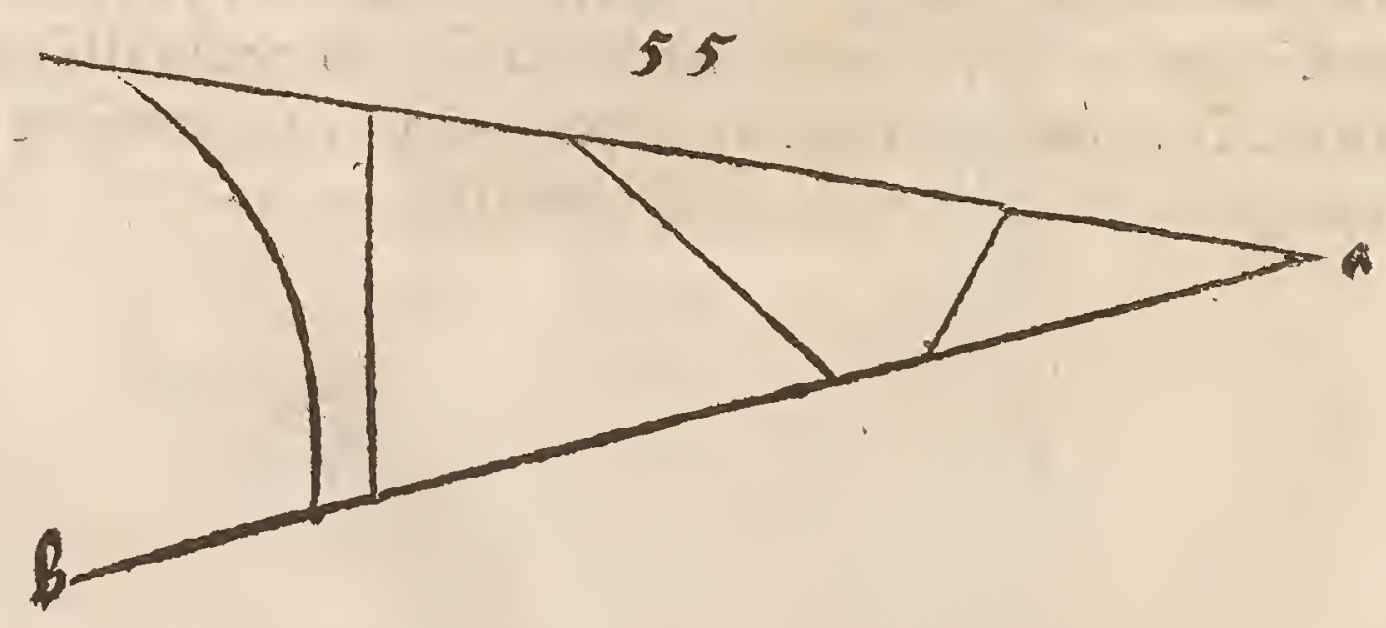


Am proximè aggredior fumoós mihi fuprà defcriptü cubum, quem admodum ftat in fuperficie fua quadrata vna cum lumine $8<\mathrm{vmbra}$, quam iacit in depreffum atque erectum fundamentum, vt paulò antè deliniaus :is igitur quum videtur, qualis vifui appareat, in hunc modùm indicabimus. Vifum in radios diuidemus diuerfos, velut vnica res prius in duas partes, depreffum fcilicet $\&$ erectum fundamentum feeta eft, $\&$ ad notanda vifus puncta oculos pingam, quos à duobus fundamentis ad latus remoueo pro arbitrio, du cọ́ per eôs lineam erectá quę xuidiftans fit lateri depreffi fundamenti.Cxterùm vifus punctum ad erectum fundamentum in ea linea ele uo, vel deprimo prout rem afpicere volo, ex eógp puncto altitudiné, humilitatê, profunditatem, atque diftantiam metior . Reliquus oculus figitur quog; in pra dicta linea fub oculo fuperiore ad quamcunque partem plani fundamenti libu. etht, vel etiam verfus medium : ex oculo hoc menfuram capio amplitudinis in vtroque latere, longè \& propè. His perfectis lineas protraho ex fuperiorepú. cto vifus ad omnes cubi partes quax videntur, quéq $q_{3}$ literis aut notis arimethicis funt fignatx. Primam lineam vifualen ex oculo iuxta erectum fundamentum produco ad punctum luminis o, fecundum inferius paulo ad finem circuli lu. minis, deinde traho duos radios fupernè ad quatuor cubi angulos:itidem facio etiă inferne. Poftea excito duas lineas ad.puncta i $8 \mathrm{~K}$. Tandem duas al has du. co ad extremitates linę tranfuerfe cui teffera incumbit, ea quadratum repręêtat, ideoóp termini èius fignati funt bis, antè quidem $g \mathrm{f}, \&$ pòlt e $\mathrm{h}$ : ita fundamentum erectum ex oculo lineis vifualibus vt oportebat notatum eft.Porro ex puncto vifus, quiiuxta planum fundamentum ponitur protrahantur rad ii vifuales ad omnia frgna fundamenti in plano : ac primo quidem ducantur linex ad quatuor angulos quadratre fuperficiei $\mathrm{f} g \mathrm{~h}$ e,poftea ad quatuor angulos fundamenti cubi qui odo denotăt, quare ante $b=28 c_{3}$ ac polt $4<\&$ a rfignati funt. Poftremo ducite tres adhuc radios ad tres angulos $1 \mathrm{~m} n$ vmbræ cubi, erúntọ line vifuales ad vtrunque fundamentum abfolutx guotquot earum opus fuerit. Quum am oculus per radios vifuales contingat id quod vider, atque hoc ipfum per picturam reprefentandum fit, conftituenda erit fuperficies pellu cida vti fupra fcriptum eft, qua omnes lineas vifuales interfecet:hac fuperficies fiue planum in noftra defignatione linea erit : ea igitur inter puncta vifus $\&$ fundamenta protrahatur cubis ipfis parallela, prope rem qua vedetur quo res vifa appareat maior, vt antè dictum elt:in hac fuperficie fiue linea locentur duo vifus puncta, in ea altitudine qua funt duo polteriora. Poffemus quog, fi $_{1}$ opus effet, hanclineam fiue fuperficiem obliqux ftatuere, vt declinet in anteriorem vel polteriorem partem:at vtcunque fituetur hoc planum, abfcind $d_{1 t}$ omnes ra dios vifuales:oculos praterea pofteriores cum anterioribus per lineas tranfuerfas coniungito. A t omnes quatuor folum vnum fignificant vifum, verùm fepa ratio hac facilitatem prabet in opere, vt fubiecta oltendit figura. 



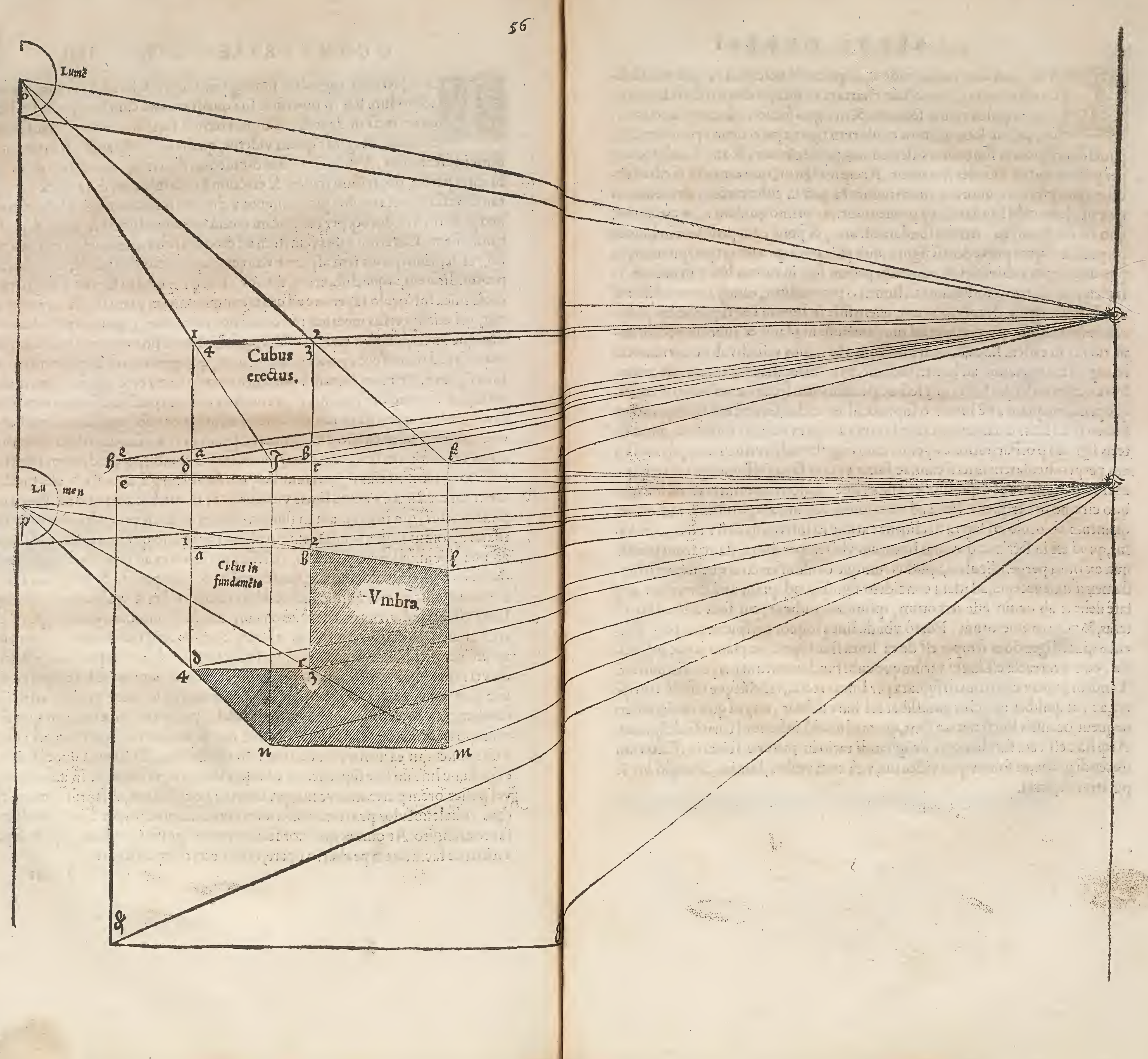




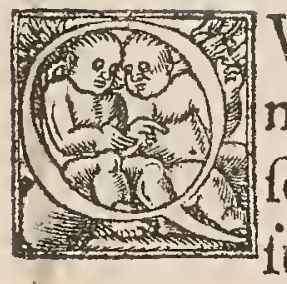

Vum iam ante oculos videas, atquè rectè percipias ea qux modò demonftrauimus, fume aliam chartam in qua producito duas lineas fefe ad angulos rectos fecantes, 8 in figno fection is locato punctum vi ius, qui hic loco quatuor oculorum figurx pręcedentis ponitur:ad cá punctum referend $x$ funt omnes altitudines, profunditates $\&$ amplitudines, que per priores radios vifuales notantur. Accipito igitur, quù errorem facilius deuites, duos circinos, quorum altero metire ea qua in cubo erecto, altero autem qux in plano cubi fundamento proueniunt. Acprimò quidern cape circinum, quo vti decreuifti ac rectum fundamentum, $\&$ pone eum pede vno in lineam perpendicularem præcedentis figuræ, quæ planum repræentat perfpicuum, in punctum vifus cubi erecti, $\&$ reliquum pedem fige in eadem linea in radium vi fualem, qui ex remotiore oculo ad lumen o protenditur, eamóg feruato diftanti am. Deinde repete alium circinum, quem fifte in lineam fiue fuperficiem pellucidam, in oculum qui pertinet ad fundamétum in plano, $\&$ alterum pedem pone rurfus in eadem linea, in eum punctum, vbi radius vifualis ab oculo remotio re atgु inferiore tranfit ad lumen p: deinceps transfer illa duo internalla ambobus circinis ad lineas binas que fefe ad punctú vifus.fecant tranfuerfè, oftendétque primum quàm altê Iumen ofupra oculum cadat, fecundum verò quantum lumen $p$ ad latus declinet:hæc duo lumina ad idem venient punctum, quod li. teris fignato po.Pariratione operare cum fingulis radiis vifualibus, qui lineá tu am perpendicularem interfecant, ac fume vt iam fape iuflimus, primo circino omnes altitudines \& depreffiones a fuperiore oculo:confimiliter examına reliquo circino in perficua linea ad inferiorem oculum amplitudines radiorum, quantum ab oculo ad latera declinent : atque ea interualla defer ad fignum vifus, quod eft in fectione duarum linearum vibi femper duo diftantiarum puncta qux ex linea perpendiculari, apud vtrum que oculum ereeti atque depreffifundamenti funt accepta, ad idem concident fignú:quod quàm alte, humiliter,atog latè debeat $a b$ oculo effe remotum, ipfummet indicat, cui fuas adfrribitoliteras, $\&$ notas numerorum. Porrò vbi de linea loquor perfpicua aut perpendiculari, intelligendum femper eft de ea linea fiue fuperficie plana atque pelluci$\mathrm{da}$, qux protracta eft iuxta vtrumque cubi fundamentum perpendiculariter. Tandem puncta continuato fignata per lineas rectas, videbíf que effectú tui ope ris, ac vbi quilibet angulus cuiuflibet rei ftare debeat, atq ea qua oculo videri nequeüt, occultis lineis notata funt, quemadmodú infernèid curiofé defignaui. A tq hac eft vera fundamenti defignandi ratio in pictura fcientia . Cxterùm. docendigratia, ea folùm qua videntur, vnà cum vmbra, lumine, \& oculo hic fe paratim depinxi. 
GEOMETRIAE LIB. III, I⿰习习

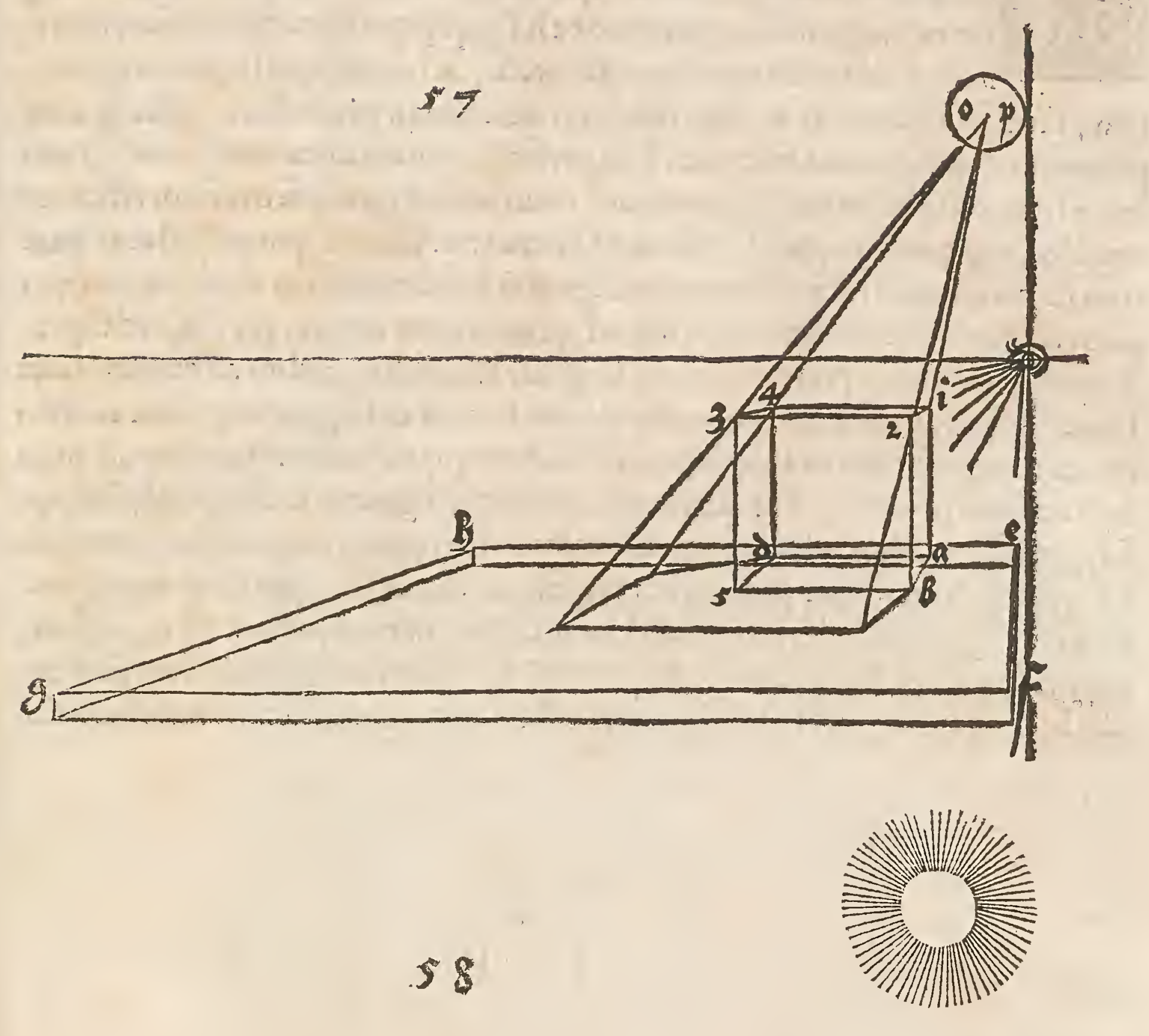

$\infty$

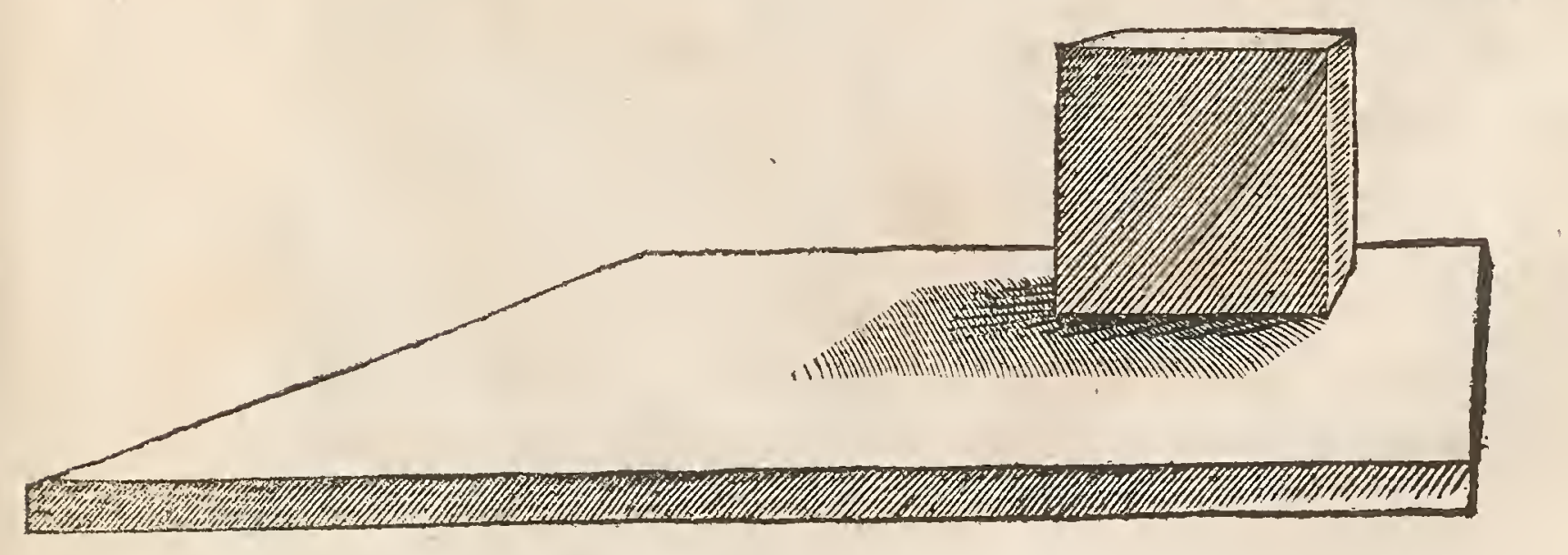


$V \mathfrak{x}$ huc vfque deliniauimus, in fequentibus breuiore faciliorég via defignare docebo, in hunc nêpe modum. Ducatur linea tranfuerfa $\mathrm{fg}$ tantæ longitudinis, quantę eft $\mathrm{e} \mathrm{fg}$, quę quadratam fignificat fuperficiem ad fundamentum cubi erecti, ac iuxtaf, eius linea extremitatem, fatis alte ftatuatur oculus, fimili ratione qua in præxcedente vifus fignum pofitum eft in linearum fectione. His perfectis trahantur ex oculo iam fignato bin $x$ linę rectę ad v trumq terminum tranfuer $x \mathrm{f} g$,ex infernè duos efficiunt angulos, atq̧ quadrati, quod deftinatur, latera tria. Cæterú quo pacto latus quar tum fit ptrahendú fie inueltigemus. Fige aliú adhuc oculú in ea altitudine qua prior eft, verùm ab codem remotum ad quantitatem eorum, qui funt ad fuprafcripta fundamenta pofteriores:ex illo igitur fcribe duos radios ad extremitates linex $f$ :deinde excitato perpendicularem lineam aa bb, qux angulum anterio rem contingat; ac vbi ea fecat lineam vifualem 'quę ex oculo rēmotiore ad angu lum acutum procedit, illicadiice $c c$. A b hoc cc figno protrahe parallelam ipfifg,per duos radios vifuales qui defcendunt à propinquiore oculo ad terminos $\mathrm{f} \& \mathrm{~g}, \&$ vbi hi radii per equidiftantem abf cinduntur, illic duo fiunt anguliliteris e $\&$ h fignandi. Sic igitur planú hoc quad ratum recte pictura eft exprefium, quemadmodum \& precedens : quocirca $\&$ eius quatuor anguli ejfdem funt notatiliteris e $\mathrm{g} \mathrm{h}$, vt in aliis factum eft:ifta omnia infernè ocul is funt fubiecta. 

cuatur,quemadmodú eum paulò anțê in plano defignauimus:id hoc pacto expeditur. Sume longitudinem lateris cubi ex fundamento in plano iam dudum facto, ac pone cam duobus punctis $x \& y$ fuper $l a-$ tus $f g$ depicti quadrati, in ea diftantia ab angulof, quantum cubus in plano fúdamento diftat à fuperficiei latere ef:deinde ex oculo ducito binas lineas vifua les ad puncta $x y$, inter quas cubus infimo fuo latere quadrato locandus erit: fed quantum à latere $\mathrm{g}$ debeat diftare hoc modo difce. Quum in iam fxpe dicto plano fundamento lcribis lineam e g diagonalem, erit ea guog diameter cu bi in plano:nam ea tranfit per angulos a $\&$ - $c$ :quod cum fic fe habeat, trahe $\&$ dimetientem e $g$ in præfenti plano $f \mathrm{~g} h$, ac vbi is difpefcit radios vifuales $\mathrm{x} \&$ $y$, llic ad radium $x$ fcribe a, \& $c$ ad radium $y$. Hoc facto trahe duas tranfuerfas à punctis a $8 \mathrm{c}$ ac fectionem tranfuerfe a cum radio y fignato litera d:at vbi trắfuerfa ctágit radium $x$, illic fcribe b:fic cubi latus a $b \mathrm{c} d$ rectè elt formaturm atq fuo loco in defignata fuperficie quad rata ef $\mathrm{h}$, guemadmodum etiam in pracedentibus. Nunc ex angulis a b c d erige quatuor lineas perpendiculares, 8 an: teriores duas tam altas fac quàm longa eft $c b$ tranfuer $\{$, atque in ea altitudine tranfuerfam protrahe ab vna ad aliam, $\&$ ad eos angulos fcribe fupra $b$ numeri, characterem2, \& fupra c notam $3:$ tandem excita duos radios ex oculo ad angu$\operatorname{los}_{2} \& 3$, ac vbi ipfiabfcind unt erectas a $\&$ d, illic adiice notas, fupra a quidem I $\&$ fupra d 4 . Ita cubus rectè defignatur in fuo plano, quod \& hic priufō ad reliqua pergerem, volui deliniare. 

GEOMETRIAE I IB, IIII.

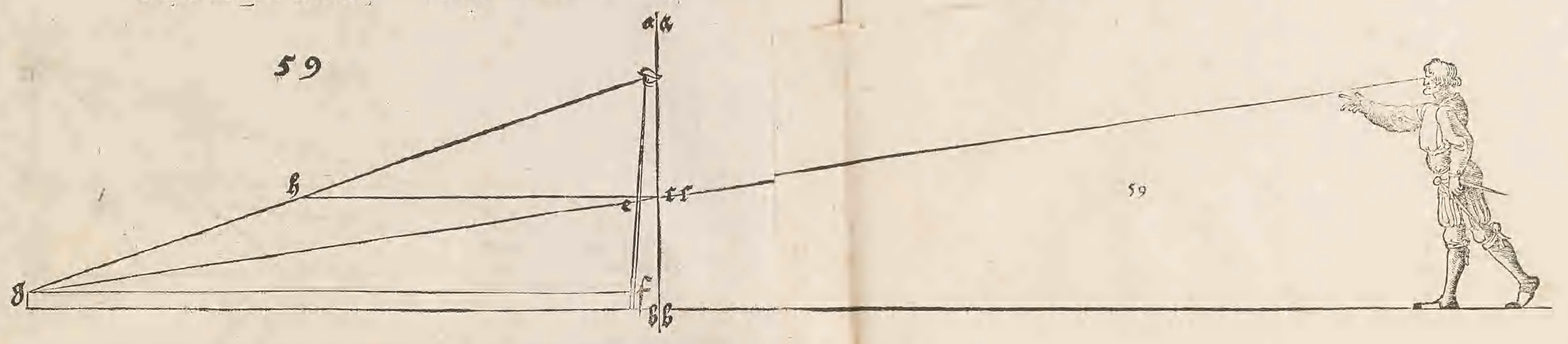

Epicta quadra ta illa fuperficie ef $\mathrm{g} \mathrm{h}$, cubus fuo loco fuper eam $\mathrm{fta}_{2}$ (- ive cuatur,quemadmodú eum paulo antế in plano defignauimus:id hoc L. 2 acto expeditur. Sumelongitudinem lateris cubi ex fundamento in i quad rati, in ea diftantia abiangulof, quantum cubus in plano fúdamento diftat à fuperficiei latere $\mathrm{ef:deinde} \mathrm{ex} \mathrm{oculo} \mathrm{ducito} \mathrm{binas} \mathrm{lineas} \mathrm{vifua}$ les ad puncta $x \& y$, inter quas cubus infimo fuo latere quadrato locandus erit: fed quiantum à latere $\mathrm{f} g$ debeat diftare hoc modo difce. Quum in iam fxpe dicto plano fundamento 1 cribis lineam e $\mathrm{g}$ diagonalem, erit ea quog diameter cu bi in plano:nam ea tranfit per angulos a $\&$ : c:quod cum fic fe habeat, trahe $\&$ dimetientem $\mathrm{g}$ in prafenti planoef $\mathrm{g} \mathrm{h}$, ac vbi is difpefcit radios vifuales $\mathrm{x} \&$ $\mathrm{y}, \mathrm{lllic}$ ad radium $\mathrm{x}$ fcribe $\mathrm{a}, \& \mathrm{c}$ ad radium $\mathrm{y}$. Hoc facto trahe duas tranfuerfas à punctis a $8 \mathrm{c}$ ac fectionem tranfuerfe a cum radio y fignato litera d:at vbi trắfuerfact ágit radium $x$, illic fcribe b:fic cubilatus a $b \mathrm{c} d$ rectè eft formatum atç fuo loco in defignata fuperficie quadrata $\mathrm{ef} \mathrm{g}$, guemadmodum etiam in pracedentibus. Nunc ex angulis a $\mathrm{b} c$ derige quatuor lineas perpendiculares, $\&$ an teriores duas tam altas fac quàm longa eft $\mathrm{c} b$ tranfuerra, atque in ea altitudine tranfuerfam protrahe ab vna ad aliam, \& ad eos angulos fcribe fupra b numeri, characterem 2 , \& fupra c notam $3:$ tandem excita duos radios ex oculo ad angu$\operatorname{los}_{2} \&$, ac vbi ipfi abfcindunt erectas a \& d, illic adiice notas, fupra a quidem 1 \& fupra d A.Ita cubus rectè defignatur in fuo plano, quod \& hic prinfö ad reliqua pergerem, volui deliniare. 


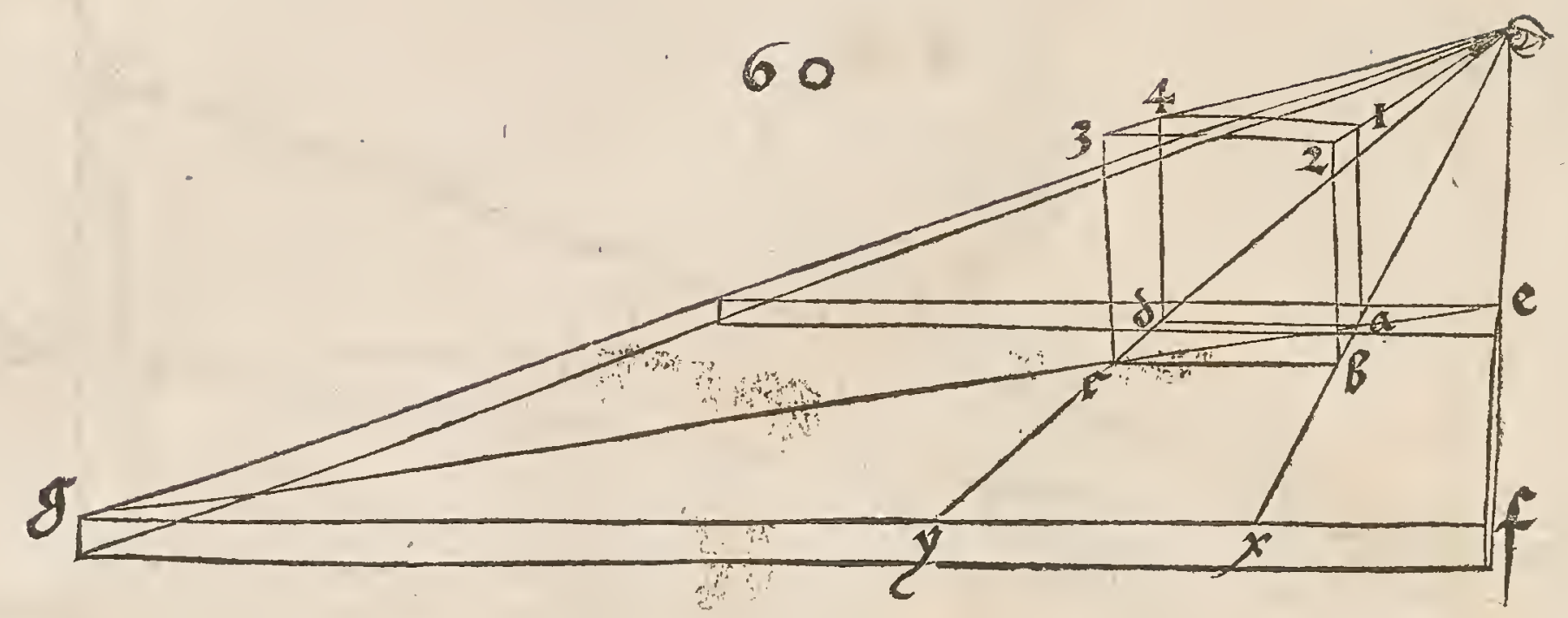

Vum iam cubus fit defignatus in fuperficie fua plana fige lumen, ac delinia in plano cubi vmbram, quod fic cómodiffimè abfolutur.Sifte lumen altè fupra oculí ad quam parté volueris,fitós illud o in co fermèloco vbi priùs fuit, deinde ex lumine o trahatur deorfú linea perpendicularis : in qua locetur punctusp, qui inferiorem reprafentet lucem. Quòd fi lumen à cubo multum libeat remouere, tunc punctum o altius pone in perpendiculari $\mathrm{p}$ o:fí verò propius adhibere lumen placeat, tunc $\mathrm{p}$ fignú ma gis deprime, at hic fortèid conftitue in ea diftantia à cubo qua in pracedétibus fuit:politis itaq po luminum punctis, ducantur tres linę recte ex o lumine per tres cubi angulos fuperiores $2,3,4$, atq 3 ex linex continuentur deorfü quoad opus fuerit. Poftea itidem protrahe tres radios ex lumine p, per tres cubi angulos inferiores $\mathrm{b} \mathrm{cd}$, ac vbi hi interfecant fuperiores illos, illic fcribe $\mathrm{m} \mathrm{n}$. His perfectis coniungantur puncta $\mathrm{b} l, 1 \mathrm{~m}, \mathrm{~m} n, \& \mathrm{n}$ d per lineas rectas: atg ita cubi vmbra bene defignata erit. Verùm vt qux prediximus clariùs intelligerentur, hic omnia oculis fubieci, eft'́p conftitutio prior.

Porròea quæ ad picturam pertinent, feparatim deliniaui exclufis lineamếtis fu peruacuis,, vt manifeftiùs viderentur. 


\section{GEOMETRIAE LIB, IIII.}
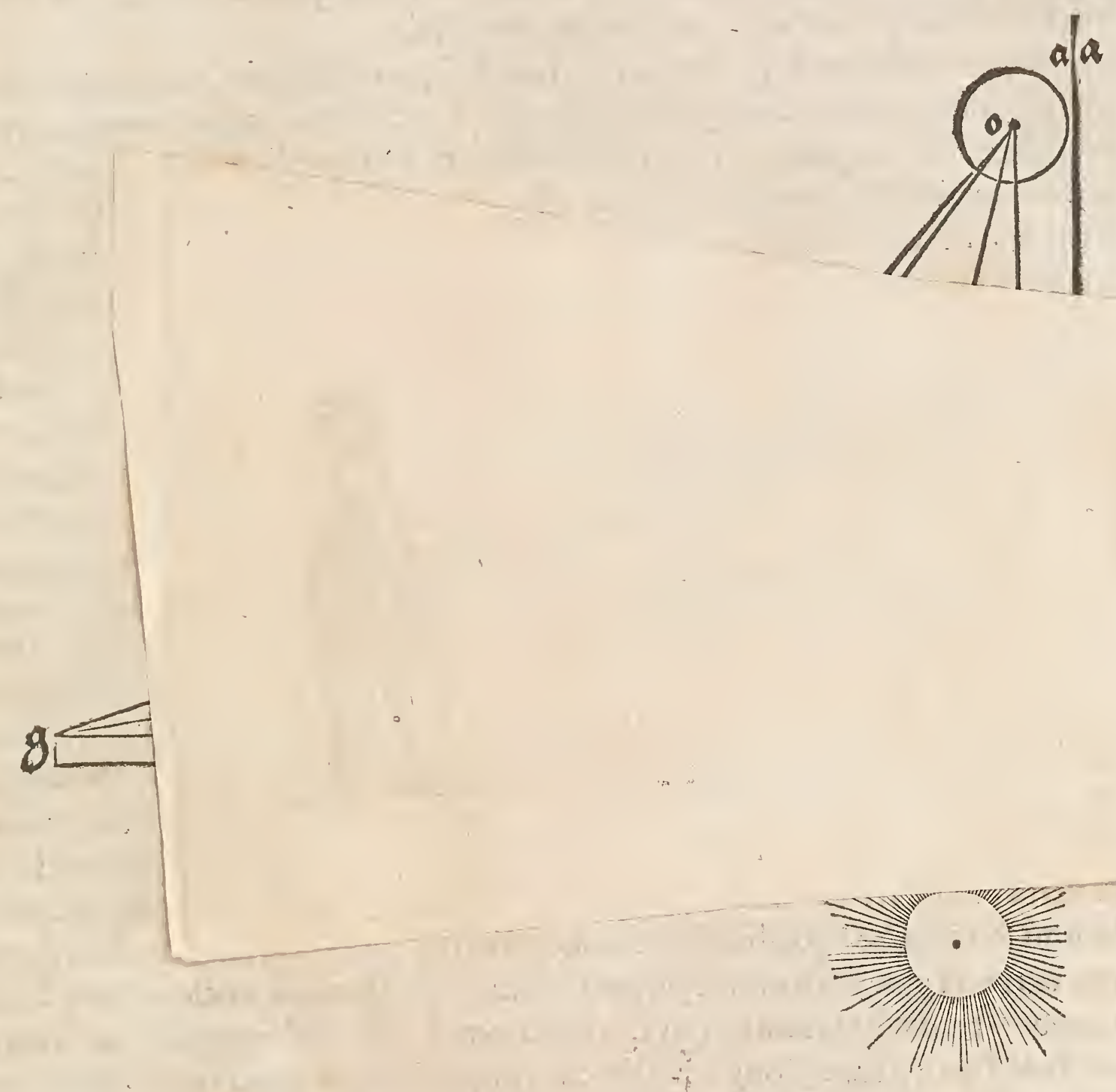

包

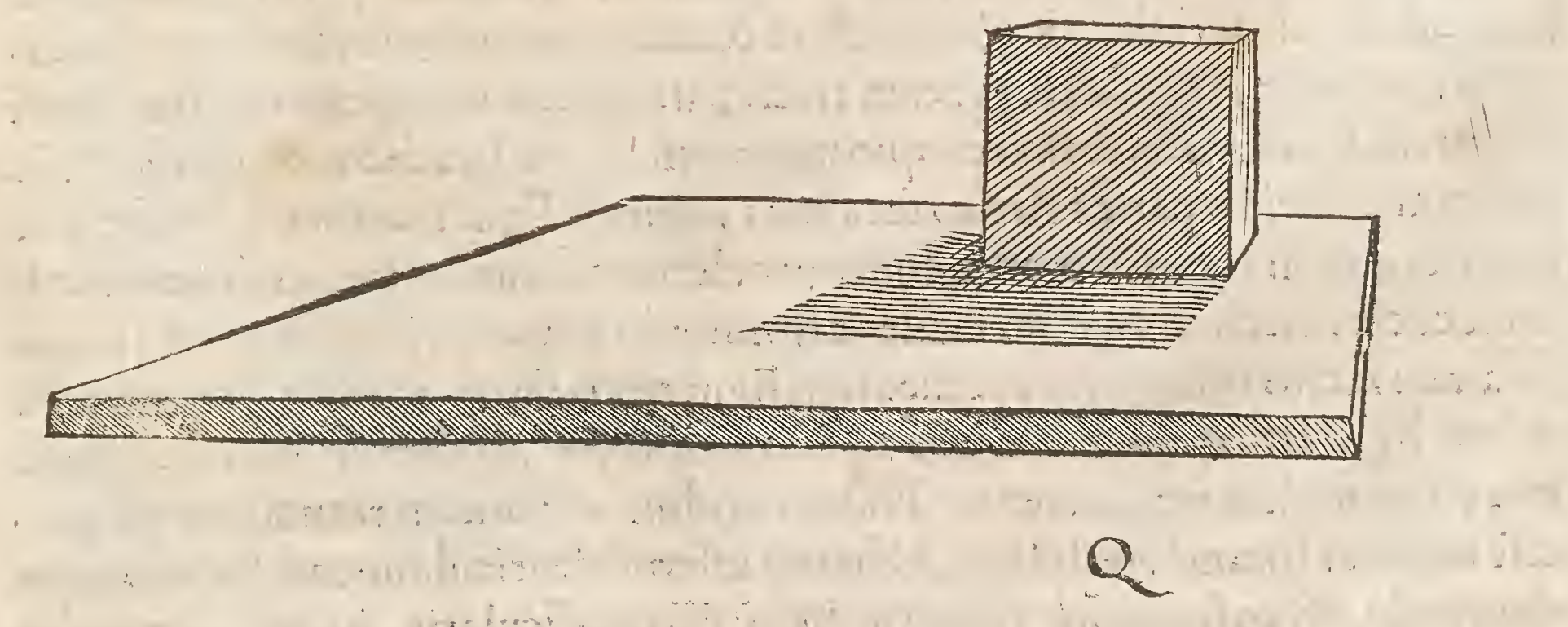





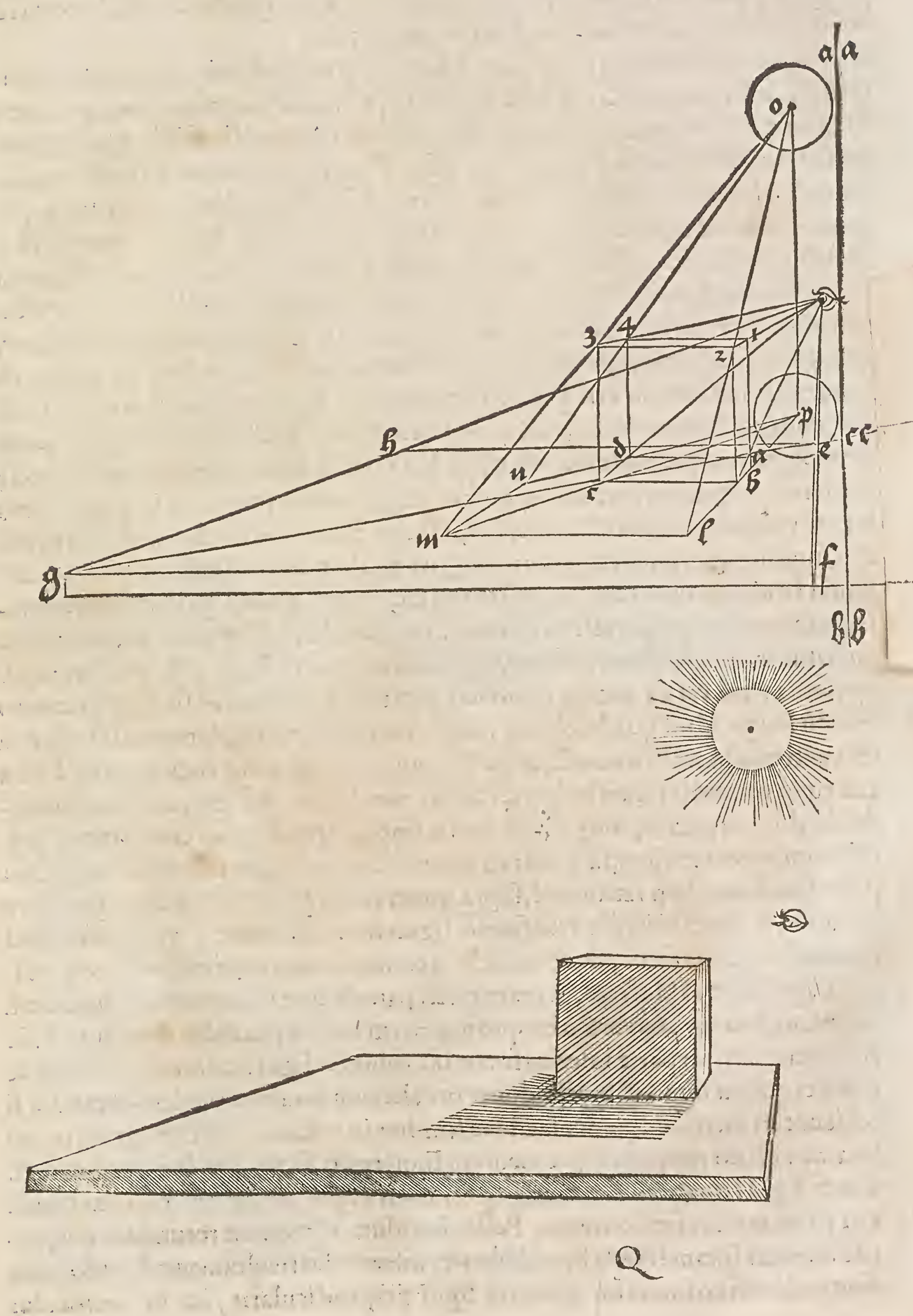

61

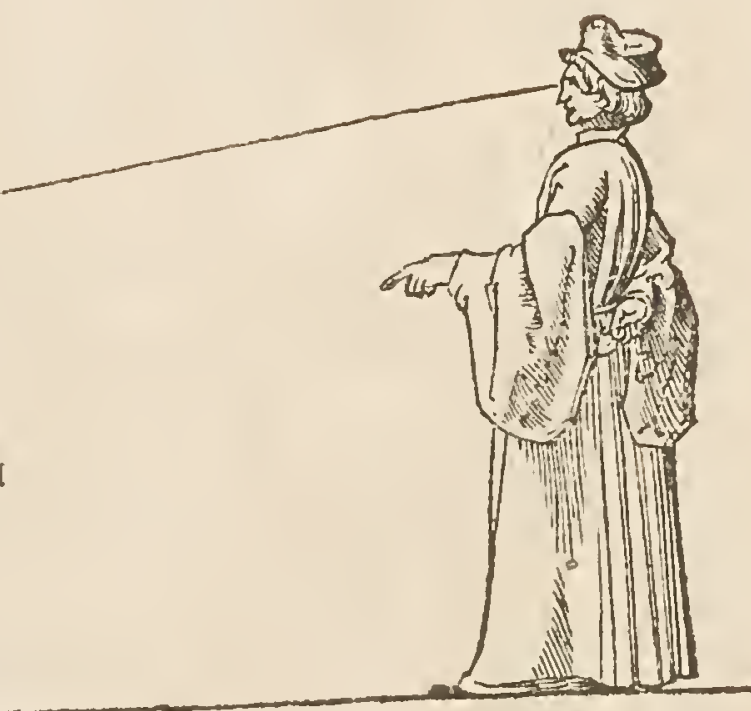


Vemadmodum cubum pictura defignaui, ita poffumus ommia cor(5). pora qux in planum fundamentum deprimi, ato in erectum eleuari 1. 5ueunt, lineamentis reprefentare: ed nunc quo pacto guod per vitrú confpicitur deliniandum fit, monftrabo.

Siergo pervitrum rem quampiam vifam defignare volueris, para prius huiufcemodinnfrumentú.Comprehêde vitrum pellucidum ac planú impage, in mo dum feneftra compacta, deinde confice afferem eius amplitudinis, qua eft impa go, fed longiorem, ac impagi quidem affige circa extremitatem vnius lateris internè duos quafir cardines, quos qum affere duobus clauis fic coniunge, vt impago in iplis facilè eleuari ac deprimi queat ad inftar tabula luforia, quo vicrú fi opus fit in afferem demitti $\&$ recondi poffit. Caterùm circa medium afferis in vtraque parce figantur duo claui qui pro capitellis annulos habeant, in quos bi. ni baslli ferrei ad catenxfimilitudinem ingrediantur, atque in ipfis moueátur, at baculi tantęint longitudinis, vt fi feneltra ad angulum rectum ab affere erigatur, eam mediam in vtraque parte contingant, $\&$ in anteriore parte, bacilli fi. ant plani, ac foramen in eis, poft quod vncus figatur mobilis. Poltea fige quoque in impagis vtraque parte aut v bi planę bacillorú extremitates eam attingút, cla uos ferreos capitibus perforatis, qubus fi applicentur foramina in planis baculorum terminis, atque vnci obdentur, impago cum vitro ftet firmiter, His perfectis, fume lignum quadrangulum longius paulo quàm affer fit amplus, cuius vnum latus pro afferis excindito latitudine, $v t$ ta men eius ligni dux extremitates maneant integr, ac afferis latitudinem excedant, 8 applica exemptum lignilatus tranfueriéfuper afferem, fiue tabulam, ita vt facilé, fi opus fit, fuper ea verfus vitrú vel $\mathrm{ab}$ eodem dimoueri queat : id lignú rurfus fupernè excauato fecúdú longitudiné quadratè, non tamé ad exremitates vf̣́ laterum. Iam duo la rera extrema pforato rotundè, ac per foramina illa immitte cocleam longá qua tamen in rotund is foraminibus circuitus non habeat, fed inter ea folùm: deinde aliud lignú parato, longú ex dimedia impagis parte quod erectú intret precilè cum parte inferiore, in quadrangulam tranfuerfi ligni concauitatem, a c im pleat eam fecundum latitudiné, fupra quam etiam parum in vtraque parte pro ducatur, \& productio illa tranfuerfo ligno iuftè incumbat, vt lignum illud ftans ad angulos rectos, fic commodè in concauitate iacentis vltro citrọ́g trăfueriè agi poffit . Hoc facto, per eam erecti partem qux in concauitate tranfuerfi cótinetur, foramen terebrato ex quo matricem coclex prædictx conficito. Nǘc cocleam anteriori parte inferito foramini rotundo ligni tranfuerfi, deinceps $\delta ?$ matrici qux in ligno eft erecto, donec per alterum foramen tandem exeat. Sic li cebit coclea erectum lignum facilè ac firmiter in vtramuis partem agere, porrò foramen facito rotundum per medium ligni ereeti fecundum longitudinem $\&$ vnum ligni latus parum excinde ad foramen vqque, atque in exus excifionis vna parte multas fecato crenas. Poltea baculum ad tornum rotundato qui precifè introeat foramé erecti ligni, habeatọ́ infernè denticulum:eum baculüi cum denticulo affixo foramini immitte ligni perpendicularis, ita vt denticulus peripram 
per ipfam incilionem defcendat. Quando hunc baculum per vnum gradum fiue crenam eleuas, ac firmiter fic manere cupis, tunc pone denticulum in crená. Sic baculus ifte in altum tolli aut deprimi pro arbitrio facilè poteft, in eius fum mitate tabellam affige paruam atque aptam, in cuius imedio foramen fit aretum admodum, vt altero fol ùm oculo per id atque vitrum in impage perfpicere certiùs queas. Quod ergo fic vides penicillo protrahe in vitro, id cùm factum erit, ex vitro tranffer hocipfum in materiam fuper quam pingere ftatuifti. $\mathrm{Hac} v a l$ de funt vtilia illis qui aliquế ad viuum deliniare volunt;cùm tamen in arte pin gédi minùs fint periti.Quocirca fi aliquem hoc pacto depingere voles, fuffulcito caput eius quo teneat firmiter, donec præcipua quæqु duxeris lineamêta, qui bus defignatis, coloribus vti licebit:at lumen adhibendum eft clarum. Poltremò fi afferi prædicto cui adhæret impago, due affigantur infernè fublcu des per tranfuerfum, atque bina in fingulis terebrentur foramina, quibus pedes inferantur infernè,ferreis cufpidibus muniti. His omnibus aptè compactis pro menfa ad hos vfus commodiffima vti poteris, ea etiam fi placuerit diffoluitur, ve aptior quo volueris fiat ad portandum. Qua fuprà pracepimus hic oculis funt fubiecta.

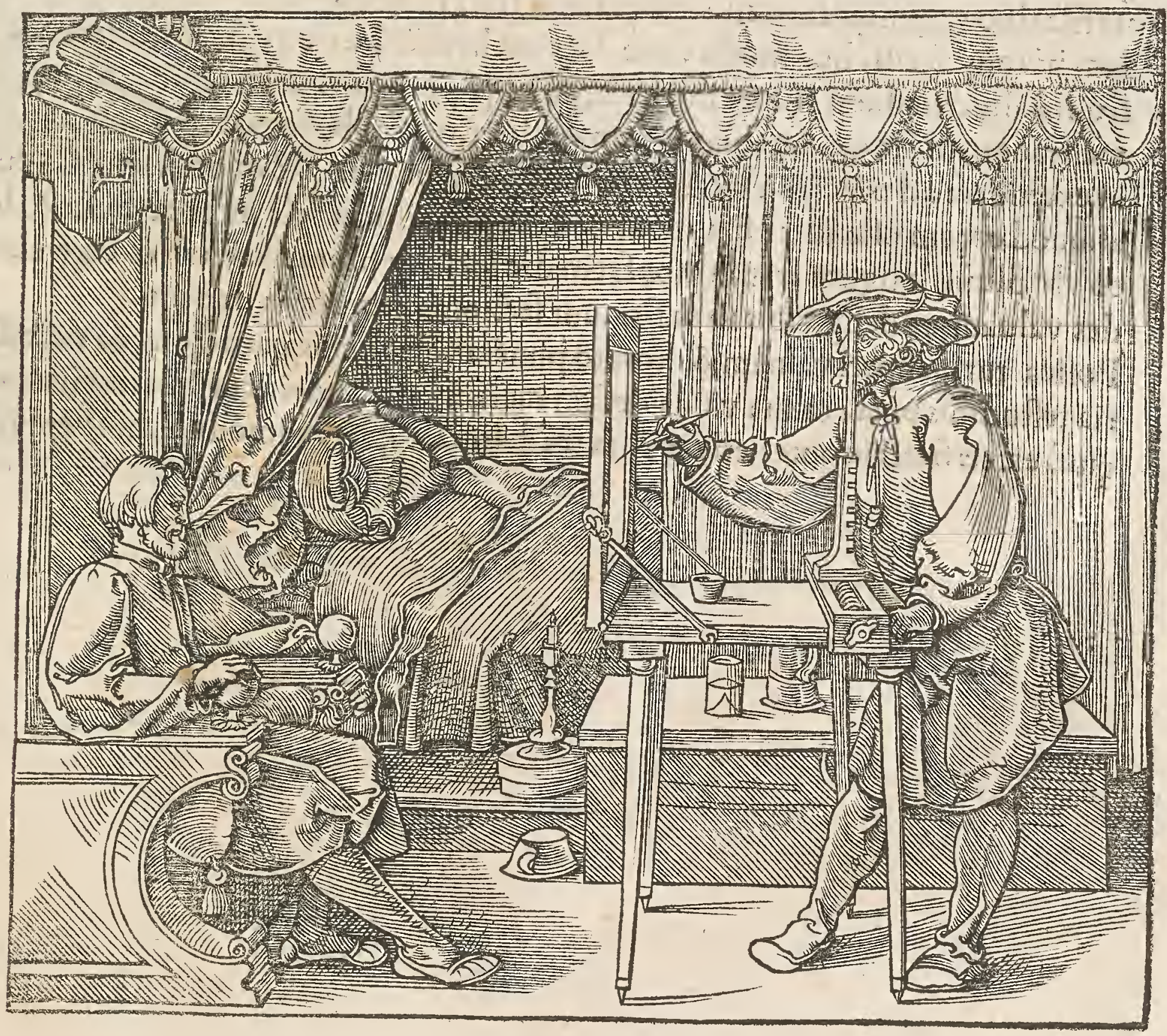


Qua via res qualibet qua videtur, \& non multum å vifu remota eft, per tria fila fit metienda, atq ita in picturam tranfferenda .

Er tr ia fila poflumus quamlibet rem, qux per ea contingitur, in pidu 1. á trăsferre, quod fic expeditur. Si in aliqua aula aut officina fueris, 1. If fige clauiculum aliquem capite perforato in parietem, fit $t^{\prime}$ llle vice 5. Lculi,per quem trahe filum forte, \& appende ei pondus quoddá plübeum : deinde pone menfam frue tabulam à fixo clauo diftantem pro arbitrio, fuper eam tabúlam erige tranfuerfè ad oculum frue dictum clauum impagem quadrangulam; altê vel humiliter, \& ad quod la tus placuerit, habeatóg ea impago valuam qua claudi $\&$ aperiri queat, atqui ea valua tabula efto in qua delini are ftatuifti rem propolitam, poltea parato alia duo fila, quorum alterú ca ra af- fige fuperiori $\&$ inferiori impagis marginibus, alterum verò erectis intraq parte regulis, fiue marginibus eiufdem impagis incarato tranfuerfê.

Nunc conficito ftl lum quemdani ferreum cum foramine in fummitate, cui filum quoque annecte quod tranfit per clauum in pariete, hunc ftilú cum filo lon go trahe per impagis feneftram, ac dato alicui focio tenendum, tu autem oblerua fila duo quę inter fe tranfuerfè extenfa funt in impage.

Iam his qua fecifti hor modo vtere. Locato hemifpharium fiue teftudinem aut fi quid aliud malueris fuper menfam, ab impage quantum libeat remotum, id' ${ }_{\mathrm{g}}$ maneat quamdiu opus habueris im mobiliter, tunc iube focio tuo fil um cum fti lo extendere ad pręcipua iacentis hemifpharii puncta, \& quoties extenfa filo aliquod corum oftendit, fila mouto in impage, donec fefe interfecent in colo. co ybi filum longum tranfit:hoc facto dic focio vi filum remittat, tu verò claudes valuam, ac defignabis in ea fignum fectionis filorum in impage.

Poftea rurfus aperivaluam, $\&$ notato puncta velut prius, quoad omnia neceffaria teltudinis púcta in tua valua defignauerissea tunc lineis cótinuato, videbisć quid ex eis fiat:Sic potes etiam alias res deliniare, quemadmodum hic depinxi. 


\section{GEOMETRIAE LIB. IIII. $s 8 \mathrm{r}$}

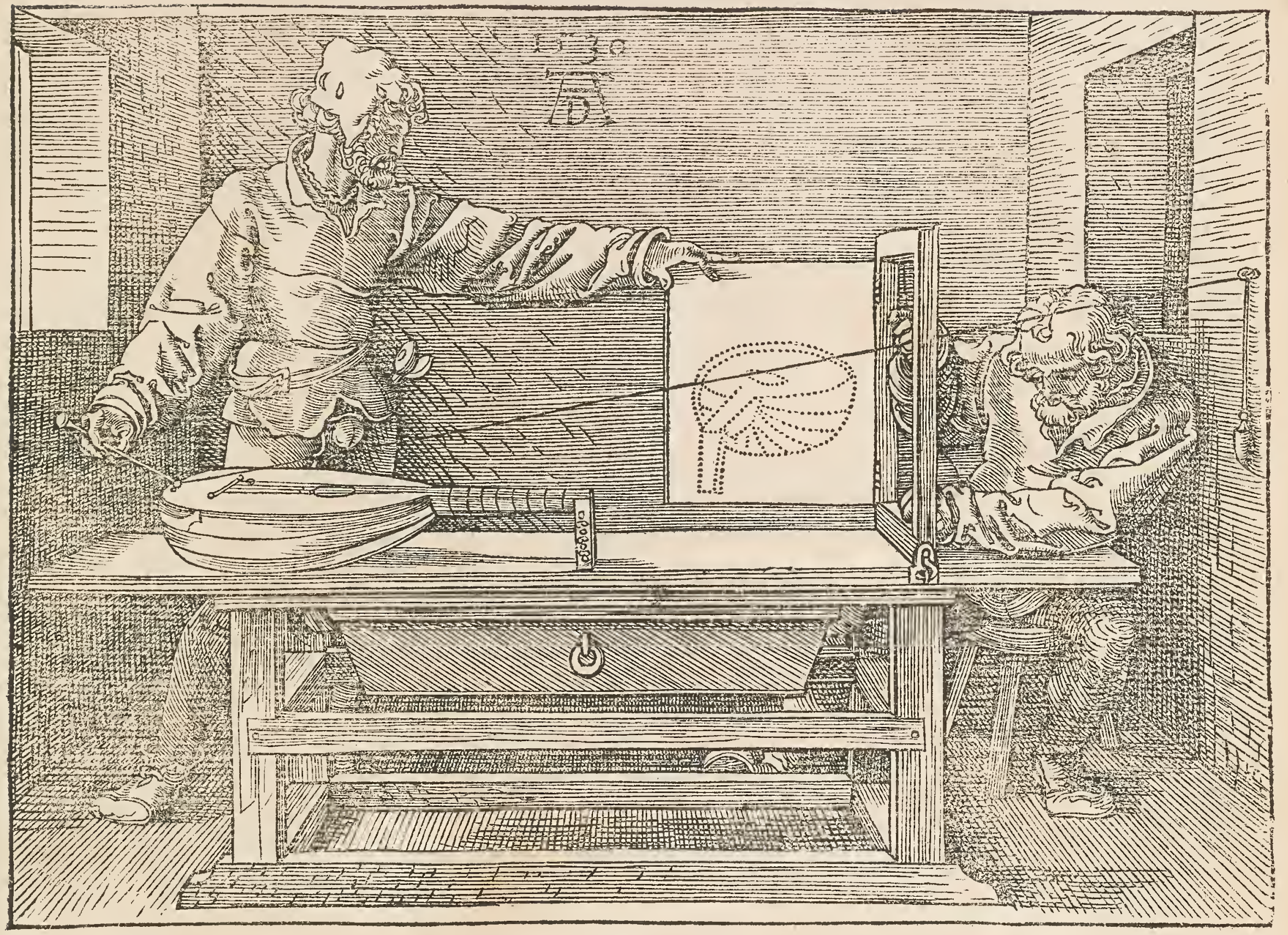

Am, Bilibalde prętantiffime amiciflimé ato deo c ptimo maximo fauente ad eos libros, quos de humana proportione confcripfi, \& alios quofdam ad idem fpectantes edédos fuo tempore me accingam.

Deo omnipotenti fit laus \& gloria imperpetuum. F \N IS. 
and

$-2$

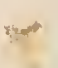

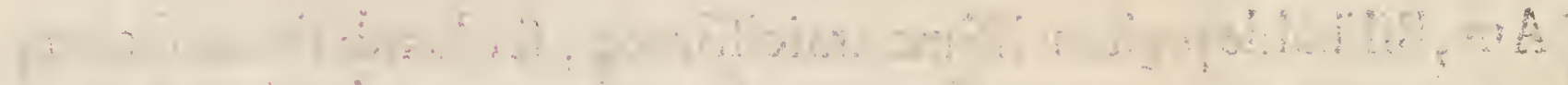

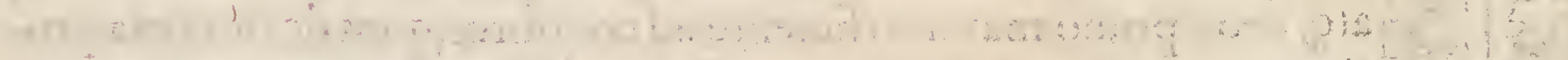

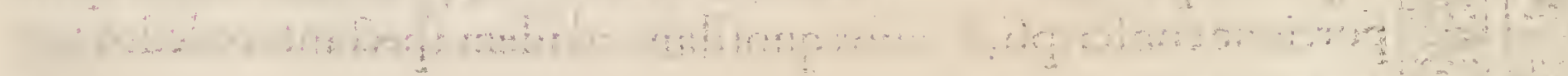

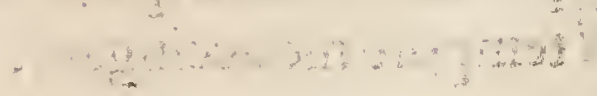

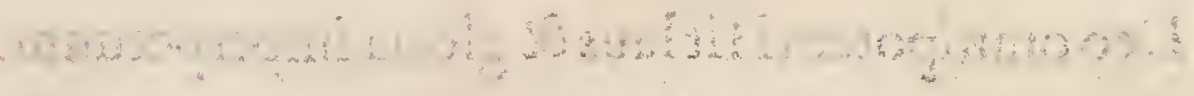

2.111 














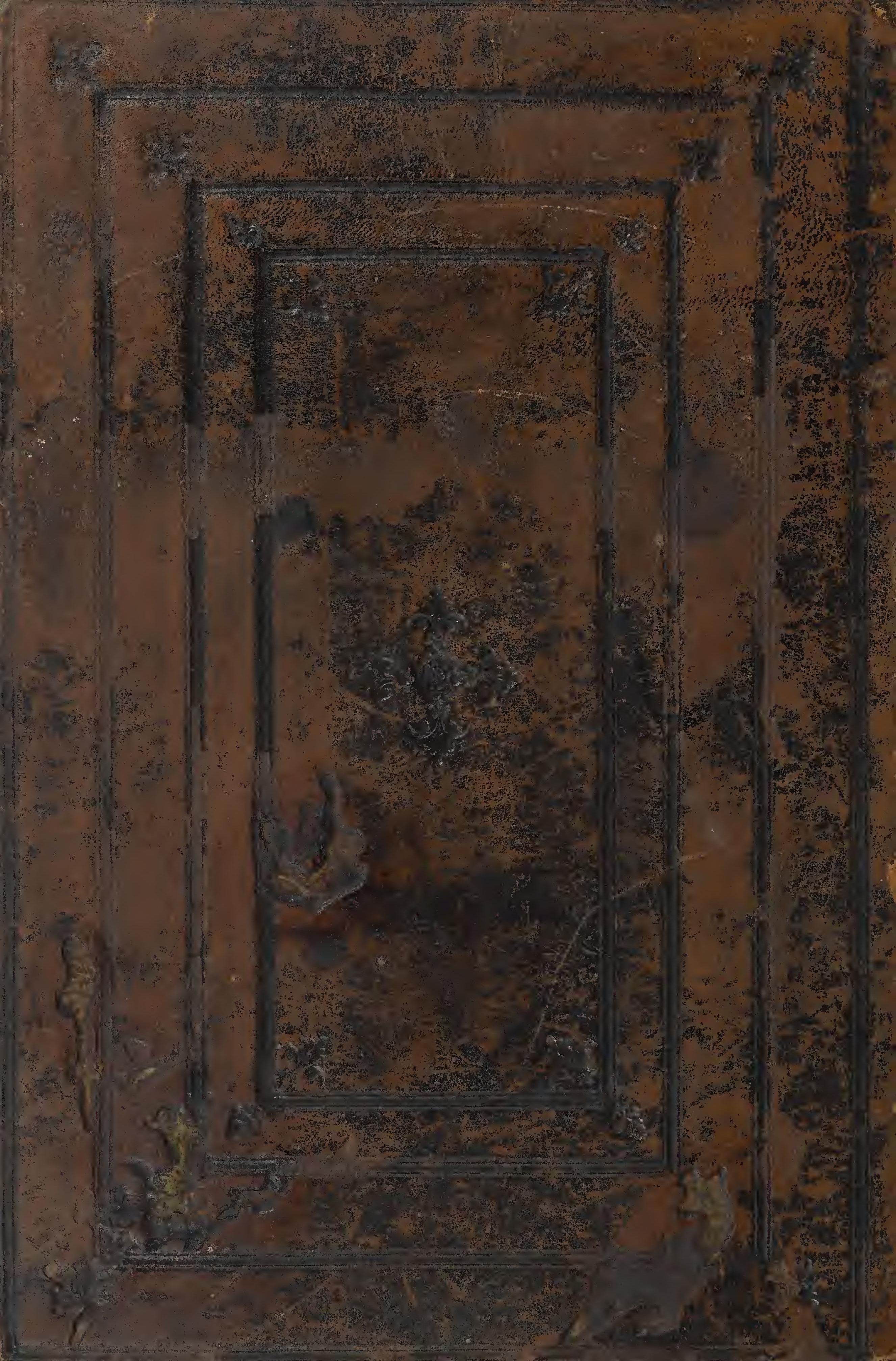

